\title{
Effect of submerged breakwater on profile development
}

Volume 1: Text

August 1995

E.W.M. Claessen

M.D. Groenewoud
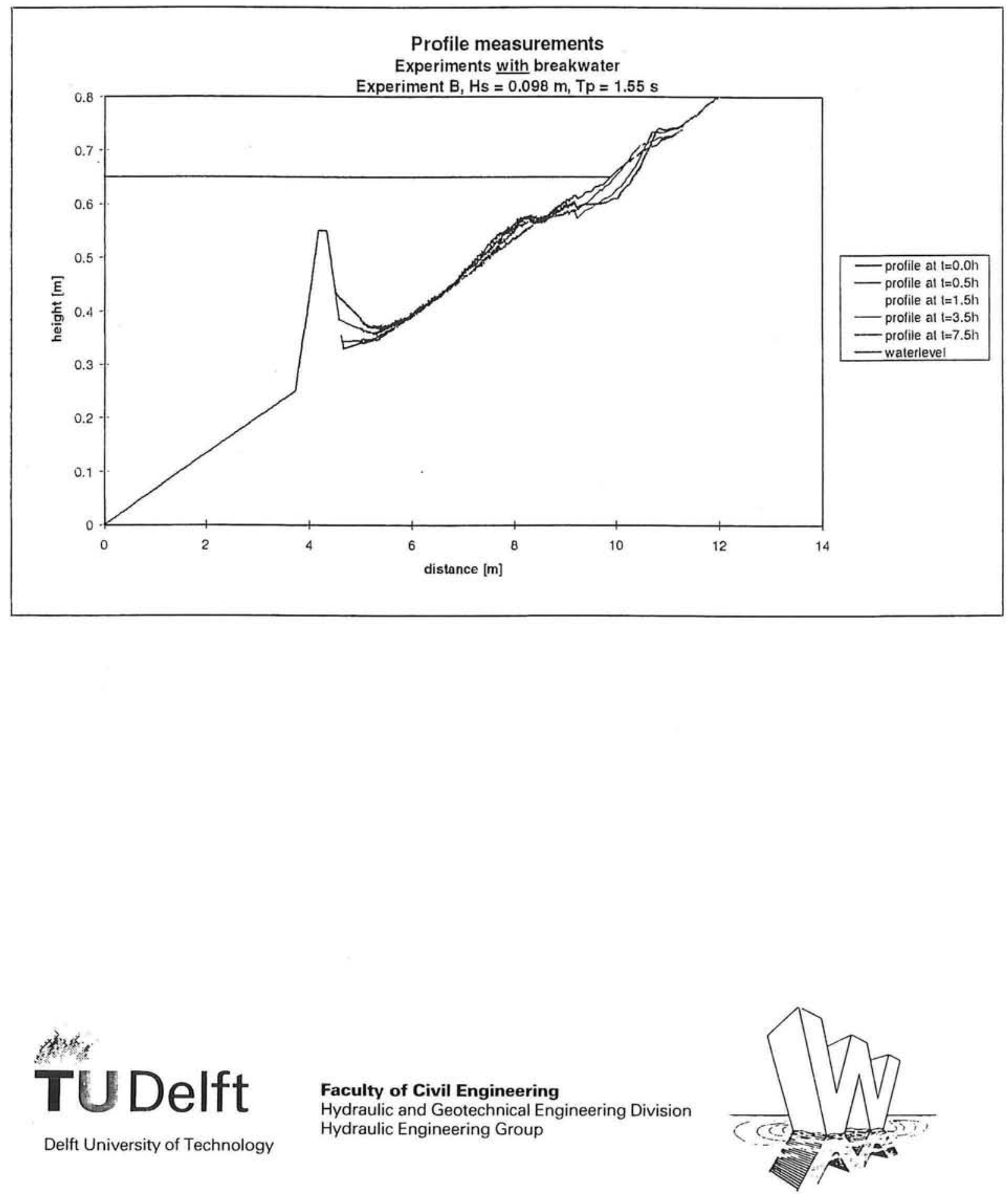


\section{EFFECT OF SUBMERGED BREAKWATER ON PROFILE DEVELOPMENT}

Delft, augustus 1995

Technische Universiteit Delft

Faculteit der Civiele Techniek

Vakgroep Waterbouwkunde en Geotechniek

Sectie Waterbouwkunde en Offshore

Afstudeerders : Edward W.M. Claessen

Martin D. Groenewoud

Hoogleraar : Prof. ir K.d'Angremond / Ir T. van der Meulen

Begeleiders : Dr ir J. van de Graaff

: Dr ir H.L. Fontijn 


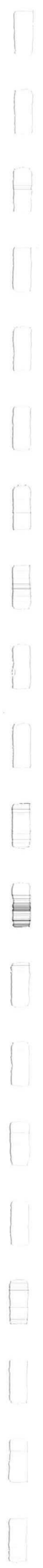




\section{PREFACE}

This paper is the master's thesis of Edward Claessen and Martin Groenewoud at Delft University of Technology.

The thesis committee consists of the following persons:

Prof. ir K.d'Angremond

Dr ir J. van de Graaff

Ir T. van der Meulen

Dr ir H.L. Fontijn

The authors wish to express their gratitude for the valuable assistance of J. van de Graaff. Gratitude is also expressed for the help of various persons, especially ir H. Klaasman, at the Laboratory of Fluid Mechanics during the experimental phase of this master's thesis. 


\section{ABSTRACT}

This study is part of a Human Capital and Mobility Project called 'Dynamics of Beaches'. This project is a co-operation of six European universities which work together in solving some unknown aspects of a submerged breakwater by means of model experiments. Several agreements about the experiments were made between the partners. These agreements consider the layout of the breakwater, the wave conditions and the initial bottom profile.

In this report the effect of a submerged breakwater on hydro- and morphodynamics has been investigated. In order to do this, six similar (2DV) tests with and without a scale model of a submerged breakwater and with a movable bed were performed in the Lange Speurwerkgoot (= wave flume) of the Laboratory of Fluid Mechanics at DUT. By means of varying wave height and wave period the influence of these parameters on hydro- and morphodynamics can be investigated. For these purposes wave heights, flow velocities, sediment concentrations and bottom profiles were measured at several locations in place and time.

The following topics are discussed in this report:

-profile development

-wave height development

-velocity distributions

-sediment concentration distributions

-sediment transport rates

-comparison with Unibest-TC

The experiments show that profile development is less pronounced in case of a breakwater present. However, it does not prevent the sediment to cross the breakwater in a seawards direction. Due to the presence of the breakwater, wave heights are reduced landwards of it. Seawards, the presence of a breakwater leads to an increase of wave heights. The breakwater also decreases the time-average velocities landwards of the breakwater. Furthermore, a comparison between the sediment transport based on measured profiles and the sediment transport based on multiplification of time-average sediment concentrations and time-average velocities shows that the last method leads to an overprediction of the sediment transport. Comparing measured profile development and wave height development with values calculated by a computer program called Unibest-TC shows that this program still has significant shortcomings in modelling the effects of a submerged breakwater. 


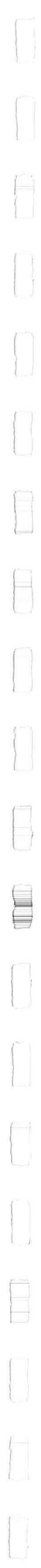




\section{TABLE OF CONTENTS}

\section{PREFACE}

\section{ABSTRACT}

TABLE OF CONTENTS

Page no.

1. Introduction 1

1.1 Introduction 1

1.2 Functioning of the submerged breakwater 2

1.3 Human Capital and Mobility Programme 2

1.4 Aims of this study 3

1.5 Layout 3

1.6 Data base 4

2. Experimental set-up 5

2.1 Introduction 5

2.2 Prototype Model and Prototype Test Matrix 5

2.2.1 Layout of the Prototype Model 5

2.2.2 Wave conditions defined in the Prototype Test Matrix 7

2.3 Scale model at DUT with matching test matrix 9

2.3.1 Scale model in the Laboratory of Fluid Mechanics at DUT 9

$\begin{array}{ll}2.3 .2 \text { Test matrix for the scale model } & 13\end{array}$

2.4 Duration of the experiments 14

$\begin{array}{ll}2.5 \text { Wave spectra } & 14\end{array}$

3. Measurements 16

$\begin{array}{ll}3.1 \text { Introduction } & 16\end{array}$

3.2 Profile measurements $\quad 16$

3.2.1 Profile measurement technique $\quad 16$

$\begin{array}{ll}3.2 .2 \text { Execution of the profile measurements } & 17\end{array}$

$\begin{array}{ll}3.2 .3 \text { Calibration of the profile measurements } & 18\end{array}$

$\begin{array}{ll}3.3 \text { Wave height measurements } & 21\end{array}$

$\begin{array}{ll}\text { 3.3.1 Wave height measurement technique } & 21\end{array}$

$\begin{array}{ll}\text { 3.3.2 Execution of the wave height measurements } & 22\end{array}$

3.3.3 Calibration of the wave height measurement equipment 23

$\begin{array}{ll}3.4 \text { Velocity measurements } & 25\end{array}$

3.4.1 Velocity measurement technique 25

3.4.2 Execution of the velocity measurements 26

3.4.3 Calibration of the velocity measurement equipment 27

3.5 Sediment concentration measurements 27

3.5.1 Sediment concentration measurement technique 27

3.5.2 Execution of the sediment concentration measurements 28

3.5.3 Calibration of the concentration measurement equipment 30

3.6 Summary of the activities during an experiment 31 
4. Profile measurement results $\quad 34$

4.1 Introduction 34

4.2 Conversions of profile measurements 34

4.2.1 Volts converted to bed heights 34

$\begin{array}{ll}4.2 .2 \text { Equal amounts of sand } & 36\end{array}$

4.2.3 Irregularities at transition point $\quad 40$

4.2.4 Reference position for sediment transport computations 41

4.2.5 Profile measurements just landwards of the submerged breakwater $\quad 43$

4.3 Profile measurement results 44

4.3.1 Comparing tests with and without breakwater 44

4.3.2 Comparing tests with equal wave height 54

4.3.3 Comparing tests with equal wave steepness $\quad 55$

4.4 Evaluation $\quad 56$

5. Wave height measurement results 58

5.1 Introduction 58

5.2 Short Review of breaking, reflection and shoaling 58

5.3 Comparing wave height development in the experiments 60

5.4 Comparing tests with and without breakwater 65

$\begin{array}{ll}5.5 & \text { Average wave heights of the experiments }\end{array}$

$\begin{array}{ll}5.6 \text { Spectra } & 69\end{array}$

5.6.1 Computation of the spectra 69

$\begin{array}{ll}5.6 .2 \text { Experiments without breakwater } & 69\end{array}$

$\begin{array}{ll}5.6 .3 \text { Experiments with breakwater } & 71\end{array}$

$6 \quad$ Velocity measurement results $\quad 73$

$\begin{array}{ll}6.1 \text { Introduction } & 73\end{array}$

6.2 Strategy for executing the velocity measurements 73

6.3 Comparing velocity measurements in all three verticals of an experiment $\quad 74$

6.4 Comparing velocity measurements per vertical of experiments with and without breakwater

7. Sediment concentrations $\quad 80$

$\begin{array}{ll}7.1 \text { Introduction } & 80\end{array}$

7.2 Calculation of sediment concentrations $\quad 80$

7.3 Calculation of the vertical position of the intake tubes 81

$\begin{array}{ll}7.4 \text { Sediment concentration distributions } & 82\end{array}$

7.5 Evaluation of the different graphs $\quad 88$

7.5.1 Comparison of the verticals per experiment (method 1) 88

7.5.2 Comparison experiments with similar wave conditions (method 2) 89

7.5.3 Comparison experiments with or without breakwater (method 3) 90

8. Sediment transport 92

8.1 Introduction $\quad 92$

8.2 Cross-shore sediment transport calculations 92

8.3 CONVERT 93

8.4 Calculation of the sediment transport based on profile measurements $\quad 94$

8.5 Results of the calculations 95 
9. Unibest-TC 97

9.1 Introduction 97

9.2 Simulation of the experiments 97

9.2.1 Modelling of the wave heights 97

9.2.2 Modelling of the profile development 97

9.3 Input parameters 98

9.3.1 Definition of the bottom profile 98

9.3.2 Definition of wave heights at offshore boundary 98

$\begin{array}{ll}\text { 9.3.3 Other parameters } & 100\end{array}$

9.4 Comparison measurements with Unibest-TC 100

9.4.1 Comparison measured and calculated wave heights 100

$\begin{array}{ll}\text { 9.4.2 Comparison measured and calculated profile } & 103\end{array}$

$\begin{array}{ll}9.5 \text { Conclusion } & 105\end{array}$

10. Conclusions and recommendations 106

10.1 Conclusions 106

$\begin{array}{ll}\text { 10.2 Recommendations } & 107\end{array}$

\section{REFERENCES}

APPENDIX A AUKE-PC

APPENDIX B RESAMPLE-CPP

APPENDIX C Wave height measurements

APPENDIX D $\quad m_{0}$-parameters of Interval 2

APPENDIX E Curve fitting method of Klaros

APPENDIX F CONVERT

APPENDIX G Unibest-TC 


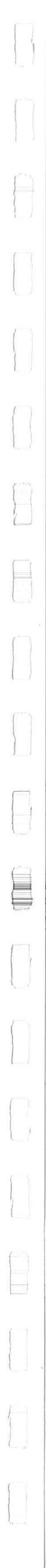




\section{CHAPTER 1 INTRODUCTION}

\subsection{Introduction}

All over the world large parts of coasts are threatened by the sea. Depending on the function of the coastal area this can be a very serious problem. Storm surges can do a lot of damage but also the influence of the day in day out working of the sea on the coast can be significant. Properly protecting threatened coasts is an important aim of coastal engineering. Those coasts can be existing coasts or new reclaimed areas. A coastal engineer has to choose a way of protecting the coast against the attacks of the sea. There are many possibilities; e.g., beach nourishment, series of groynes, revetments, seawalls, offshore breakwaters, submerged breakwaters, etc. The important thing is to know what kind of effect a certain method of coast protection will have in the given circumstances.

The Dutch coast is a good example of a (sandy) coast that is threatened by the sea. Every year the sea tries to gain land. Especially in the winter a lot of beach and dune erosion takes place. The economic and human interests are very high and the Dutch gouvernment has decided that a further retreat of the shoreline is not acceptable (since 1990). At the moment beach nourishment is the most used method to prevent a retreat of the shoreline. For the Netherlands it is estimated that a volume of 6 million cubic meters of sand disappears in the sea every year. The gouvernment spends about 60 million guilders every year to deal with this problem.

Beach nourishment is not a long term solution, it has to be repeated endlessly. Nowadays the question is whether there is an alternative to defend the coastline in a cheaper way. Such an alternative must be judged on many criteria. Apart from the safety criterion it is very important that a beach keeps its recreational function. Aesthetics plays an important role. Protecting the entire coast by a concrete wall is not the most elegant method. One of the promising alternatives is the use of submerged breakwaters along the coast. In the framework of the Human Capital and Mobility program of the European Union six universities work together in solving some of the unknown aspects related to the use of submerged breakwaters along sandy coasts. Many experiments are being or have been carried out by the partners with the main emphasis on the morphodynamical, hydrodynamical and structural effects of a submerged breakwater (see Section 1.3).

Experiments with a scale model of a submerged breakwater with a movable bed were performed in the Lange Speurwerkgoot (= wave flume) of the Laboratory of Fluid Mechanics at Delft University of Technology (DUT). These experiments have been carried out by the authors of this report. The main emphasis was on the development with time of the bed. Tests were performed with and without breakwater on an initial slope of 1 in 15 . Different combinations of wave heights and periods were used. For further details about the experiments see Chapter 2. 


\subsection{Functioning of the submerged breakwater}

Theoretically it is assumed that every natural cross-shore beach profile eventually will reach an equilibrium shape. The shape of the profile depends on waves, tides, winds, currents and sediment parameters. In the winter the profile will be different as in the summer due to different wave conditions, for instance heavy storms. If you would take a look at a longer period of time, for instance about ten years, you would discover that the profile shape fluctuates around an 'average profile', the so called equilibrium profile. The equilibrium profile is dynamic. Changes in wind and/or sea conditions on the long term will effect the shape of the equilibrium profile. If one assumes that in a certain case, for example the Dutch coast, no dramatic changes in the natural conditions occur, the profile will reach its equilibrium shape and thus it would have a dynamically stable position. In case of the Dutch coast every year a lot of beach and dune erosion takes place. Normally you would expect that erosion would be restored by nature itself. This certainly happens but not for the full one hundred percent. This is often due to a gradient in the longshore transport. If at one side of a certain control volume (read: part of the coast) less sediment goes in than at the other side goes out the total amount of sediment in the control volume will decrease. The profile is shifted landwards, in other words the shoreline retreats. As stated earlier the common method to solve this problem is artificial beach nourishment. This costs a lot of money and it has to be repeated endlessly. The general idea behind the use of a submerged breakwater is simple. The submerged breakwater must prevent or reduce erosion of the profile landward of it. The breakwater forms a barrier that must prevent or reduce the transport of sediment offshore. In this way the costs of coast protection can be reduced.

\section{$\underline{1.3 \text { Human Capital and Mobility Programme }}$}

The tests are carried out in the framework of the Dynamics of Beaches project. The Dynamics of Beaches project is a part of the HCM-framework This particular project is a cooperation of six European universities, viz.:

- Aristotle University of Thessaloniki (Greece)

- University of Ghent (Belgium)

- University of Liverpool (United Kingdom)

- University College of Cork (Ireland)

- Delft University of Technology (the Netherlands)

- Universitat Politecnico de Catalunya (Spain)

The objectives of the Dynamics of Beaches project are to improve the existing knowledge on physical processes pertaining to the nearshore region (including the surf zone). Four of the above mentioned universities will carry out tests within their experimental facilities. These tests will cover three areas: 
1 - Hydrodynamics: water motion

2 - Morphodynamics: sediment motion - bed and suspended transport and resulting bottom evolution.

3 - Structural effects: all tests will be performed with and without a submerged breakwater with a view to assess the physical (hydro- and morpho-) impact of this structure on the nearshore zone

At the DUT two different series of movable bed tests will be carried out. The first series of tests will be $2 \mathrm{DV}$ tests in the wave flume of the DUT. The second series of tests will be 3D tests in the large wave basin of the DUT. This report will discuss the $2 \mathrm{DV}$ tests and its results.

\subsection{Aims of this study}

This study is part of a Human Capital and Mobility Project called Dynamics of Beaches. This project is a co-operation of six European universities which work together in solving some unknown aspects of a submerged breakwater. Therefore several agreements have been made by the partners about the way of performing the tests. Every partner has its own test set-up and therefore its own aspects to investigate. In Delft the main emphasis will be on bottom evolution. This leads to the primary aim of the study.

The primary aim of this study is to investigate the effect of a submerged breakwater on the profile development landward of this submerged breakwater.

In order to do this, similar tests are performed with and without breakwater in a wave flume. By means of varying wave height and wave period the influence of these parameters on hydro- and morphodynamics can be investigated. For these purposes wave heights, flow velocities, sediment concentrations and bottom profiles were measured at several locations in place and time. These results have been interpreted, edited by and compared with computer programs like Auke/PC, Unibest-TC, Convert and Klaros. This leads to the secondary aim of this study.

The secondary aim of this study is to compare the results found in our tests with several existing ideas often mathematically modelled in computer programs.

\section{$\underline{1.5 \text { Layout }}$}

This section gives a short review of the contents of this report.

The report consists of two separate volumes. This volume (Volume 1: Text) contains the text of this report and the appendices. The other volume (Volume 2: Graphics) contains only graphs of the results. The text of the first volume refers (if necessary) to the graphs presented in the second volume. A list of all the graphs is presented in Volume 2: Graphics.

The contents of this volume (Volume 1: Text) is described below.

Chapter 2 describes the experimental set-up for the tests that are performed in the 'Dynamics of Beaches' project. In the Test Definition Report the wave conditions, layout of the 
breakwater and the initial bottom slope are prescribed for all test series. For the test series at DUT these conditions are scaled down to match the dimensions of the wave flume in the Laboratory of Fluid Mechanics. The last section of this chapter describes the spectra that were made in order to perform the different wave conditions.

The next chapter describes the measurements that were performed during the test series. Four different kind of measurements were performed, viz.:

1- Profile measurements

2- Wave height measurements

3- Velocity measurements

4- Sediment concentration measurements

Explanations are given on how these measurements were performed, how many measurements were performed and the calibration procedures of all measuring methods. The next chapters will discuss these measurements in the same order of appearance.

Chapter 4 discusses the profile measurements and the results that were found from the test series. Chapter 5 and 6 do the same for respectively the wave height measurements and the velocity measurements. The next chapter describes the sediment concentration measurements. A computer program called 'Klaros' was used to present the sediment concentration distributions. This program is discussed as well in this chapter.

Chapter 8 describes the sediment transport. Comparisons were made between sediment transport as was found in the test series and the sediment transport rates as the computer program Convert calculates it. Furthermore, this computer program is discussed.

The next chapter describes the simulations of the experiments made with the computer program called Unibest-TC. This computer program does calculate wave heights and profile development. The results are compared with the results found during the test series.

Finally, in Chapter 10 conclusions are drawn and recommendations are made.

\subsection{Data base}

The experiments that were performed resulted in many data. These data are available for further analysis. Persons who are interested in this data base can contact the following person:

Dr ir J. van de Graaff

Faculty of Civil Engineering

Stevinweg 1

2826 CN Delft

The Netherlands

tel.: + 3115784846

fax.: + 3115785124

e-mail: graaff@dutcvs5.tudelft.nl 


\section{CHAPTER 2 EXPERIMENTAL SET-UP}

\subsection{Introduction}

Several agreements concerning the experiments were made between the partners of the Dynamics of Beaches project. These have been defined in the Test Definition Report. Those agreements that are relevant to the 2DV experiments in Delft will be discussed in the first two sections of this chapter.

The experiments can be divided in two series, namely a series of experiments with breakwater present and a series of experiments without breakwater present.

The layout of the breakwater and the layout of the (initial) bottom profile are defined in the so called 'Prototype Model'.

The wave conditions for the experiments are also defined and they are listed in the 'Prototype Test Matrix'.

Together the Prototype Model and the Prototype Test Matrix form the basic concept for all the experiments that will be or have been carried out by each of the partners of this Dynamics of Beaches project.

Section 2.2 discusses the layout of the Prototype Model and the wave conditions defined in the Prototype Test Matrix. In Section 2.3 the dimensions of the Prototype Model and the values of the parameters of the Prototype Test Matrix will be scaled to new values to match the dimensions of the wave flume at DUT. Section 2.4 discusses the duration of the experiments. Section 2.5 discusses the wave spectra.

\subsection{Prototype Model and Prototype Test Matrix}

\subsubsection{Layout of the Prototype Model}

The Prototype Model consists of of a submerged breakwater on a 1 in 15 slope. The design of the submerged breakwater itself has been done by the University of Ghent (Belgium). The prototype breakwater has been designed with the steps outlined below.

1. The breakwater has been designed with a permeable core, but will be modelled in the experiments, for simplicity, with an impermeable core. From a hydrodynamic point of view this has been shown by several authors to be a reasonable approach (Van der Meer and Daemen (1994) and Davies and Kriebel (1992)).

2. The armour layer, consisting of 2 layers of rock stones, has been designed according to the formula presented in, Van der Meer (1990), Van der Meer (1991), Van der Meer (1993), and Van der Meer and Daemen (1994):

$\frac{h_{c}}{h}=(2.1+0.1 * S) * \exp \left(-0.14 * N_{s}^{*}\right)$

where the special stability number $N_{s}^{*}$ is defined by 


$$
N_{S}^{*}=\frac{H_{S}}{\Delta D_{n s 0}} * S_{p}^{-1 / 3}
$$

with:

$\mathrm{h}=$ water depth at seaward toe

$\mathrm{h}_{\mathrm{c}} \quad=$ crest height

$\mathrm{S} \quad=$ damage level (here chosen as 2, corresponding to start of damage)

$\Delta \quad=$ bouyant mass density (here 1.573)

$\mathrm{D}_{\mathrm{n} 50}=$ nominal diameter

$\mathrm{s}_{\mathrm{p}} \quad=$ local wave steepness

Although the formula presents some uncertainties, mainly due to the scatter in the data to which it was fitted, it is normally presented as being on the safe side. From the test matrix (see Table 2.1) it is seen that the ratio between the crest height and the water depth at the seaward toe is constant for all experiments, that is $h_{c} / h=0.75$. Two local wave steepnesses are being investigated: a design wave height of $4.0 \mathrm{~m}$ and corresponding wave periods of 8 to $9 \mathrm{~s}$, respectively. The calculations lead to an average rock mass of $\mathrm{W}_{50}=1.416$ ton and $\mathrm{D}_{\mathrm{n} 50}=$ $0.812 \mathrm{~m}$, for a rock density of $2.65 \mathrm{ton} / \mathrm{m}^{3}$.

3. The crest width is chosen as $3^{*} D_{n 50}$ and the thickness of the armour layer is estimated to $2 * D_{\text {n } 50}$.

4. The gradation of the stones for the armour layer must follow the log-linear relationship:

$$
W_{y}=W_{0} *\left(W_{100} / W_{0}\right)^{y / 100}
$$

where the mass of the lightest block is $\mathrm{W}_{0}=1$ ton, and the mass of the heaviest block is $\mathrm{W}_{100}=$ 2 ton.

The resulting prototype design is shown in Figure 2.1.

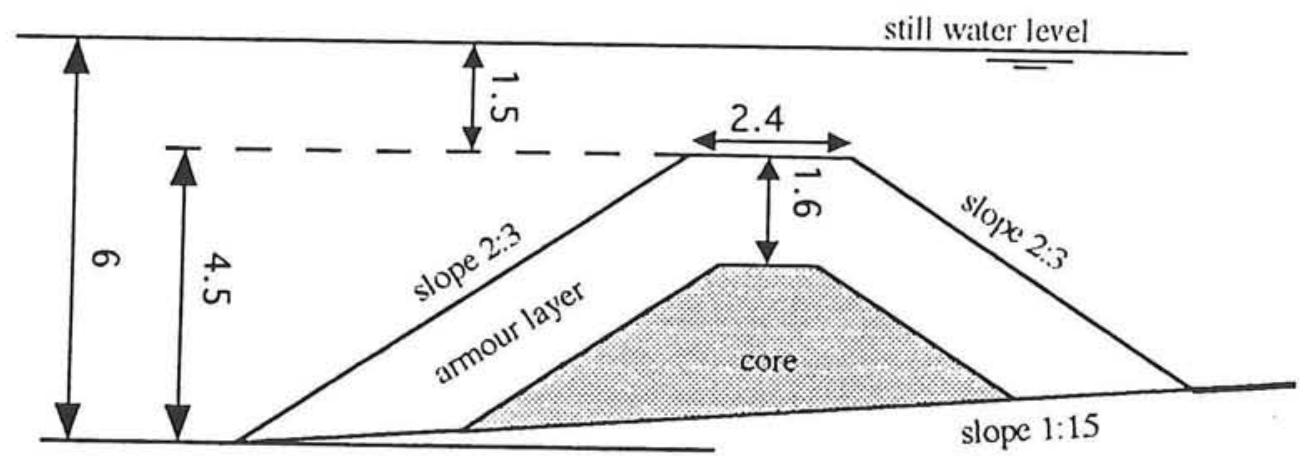

All dimensions are in meters

Figure 2.1 Prototype Model of the submerged breakwater. 


\subsubsection{Wave conditions defined in the Prototype Test Matrix}

A test matrix (Table 2.1) was made which defines the conditions for the several tests. The ideas behind the different combinations of parameters in the test matrix will be explained in the following section taken from the Test Definition Program.

\begin{tabular}{|l|l|l|l|l|l|l|l|}
\hline $\begin{array}{l}\text { Test- } \\
\text { case }\end{array}$ & $\begin{array}{l}\mathbf{F} / \mathbf{H}_{\mathbf{s}} \\
-\end{array}$ & $\begin{array}{l}\mathbf{H}_{\mathbf{s}} / \mathbf{L}_{\mathbf{0 p}} \\
\mathbf{\%}\end{array}$ & $\begin{array}{l}\mathbf{T}_{\mathbf{p}} \\
{[\mathbf{s}]}\end{array}$ & $\begin{array}{l}\mathbf{H}_{\mathbf{s}} \\
{[\mathbf{m}]}\end{array}$ & $\begin{array}{l}\mathbf{h} \\
{[\mathbf{m}]}\end{array}$ & $\begin{array}{l}\mathbf{F} \\
{[\mathbf{m}]}\end{array}$ & $\begin{array}{l}\mathbf{H}_{\mathbf{s}} / \mathbf{L}_{\mathbf{p}} \\
\mathbf{\%}\end{array}$ \\
\hline $\mathbf{A}$ & 1.00 & 1.50 & 8.0 & 1.5 & 6.0 & 1.5 & 2.61 \\
$\mathbf{B}$ & 1.00 & 2.67 & 6.0 & 1.5 & 6.0 & 1.5 & 3.67 \\
$\mathbf{C}$ & 1.00 & 3.84 & 5.0 & 1.5 & 6.0 & 1.5 & 4.66 \\
$\mathbf{D}$ & 0.75 & 2.61 & 7.0 & 2.0 & 6.0 & 1.5 & 4.06 \\
$\mathbf{E}$ & 1.50 & 2.56 & 5.0 & 1.0 & 6.0 & 1.5 & 3.01 \\
\hline
\end{tabular}

Table 2.1 Prototype Test Matrix for all experiments (HCM-program).

with:

$\mathrm{F}=$ freeboard, $\mathrm{F}=\mathrm{h}-\mathrm{h}_{\mathrm{c}}$

$\mathrm{H}_{\mathrm{s}}=$ significant wave height

$\mathrm{L}_{0}=$ offshore wave length $\mathrm{gT}^{2} /(2 \pi)$, where $\mathrm{g}$ is the gravitational accelleration

Substituting $\mathrm{T}$ by $\mathrm{T}_{\mathrm{p}}$ defines $\mathrm{L}_{\mathrm{op}}$ :

$\mathrm{T}_{\mathrm{p}}=$ spectral peak period

$\mathrm{h}=$ water depth at toe of structure

$\mathrm{h}_{\mathrm{c}}=$ crest height

$\mathrm{L}_{\mathrm{p}}=$ local wave length (Airy wave theory)

Two non-dimensional parameters have been considered to be the most significant for the hydrodynamics around a submerged breakwater: a wave parameter (the wave steepness) and a geometry parameter (the relative freeboard, $\mathrm{F} / \mathrm{H}_{s}$ ). In the following the wave steepness is defined by $\mathrm{H}_{s} / \mathrm{L}_{0 \mathrm{p}}$, which relates to the Irribarren number, $\tan \alpha / \sqrt{ }\left(\mathrm{H} / \mathrm{L}_{0 \mathrm{p}}\right)$. The correct local wave steepness at the position of the breakwater is also calculated for comparison. To cover a reasonable range of normal wave steepnesses, that is from moderately long, to relatively short waves, $\mathrm{H}_{s} / \mathrm{L}_{0 \mathrm{p}}$ is suggested to vary within the range $1 \%$ to $4 \%$. Likewise the relative freeboard must be chosen so that the submerged breakwater affects significantly wave transmission without acting as a non-submerged breakwater. $\mathrm{F} / \mathrm{H}_{\mathrm{s}}$ is suggested to vary within the range -0.75 to -1.5 . The general idea is to cover a reasonable range of variation of the aforementioned variables with 5 test cases: a central case and four additional cases, in which $\mathrm{H}_{s} / \mathrm{L}_{0 \mathrm{p}}$ is increased and decreased for a fixed value of $\mathrm{F} / \mathrm{H}_{\mathrm{s}}$ and vice versa (i.e. defining a diamond shaped set of test conditions). As the two non-dimensional parameters, $\mathrm{F} / \mathrm{H}_{\mathrm{s}}$ and $\mathrm{H}_{\mathrm{s}} / \mathrm{L}_{0 \mathrm{p}}$ consist of three dimensional parameters (two wave parameters and one geometry parameter) the possible solutions are:

1. Fixing $L_{0 p}$ (i.e. $T_{p}$ ) and varying $H_{s}$ and $F$ (i.e. $h$ ).

2. Fixing $F\left(\right.$ i.e. $h$ ) and varying $H_{s}$ and $L_{0 p}$ (i.e. $T_{p}$ ). 
3. Fixing $H_{s}$ and varying $L_{0 p}$ (i.e. $T_{p}$ ) and $F$ (i.e. h).

It was decided to fix the breakwater cross-section in all experiments. Therefore a variation of $\mathrm{F}$ should be obtained by varying $\mathrm{h}$. In theory this is not a problem, but it is generally a more reasonable solution to fix the water depth for all experiments for practical reasons, e.g. the tuning of the active absorption limit for reflected waves and the time consuming process of adding or removing water specially in the large scale wave flumes. Hence, it was decided to carry out the experiments for a wave matrix following solution 2. The Prototype Test Matrix which applies to all experiments to be conducted is shown in Table 2.1. This provides a nearly regular "diamond pattern" in the $\left(\mathrm{F} / \mathrm{H}_{s}, \mathrm{H}_{s} / \mathrm{L}_{0 \mathrm{p}}\right)$-plane, a distorted 'diamond pattern' in the $\left(\mathrm{F} / \mathrm{H}_{s}\right.$ $\mathrm{H}_{s} / \mathrm{L}_{\mathrm{p}}$ )-plane and a vertical line in the $\left(\mathrm{H}_{s}, \mathrm{~F}\right)$-plane (see Figure 2.2).

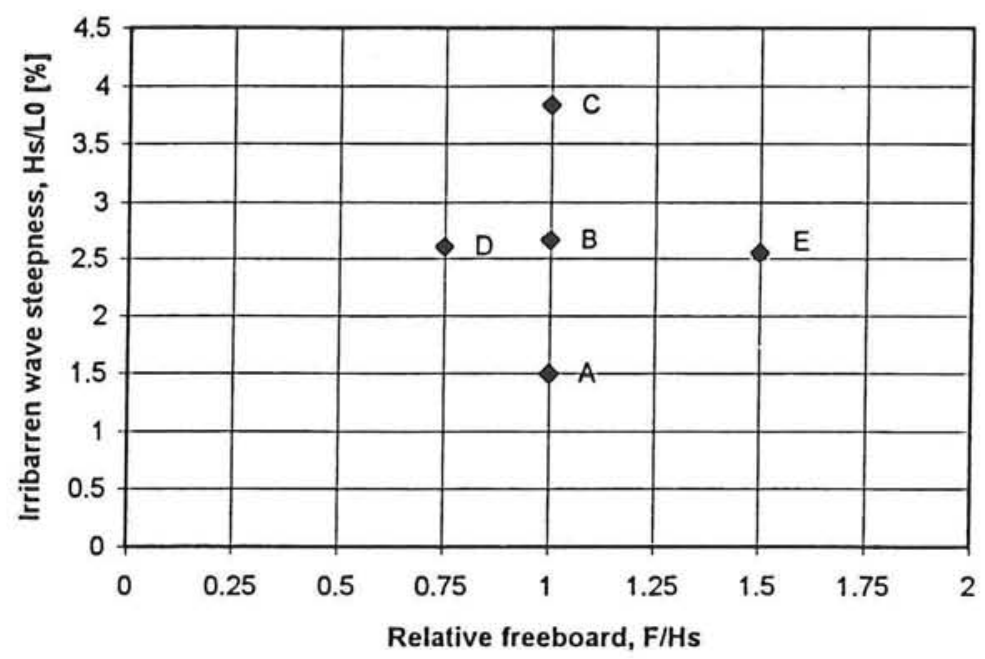

Figure 2.2 Diamond pattern of the Prototype Test Matrix. 


\section{$\underline{2.3 \text { Scale model at DUT with matching test matrix }}$}

\subsubsection{Scale model in the Laboratory of Fluid Mechanics at DUT}

The experiments were carried out in the Lange Speurwerkgoot ( = wave flume) of the Laboratory of Fluid Mechanics at Delft University of Technology (DUT). This is a medium scale wave flume (see Figure 2.3 and Picture 2.1) with the following dimensions:

Length: $\quad 32.0 \mathrm{~m}$

Width: $\quad 0.8 \mathrm{~m}$

Height: $\quad 1.0 \mathrm{~m}$

The wave board (see Picture 2.2) can generate irregular waves. Because of the dimensions of the wave flume it was decided to perform the experiment at scale 1:15. According to the test program tests were done with and without breakwater with a 1 in 15 slope and a movable bed. The bed consisted of sand with a $D_{50}=95 \mu \mathrm{m}\left(D_{10}=76 \mu \mathrm{m}, D_{90}=131 \mu \mathrm{m}\right)$ which is a relatively small diameter for sand along beaches. The reason for this choice is that in this way the ratio between bed transport and suspension transport will be closer to the value that would occur if the experiments were done at a scale 1:1. Using scaled down wave parameters and unscaled sand parameters instead would decrease the amount of suspension transport in proportion to the amount of bed transport. The explanation for this is that smaller waves have less energy to stir up the sand so there will be less sand in suspension. Sand with a smaller diameter can more easily be stirred up. The first part of the slope is a non-movable bed made out of concrete (see Figure 2.3). This was done for two reasons. First, our primary aim was to measure the effect of the breakwater on the bed profile landward of the breakwater in comparison with the situation without a breakwater present. A movable bed in front of the breakwater could for instance lead to scour holes in front of the breakwater and thereby also strongly affect the hydrodynamics and morphodynamics on both sides of the breakwater. If such a scour hole would occur, the stability of the breakwater would be endangered. The development of a scour hole in front of the breakwater must be prevented. In reality the development of a scourhole will not be allowed. Secondly the advantage of pouring a part of the slope in concrete is that in case of a test with the breakwater, the amount of sediment that passes the breakwater in offshore direction can be measured very accurately. Transport of sediment over or through the breakwater in seaward direction can be considered as a loss of sediment. The main purpose of the breakwater is to prevent sediment loss to the sea.

Finally, the scaled breakwater is shown in Figure 2.4. 


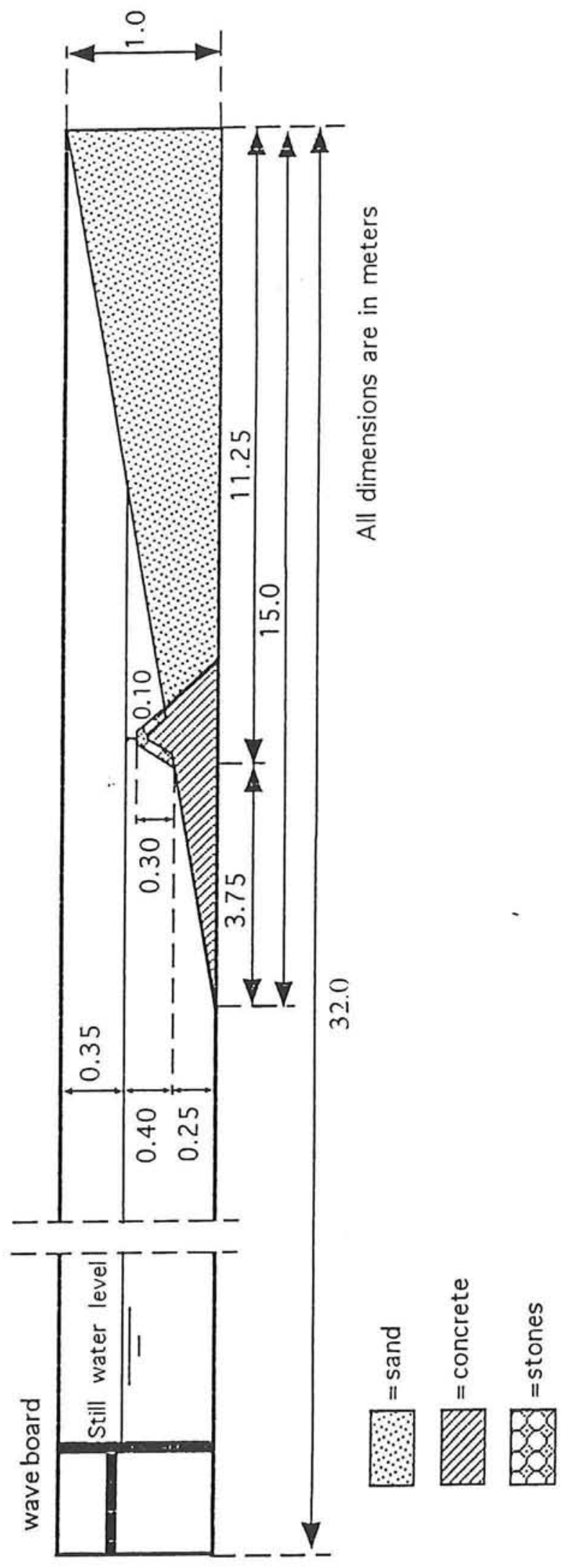

Figure 2.3 The 'Lange Speurwerkgoot' (= wave flume) in the Laboratory of Fluid Mechanics at Delft University of Technology (TUD). 


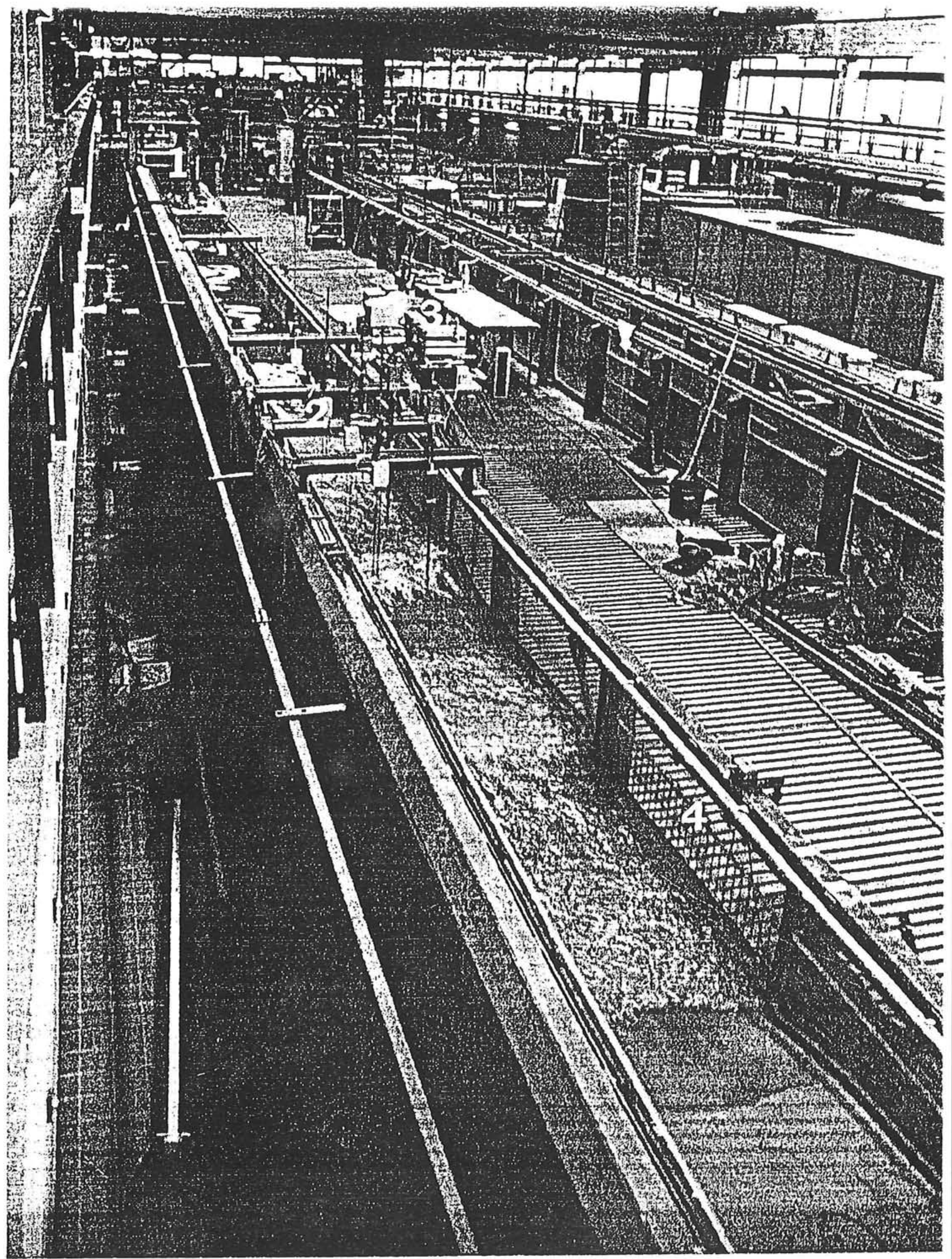

Picture 2.1 (see next page) 
$1=$ Position of the wave board

$2=$ Measurement equipment

3 = Computers and electronic equipment for controlling the wave board and registering the measurements

$4=$ Visual measurement area

Picture 2.1 (previous page) The 'Lange Speurwerkgoot' (= wave flume) in the Laboratory of Fluid Mechanics at Delft University of Technology (TUD).

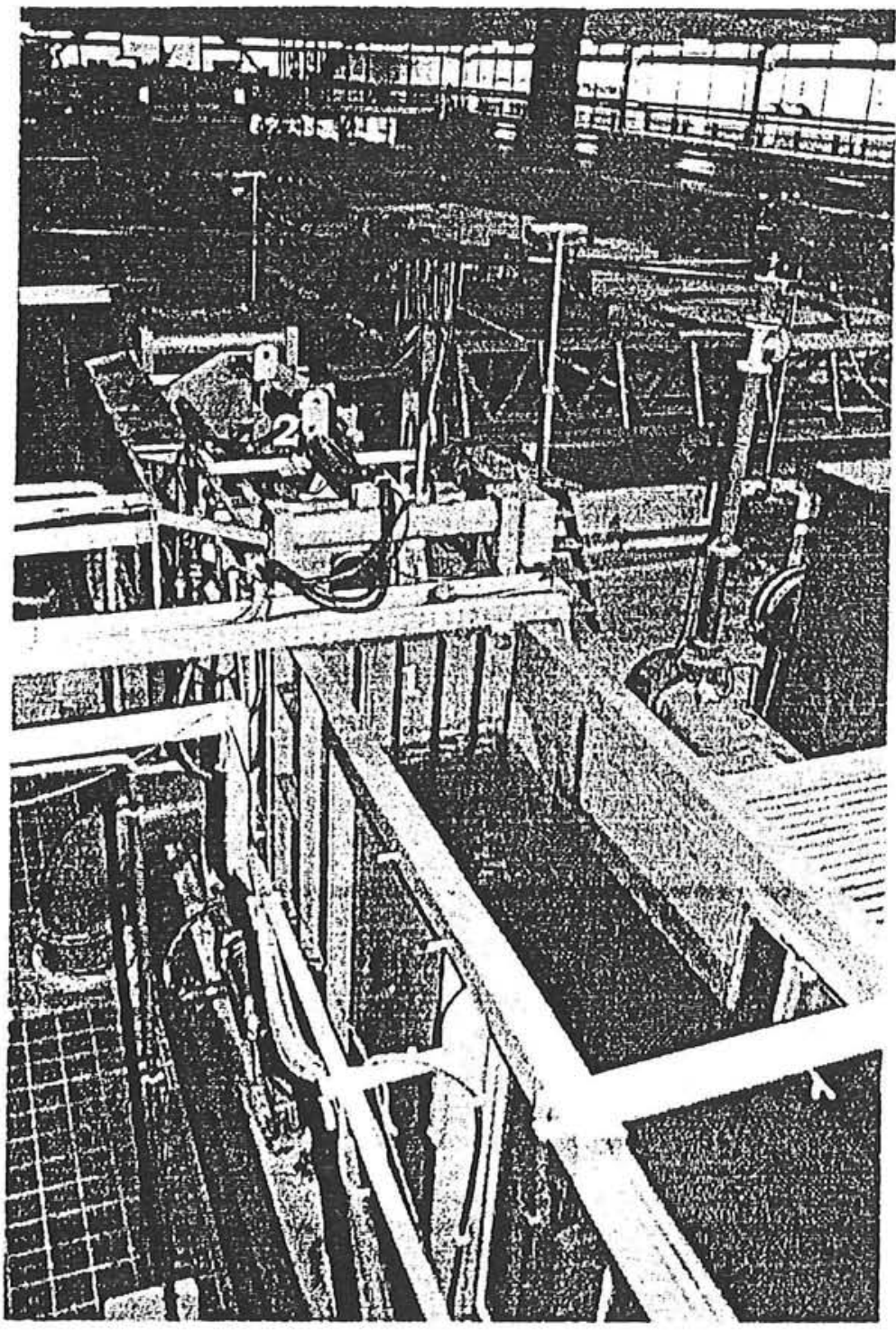

$$
\begin{array}{ll}
1 & =\text { Waveboard } \\
2 & =\text { Hydraulic cylinder for moving the wave board }
\end{array}
$$

Picture 2.2 Waveboard of the 'Lange Speurwerkgoot'. 


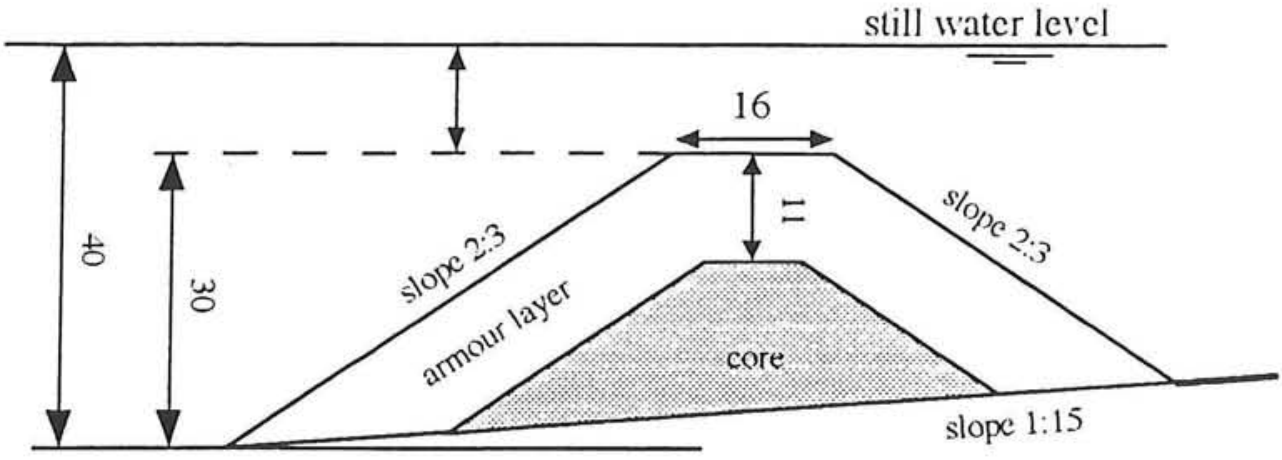

All dimensions are in centimeters

Figure $2.4 \quad$ Scale model of the submerged breakwater.

\subsubsection{Test matrix for the scale model}

The prototype wave conditions prescribed in the Test Definition Report had to be scaled down to match the scale of the model in the wave flume. The results (the new wave parameters) are presented in Table 2.2. One wave condition was added to the test program called $F$. Wave condition $\mathrm{F}$ consists of a regular sinusoidal wave $(\mathrm{H}=0.1 \mathrm{~m}, \mathrm{~T}=1.55 \mathrm{~s})$. This was done to make a coupling between the results of these $2 \mathrm{DV}$ tests and the $3 \mathrm{D}$ tests with regular waves that will take place in the large wave basin at DUT at a later stage. In total twelve successful experiments have been done. Six experiments were done without the breakwater present, each of them with one of the wave conditions (A, B, C, D, E, F). The same strategy was repeated with the breakwater present; six experiments and each of them with a different wave condition. By using the same conditions for the tests with and the tests without breakwater it is possible to make a true comparison between both situations.

\begin{tabular}{|l|l|l|l|l|l|l|l|}
\hline $\begin{array}{l}\text { Test- } \\
\text { case }\end{array}$ & $\begin{array}{l}\mathbf{F} / \mathbf{H}_{\mathbf{s}} \\
-\end{array}$ & $\begin{array}{l}\mathbf{H}_{\mathrm{s}} / \mathrm{L}_{0 \mathrm{p}} \\
\%\end{array}$ & $\begin{array}{l}\mathrm{T}_{\mathrm{p}} \\
{[\mathrm{s}]}\end{array}$ & $\begin{array}{l}\mathbf{H}_{\mathbf{s}} \\
{[\mathbf{m}]}\end{array}$ & $\begin{array}{l}\mathbf{h} \\
{[\mathbf{m}]}\end{array}$ & $\begin{array}{l}\mathbf{F} \\
{[\mathrm{m}]}\end{array}$ & $\begin{array}{l}\mathbf{H}_{\mathrm{s}} / \mathrm{L}_{\mathrm{p}} \\
\mathbf{\%}\end{array}$ \\
\hline A & 1.00 & 1.50 & 2.07 & 0.1 & 0.4 & 0.1 & 2.61 \\
B & 1.00 & 2.67 & 1.55 & 0.1 & 0.4 & 0.1 & 3.67 \\
C & 1.00 & 3.84 & 1.29 & 0.1 & 0.4 & 0.1 & 4.66 \\
D & 0.75 & 2.61 & 1.81 & 0.133 & 0.4 & 0.1 & 4.06 \\
E & 1.50 & 2.56 & 1.29 & 0.067 & 0.4 & 0.1 & 3.01 \\
\hline
\end{tabular}

Table 2.2 Model test matrix for experiments at DUT.

with:

$\mathrm{F}=$ freeboard, $\mathrm{F}=\mathrm{h}-\mathrm{h}_{\mathrm{c}}$

$\mathrm{H}_{\mathrm{s}}=$ significant wave height

$\mathrm{L}_{0}=$ offshore wave length $\mathrm{gT}^{2} /(2 \pi)$, where $\mathrm{g}$ is the gravitational accelleration 
Substituting $T$ by $T_{p}$ defines $L_{0 p}$ :

$\mathrm{T}_{\mathrm{p}}=$ spectral peak period

$\mathrm{h}=$ water depth at toe of structure

$\mathrm{h}_{\mathrm{c}}=$ crest height

$\mathrm{L}_{\mathrm{p}}=$ local wave length (Airy wave theory)

\subsection{Duration of the experiments}

Each experiment lasted for 7.5 hours. It occured that after 7.5 hours of exposing the bottom profile to waves enough bottom profile evolution has taken place to draw conclusions. This period of 7.5 hours was divided in four intervals, viz.:

$\begin{array}{llll}\text { Interval } 1 \text { from: } & t=0 \text { hours till: } t=0.5 \text { hours } & \Delta t=0.5 \text { hours } \\ \text { Interval } 2 \text { from: } & t=0.5 \text { hours till: } t=1.5 \text { hours } & \Delta t=1.0 \text { hours } \\ \text { Interval } 3 \text { from: } & t=1.5 \text { hours till: } t=3.5 \text { hours } & \Delta t=2.0 \text { hours } \\ \text { Interval } 4 \text { from: } & t=3.5 \text { hours till: } t=7.5 \text { hours } & \Delta t=4.0 \text { hours }\end{array}$

Before the start of Interval 1, between the different intervals and after Interval 4 the bottom profile has been measured. The duration of each following interval doubles. This is done because morphological processes often happen on a logarithmic time scale. During the different intervals wave height, velocity and sediment concentration measurements have been performed. All the measurements are further discussed in Chapter 3.

\subsection{Wave spectra}

The wave spectra of the irregular waves (experiments A, B, C, D and E) were created using the program STIR. STIR is part of the software package AUKE/PC which has been developed by Delf Hydraulics. STIR was used to create the required Jonswap 3.3 spectra with the right wave height and period. The program GOLFHYD (also part of AUKE/PC) is able to use these spectra and translate them to output signals for controlling the wave board.

A description of the program STIR can be found in Appendix A.

The duration of each spectrum made was 15 minutes. The number of repetitions of such a spectrum can be defined in GOLFHYD so that it is possible to generate waves for a longer period of time without any interruptions. The reason for creating spectra with durations of 15 minutes will be explained in the next paragraphs.

A spectrum needs a certain (minimum) amount of waves to be reliable. The longer the duration of a created spectrum is (or in other words the more waves one spectrum contains) the more reliable this spectrum is. From this point of view a spectrum with a duration of 7.5 hours would be excellent for these experiments. The reason for not creating a spectrum of 7.5 hours has to do with the measurements that have to be performed. One of the aims of this project is to do research on hydrodynamics by means of velocity and wave height measurements. Velocities have to be measured on different vertical positions to get an insight in the distribution of the velocity in a certain vertical position. Each of these velocity measurements in a certain vertical position has a certain duration. At this point there is a conflict of interest. The longer the duration the more reliable the measurement is (influence of bottom changes in the meantime on hydrodynamics not taken into account), but a long 
duration also results in less time to measure in other positions of a vertical. A choice has to be made between a vertical velocity distribution based on a (relatively) small number of measured points with each a (relatively) high reliability and a vertical velocity distribution based on a larger number of measured points with each a relatively less reliability. The conclusion of this evaluation is that a compromis has to be found between those two interests.

For these types of experiments it is generally assumed that a number of approximately 500 waves is sufficient to give reliable measurement results. Multiplying this number by the average wave period gives an indication of the required duration. For example an average wave period of $1.75 \mathrm{~s}$ would result in a duration of $875 \mathrm{~s}$. The choice was made to use a duration of 15 minutes $(900 \mathrm{~s})$ for the wave height, velocity and sediment concentration measurements.

This leads us back to the relation between the duration of the measurements and the spectrum to be created. When a measurement of 15 minutes would be performed and the used spectrum would have a duration of, for instance, 7.5 hours the measurement would represent only a part of this spectrum. The parameters that characterize the total of this particular spectrum (e.g., wave height, wave period) are not necessarily the same for a part of this spectrum. The best way to avoid this problem is to create a spectrum that has exactly the same duration as the measurement. This has the advantage that whenever a measurement is started it always lasts exactly one entire spectrum. Therefore it was decided to create spectra with durations of 15 minutes. The duration of each of the four intervals is also a multiple of the duration of one spectrum. In that way the profile measurements performed after the intervals are always after a whole number of (repeated) spectra.

The spectrum of the two experiments with regular waves (experiment $F$, with and without breakwater) was also created with the program STIR. Such a spectrum is nothing else than an 'endlessly' continuous sinusoidal wave with the same height and period. 


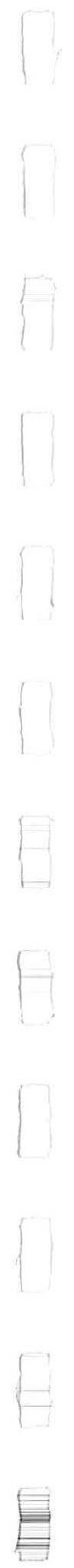

䓃 


\section{CHAPTER 3 MEASUREMENTS}

\section{$\underline{3.1 \text { Introduction }}$}

Apart from the layout of the scale model and the wave conditions the Test Definition Program did not prescribe the exact way of performing the experiments. The decision about the way of performing the experiments was up to each of the individual partners.

Four types of measurements have been carried out in the medium scale wave flume of the Laboratory of Fluid Mechanics at DUT, viz:

1 - Profile measurements

(Section 3.2)

2 - Wave height measurements

(Section 3.3)

3 - Velocity measurements

(Section 3.4)

4 - Sediment concentration measurements

(Section 3.5)

Each of these sections is built up in the same way. They start with a subsection that explains the used measurement technique. This subsection is followed by another one which explains how and when these measurements have been performed. Each final subsection discusses the method of calibration for each measurement technique. Calibration of the often very sensitive measurement equipment is of vital importance for getting reliable measurement results.

Section 3.6 gives a summary of all the activities that were necessary during a single experiment.

\subsection{Profile measurements}

\subsubsection{Profile measurement technique}

To measure bed levels an electronic profile-follower (=PROFO) (see Picture 3.1) was used. This instrument consists of a probe placed vertically in the water. A servomechanism maintains the tip of the probe at a constant distance above the bed. When the instrument is being displaced in a horizontal direction, the probe (needle) will follow the configuration of the bed continuously. The principle of operation is the appreciable difference between the electric conductivity of water and bed material.

The probe is a stainless-steel tube completely insulated. At a distance of approximately $15 \mathrm{~mm}$ from its tip, a stainless-steel ring interrupts the insulation. This is the compensation electrode. At the tip of the tube is the measuring electrode, completely insulated. When placing the probe in a fluid, the electrical resistance between the electrodes and a plate-electrode (in the fluid) is a function of the area of the electrodes and the conductivity of the fluid. As soon as the measuring electrode is in the vicinity of a non-conductive bed, the resistance of the measuring electrode increases rapidly. The resistance of the measuring electrode remains almost constant. A potentiometer attached to the probe enables the position of the probe to be indicated. The analog output signal of the potentiometer is digitized by an analog-digital converter. The digitized signal can be registered by a computer.

The PROFO has an accuracy of $0.2 \mathrm{~mm}$. 


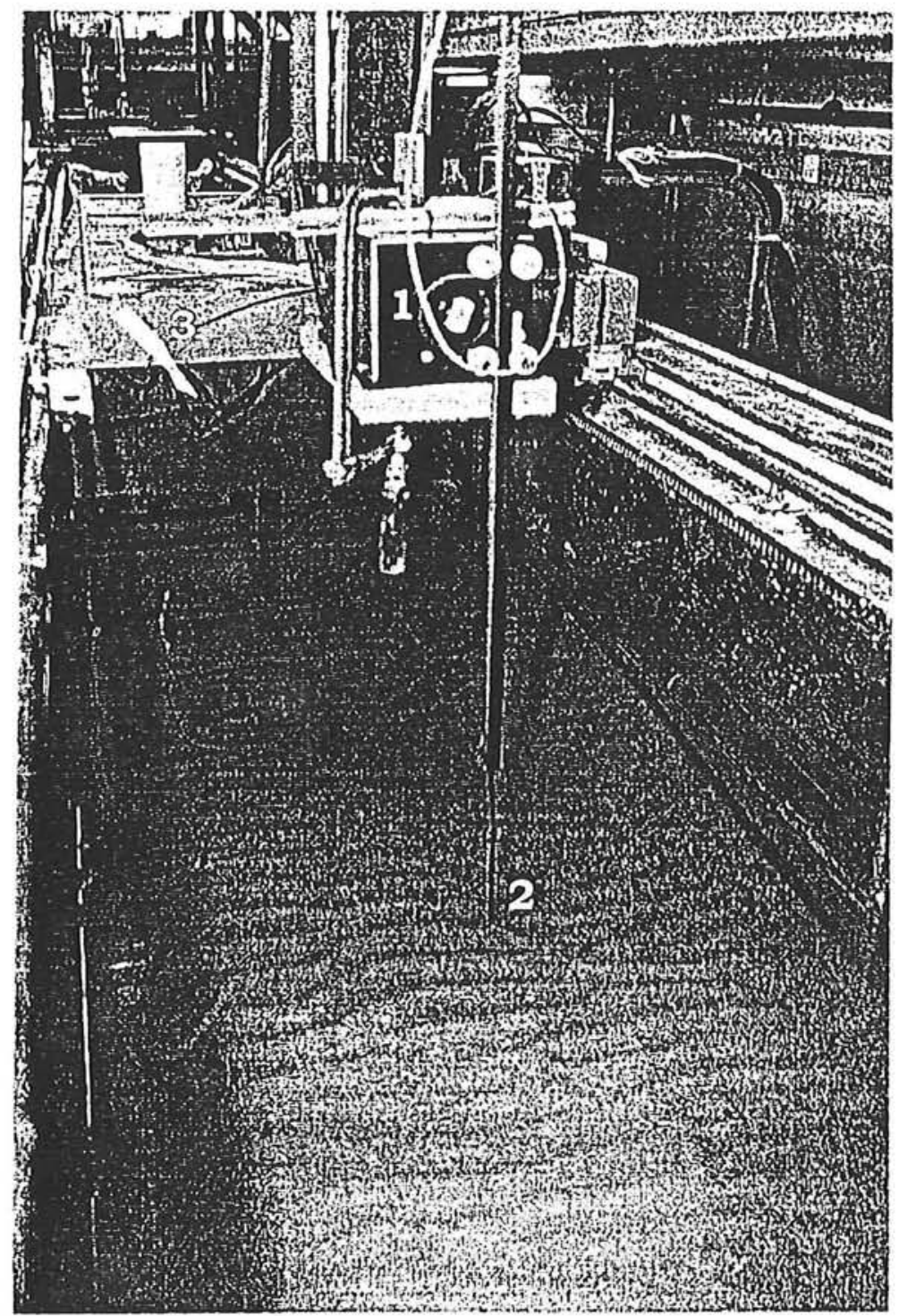

$1=$ Elcctronic profile-indicator

$2=$ Point of the probe following the bed

$3=$ Trolley for moving the clectric profilc-indicator horizontally over the bed

\section{Picture 3.1 Electronic profile-indicator.}

\subsubsection{Execution of the profile measurements}

During one experiment five times a profile measurement was performed. In order to perform a profile measurement a trolley with a PROFO attached to it, was moved in a horizontal direction over the flume. To start the profile measurement at exactly the same position every time, a trigger is attached to the side wall of the flume. The very moment the trolley passes the trigger, an electric signal is sent to the computer (see Picture 3.2 and 3.3) that immediately starts registering the incoming signal of the electronic profile-indicator. To end a PROFO measurement at the same position every time, another trigger is installed at the end of the bed too.

The trolley moves at a speed of about $0.061 \mathrm{~m} / \mathrm{s}$. A servomechanism controls this speed. 
The PROFO is only able to measure the bed under water (a minimum water depth of about 2 $\mathrm{cm}$ is necessary). The PROFO measurement covered the larger part of the bed. The rest of the bed, near the shoreline, was visually measured. These visual measurements were performed in the following way. At the side wall of the flume vertical centimeter distributions were attached to the glass every five centimeters (total number of 50 centimeter distributions, see Picture 3.4). During every profile measurement the bed height was measured at every centimeter distribution. An overall view of the bed profile is obtained by pasting these two measurements next to each other.

In case of the tests without breakwater the PROFO measurement started at the beginning of the slope at position $\mathrm{x}=0.00 \mathrm{~m}$ and ended near the shoreline at position $\mathrm{x}=9.20 \mathrm{~m}$. From there on the profile was visually measured.

In case of the tests with breakwater the PROFO measurement started behind the submerged breakwater at position $\mathrm{x}=5.00 \mathrm{~m}$ and ended near the shoreline at position $\mathrm{x}=9.20 \mathrm{~m}$. From there on the profile was visually measured.

During a test the following profile measurements were performed:

profile measurement no. 0 at $\mathrm{t}=0.0$ hours (before Interval 1 )

profile measurement no. 1 at $\mathrm{t}=0.5$ hours (after Interval 1 )

profile measurement no. 2 at $t=1.5$ hours (after Interval 2)

profile measurement no. 3 at $t=3.5$ hours (after Interval 3 )

profile measurement no. 4 at $t=7.5$ hours (after Interval 4 )

\subsubsection{Calibration of the profile measurement equipment}

A PROFO has to be calibrated every day. It does not have a warm-up period. In order to calibrate the PROFO a special construction has been made. This construction (see Figure 3.1) was placed under water in the wave flume. Next the PROFO was positioned above each 'step of the ladder'. The output signals (Volts) at each of these positions were registered. In this way the relation between the vertical position and output signal could be determined. The vertical position of the calibration construction itself was measured with a gauge. 


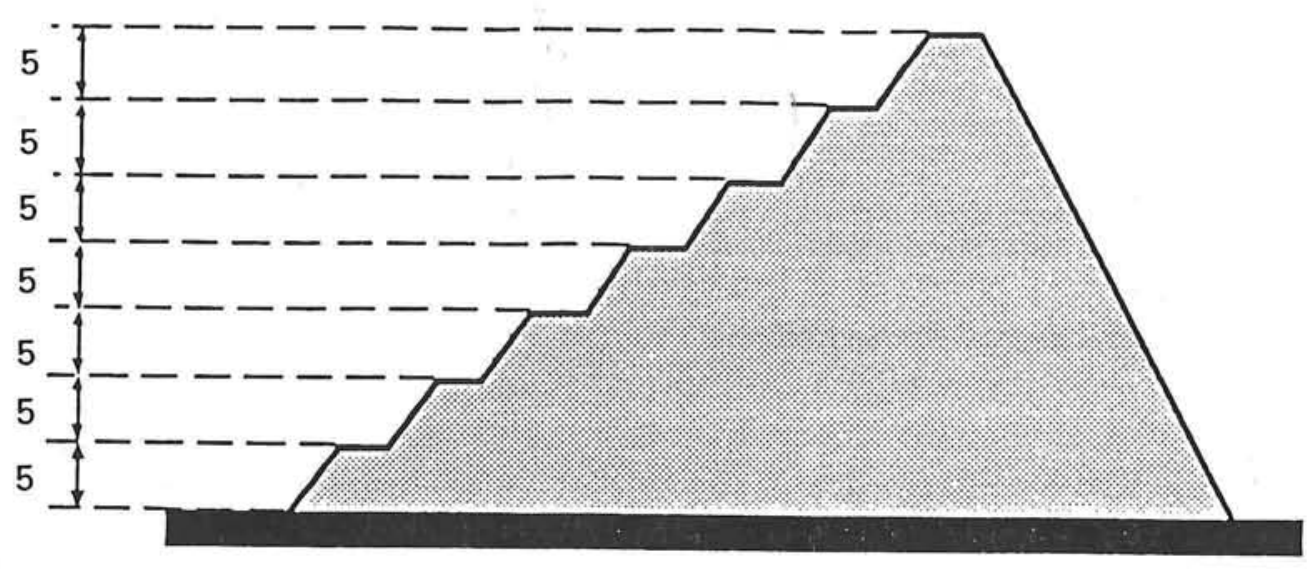

All dimensions are in centimeters

Figure 3.1 Calibration construction of the PROFO.

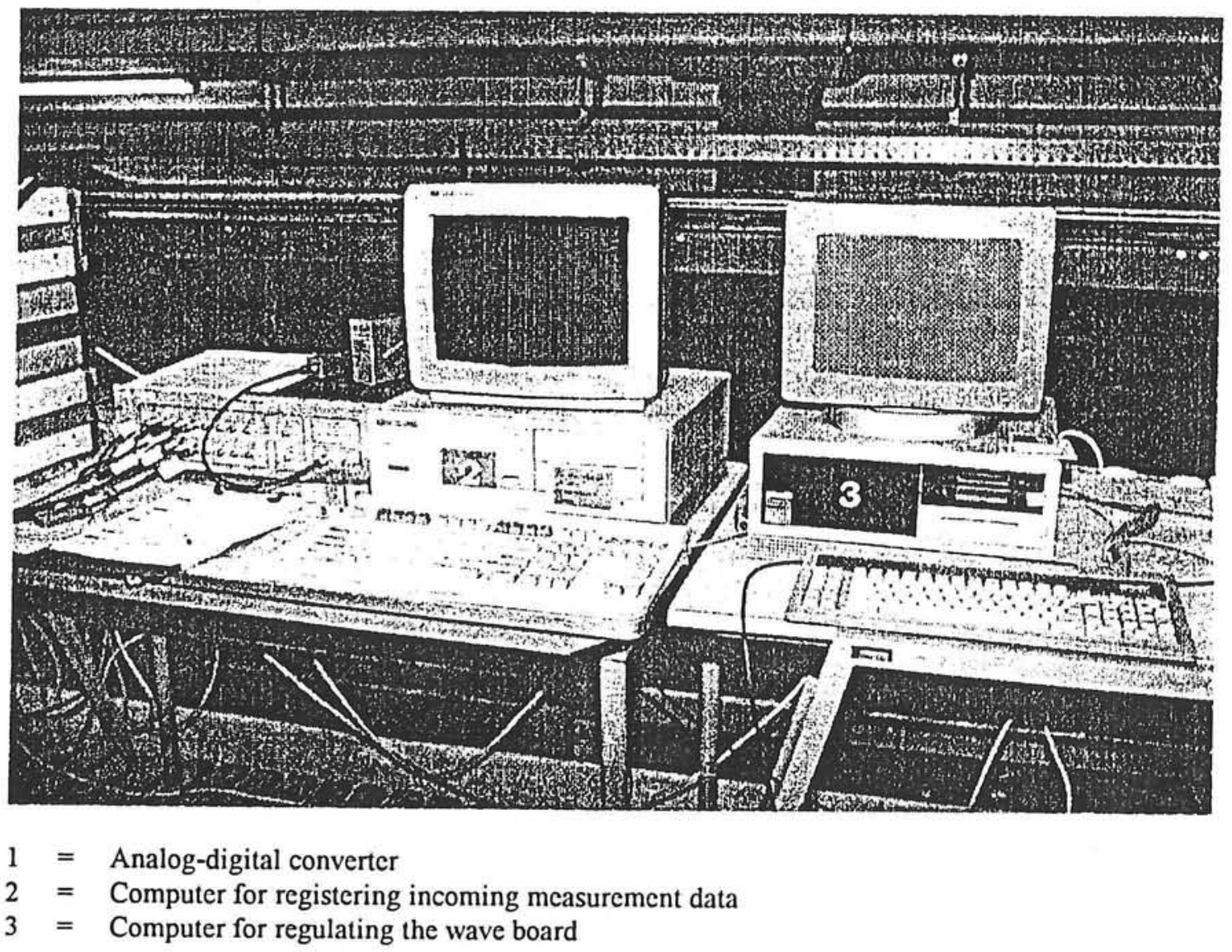

Picture 3.2 Measurement registration and controlling equipment (I). 


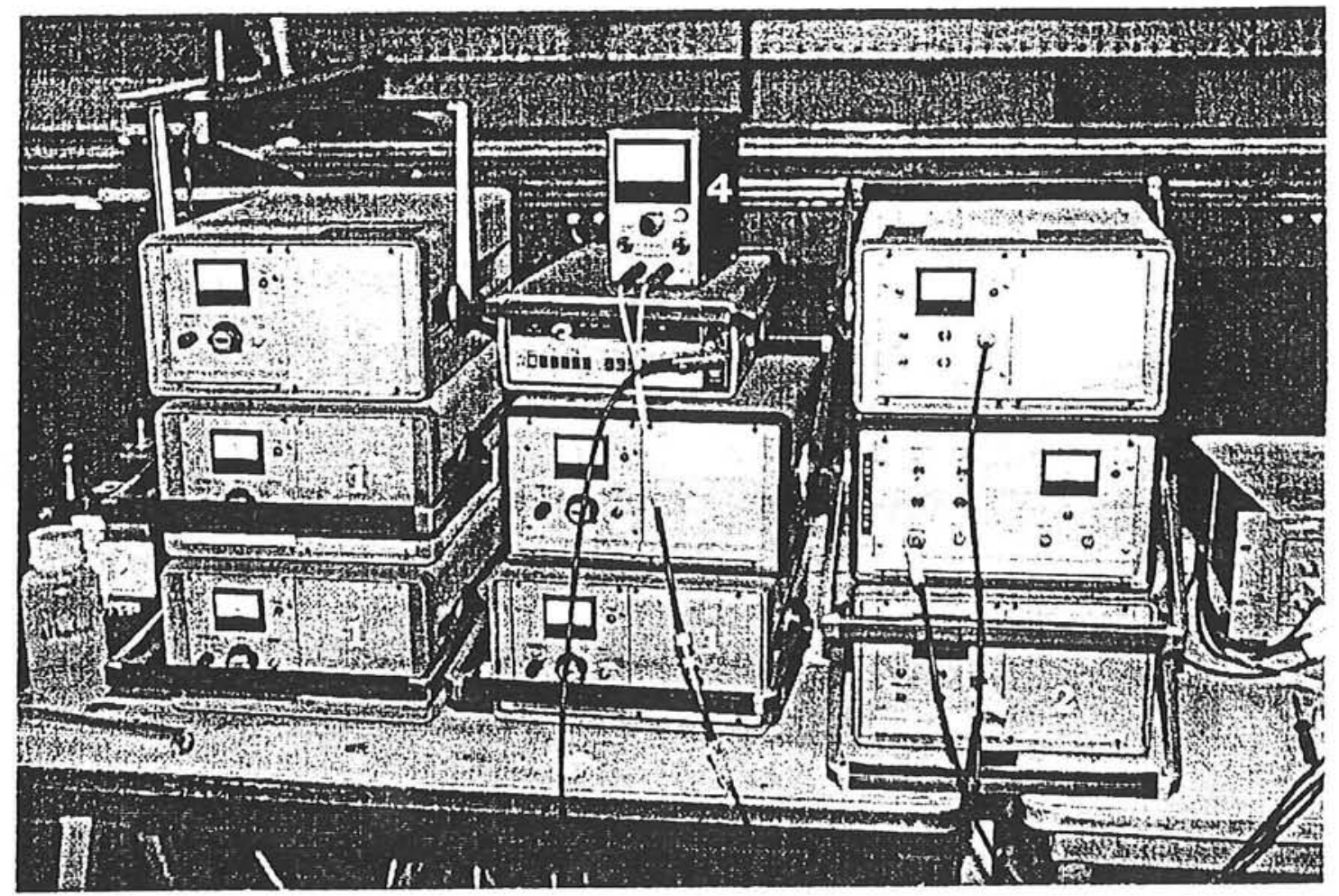

$1=$ Main amplifiers of the wave height meters

$2=$ Amplifiers of the electronic fluid-velocity meters

$3=$ Voltmeter used for the calibration of the electronic profile-indicator and the electronic fluid-velocity meters

$4=$ Amplifier for putting potential difference over trigger circuit

Picture 3.3 Measurement registration and controlling equipment (2).

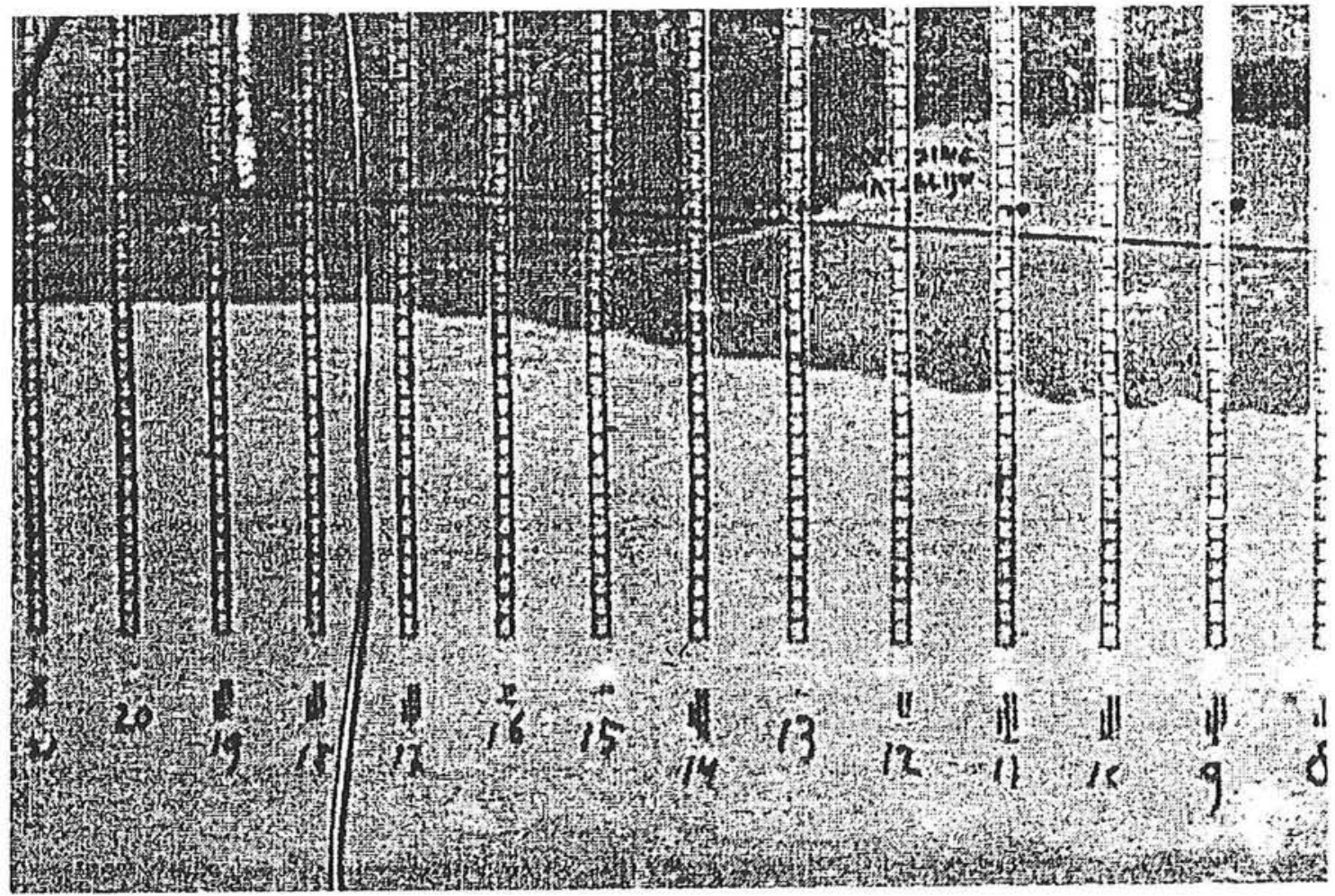

Picture 3.4 Visual bed profile measurement method. 


\subsection{Wave height measurements}

\subsubsection{Wave height measurement technique}

In order to measure wave heights we used wave height meters (WHM) (see Picture 3.5). Every WHM used was supplied by Delft Hydraulics. It is composed of two parts, a gauge with an integral pre-amplifier and a separate main-amplifier. The gauge consists of two parallel stainless steel rods, mounted underneath a small box, containing the pre-amplifier. The rods act as electrodes of an electric resistance meter.

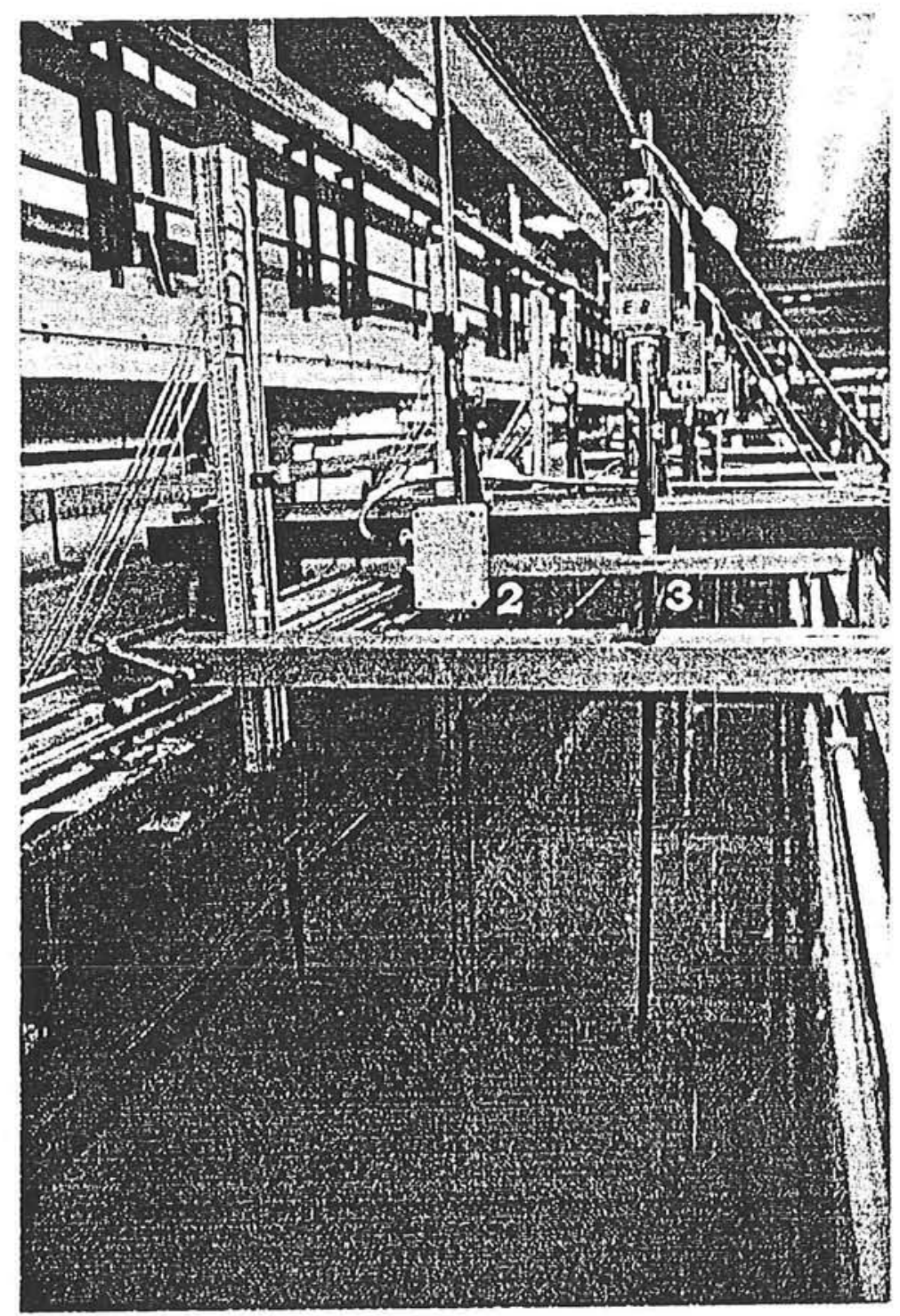

$$
\begin{aligned}
& 1=\text { Sediment concentration meter } \\
& 2=\text { Wave height meter (WHM) } \\
& 3=\text { Electromagnetic fluid-velocity meter (EMS) }
\end{aligned}
$$

Picture 3.5 Measurement equipment. 
The electric resistance measured between the electrodes is inverse proportional to the instantaneous depth of immersion and the specific conductivity of the water. To avoid the effect of conductivity fluctuations, a platinum reference-electrode is mounted between the rods at the lower end of the gauge.

At the main amplifier several ranges can be selected, namely ranges of $5,10,20$ and $50 \mathrm{~cm}$. We used the $50-\mathrm{cm}$ range. Each range has an error of less than $+/-0.5 \%$.

If the gauge has been dry for a long period, it should be cleaned and the electrodes should be placed under water for a few hours before starting the actual measurement. At the beginning of each experiment it is recommended to clean the rods and the reference-electrode of the gauge with a piece of cloth soaked in a solution of alcohol. Furthermore it is recommended to allow the instrument a warm-up period of at least half an hour. After the electronics has warmed up, the instrument must be calibrated. For that purpose the probe is attached to a point-gauge. The depth of the probe is chosen in such a way that during the calibration and the actual measurement the top of the reference electrode is immersed for at least $4 \mathrm{~cm}$. Then the pointer of the indicating meter on the front panel of the main amplifier and also the output voltage must be adjusted to its center-scale position which corresponds with 0.000 Volt output. After these preparations the calibration of the instrument can be carried out, i.e., changing the immersion-depth of the probe by means of the point-gauge and measuring the change in the output voltage. At least five calibration points must be determined. Then the best straight line through these points is calculated. In order to do this we used the program EDFM, which is generally used in the Laboratory of Fluid Mechanics.

When several WHM are used simultaneously and close to each other, they might interfere. However, for distances larger than $20 \mathrm{~cm}$, the interference is negligible.

\subsubsection{Execution of the wave height measurements}

We used five wave height meters in every experiment we performed. The positions of the wave height meters were (see Figure 3.2):

$\begin{array}{lll}\text { Position WHM 1: } & x=-3.00 \mathrm{~m} & \\ \text { Position WHM 2: } & \mathrm{x}=3.50 \mathrm{~m} & \\ \text { Position WHM 3: } & \mathrm{x}=5.25 \mathrm{~m} & \text { (referred to as Vertical (a)) } \\ \text { Position WHM 4: } & \mathrm{x}=6.15 \mathrm{~m} & \text { (referred to as Vertical (b)) } \\ \text { Position WHM 5: } & \mathrm{x}=7.05 \mathrm{~m} & \text { (referred to as Vertical (c)) }\end{array}$

The position of WHM 1 was chosen in order to measure the generated wave height by the wave paddle. The position of WHM 2 was chosen in order to measure the wave height just seaward of the submerged breaker. The positions of WHM 3, WHM 4 and WHM 5 were chosen in order to measure the wave height landward of the breakwater. At the last three positions also velocities and sediment concentrations have been measured. Because these positions play an important role in this study it was decided to give them special names for clearness' sake, viz. Vertical (a), (b) and (c).

It was not possible to measure wave heights even further landward than Vertical (c) because of shallowness. 


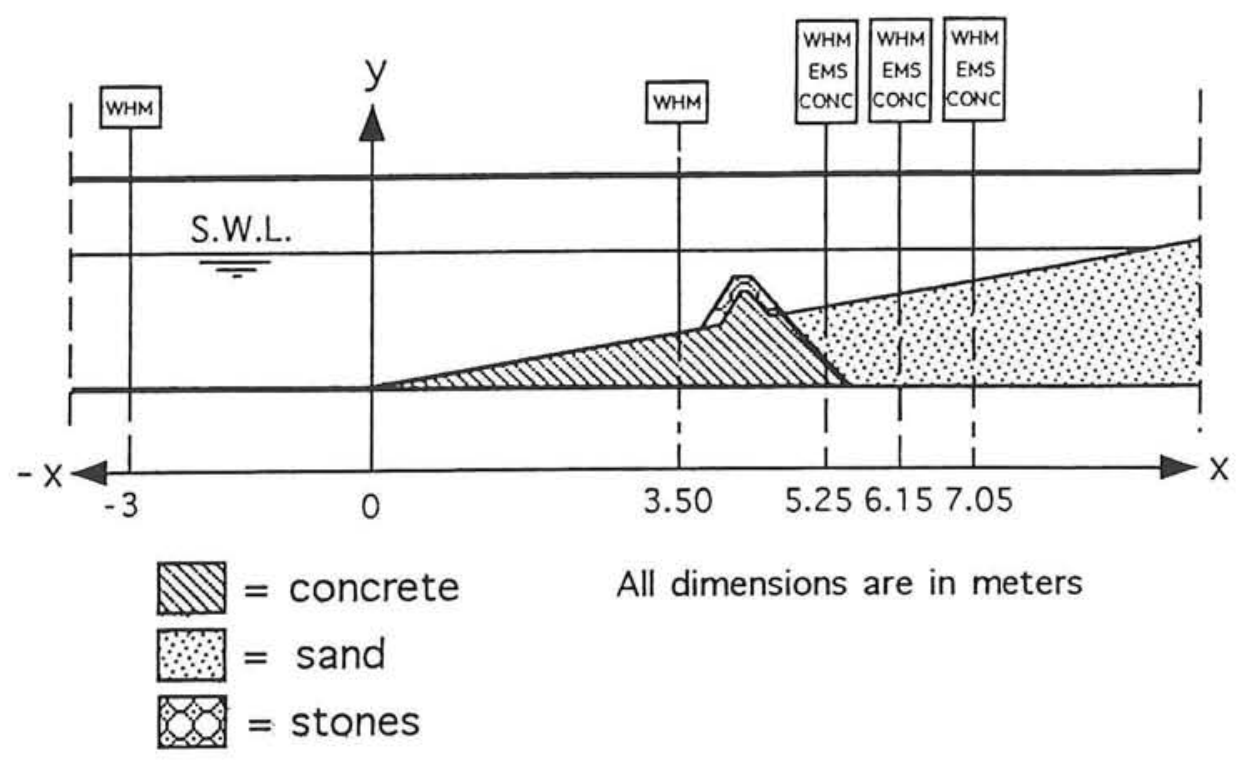

Figure 3.2 Cross-section of the flume and measuring positions.

During an experiment the following wave height measurements were performed:

Interval 1: 1 measurement

Interval 2: 2 measurements

Interval 3: 4 measurements

Interval 4: 6 measurements

The number of measurements might seem rather large especially because they were all performed at the same positions. The advantage of having relatively a lot of measurements is that by comparing the results for each of these measurements a good impression of the vulnerability and reliability of the measurement results (e.g., wave parameters) can be gained. The time-point of starting each of the measurements depended also strongly on the velocity measurements. Due to the technical operation of the measurement registration equipment it is not possible to simultaneously register input signals with different starting and stopping times. Whenever a wave height measurement was started also a velocity measurement was started. The time-points of starting the measurements will be further explained in Section 3.4.

\subsubsection{Calibration of the wave height measurement equipment}

A WHM has to be calibrated every day. The electronics of a WHM has a warm-up period of half an hour, while its gauge has to be in the water for at least a few hours. Calibration of a WHM is performed after this period:

1. The platinum reference-electrode at the lower end of the gauge is positioned at least $4 \mathrm{~cm}$ below the lowest expected water level (zero-position). 
2. The $50-\mathrm{cm}$ range is selected at the main amplifier. Then the pointer of the indicating meter on the front panel of the main amplifier (see Picture 3.3) must be adjusted to its center-scale position which corresponds with 0.000 Volt output.

3. The immersion-depth of the probe is changed to five different depths, for example:

$-60 \mathrm{~mm},-40 \mathrm{~mm}, 0 \mathrm{~mm},+40 \mathrm{~mm}$ and $+60 \mathrm{~mm}$.

The measured values at these depths are written to five different files, for example:

-CWHMU(up)000.LOG
-CWHMU040.LOG
-CWHMU060.LOG
-CWHMD(down)040.LOG
-CWHMD060.LOG.

These files are written to DACON (Digital Analog CONverter). At every depth DACON takes a sample every $1000 \mathrm{~ms}$ for 20 seconds.

4. These five measurements are put in a graph. The best straight line through these points is calculated with the least square method.

This method results in a line with the following equation:

$y=a * x+b$

with:

$\mathrm{x}=$ WHM position in $\mathrm{mm}$

$\mathrm{y}=\mathrm{DACON}$ value for this WHM-position

With the EDFM-program (Elaboration Data File Manually) factors a and b can easily be computed. Notice that EDFM uses real DACON values. These values have to be written in real Volts and centimetres. Therefore the EDFM-equation is written as:

Storevalue $=\frac{a}{20.48} *$ Value $+\frac{b}{204.8}$

In $\mathrm{AUKE} / \mathrm{PC}$ ( $\mathrm{AUKE} / \mathrm{PC}$ is a processing program that computes wave parameters) this equation is used in a different way. Viz.:

Value $=C_{0}+C_{1} *$ Storevalue

These two different equations are related to each other:

$$
\begin{array}{ll}
C_{0}=-\frac{b}{204.8} & (\mathrm{~cm}) \\
C_{1}=\frac{20.48}{a} & (\mathrm{~cm} / \mathrm{V})
\end{array}
$$


5. After the calibration values are known, the calibration of a WHM is complete and measurements can be performed.

\section{$\underline{3.4 \text { Velocity measurements }}$}

\subsubsection{Velocity measurement technique}

In order to measure flow velocities we used electromagnetic fluid-velocity meters (EMS) (see Picture 3.5). The EMS is in fact the inside-out version of the electromagnetic pipe flow meter employing Faraday's Induction Law for measuring the velocity of a conductive fluid moving across a magnetic field. This field is generated by a pulse current through a small coil inside the body of the sensor. Two pairs of diametrically opposed platinum electrodes sense the voltages produced by the flow passing the sensor. These voltages are proportional to the sine and cosine of velocities parallel to the plane of the electrodes. The low level output signals are converted to a high-level output signal by means of an amplifier. The magnitude of the velocity and its direction, with respect to a reference, can be derived by application of common geometry.

The sensor has an ellipsoidal shape $(11 \times 33 \mathrm{~mm})$ and a small sensing area. The sensing area is a cylinder just below the ellipsoidal sensor with diameter $33 \mathrm{~mm}$ and height $5 \mathrm{~mm}$. This makes it possible to measure velocities up to $5 \mathrm{~mm}$ from the bottom.

The range is variable; 0 to $+/-1 \mathrm{~m} / \mathrm{s}$ or 0 to $+/-5 \mathrm{~m} / \mathrm{s}$. The maximum error is $+/-1 \%$ of the selected full scale. These specified accuracies apply to reference conditions after a calibration. Every EMS used was calibrated by Delft Hydraulics. A calibration certificate is supplied with every EMS by Delft Hydraulics. The zero-flow stability is better than one $\mathrm{cm} / \mathrm{s}$ per day.

The probe must be kept as clean as possible which means it must be cleaned regularly with a wet sponge or a soft cloth (never use chemicals). Before starting a measurement it is recommended to immerse the probe for at least half an hour in the medium in which it will be used. If the probe has been dry for a long time, it is advised to place it in water for several days.

If several electromagnetic flow meters are employed in an experiment, they may interfere if they are employed close to each other. However, in our experiments the interference is negligible because the distance between the probes is much more than $15 \mathrm{~cm}$.

Prior to using an EMS, the X (or Y) output is adjusted for 0.000 Volt in still water by means of the potentiometers on the front panel.

Furthermore it is recommended to use an isolation-transformer to reduce the effects of ground loops when data from several velocity meters are logged on a personal computer. 


\subsubsection{Execution of the velocity measurements}

In our experiments we used three velocity meters which were positioned at three different locations in the flume. These positions were (see Figure 3.2):

$\begin{array}{lll}\text { Position EMS 1: } & \mathrm{x}=5.25 \mathrm{~m} & \text { (referred to as Vertical (a)) } \\ \text { Position EMS 2: } & \mathrm{x}=6.15 \mathrm{~m} & \text { (referred to as Vertical (b)) } \\ \text { Position EMS 3: } & \mathrm{x}=7.05 \mathrm{~m} & \text { (referred to as Vertical (c)) }\end{array}$

All three positions are landwards of the breakwater. This was done because this study focuses mainly on that area. It was not possible to measure velocities even further landward of the breaker than Vertical (c) because of shallowness.

One velocity measurement had a duration of fifteen minutes. A minimum number of about 500 waves had to be measured to obtain fair results (see also Section 2.5 ). The following velocity measurements were performed:

Interval 1: one measurement, approximately $2.5 \mathrm{~cm}$ above the bed

Interval 2: two measurements, approximately $2.5 \mathrm{~cm}$ and $5 \mathrm{~cm}$ above the bed

Interval 3: four measurements, from the wave trough down to approximately $2.5 \mathrm{~cm}$ above the bed

Interval 4: six measurements, from the wave trough down to approximately $2.5 \mathrm{~cm}$ above the bed

During Interval 1 only one velocity measurement was performed. This measurement was performed in the middle of the interval.

Two velocity measurements were performed during Interval 2. One at the start of the interval and one at the end of the interval. These measuring times were chosen to be as near as possible to the profile measurements that are performed between all intervals. This way a high accuracy is obtained to the position of the bed and thus of the position of the velocity measurement in the vertical.

During Interval 3 four velocity measurements were performed. To get the best insight of the velocities in one Vertical these measurements were performed continuously after each other. Halfway during the interval we started the first measurement close to the wave trough level. Immediately after this measurement the next measurement was performed a few centimeters lower in the same Vertical. (The number of centimeters we lowered the measuring position depended on the wave spectrum and the developed bed height. Our goal was to measure as much as possible of one Vertical. Therefore our first measurement was started as high as possible in the Vertical and in three equal steps we lowered the measuring position.) After the second measurement a third measurement was performed a few centimeters lower in the Vertical. Finally a fourth measurement was performed at the end of the interval. This measurement was performed approximately $2.5 \mathrm{~cm}$ above the bed.

In Interval 4 we performed six velocity measurements. The same strategy as in Interval 3 was applied during Interval 4, only this time with six measuring positions. Again the measurements were started near the wave trough level and ended at approximately $2.5 \mathrm{~cm}$ above the bed.

The velocity measurements are all performed simultaneously to the wave height measurements. In other words, whenever a velocity measurement was started also a wave height measurement was started. 


\subsubsection{Calibration of the velocity measurement equipment}

An EMS also has to be calibrated every day and also has a warm-up period of at least half an hour. If the probe has been dry for a long time, it is advised to place it in the water several days before using it. After the warm-up period the EMS is calibrated. Unlike a WHM, every EMS has standard calibration formulas, supplied by Delft Hydraulics. This means that no calibration factors have to be computed.

An example of such a calibration formula is:

E-probe EMS, serial no.127, used at Vertical (a):

X-channel: $|v|=-4.8729 * 10^{-4} * u^{2}+1.0371 * 10^{-1} *|u|+5.9365 * 10^{-3} \quad \mathrm{~m} / \mathrm{s}$

This formula is used in AUKE/PC.

Experiments, however, show that there is a shifting of the zero setting proportional to time and this is on an average 0.005 Volt per hour. Therefore it is recommended to measure the average output in the stagnant water before and after the actual measurement. With these zero measurements and their points in time, the actual measurements can easily be compensated for the shifting of the zero setting if the point of time of the measurement is known. During the experiments the output of an EMS was checked and if necesarry adjusted to 0.000 Volt between every Interval. Therefore, hardly any shifting of the zero setting was measured. Only in Interval 4, which is the longest interval (4 hours), some shifting was measured during some tests.

\subsection{Sediment concentration measurements}

\subsubsection{Sediment concentration measurement technique}

In order to measure concentrations in the flume the transverse suction method with intake tubes was used (see Picture 3.5 and 3.6). One concentration meter consists of eight intake tubes. The distance between two intake tubes (center to center) is exactly $2.5 \mathrm{~cm}$. To obtain fair results, suction tubes with an inner diameter of $3 \mathrm{~mm}$ were used and a suction velocity of approximately $0.78 \mathrm{~m} / \mathrm{s}$, which is more than three times the maximum flow velocity. However, the samples still differ by a constant factor from the actual concentrations. This factor is to some extent dependent on the particle diameter. In these experiments the ratio for $\mathrm{c}_{\text {measured }} / \mathrm{c}_{\text {real }}$ is 0.80 . In case of concentration measurements under wave conditions the tube has to be located perpendicular to the direction of wave propagation.

After the samples have been obtained, the concentration has to be determined. First, the weight of the sediment-water mixture is determined with a balance (see Picture 3.7). Then, the surplus of water is poured off. After this, the sand is weighed with a so called 'underwater balance' (see Picture 3.8). The wet sand is poured down through a funnel into a reservoir that is attached to the balance. This balance weighs the amount of sand under water. The sand remains in this reservoir. This pouring process must be done very slowly and carefully otherwise the sand will not settle and flow over the edge of the reservoir. The water level in the bucket, in which the balance is placed, remains constant because of an overflow. The total weight of the sand is measured with an accuracy of 0.1 grams. 


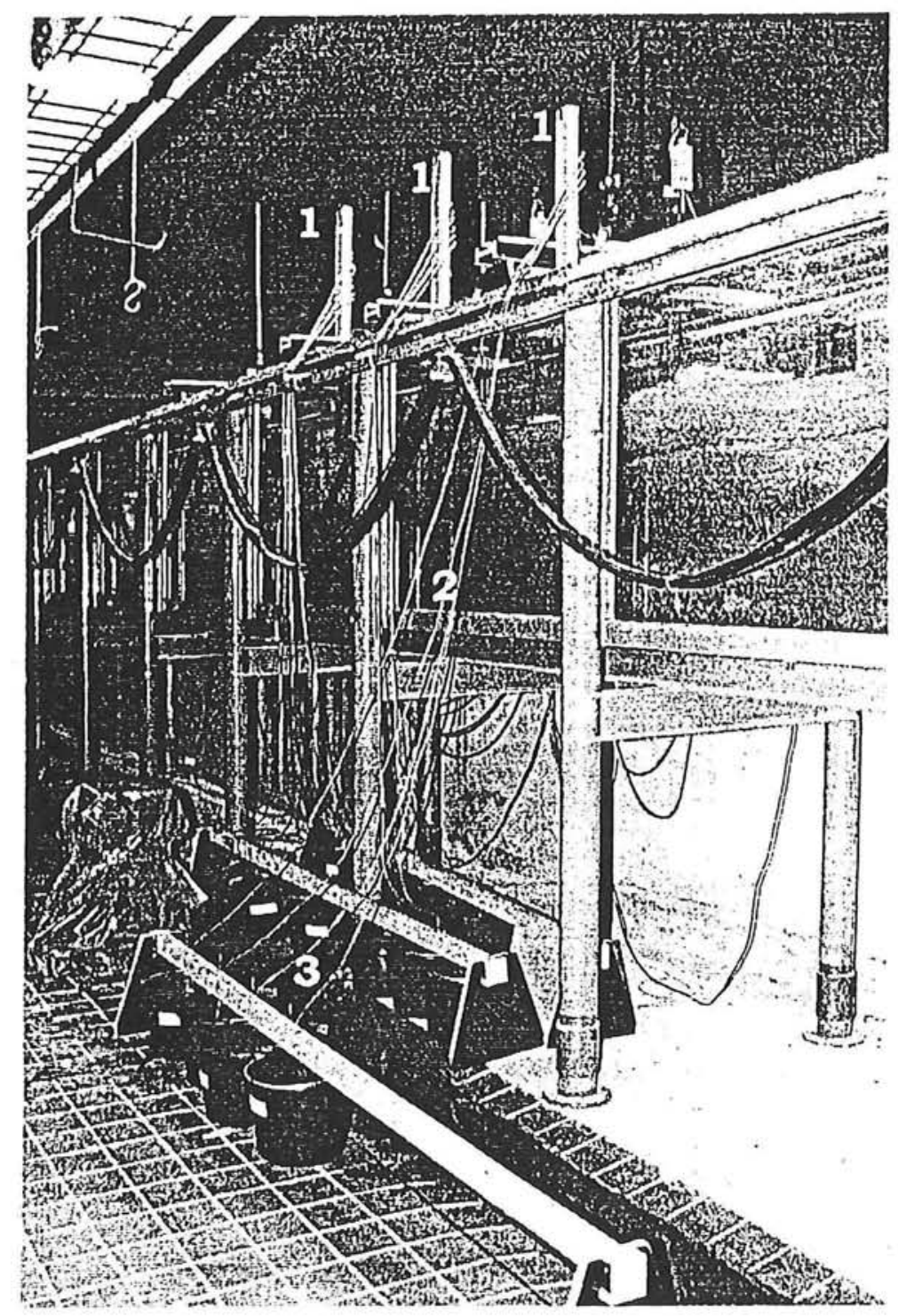

$1=$ Sediment concentration meters
$2=$ Tubcs
$3=$ Buckets for collecting samples

Picture 3.6 Sediment concentration measurement equipment.

\subsubsection{Execution of the sediment concentration measurements}

During every experiment two concentration measurements were performed. One at the start of the experiment and one at the end of an experiment. The first one was performed in order to compare the difference in concentrations generated by the different wave conditions while the bed profile still was in tact. The second one was performed in order to compare the difference in concentrations generated by the different wave conditions while the bed had undergone maximum changes.

The intake tubes were placed at the following positions (see Figure 3.2): 
Position conc. 1:

Position conc. 2:

Position conc. 3: $\mathrm{x}=5.25 \mathrm{~m}$

$\mathrm{x}=6.15 \mathrm{~m}$

$\mathrm{x}=7.05 \mathrm{~m}$ (referred to as Vertical (a))

(referred to as Vertical (b))

(referred to as Vertical (c))

Because the water depth was limited at Vertical (b) and (c), it was not possible to measure concentrations in all eight vertically placed tubes. Therefore at Vertical (b) only six tubes and at Vertical (c) only four tubes were measured.

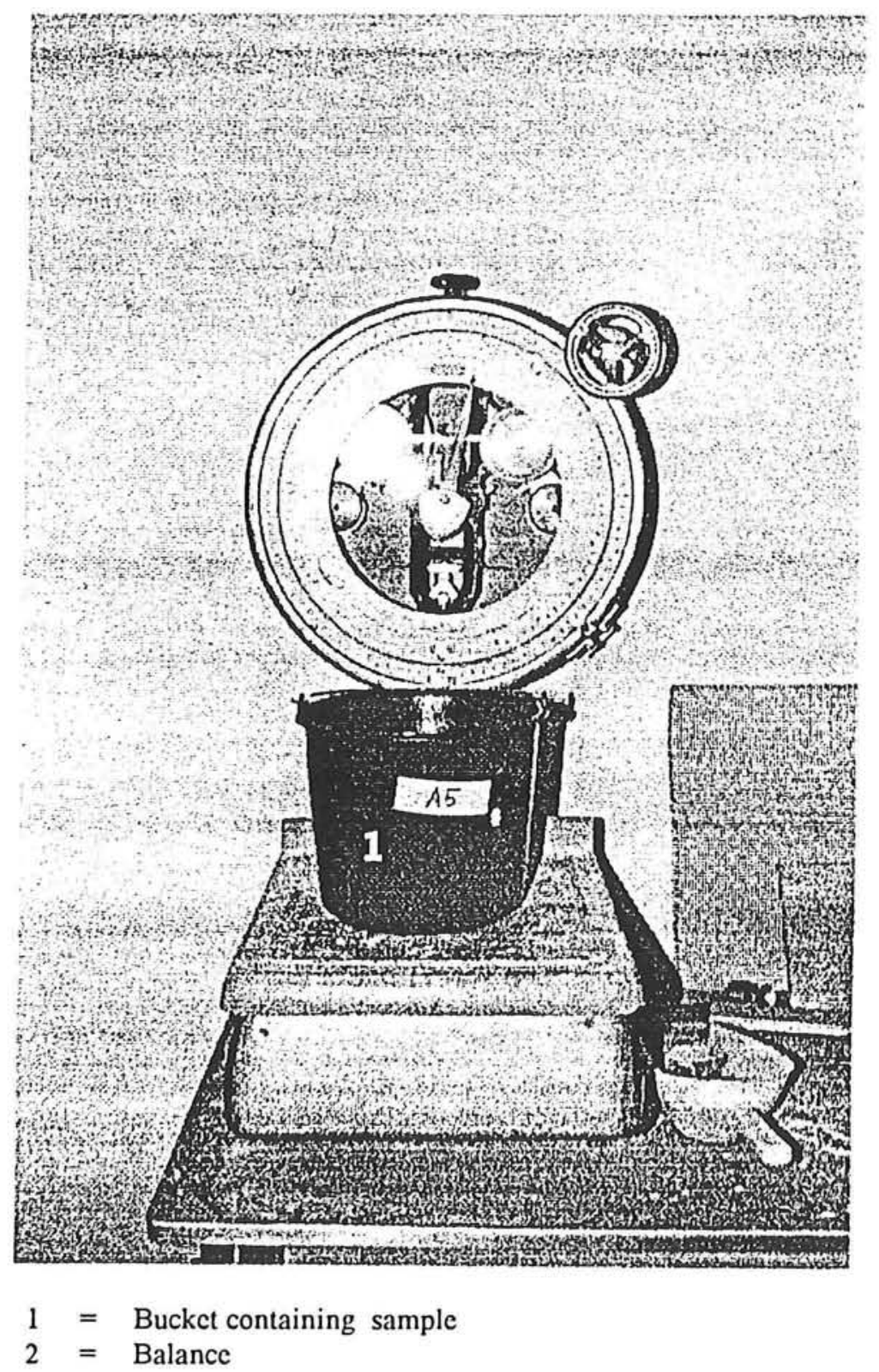

Picture 3.7 Balance for weighing sediment-water mixture. 


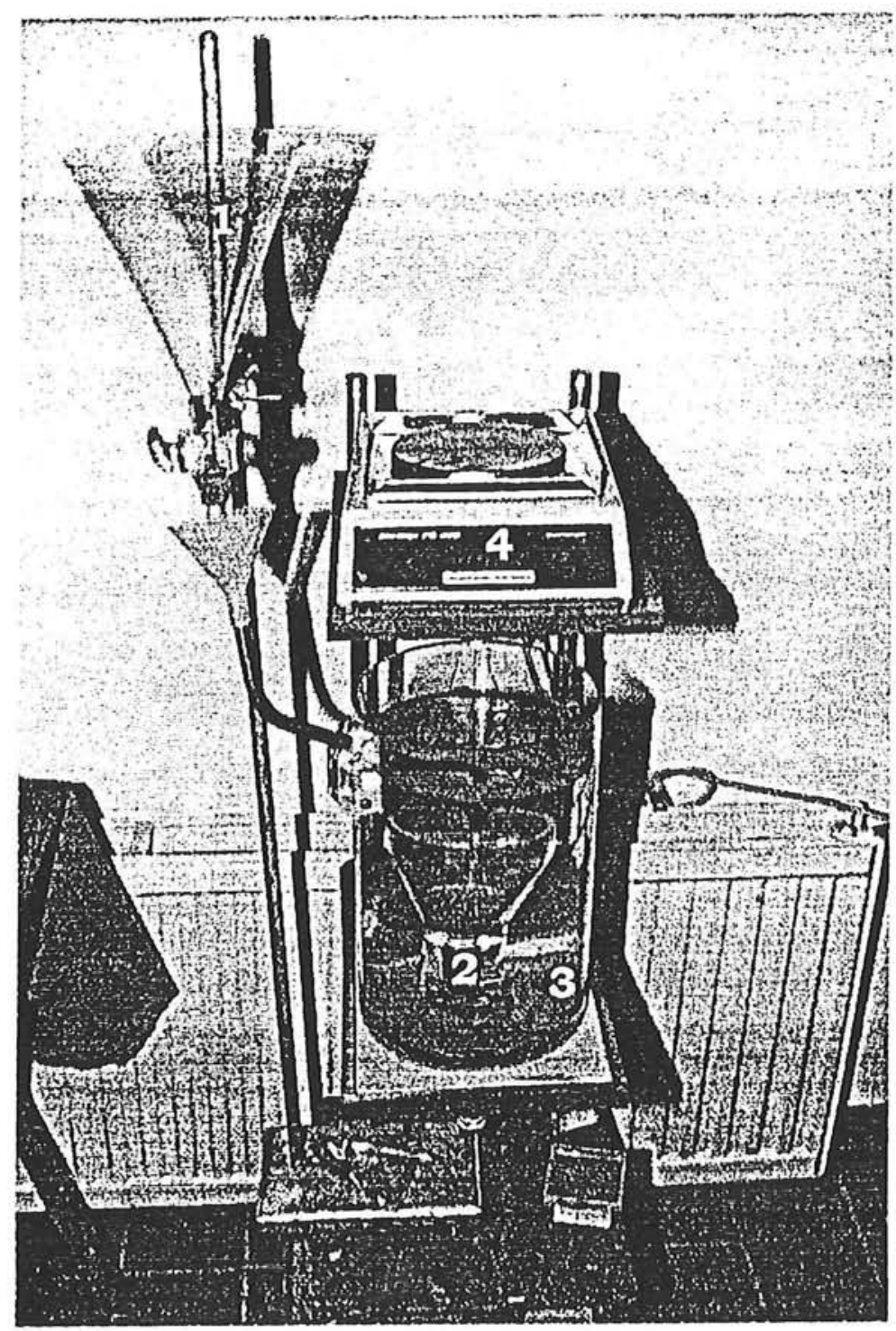

$1=$ Funnel for pouring in water-sediment mixture

$2=$ Inner reservoir for settling of the sediment

$3=$ Outer reservoir with constant level of water height

$4=$ Balance

\section{Picture $3.8 \quad$ Under water balance method.}

\subsubsection{Calibration of the sediment concentration measurement equipment}

The relation between the real time-averaged sediment concentration and the average sediment concencentration in the buckets has already been investigated. This has already been discussed in Section 3.5.1. No further calibration of the sediment concentration measurement equipment was necessary. 


\subsection{Summary of the activities during an experiment}

In this section the activities during one test are described.

1. Before a test is started the slope is adjusted to a 1 in 15 slope with great care. This is done with the help of two steel rulers and a wooden board. The steel rulers are hung up at the side walls of the wave flume. The wooden board is manufactured in such a way that if it is shoved over the bed while pressing down on the board, an almost perfect 1 in 15 slope is obtained. duration: 1.5 hours

2. The wave flume is carefully filled up to $65.0 \mathrm{~cm}$ precisely with water from the water system in the laboratory. This can not be done too fast, otherwise the sand goes in suspension and the slope is disturbed. To check the water level accurately the water must be stagnant. This takes some time. The last few millimetres are filled with a hose to be in better control of the water supply.

duration: 20 minutes

3. All electronic apparatus (like EMS and WHM) are cleaned and then turned on. They have a warm-up period of half an hour. This can not be done earlier because their probes have to be in the water (while adjusting the bed the flume was dry and the probes were positioned elsewhere). During this warm-up period the computers are put on in order to generate a signal for the wave board. The correct wave-spectrum is selected. Directories are created to write the incoming signals from the EMS, WHM and PROFO.

duration: 30 minutes

4. The PROFO is calibrated. The calibration procedure is described in Section 3.2.3. duration: 10 minutes

5. The wave machine is turned on. For about one minute the wave board generates waves in the flume. This is done to create a better slope with some ripples and without air or other irregularities in the bed. After these few waves a profile measurement (no.0) is performed. A profile measurement consists of an electronic measurement part ( $=$ PROFO) and of a visual measurement part.

duration: 20 minutes

6. All EMS are placed in their first measuring position. For Interval 1 this is approximately $2.5 \mathrm{~cm}$ above the bed. The WHM and EMS are calibrated. How these calibrations are done is explained in sections 3.3.3 and 3.4.3. The concentration meters are placed in their measuring position which also is approximately $2.5 \mathrm{~cm}$ above the bed.

duration: 30 minutes

7. After this Interval 1 is started (this is a 30 minute period of waves). Immediately after the start of Interval 1 the buckets are positioned under the matching outlet of an intake tube. All intake tubes are checked on functioning and if necesarry they are sucked again. After finishing this check the concentration measurements are started. At the same time the wave height and velocity measurements are started. Approximately fifteen minutes later all these measurements are finished. The intake tubes, WHM and EMS are removed from the wave flume (the EMS and WHM are positioned in the wave flume at an other position, but in the water).

duration: 35 minutes 
8. After Interval 1 has finished and all the sediment in the flume is settled a profile measurement (no.1) can be performed. After this measurement the WHM and EMS are replaced to their original position. Again the wave flume is carefully filled up to $65.0 \mathrm{~cm}$ precisely (the waterlevel is approximately $3 \mathrm{~mm}$ lower than at the start of the test due to the concentration measurement). Finally, the EMS are adjusted for 0.000 Volt output.

duration 25 minutes

9. Interval 2 is started (this is an one hour period of waves). Immediately after Interval 2 is started, the first WHM and EMS measurements of this interval are started also. After these measurements are finished, the EMS probes are positioned $2.5 \mathrm{~cm}$ higher above the bed. This is done in order to measure velocities at two different heights in one vertical. About twenty minutes before Interval 2 finishes, the second WHM and EMS measurements are started. After these measurements are finished the WHM and EMS are removed from the wave flume.

duration: 1 hour

10. After Interval 2 has finished and all sediment is settled a profile measurement (no.2) can be performed. After this profile measurement is finished the WHM and EMS are replaced to their original position. The EMS are adjusted for 0.000 Volt output.

duration 15 minutes

11. Interval 3 is started (this is a two hour period of waves). In this interval four EMS and WHM measurements are performed, all in the last hour and a quarter of the Interval. After the start of this Interval the sediment in some of the buckets (concentration measurements from Interval 1) is weighed (duration 1.5 hours). The remainder of the buckets with sediment have to wait until Interval 4. After the WHM and EMS measurements are finished their probes are removed from the wave flume.

duration: 2 hours

12. After Interval 3 has finished and all sediment is settled a profile measurement (no.3) can be performed. After this profile measurement is finished the WHM and EMS are replaced to their original position. The EMS are adjusted for 0.000 Volt output.

duration: 15 minutes

13. Interval 4 is started (this is a four hour period of waves). The sediment that still remains in the buckets is weighed and the buckets are cleaned afterwards for the concentration measurement at the end of this interval. Furthermore in this Interval six WHM and EMS measurements are performed, all during the last two hours of the Interval. In the last twenty minutes there is a concentration measurement. In order to perform a concentration measurements the buckets are positioned under the matching outlet of an intake tube. All intake tubes are checked and if necesarry they are sucked again. After finishing this check the concentration measurements are started. At the same time the last wave height and velocity measurement are started also. Approximately fifteen minutes later all these measurements are finished. The intake tubes, WHM and EMS are removed from the wave flume (the EMS and WHM are positioned in the wave flume at an other position, but in the water).

duration: 4 hours 
14. After Interval 4 has finished and all sediment is settled the last profile measurement (no.4) can be performed. Finally the sediment in all the buckets have to be weighed (duration 1.5 hours) and the buckets are cleaned afterwards for the next test.

duration: 2 hours

One test is completed.

If a test is performed in one day the duration is approximately 14 hours. This means that one test can not be performed in one day, an additional day is needed to perform one test. On this additional day of a test, some activities have to be repeated before a test can continue. These activities are:

- calibration of WHM, EMS and PROFO

- checking of the waterlevel (some water has been vaporized during the night)

These activities take an extra hour.

The total duration of one test is then about 15 hours. Usually this came down to one test per two days. 


\section{CHAPTER 4 PROFILE MEASUREMENT RESULTS}

\subsection{Introduction}

In the previous chapter it is discussed, among other things, how the profile measurements were performed. In this chapter the results of these profile measurements are discussed.

Section 4.2 discusses some difficulties that had to be solved in order to convert the profile measurements. Section 4.2.1 describes the translation of measured Volt values to bed heights. An example of a conversion formula is given. During an experiment, different amounts of sand are found in the profile measurements. Section 4.2.2 explains this problem. The next section describes the irregularities that can be noticed at the transition point in almost every profile measurement graph (see Volume 2: Graphics, Part 1, Graph 1.1 to 1.26). In Section 4.2 .4 it is described which reference position is defined for sediment volume transport graphs (see Volume 2: Graphics, Part 1, Graph 1.26 to 1.86). Section 4.2 .5 describes the profile measurements just landwards of the submerged breakwater.

In Section 4.3 the final results of the profile measurements are discussed. Section 4.3.1 compares all tests with and without a submerged breakwater present (see also Volume 2: Graphics, Part 1, Graph 1.1 to 1.18 and 1.27 to 1.62). In the next section comparisons are made between tests with an almost equal significant wave height (see also Volume 2: Graphics, Part 1, Graph 1.19 to 1.22 and 1.63 to 1.74). The last section of this chapter describes the results of tests performed with an almost equal wave steepness (see also Volume 2: Graphics, Part 1, Graph 1.23 to 1.26 and 1.75 to 1.86 ).

\subsection{Conversion of the profile measurements}

\subsubsection{Volts converted to bed heights.}

The electronic profile-follower (= PROFO) measures the bed height in Volts. This signal is registered by a computer. Afterwards, these measured Volt values have to be translated to bed heights (y-value $[\mathrm{m}])$ and to a corresponding position in the flume (x-value $[\mathrm{m}])$. Therefore, two problems have to be solved. Viz:

1. Relation between measured Volt values and bed heights.

2. Relation between measured Volt values and distances in the flume.

\section{ad.1 Relation between measured Volt values and bed heights}

This relation is derived from the calibration of the PROFO. In this calibration a few points in the flume are measured. For example:

- the bottom

- a construction of standard size (see Figure 3.1)

At all these points the real height is known and the corresponding Volts can be measured. Now, a relation can be derived between Volts and heights. An example will be given: 
The following positions have been measured during a certain calibration:

\begin{tabular}{|c|c|c|}
\hline \multicolumn{3}{|c|}{ - the bottom of the flume: } \\
\hline & $0.0 \mathrm{~cm}$ & 0.244 Volt \\
\hline \multicolumn{3}{|c|}{ - seven positions of the construction: } \\
\hline nr. 1 & $20.0 \mathrm{~cm}$ & $1.184 \mathrm{Vo}$ \\
\hline nr. 2 & $25.0 \mathrm{~cm}$ & 1.416 \\
\hline $\mathrm{nr} .3$ & $30.0 \mathrm{~cm}$ & $1.653 \mathrm{Vo}$ \\
\hline $\mathrm{nr} .4$ & $35.0 \mathrm{~cm}$ & 1. \\
\hline nr. 5 & $40.0 \mathrm{~cm}$ & \\
\hline $\mathrm{nr} .6$ & $45.0 \mathrm{~cm}$ & $2.361 \mathrm{Vol}$ \\
\hline nr.7 & $50.0 \mathrm{~cm}$ & $2.604 \mathrm{Vo}$ \\
\hline
\end{tabular}

With these calibration positions a formula can be derived between height in the flume and the measured Volt values. In this case:

$$
0.00 \mathrm{~cm}=0.244 \text { Volt and } \quad \Delta 5 \mathrm{~cm}=0.236 \text { Volt } \quad \text { (4.1) and (4.2) }
$$

\section{ad.2 Relation between measured Volt values and distances in the flume.}

In order to measure the entire bed the PROFO has to be moved along the bed. In order to do this the PROFO is attached to a trolley and this trolley is moved along the flume in a horizontal direction. This is done at a constant speed of about $0.061 \mathrm{~m} / \mathrm{s}$. The PROFO takes a sample every $50 \mathrm{~ms}$. By multiplying these two numbers the sampling distance is obtained. In this case the sampling distance is $0.003 \mathrm{~m}$.

In case of no breakwater present, the entire bed covered by the PROFO was $9.200 \mathrm{~m}$ (the rest of the bed was measured visually). So, it should take the trolley $150 \mathrm{~s}$ to ride across the bed. If this was true, then a relation between a measured Volt value and its position in the flume could easily be obtained. In reality, however, the trolley took between 147 and $153 \mathrm{~s}$ to ride across the bed. This was due to irregularities of the rails. Therefore, a PROFO measurement contained between 2940 and 3060 samples. A solution had to be found to compare these different measurements. These measurements all covered the entire bed. Electronic profile measurements are started and ended by an electric trigger. This trigger is an electric device that sends a signal to the computer when the trolley passes by. These triggers were positioned at the beginning and at the end of the bed. Therefore sample 1 was taken at $x=0.000 \mathrm{~m}$ and the last sample was taken at $x=9.200 \mathrm{~m}$ during every measurement. The exact location of the intermediate samples was not known. It is not possible to compare profile measurements with a different number of samples. Therefore all profile measurements were resampled to 1840 samples (in case of no breakwater present). This resampling is done with the program RESAMP.CPP (see Appendix B for a listing of this program). This program resamples a certain file with a various number of samples (between 2800 and 3200) to a file with a constant (1840) number of samples. These resampled bedprofile files are now ready for further computations.

In case of a test with a breakwater present, the length of the bed measured with a PROFO is much shorter (total length $=4.200 \mathrm{~m}$ ). But in this case still a different number of samples was measured. These measurements are resampled to 840 samples (the same resampling program is used, but in this case adapted to resample to 840 samples instead of 1840 samples).

In both cases the input files are resampled to a sampling distance of $0.005 \mathrm{~m}$. 


\subsubsection{Equal amounts of sand}

Before a test starts the bed is adjusted to the original slope of 1 in 15 very carefully. This is done with help of some specially made steel lineals and a wooden board. Every test starts with approximately an equal amount of sand in the flume. During a test some profile measurements are performed. For each profile measurement the total amount of sand is calculated afterwards. This is done with the following formula (see Figure 4.1):

$$
\sum_{n=0}^{n=N}\left[\frac{\{P(n+1)+P(n)\}}{2} *\{(n+1)-n\}\right]=\text { total amount of sand }
$$

with:

$N=1840$

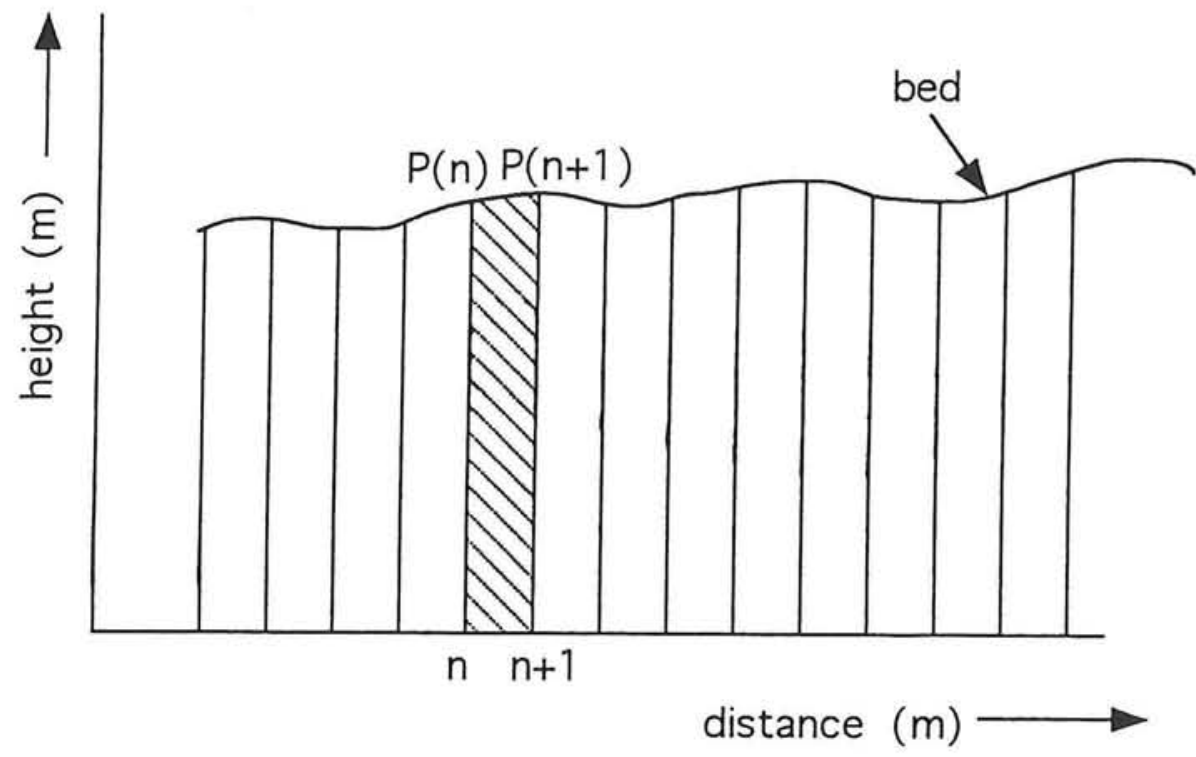

Figure 4.1 Total amount of sand.

The total amount of sand should be equal for every profile measurement during a test. However, this is not the case in many tests. In many tests, small amounts of sand are lost or small amounts of sand are in surplus. Some explanations for this result are:

1. different packing of sand

2. concentration measurements

3. sand is transported seawards of the test area

4. inaccuracies of the measuring methods 


\section{ad.1 different packing of sand}

During an entire test the bed is exposed to well over 10000 waves. A lot of sand particles are stirred up and going into suspension. These particles are transported and settle elsewhere. There is a good possibility that the pore volume between the sand particles will change during this process. In other words, the packing of sand can change during a test. If the packing of sand changes during a test, different amounts of sand will be found for every profile measurement during that test. If, for example, the sand is packed more loosely after half an hour of wave action. (more pore volume in between the sand particles) the bed will raise to a higher level, and it will appear as if there is more sand in the flume. The other way around is also possible. In that case the sand is packed more tightly after a few hours of wave action and it will appear that there is less sand present in the flume.

\section{ad.2 sediment concentration measurements}

During every test two concentration measurements are performed. This means that a small amount of sand is sucked out of the wave flume two times during every test. This amount varied from about 10 gram for experiment $\mathrm{E}$ (smallest waves) up to 350 gram for experiment $\mathrm{D}$ (highest waves). This effect is so little that it can be neglected in further computations.

\section{ad. 3 sand is transported seawards of the test area}

A third possibility is that some amount of sand is transported out of the test area. Two different cases can be distinguished:

CASE 1: no breakwater present (see Figure 4.2)

The profile measurements started at the beginning of the slope and ended landwards of the maximum wave run-up. This means sand can only 'disappear' if it is transported landwards or seawards of these borders (see Figure 4.2). The landward border $(x=12.00 \mathrm{~m})$ was chosen in such a way, that no sand transport took place near this border. The seaward border was located at the beginning of the slope $(x=0.00 \mathrm{~m})$. In all the tests there was no transport through this border, as is shown in the profile graphs (see Volume 2: Graphics, Part 1, Graph 1.1 to 1.6). In these graphs it is clear that transported sand never came near this seawards testing border. Although no sand was transported through this border, there was some transport of very light material (very small particle diameter) through this border. The amount of this transported material was not measured, because it was too less (approximately a few hundred grams maximum). 


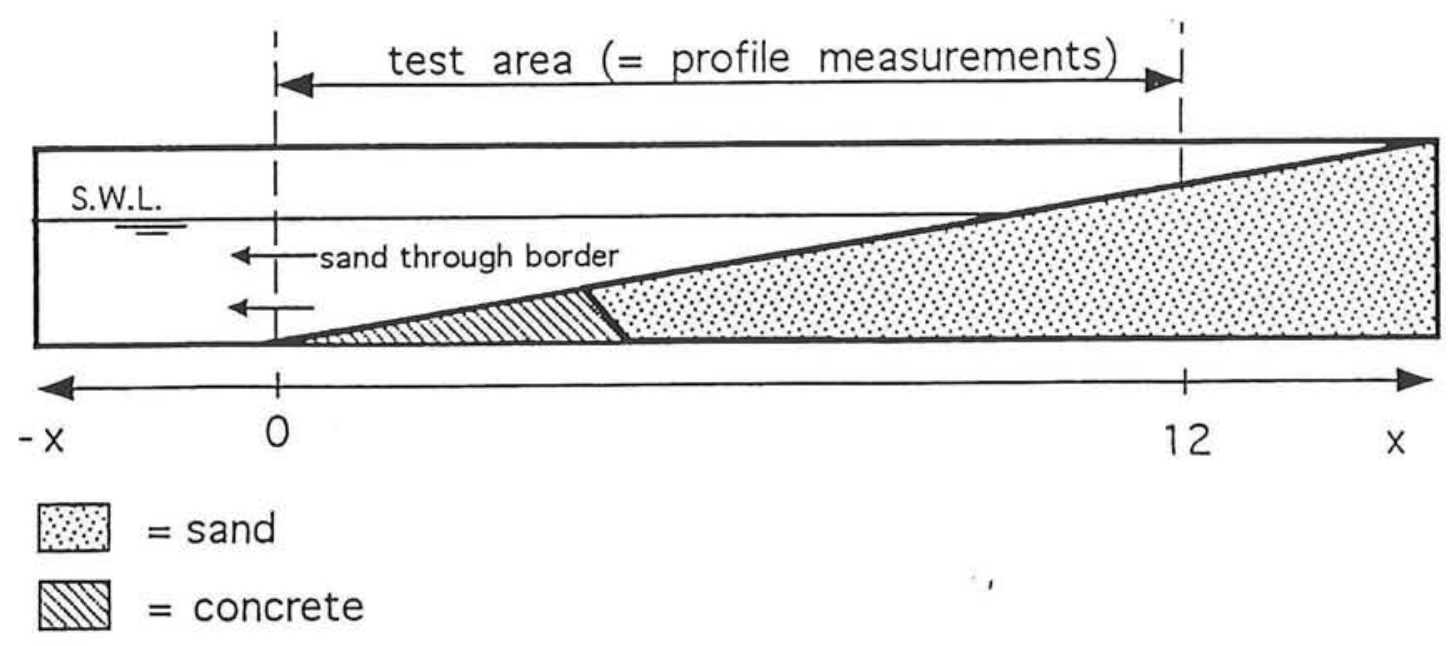

\section{Figure $4.2 \quad$ Test area without breakwater present.}

CASE 2: with breakwater present (see Figure 4.3)

In a test with breakwater the profile measurements did not start at the beginning of the slope, but landwards of the submerged breakwater (see Figure 4.3). This was done because of the following reason. During the first three intervals there was no transport over the breakwater (only during Interval 4 there was some sand transport over/through the breakwater (see Table 4.1)). If there is no transport over the breakwater, there are no profile changes that can be measured. Therefore, we decided to weigh the sand that settled on the slope seawards of the breakwater at the end of every test. In Table 4.1 the amounts of sand seawards of the breakwater are given. The elevation of the bed seawards of the breakerwater can easily be calculated. First, the amount of sand $[\mathrm{kg}]$ is divided by its mass density $\left[\mathrm{kg} / \mathrm{m}^{3}\right]$. Secondly, the pore volume is accounted for. Thirdly, the outcome $\left[\mathrm{m}^{3}\right]$ is divided by the total area seawards of the breakwater $\left[\mathrm{m}^{2}\right]$. Then the average elevation of this area (bed) is found.

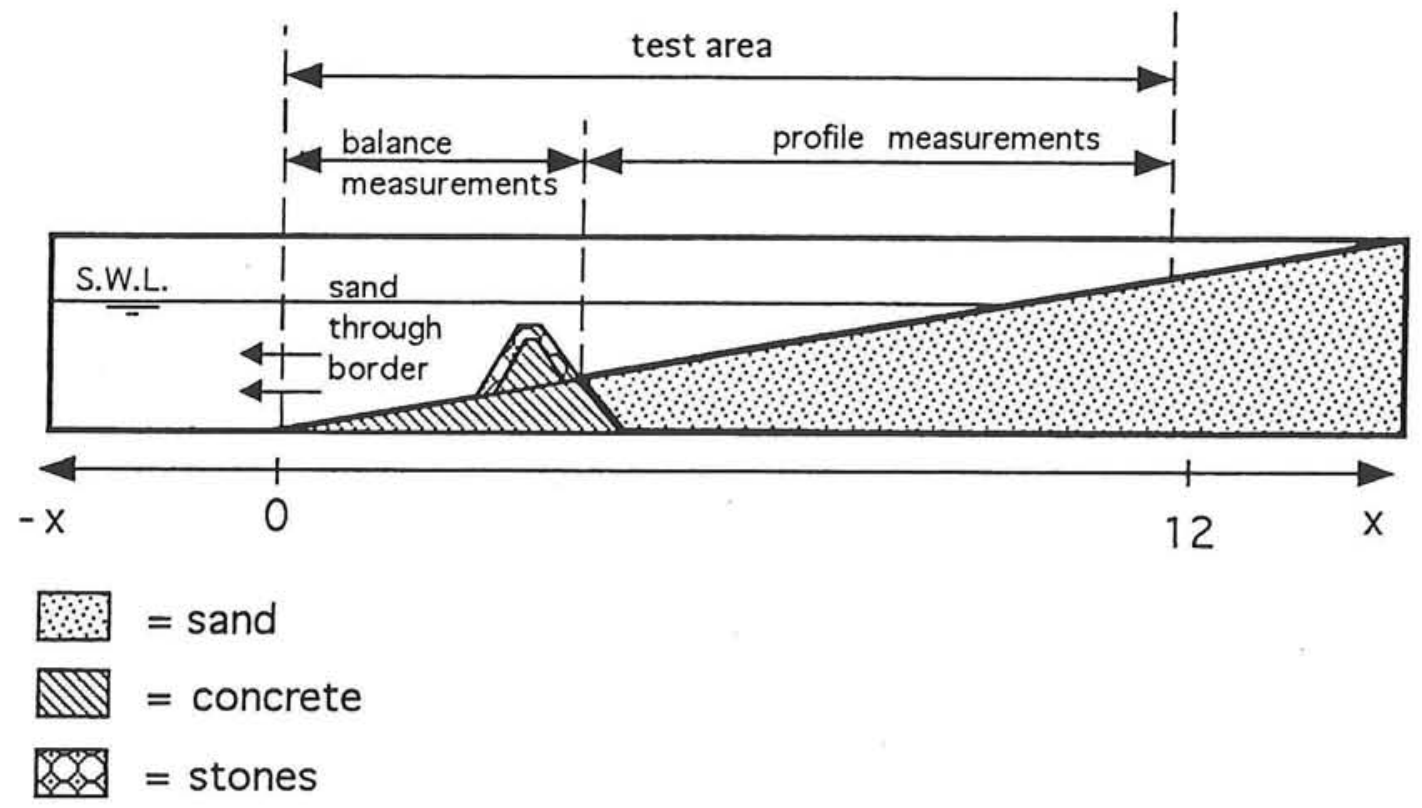

Figure 4.3 Test area with breakwater present. 


\begin{tabular}{|c|c|c|c|c|c|}
\hline $\begin{array}{c}\text { Test- } \\
\text { case }\end{array}$ & $\begin{array}{c}\text { Sand } \\
\text { seawards of } \\
\text { breakwater } \\
{[\mathrm{kg}]}\end{array}$ & $\begin{array}{c}\text { Mass } \\
\mathbf{d e n s i t y} \\
{\left[\mathrm{kg} / \mathrm{m}^{3}\right]}\end{array}$ & $\begin{array}{c}\text { Pore } \\
\text { Volume } \\
\%\end{array}$ & $\begin{array}{c}\text { Area } \\
\text { seawards of } \\
\text { breakwater } \\
{\left[\mathbf{m}^{\mathbf{2}}\right]}\end{array}$ & $\begin{array}{c}\text { Calculated } \\
\text { average } \\
\text { elevation of the } \\
\text { bed }[\mathbf{m}]\end{array}$ \\
\hline $\mathbf{A}$ & $\approx 4.9$ & 2650 & 40 & 3.0 & $=0.0010$ \\
$\mathbf{B}$ & $\approx 4.2$ & 2650 & 40 & 3.0 & $=0.0009$ \\
$\mathbf{C}$ & $\approx 1.2$ & 2650 & 40 & 3.0 & $=0.0003$ \\
$\mathbf{D}$ & $\approx 27$ & 2650 & 40 & 3.0 & $=0.0057$ \\
$\mathbf{E}$ & $\approx 0.1$ & 2650 & 40 & 3.0 & $=0.0000$ \\
$\mathbf{F}$ & $\approx 48$ & 2650 & 40 & 3.0 & $=0.0101$ \\
\hline
\end{tabular}

Table 4.1 Amount of sand seawards of the breakwater.

It can be concluded that very little sand was transported outside the testing area.

ad. 4 inaccuracies of the measuring methods

During every experiment that is performed there will always be inaccuracies in the measuring methods. In our tests measuring inaccuracies occurred too. Viz.:

- The PROFO measures the bed with an accuracy of $0.2 \mathrm{~mm}$ (see technical description electronic profile indicator)

- The PROFO does not move along the bed at a constant speed (see Section 4.2.1). Therefore, the profile measurements are resampled with the program RESAMP.CPP. With this method we assume that the PROFO does move at a constant speed over the bed, because the program resamples the measurements of the entire bed. In reality, however, this is not completely true. The irregularities of the rails force the trolley (and thus the PROFO) to ride across the bed with small hitches, but it is not known where the hitches take place. To be more accurate only the areas where the hitches take place should be resampled. It was not possible to get a higher accuracy with this rails and trolley.

- Profile measurements around the submerged breakwater were difficult to perform, due to the stones of the breakwater. Sediment was settling between these stones, which was difficult to measure.

- The visual measurements were performed every $5 \mathrm{~cm}$. Between two visual measurements the bed was assumed to be straight. However, this is not completely true. There were some ripples in the bed (see Figure 4.4).

- It is assumed that the height of the bed is constant over the width of the flume. This is not true. Due to boundary effects caused by the side walls, profile development is not the same over the width of the flume. At the beginning of a test the bed was horizontal over the width of the flume. During a test this horizontal bed changes to a slightly hollow- or roundshaped bed. 


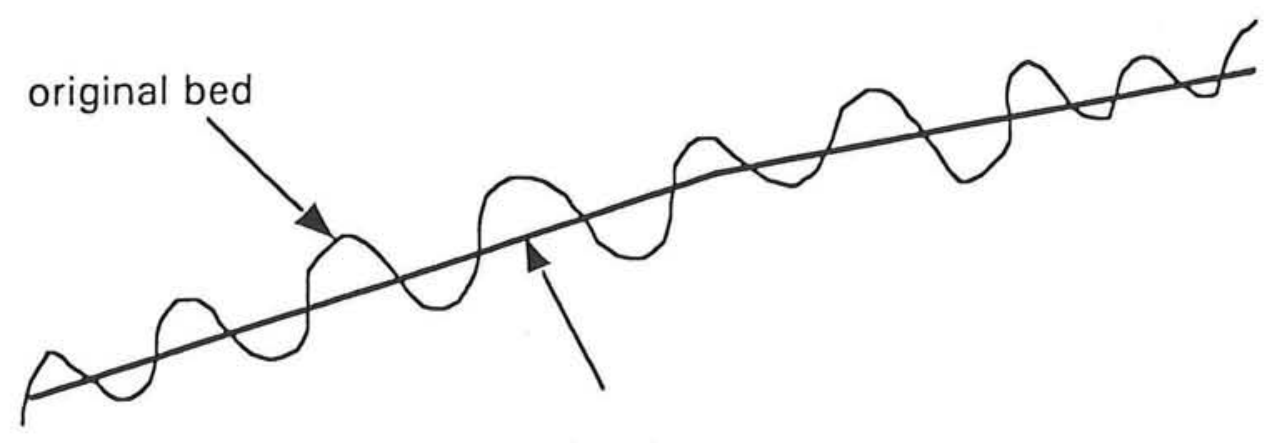

visual measurement

Figure 4.4 Ripples in bed at visual profile measurement area.

All these measuring inaccuracies may be possible explanations for the loss or gain of some small amounts of sediment.

So, in conclusion, a difference in profile development over the width of the flume is mainly responsible for the difference in total sand amounts during one test. To obtain fair results all computations with all profile measurements during an experiment have to start with the same amount of sand. Therefore some profiles were lifted up or lowered down approximately one millimetre (average) to have an equal amount of sand during the entire test.

\subsubsection{Irregularities at the transition point}

The transition point $(x=9.200 \mathrm{~m})$ is the position where the PROFO measurements end and the visual profile measurements take over. At this position it can be noticed that in almost every profile graph there is a jump in the bed (see Volume 2: Graphics, Part 1, Graph 1.1 to 1.26). This is a result of measuring the bed profile at different positions in the flume. The PROFO measures the profile in the middle of the wave flume, while the visual measurements were taken at the side wall of the wave flume (see Figure 4.5). So, apparently there is a difference in profile development over the width of the wave flume. In most profile measurements this downwards jump occurs and the result is always the same. The visual profile measurement taken of the bed at the side wall is a bit lower than the PROFO measurement taken of the bed in the middle of the flume. This is a result of boundary-effects caused by the side walls. The average drop in bed height is about $1 \mathrm{~cm}$. 


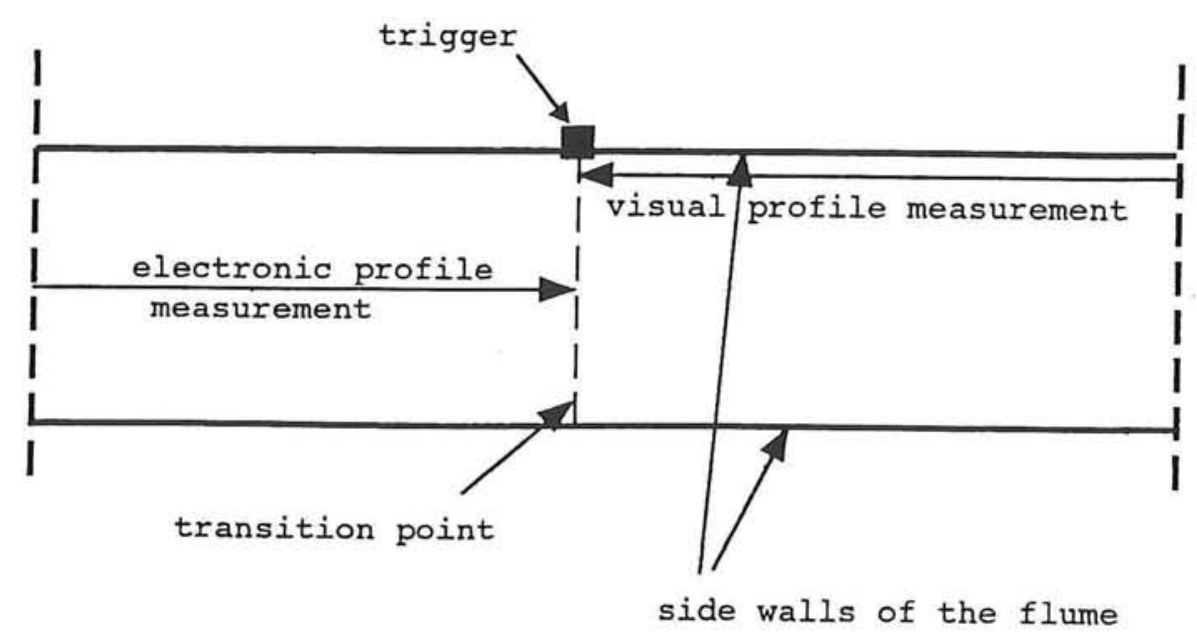

Figure 4.5 Transition position.

\subsubsection{Reference position for sediment transport computations}

In order to compare the results from different tests the profile measurements have been computed with a number of formulas and meet some criteria.

These criteria are (see Figure 4.6):

- sediment transportation through a defined vertical cross-section.

These cross-sections are: Vertical (a): $x=5.25 \mathrm{~m}$

Vertical (b): $x=6.15 \mathrm{~m}$

Vertical (c): $x=7.05 \mathrm{~m}$

- sediment transportation through a defined horizontal level.

These levels are: $\quad$ horizontal (a): $y=0.45 \mathrm{~m}$

horizontal (b): $y=0.55 \mathrm{~m}$

horizontal (c): $y=0.65 \mathrm{~m}$ 


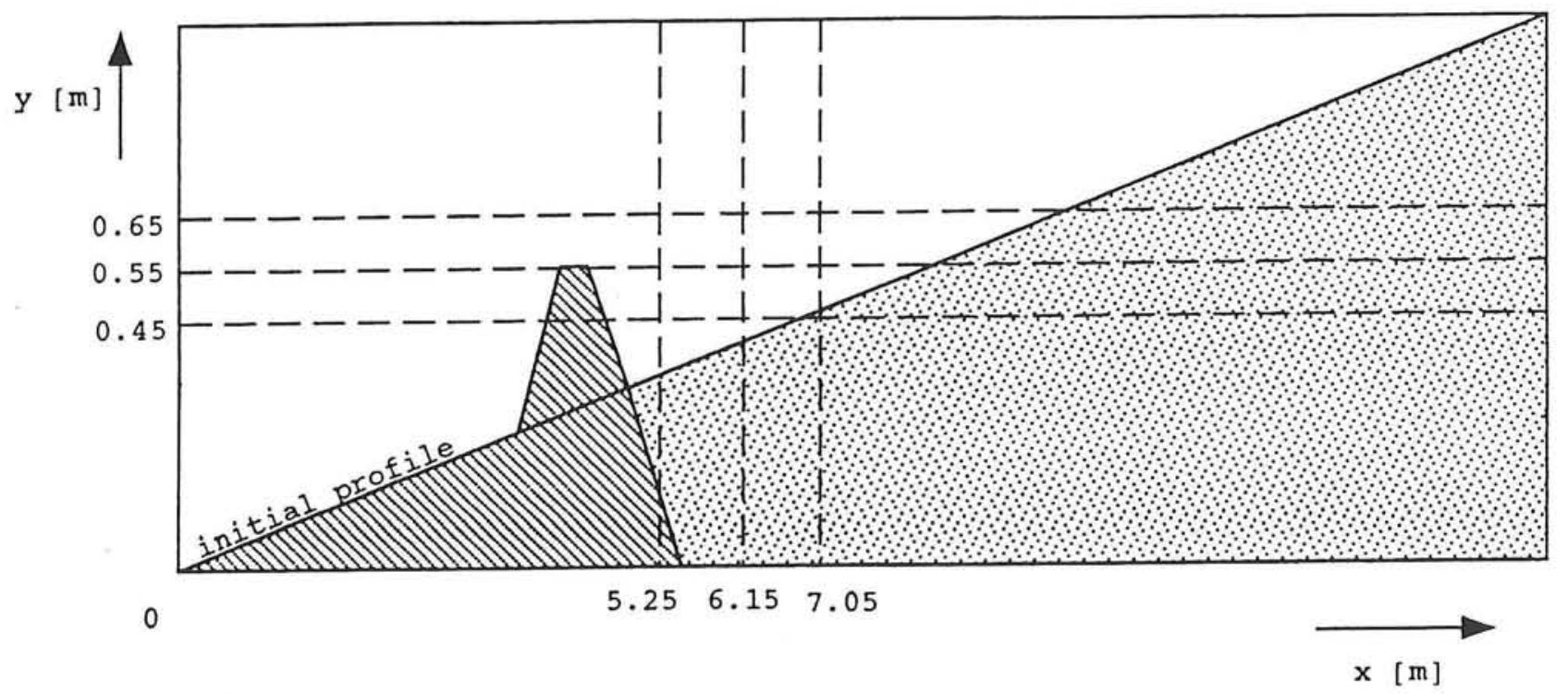

Figure 4.6 Reference levels for further computations.

These criteria are not chosen randomly. The vertical cross-sections coincide with the positions of the wave height, velocity and concentration measurements. The horizontal levels coincide with the still water level, the crest height of the submerged breakwater and $20 \mathrm{~cm}$ below still water level. These levels are chosen to investigate the effect of the breakwater at different depths below the still water level.

The sediment transports through a vertical cross-section are easy to compute. There is only one position where the bed crosses a section. This is different in case of sediment transport through a horizontal level. In most tests the bed crosses a certain horizontal level several times. Sometimes this is due to big fluctuations of the bed, but in most cases it is due to the presence of ripples in the bed (see Figure 4.7). Now, a problem arises which position has to be chosen for further computations. There are various possibilities to define different crossing positions. For example, three different definitions might be (see Figure 4.7):

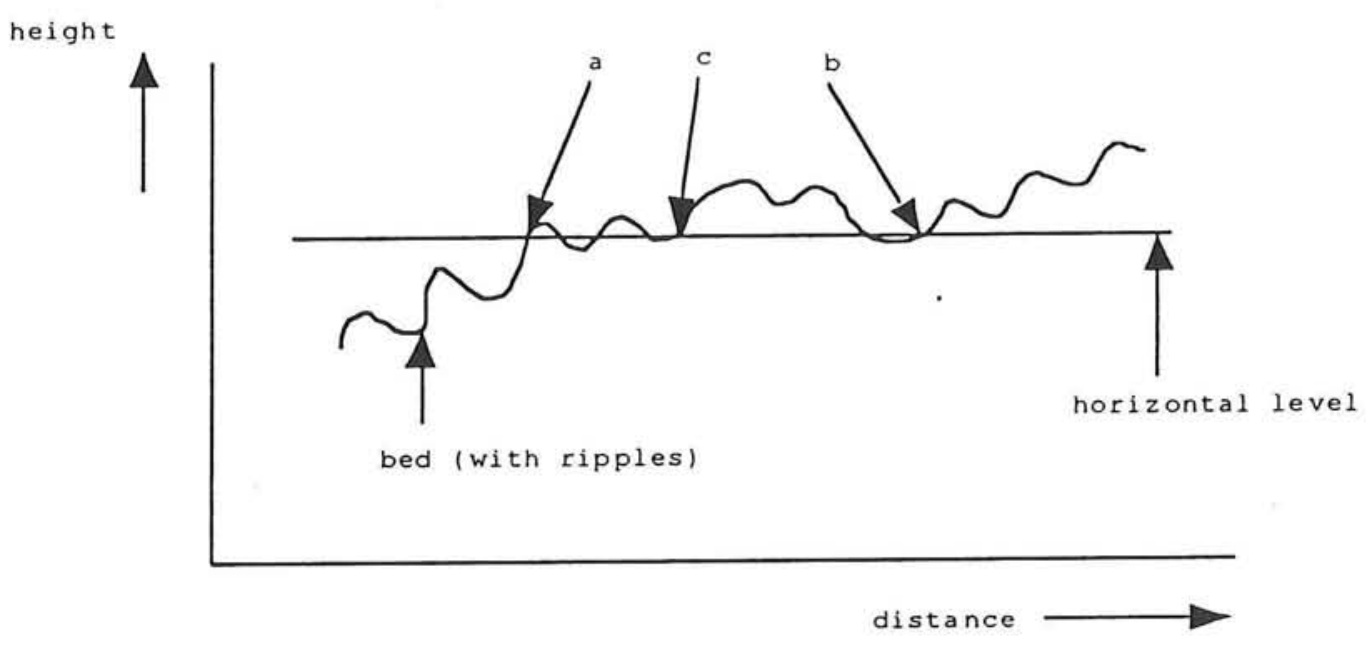

Figure 4.7 Different definitions for a reference position. 
1. the most seaward position where the bed crosses a defined horizontal level (position a)

2. the most landward position where the bed crosses a defined horizontal level (position b)

3. an intermediate position between position a and position b (position $\mathrm{c}$ )

It is decided to define position b (most landward crossing position) as a reference position for further computations.

\subsubsection{Profile measurements just landwards of the submerged breakwater}

As mentioned before, during tests with a breakwater present profile measurements started just landwards of this breakwater. The trigger was attached to the side wall of the flume as close as possible to the breakwater (see Figure 4.8). At this position all the profile measurements started. However, after a few hours of waves sediment accumulated just landward of the breakwater. This amount of accumulated sand is different for every test. Therefore it was not possible to move the trigger forward to pre-set positions. Another difficulty is the trolley. This trolley has to be in motion when a measurement starts. This has to be done to avoid irregularities in speed. Therefore, the trolley needs some distance to move along the bed before a measurement can start. These are the reasons for not measuring the bed near the submerged breakwater with a PROFO. During the tests drawings were made of the accumulation of sand landward of the breakwater. These drawings were 'pasted' next to the PROFO measurements. In the Volume 2: Graphics, Part 1 these pasted areas can be noticed (the straight lines just landward of the breakwater).

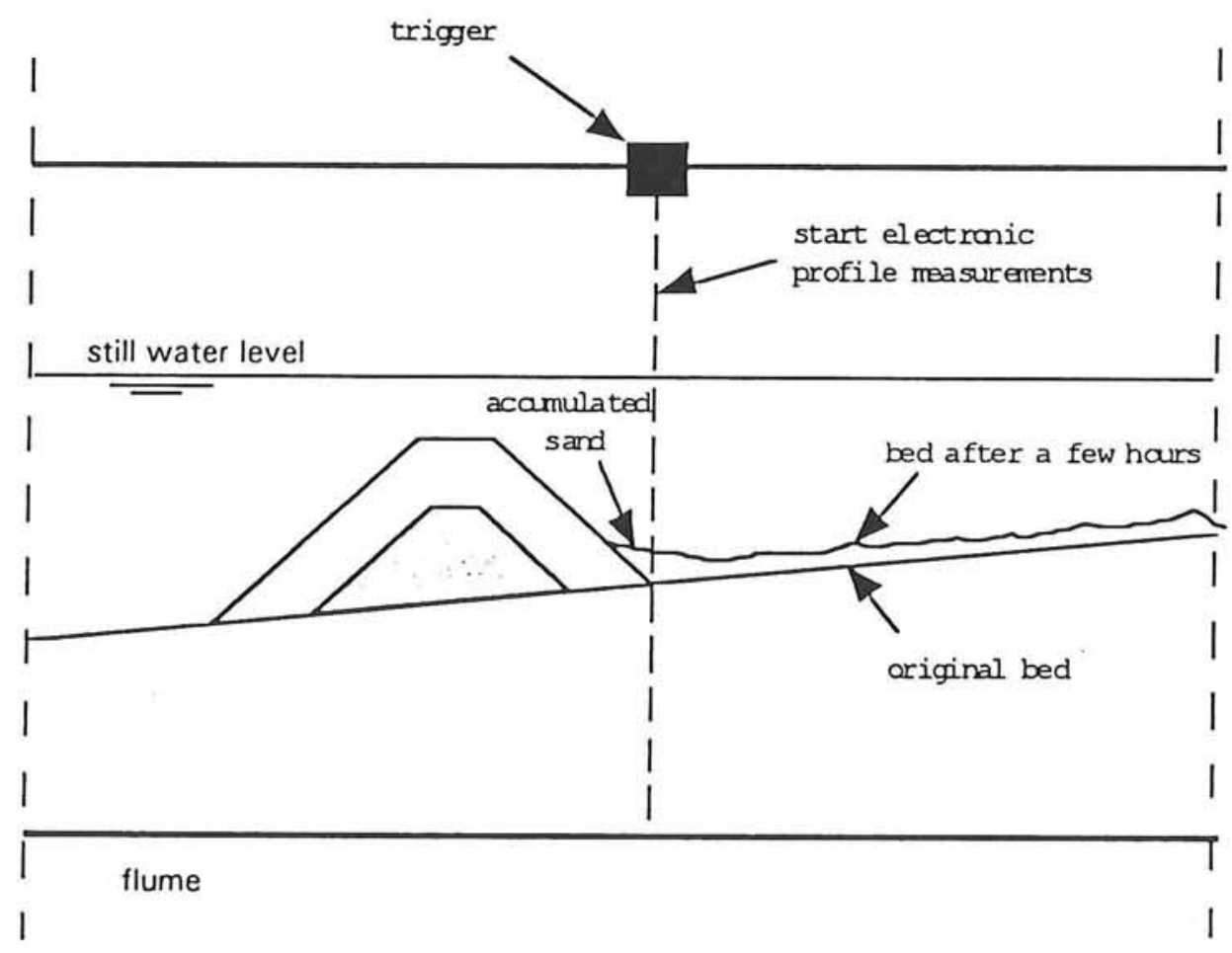

Figure 4.8 Starting position of PROFO measurements in case of a breakwater present. 


\subsection{Profile measurement results (see Volume 2: Graphics, Part 1)}

To increase the readability of this section the following shortscript will be used:

experiment with breakwater $\quad \rightarrow \quad$ test +

experiment without breakwater $\rightarrow$ test -

\subsubsection{Comparing experiments with and without breakwater}

In this section the final results of all profile measurements during the test series are discussed. As an example, one of the tests is discussed in detail in this section and all its graphs are printed in this section too. All the other tests are discussed more briefly and the corresponding graphs are printed in Volume 2: Graphics, Part 1.

experiment $\mathrm{B}$ is chosen for detailed discussion because of its average wave height and period. experiment $\mathrm{B}$ has a central position in the diamond pattern of the Model Test Matrix (see Section 2.2.2).

Experiment $B\left(H_{s}=0.098 \mathrm{~m} ; \mathrm{T}_{\mathrm{p}}=1.55 \mathrm{~s}\right)$

Profile measurements without breakwater (see Figure 4.9)

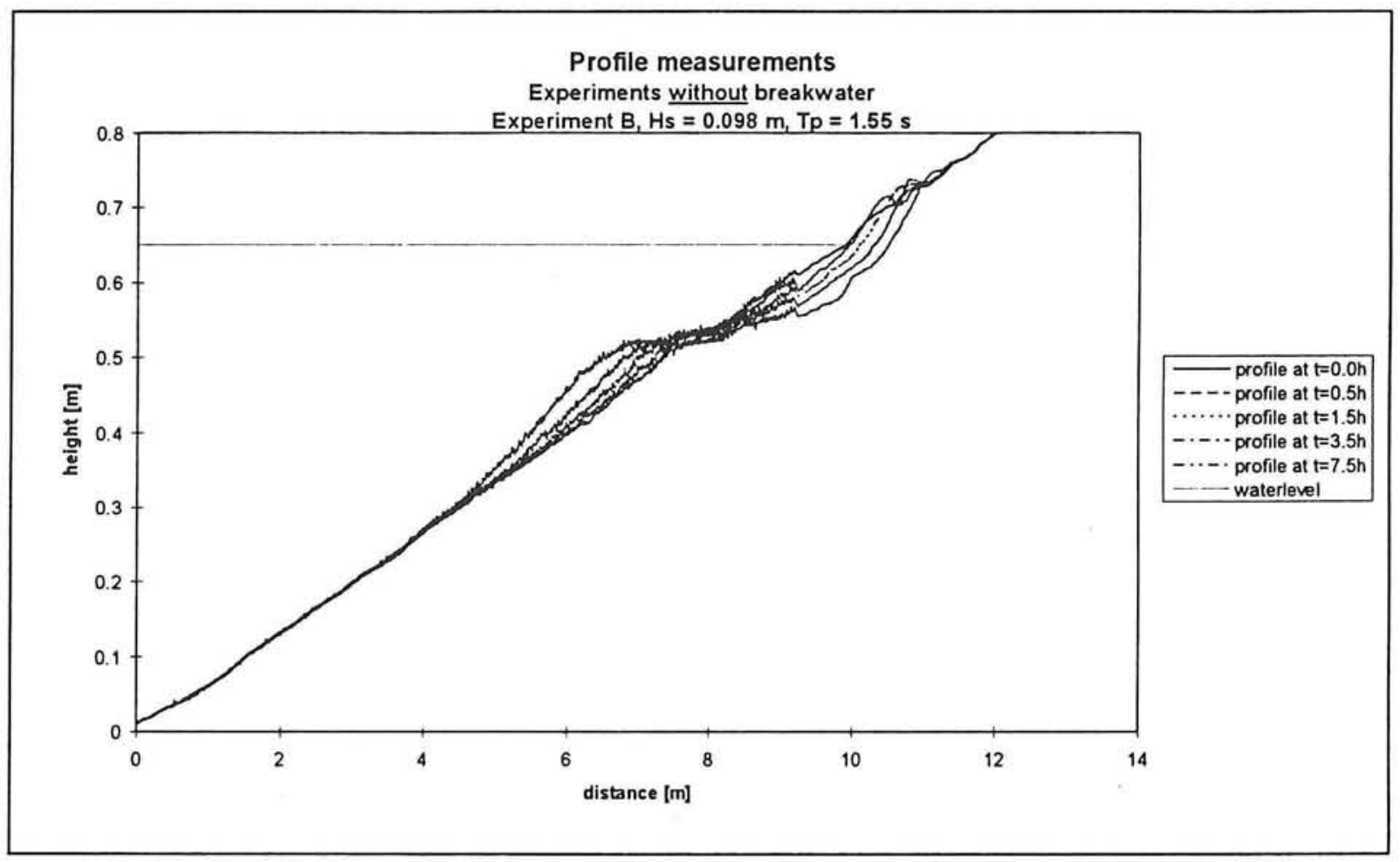

Figure 4.9 Measured profiles of experiment B without breakwater. 
Figure 4.9 shows all profile measurements performed during this test.

After the start of the experiment it can be noticed that some sediment is starting to move. In the first interval the sediment that is positioned around $\mathrm{x}=9.5 \mathrm{~m}$ is moved into two directions. Some of it is going landwards, due to wave run-up, and some of it is going seawards due to water motion near the bed. During Interval 2 a retreat of the waterline is visible. Most of all transported sediment is going seawards. An underwater barrier is formed around $\mathrm{x}=7.0 \mathrm{~m}$. Between $x=7.5 \mathrm{~m}$ and $\mathrm{x}=8.5 \mathrm{~m}$ a much flatter bed is created than the initial slope. During Interval 3 the scour of the bed continues around $\mathrm{x}=8.5 \mathrm{~m}$ to $\mathrm{x}=10.5 \mathrm{~m}$ and sediment is still going seawards. The barrier increases and moves seawards. The waterline is still retreating. The flattening of the bed increases in length and is now positioned between $\mathrm{x}=7.0 \mathrm{~m}$ and $\mathrm{x}=$ $9.0 \mathrm{~m}$. During Interval 4 all these processes continue. The barrier still increases and moves seawards to around $\mathrm{x}=6.5 \mathrm{~m}$, while the waterline still retreats and is positioned around $\mathrm{x}=$ $10.40 \mathrm{~m}$ (the initial position of the waterline at the start of the experiment is $\mathrm{x}=9.75 \mathrm{~m}$ ). Between $x=7.0 \mathrm{~m}$ and $\mathrm{x}=8.25 \mathrm{~m}$ an almost horizontal bed can be noticed. This wave condition has changed the bed in between approximately $\mathrm{x}=4.50 \mathrm{~m}$ and $\mathrm{x}=11.25 \mathrm{~m}$.

\section{Profile measurements with breakwater (see Figure 4.10)}

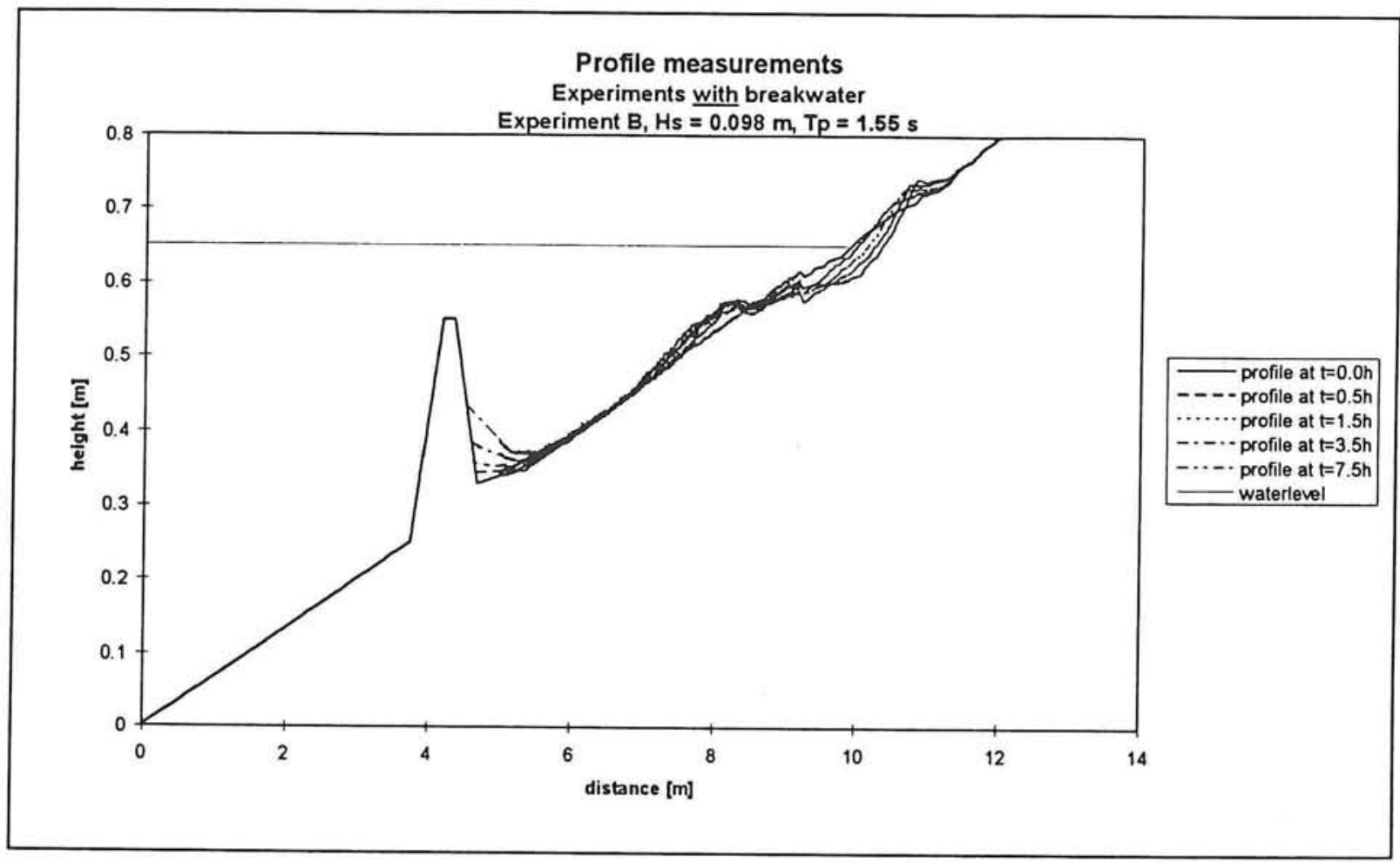

Figure 4.10 Measured profiles of experiment B with breakwater.

Figure 4.10 shows all profile measurements performed during this test.

After the start of the experiment it can be noticed that some sediment is starting to move. In the first interval the sediment that is positioned around $\mathrm{x}=9.5 \mathrm{~m}$ is moved into two directions. Some of it is going landwards and some of it is going seawards. A slight retreat of the waterline can be noticed during the first interval. During Interval 2 the retreat of the waterline continues. Most of all sediment is still transported seawards. An underwater barrier is formed around $\mathrm{x}=7.0 \mathrm{~m}$. Between $\mathrm{x}=8.0 \mathrm{~m}$ and $\mathrm{x}=8.5 \mathrm{~m}$ a much flatter bed is created than the 
initial slope. Some sediment is accumulating landwards of the submerged breakwater. During Interval 3 all these sediment transport processes continue. The scour of the bed continues around $\mathrm{x}=9.0 \mathrm{~m}$ to $\mathrm{x}=10.25 \mathrm{~m}$ and sediment is still transported seawards. The waterline is still retreating. The underwater barrier increases and moves slightly seawards. Some more sediment is accumulating landwards of the submerged breakwater. During Interval 4 the barrier still increases slightly, while the waterline still retreats and is now positioned around $\mathrm{x}$ $=10.30 \mathrm{~m}$. Even more sediment has accumulated landwards of the submerged breakwater, but hardly any sediment is transported seawards of the breakwater (see Table 4.1). Between $x=$ $8.0 \mathrm{~m}$ and $\mathrm{x}=9.0 \mathrm{~m}$ an almost horizontal bed can be noticed. Between $\mathrm{x}=5.5 \mathrm{~m}$ and $\mathrm{x}=7.0$ $\mathrm{m}$ hardly any changes in the bed occurred.

Transported volume of sediment through $x=5.25 \mathrm{~m}$ (see Figure 4.11).

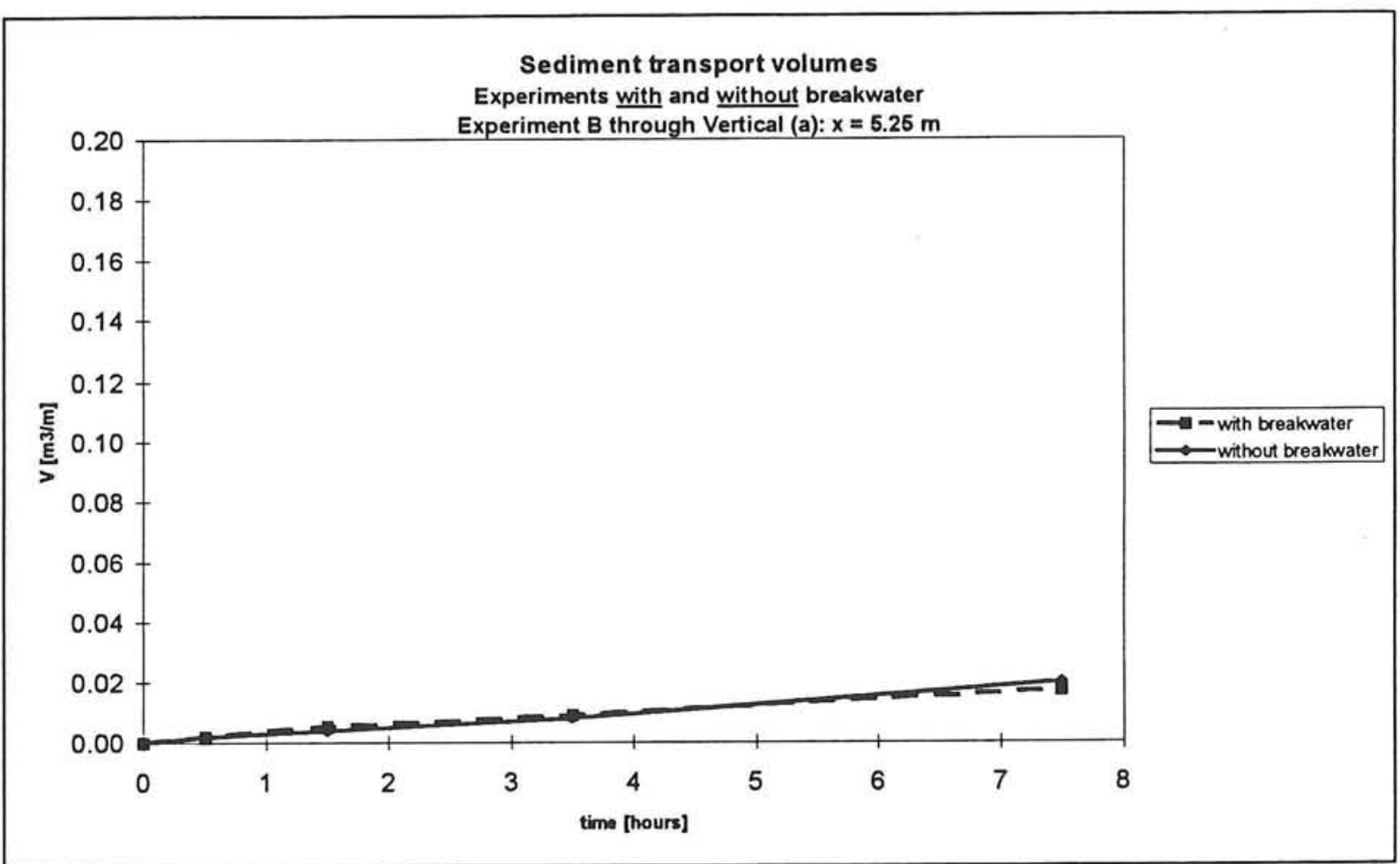

Figure 4.11 Transported volume of sediment through $x=5.25 \mathrm{~m}$ during experiment $B$.

Figure 4.11 shows the transported volume of sediment through Vertical (a): $x=5.25 \mathrm{~m}$ in a seawards direction. In this figure it can be noticed that a small amount of sediment crosses the vertical position $\mathrm{x}=5.25 \mathrm{~m}$ in a seawards direction, in both experiments. This transport is almost equal in both cases (with and without breakwater) and practically constant during both tests. In case of a breakwater present the transport rates decrease from $0.0036 \mathrm{~m} / \mathrm{m} /$ hour during Interval 1 and 2 to $0.0021 \mathrm{~m}^{3} / \mathrm{m} /$ hour during the last interval. In case of no breakwater present these transport rates increase from $0.0018 \mathrm{~m}^{3} / \mathrm{m} /$ hour in Interval 1 and 2 to 0.0030 $\mathrm{m}^{3} / \mathrm{m} /$ hour in Interval 4 . 
Transported volume of sediment through $x=6.15 \mathrm{~m}$ (see Figure 4.12).

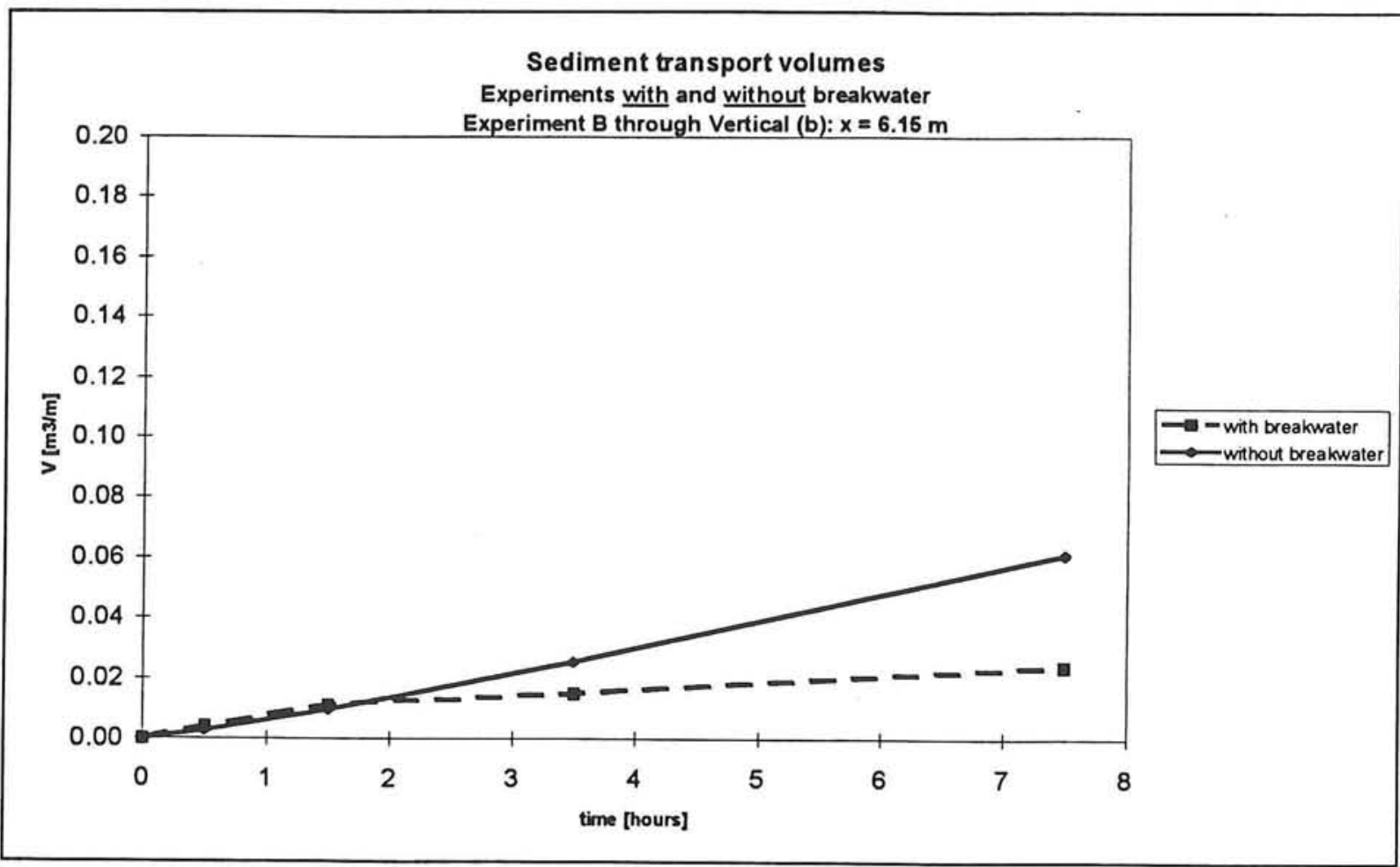

Figure 4.12 Transported volume of sediment through $x=6.15 \mathrm{~m}$ during experiment $B$.

Figure 4.12 shows the transported volume of sediment through Vertical (b): $x=6.15 \mathrm{~m}$ in a seawards direction. In this figure a slightly bigger difference in sediment transport can be noticed between test + and test -. During the first two intervals there is no difference between these tests. The rate of transport in these intervals is approximately $0.0075 \mathrm{~m}^{3} / \mathrm{m} / \mathrm{hour}$. In the third interval a difference occurs. While in test - the rate of transport remains constant, test + shows a decrease in transport rates (down to approximately $0.0019 \mathrm{~m} / \mathrm{m} / \mathrm{hour}$ ). During Interval 4 this difference remains constant as the rates differ from approximately 0.0090 $\mathrm{m}^{3} / \mathrm{m} /$ hour in test - to approximately $0.0021 \mathrm{~m}^{3} / \mathrm{m} /$ hour in test + . 


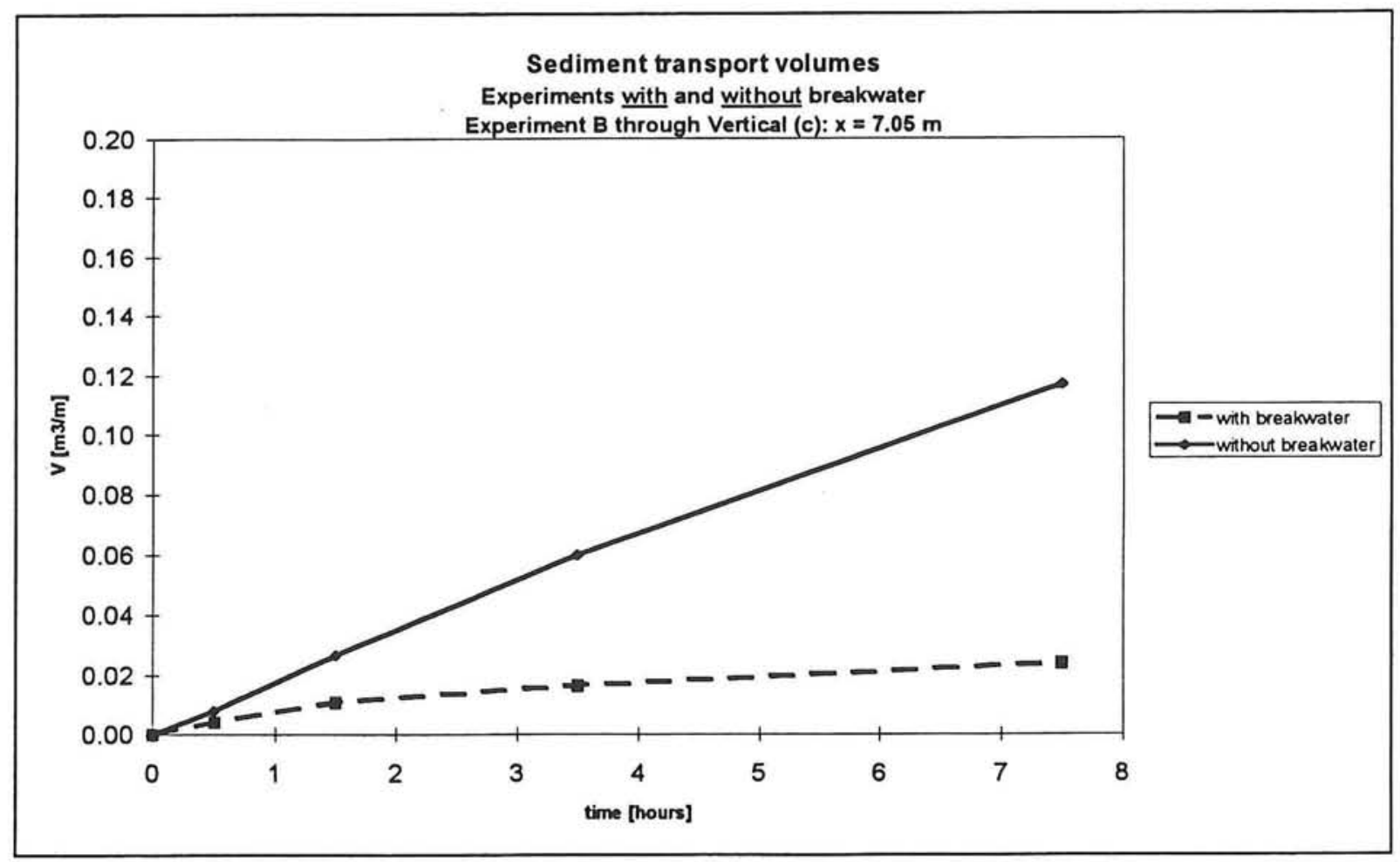

Figure 4.13 Transported volume of sediment through $x=7.05 \mathrm{~m}$ during experiment $B$.

Figure 4.13 shows the transported volume of sediment through Vertical (c): $x=7.05 \mathrm{~m}$ in a seawards direction. It this figure an even bigger difference can be noticed between test + and test -. In the first interval this difference is noticeable already as the transport rates differ a factor two, $0.0082 \mathrm{~m}^{3} / \mathrm{m} /$ hour in case of test + to $0.0160 \mathrm{~m}^{3} / \mathrm{m} /$ hour in case test -. During Interval 2 this difference increases from $0.0067 \mathrm{~m} / 3 / \mathrm{m} /$ hour in case of test + to 0.0185 $\mathrm{m}^{3} / \mathrm{m} /$ hour in case of test -. During Interval 3 the difference between the two tests still increases as both transport rates decrease but at a faster rate in case there is a breakwater present. The rates now differ from $0.0029 \mathrm{~m}^{3} / \mathrm{m} / \mathrm{hour}$ in case of test + to $0.0166 \mathrm{~m}^{3} / \mathrm{m} / \mathrm{hour}$ in case of test -. During the last Interval the transport rates still decrease, but there is still a very big difference in transport rates. The rates now differ by a factor 7.5. These rates are 0.0019 $\mathrm{m}^{3} / \mathrm{m} /$ hour in case of test + and $0.0143 \mathrm{~m}^{3} / \mathrm{m} /$ hour in case of test - . 
Transported volume of sediment through $y=0.45 \mathrm{~m}$ (see Figure 4.14).

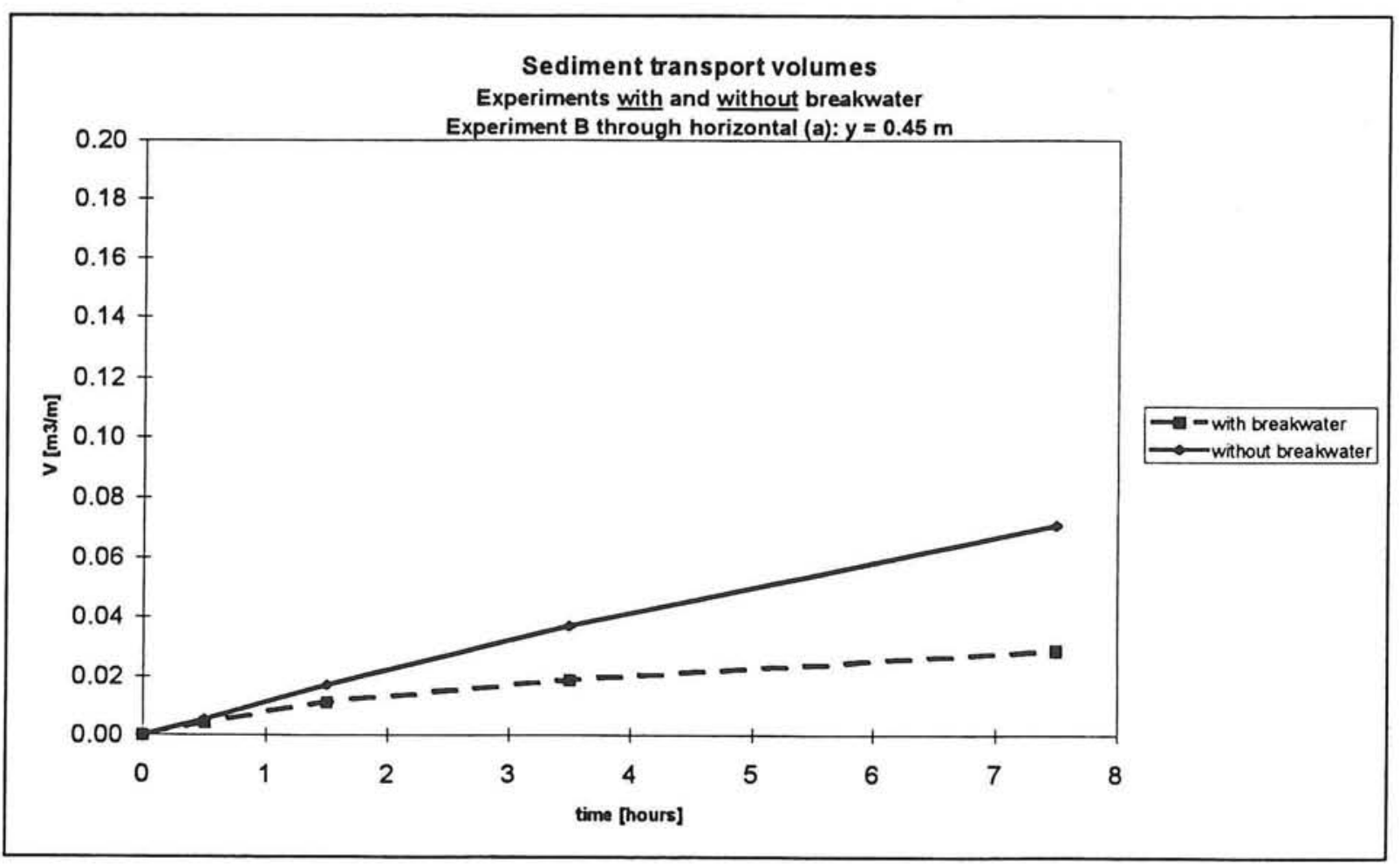

Figure 4.14 Transported volume of sediment through $y=0.45 \mathrm{~m}$ during experiment $B$.

Figure 4.14 shows the transported volume of sediment through horizontal (a): $y=0.45 \mathrm{~m}$ in a seawards direction. In this figure a clear difference can be noticed between test + and test During Interval 1 the transport rates in a downwards (= seawards) direction do not differ very much. In Interval 2 the difference in sediment transport rates is noticeable. In case of test + the transport rate slightly decreases to $0.007 \mathrm{~m}^{3} / \mathrm{m} /$ hour, while in case of test - the transport rate slightly increases to $0.012 \mathrm{~m}^{3} / \mathrm{m} /$ hour. During Interval 3 the sediment transport in case of test + decreases further to $0.004 \mathrm{~m}^{3} / \mathrm{m} /$ hour. The transport in case of test - decreases too, but to $0.010 \mathrm{~m}^{3} / \mathrm{m} /$ hour, which is a much higher rate. In Interval 4 these transport rates decreases further to respectively $0.0025 \mathrm{~m}^{3} / \mathrm{m} /$ hour and $0.0086 \mathrm{~m}^{3} / \mathrm{m} /$ hour. 


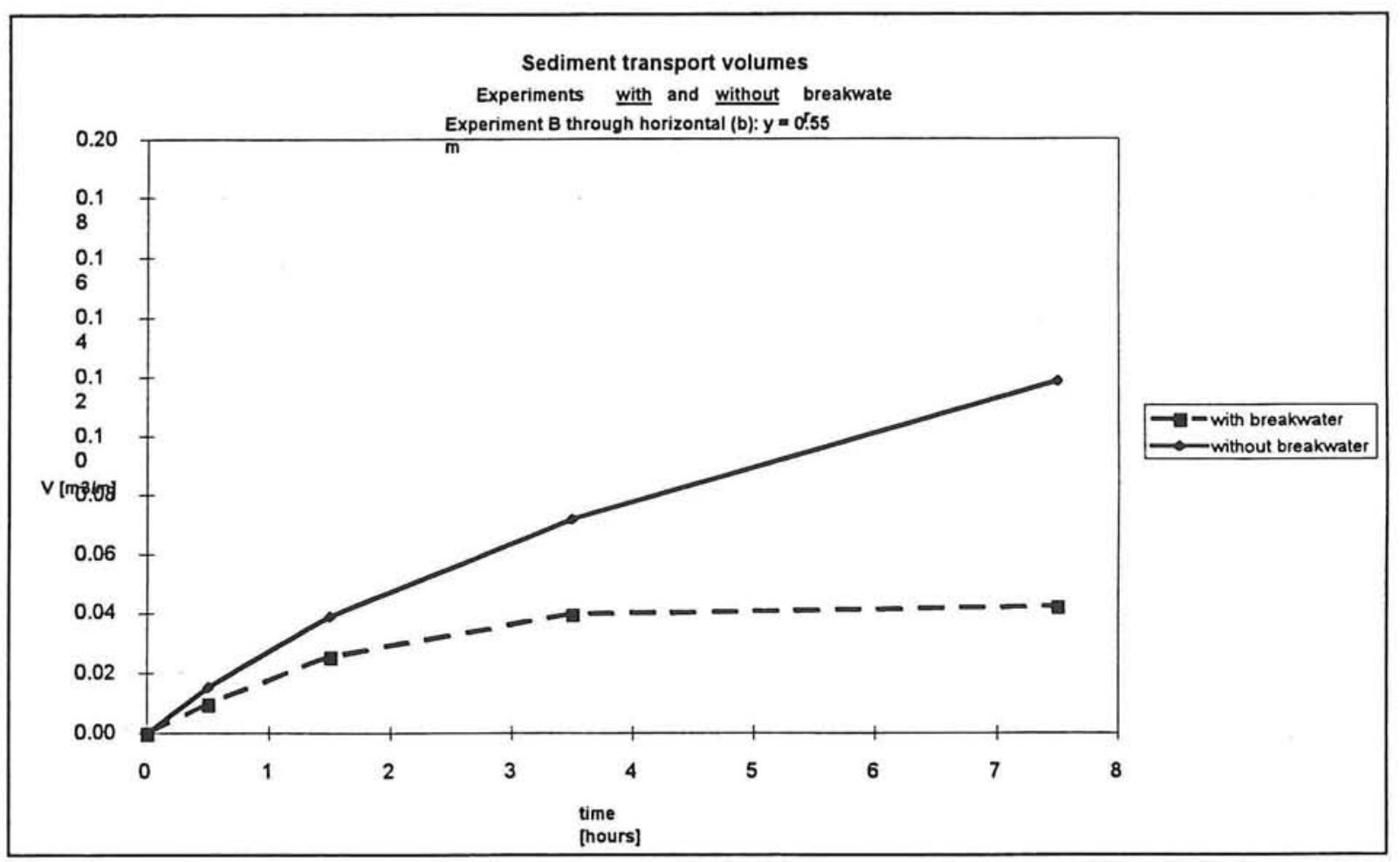

Figure 4.15 Transported volume of sediment through $y=0.55 \mathrm{~m}$ during experiment $B$.

Figure 4.15 shows the transported volume of sediment through horizontal (a): $y=0.55 \mathrm{~m}$ in a seawards direction. In this figure, again, a clear difference can be noticed between test + and test -. In Interval 1 the difference in sediment transport in a downward direction already is noticeable. In case of test + there is a sediment transport of $0.020 \mathrm{~m}^{3} / \mathrm{m} /$ hour, while in case of test - this rate is $0.031 \mathrm{~m}^{3} / \mathrm{m} /$ hour, which is considerably higher. During Interval 2 both these transport rates decrease to respectively $0.016 \mathrm{~m}^{3} / \mathrm{m} /$ hour and $0.024 \mathrm{~m}^{3} / \mathrm{m} /$ hour. During the next Interval again the sediment transport rates decrease. These values are now respectively $0.007 \mathrm{~m}^{3} / \mathrm{m} /$ hour and $0.016 \mathrm{~m}^{3} / \mathrm{m} /$ hour. During the last Interval the sediment transport in case of test + seems to have stopped, while the transport rate in the other case is still considerable at a value of $0.012 \mathrm{~m}^{3} / \mathrm{m} /$ hour. 


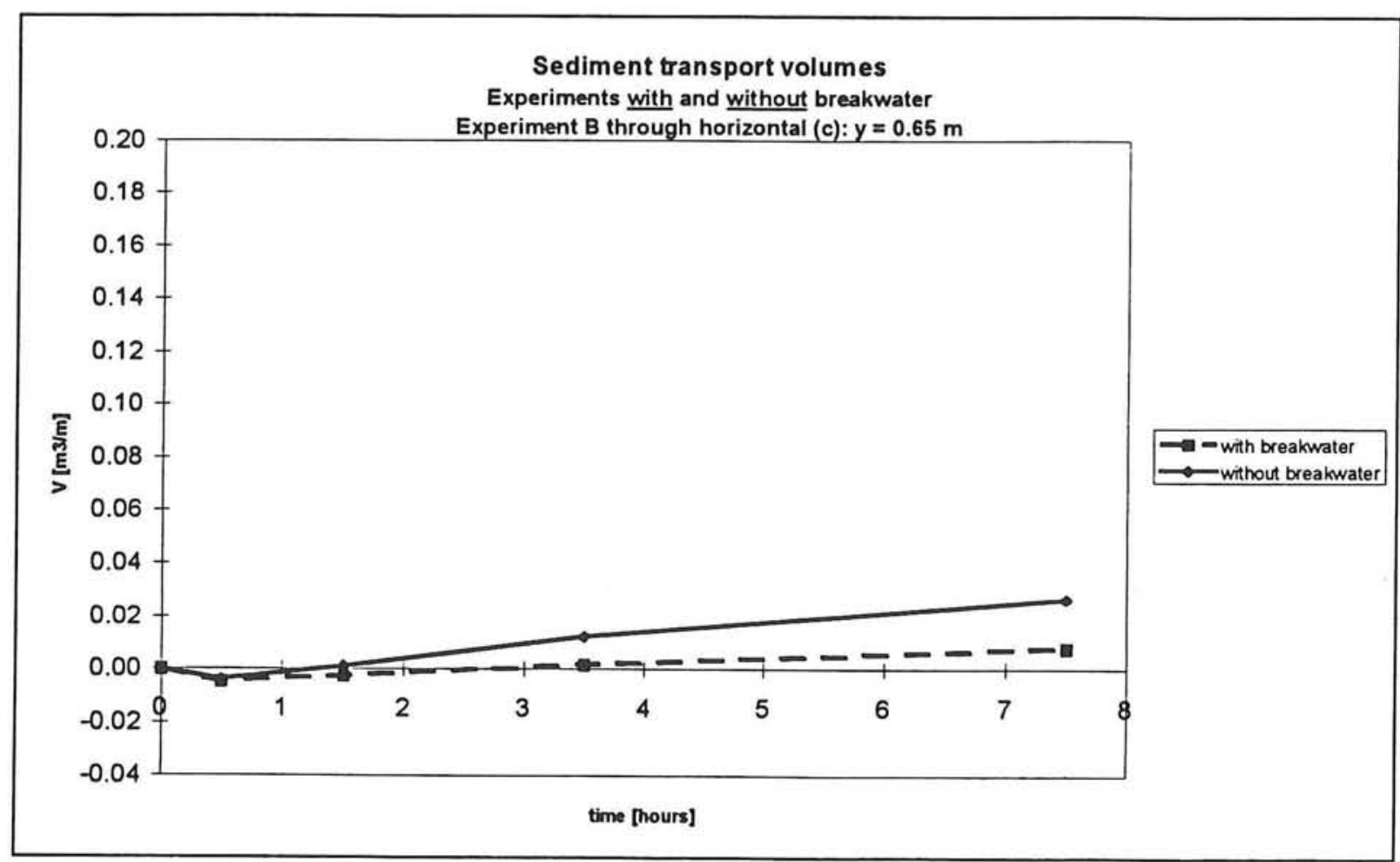

Figure 4.16 Transported volume of sediment through $y=0.65 \mathrm{~m}$ during experiment $B$.

Figure 4.16 shows the sediment transport volume through horizontal (a): $y=0.65 \mathrm{~m}$ in a seawards direction. In this figure there is only a slight difference in sediment transport rate between test + and test -. In Interval 1 there is a small net transport in an upwards direction. In both tests this is a small transport with a value of approximately $0.004 \mathrm{~m}^{3} / \mathrm{m} /$ hour. During Interval 2 there is hardly any net transport in any direction. In this Interval the net transport in case of test + is still slightly upwards, while in case of test - the net transport is in a downwards direction. During Interval 3 both tests have reached a net transport in a downwards direction. In case of test - this transport is approximately $0.005 \mathrm{~m}^{3} / \mathrm{m} /$ hour and in test + this value is only $0.002 \mathrm{~m}^{3} / \mathrm{m} /$ hour. In Interval 4 these values decrease slightly to values of respectively $0.0035 \mathrm{~m}^{3} / \mathrm{m} /$ hour and $0.0016 \mathrm{~m}^{3} / \mathrm{m} /$ hour. 


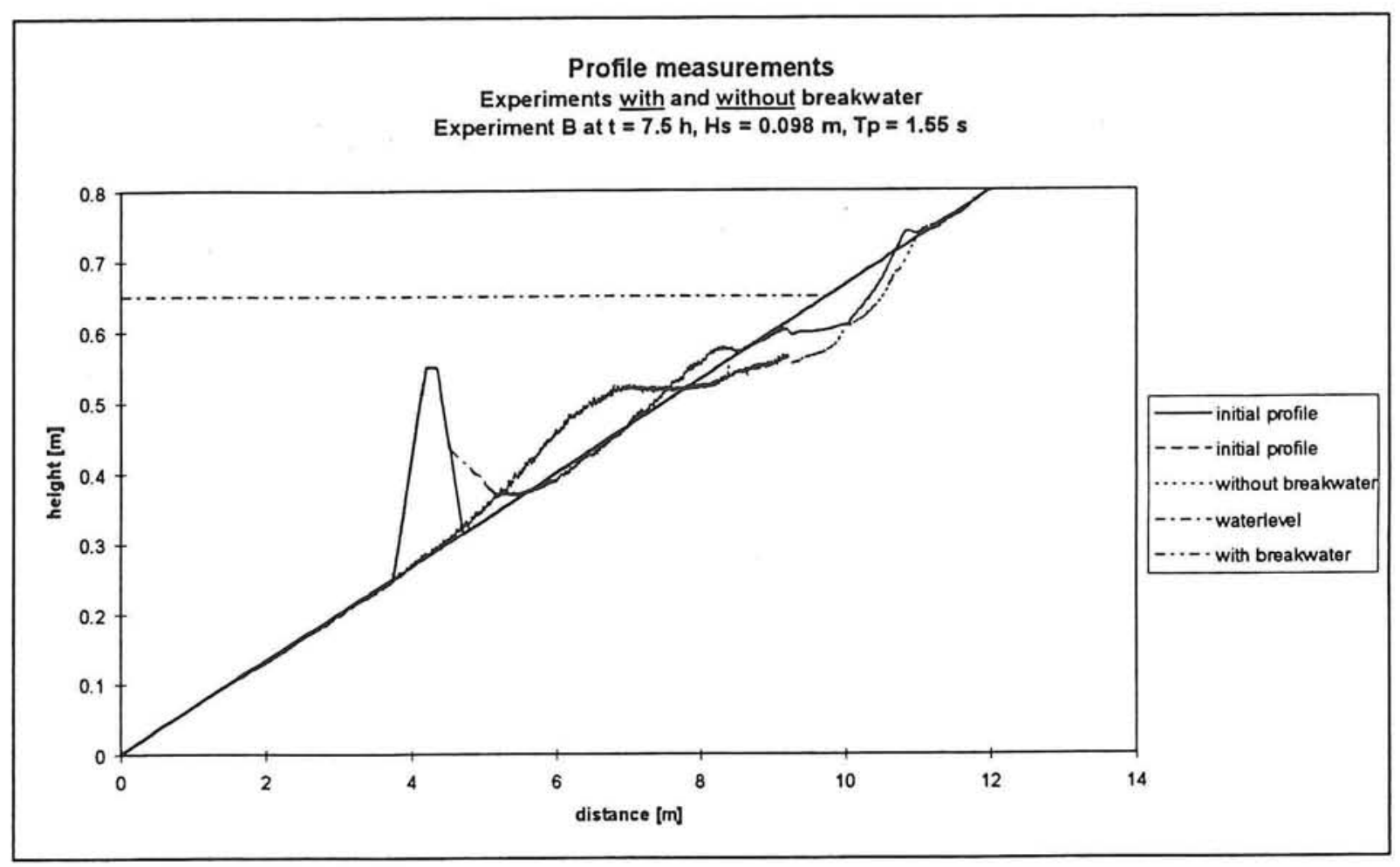

Figure 4.17 Measured profiles of experiment $B$ at $t=7.5 \mathrm{~h}$.

In this test a significant difference can be noticed in the profile development. In case of test the initial slope has changed much more than in case of test + . Also, the underwater barrier is much bigger and positioned more seawards in case of test - than in case of test + . In case of test + the transported sediment near the breakwater has accumulated landwards of this submerged breakwater. The shoreline retreat in case of test - is a little more severe than in case of test + . Another difference that can be noticed is the difference in sediment transport during the last two intervals. In most of all sediment transport volume figures it is clear that in case of test - the transport of sediment (gradient of transported volume of sediment lines) is greater than in case of test + . Therefore it can be concluded that in case of test + an equilibrium state shall be reached sooner than in case of test -.

All the other tests are discussed more briefly. The corresponding graphs are printed in Volume 2: Graphics.

Experiment A $\left(\mathrm{H}_{\mathrm{s}}=\mathbf{0 . 0 9 5} \mathrm{m} ; \mathrm{T}_{\mathrm{p}}=\mathbf{2 . 0 7} \mathrm{s}\right)$

(see Volume 2: Graphics, Part 1, Graph 1.1, 1.7 and 1.27 to 1.32)

In this test there is not much difference in the profile development between an experiment with or without breakwater. Some differences, however, can be noticed. In case of test - the slope has undergone more changes than in case of test + . Another noticeable difference is the barrier that is formed underwater. In case of test - this barrier is bigger than in case of test + . In case of test + the transported amount of sediment near the breakwater has accumulated landwards of this submerged breakwater. The shoreline retreat is in both cases almost equal. Another 
difference that can be noticed is the difference in sediment transport during the last interval. In all the volume transport figures it is clear that in case of test - the transport of sediment (gradient of transported volume of sediment lines) is smaller than in case of test + . Therefore it can be concluded that in case of test - an equilibrium state shall be reached sooner than in case of test + .

Experiment $\mathrm{C}\left(\mathrm{H}_{\mathrm{s}}=0.097 \mathrm{~m} ; \mathrm{T}_{\mathrm{p}}=1.29 \mathrm{~s}\right)$

(see Volume 2: Graphics, Part 1, Graph 1.3, 1.9 and 1.39 to 1.44)

In this test, a more significant difference can be noticed in the profile development. In case of test - the slope is much more disturbed than in case of test + . Also the barrier, which is formed under water, is much bigger and positioned more seawards in case of test -. In this test the sediment transport at greater depth ( 30 to $35 \mathrm{~cm}$ ) is very small. So, in case of test + there is hardly any sediment settling landwards of this breakwater. The shoreline retreat in case of test - is a little more severe than in case of test + . Another difference that can be noticed is the difference in sediment transport during the last two intervals. In most of all of the transported volume of sediment figures it is clear that in case of test - the rate of sediment transport (gradient of transported volume of sediment lines) is greater than in case of test + . Therefore it can be concluded that in case of test + an equilibrium situation shall be reached sooner than in case of test -.

Experiment $\mathrm{D}\left(\mathrm{H}_{\mathrm{s}}=0.137 \mathrm{~m} ; \mathrm{T}_{\mathrm{p}}=1.81 \mathrm{~s}\right)$

(see Volume 2: Graphics, Part 1, Graph 1.4, 1.10 and 1.45 to 1.50 )

The results of this test show that there is a clear difference in profile development during the time these tests lasted. In test - the profile is changing much faster in the first three intervals than in test + . The last interval, however, shows a turnabout in sediment transport. In this interval the sediment transport is, in case of test -, much smaller than in case of test + .

Furthermore, the profile is slightly more disturbed in case of test - than in case of test + . In case of test - the barrier is bigger and positioned more seawards than in case of test + . It can be noticed that in test + the sediment transported near the landwards side of the breakwater has accumulated against the breakwater. During Interval 4 there was a considerable amount of sediment transported over the breakwater (see Table 4.1 on page 39). In case of test + the retreat of the waterline is less than in case of test -. In conclusion, in case of test - an equilibrium state shall be reached sooner than in case of test + .

If the test had lasted a few hours longer there is a good possibility that the sediment transport in case of test - will be less than in case of test + (the transported volume of sediment lines would cross).

Experiment $\mathrm{E}\left(\mathrm{H}_{\mathrm{s}}=0.066 \mathrm{~m} ; \mathrm{T}_{\mathrm{p}}=1.29 \mathrm{~s}\right)$

(see Volume 2: Graphics, Part 1, Graph 1.5, 1.11 and 1.51 to 1.56 )

In this test it is clear that there is a big difference in sediment transport and thus in profile development between these two tests. Although the waterline retreat is in both cases practically equal, the rest of the profile is much more disturbed in case of test -. The barrier that is formed underwater is much bigger and positioned more seawards in case of test -. At approximately $15 \mathrm{~cm}$ below still water level there is hardly any sediment transport in case of test + . In all profile transport figures it is noticed that in the last Interval there is a big 
difference in sediment transport between the two tests. In case of test - the sediment transport is continuing at a much faster rate than in case of test + . In the latter case there is hardly any sediment transport at all during this interval. Therefore, it can be concluded that in case of test + , an equilibrium state is reached much sooner than in case of test -.

Experiment $\mathrm{F}(\mathrm{H}=\mathbf{0 . 1 0 4} \mathrm{m} ; \mathrm{T}=\mathbf{1 . 5 5} \mathrm{s})$

(see Volume 2: Graphics, Part 1, Graph 1.6, 1.12 and 1.57 to 1.62)

In this test, with regular waves, the sediment transport rates do not differ that much from each other, although there is slightly more sediment transport in case of test + . Another difference between the two tests is the formation of one big barrier in test -, and the formation of two smaller barriers in case of test + . In most sediment transport volume graphs ( 5 out of 6 ) the sediment transport in case of test - is less than in case of test + . There is hardly any difference in sediment transport during Interval 4 between these two tests. The sediment transported near the submerged breakwater has accumulated landwards of this breakwater. During Interval 4 there was, like in experiment D, a considerable amount of sediment transported over the breakwater (see Table 4.1). In case of test - the retreat of the waterline was slightly more severe than in case of test + . An equilibrium state will take much more time in both tests.

\subsubsection{Comparing experiments with equal wave height}

In the Model Test Matrix of the experiments three wave conditions have more or less an equal significant wave height. In Table 5.4 (on page 68) these wave conditions are presented. Test cases $\mathrm{A}, \mathrm{B}$ and $\mathrm{C}$ have more or less an equal significant wave height of approximately $0.10 \mathrm{~m}$, but differ in spectral peak period. Test case $\mathrm{A}$ is a wave condition with moderately long waves and a wave steepness $\left(=\mathrm{H}_{s} / \mathrm{L}_{0 \mathrm{p}}\right)$ of $1.50 \%$. Test case B is a normal wave condition with a wave steepness of $2.67 \%$. Test case $\mathrm{C}$ is a wave condition with moderately short waves and a wave steepness of $3.84 \%$. These tests are compared with each other in order to investigate the effect of the submergd breakwater on these different wave steepnesses. One would expect that wave condition $\mathrm{C}$, with the short waves, has less effect on profile development at a greater depth than wave condition B. Wave condition A should have more effect on profile development at a greater depth than wave condition B.

Tests without a breakwater present (see Volume 2: Graphics, Part 1, Graph 1.19, 1.20 and 1.63 to 1.68 ).

Graph 1.19 in Volume 2: Graphics shows the profile measurements performed after Interval 2 of these tests. It can be noticed that there is hardly any difference between these tests on such a short term. In all tests the waterline retreats and a small underwater barrier is formed already.

Graph 1.20 in Volume 2: Graphics shows the profile measurements performed at the end of these tests. There is not much difference between these measurements. In every experiment there is an almost equal retreat of the waterline. Furthermore, every experiment has an almost horizontal slope around $x=8.0 \mathrm{~m}$. The only big differences that can be noticed is the considerably smaller barrier formed in experiment $\mathrm{C}$ and the uneffected bed seawards of $\mathrm{x}=$ $5.0 \mathrm{~m}$ in this experiment. In both experiments $\mathrm{A}$ and $\mathrm{B}$ some sediment settled at around $\mathrm{x}=$ $4.0 \mathrm{~m}$. An explanation for this could be that the shorter waves of experiment $\mathrm{C}$ do not 'reach' to this great depth. 
Tests with a breakwater present (see Volume 2: Graphics, Part 1, Graph 1.21, 1.22 and 1.69 to 1.74).

Graph 1.21 in Volume 2: Graphics shows the profile measurements performed after Interval 2 of these tests. Again, there is not much difference between these tests. All three tests show an equal retreat of the waterline. A small underwater barrier is formed in all three experiments. However, experiment A has the smallest barrier and is positioned more landwards than both other tests. In all three tests some sediment has already accumulated landwards of the submerged breakwater.

Graph 1.22 in Volume 2: Graphics shows the profile measurements performed at the end of these tests. In this figure a much bigger difference between these tests can be noticed. The waterline retreat in experiment $\mathrm{A}$ is considerably more than in both other tests. Another big difference is the different shape of the barrier in experiment $\mathrm{A}$. The amount of transported sediment in a seaward direction is so much, that the bed between $x=5.0 \mathrm{~m}$ and $\mathrm{x}=7.0 \mathrm{~m}$ is lifted a few centimeters. Experiment $\mathrm{C}$ has considerably less accumulation of sediment landwards of the submerged breakwater than both other experiments.

\subsubsection{Comparing experiments with equal wave steepness}

Three wave conditions have been executed with a more or less equal wave steepness. These tests are: B, D and E. Testcase B has a wave steepness of $2.67 \%$, Testcase D has a wave steepness of $2.61 \%$ and Testcase $\mathrm{E}$ has a wave steepness of $2.56 \%$.

Tests without a breakwater present (see Volume 2: Graphics, Part 1, Graph 1.23, 1.24 and 1.75 to 1.80$)$.

Graph 1.23 in Volume 2: Graphics shows the profile measurements performed after Interval 2 of these tests. Already a few differences occur between these measurements. Experiment D has the most severe waterline retreat. In experiment B this retreat is less than in experiment D. In experiment $\mathrm{E}$ hardly any retreat of the waterline has occurred yet. In all three experiments an underwater barrier is formed already. In experiment $\mathrm{D}$ this barrier is located more seawards and thus at greater depth, than in both other experiments. This is due to the higher waves in experiment $\mathrm{D}$. Another noticeable difference is the wave run-up. Experiment $\mathrm{E}$ has the lowest wave run-up. Experiment $\mathrm{D}$ has the highest wave run-up. Furthermore, it is very clear that experiment $\mathrm{D}$ has already far more sediment transport in a seawards direction than both other tests. Experiment $\mathrm{E}$ has less sediment transport than experiment B. All these differences are consequences of the difference in wave height.

Graph 1.24 in Volume 2: Graphics shows the profile measurements performed at the end of these tests. In this graph still a lot of differences are visible between these experiments. Experiment D still has the most severe waterline retreat. In experiment B this retreat is less than in experiment $\mathrm{D}$. In experiment $\mathrm{E}$ the waterline has retreated, but much less than in both other tests. In experiment D the underwater barrier is located more seawards than in experiment B and E. Again, a considerable difference between these tests can be noticed in wave run-up. Experiment $\mathrm{E}$ still has the lowest wave run-up, while experiment $\mathrm{D}$ still has the highest wave run-up. 

to 1.86).

Graph 1.25 in Volume 2: Graphics shows the profile measurements performed after Interval 2 of these tests. The differences between these tests are smaller than in the tests with no breakwater present (see Graph 1.20 in Volume 2: Graphics). Nevertheless, some differences can already be noticed. The retreat of the waterline in experiment $\mathrm{D}$ is slightly bigger than in experiment $\mathrm{B}$. Experiment $\mathrm{E}$ shows hardly a retreat of the waterline on such a short term. The formation of an underwater barrier is in a further stadium in experiment $B$ and $D$ than it is in experiment E. Furthermore, in experiment B and D some sediment has been transported near the submerged breakwater and has accumulated landwards of this breakwater. Finally, considerable differences between these tests can be noticed in wave run-up. Experiment E again has the lowest wave run-up, while experiment $D$ has the highest wave run-up.

Graph 1.26 in Volume 2: Graphics shows the profile measurements performed at the end of these tests. From this figure it is clear there are more differences between these tests. Experiment $\mathrm{D}$ has the most severe waterline retreat of these tests. Experiment $\mathrm{E}$ has less retreat of waterline than experiment $B$. The underwater barrier is practically the same in experiment B and E. A big difference between these last two tests is the accumulation of sediment landwards of the submerged breakwater. Experiment B shows a considerable amount of sediment accumulated near this breakwater, where experiment $\mathrm{E}$ shows none at all. Experiment D shows by far the most accumulation of sediment near this breakwater. In this experiment the barrier is positioned so much seawards, that it is difficult to distinguish where the barrier ends and the accumulation of sediment near the breakwater starts. Finally, considerable differences between these tests can be noticed in wave run-up. Experiment E again has the lowest wave run-up, while experiment $\mathrm{D}$ has the highest wave run-up.

\subsection{Evaluation}

When an evaluation is made of all these profile measurement results, some remarks must be made.

- The initial profile of all experiments was a 1 in 15 slope which is not a very natural slope for a sand beach. Therefore, there was very much transport of sediment during the experiments. An equilibrium profile was not reached during the short duration of these experiments. But that was not an aim of this study. This study only investigated profile development of these experiments on a short term basis.

- The presence of a submerged breakwater leads to different results in the six different experiments that were executed. In some experiments there is a clear difference in profile development during the 7.5 hours of wave action in an experiment. Other experiments show considerably less differences between tests with and without a breakwater. Therefore, it is advised to perform experiments in which an equilibrium profile is reached during the experiments. This way also the long term profile development can be investigated.

- Other very important factors for the effect of the submerged breakwater on profile development are e.g., the freeboard, tides, particle diameter of the bed and waves that attack the coast under an angle (3DV effects). All these factors (and others) will also have to be investigated in order to evaluate the effectiveness of a submerged breakwater. 
- Taking all these restrictions into consideration the following conclusions can be drawn. In some experiments the transported volume of sediment in seaward direction is considerably reduced (experiment $\mathrm{B}, \mathrm{C}$ and $\mathrm{E}$ ). This is an important advantage of the breakwater. Other experiments show less difference or hardly any difference at all in transported volume of sediment (experiment $\mathrm{A}, \mathrm{D}$ and $\mathrm{F}$ ). In experiments $\mathrm{D}$ and $\mathrm{F}$ considerable amounts of sediment are transported over the breakwater in seaward direction. Thus, the breakwater does not totally block the sediment transport in seaward direction.

Finally, it can be concluded that the presence of a breakwater reduces the transported volume of sediment in some experiments and has much less effect on sediment transport in other experiments. 


\section{CHAPTER 5 WAVE HEIGHT MEASUREMENT RESULTS}

\subsection{Introduction}

In this chapter the wave height measurement results are described. All wave height measurement results have been computed by WAVES, a program of AUKE/PC. The positions of the WHM's (= Wave Height Meters) are:

Position WHM 1:

Position WHM 2:

Position WHM 3:

Position WHM 4:

Position WHM 5:

$$
\begin{aligned}
& x=-3.00 \mathrm{~m} \\
& x=3.50 \mathrm{~m} \\
& x=5.25 \mathrm{~m} \\
& x=6.15 \mathrm{~m} \\
& x=7.05 \mathrm{~m}
\end{aligned}
$$

These WHM positions can also be found in Figure 3.2.

In Appendix $\mathrm{C}$ a table is printed with all significant wave heights measured during the test series (see Table C.1).

Section 5.2 gives a short review of different situations that occur when waves approach the coast during the test series.

Section 5.3 compares some wave height measurements performed during a test. Three different wave height measurements are compared with each other. These measurements are taken in Interval 1, halfway Interval 3 and at the end of Interval 4. This is done to investigate the wave height development during an entire test.

Section 5.4 compares wave height measurements performed during tests with and without breakwater in Interval 1.

Section 5.5 gives a summary of all average wave heights of every experiment.

Finally, Section 5.6 describes the developments of the spectra during the experiments with and without breakwater.

\subsection{Short review of breaking, reflection and shoaling}

\section{Breaking}

When analysing the measured wave fields, suddenly decreasing wave heights can be observed. This can be a result of wave breaking. Wave breaking occurs when a wave approaches water which is gradually becoming shallower. The wave will be affected by the bottom when the water depth becomes less than half the wave length. Nearing the breaker line the wave celerity (c) and therefore the wave length (L) decreases while the wave height $(\mathrm{H})$ increases (approaching from deep water there will be initially a slight decrease in wave height). Therefore, a steeper wave profile develops. At a certain water depth the wave height (or in some cases the wave steepness) becomes so large that the wave will break and the wave energy will be dissipated. There is an upper limit for the wave height:

- due to a maximum wave steepness (H/L)

- due to a maximum wave height water depth ratio $(\mathrm{H} / \mathrm{h})$ 
For the first criterion, wave steepness, a limiting steepness is found to be :

$$
(H / L)_{\max }=(1 / 7) \tanh \left[\frac{2 \pi h}{L}\right]
$$

with:

$\mathrm{H}$ = wave height

L = wave length

h = water depth

In shallow water equation 5.1 becomes:

$$
(H / L)_{\max }=(1 / 7) 2 \pi h / L \approx 0.9 h / L
$$

Therefore $\mathrm{H}_{\max } \approx 0.9 \mathrm{~h}$ from which an upper limit for the second criterion (the wave height to water depth ratio) is found more or less automatically. The water depth at which the wave breaks is called the breaker depth. The ratio breaker wave height to breaker depth is often called the breaker index, denoted by $\gamma$.

$$
\gamma=H_{b} / h_{b}
$$

Solitary wave theory gives:

$$
H_{\max } / h=0.78
$$

A more practical and often used value for this breaker index is 0.6 .

\section{Reflection}

If the bottom slope has a steep profile like a submerged breakwater, reflection of waves can occur. Waves are partly reflected and partly transmitted. Dissipation of energy often occurs in such a situation. The steeper the bottom profile, the greater the wave reflection. A vertical wall will reflect practically all the wave energy and a standing wave will develop in front of the wall.

The following equation is valid for incoming waves:

Incoming wave energy $=$ Reflection + Transmission + Dissipation 


\section{Shoaling}

When waves approach the coast and the water depth becomes more shallow, wave heights increase. This phenomenon is called shoaling.

A more extensive and theoretical background on these phenomena can be found in Short Waves (in Dutch), Lecture Notes B76 by Battjes (1986).

\subsection{Comparing wave height development in the experiments}

This section describes wave height developments of some of the experiments performed. First, experiment $\mathrm{B}$ is described extensively. The reason for choosing this experiment is given in Chapter 4. Secondly, experiment A is described somewhat briefer. All other experiments are not described because the explanations would be the same as for the above mentioned experiments. All graphs of these wave height measurements are printed in Volume 2: Graphics, Part 2.

\section{Experiment B}

Experiment without breakwater (Figure 5.1)

Figure 5.1 shows three different wave height measurements during this experiment.

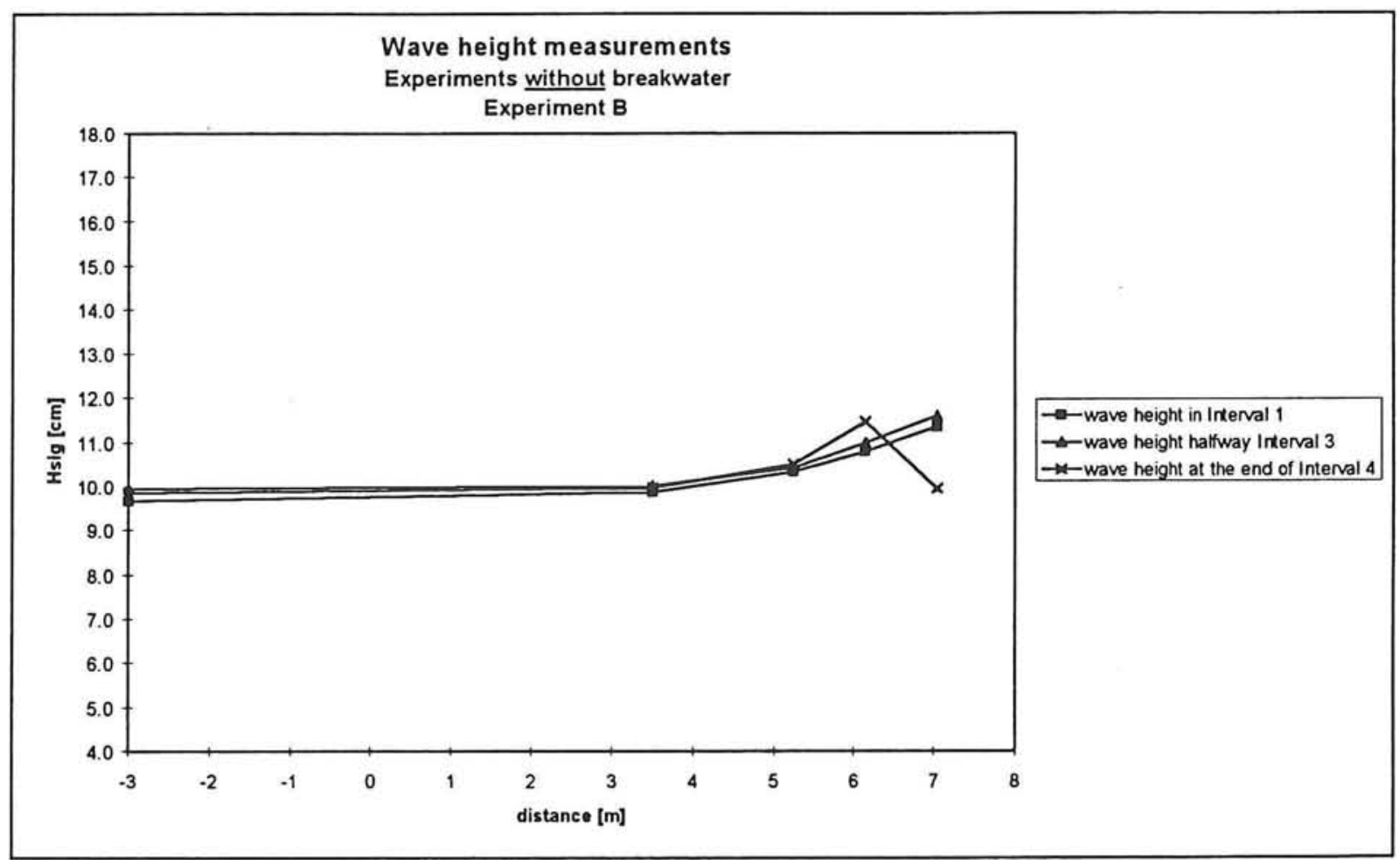

Figure 5.1 Wave height measurements during experiment B without breakwater. 
Wave heights in Interval 1 (measurement $\mathrm{t} 11$ )

At WHM 1 an incoming significant wave height of $9.7 \mathrm{~cm}$ is measured. When these waves further approach the coast to WHM 2 a slight increase in significant wave height to $9.9 \mathrm{~cm}$ can be noticed. This is due to the decreasing water depth when waves approach the coast. This decreasing water depth generates shoaling which increases the wave height. Between WHM 2 and WHM 3 the average wave height increases to $10.3 \mathrm{~cm}$ due to shoaling. The effect of shoaling increases as the water depth decreases. When the waves further approach the coast, shoaling increases the wave height to $10.8 \mathrm{~cm}$ at WHM 4 and to $11.4 \mathrm{~cm}$ at WHM 5 .

Wave heights halfway Interval 3 (measurement $\mathrm{t} 31$ )

After two and a half hours almost the same wave height development can be noticed. However, some differences can be noticed. The wave heights at WHM 4 and WHM 5 have slightly increased. This can be a result of a decreasing water depth at these WHM-positions. Sediment transport is in a seawards direction and therefore the bottom height increases (see Chapter 4) and shoaling occurs. Another explanation can be the slightly increased incoming wave height (from $9.7 \mathrm{~cm}$ in Interval 1 to $9.9 \mathrm{~cm}$ halfway Interval 3).

Wave heights at the end of Interval 4 (measurement t46)

After almost seven and a half hours the incoming wave height is still almost the same. At WHM 2 and WHM 3 there are hardly any differences with earlier measurements to be noticed. At WHM 4 the wave height increases to $11.5 \mathrm{~cm}$, which is considerably higher than earlier measurements at WHM 4. This is due to an increase in bottom height during this experiment. The bottom height increases approximately $5 \mathrm{~cm}$ and therefore the water depth decreases from approximately $23 \mathrm{~cm}$ halfway Interval 3 to $18 \mathrm{~cm}$ at the end of Interval 4 . This means that shoaling, and thus the wave height, will increase considerably. The breaker index $\gamma$ at the position of WHM 4 is:

WHM 4 (= Vertical (b): $\mathrm{x}=6.15 \mathrm{~m}$ )

Water depth $\quad=18.0 \mathrm{~cm}$

Average significant wave height $\quad=11.5 \mathrm{~cm}$

$\gamma=\frac{H s}{h}=11.5 / 18.0=0.63$

This is very close to the breaking criterion. Some (high) waves might be breaking already.

After WHM 4 the water depth decreases further due to sediment accumulation at the bottom. This smaller water depth leads to wave breaking due to exceeding the maximum wave height water depth ratio $(\mathrm{H} / \mathrm{h})$. Therefore, at WHM 5 the wave height has decreased considerably. The actual wave height water depth ratio can be calculated as follows: if the wave height at WHM 4 would also occur at WHM 5 the breaker index would be:

WHM 5 (=Vertical (c): $\mathrm{x}=7.05 \mathrm{~m}$ )

Water depth $\quad=13.0 \mathrm{~cm}$

Average significant wave height $\quad=11.5 \mathrm{~cm}$

$\gamma=H s / h=11.5 / 13.0=0.88$ 
This is not possible and therefore most waves are breaking between WHM 4 and WHM 5 . This leads to energy dissipation and thus to a lower wave height. Therefore a much lower wave height is measured at WHM 5.

\section{Experiment with breakwater (Figure 5.2)}

Figure 5.2 shows three different wave height measurements during this experiment.

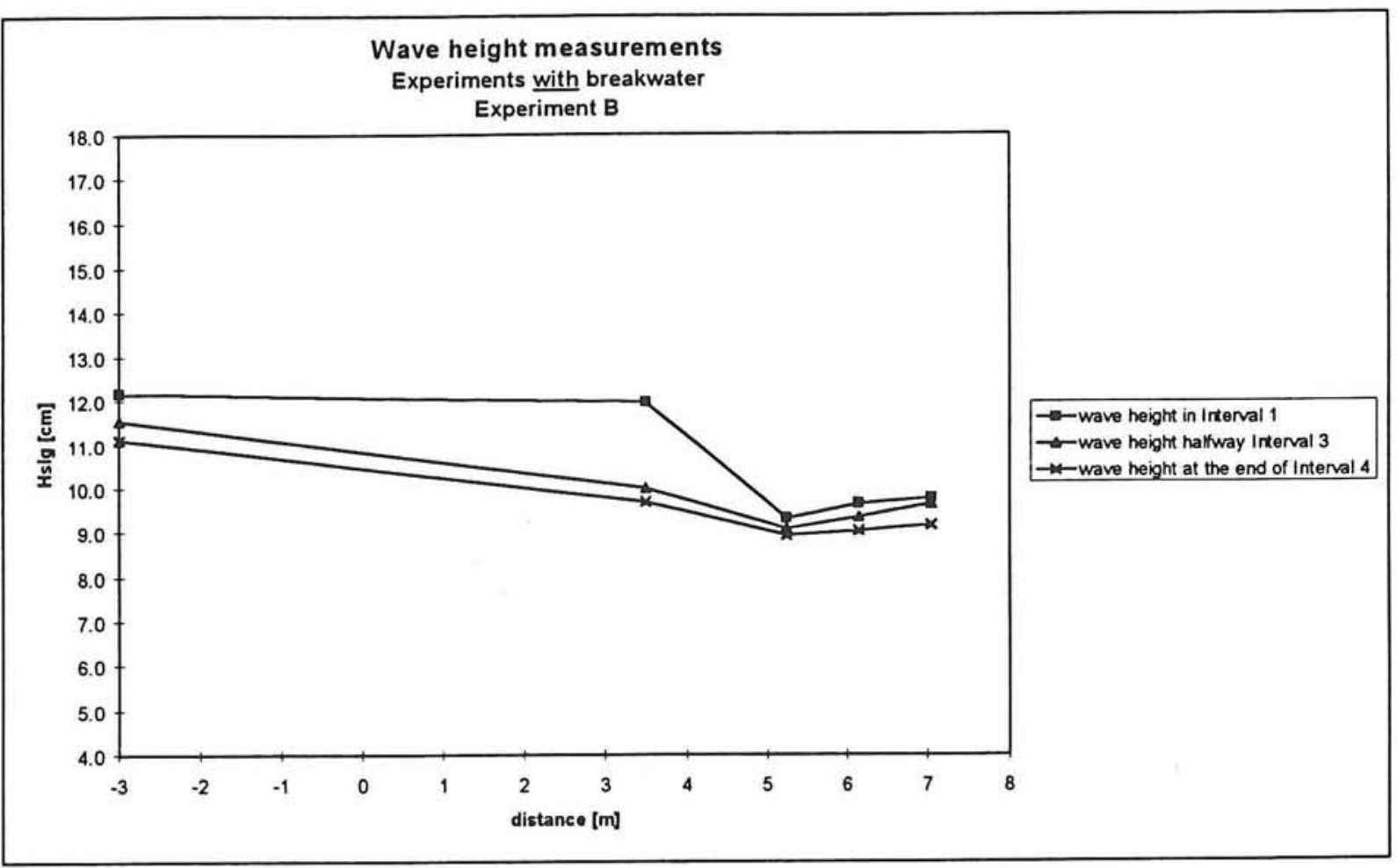

Figure 5.2 Wave height measurements during experiment B with breakwater.

Wave heights in Interval 1 (measurement t11)

At WHM 1 an average incoming wave height of $12.2 \mathrm{~cm}$ is measured. At WHM 2 this wave height has hardly changed. Between WHM 2 and WHM 3 the waves pass the submerged breakwater. Therefore, the incoming waves will partly reflect and partly transmit. The wave height will decrease considerably. This can be noticed in the wave height measurements at WHM 3. After WHM 3 the waves start to increase again due to shoaling.

Wave heights halfway Interval 3 (measurement $\mathrm{t} 31$ ) WHM 1 measured an average incoming wave height of $11.6 \mathrm{~cm}$. WHM 2 measured a wave height of $10.0 \mathrm{~cm}$. This wave height is much lower than the wave height at WHM 1 or the wave height at WHM 2 in Interval 1. An explanation for this could be as follows: the position of WHM 2 is just seaward of the submerged breakwater. Therefore, there is some interference of incoming and reflected waves. This, of course, affects the wave height measurements.

Between WHM 2 and WHM 3 the wave heights reduce again due to the effect of the breakwater on wave energy. Shoaling increases the wave heights after WHM 3. 
Wave heights at the end of Interval 4 (measurement t46)

At the end of Interval 4 the incoming significant wave height is $11.1 \mathrm{~cm}$ at WHM 1 . This wave height has decreased at WHM 2. Again, there is a big difference between measurements at WHM 1 and WHM 2. An explanation for this is given above. Over the breakwater the wave height further decreases. After WHM 3 the wave heights increase again due to shoaling.

A remark can be made on the similarity between wave height measurements at WHM 1 and WHM 5. The measurement with the highest incoming wave height at WHM 1 has also the highest wave height at WHM 5.

\section{Experiment A}

Experiments without breakwater (see Volume 2: Graphics, Part 2, Graph 2.1)

Graph 2.1 shows three different wave height measurements during this experiment.

Wave heights in Interval 1 (measurement $\mathrm{t} 11$ )

At WHM 1 an incoming wave height of $9.7 \mathrm{~cm}$ is measured. WHM 2 measures an almost equal wave height. After WHM 2 the wave height increases due to shoaling.

Wave heights halfway Interval 3 (measurement $\mathrm{t} 31$ )

This wave height measurement shows an almost identical wave height development as in Interval 1, except for wave heights, which are higher in Interval 3. These higher wave heights are a result of an increased bottom height, which increases shoaling.

Wave heights at the end of Interval 4 (measurement t46)

Again, the wave height development is similar to the first two measurements presented. The wave heights are higher than in Interval 3. A big difference occurs at WHM 5. Here, the wave height has decreased considerably. This is due to breaking of many waves between WHM 4 and WHM 5 (for an explanation, see Experiment B earlier in this section).

Experiments with breakwater (see Volume 2: Graphics, Part 2, Graph 2.7).

Graph 2.6 shows three different wave height measurements during this experiment.

Wave heights in Interval 1 (measurement $\mathrm{t} 11$ )

An incoming wave height of $10.1 \mathrm{~cm}$ is measured at WHM 1. Between WHM 2 and WHM 3 the waves pass the submerged breakwater and this decreases the wave heights at WHM 3 considerably. This is due to the reflection of waves in front of the breakwater. After WHM 3 wave heights increase again due to shoaling.

Wave heights halfway Interval 3 (measurement $\mathrm{t} 31$ )

In this interval the wave heights are almost equal to the wave heights in Interval 1. Only at WHM 4 and WHM 5 a very slight increase in wave heights can be noticed. This might be due to a slightly decreasing water depth. 
Wave heights at the end of Interval 4 (measurement t46)

In this interval the wave heights are considerably higher than in the previous intervals.

A remark can be made about the incoming wave heights at WHM 1 in every experiment with a breakwater present. When these wave heights are compared to incoming wave heights in experiments without a breakwater present, it can be noticed that in case of a breakwater present the incoming wave heights vary considerably during an experiment (see Figure 5.3 and Figure 5.4).

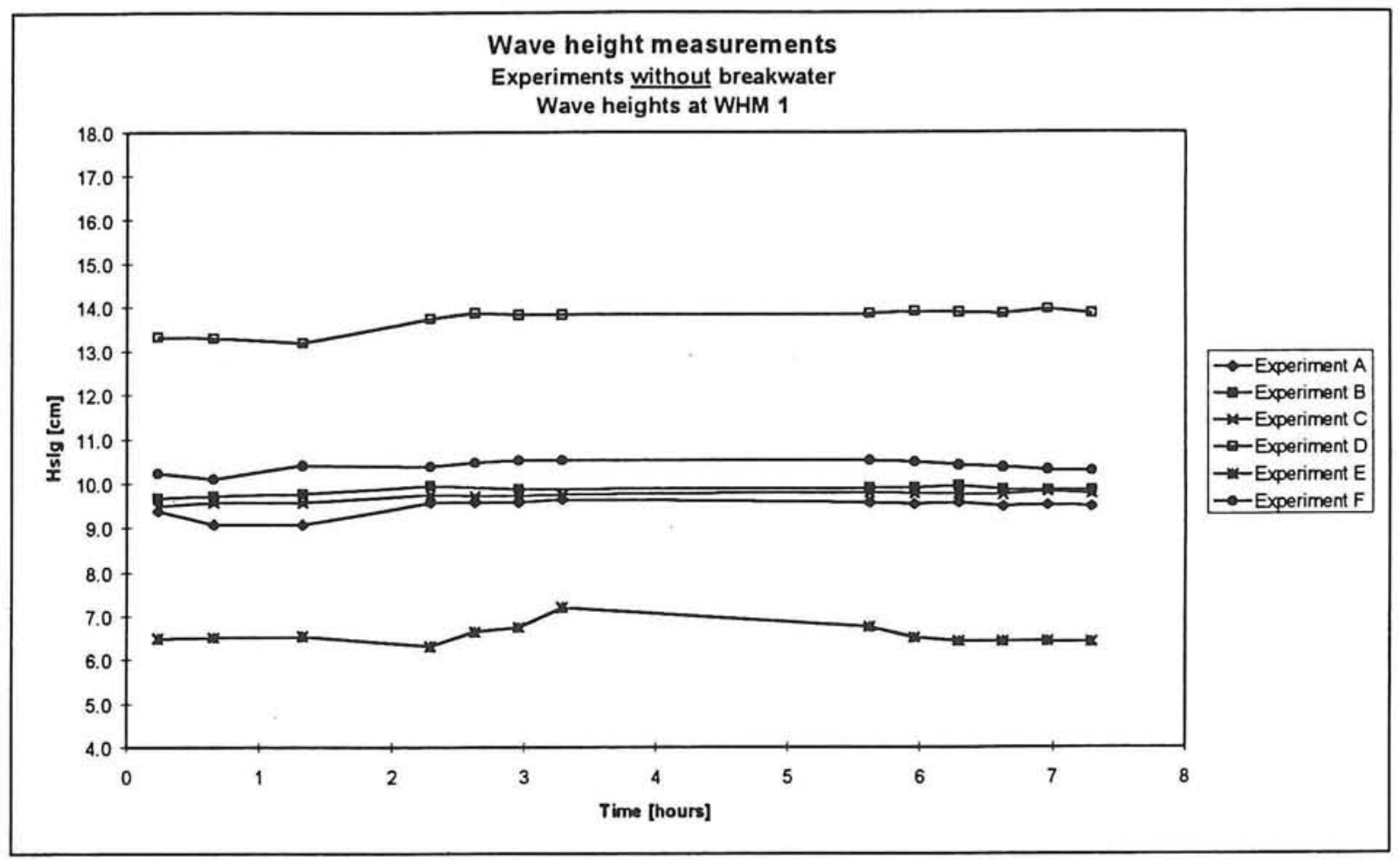

Figure 5.3 Wave height measurements at WHM 1 in experiments without breakwater. 


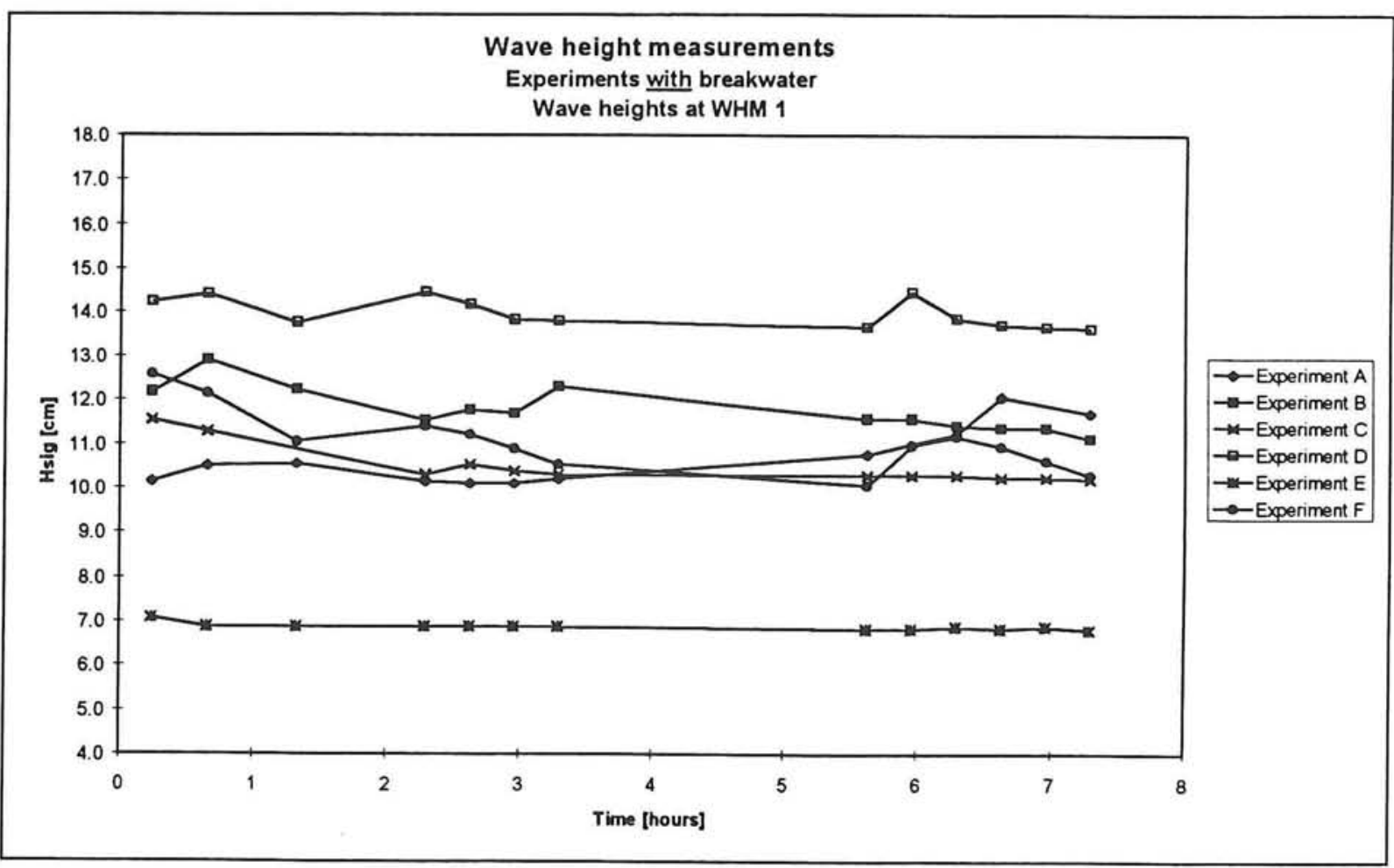

Figure 5.4 Wave height measurements at WHM 1 in experiments with breakwater.

\section{Other Experiments}

All other experiments are not described because of similarity with the above described experiments. The wave height graphs of these experiments are printed in Volume 2: Graphics, Part 2. These graphs will appear according to Table 5.1:

\begin{tabular}{|l|l|l|}
\hline Experiment: & Without breakwater & With breakwater \\
\hline C & Graph 2.3 & Graph 2.9 \\
D & Graph 2.4 & Graph 2.10 \\
E & Graph 2.5 & Graph 2.11 \\
F & Graph 2.6 & Graph 2.12 \\
\hline
\end{tabular}

Table 5.1 Index of graphs of other experiments in Volume 2: Graphics, Part 2.

\section{$\underline{5.4 \text { Comparing tests with and without breakwater }}$}

This section describes the comparisons made between a test performed with and without a breakwater present. These comparisons are made only in Interval 1 because during this interval the bed has undergone only minor changes. Therefore a true comparison can be made between two experiments. Again, only Experiments B and A are described. Other experiments have corresponding similarities and differences between the experiments with and without breakwater present. All graphs of these other experiments are printed in Volume 2: Graphics, Part 2. 


\section{Experiment B}

Experiments with and without breakwater at Interval 1 (see Figure 5.5)

Figure 5.5 shows the wave height measurements performed at Interval 1.

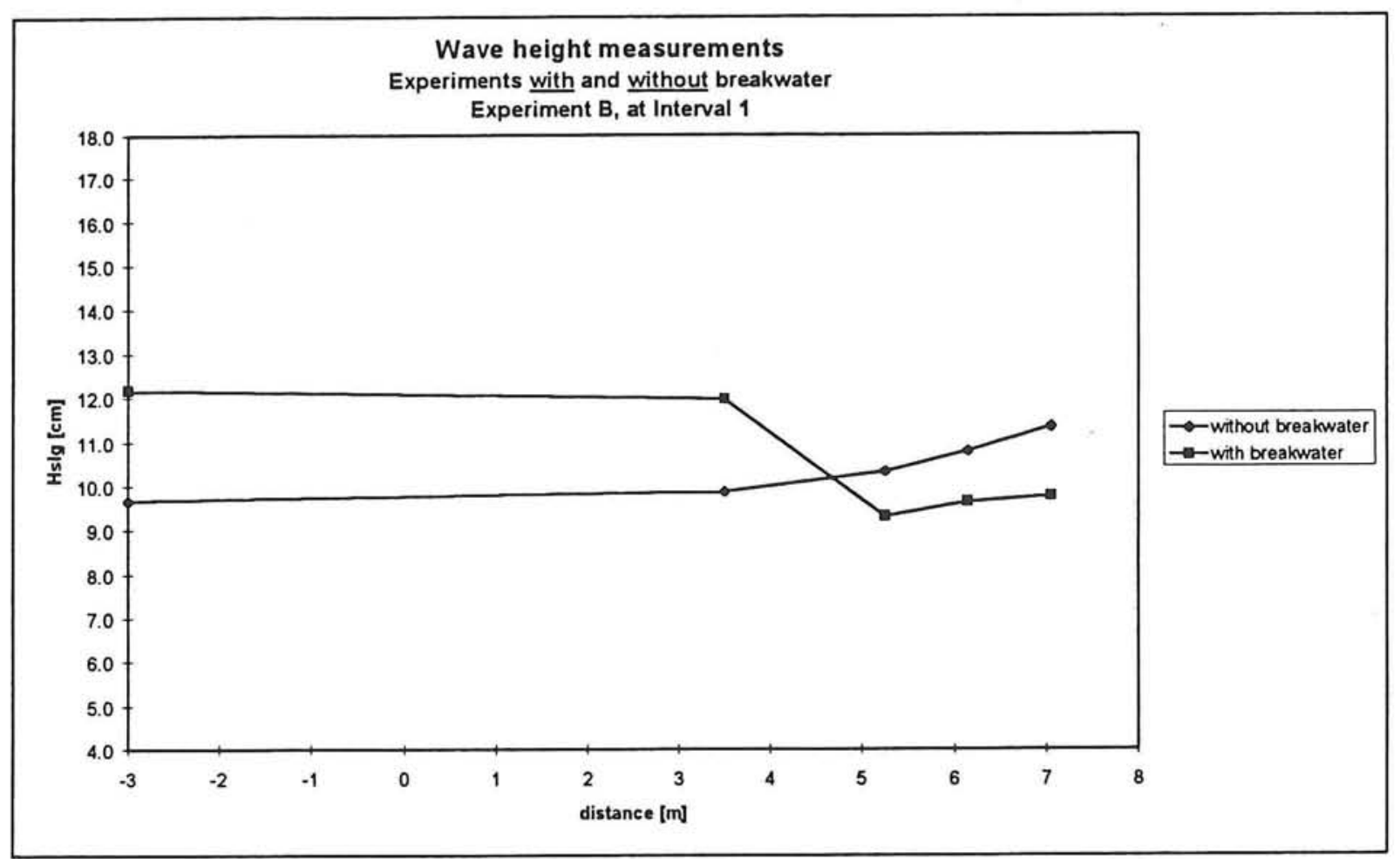

Figure 5.5 Wave height measurements during Experiment B with and without breakwater in Interval 1.

Although the generated wave board signal is equal, there is a big difference in incoming wave heights at WHM 1. In case of a breakwater present this average incoming wave height is 12.2 $\mathrm{cm}$. In the other case the wave height is $9.7 \mathrm{~cm}$. The reason for this is the presence of the submerged breakwater in one test. When this breakwater is present in the wave flume, there is much reflection of wave energy. This reflected wave energy increases the wave height seawards of the breakwater. This explains the big difference in incoming wave height between these two experiments. Between WHM 2 and WHM 3 big differences also occur, again this is due to the presence of the breakwater in one test. In this test the wave height decreases considerably due to wave energy reflection and wave energy dissipation caused by the breakwater. Therefore less energy is transmitted across the breakwater. This decreases the wave height landwards of this breakwater, while in the other test the wave height increases due to shoaling. After WHM 3 the wave height increases in both tests due to shoaling. During the test series, no specific reflection measurements were performed. 


\section{Experiment A}

Experiments with and without breakwater at Interval 1 (see Volume 2: Graphics, Part 2, Graph 2.13)

Graph 2.13 shows the wave height measurements performed at Interval 1.

Again, there is a big difference in incoming wave heights at WHM 1. In case of a breakwater present this average incoming wave height is $10.1 \mathrm{~cm}$. In the other case the wave height is 9.4 $\mathrm{cm}$. Like in Experiment B, this difference is a result of wave energy reflection in case of a breakwater present. This reflection of wave energy considerably increases the wave height seawards of the breakwater. Between WHM 2 and WHM 3 another difference can be noticed. This is due to the presence of a breakwater in one test. In this test the wave height decreases considerably due to less wave energy landwards of the breakwater (see explanation Experiment B), while in the other test the wave height increases due to shoaling. After WHM 3 the wave height increases in both tests due to shoaling.

\section{Other Experiments}

The graphs of all other experiments are printed in Volume 2: Graphics, Part2. These graphs will appear according to Table 5.2:

\begin{tabular}{|l|l|}
\hline Experiment: & With and without breakwater \\
\hline C & Graph 2.15 \\
D & Graph 2.16 \\
E & Graph 2.17 \\
F & Graph 2.18 \\
\hline
\end{tabular}

Table 5.2 Index of graphs of other experiments in Volume 2: Graphics, Part 2.

\subsection{Average wave heights of the experiments}

An average incoming significant wave height at WHM 1 can be calculated for every experiment. During an experiment, 13 wave height measurements are performed. All these wave height measurements have to be taken into account in order to get a true average wave height. The following method of calculation is used.

During all four intervals wave heights are measured (see Graph 2.19 and Graph 2.20). Every interval has a different duration. It is important to acknowledge the difference of wave heights measured in a four hour lasting interval and wave heights measured in a one hour lasting interval. Therefore, a weight distribution to the different intervals was given according to Table 5.3. 


\begin{tabular}{|l|l|l|}
\hline Interval: & Duration: & Weight: \\
\hline $\mathbf{1}$ & 0.5 hour & 1 \\
$\mathbf{2}$ & 1.0 hour & 2 \\
$\mathbf{3}$ & 2.0 hours & 4 \\
$\mathbf{4}$ & 4.0 hours & 8 \\
\hline
\end{tabular}

\section{Table 5.3 Weight of the different intervals.}

First, the average wave height of every interval is calculated. This is done by adding all wave height measurements performed during an interval and dividing the outcome by the number of measurements. This results in an average wave height per interval. These average wave heights per interval are multiplied by the weight of that interval. This is done for every interval. These outcomes are added and the overall outcome is divided by 15 (total weight of an experiment).

The calculated average wave heights are listed in Table 5.4 and Table 5.5.

These tables also present the standard deviations of the average wave heights. (To calculate these standard deviations each measured wave height was given the same weight). As stated earlier, the measured wave heights of the experiments with breakwater vary more than the wave heights of the experiments without breakwater (see Figure 5.3 and Figure 5.4). Therefore the standard deviations of the average wave heights of the experiments with breakwater are relatively large.

\begin{tabular}{|l|c|c|}
\hline Experiment: & Average $\mathbf{H}_{\text {sig }}$ [m] at WHM 1 & Standard deviation [m] \\
\hline A without breakwater & 0.095 & 0.0018 \\
B without breakwater & 0.098 & 0.0009 \\
C without breakwater & 0.097 & 0.0010 \\
D without breakwater & 0.137 & 0.0025 \\
E without breakwater & 0.066 & 0.0022 \\
& & \\
A with breakwater & 0.108 & 0.0066 \\
B with breakwater & 0.118 & 0.0048 \\
C with breakwater & 0.105 & 0.0043 \\
D with breakwater & 0.140 & 0.0031 \\
E with breakwater & 0.069 & 0.0006 \\
\hline
\end{tabular}

Table $5.4 \quad$ Average significant wave heights and standard deviations of the experiments with irregular waves at WHM 1.

\begin{tabular}{|l|l|l|}
\hline Experiment: & Average $\mathbf{H}[\mathrm{m}]$ at WHM 1 & Standard deviation $[\mathrm{m}]$ \\
\hline F without breakwater & 0.104 & 0.0012 \\
F with breakwater & 0.111 & 0.0067 \\
\hline
\end{tabular}

Table 5.5 Average wave heights and standard deviations of the experiments with regular waves at WHM 1.

A summary of all wave height measurements is printed in Appendix C (see Table C.1). 


\subsection{Spectra}

\subsubsection{Computation of the spectra}

The spectral variance density functions $E(f)$ of the experiments with irregular waves have been computed with the program SPECTPER. The program uses a periodogram, being an amplitude spectrum, as input to compute a spectrum. The periodogram is computed with the program FILTER. FILTER uses a FFT (Fast Fourier Transformation) to create the periodogram. SPECTPER and FILTER are part of AUKE/PC software package. A description of the theoretical background of these programs can be found in Appendix A.

To investigate the influence of the breakwater it was decided to compare the spectra at two positions, viz. at the positions of WHM 1 and WHM 3 (see Figure 3.2). WHM 1 is located 3 meters in front of the beginning of the slope $(x=-3.00 \mathrm{~m})$. WHM 3 is located landward of the breakwater (if the experiment is with breakwater of course). By comparing the spectra at these two locations the effect of the breakwater can be investigated.

The measurements during Interval 1 were used to calculate the spectral densities. At Interval 1 the bottom profile changes are so small that the effect on the wave height measurements can be neglected.

Graphs have been made of the spectra. They are presented in Volume 2: Graphics, Part 2.

Table 5.6 presents a list of these graphs. It should be stressed that the scale of the vertical axis is different per experiment. This was done to maintain the surveyability because the differences between the two curves in a graph are often very small. In the next sections the development of the spectra is discussed.

\begin{tabular}{|l|l|}
\hline & Experiment \\
\hline Graph 2.21 & experiment A without breakwater \\
Graph 2.22 & experiment B without breakwater \\
Graph 2.23 & experiment C without breakwater \\
Graph 2.24 & experiment D without breakwater \\
Graph 2.25 & experiment E without breakwater \\
Graph 2.26 & experiment A with breakwater \\
Graph 2.27 & experiment B with breakwater \\
Graph 2.28 & experiment C with breakwater \\
Graph 2.29 & experiment D with breakwater \\
Graph 2.30 & experiment E with breakwater \\
\hline
\end{tabular}

Table 5.6 Review of the graphs that are made of the spectra of the experiments. Graphs are presented in Volume 2: Graphics, Part 2.

\subsubsection{Experiments without breakwater}

The spectra of the experiments without breakwater are almost the same at WHM 1 and WHM 3. There are, however, changes at the higher frequencies. This will be explained by the graph of experiment B that is shown in Figure 5.6. 


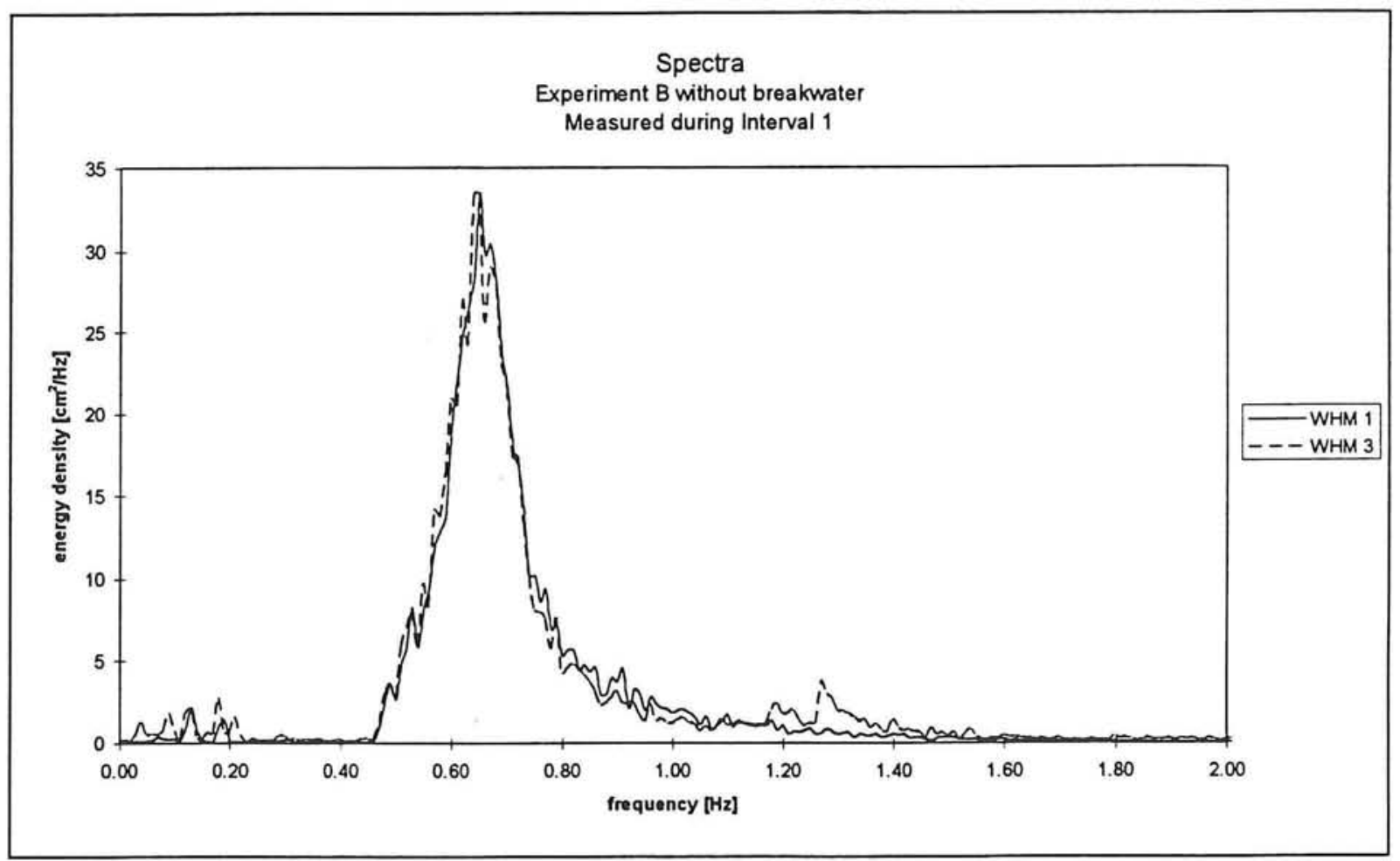

Figure 5.6 Spectra of experiment B without breakwater.

From a frequency of about $0.75 \mathrm{~Hz}$ till a frequency of about $1.10 \mathrm{~Hz}$ a slight decrease in spectral density can be noticed from WHM 1 to WHM 3. From approximately $1.10 \mathrm{~Hz}$ till $1.60 \mathrm{~Hz}$ the spectral density increases from WHM 1 to WHM 3. The peak period does not change. The other experiments show the same type of development.

Table 5.7 presents a list of the $m_{0}$-parameters. The $m_{0}$-parameter is directly related to the total amount of energy of the waves. The $m_{0}$-parameter is the first order moment of the spectrum, in other words, it is the area beneath the spectral variance density curve (see Equation 5.5). The total average energy of the wave field per unit surface area itself can be found by multiplying the $\mathrm{m}_{0}$-parameter by $0.5 \rho \mathrm{g}$ (see Equation 5.6). Therefore the spectral variance density function is often called the wave energy spectrum.

$m_{0}=\int_{0}^{\infty} E(f) \cdot d f$

with:

$\mathrm{m}_{0} \quad=$ first order moment of the spectrum

$\mathrm{E}(\mathrm{f}) \quad=$ spectral variance density function

$\mathrm{f}=$ frequency in cycles per unit time

$$
\bar{E}=0.5 \cdot \rho \cdot g \int_{0}^{\infty} E(f) \cdot d f
$$

with:

$\overline{\mathrm{E}} \quad=$ total average energy per unit surface area 


\begin{tabular}{|l|c|c|}
\hline & $\mathbf{m}_{\mathbf{0}}$ at WHM 1 $\left[\mathrm{cm}^{2}\right]$ & $\mathbf{m}_{0}$ at WHM 3 $\left[\mathrm{cm}^{2}\right]$ \\
\hline experiment A without breakwater & 5.7 & 5.9 \\
experiment B without breakwater & 6.1 & 6.3 \\
experiment C without breakwater & 5.8 & 5.1 \\
experiment D without breakwater & 11.6 & 12.2 \\
experiment E without breakwater & 2.8 & 2.6 \\
\hline
\end{tabular}

Table 5.7 List of values of first order moments of the spectra of the measurements during Interval 1.

The amount of energy increases a little from WHM 1 to WHM 3 for the experiments A, B and $D$. This is caused by shoaling. The wave height increases and as a result of that the average energy per surface area increases. Experiment $\mathrm{C}$ and $\mathrm{E}$ show a little decrease of the total amount of energy. The effect of dissipation of energy of the waves must have effected the average amount of energy per surface area more than the shoaling process.

To check whether the values of the $m_{0}$-parameter are reliable, also the $m_{0}$-parameters of the two measurements during Interval 2 have been computed. These values also show higher values for the $\mathrm{m}_{0}$-parameter at WHM 3 for the experiments A, B and D (see Appendix D).

\subsubsection{Experiments with breakwater}

The differences between the spectral densities at WHM 1 and WHM 3 of the experiments with breakwater are much bigger. There is a sharp decrease in spectral density around the peak frequencies. The breakwater fulfils its task and causes a lot of dissipation. Figure 5.7 shows the graph of experiment B with breakwater.

It should be mentioned that the effect of the reflection of waves against the breakwater and the wave board have a significant effect on the total amount of energy measured at WHM 1. Table 5.8 presents the values of the $\mathrm{m}_{0}$-parameter. 


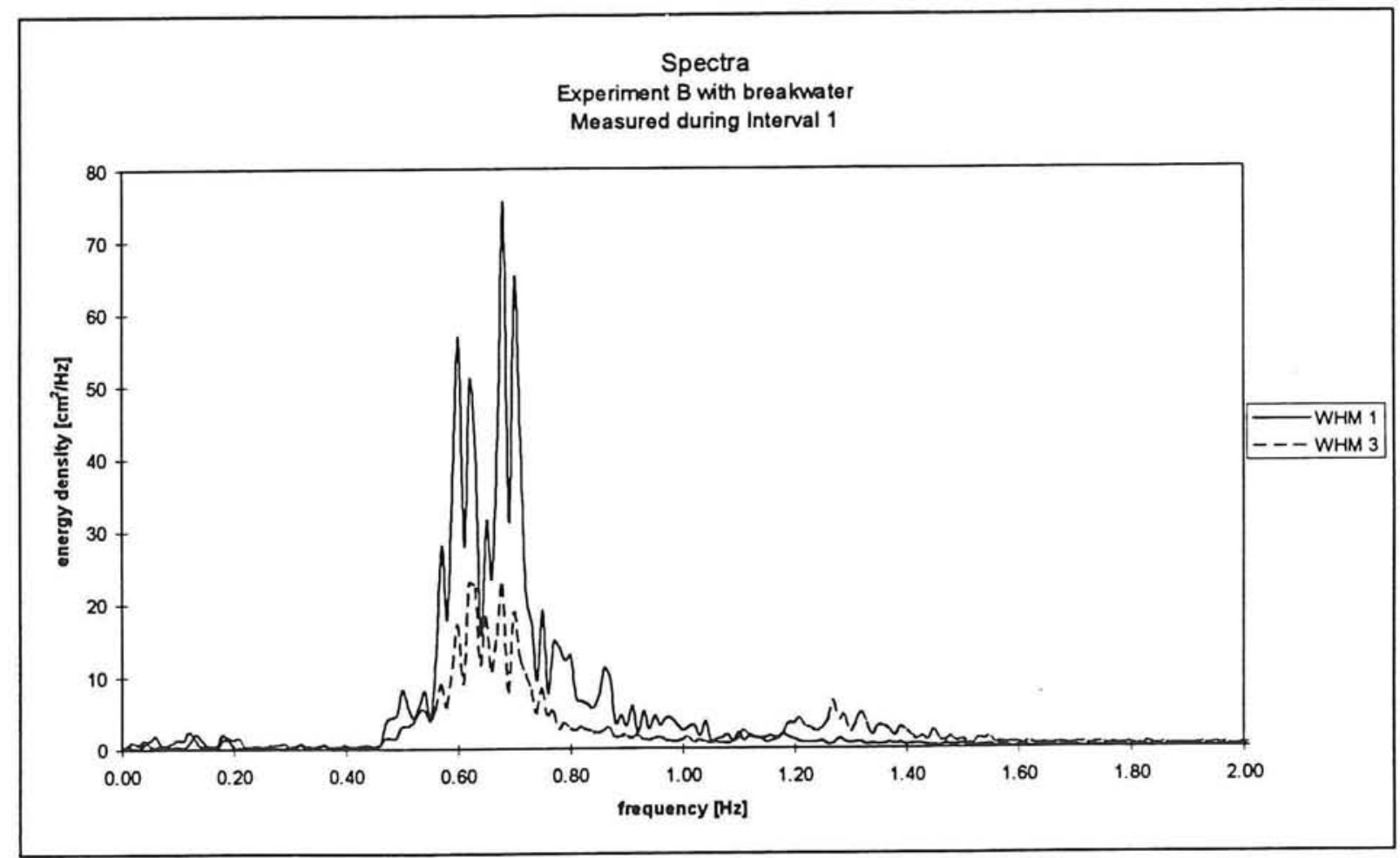

Figure 5.7 Spectra of experiment B with breakwater.

\begin{tabular}{|l|c|c|}
\hline & $\mathbf{m}_{\mathbf{0}}$ at WHM $\mathbf{1}\left[\mathbf{c m}^{2}\right]$ & $\mathbf{m}_{\mathbf{0}}$ at WHM 3 $\left[\mathbf{c m}^{2}\right]$ \\
\hline experiment A with breakwater & 6.9 & 4.4 \\
experiment B with breakwater & 9.5 & 4.9 \\
experiment C with breakwater & 8.3 & 4.2 \\
experiment D with breakwater & 13.2 & 6.8 \\
experiment E with breakwater & 3.3 & 2.3 \\
\hline
\end{tabular}

Table 5.8 List of values of first order moments of the spectra of the measurements during Interval 1.

A comparison between the experiments with and without breakwater under similar wave conditions (e.g., experiment B with breakwater versus experiment B without breakwater) shows that the total amount of energy measured at WHM 1 is significantly more in case of the experiments with breakwater.

At WHM 3 the amount of energy is less in case of the experiments with breakwater. This is caused by the dissipation of the energy due to the breakwater.

Like the experiments without breakwater there is a strong increase in spectral density at the higher frequencies.

In case of experiment B there is an increase in spectral density from a frequency of about 1.15 $\mathrm{Hz}$. The increase in frequency after the breakwater could be noticed very well during the experiments.

The spectral density around the peak frequency is very irregular. This could be caused by the interference of the waves. Waves that propagate in two directions can either amplify or dampen each other. 


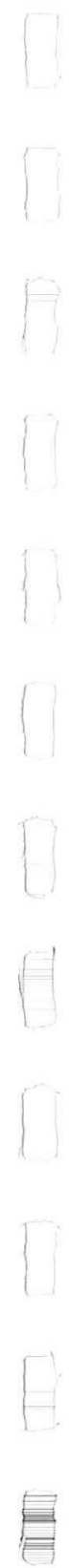

㞏 


\section{CHAPTER 6 VELOCITY MEASUREMENT RESULTS}

\subsection{Introduction}

In this chapter the velocity measurement results are described. All velocity measurement results have been computed by STATIST, a program of AUKE/PC. The positions of the EMS's (= electromagnetic fluid-velocity meters) are:

$\begin{array}{lll}\text { Position EMS 1: } & \mathrm{x}=5.25 \mathrm{~m} & \text { (referred to as Vertical (a)) } \\ \text { Position EMS 2: } & \mathrm{x}=6.15 \mathrm{~m} & \text { (referred to as Vertical (b)) } \\ \text { Position EMS 3: } & \mathrm{x}=7.05 \mathrm{~m} & \text { (referred to as Vertical (c)) }\end{array}$

These EMS positions can also be found in Figure 3.2.

All velocity measurement results showed in this chapter (and in Volume 2: Graphics, Part 3) are performed during Interval 4. This interval was choosen because of its many measuring positions. During this interval, six velocity measurements per vertical are performed over the entire cross-section. More detailed information about the performance of these measurements can be found in Chapter 3.

Section 6.2 describes the strategy concerning the execution of the velocity measurements.

Section 6.3 compares the velocity measurements performed in all three verticals per experiment.

The next section describes the comparisons made, per vertical cross-section, between an experiment with and an experiment without a breakwater.

\subsection{Strategy for performing the velocity measurements}

All graphs show distributions of the time-average velocities, which were measured during Interval 4 . In this interval, six velocity measurements were performed over the entire crosssection. An attempt was made, to measure the velocity distribution over the entire water depth. The highest measuring position was just below the deepest wave troughs (an EMS only measures accurately if its probe is submerged all the time). The lowest position was approximately $2.5 \mathrm{~cm}$ above the bed. In order to do this the EMS-probes are positioned at different locations across the entire vertical cross-sections. From the sidewall of the flume these two horizontal levels, between which the probes can be positioned, are determined.

Before the first velocity measurement starts these two levels are determined, this in order to calculate the step-size between two measurements beforehand. The step-size is calculated as follows.

The lowest measuring position is substracted from the highest measuring position. This outcome is divided by 5 . This results in the step-size.

The highest position is determined by the wave trough. For a few minutes the wave troughs were observed and the lowest wave trough determined the position for the probe. The lowest position is determined by the bottom height (see Figure 6.1). 


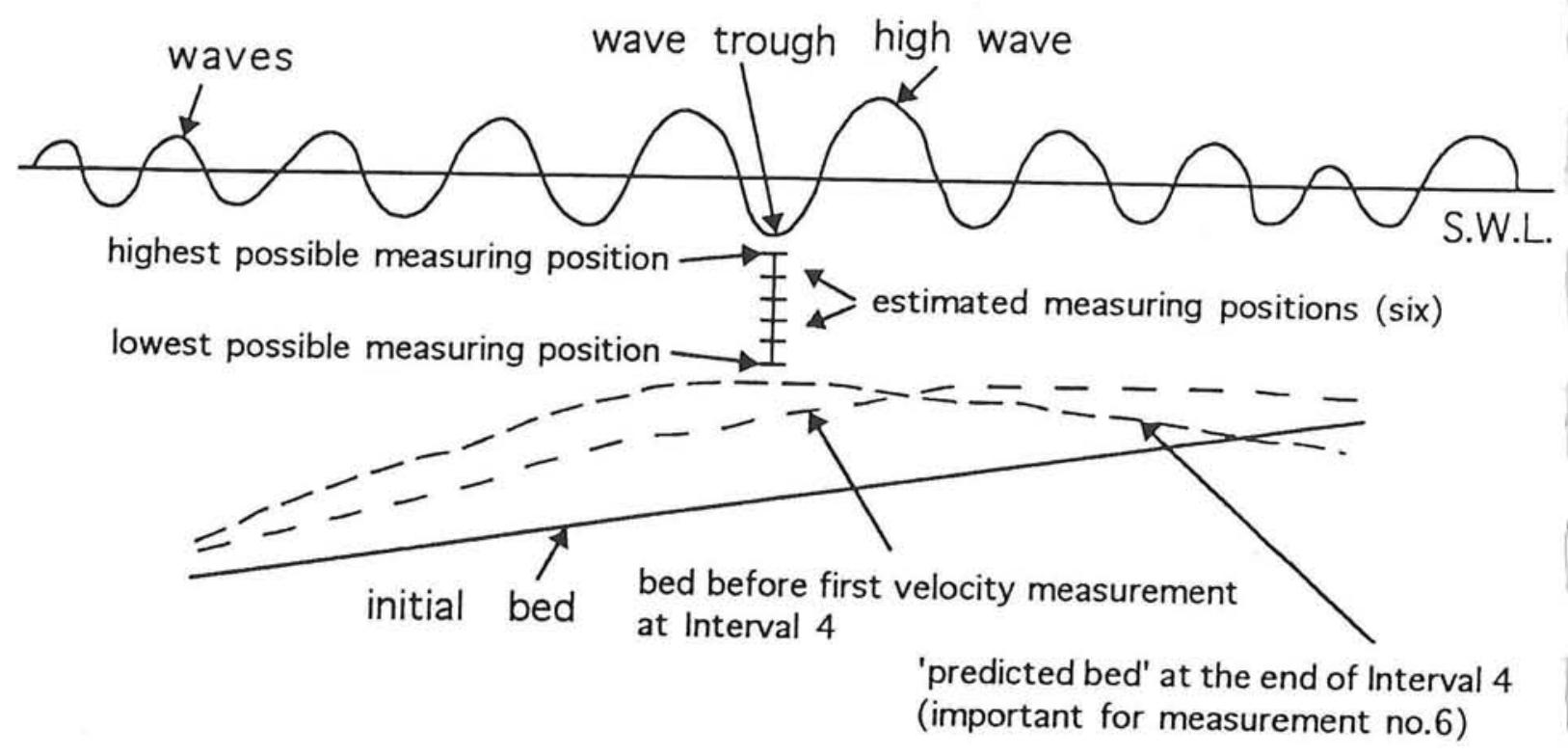

Figure 6.1 Estimating the highest and lowest possible levels for velocity measurements.

A problem with this lowest position is to predict the bed elevation on beforehand (it takes about 2 hours to perform 6 consecutive velocity measurements, therefore one has to predict the bed elevation approximately 2 hours ahead).

This attempt to measure as much as possible of every cross-section leads to different vertical measuring positions in every experiment. The lower measuring positions vary due to different bottom elevations in every experiment. The higher measuring positions vary due to differences in the pattern of wave breaking, which is located at different positions in every experiment. Especially when waves are breaking (with lots of air bubbles in the water) nearby a vertical cross-section of measurements this can lead to very small verticals for measurements. Therefore, all graphs show a different pattern of measuring positions and this makes it very difficult to compare velocity distributions from different verticals with each other. It is even harder to compare different experiments with each other. However, an attempt is made in the next sections to describe the velocity distributions.

\subsection{Comparing velocity measurements in all three verticals of an experiment}

This section describes the velocity distributions in all three verticals of Experiment B. All other experiments are not described. The reason for choosing Experiment B is given in Chapter 4 . All graphs of the other experiments are printed in Volume 2: Graphics, Part 3 (see Table 6.1). All graphs show a velocity distribution, which is measured during Interval 4. 


\section{Experiment B}

Experiment without breakwater (Figure 6.2)

Figure 6.2 shows the time-averaged velocity distributions in all three verticals during Interval 4 in this experiment.

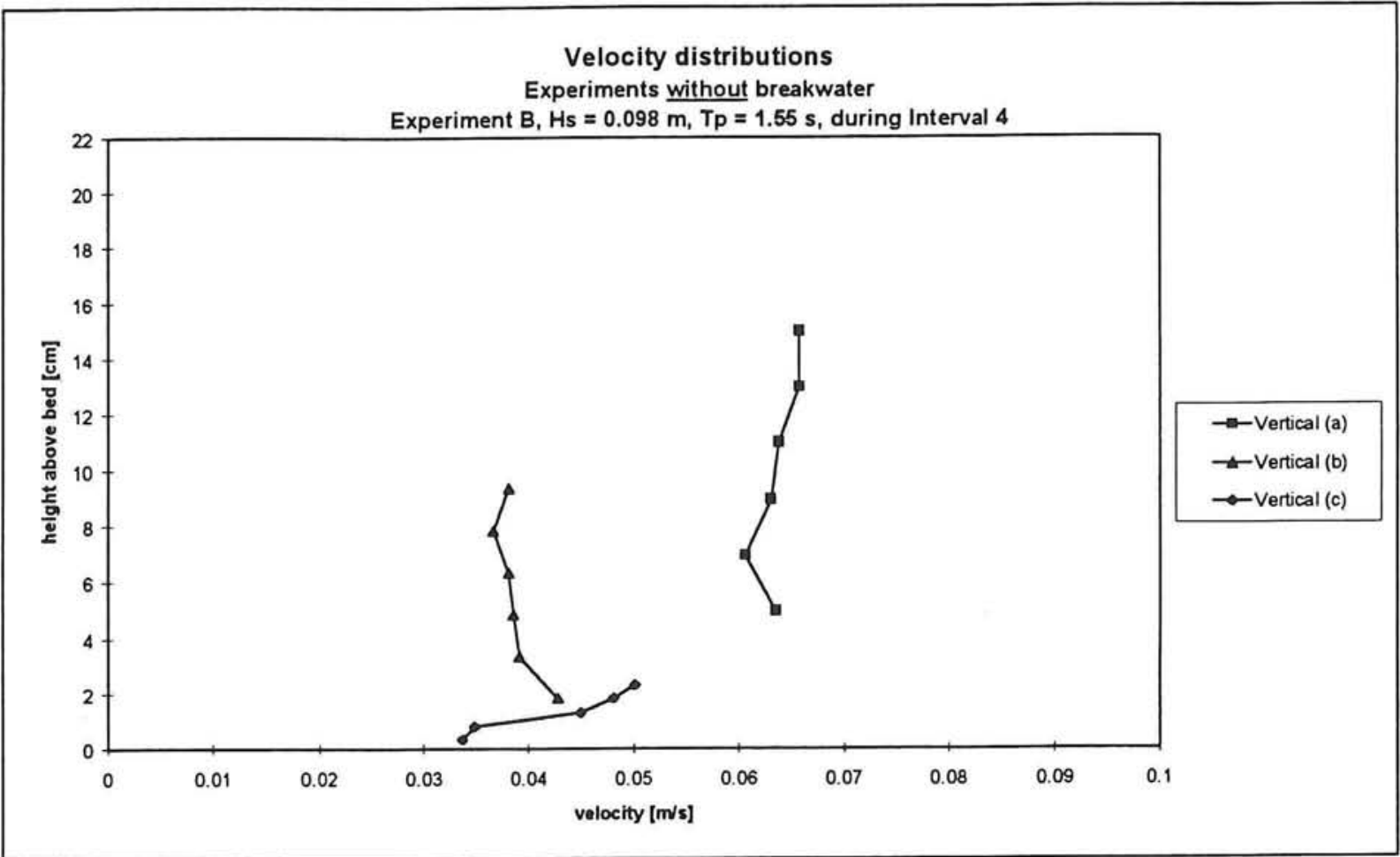

Figure 6.2 Velocity distributions of Experiment B without breakwater (positive values of average velocities are in seaward direction).

Vertical (a) shows a constant average velocity of approximately $0.065 \mathrm{~m} / \mathrm{s}$. In Vertical (b) the velocities have decreased to approximately $0.038 \mathrm{~m} / \mathrm{s}$. Vertical (c) shows a decrease in velocities over the measured depth, going from the wave trough down to the bottom. These measurements are taken very close to the bed and the EMS may be effected by the bed transport and/or the bottom.

Experiment with breakwater (Figure 6.3)

Figure 6.3 shows the velocity distributions in all three Verticals during Interval 4 in this experiment. 


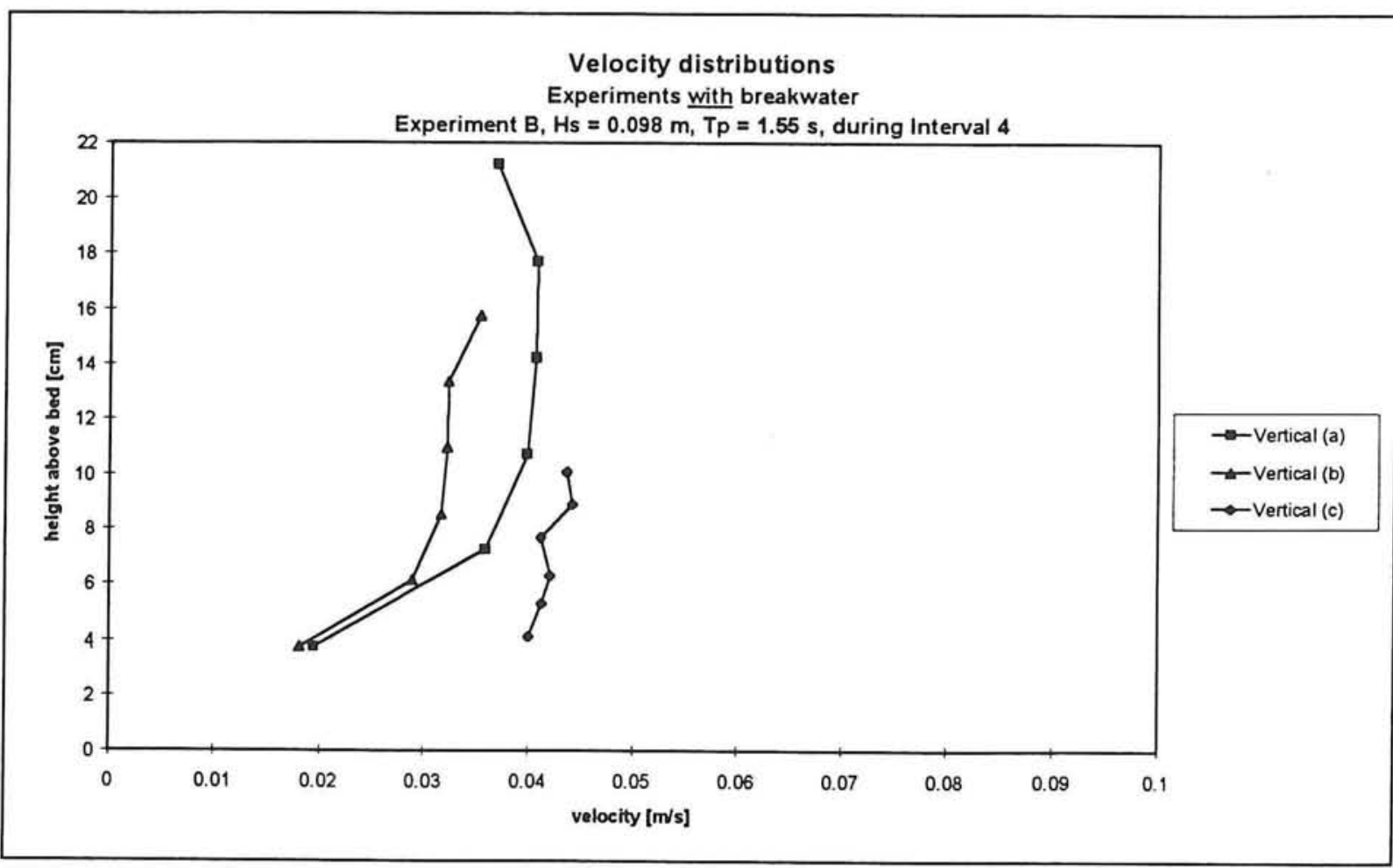

Figure 6.3 Velocity distributions of experiment B with breakwater (positive values of average velocities are in seaward direction).

Vertical (a) shows an almost constant average velocity in the upper four measuring positions of approximately $0.039 \mathrm{~m} / \mathrm{s}$. The two lower measuring positions show a decrease in velocities. This may be an effect of the presence of the breakwater (Vertical (a) is located just landwards of the breakwater). Vertical (b) shows a similar velocity distribution, with slightly lower values. In Vertical (c) there is a constant average velocity over the measured depth. Velocities in this Vertical have a value of about $0.042 \mathrm{~m} / \mathrm{s}$.

\section{Other Experiments}

All graphs of other experiments are printed in Volume 2: Graphics, Part 3. These graphs will appear according to Table 6.1.

\begin{tabular}{|l|l|l|}
\hline Experiment: & Without breakwater & With breakwater \\
\hline A & Graph 3.1 & Graph 3.6 \\
B & Graph 3.2 & Graph 3.7 \\
C & Graph 3.3 & Graph 3.8 \\
D & Graph 3.4 & Graph 3.9 \\
E & Graph 3.5 & Graph 3.10 \\
\hline
\end{tabular}

Table 6.1 Index of graphs of all experiments in Volume 2: Graphics, Part 3. 
6.4 Comparing velocity measurements per vertical of experiments with and without breakwater

This section compares the velocity distributions per Vertical of experiment B with and without breakwater.

\section{Experiment B}

Vertical (a)

Figure 6.4 shows the velocity measurements with and without breakwater at Vertical (a)

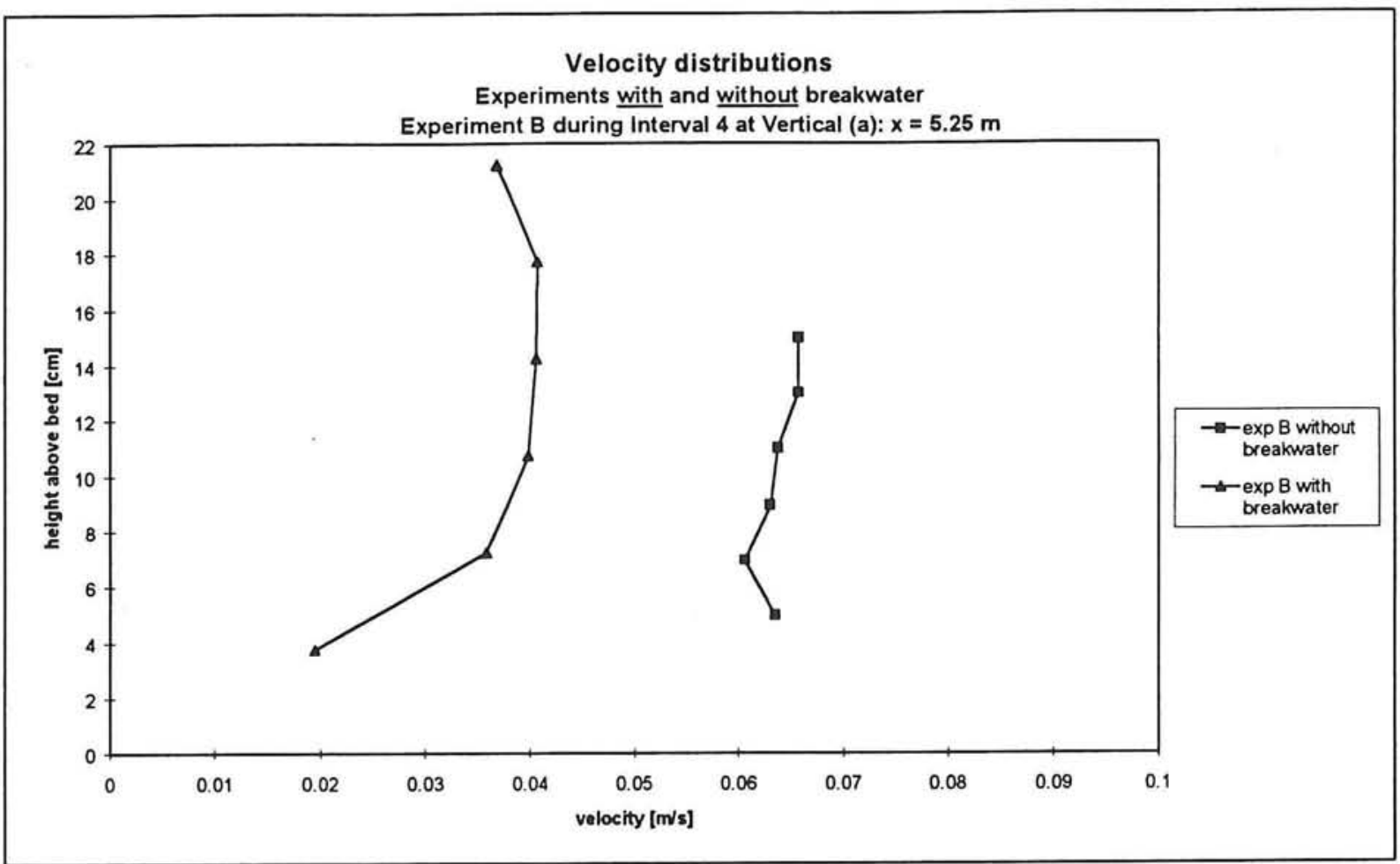

Figure 6.4 Velocity distributions at Vertical (a) during experiment B with and without breakwater (positive values of average velocities are in seaward direction).

This figure shows there is a difference in average velocities between an experiment with and an experiment without a breakwater present. The presence of a breakwater reduces the average velocities in Vertical (a). Another difference is the upper level of positioning the EMS for measuring the velocities. In case of a breakwater present this position is located much higher than in case of no breakwater present. This is due to the reducing effect of the breakwater on wave heights. Reduced wave heights lead to an increasing waterdepth below the wave trough. Therefore, it is possible to measure at a higher level in case of a breakwater present.

Vertical (b)

Figure 6.5 shows the velocity measurements with and without breakwater at Vertical (b) 


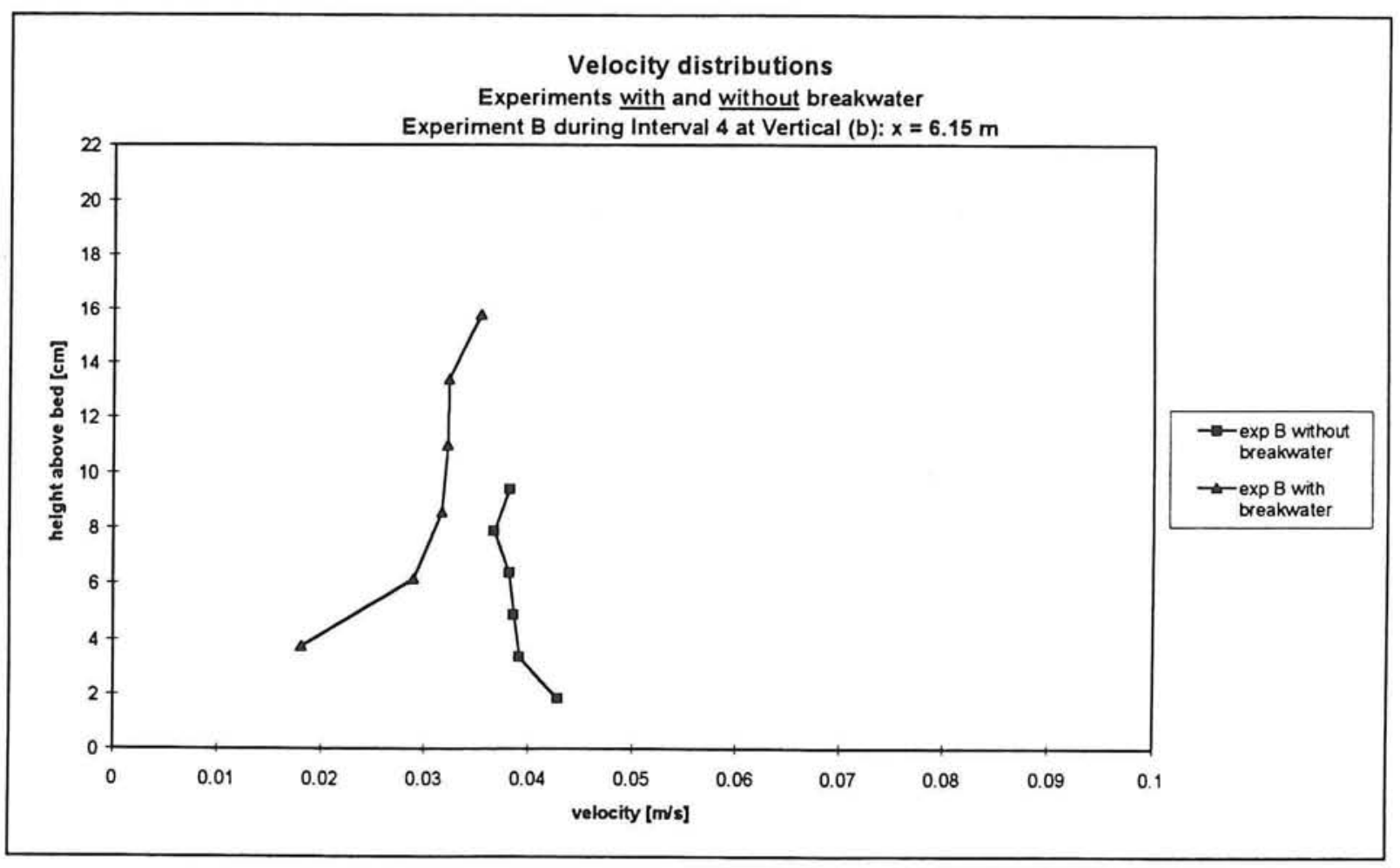

Figure 6.5 Velocity distributions at Vertical (b) during experiment B with and without breakwater (positive values of average velocities are in seaward direction).

Again, there is a difference between the experiment with and the experiment without a breakwater. The presence of a breakwater reduces the average velocities in Vertical (b) also. However, this difference is much less than at Vertical (a). This is due to the fact that Vertical (b) is located further away from the breakwater than Vertical (a). Like at Vertical (a) it is, in case of a breakwater present, possible to start the measurements at a higher level than in case of no breakwater present (for explanation, see Vertical (a)).

Vertical (c)

Figure 6.6 shows the velocity measurements with and without breakwater at Vertical (c) 


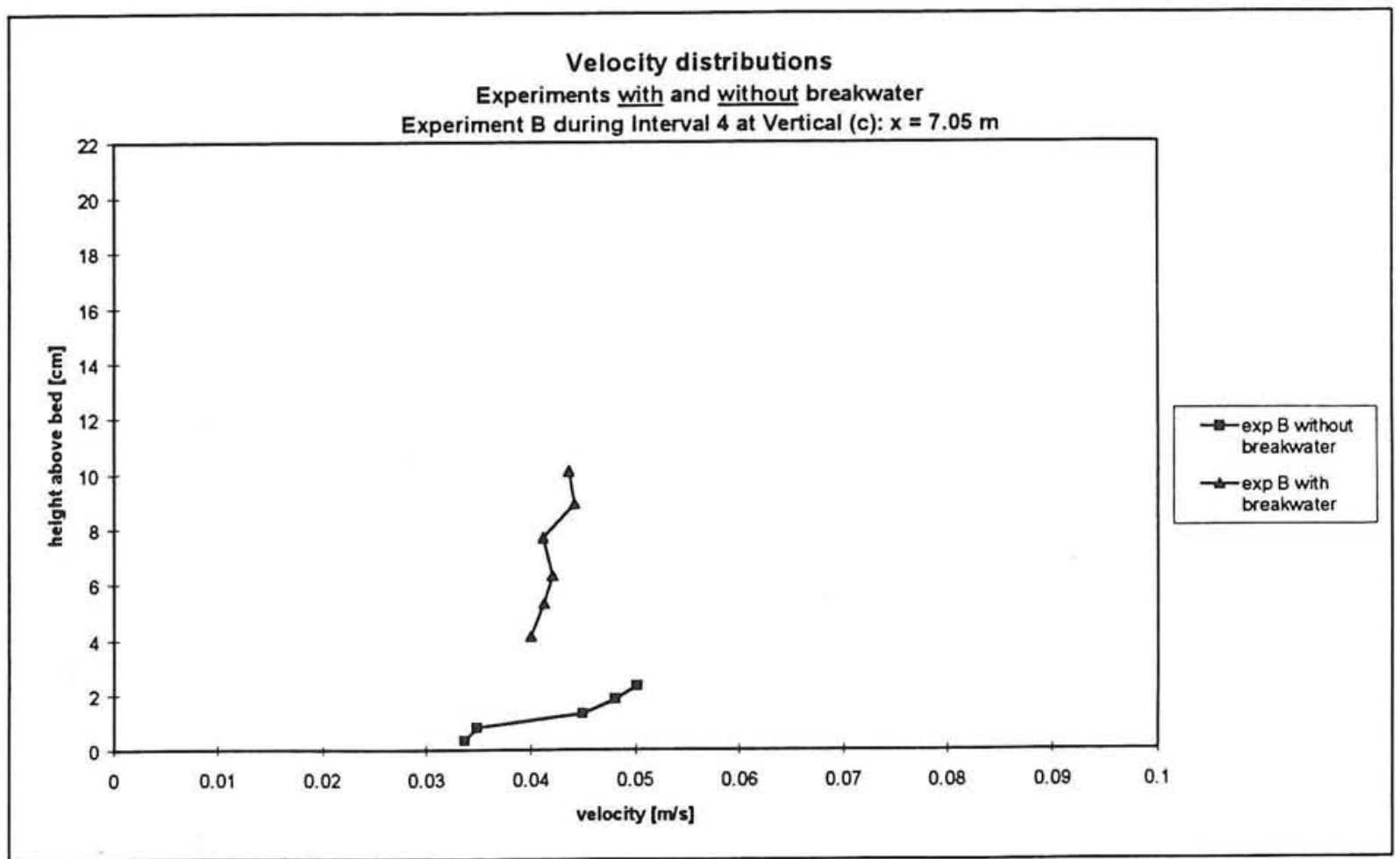

Figure 6.6 Velocity distributions at Vertical (c) during experiment B with and without breakwater (positive values of average velocities are in seaward direction).

From this figure it is clear that hardly any comparisons can be made between these two experiments, because the measured velocity distributions have different distances to the bed. Nevertheless, it can be noticed again that in case of a breakwater present it is possible to measure at higher levels than in case of no breakwater present (for explanation, see Vertical (a)).

\section{Other Experiments}

All graphs of other experiments are printed in Volume 2: Graphics, Part 3. These graphs will appear according to Table 6.2 .

\begin{tabular}{|l|l|c|l|}
\hline Experiment: & Vertical (a) & Vertical (b) & Vertical (c) \\
\hline A & Graph 3.11 & Graph 3.12 & Graph 3.13 \\
B & Graph 3.14 & Graph 3.15 & Graph 3.16 \\
C & Graph 3.17 & $*$ & Graph 3.18 \\
D & Graph 3.19 & $*$ & Graph 3.20 \\
E & Graph 3.21 & $*$ & Graph 3.22 \\
\hline
\end{tabular}

* Measurements failed during these experiments due to a not properly working EMS.

Table 6.2 Index of graphs of all experiments in Volume 2: Graphics, Part 3. 
. 


\section{CHAPTER 7 SEDIMENT CONCENTRATIONS}

\section{$\underline{7.1 \text { Introduction }}$}

Chapter 3 already discussed the sediment concentration measurements that have been executed. This chapter evaluates the sediment concentration distributions at the positions of Vertical (a), Vertical (b) and Vertical (c). The real sediment concentrations had to be calculated using the measurements. The method to do this is explained in Section 7.2. The height of the bottom at the different positions of the measurement equipment also had to be calculated, this is explained in Section 7.3. Section 7.4 discusses three methods of presentation of the different sediment concentration distributions which were made using a computer program called 'Klaros'. This computer program is discussed as well in Section 7.4. This way of presentation resulted in a number of graphs which can all be found in Volume 2: Graphics, Part 4. Some examples of the graphs of the sediment concentration distributions are presented in this chapter. Section 7.5 finally evaluates the results.

\subsection{Calculation of sediment concentrations}

In Chapter 2 the method of execution of the sediment concentration measurements has already been explained. This section explains the method of translating the measurements into real sediment concentrations.

After performing the sediment concentration measurement the buckets contain a watersediment mixture. First of all the total weight of each bucket filled with this mixture is determined. After this, the weight of the sand under water is determined using the so called 'under water balance' (see Picture 3.8).

The weight of each empty bucket was already measured. The weight of a filled bucket minus its empty weight is the weight of the water-sand mixture.

The weight of the sand measured with the under-water-balance is the weight of the sand under water. The real weight of the sand can be calculated by multiplying this weight with a factor to compensate for the lifting force on the sediment caused by the replaced water. This factor is:

$$
\frac{\rho_{s}}{\rho_{s}-\rho_{w}}=\frac{2650}{2650-1000} \approx 1.61
$$

with:

$\rho_{s} \quad=$ mass density of the sediment $\left[\mathrm{kg} / \mathrm{m}^{3}\right]$

$\rho_{\mathrm{w}} \quad \quad=$ mass density of the water $\left[\mathrm{kg} / \mathrm{m}^{3}\right]$

Dividing the weight of the sediment by the weight of the water-sand mixture gives the average sediment concentration in the bucket. Research has showed that this is about $80 \%$ of the real sediment concentration in the wave flume. Equation 7.2 shows the entire equation needed to determine the sediment concentration.

$$
c[g / 1]=\frac{1}{c_{\text {measured }} / c_{\text {real }}} \cdot \frac{\rho_{\mathrm{s}}}{\rho_{\mathrm{s}}-\rho_{\mathrm{w}}} \cdot \mathrm{F}_{\text {balance }} \cdot \frac{1}{\mathrm{~F}_{\text {bucket }}-\mathrm{G}_{\text {bucket }}} \cdot 1000 \mathrm{~kg} / 1=
$$




$$
=\frac{1}{0.8} \cdot \frac{2650}{2650-1000} \cdot F_{\text {balance }} \cdot \frac{1}{F_{\text {bucket }}-G_{\text {bucket }}} \cdot 1000 \mathrm{~kg} / 1
$$

with:

$\begin{array}{ll}c & =\text { sediment concentration }[\mathrm{g} / \mathrm{l}] \\ \mathrm{c}_{\text {measured }} / \mathrm{c}_{\text {real }} & =\text { ratio measured and real concentration }[-/-] \\ \rho_{\mathrm{s}} & =\text { mass density of the sediment }\left[\mathrm{kg} / \mathrm{m}^{3}\right] \\ \rho_{\mathrm{w}} & =\text { mass density of the water }\left[\mathrm{kg} / \mathrm{m}^{3}\right] \\ \mathrm{F}_{\text {balance }} & =\text { mass of sand on under-water-balance }[\mathrm{kg}] \\ \mathrm{F}_{\text {bucket }} & =\text { mass of filled bucket }[\mathrm{kg}] \\ \mathrm{G}_{\text {bucket }} & =\text { mass of empty bucket }[\mathrm{kg}]\end{array}$

\section{$\underline{7.3 \text { Calculation of the vertical position of the intake tubes }}$}

The placement of the intake tubes can have effect on the measurements. It can make a difference whether the intake tubes are positioned above the crest of a ripple or above the trough of a ripple. Therefore sediment concentration measurements are sometimes executed by equipment attached to a trolley that is moving constantly above a certain part of the bed. In this way the problem of a possible influence of the position of the intake tubes on the measured sediment concentrations can be avoided.

In case of this particular project this strategy would cause a lot of technical problems. A 1 in 15 slope is quite steep. The vertical distance of the intake tubes to the bottom should remain more or less the same so the intake tubes should have to move parallel to the slope. Secondly it was decided to perform sediment concentrations measurements at three different positions in the flume at one time. This has the advantage that in this way a good view of the situation at a certain time-point can be obtained. Bottom evolution takes place rather quickly. Performance of the measurements at different time-points would make a comparison of sediment concentrations distributions at the different horizontal positions in the flume less reliable because the bottom profile has a big effect on the measurements. Also wave height and flow velocity measurements were performed at the same time. It would technically be almost impossible to let the concentration measurement equipment move across the bed under an angle at three different positions at one time and also perform other measurements at fixed positions simultaneously. The disadvantages of performing sediment concentration measurements while making use of moving equipment would be much bigger than the benefits. Because of these reasons it was decided to perform the sediment concentration measurements with equipment at fixed positions.

Further it has to be said that the ripples of the bed move in time which results in a sort of natural 'moving' of the equipment over the ripples. The influence of the placement of the intake tubes above the ripples on the sediment concentration measurements also decreases with increasing distance between a particular intake tube and the bottom.

In order to present the sediment concentration distributions over the vertical there is a need for a definition of the bottom height. It was decided to use an average bottom height to relate the positions of the intake tubes to. These average bottom heights were calculated using the profile measurement results. To take into account the effect of the ripples of the bed every time the average value of the bottom height over a distance of $10 \mathrm{~cm}$ was calculated, viz. from 
$5 \mathrm{~cm}$ seaward of the 'horizontal position' (=x-value) of the measurement equipment, till $5 \mathrm{~cm}$ landward of the horizontal position of the measurement equipment.

To calculate the average bottom heights for the sediment concentration measurements at the beginning of Interval 1 the profile measurements made before this interval (profile measurement $\mathrm{nr} .0$ ) were used. The profile measurements after Interval 4 (profile measurement nr. 4) were used to calculate the average bottom heights for the measurements at the end of Interval 4.

The calculated values of the bottom heights are of course estimations of the real bottom heights. To make these calculations as reliable as possible the sediment concentration measurements were always executed right after (Interval 1) or right before (Interval 4) a profile measurement.

By means of a vertical ruler system attached to the concentration measurement equipment the exact vertical position of each of the intake tubes could be determined.

\subsection{Sediment concentration distributions}

To present the sediment concentration distributions a computer program called Klaros has been used. Klaros is a computer program developed at the Delft University of Technology. Klaros is (among other things) capable to find a mathematical description of the sediment concentration over the entire water depth by means of fitting a curve as good as possible through a number of measured points. The mathematical method for this curve-fitting process is presented in Appendix E.

Sediment concentration measurements have been performed at the beginning and at the end of each experiment. The results for some of the experiments are presented. These experiments, the matching positions of the measurement equipment and the time of execution of the measurements are listed in Table 7.1:

\begin{tabular}{|l|l|}
\hline Experiments & A,B,C,D (with and without breakwater) \\
\hline Positions & Vertical (a): $x=5.25 \mathrm{~m}$ \\
& Vertical (b): $x=6.15 \mathrm{~m}$ \\
& Vertical (c): $\mathrm{x}=7.05 \mathrm{~m}$ \\
\hline Time-points & $\begin{array}{l}\text { Begin of Interval 1 } \\
\text { End of Interval 4 }\end{array}$ \\
\hline
\end{tabular}

Table 7.1 Review of the sediment concentration measurements.

The sediment concentrations of experiment $\mathrm{E}$ (with and without breakwater) were often so low that they could not be measured with the under-water-balance. Therefore not enough measurement points were available to present a reliable curve fit. It was decided not to present the results for experiment $\mathrm{E}$.

Using Klaros, graphs were made of the sediment concentration distributions of these experiments. These graphs are presented in Volume 2: Graphics, Part 4. Part 4 is divided in two different sections, namely Section 1 for the results of the measurements during Interval 1 and Section 2 for the results of the measurements during Interval 4.

In general the curve fits made by Klaros look very natural, only the extrapolation of the curve from the upper measurement point to the mean waterline seems sometimes not to be so well 
(see for instance Graph 4.1.1, Vertical (b)). A similar problem occurs with the extrapolation of the curve from the lowest measurement point to the bottom. The curve-fitting method of Klaros is a mathematical one and has its shortcomings but as long as one bears that in mind Klaros can be a very good tool.

In each of these two sections three different methods of presentation are used to show the different sediment concentration distributions:

\section{Method 1}

The sediment concentration distributions in the three verticals are presented for each experiment separately. In this way the sediment concentrations at the three different verticals of one experiment can be compared.

This results in a number of graphs which are listed in Table 7.2. The first number of each graph indicates in which part of Volume 2 the graphs can be found (all the graphs of the sediment concentrations begin with the number 4). The second number indicates in which of the two sections the graph can be found.

As an example the graph of the sediment concentration distributions of the three verticals of experiment B without breakwater is presented in Figure 7.1.

\begin{tabular}{|l|c|l|l|}
\hline & Time-point & Experiments & Position \\
\hline Graph 4.1.1 & Interval 1 & A (without breakwater) & Vertical (a), (b) and (c) \\
Graph 4.1.2 & Interval 1 & B (without breakwater) & Vertical (a), (b) and (c) \\
Graph 4.1.3 & Interval 1 & C (without breakwater) & Vertical (a), (b) and (c) \\
Graph 4.1.4 & Interval 1 & D (without breakwater) & Vertical (a), (b) and (c) \\
Graph 4.1.5 & Interval 1 & A (with breakwater) & Vertical (a), (b) and (c) \\
Graph 4.1.6 & Interval 1 & B (with breakwater) & Vertical (a), (b) and (c) \\
Graph 4.1.7 & Interval 1 & C (with breakwater) & Vertical (a), (b) and (c) \\
Graph 4.1.8 & Interval 1 & D (with breakwater) & Vertical (a), (b) and (c) \\
& & & \\
Graph 4.2.1 & Interval 4 & A (without breakwater) & Vertical (a), (b) and (c) \\
Graph 4.2.2 & Interval 4 & B (without breakwater) & Vertical (a), (b) and (c) \\
Graph 4.2.3 & Interval 4 & C (without breakwater) & Vertical (a), (b) and (c) \\
Graph 4.2.4 & Interval 4 & D (without breakwater) & Vertical (a), (b) and (c) \\
Graph 4.2.5 & Interval 4 & A (with breakwater) & Vertical (a), (b) and (c) \\
Graph 4.2.6 & Interval 4 & B (with breakwater) & Vertical (a), (b) and (c) \\
Graph 4.2.7 & Interval 4 & C (with breakwater) & Vertical (a), (b) and (c) \\
Graph 4.2.8 & Interval 4 & D (with breakwater) & Vertical (a), (b) and (c) \\
\hline
\end{tabular}

Table 7.2 Review of the graphs made of the sediment concentration distributions of each experiment separately. Graphs are presented in Volume 2: Graphics, Part 4, Section 1. 


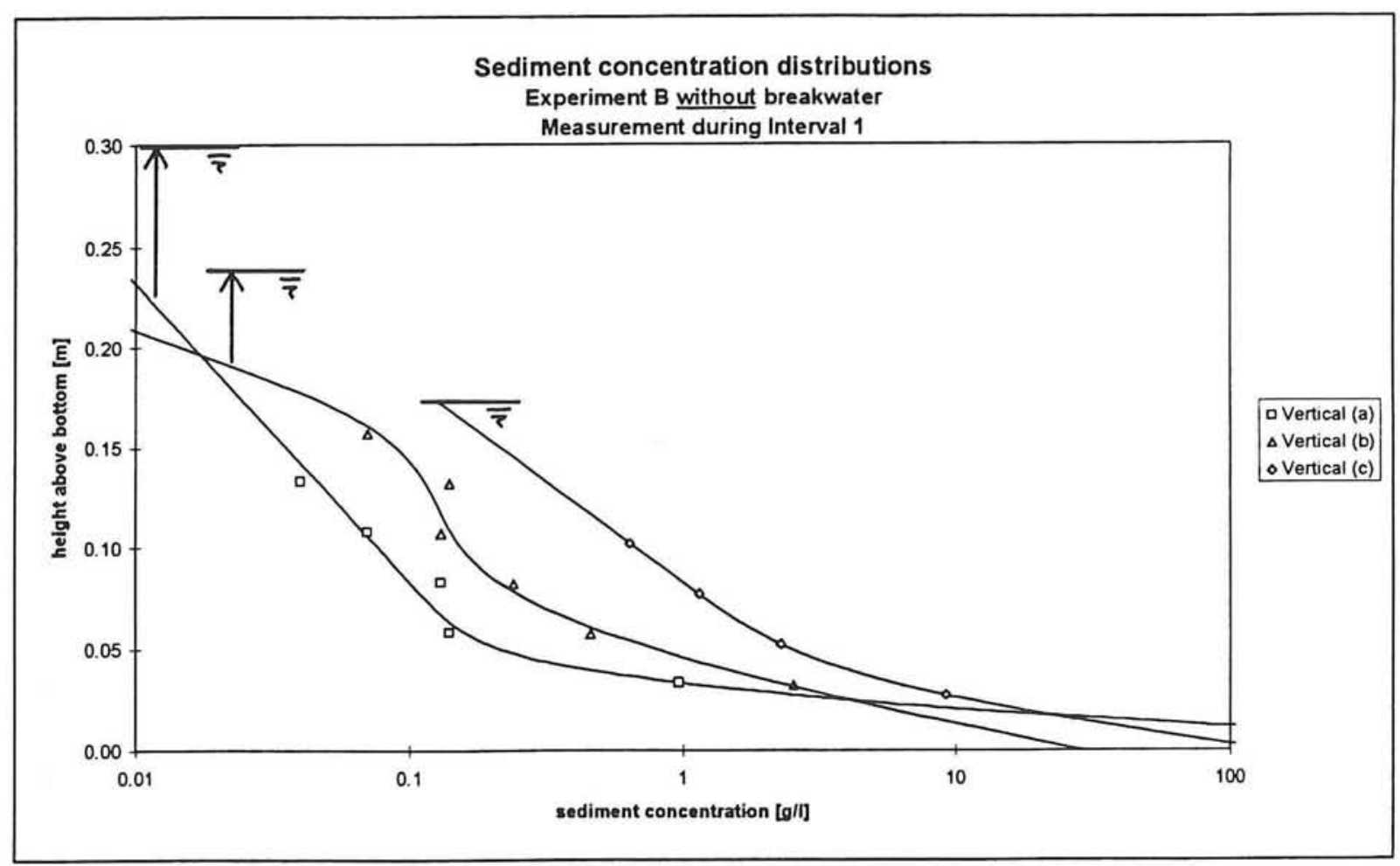

Figure 7.1 Example of a graph of a sediment concentration distribution (Graph 4.1.2).

\section{Method 2}

The sediment concentration distributions of the experiments with and without breakwater with similar wave conditions are presented together in one graph. Each vertical is presented separately. In this way the influence of the breakwater on the sediment concentrations can be compared to the situation without breakwater.

Table 7.3 gives a review of the graphs that are presented in Volume 2: Graphics, Part 4. As example of this, the sediment concentration distribution of experiment B (with and without breakwater) during Interval 1 at Vertical (a) is presented in Figure 7.2. 


\begin{tabular}{|l|c|c|c|}
\hline & Time-point & Experiments & Position \\
\hline Graph 4.1.9 & Interval 1 & A (with and without breakwater) & Vertical (a) \\
Graph 4.1.10 & Interval 1 & A (with and without breakwater) & Vertical (b) \\
Graph 4.1.11 & Interval 1 & A (with and without breakwater) & Vertical (c) \\
Graph 4.1.12 & Interval 1 & B (with and without breakwater) & Vertical (a) \\
Graph 4.1.13 & Interval 1 & B (with and without breakwater) & Vertical (b) \\
Graph 4.1.14 & Interval 1 & B (with and without breakwater) & Vertical (c) \\
Graph 4.1.15 & Interval 1 & C (with and without breakwater) & Vertical (a) \\
Graph 4.1.16 & Interval 1 & C (with and without breakwater) & Vertical (b) \\
Graph 4.1.17 & Interval 1 & C (with and without breakwater) & Vertical (c) \\
Graph 4.1.18 & Interval 1 & D (with and without breakwater) & Vertical (a) \\
Graph 4.1.19 & Interval 1 & D (with and without breakwater) & Vertical (b) \\
Graph 4.1.20 & Interval 1 & D (with and without breakwater) & Vertical (c) \\
& & & \\
Graph 4.2.9 & Interval 4 & A (with and without breakwater) & Vertical (a) \\
Graph 4.2.10 & Interval 4 & A (with and without breakwater) & Vertical (b) \\
Graph 4.2.11 & Interval 4 & A (with and without breakwater) & Vertical (c) \\
Graph 4.2.12 & Interval 4 & B (with and without breakwater) & Vertical (a) \\
Graph 4.2.13 & Interval 4 & B (with and without breakwater) & Vertical (b) \\
Graph 4.2.14 & Interval 4 & B (with and without breakwater) & Vertical (c) \\
Graph 4.2.15 & Interval 4 & C (with and without breakwater) & Vertical (a) \\
Graph 4.2.16 & Interval 4 & C (with and without breakwater) & Vertical (b) \\
Graph 4.2.17 & Interval 4 & C (with and without breakwater) & Vertical (c) \\
Graph 4.2.18 & Interval 4 & D (with and without breakwater) & Vertical (a) \\
Graph 4.2.19 & Interval 4 & D (with and without breakwater) & Vertical (b) \\
Graph 4.2.20 & Interval 4 & D (with and without breakwater) & Vertical (c) \\
\hline
\end{tabular}

Table 7.3 Review of the graphs made of the sediment concentration distributions of the experiments with and without breakwater per time-point and per position. Graphs are presented in Volume 2: Graphics, Part 4. 


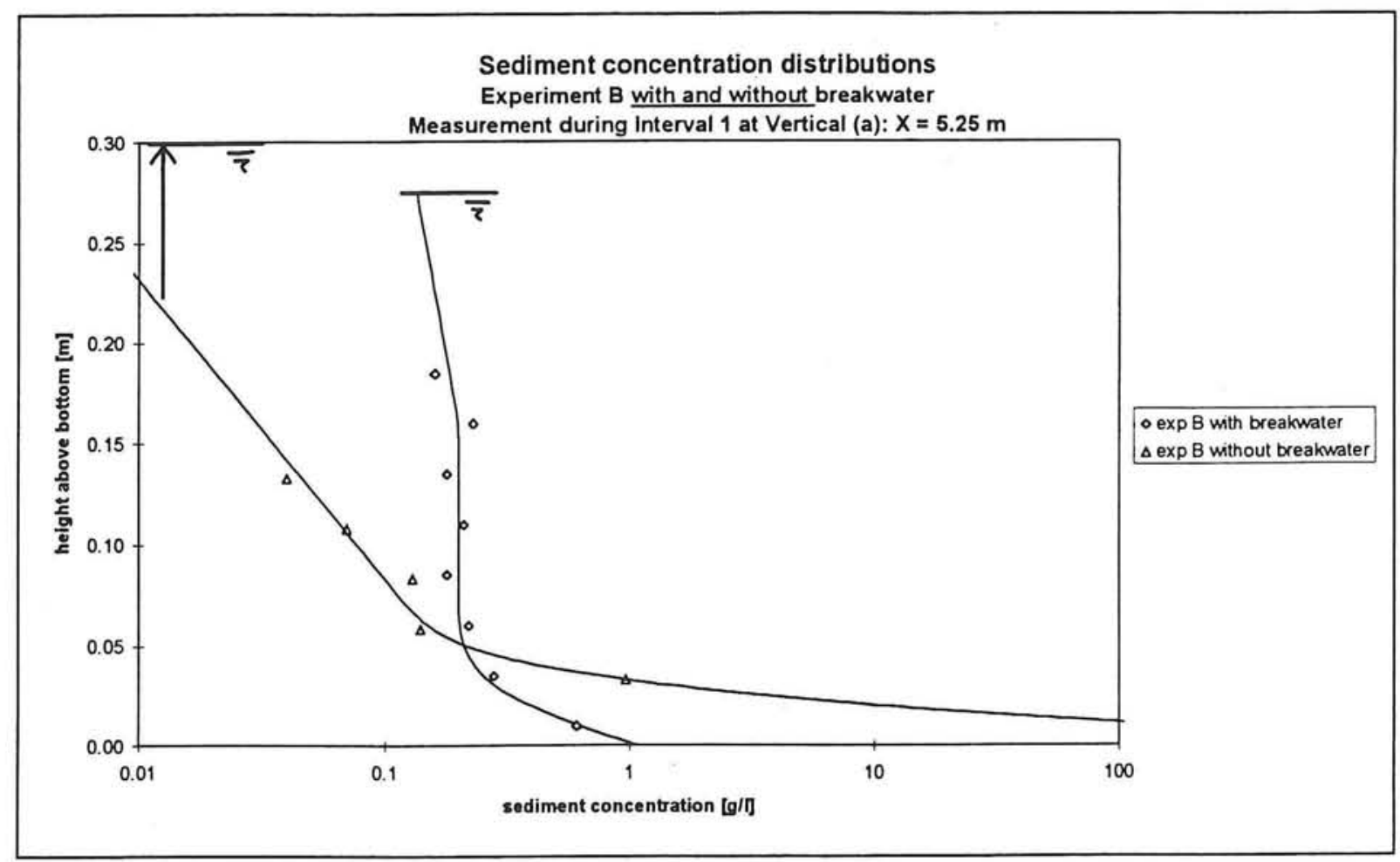

Figure 7.2 Example of a graph of a sediment concentration distribution (Graph 4.1.12).

\section{Method 3}

The sediment concentration distributions of the experiments without breakwater are presented separately from those with breakwater. The concentration distributions of the four different experiments (A, B, C and D) are presented together in one graph. Each vertical is presented separately. In this way the influence of wave height and period on the sediment concentrations can be investigated.

An example of this, a graph is presented in Figure 7.3 (Experiments A, B, C and D with breakwater at Interval 1, Vertical (a)). 


\begin{tabular}{|l|c|l|l|}
\hline & Time-point & Experiments & Position \\
\hline Graph 4.1.21 & Interval 1 & A,B,C,D (without breakwater) & Vertical (a) \\
Graph 4.1.22 & Interval 1 & A,B,C,D (without breakwater) & Vertical (b) \\
Graph 4.1.23 & Interval 1 & A,B,C,D (without breakwater) & Vertical (c) \\
Graph 4.1.24 & Interval 1 & A,B,C,D (with breakwater) & Vertical (a) \\
Graph 4.1.25 & Interval 1 & A,B,C,D (with breakwater) & Vertical (b) \\
Graph 4.1.26 & Interval 1 & A,B,C,D (with breakwater) & Vertical (c) \\
& & & \\
Graph 4.2.21 & Interval 4 & A,B,C,D (without breakwater) & Vertical (a) \\
Graph 4.2.22 & Interval 4 & A,B,C,D (without breakwater) & Vertical (b) \\
Graph 4.2.23 & Interval 4 & A,B,C,D (without breakwater) & Vertical (c) \\
Graph 4.2.24 & Interval 4 & A,B,C,D (with breakwater) & Vertical (a) \\
Graph 4.2.25 & Interval 4 & A,B,C,D (with breakwater) & Vertical (b) \\
Graph 4.2.26 & Interval 4 & A,B,C,D (with breakwater) & Vertical (c) \\
\hline
\end{tabular}

Table 7.4 Review of the graphs made of the sediment concentration distributions of the experiments with or experiments without breakwater per time-point and per position. Graphs are presented in Volume 2: Graphics, Part 4.

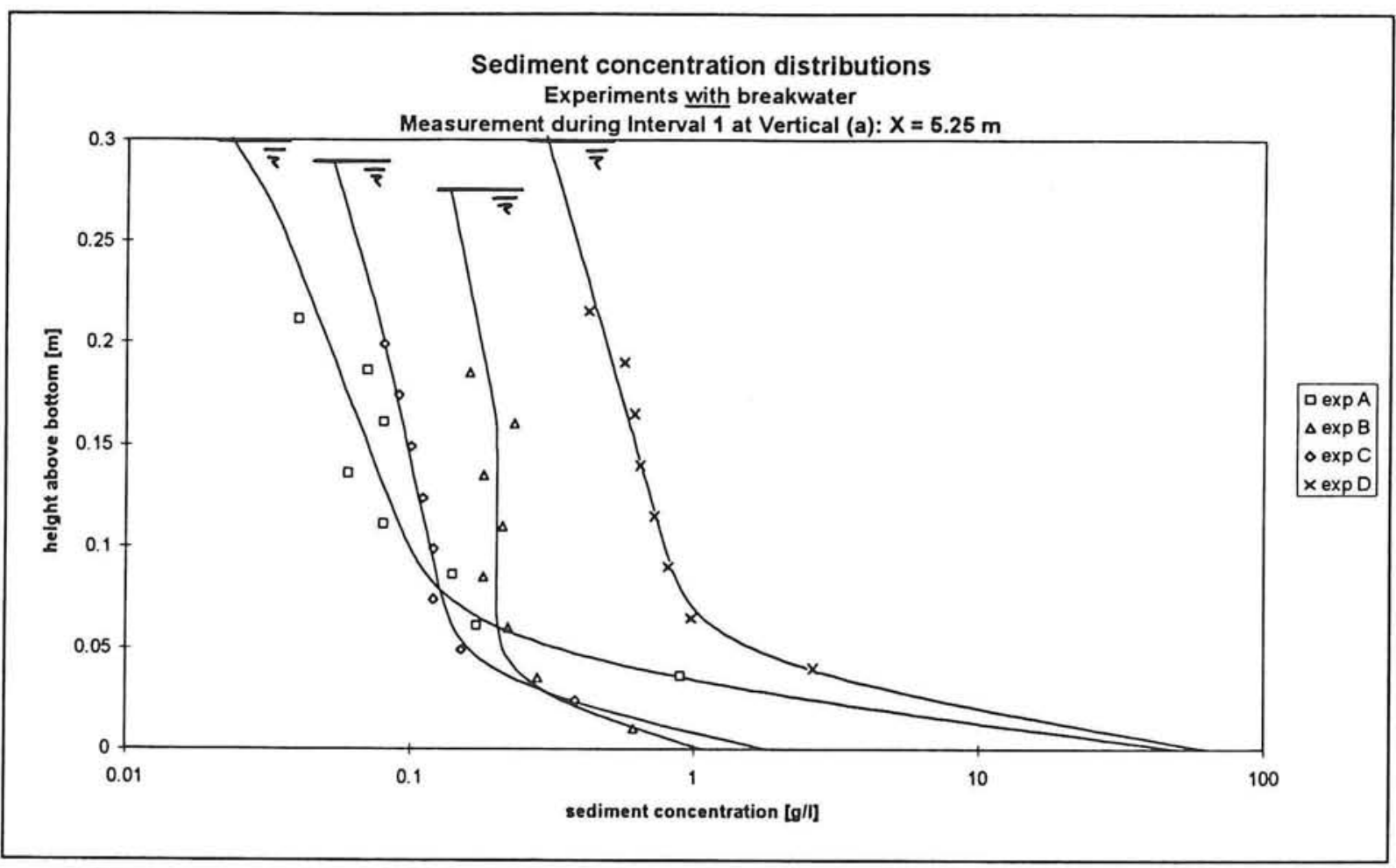

Figure 7.3 Example of a graph of a sediment concentration distribution (Graph 4.1.24). 


\subsection{Evaluation of the different graphs}

\subsubsection{Comparison of the verticals per experiment (method 1)}

This section evaluates the results of the sediment concentration measurements by comparing the sediment concentrations of the different verticals. Only the sediment concentration distributions of the verticals of one and the same experiment are compared to each other. First, the measurements without breakwater and next the measurements with breakwater will be discussed.

\section{Experiments without breakwater}

Relevant graphs of Interval 1:

Graph 4.1.1 - Graph 4.1.4

Relevant graphs of Interval 4:

Graph 4.2.1 - Graph 4.2.4

The graphs of the experiments without breakwater at Interval 1 show all a quite logical picture. The sediment concentrations increase as the water depth decreases. Vertical (a) always has the lowest and Vertical (c) always has the highest sediment concentrations. The orbital motion of the water caused by the waves increases near the bottom with decreasing water depth. In other words, there is more energy to stir up the sediment when the water depth reduces. At Interval 1 almost all the waves were breaking landward of Vertical (c).

The situation for the experiments without breakwater at Interval 4 is similar to the situation at Interval 1. The sediment concentration distribution curves of Vertical (c) are even steeper because of the decreased water depth and the increased amount of breaking waves at that position. The relative high waves of experiment $\mathrm{D}$ caused much sediment transport in offshore direction with the result that the bottom height at the end of Interval 4 at Vertical (b) had increased much and was almost the same as at Vertical (c). The reduced water depth caused an increasing amount of breaking waves which explains the high sediment concentrations at Vertical (b) for this experiment.

\section{Experiments with breakwater}

Relevant graphs of Interval 1: Relevant graphs of Interval 4:

The graphs of the experiments with breakwater at Interval 1 show a different picture. The curves of the sediment concentration distributions of Vertical (a) are all very steep in the upper part. Near the bottom the sediment concentration increases rapidly with decreasing distance to the bottom; the curves become more levelled. The curves of Vertical (b) and Vertical (c) are less steep and the sediment concentrations near the water level are less than at Vertical (a). The sediment concentrations in the lower part of the verticals are slightly higher than at Vertical (a).

The situation for the experiments with breakwater at Interval 4 is very similar to the situation at Interval 1. 


\subsubsection{Comparison experiments with similar wave conditions (method 2)}

In this section the experiments with breakwater are compared to the experiments without breakwater in case of similar wave conditions. Each of the three measurement positions (Vertical (a), Vertical (b) and Vertical (c)) is discussed separately.

\section{$\underline{\operatorname{Vertical}(a)}$}

$\begin{array}{ll}\text { Relevant graphs of Interval 1: } & \text { Graph 4.1.9 } \\ & \text { Graph 4.1.12 } \\ & \text { Graph 4.1.15 } \\ & \text { Graph 4.1.18 } \\ \text { Relevant graphs of Interval 4: } & \text { Graph 4.2.9 } \\ & \text { Graph 4.2.12 } \\ & \text { Graph 4.2.15 } \\ & \text { Graph 4.2.18 }\end{array}$

If a comparison is made between the experiments with breakwater and the experiments without breakwater in case of similar wave conditions at Interval 1, it can be concluded that at Vertical (a) the sediment concentration distributions for the experiments with breakwater are all much steeper and higher than for the experiments without breakwater.

Near the bottom the concentrations seem to be a little higher in case of the tests without breakwater. The method of interpolation between the lowest measurement point and the bottom has much influence on the impression one gets about the concentration distribution near the bottom. The way Klaros interpolates between these points is not necessarily the right way.

The breakwater causes a lot of dissipation. A lot of turbulence occurs behind the breakwater which is the cause of these relative high and steep concentration distributions.

At Interval 4 the picture is more or less the same, except for experiment D. Experiment D is the only experiment where the sediment concentrations of the experiment without breakwater are higher in every point.

\section{$\underline{\text { Vertical (b) }}$}

Relevant graphs of Interval 1: $\quad$ Graph 4.1.10

Graph 4.1.13

Graph 4.1.16

Graph 4.1.19

Relevant graphs of Interval 4: $\quad$ Graph 4.2.10

Graph 4.2.13

Graph 4.2.16

Graph 4.2.19

The differences between the experiments with and without breakwater with similar wave conditions at Interval 1 are much less than at Vertical (a). The dissipation of the energy in the form of turbulence caused by the breakwater has probably lost most of its power to stir up the sand. Even though the wave heights and periods differ a lot (the experiments with breakwater have lower wave heights and the average frequency is higher), the sediment concentration 
curves have lot in common, like the shape of the curve. Again, it is emphasized that the extrapolation from the highest measurement point to the mean water level does not always seem to be very good (see for instance Graph 4.1.13).

The sediment concentrations of the experiments B, C and D without breakwater at Interval 4 have increased and are higher than experiments B, C and D with breakwater. The water depths of the experiments B, C and D without breakwater are smaller than those with breakwater which can be one of the causes of the difference in sediment concentrations. The sediment concentrations of experiment $\mathrm{A}$ are very much alike.

$\underline{\operatorname{Vertical}(c)}$

Relevant graphs of Interval 1: $\quad$ Graph 4.1.11

Graph 4.1.14

Graph 4.1.17

Graph 4.1.20

Relevant graphs of Interval 4: $\quad$ Graph 4.2.11

Graph 4.2.14

Graph 4.2.17

Graph 4.2.20

The sediment concentrations of the experiments without breakwater at Interval 1 are all higher than those of the experiments with breakwater. The difference is rather large for experiment D. In case of the experiments with breakwater the waves have already lost some of their energy when passing the breakwater. The reduced wave height could very well be the cause of the lower sediment concentrations at Vertical (c).

The sediment concentration distributions of the experiments at Interval 4 show also higher sediment concentrations for the experiments without breakwater.

\subsubsection{Comparison experiments with or without breakwater (method 3)}

The concentration distributions of the experiments without breakwater are presented separately from those with breakwater. The concentration distributions of the four different experiments (A, B, C, D) are presented together in one graph. Each vertical is presented separately. First the experiments without breakwater and then the experiments with breakwater are discussed. Each vertical is discussed separately.

Experiments without breakwater

$\begin{array}{lll}\text { Vertical (a) } & \text { Relevant graph of Interval 1: } & \text { Graph 4.1.21 } \\ & \text { Relevant graph of Interval 4: } & \text { Graph 4.2.21 }\end{array}$

Very low sediment concentrations occur at Vertical (a) at both Interval 1 and Interval 4. The curves are not steep. Experiment D has the highest sediment concentrations; the influence of the wave height is great. Experiment $\mathrm{C}$ has the lowest sediment concentrations. Experiment $\mathrm{C}$ has the shortest waves and will in theory have less influence on the bottom at deeper water than waves with a longer period. 
$\underline{\text { Vertical (b) }}$

Relevant graph of Interval 1:

Graph 4.1.22

Relevant graph of Interval 4:

Graph 4.2.22

The sediment concentrations at both Interval 1 and 4 are somewhat higher than at Vertical (a), probably because of the smaller water depth. The influence of the period of the waves on the sediment concentrations is hard to describe because no obvious changes occur. Again the sediment concentrations of experiment $\mathrm{D}$ are high.

Vertical (c)

Relevant graph of Interval 1:

Graph 4.1.23

Relevant graph of Interval 4:

Graph 4.2.23

Very high sediment concentrations occur at this vertical especially during Interval 4. This is caused by the breaking of the waves which stirs up a lot of sediment. During Interval 1 almost all the waves were breaking more landward of Vertical (c) but during Interval 4 also a lot of waves broke close to Vertical (c), due to the decreased water depth.

Experiments with breakwater

Vertical (a)

Relevant graph of Interval 1:

Graph 4.1.24

Relevant graph of Interval 4:

Graph 4.2.24

At Interval 1 and at Interval 4 all the sediment concentration distribution curves are very steep in the upper part and more levelled in the lower part. The sediment concentrations of experiment $\mathrm{D}$ are the largest.

$\underline{\text { Vertical (b) }}$

Relevant graph of Interval 1:

Graph 4.1.25

Relevant graph of Interval 4:

Graph 4.2.25

At Interval 1 and at Interval 4 the sediment concentration distribution curves are less steep than at Vertical (a). Again, experiment D has the highest sediment concentrations. The sediment concentrations of the experiments A, B and C are almost the same at Interval 1. The effect of the variation of the wave periods on the sediment concentrations seems not to be very large. The wave height has much more influence.

Vertical (c) Relevant graph of Interval 1: Graph 4.1.26

Relevant graph of Interval 4: $\quad$ Graph 4.2.26

The sediment concentration distribution curves are a little less steep than at Vertical (b). Again experiment $\mathrm{D}$ has the highest sediment concentrations. No major differences occur between the experiments with (almost) equal wave heights and varying wave periods. 


\section{CHAPTER 8 SEDIMENT TRANSPORT}

\subsection{Introduction}

The amount of sediment transport through Vertical (a), Vertical (b) and Vertical (c) was calculated by comparing the differences in bottom profiles of an experiment. Chapter 4 already presented these results. Another method of calculating sediment transport is the multiplication of the time-average velocities and the time-average sediment concentrations. Section 8.2 discusses this method of sediment transport calculation. In order to do this the program CONVERT was used. CONVERT is a program developed by Delft Hydraulics and is discussed in Section 8.3. In Section 8.4 the sediment transport based on the profile measurements is discussed. Section 8.5 presents the results of the calculations of the sediment transport by CONVERT and compares them with the sediment transport calculations based on the profile measurements.

\subsection{Cross-shore sediment transport calculations}

In general sediment transport in water can be defined as the product of a sediment concentration (c) and a velocity (V):

$$
S=c \cdot V
$$

The interest is in the sediment transport through a unit width. Because of the waves, the water velocity $(\mathrm{V})$ and the sediment concentration (c) vary strongly as a function of time (on a scale comparable to the wave period). The sediment transport can be expressed by:

$$
S=\frac{1}{t^{\prime}} \int_{0}^{h+\eta} \int_{0}^{t^{\prime}} c(z, t) \cdot V(z, t) \cdot d t \cdot d z
$$

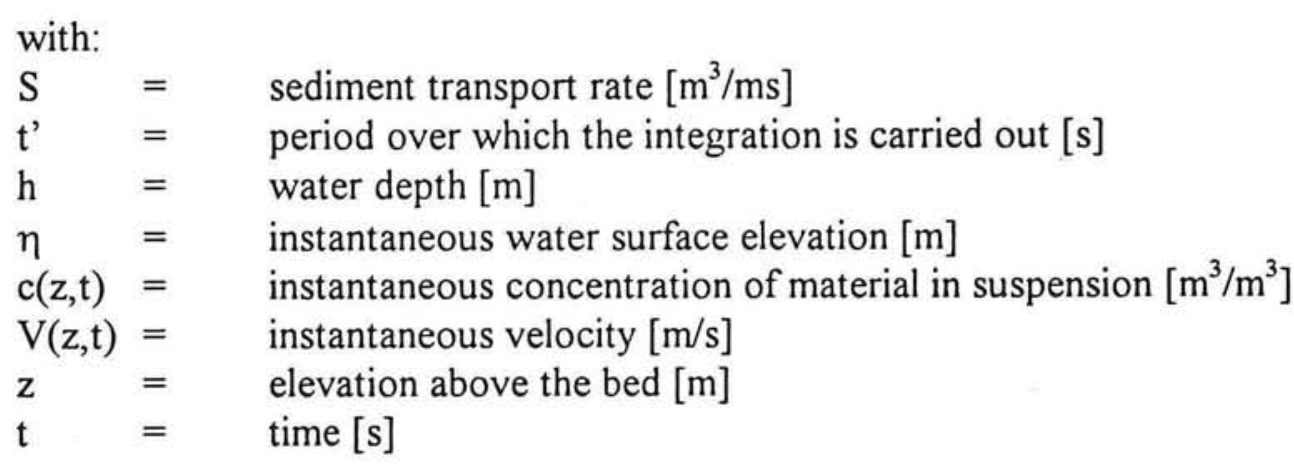

In Equation 8.2, any variations of the parameters over the unit width have been averaged out. The time ( $t$ ') should be sufficiently long to average out irregularities in the waves, therefore t' should be much longer than one wave period.

The difficulty of Equation 8.2 is that information is needed about $\mathrm{c}(\mathrm{z}, \mathrm{t})$. The sediment concentration measurements that were performed give only information about the timeaverage sediment concentrations. Therefore it is not possible to use Equation 8.2. 
A considerable simplification is to use this time-average sediment concentration $c(z)$ instead of the time-dependent sediment concentration $\mathrm{c}(\mathrm{z}, \mathrm{t})$. As a consequence of this also the timeaverage velocity $\mathrm{V}(\mathrm{z})$ has to be taken instead of the time-dependent velocity. This makes the problem much easier to solve. The total transport can now be found by integrating the transport at each level in the water:

$$
S=\int_{0}^{h+\eta} \overline{c(z)} \cdot \overline{V(z)} \cdot d z \approx \int_{0}^{h} \overline{c(z)} \cdot \overline{V(z)} \cdot d z
$$

with:

$\mathrm{S}=$ sediment transport rate $\left[\mathrm{m}^{3} / \mathrm{ms}\right]$

$\mathrm{h} \quad=\quad$ water depth $[\mathrm{m}]$

$\eta=$ instantaneous water surface elevation $[\mathrm{m}]$

$\overline{c(z)}=$ time-average sediment concentration $\left[\mathrm{m}^{3} / \mathrm{m}^{3}\right]$

$\overline{\mathrm{V}(\mathrm{z})}=$ time-average water velocity $[\mathrm{m} / \mathrm{s}]$

A computer program called CONVERT is capable to do this. CONVERT is discussed in the next section.

\subsection{CONVERT}

CONVERT can be used to calculate sediment transport in the way as described above. Therefor Convert needs some input parameters. To calculate the sediment transport at a certain vertical, the distribution of the time-average velocities and the time-average sediment concentrations over this vertical are needed.

In order to do this the measured average velocities and their height above the bottom must be defined. CONVERT uses linear interpolation between these points. The velocity at the bottom is assumed to be zero. Between the lowest measurement point and the bottom an exponential function is used. The velocity between the highest measurement point and the still water line is assumed to be equal to the velocity in the highest measurement point. In this way a distribution of the velocity over the entire water depth is obtained.

The average sediment concentrations are defined in a similar way. From the lowest measurement point to the bottom three different methods of extrapolation can be used. The first two methods give an underlimit and an upperlimit, respectively, for the sediment concentrations near the bottom. The other method gives a much more natural extrapolation. It was decided to use this last method of extrapolation. Appendix F presents the assumptions of this method.

CONVERT divides the water depth in a number of pieces $(\Delta \mathrm{h})$ and calculates for each of these pieces the average velocity and the average sediment concentration. The sum of all the multiplications per level of the average velocity and the average sediment concentration gives the total sediment transport.

CONVERT calculates the amount of sediment transport in $\mathrm{kg} / \mathrm{sm}$. This has been converted to $\mathrm{kg} / \mathrm{m} / \mathrm{hr}$ (Table 8.1 in Section 8.5). 


\subsection{Calculation of the sediment transport based on profile measurements}

The amount of sediment transport through the three verticals during Interval 4 was already calculated in Chapter 4. This amount was expressed in change of volume per unit width $\Delta \mathrm{V}$ $\left[\mathrm{m}^{3} / \mathrm{m}\right]$. Multiplication of this change of volume by the mass density and a factor to account for the porosity gives the amount of sediment transport in $\mathrm{kg} / \mathrm{m}$. Dividing this amount of sediment transport by the duration of Interval 4 gives the average amount of sediment transport per unit time $[\mathrm{kg} / \mathrm{hr} / \mathrm{m}]$ :

$$
S=\frac{(1-n) \cdot \rho \cdot \Delta V}{\Delta t}=\frac{(1-0 . \overline{4}) \cdot 2650 \cdot \Delta V}{4}=397.5 \cdot \Delta V
$$

with:

$\mathrm{S}, \quad=$ sediment transport $[\mathrm{kg} / \mathrm{hr} / \mathrm{m}]$

$\mathrm{n} \quad=$ porosity $[-/-]$

$\rho \quad=$ mass density of sediment $\left[\mathrm{kg} / \mathrm{m}^{3}\right]$

$\Delta \mathrm{t} \quad=$ duration of Interval $4[\mathrm{hr}]$

$\Delta \mathrm{V}=$ change of volume per unit width during Interval $4\left[\mathrm{~m}^{3} / \mathrm{m}\right]$

\subsection{Results of the calculations}

The sediment transports of the experiments A, B, C and D (all with and without breakwater) during Interval 4 have been calculated. The velocity distributions have been discussed in Chapter 6. The sediment concentration distributions have been discussed in Chapter 7. The results of the computations with the help of CONVERT are presented in Table 8.1. A list of the calculated sediment concentrations near the bed is presented in Appendix F (see Table F.1).

Table 8.1 also presents the calculation of the sediment transport based on the profile measurements. As example the measured sediment transport versus the calculated transport of experiment B without breakwater are presented in Figure 8.1.

The results of the calculations show that an approach based on time-averaged velocities and time-averaged sediment concentrations often leads to large differences with the sediment transport calculations based on the profile measurements. The reality is oversimplified. The strong fluctuations of the velocity and of the sediment concentrations in time are not taken into account. Neglecting these fluctuations can be one of the causes for the differences.

Another cause can be the way the transport near the bottom is modelled by CONVERT. In case of ripples the bottom transport is often in the opposite direction of the suspended transport. The time-average velocities very close to the bottom can be in the direction of the wave propagation. CONVERT assumes that the velocity near the bottom decreases to zero. The extrapolation of the sediment concentration curve near the bottom also has a large effect on the calculated sediment transport. It can be concluded that the reliability of the calculations of the sediment transport near the bottom is small. Unfortunately bottom transport is technically very hard to measure. The bottom transport is of course included in the calculations based on the profile measurements.

The different time-points of the measurements can also cause differences. The sediment concentrations were measured at the end of Interval 4. The results of CONVERT are based on this situation. The calculations based on the profile measurements give the amount of sediment 
transport that took place during Interval 4. During the four hours of Interyal 4 the sediment concentrations change and as a result of that the sediment transport changes. However, the differences between the two methods of calculation are often so large that a relatively small change of the sediment concentrations will not be the cause of the large differences between the two methods.

\begin{tabular}{|l|c|c|c|}
\hline Experiment & Vertical & $\begin{array}{c}\text { Sediment transport } \\
\text { calculated by } \\
\text { CONVERT (kg/hr/m) }\end{array}$ & $\begin{array}{c}\text { Sediment transport } \\
\text { calculated using profile } \\
\text { measurements (kg/hr/m) }\end{array}$ \\
\hline A without breakwater & (a) & 9.9 & 6.6 \\
B without breakwater & (a) & 29.0 & 4.8 \\
C without breakwater & (a) & 1.4 & -0.6 \\
D without breakwater & (a) & 136.8 & 9.9 \\
A with breakwater & (a) & 18.4 & 10.2 \\
B with breakwater & (a) & 5.1 & 3.3 \\
C with breakwater & (a) & 2.1 & 2.6 \\
D with breakwater & (a) & 43.3 & 17.9 \\
A without breakwater & (b) & 22.9 & 12.5 \\
B without breakwater & (b) & 45.9 & 14.2 \\
C without breakwater & (b) & $-*$ & 5.7 \\
D without breakwater & (b) & $-*$ & 14.7 \\
A with breakwater & (b) & 26.2 & 18.6 \\
B with breakwater & (b) & 5.6 & 3.6 \\
C with breakwater & (b) & 4.2 & 0.1 \\
D with breakwater & (b) & 39.3 & 26.0 \\
A without breakwater & (c) & 145.0 & 18.8 \\
B without breakwater & (c) & 85.9 & 22.7 \\
C without breakwater & (c) & 86.4 & 16.7 \\
D without breakwater & (c) & 97.3 & 3.3 \\
A with breakwater & (c) & 47.7 & 2.3 \\
B with breakwater & (c) & 138.5 & 2.3 \\
C with breakwater & (c) & 25.5 & 4.3 \\
D with breakwater & (c) & 44.4 & \\
& & & \\
\hline
\end{tabular}

* The sediment transport of experiment $\mathrm{C}$ and $\mathrm{D}$ without breakwater at Vertical (b) could not be calculated because the velocity distributions were not available due to errors in the measurements.

Table 8.1 Calculated sediment transport by CONVERT versus measured sediment transport. 


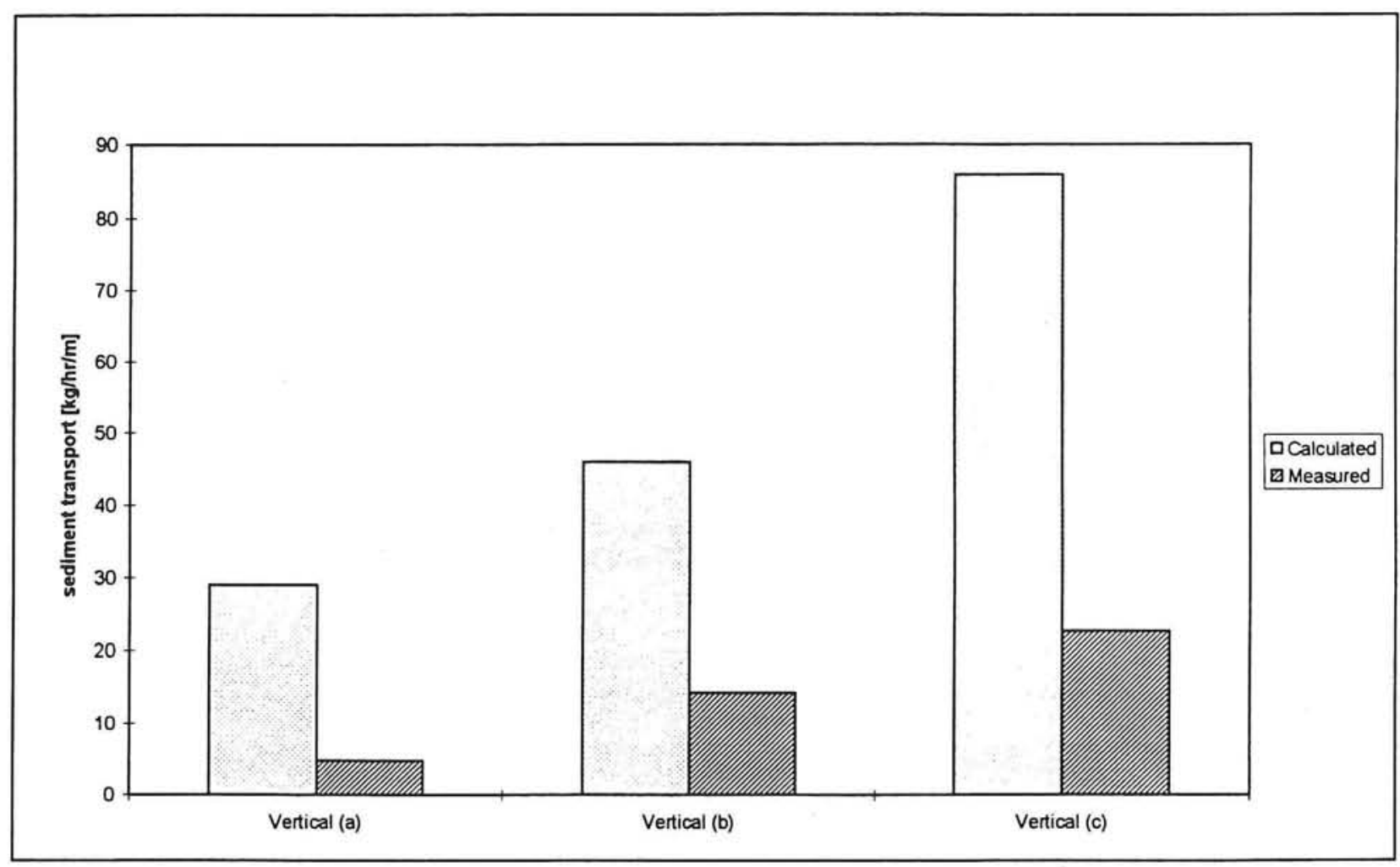

Figure 8.1 Calculated sediment transport by CONVERT versus measured sediment transport of experiment B without breakwater. 


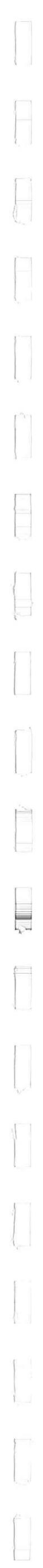




\section{CHAPTER 9 UNIBEST-TC}

\subsection{Introduction}

This chapter discusses the question whether the effect of the submerged breakwater on the morpho- and hydrodynamic behaviour can be simulated by an already existing computer program called Unibest-TC. Unibest stands for UNIform BEach Sediment Transport, TC stands for Time-dependent Cross-shore.

The Unibest-TC module is a computer package developed by Delft Hydraulics with the purpose of computing the profile changes of alongshore uniform beaches due to wave-induced cross-shore sediment transport. Unibest-TC is part of the total Unibest software package which amongst others also deals with longshore transport. In this project the focus is on the 2 DV cross-shore profile, therefore only use was made of Unibest-TC. Full information on the mathematical-physical and numerical background of Unibest-TC will be given in Appendix G (a partial reprint of the user manual).

Section 9.2 discusses which methods were used to investigate how well Unibest-TC can simulate the experiments. In order to use Unibest-TC certain parameters have to be defined. These parameters are listed in Appendix G. However some input values need to be explained, this is done in Section 9.3. Section 9.4 compares the measurement results with the results obtained with Unibest-TC. In Section 9.5 some conclusions are drawn.

\subsection{Simulation of the experiments}

\subsubsection{Modelling of the wave heights}

The experiments with irregular waves (A, B, C, D and E) have been simulated by using Unibest-TC. In order to investigate how well Unibest-TC models the development of the wave height, the measured wave heights at Interval 1 have been compared with wave heights calculated by Unibest-TC. The wave propagation program ENDEC (ENergy DECay) is implemented in Unibest-TC. This program ENDEC is used to calculate wave heights.

First of all the wave heights in case of the experiments without breakwater are calculated. Secondly the wave heights in case of the experiments with breakwater are calculated. A good modelling of the propagation of the waves is the basis for a model that calculates profile changes.

Graphs have been made which are presented in Volume 2: Graphics, Part 5.

The results are evaluated in Section 9.4.

\subsubsection{Modelling of the profile development}

In order to investigate the modelling of the profile development the measured profiles at the end of the experiments (after 7.5 hours) are compared to profiles calculated by Unibest-TC. This was done for both the experiments with, and the experiments without breakwater.

Graphs have been made which are presented in Volume 2: Graphics, Part 5. The results are evaluated in Section 9.4. 


\subsection{Input parameters}

\subsubsection{Definition of the bottom profile}

By editing a number of co-ordinates a bottom profile can be defined. Such a co-ordinate is a combination of a value for distance $\mathrm{x}[\mathrm{m}]$ and a value for bottom height $\mathrm{z}[\mathrm{m}]$. The program uses linear interpolation between two following co-ordinates.

There are two types of bottom profiles that can be defined in Unibest-TC, namely a 'movable bottom' and a 'fixed bottom'. The movable bottom simply is the sandy bottom on which erosion and/or accretion can occur. The fixed bottom is a definition of a minimum value for the bottom height. Above the level of the fixed bottom there can be a layer of sand on which erosion and/or accretion of sediment can occur, in other words, above a fixed bottom there can also be a movable bottom. An example of a 'fixed bottom' is the bottom of a wave flume. The possibility of defining a fixed bottom is of vital importance for the simulation of the experiments. The bottom of the wave flume, the first part of the slope made out of concrete and the submerged breakwater have been defined as fixed bottom (see Figure 2.3)

The bottom profile has been defined from location $x=-3.00 \mathrm{~m}$ till $\mathrm{x}=12.00 \mathrm{~m}$. The location $\mathrm{x}$ $=-3.00 \mathrm{~m}$ is the seaward boundary and is also the location of WHM 1. The location of the landward boundary $(x=12.00 \mathrm{~m})$ has been chosen because at that location certainly no profile changes will occur.

To calculate the wave heights and compare them with the measured wave heights of Interval 1 , the bottom profile was assumed to be still 1 in 15 (undisturbed bed). In reality, the bottom profile already would have undergone some changes during the measurement at Interval 1. However, these changes are so small that they would not significantly effect the wave heights at the measurement positions. Therefore small bottom changes during Interval 1 were not taken into account.

\subsubsection{Definition of wave heights at the offshore boundary}

Unibest-TC needs a couple of input parameters for the wave conditions, one of them is the $\mathrm{H}_{\mathrm{mms}}$ (= root-mean-square wave height) at the offshore boundary. The input value of the $\mathrm{H}_{\mathrm{rms}}$ needs some explanation.

There are two definitions for the $\mathrm{H}_{\text {ms }}$. One of them is the $\mathrm{H}_{\text {ms }}$ based on the total amount of energy in the spectrum (the $\mathrm{m}_{0}$ parameter). The definition of this $\mathrm{H}_{\mathrm{ms}}$ is:

$$
H_{m s}=2 \sqrt{2 m_{0}}
$$

The program SPECTPER (see Section 5.6) is able to calculate the values for the $m_{0}$-parameter of the registered signal of the wave height.

The other definition of the $\mathrm{H}_{\mathrm{ms}}$ is based on the sum of the square of the measured wave heights $\left(\mathrm{H}_{\mathrm{i}}\right)$. This sum is divided by the total number of waves $(\mathrm{N})$. The root of this value is the $\mathrm{H}_{\mathrm{rms}}$ (see Equation 9.2).

$$
H_{m s}=\sqrt{\frac{\sum H_{i}^{2}}{N}}
$$


The computer program WAVES can calculate this parameter out of the registered signal (see Chapter 5).

It is stressed that Unibest-TC uses the $\mathrm{H}_{\text {ms }}$ based on the total amount of energy in the spectrum.

As stated earlier the offshore boundary has the same location as WHM 1.

In case of the experiments without submerged breakwater the reflection of waves on the beach is relatively very small and the effect of this reflection on the wave height measurements is therefore neglected.

In case of the experiments with submerged breakwater the wave reflection caused by the breakwater is no longer negligible. When the reflected waves reach the wave board they are reflected again. The waveboard is equipped with a facility to dampen reflected long waves but there is no such facility to avoid the reflection of short waves against the wave board. Due to these circumstances the waves contain more energy than in case of the experiments without breakwater.

The wave heights measured at $x=-3.00 \mathrm{~m}$ were effected by reflected waves. Unfortunately it was technically not possible to determine the effect of waves reflected by the breakwater and waves reflected by the wave board on the measured wave height.

In case of the experiments with breakwater there are per experiment quite big differences in the measured values of the wave heights at WHM 1 at the different time-points. The differences were much less in case of the experiments without breakwater. To illustrate this, two graphs were made of the $\mathrm{H}_{\text {rms }}$ at WHM 1 , one of the experiments without breakwater and another of the experiments with breakwater. The graphs are presented in Volume 2: Graphics, Part 5. A list of these wave heights is presented in Appendix G.

To present the modelling of the $\mathrm{H}_{\text {ms }}$ by Unibest-TC the measured values at Interval 1 have been compared to the values calculated by Unibest-TC. As input value for the $\mathrm{H}_{\mathrm{mm}}$ the value measured by WHM 1 at Interval 1 was taken. In this way the measured values during Interval 1 of the other wave height meters are related to the measured value of WHM 1 .

To calculate the profile changes it was decided to use the 'average' $\mathrm{H}_{\mathrm{ms}}$ as input value for Unibest-TC. The 'average' $\mathrm{H}_{\text {ms }}$ is calculated in the same way as the average $\mathrm{H}_{\text {sig }}$ (see also Chapter 5). The average wave height of each interval was calculated. The value of the average wave height of each interval gets a weight depending on the duration of that interval.

This is shown in Table 9.1:

\begin{tabular}{|l|c|r|}
\hline & Duration of interval & $\begin{array}{l}\text { Weight of average } \\
\text { wave height }\end{array}$ \\
\hline Interval 1 & 0.5 hours & 1 \\
Interval 2 & 1.0 hours & 2 \\
Interval 3 & 2.0 hours & 4 \\
Interval 4 & 4.0 hours & 8 \\
& & Total: 15 \\
\hline
\end{tabular}

Table 9.1 Weights of the average wave heights of the different intervals. 
The average wave height of each interval is multiplied by its weight and the total sum is divided by 15 . The resulting value, the averaged $\mathrm{H}_{\mathrm{rms}}$ at WHM 1 , is taken as input value for Unibest-TC to calculate the bottom changes. This strategy has been followed for both the experiments with and without breakwater. The average wave heights are presented in Table 9.2.

A comparison of the values of the average $\mathrm{H}_{\mathrm{rms}}$ of the experiments with similar wave conditions (e.g., experiment B with and without breakwater) shows that the values of the average $\mathrm{H}_{\mathrm{rms}}$ of the experiments with breakwater are all higher than the values of the $\mathrm{H}_{\mathrm{mm}}$ of the experiments without breakwater. This could be expected because in case of the experiments with breakwater the waves contain more energy due to reflection.

\begin{tabular}{|l|c|c|}
\hline Average $\mathbf{H}_{\text {rms }}$ at WHM 1 & without breakwater & with breakwater \\
\hline Experiment A & $0.068 \mathrm{~m}$ & $0.080 \mathrm{~m}$ \\
Experiment B & $0.071 \mathrm{~m}$ & $0.084 \mathrm{~m}$ \\
Experiment C & $0.069 \mathrm{~m}$ & $0.074 \mathrm{~m}$ \\
Experiment D & $0.100 \mathrm{~m}$ & $0.101 \mathrm{~m}$ \\
Experiment E & $0.048 \mathrm{~m}$ & $0.049 \mathrm{~m}$ \\
\hline
\end{tabular}

Table 9.2 Average values of the $H_{\text {rms }}$ at WHM 1.

\subsubsection{Other parameters}

For the calculations it was necessary to divide the domain $(x=-3.0 \mathrm{~m}$ till $\mathrm{x}=12.0 \mathrm{~m})$ in a number of pieces with length $\Delta \mathrm{x}$ (see Appendix $\mathrm{G}$ ).

It was also necessary to define a time-step. In case of the calculation of the wave heights at the beginning of the experiments only one short time-step was taken.

In case of the calculation of the profile changes after 7.5 hours a timestep of 5 minutes was chosen which resulted in a total of 90 timesteps.

The remaining parameters that are needed for the wave and the sediment transport model can also be found in Appendix G.

\subsection{Comparison measurements with Unibest-TC}

\subsubsection{Comparison measured and calculated wave heights}

All the experiments with irregular waves (A, B, C, D and E) have been simulated by using Unibest-TC. The wave heights measured at Interval 1 have been compared to the wave heights calculated by Unibest-TC. In order to do this some graphs were made. These graphs, listed in Table 9.3, are presented in Volume 2: Graphics, Part 5.

First of all the experiments without breakwater have been investigated. Figure 9.1 shows as an example the graph of the results of experiment B without breakwater. The wave propagation model ENDEC, which is included in Unibest-TC, describes the propagation of the 


\begin{tabular}{|l|l|}
\hline & Experiment \\
\hline Graph 5.3 & experiment A without breakwater \\
Graph 5.4 & experiment B without breakwater \\
Graph 5.5 & experiment C without breakwater \\
Graph 5.6 & experiment D without breakwater \\
Graph 5.7 & experiment E without breakwater \\
Graph 5.8 & experiment A with breakwater \\
Graph 5.9 & experiment B with breakwater \\
Graph 5.10 & experiment C with breakwater \\
Graph 5.11 & experiment D with breakwater \\
Graph 5.12 & experiment E with breakwater \\
\hline
\end{tabular}

Table 9.3 Review of the graphs made of the measured $H_{m s}$ at WHM 1 during Interval 1 and the calculated $H_{m s}$. Graphs are presented in Volume 2: Graphics, Part 5.

waves very well. The graph of experiment A shows that the calculated increase of the wave height towards the shore (as effect of shoaling) is larger than the measured increase of the wave height. The graphs of experiments $\mathrm{C}$ and $\mathrm{E}$ also show a small overestimation of the increase of the wave height. A possible explanation for this fact could be that the amount of energy dissipation due to bottom friction and internal dissipation is somewhat higher in reality than was calculated. Apart from this, the agreement between measured and calculated wave heights is very good.

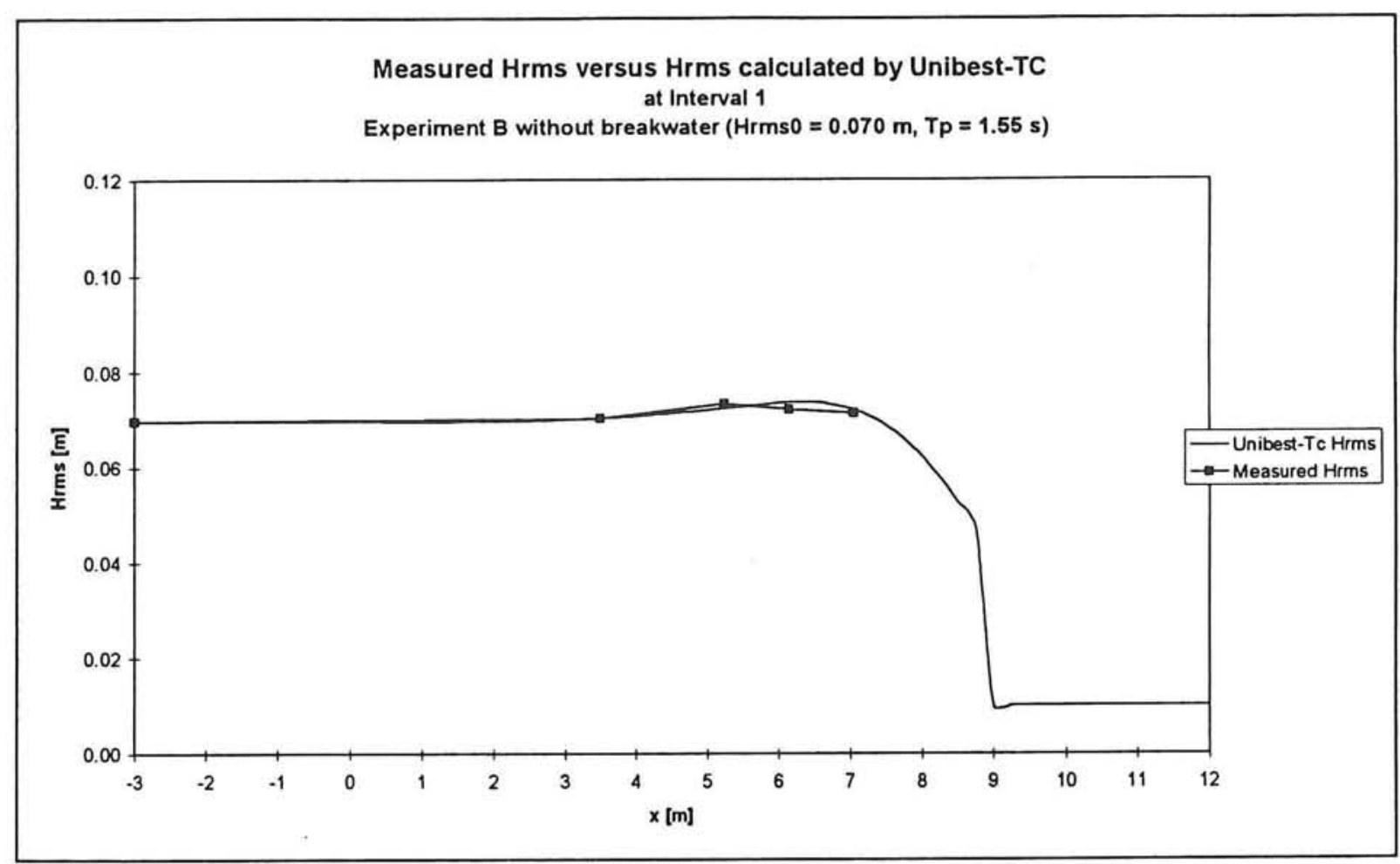

Figure 9.1 Example of a graph of the comparison between measured and calculated wave heights. 
The next step was to evaluate how well the effect of the submerged breakwater on hydrodynamics was modelled. Figure 9.2 shows as an example the graph of the results of experiment B with breakwater.

The results of the experiments with breakwater are less good compared to the results of the experiments without breakwater but are still acceptable. The calculated wave heights behind the breakwater are for all experiments slightly higher than the measured wave heights. The amount of energy dissipation due to the passing of the breakwater seems to be underestimated. A cause for the larger differences between measured and calculated wave heights of the experiments with breakwater can be the way the submerged breakwater is modelled in Unibest-TC. The breakwater is modelled as 'fixed bottom', therefore the effect of the porosity and the roughness of the armour layer on hydrodynamics is not taken into account.

At the position of the breakwater $(x \cong 4.2 \mathrm{~m})$ the calculated wave heights suddenly increase a lot. The way in which ENDEC calculates wave propagation does not reckon with the fact that wave height and wave form in reality can not react instantaneously on abrupt changes in the bottom profile. The breakwater is such an abrupt change in the bottom profile.

Also the fact that the influence of the reflected waves on the measured wave heights of WHM 1 could not be determined precisely, might cause differences between measured and calculated wave heights. The input value of the $\mathrm{H}_{\text {rms }}$ at the offshore boundary determines the wave heights at the other positions.

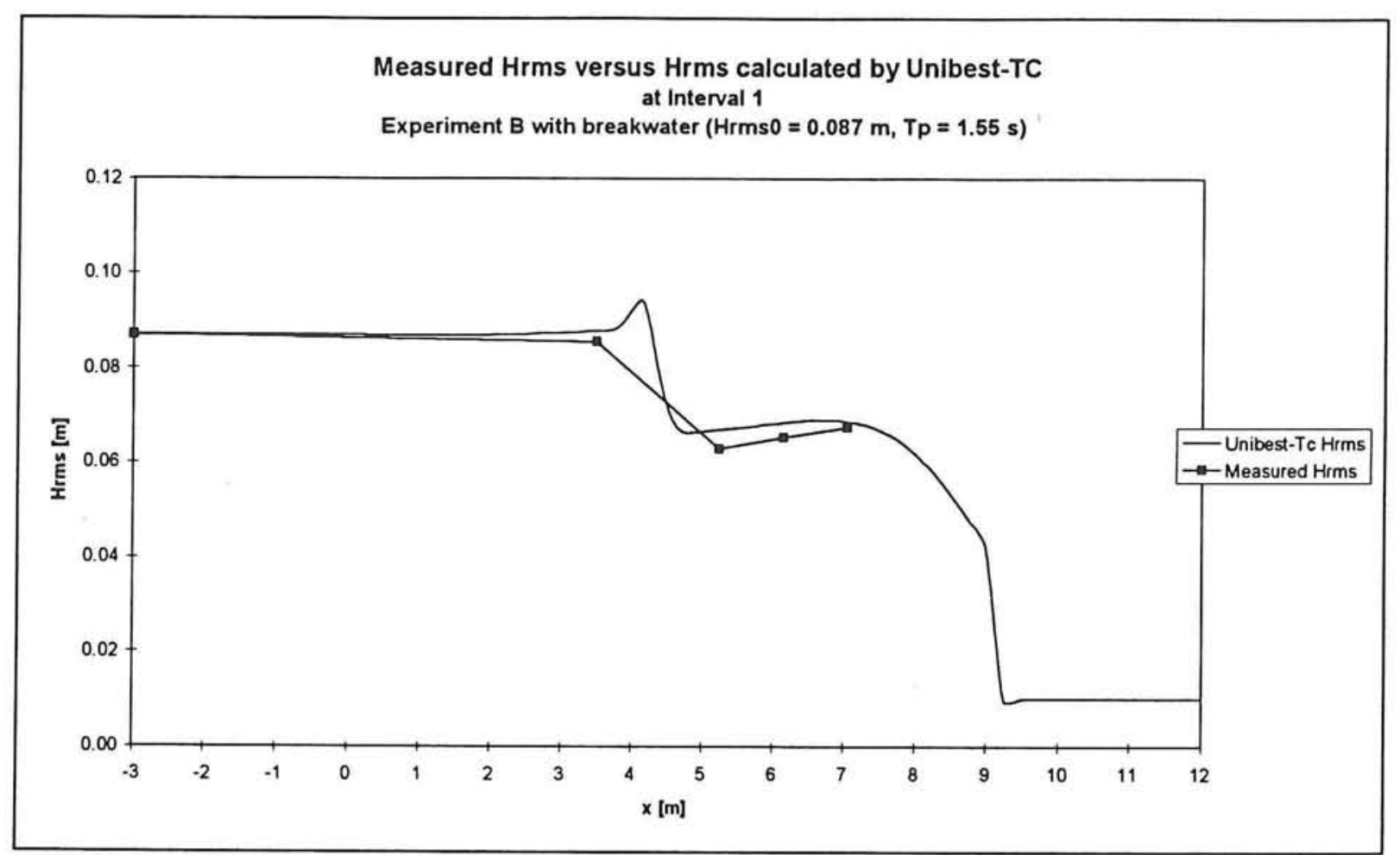

Figure 9.2 Example of a graph of the comparison between measured and calculated wave heights. 


\subsubsection{Comparison measured and calculated profile}

All the experiments with irregular waves (A, B, C, D and E) have been simulated by UnibestTC. The measured profiles and the calculated profiles after 7.5 hours of waves are presented in graphs. Each experiment is presented separately. Table 9.4 gives a list of the graphs that are presented in Volume 2: Graphics, Part 5.

\begin{tabular}{|l|l|}
\hline & Experiment \\
\hline Graph 5.13 & experiment A without breakwater \\
Graph 5.14 & experiment B without breakwater \\
Graph 5.15 & experiment C without breakwater \\
Graph 5.16 & experiment D without breakwater \\
Graph 5.17 & experiment E without breakwater \\
& \\
Graph 5.18 & experiment A with breakwater \\
Graph 5.19 & experiment B with breakwater \\
Graph 5.20 & experiment C with breakwater \\
Graph 5.21 & experiment D with breakwater \\
Graph 5.22 & experiment E with breakwater \\
\hline
\end{tabular}

Table 9.4 Review of the graphs of the measured and calculated profiles after 7.5 hours. Graphs are presented in Volume 2: Graphics, Part 5.

In case of the experiments without submerged breakwater Unibest-TC predicts the profile development reasonable. As an example the graph of experiment B without breakwater is presented in Figure 9.3.

The trend of erosion in the breakerzone and accretion more offshore is well predicted.

Only in the region near the waterline differences between measured and calculated profile become large. This is due to the ' $\mathrm{T}_{\mathrm{dry}}$-parameter' (see Appendix $\mathrm{G}$ ) of Unibest-TC. The $\mathrm{T}_{\mathrm{dry}}$ parameter is a parameter that indicates the water depth beyond which the normal calculations are stopped because the water depth gets so small that the used formulas are not valid any more. The sediment transport process near the waterline simply is not understood well enough to put this in a (reliable) model. The bottom profile landward of the shoreline calculated by Unibest-TC shows therefore hardly any development contrary to the measured profile. In other words, the shoreline remains at the same place. This is a serious shortcoming of the model because in reality the interest often is in the profile development near the shoreline.

In case of experiment $\mathrm{A}$ and $\mathrm{E}$ the calculated profile changes are considerably smaller than the measured profile changes. The results for experiment $\mathrm{B}, \mathrm{C}$ and $\mathrm{D}$ are much better.

All together it can be concluded that Unibest-TC predicts the trend of the profile development quite well. The time scale on which the profile changes occur are well predicted for the experiments $\mathrm{B}, \mathrm{C}$ and $\mathrm{D}$ and less good for experiment $\mathrm{A}$ and $\mathrm{E}$. 


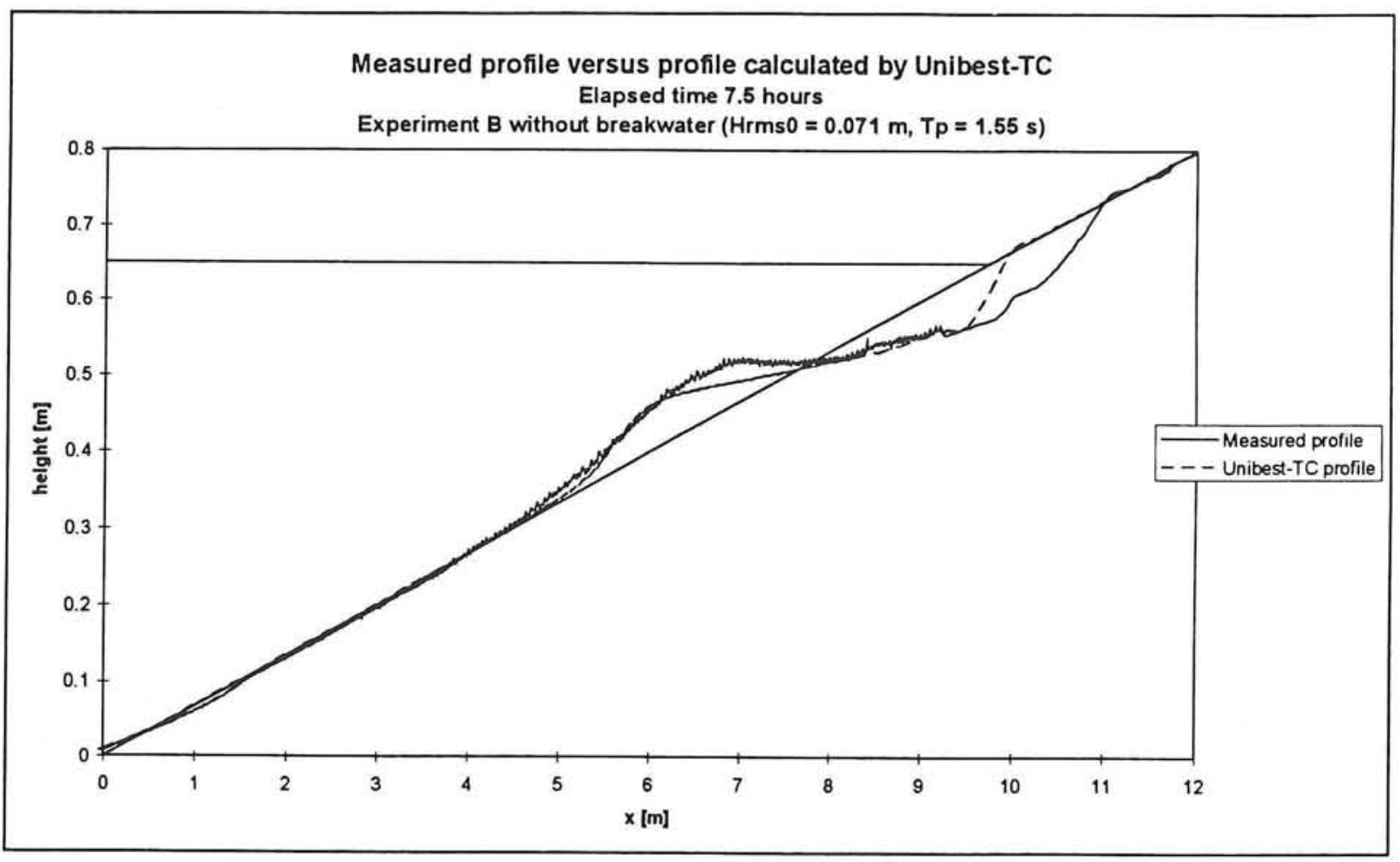

Figure 9.3 Example of a graph of the measured profile versus the calculated profile.

In case of the experiments with submerged breakwater the differences between measured and calculated profiles are larger. As an example the graph of experiment B with breakwater is presented in Figure 9.4.

Near the shoreline the same problem as with the experiments without breakwater occurs. Near the breakwater the differences also become large. The accretion of sediment at the back of the breakwater is underestimated by Unibest-TC.

In general Unibest-TC seems to underestimate the profile changes behind the breakwater. This seems strange because the calculated wave heights behind the breakwater are larger than the measured wave heights (see previous section). In case of the experiments without breakwater the calculated wave heights were just slightly bigger and for those experiments the profile development was predicted quite well.

An explanation for the underestimated profile changes can be that the increase in turbulence and the increase of the average wave frequency caused by the breakwater are not modelled in Unibest-TC.

The calculated bottom profiles of the experiments with breakwater have a lot in common with the calculated bottom profiles of the experiments without breakwater. Their shape is more or less the same, only the amount of accretion and erosion in case of the experiments with breakwater is less due to the smaller wave heights. 


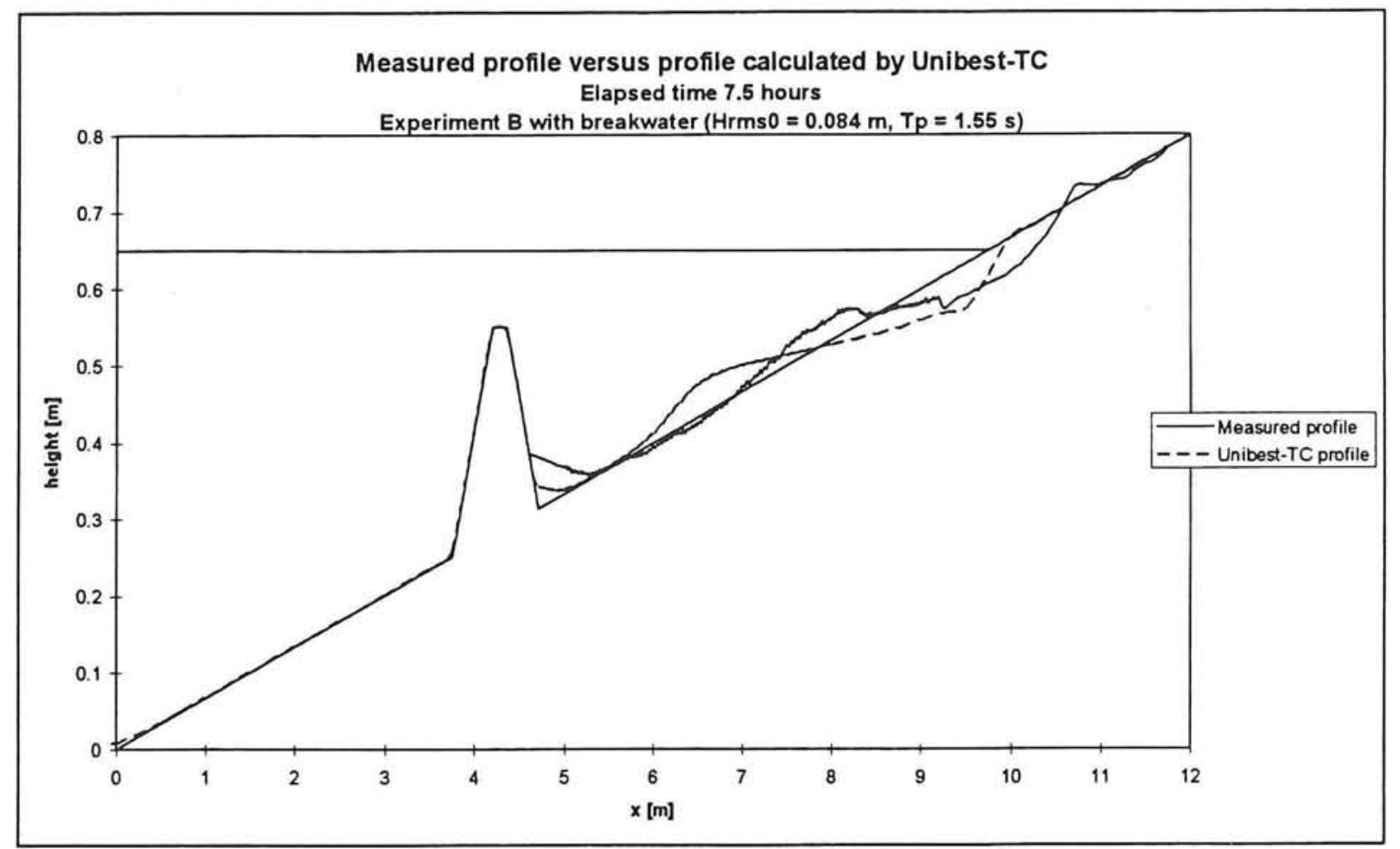

Figure 9.4 Example of a graph of the measured profile versus the calculated profile.

\subsection{Conclusion}

The sediment transport process cross-shore is very complicated. It is influenced by a lot of factors (wave conditions, sediment parameters etc.). How all these factors together determine the sediment transport is still not fully understood. A computer model like Unibest-TC is a simplification of the reality. Like every other model it has its shortcomings but as long as we keep those shortcomings in mind it can be a very good tool. In case of the experiments without breakwater the results are quite reasonable. The results for the experiments with breakwater are less good. This could be expected because the model was not specifically designed to take into account the effect of very abrupt changes of the bottom profile on the hydro- and morphodynamics. The simulation of the experiments with breakwater with Unibest-TC is an even further simplification of the reality. The results are nevertheless hopeful but still a lot of research has to be done to improve the knowledge about sediment transport processes and thereby improve the reliability of models like Unibest-TC. 


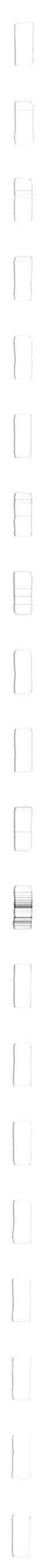




\section{CHAPTER 10 CONCLUSIONS AND RECOMMENDATIONS}

\section{$\underline{10.1 \text { Conclusions }}$}

The aim of building a submerged breakwater is to protect the coast behind it. The experiments have been performed in order to investigate how well this aim can be achieved by such a submerged breakwater. The experiments with breakwater showed that in general the amount of sediment transport offshore was reduced compared to the tests without breakwater. However, there was sediment transport over the breakwater in offshore direction. This sediment can be considered as lost sediment. It is not to be expected that under natural conditions a significant amount of this 'lost' sediment will pass the breakwater again in onshore direction.

The breakwater causes much dissipation of wave energy. The wave heights behind the breakwater are reduced. The wave heights offshore of the breakwater have increased due to reflection.

The breakwater decreases the time-average velocities landwards of the breakwater. This is of course related to the smaller orbital motions as result of the smaller wave heights.

The sediment concentration measurements showed a relatively high and steep sediment concentration curve in the first vertical behind the breakwater (Vertical (a)). A lot of turbulence occurs behind the breakwater which stirs up the sand. At the most landward vertical (Vertical (c)) the sediment concentrations of the experiments with breakwater were smaller compared to the experiments without breakwater. This is a result of the smaller wave heights.

If a comparison is made between the experiments A, B, C, D and E some conclusions can be made. A change in wave height (compare experiment $\mathrm{B}, \mathrm{D}$ and $\mathrm{E}$, all with a more or less equal wave steepness) has more effect on the hydro- and morphodynamics than a change in wave period (compare experiment A, B and C, all with a more or less equal wave height). This was the case for both the series of experiments with and without breakwater.

A comparison between the sediment transport based on measured profiles and the sediment transport based on multiplification of time-average sediment concentrations and time-average velocities, shows that the last method leads to an overprediction of the sediment transport.

The results of the calculations with Unibest-TC show the importance of experiments like this. The sediment transport process cross-shore is very complicated. It is influenced by a lot of factors (wave conditions, sediment parameters etc.). This process is still not fully understood. A computer program based on the knowledge of today will therefore have its shortcomings. Implementation of the effects of a submerged breakwater is a step further. First the effects of a submerged breakwater on hydro- and morphodynamics will have to be known better to be able to implement them in a (reliable) computer program. These experiments are a good way to increase this knowledge.

To judge about the effectiveness of a submerged breakwater as a protection of the coast also $3 \mathrm{DV}$ effects must be taken into account. The performed 2DV experiments are one part of the story. The influence of waves coming in under an angle and the thereby caused longshore current and longshore transport will strongly effect the hydro- and morphodynamics. 


\section{$\underline{10.2}$ Recommendations}

To draw conclusions about the profile development it is recommended that also some experiments should be performed over a longer period of time. During the now performed experiments the equilibrium profile was not reached.

For wave height measurements a non-movable bed would be preferable. The advantage of a non-movable bed is that the wave heights can not be effected by a change in the bed profile. A comparison of the wave heights of different experiments will become more reliable.

To draw conclusions about the effect the breakwater has on velocity distributions the experiments can also better be performed with a non-movable bed for more or less the same reasons. Another advantage is that a non-movable bed gives the oppurtunity to increase the number of measerement points. The situation remains stable (no profile changes).

Experiments with non-movable beds will be performed by other partners of this Dynamics of Beaches project in the near future. A comparison of all the results of the experiments (2DV and $3 \mathrm{DV}$, non-movable and movable bed) will certainly increase the understanding of the effects of a submerged breakwater and the role it can play as a proper protection tool of the coast.

At last it is stressed that the experiments that were performed during this project resulted in a large data base. This data base is available for further analysis (see Section 1.6). 


\section{REFERENCES}

- $\quad$ Battjes, J.A., 1986

Korte golven (short waves, in Dutch)

Collegedictaat B76, korte golven

Delft University of Technology, Faculty of Civil Engineering

- $\quad$ Battjes, J.A., 1990

Windgolven (Wind-waves, in Dutch)

Collegedictaat B78, windgolven

Delft University of Technology, Faculty of Civil Engineering

- $\quad$ Daemen, I.F.R., 1991

Wave transmission at low-crested breakwaters

Master's thesis

Delft University of Technology, Faculty of Civil Engineering

- $\quad$ Davies, B.L. and Kriebel, D.L., 1992

Model testing of wave transmission past low-crested breakwaters

In Proceedings of 23rd International Conference on Coastal Engineering, volume 1, pp 1115-1128

- $\quad$ Van der Meer, J.W. and Daemen, I.F.R., 1994

Stability and wave transmission at low-crested rubble-mound structures

Waterway, Port, Coastal and Ocean Engineering, pp 1(1):1-19

- $\quad$ Van der Meer, J.W., 1990

Low-crested and reef breakwaters

Technical Report, Delft Hydraulics, DH H-986

- $\quad$ Van der Meer, J.W., 1991

Stability and transmission at low-crested structures

Technical Report, Delft Hycraulics, DH H-453 


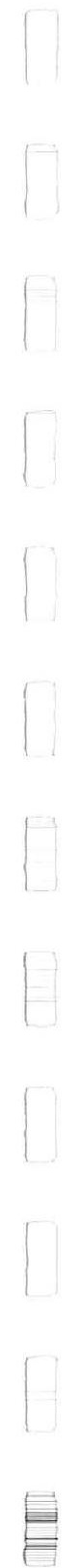

咒 


\section{APPENDIX A}

AUKE/PC

$$
\begin{array}{ll}
- & \text { STIR } \\
- & \text { FILTER } \\
- & \text { SPECTPER } \\
- & \text { STATIST } \\
- & \text { WAVES }
\end{array}
$$




\section{AUKEPC}

Program : STIR

Version 1.00

Licensed user : DELFT HYDRAULICS

Copyright (C) DELFT HYDRAULICS 1989 


\section{Program description}

\section{Introduction}

In this part of the user's manual the theoretical background is described. With this background the use of program STIR is described.

Secondly, the use of the commands required to generate a control signal is explained.

\section{Theoretical backgrounds}

Wave types.

In STIR three wave types are available :

-regular waves

-deterministic waves

-random waves

They are described in the given order. The formulae which are used represent the wave field in the flume or basin. These formulae have nothing to do with the signal for the wave board movement.

Regular waves.

This wave type has only one frequency. The formula used to compute the water elevation $\mathrm{x}$ at time $\mathrm{t}$ for a regular wave :

$x(t)=A \cos (2 \pi f t+\psi)$

with A the wave amplitude $(\mathrm{m})$ being half the height between top and trough and $\mathrm{f}$ the wave frequency $(\mathrm{Hz})$, being the reciprocal of the wave period. In the formula $\psi$ represents the phase of the signal at $t=0$. In STIR this phase is set to zero, as any other value is of no influence on the wave field.

\section{Deterministic waves.}

This wave type is a summation of a number of regular waves, expressed by the formula :

$$
x(t)=\sum_{i=1}^{n} A_{i} \cos \left(2 \pi f_{i} t+\Psi_{i}\right)
$$

For all frequencies the $\psi_{i}$ phases are set to 0 .

From the formula it will be obvious that, for instance it is possible to generate a bichromatic wave field with two frequencies close to each other. The actual periods used are accurate within $.001 \mathrm{~s}$ of the required value. 
Random waves.

A random signal is in fact a superposition of regular waves with varying frequencies according to the following formula :

$x(t)=\sum_{i=1}^{\infty} A_{i} \sin \left\{2 \pi f_{i} t+\psi\left(f_{i}\right)\right\}$

with $A_{i}$ the wave amplitude for the $i$-th frequency component and $\psi$ the phase of the corresponding frequency $f_{i}$ at $t=0$.

We can rewrite this to

$x(t)=\sum_{i=1}^{\infty}\left\{a_{i} \cos \left(2 \pi f_{i} t\right)+b_{i} \cos \left(2 \pi f_{i} t\right)\right\}$

where $a_{i}=-A_{i} \sin \left(\psi_{i}\right)$ and $b_{i}=A_{i} \cos \left(\psi_{i}\right)$. Here $A_{i}$ is again the wave amplitude for the $\mathrm{i}$-th frequency component.

The variable $A_{i}$ is related to spectral densities according to :

$A_{i}=\sqrt{2 S\left(f_{i}\right) \Delta f}$

where $\mathrm{S}\left(\mathrm{f}_{\mathrm{i}}\right)$ is the spectral density at frequency component $i$.

Now Fourier analysis can be applied according to the formula :

$x(n)=\frac{1}{N} \sum_{k=0}^{N-1} X(k) e^{i \frac{2 \pi}{N} n k}$

where $X(k)$ is the complex variable with real part $a_{n}$ and imaginary part $b_{n}$ from Eq. 4 and $x(n)$ the value of the real signal at the discrete point $n$, with $0 \leq \mathrm{n} \leq \mathrm{N} / 2+1$.

As the frequency values are chosen such that they are equidistant at $\Delta \mathrm{f}$ it is possible to use a Fast Fourier Transform to transform the values to a signal in the time domain.

The spectral density at each frequency, $S(f)$, is either computed using existing formulae or according to a spectral shape prescribed by the user.

With the latter option the densities are computed by linear interpolation between the given values at the prescribed frequency values.

When a formula is selected, the density at a certain frequency is computed according to this formula. In STIR two spectral types are applied, the JONSWAP spectrum and the PIERSON-MOSKOVITZ spectrum.

The formula of the JONSWAP spectrum : 


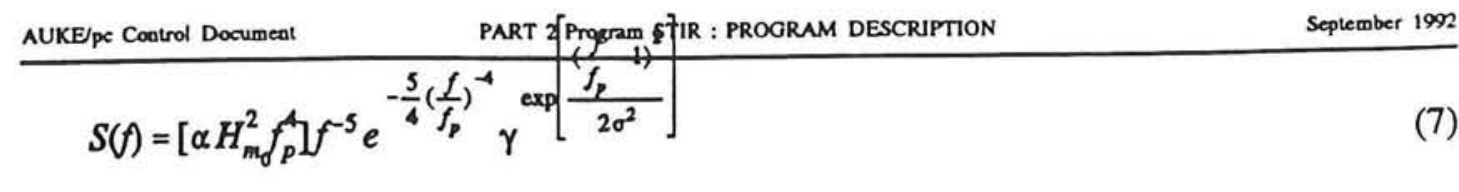

where $\gamma=3.3$ for the JONSWAP spectrum and any other positive value is allowed. The $\alpha$ is a scaling factor to obtain the significant wave height $\mathrm{H}_{\mathrm{mo}}=4 \sqrt{m_{0}}$.

The frequency $f_{p}$ is the frequency with the greatest density in the spectrum. In the program the values for $\sigma$ are set to $\left\{0.07 ; \mathrm{f} \leq \mathrm{f}_{\mathrm{p}}\right\}$ and to $\left\{0.09 ; \mathrm{f}>\mathrm{f}_{\mathrm{p}}\right\}$.

The formula for the Pierson-Moskovitch spectrum is almost identical to the JONSWAP formula. Now $\gamma=1$ is used, so the JONSWAP formula transforms to a simpler one :

$S(f)=\left[\alpha H_{m_{f}}^{2} f_{p}^{4} f^{-5} e^{-\frac{5}{4}\left(\frac{f}{f_{p}}\right)^{-4}}\right.$

In STIR $\infty$ is not used, the upper limit is default set to $5 \mathrm{~Hz}$ as long as the user does not overrule this value. Most of the time the user will set it to a lower limit. The program tries to find a result such that $\mathrm{H}_{\mathrm{m} 0}$ is accurate within $1 \%$ of the asked $\mathrm{H}_{\mathrm{m} 0}$ where $H_{m 0}=4 \sqrt{m_{0}}$ and $\mathrm{m}_{0}$ the area of the spectral function. However, an upper frequency limit also implies that part of the frequencies are cut off. If the $1 \%$ accuracy cannot be obtained the program tries to find a user-supplied accuracy. It scales all spectral density values such that the required $\mathrm{H}_{\mathrm{mo}}$ value is obtained.

In STIR $\psi\left(\mathrm{f}_{\mathrm{i}}\right)$ in Eq. 3 or the $\mathrm{a}_{\mathrm{i}}$ and $\mathrm{b}_{\mathrm{i}}$ coefficients in Eq. 4 are selected at random with two possible available methods :

1. Random Phase Method.

2. Random Coefficient Method.

With the Random Phase Method the phase $\psi(0 \leq \psi \leq 2 \pi)$ is selected at random with a uniform random number generator.

When the Random Coefficient Method is used both $\mathrm{a}_{\mathrm{n}}$ and $\mathrm{b}_{\mathrm{n}}$ are selected at random with a random number generator selecting values according to the Normal distribution function with the mean $=0$, and the standard deviation 1 . Then

$$
a_{n} ; b_{n}=N(0,1) \cdot \sqrt{S\left(f_{n}\right) \Delta f}
$$

with $\mathrm{N}$ a random number out of the normal distribution and a successive selection for $a_{n}$ and $b_{n} . S\left(f_{n}\right)$ : the spectral density for frequency is $f_{n}$ and $\Delta f$ is the equidistant step in the frequency band.

As the amplitude $A_{n}$ at frequency $f_{n}$ is related to the coefficients according to 


$$
A_{n}=\sqrt{\left(a_{n}^{2}+b_{n}^{2}\right)}
$$

then the values $A_{i}$ are $\chi^{2}$ distributed with two degrees of freedom. Now a new density value is used:

$$
S\left(f_{n}\right)=\frac{A_{n}^{2}}{2 \cdot \Delta f}
$$

The Random Coefficient Method meets nature best. The resulting $\mathrm{H}_{\mathrm{m} 0}$ may deviate from the required value. This depends on the total number of frequencies used in the spectrum : the longer the signal the better $\mathrm{H}_{\mathrm{m} 0}$ will be met.

Second order transformation is described in Appendix A of this manual. We refer to this Appendix to learn about its use and application.

\section{Transfer functions.}

A signal sent to the wave board system is transformed before it becomes a wave field. Two transfer functions apply :

- the transfer of the wave board electronics and mechanics, the so-called hardware filter.

- the transfer between the wave board and water, the so-called Biesel function.

Both functions are defined in the frequency domain. The Biesel function only transforms the amplitude whereas the hardware filter transforms both the amplitude and the phase.

The Biesel function is independent of the used hardware. The hardware filter is not defined and must be measured for various frequencies to determine its proportions and to fix the parameters of the function.

For a certain frequency the value of the functions is used to transform a wave amplitude to a wave board movement.

\section{Biesel function}

This function gives the relation between the amplitude $A_{x}$ of the wave board at a given frequency $f_{n}$ and the amplitude $A$ of the water for this frequency.

$$
\frac{A_{x}\left(f_{n}\right)}{A\left(f_{n}\right)}=\frac{C_{g}\left(f_{n}\right)}{C\left(f_{n}\right) \tanh \left(k_{n} h\right)}
$$

where $C$ is the phase velocity and $\mathrm{C}_{\mathrm{g}}$ the group velocity. For the given water depth $\mathrm{h}$ the wave number $\mathrm{k}$ is computed. The right hand part of Eq. 9 is the Biesel function. This transfer is performed before the hardware filter is used as the system needs the 
real movement of the wave board.

\section{Hardware filter}

The hardware filter is the transfer function of the wave board, both mechanical and electronical. It presents the relation between the expected deflection for a given voltage and the real, resulting deflection. At the lower frequencies this will be 1 , with increasing frequencies the reciprocal of this factor will decrease.

Along the frequency band there is a phase lag (shift). With first order control this phase lag does not unacceptably influence the wave field. With second order controlling the phase lag has to be accounted for. In STIR the phase shift is corrected for both control modes.

These two functions of the frequency form a complex hardware filter.

The formula for the filter :

$$
H(s)=\frac{\underset{i=1}{m}\left(Z_{i}-s\right)}{\prod_{j=1}^{n}\left(P_{j}-s\right)}
$$

with $\mathrm{P}_{\mathrm{j}}$ the complex poles and $\mathrm{Z}_{\mathrm{i}}$ the complex zero points.

The $s$ domain is related to the frequency with $s_{n}=2 \pi f_{n}$.

The filter functions are presented in Fig. 1.

\section{Resampling.}

Resampling is only done with deterministic waves and random waves. Regular waves are not resampled, here the exact control ratio is computed.

Initially, the control signal is generated such that frequencies which contain energy are in the active range of the frequency domain. In the second order mode this active range is doubled.

A maximum frequency is selected, above which no energy is taken into account. This maximum frequency is used to compute the Nyquist frequency which is equal or greater than the maximum frequency.

The program is not free in selecting this frequency because in Fourier analysis there is also a relation between the Nyquist frequency and the time step in the time domain :

Nyquist frequency $=.5 / \Delta t$

As for the control signal the user can set a control ratio such that either the generated wave or the wave board motion is so smooth that no undesirable acceleration will be 
introduced. The time step of the Nyquist frequency has to be an integer multiple of the control ratio used. Program STIR provides this condition.

The Nyquist frequency used will also prevent the effect of unwanted modulation which may occur with random wave generation.

Resampling is performed in the frequency domain with the use of an FFT. When performed in the time domain it is possible that unwanted accelerations will be introduced.

For this principle the formula used is :

$\hat{F}\left(f_{j}\right)=F\left(f_{j}\right) e^{2 \pi i \frac{\pi f_{j}}{\Delta \Delta \Delta^{N}}}$

where $\Delta_{t}$ and $\Delta_{f}$ are the equally spaced steps both in the time and in the frequency domain. Provided that inverse FFT is used for transforming $\hat{F}$, the signal values at time $t=t_{k}+\tau$ are found. 


\section{FILTER}

\section{Introduction.}

The FILTER program can be applied for :

-elimination of harmonics with given frequencies

-making the first derivative of a series

-performing a Hilbert transform

-processing a periodogram

One or more series from one data file can be transformed. The resulting new series are stored in a new series data file.

\section{Theory}

A complete series or a part of a series from a series file is selected. Then, for analysis, an FFT is used according to Eq. 1 in Part 1 of the Process document. The $\hat{X}(k)$ will be transformed to $\tilde{X}(k)$, according to the selected mode. With an inverse FFT according to Eq. 7 the result again is transformed to the time domain.

The FFT used can handle a number of values according to $n=2.2^{i} \cdot 3^{\mathrm{j}} \cdot 5^{\mathrm{k}}$ with $\{\mathrm{i} ; \mathrm{j} ; \mathrm{k}\} \geq 0$. The $\mathrm{n}$ is set closest to the number of selected values but must always be equal or higher.

Note : the maximum number of available values amounts to 60,000 .

\section{Filtering}

Filtering of harmonics means that in the complex series $\hat{X}\left(0: \frac{N}{2}\right)$ values are set at zero. With special commands in the PCF file the following filtering options can be selected :

- low pass, all series values with a location value which is higher than or equal to the low value are set at zero.

Example : suppose that the low value is 1.3 and the equidistant step value in $\hat{X}\left(0: \frac{N}{2}\right)$ is .15 , then all series values in locations $\operatorname{ENTIER}\left(\frac{1.3}{.15}\right)=8$ and higher are made zero. The location value is $8^{*} .15=1.2$.

- high pass, all series values with a location value which is smaller than or equal to the high value are set at zero.

- band pass, two values are given being the lower and upper limits of a frequency band. Two options are available :

1. The values pertaining to the frequencies which lie between the given 
values are passed.

2. The values pertaining to the frequencies which lie outside given values are passed.

Where passing is not permitted the values are set at zero.

One or more of these band pass commands may be used, the commands are executed one after another. For each filter operation the result of the previous filter operation is used.

\section{First derivative}

The first derivative of a series is computed according to the formula

$$
\tilde{X}(k)=i(2 \pi k \Delta f) \hat{X}(k) \text { with } \mathrm{k}=0,1, \ldots, \mathrm{N} / 2 .
$$

The derivative of the resulting series may be computed again and again in order to obtain the $\mathrm{n}^{\text {th }}$ derivative of a series. This can be done by giving as many DERIVATIVE type commands as the magnitude of the required power number of the derivative. The higher frequencies will be influenced by this operation.

\section{Integration}

Integration is the reciproke of derivative computation. The formula :

$$
\tilde{X}(k)=\hat{X}(k) / i(2 \pi k \Delta f) \text { with } \mathrm{k}=0,1, \ldots, \mathrm{N} / 2 .
$$

Here too integration can be done repeatedly. The lower frequencies are influenced.

\section{Hilbert transform}

With the Hilbert transform the phases of the various frequencies are set $90^{\circ}$ backwards. The used transform is :

$$
\tilde{X}(k)=-i \hat{X}(k) \quad \text { with } \mathrm{k}=0,1, \ldots, \mathrm{N} / 2 \text {. }
$$

All types of transformation mentioned before can be used one after another on the result of a previous transformation.

The following operation to obtain a periodogram reacts to the final result of the filtering. If no filtering is done the original selected series values will be used. 


\section{Periodogram}

After the FFT is completed the amplitude for all complex $\hat{X}=(k)$ values is computed. In this way, the spectral information for all possible frequencies is computed. No inverse FFT will be performed and the resulting series is frequency-based.

The phase pertaining to each frequency is also computed so that it can be used or examined by the user.

Commands summary

General commands

DATA,string

DATAFILE, string

SCALE, $\{[$ YES $], \underline{\text { NO }}\}$

SELECT, $\{[\underline{A L L}] \mid$

$<$ EQ-STEP, LOW $=$ value, $\mathbf{H I G H}=$ value, $\mathbf{S T E P}=$ value $>\mid$

$<$ EQ-NYQ,LOW $=$ value, $\mathbf{H I G H}=$ value,$N Y Q=\underline{\text { value }}>\mid$

$<\underline{\text { SERIES}}$, NAME $=\underline{\text { string, }}$ LOW $=\underline{\text { value }}, \mathbf{H I G H}=\underline{\text { value }}>\mid$

$<\underline{\text { \#}}, \mathrm{LOW}=$ intval, HIGH $=$ intval, STEP $=$ intval $>$ \}

SERIES, $\{[\underline{A L L}] \mid$

$<$ CODE, CODEPART $=$ string, EXTENSION $=$ string $>\mid$

$<$ RANGE, CODENAME $=$ string, LOW $=$ intval, $\mathbf{H I G H}=$ intval $>\mid$

$<$ UNIOUE, NAME $=$ string $>\}$

ZEROLEVEL, $\{[\underline{\text { YES }]}|\underline{\text { NO }}|\}$

Special USE commands

USE

POLY0, $\{$ CALCULATE $\mid\}$

POLY1,\{CALCULATE $\mid\}$

POLY2,\{CALCULATE $\mid\}$

POLY3, \{CALCULATE $\}$

END:USE

Program FILTER commands

TYPE, $\{$ NONE $\mid$

$<$ LOW-PASS,FREQ $=$ freq $>$

$<$ HIGH-PASS,FREQ $=$ freg $>$ ।

$<$ BAND-PASS, LOWER $=$ freq,, UPPER $=$ freq,,$F R E Q=\{[$ INSIDE $] / /$

OUTSIDE $\}>$ |

DERIVATIVE 


\section{INTEGRATION}

HILBERT\}

SEQUENCE, CODE-OLD $=$ name,, ODE-NEW $=$ name, UNIT $=$ string

STORE, $\{<$ PERIODOGRAM|[SERIES]\}

\section{Commands description}

The TYPE command reflects the possibilities as described in the theory section. The SEQUENCE command is used for administration purposes. It is obligatory to use the general command DATA because the purpose of the program is both to generate adjusted signals and to store them.

\section{TYPE, \{NONE $\mid$}

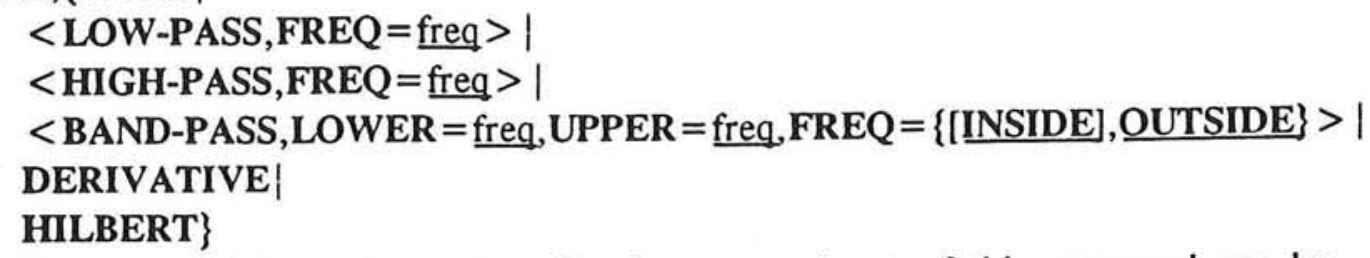

The command is used to select filtering types. A set of this command can be used. If there is more than one type in the input field all types work together to generate a new signal in the sequence they are incorporated in the command file.

NONE let the program know that no filtering is required. This option overrules other TYPE options in the command file.

LOW-PASS is used to set a frequency freq. This frequency is a limit above which all amplitudes at the computed frequencies are set at zero.

HIGH-PASS is the opposite of the previous mode. Here all amplitudes of the frequencies below freg are set at zero.

BAND-PASS is used to set frequencies ranging from the LOWER to the UPPER frequency, the band. The choice FREQ lets the program know whether the amplitudes OUTSIDE the band or INSIDE the band are passed and so the remaining amplitudes are set at zero. Default the INSIDE is not changed.

DERIVATIVE of the signal is computed.

INTEGRATION of the signal.

HILBERT transform of the signal is computed.

\section{STORE, $\{<$ PERIODOGRAM $\mid[$ SERIES] $\}$}

This command lets the program know what result must be stored in a new series file. If SERIES is set the series as a result of the filtering will be stored. When PERIODOGRAM is included in the file the periodogram of the signal is computed, no inverse transformation to the time domain is performed. Such a periodogram makes it possible to assess quickly the influence of filtering operations.

The storage type selected always uses the result after possible filtering of the original series.

Only the first STORE command is used. 


\section{SEQUENCE, CODE-OLD $=$ name, CODE-NEW $=$ name, UNIT $=$ string}

With this command the original sequence can be renamed. The old name, given with the CODE-OLD name, is renamed to the CODE-NEW name.

New series can be given a new UNIT. In string the units described below can be used. If a newly given unit does not correspond with one from the table an error is will be generated and the program stops.

The following units are available :

$$
\begin{aligned}
& \mathrm{N}, \mathrm{kN} \\
& \mathrm{N} / \mathrm{m}, \mathrm{Kn} / \mathrm{m} \\
& \mathrm{Nm}, \mathrm{kNm} \\
& \mathrm{Nm} / \mathrm{m}, \mathrm{Knm} / \mathrm{m} \\
& \mathrm{N} / \mathrm{m} 2, \mathrm{Kn} / \mathrm{m} 2 \\
& \mathrm{~km}, \mathrm{~m}, \mathrm{dm}, \mathrm{cm} \\
& \mathrm{mile}, \text { feet, inch } \\
& \mathrm{s}, \mathrm{Hz} \\
& \mathrm{m} / \mathrm{s} \\
& \mathrm{m} / \mathrm{s} 2 \\
& \mathrm{~m} 3 / \mathrm{s} \\
& \text { graden, degrees } \\
& \text { procent, percent }
\end{aligned}
$$

The used string may be in capitals, lower case or occur in any combination. So $\mathrm{knm} / \mathrm{M}$ is identical to $\mathrm{Knm} / \mathrm{m}$ and so on.

A SEQUENCE command can be used for each selected series, but the moment OLD_CODE is not used the old name is used for the new series.

When the PERIODOGRAM command is active then an adapted SEQUENCE command is used. No unit directive (DIMENSION) is stored, nor the old nor the new one. The name of the amplitude series is according to the name conventions as described. If the phase series is computed this name will also be used with the addition of PHASE. 


\title{
SPECTPER
}

\section{Introduction}

The program uses a periodogram, being an amplitude spectrum, as input to compute a spectrum. The periodogram can be computed with program FILTER. An amplitude spectrum computed with another program can also be used when it is stored in file that can be transformed to an AUKE/pc series file.

\section{Theory}

The user must set a $\Delta$-frequency and the periodoigram information is used to compute the energy density spectrum for that $\Delta$-frequency. The program uses the $\Delta$-frequency in the periodogram as a minimum. The formula used for transformation to a spectral value:

$$
\tilde{S}(k . \Delta f)=\frac{1}{2 \Delta f} \sum_{i=-j / 2}^{i=j / 2} p^{2}\left(i . \Delta f^{\prime}\right)
$$

where $\mathrm{k}$ is the $\mathrm{k}$-th spectral value in the $\Delta \mathrm{f}$ scheme. The frequency $\mathrm{k} . \Delta \mathrm{f}$ of this estimate equals the frequency for $\mathrm{i}=0$ in the $\Delta \mathrm{f}^{\prime}$ scheme. The range $\mathrm{i}=\langle-\mathrm{j} / 2, \mathrm{j} / 2\rangle$ equals $\Delta \mathrm{f}$.

Once the new spectrum is computed all the parameters described in subsection 4.2.2. Spectral parameters are computed and send to output.

\section{Commands summary}

\section{General commands}

DATA, string

DATAFILE, string

SERIES,[ALL] $<$ CODE, CODEPART $=$ string, EXTENSION $=$ string $>$ $<$ RANGE, CODENAME $=$ string, LOW $=$ intval, HIGH $=$ intval $>\mid$

$<$ UNIQUE, NAME $=$ string $>$

\section{Program SPECTPER commands}

\author{
PRINTPLOT,$\{$ XES $\mid[$ NO $]\}$, CUTFACTOR $=$ factor \\ SHAPE, $\{$ XES $\mid[$ NO $]\}$ \\ DELTA-F, df_new[df]
}

\section{Commands description}

In this program there is a complication: all available general commands can be used but not all should be used. The reason for this is that program FILTER stores all data and information that must be used in the series data and all data must be used as it represents the complete information from the original series. In the summary the commands are presented that should be used, do not use the possibilities not given here.

Program SPECTPER can not check whether the used series from the series file defined with DATAFILE really are amplitude spectra values, it is the responsibility of the user. The resulting 
spectra is stored in the file from the DATA command.

descr

PRINTPLOT,$\{$ YES $\mid[$ NO $]\}$, CUTFACTOR $=$ factor

All the computed spectral values can be presented on output. The values themselves are in a column and on the same line of a value a sign is printed in the plot area such that the position indicates the relation to the minimum and maximum values in the complete series. This is done for all spectral series values.

When a printer plot is activated the printer output of the spectral density values may be stopped on a given point. This point is computed with the CUTFACTOR value. The value is multiplied by the maximum value in the series and when this product is reached and there is no higher value available to at the end of the series, then the output of that series is stopped.

The cutfactor value must be between 0 . and 1 .

descr

SHAPE, $\{$ XES $\mid[N O]\}$

Computed spectra are stored with there energy density values. However, it is possible to store them in a normilaized way: the maximum value found is set to 1 . and all other values are scaled to this maximum value. When the SHAPE command is found, possibly with XES the spectra are normalized. In all other cases, no SHAPE command or with the parameter NO, the energy density values are stored.

The computed parameter values are always for the non-normalized spectrum.

In this way the spectra of various series can be plotted in one graph allowing a comparison of the shape of these spectra.

descr

DELTA-F,df_new[df]

The $\Delta \mathrm{f}$ for the frequency is set. When it is not a multiple of the $\Delta \mathrm{f}$ frequency step in the periodogram (df) the $\Delta \mathrm{f}$ is broadened to a multiple. When the command is not found the $\Delta \mathrm{f}^{\prime}$ step of the periodogram series. 


\section{STATIST}

\section{Introduction}

STATIST enables computation and display of some statistical quantities for the selected series.

Also the values of the USE parameters can be computed after which they are stored in the information .SEQ file. Program STATIST is the only program that stores these values. All programs, including STATIST, can read USE values from the data base or calculate them for the selected series part.

\section{Theory}

Program STATIST is designed to compute some statistical characteristics on series for the selected part. These statistical characteristics are :

$$
\begin{aligned}
& \text {-mean value }: \frac{1}{m} \sum_{j=1}^{m} x_{j} \\
& \text {-standard deviation }: \sqrt{\frac{1}{m-1} \sum_{j=1}^{m}\left(x_{j}-\bar{x}\right)^{2}}
\end{aligned}
$$

-minimum and maximum series value together with their position in the series

At the request of the client, other specific parameters can be built in STATIST, provided that they do not conflict with the program's structure, where the statistical characteristics are computed for the sample values and not for any combination of samples or series. If a USE parameter is selected the statistical result includes its influence.

\section{Commands summary}

\section{General commands}

DATA, string

DATAFILE, string

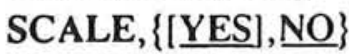

SELECT, $\{[\underline{A L L}] \mid$

$<$ EQ-STEP, LOW $=$ value, $\mathrm{HIGH}=$ value, $\mathrm{STEP}=$ value $>\mid$

$<$ EQ-NYQ, LOW $=$ value, $\mathbf{H I G H}=\underline{\text { value }}, \mathrm{NYQ}=\underline{\text { value }}>$ |

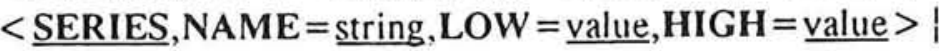

$<\underline{\#}, \mathrm{LOW}=$ intval, $\mathbf{H I G H}=$ intval, STEP $=$ intval $>\}$

SERIES, $\{$ | $\underline{\mathrm{ALL}} \mid\}$ 
$<$ CODE, CODEPART $=$ string, EXTENSION $=$ string $>\mid$

$<\underline{\text { RANGE }}$, CODENAME $=$ string, LOW $=$ intval, HIGH $=$ intval $>\mid$

$<\underline{\text { UNIQUE}}$, NAME $=\underline{\text { string }}>\}$

ZEROLEVEL, $\{[\underline{\text { YES }]}|\underline{\text { NO }}|\}$

Special USE commands

USE

POLY0, \{CALCULATE $\}$

POLY1, \{CALCULATE $\}$

POLY2, CALCULATE $\}$

POLY $3,\{$ CALCULATE $\}$

END:USE

Program STATIST command

HEADING

END:HEADING

STATISTICS, $\{[\mathrm{YES}] \mid$ NO $\}$

STORE-USE, $\{$ YES, $\mid[\underline{\text { NO }]\}}$

TABLE, NAME $=\underline{\text { string }}$

Command and extra run parameters.

Commands

DATA, string

A special option of program STATIST is the possibility to store statistical values from the series in a series file in order to plot them with AUKEPLOT. The storage type of the series is RELATED.

The name of this file is string.

For the selected series part the following parameters are stored as series :

\#SIGNAL the sequence number of the selected series in the series file used for computation.

MEAN the mean values

ST-DEV standard deviations

MINIMUM minimum values

MAXIMUM maximum values

To be able to connect the parameter values to the original series names, these names are stored in a SCAN-NAMES block in the information SEQ file of the created file. 
Example: $\quad$ Suppose that three series are selected, named SER2, SER5, SER10, where the number in the name corresponds to the sequence in the original series file.

The numbers 2, 5 and 10 will subsequently be stored as \#SIGNAL together with, for instance, the mean values of the three original series in the new series MEAN.

Also stored in the information file is the block:

\section{SCAN-NAMES \\ SER2 \\ SER5 \\ SER 10 \\ END:SCAN-NAMES}

In program AUKEPLOT both these scan names can be selected for use on the $\mathrm{x}$ axis. Note that this is the selection of a master series.

\section{HEADING}

HEADING can be regarded as a special command, rather a directive, which is exclusively used in combination with the TABLE command.

HEADING is a block command that ends with END:HEADING. All lines in between are lines to be used as a heading which is printed as the heading of a table.

\section{STATISTICS, $\{[\mathrm{YES}] \mid \mathrm{NO}\}$}

You may decide whether the statistics should be computed and sent to output. This is especially useful if you only want to store the use values.

\section{STORE-USE, $\{$ YES,$\{$ NO] $\}$}

In STATIST this command is used to activate the storage of USE parameters values. Only this program enables the storage of USE values in the information .SEQ file of a series file. These parameters are described in Part 1 of the Process Document and we do not repeat it here for this reason.

When this command is available all USE parameters are computed for all the selected series part. They are stored in the information. SEQ file in the various SERIES blocks. If there are already one or more USE parameters included in the block that value will be overwritten.

No other program but STATIST offers the possibility to store USE parameters. The regular USE commands are used to select what parameter must be computed. However, the command is only activated when YES is included in the parameter field, other combinations are regarded as NO.

TABLE, NAME $=\underline{\text { string }}$

This command instructs the program to send information to a file with the name 
string. The information is arranged in a table. The heading of this table has an output that includes the lines from a LOGGING block in the SEQ file of the selected series file and the lines from the HEADING block in the command file.

Before that fixed output informs the user what data file is used and what part of the series is used for processing.

The LOGGING block in the SEQ information file is not obligatory. If it is in the file the lines between LOGGING and END:LOGGING are printed.

The same holds for the HEADING block which must be present in the command file.

If the file string exists it will be opened and the new table is being added with the new page character printed before the table is printed. The file may be imported in wordprocessor programs such as Word Perfect ${ }^{\infty}$ and the like.

In one table information is presented for all the selected series. The parameters are :

mean

standard deviation

minimum

maximum

\section{Run string}

Program STATIST enables the use of some extra run parameters to facilitate its use. In the following we describe these extra run parameters.

DATA $=\underline{\text { filedescriptor }}$

The filedescriptor is the general name for the basic series data files. The statistics and possibly USE parameters of the series selected are computed when using this run parameter.

\section{PROC $=$ program}

For computation purposes the command file program.PCF is used. It enables computation according to the commands in that command file. Note that when the command STORE-USE is included in the command file the USE parameters are stored according to the described function of this command.

When both run parameters are incorporated in the run string, DATA is preferred. It is obvious that the command file STATIST.PCF will be applied when neither of the two run parameters is in the run string. 


\section{WAVES}

\section{Introduction}

With WAVES the development of series values between two positive zero crossings is determined. Some of the results can be stored in a new series file. In the description the term waves and wave signal is used. However, any signal that acts like a wave signal may be used.

\section{Theory}

A definition of the various quantities in a wave series, or in any series with the behaviour like in a wave signal, is presented by the next illustration, derived from the 'List of sea state parameters' from IAHR.

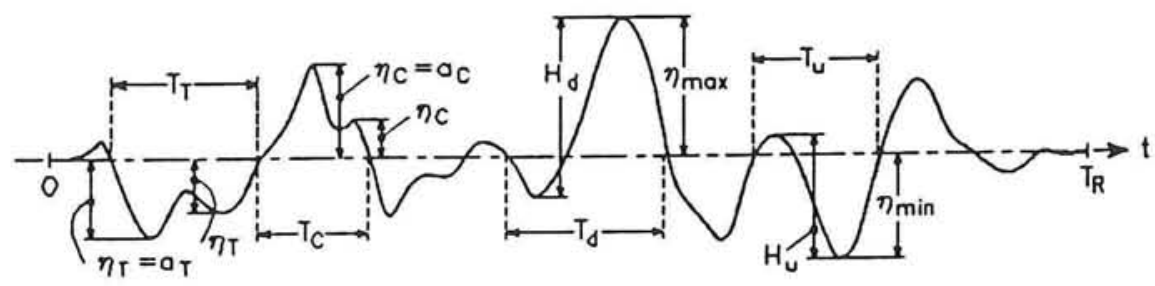

The wave parameters in the illustration are :

$\eta \quad$ the distance to a base line, normally zero

$\mathrm{H}$ the height between the minimum and maximum values found between two base line crossings with the same direction.

$T$ the difference of the values of two base line crossings with the same direction.

a the amplitude being the greatest $\eta$ between two successive base line crossings, so with different direction.

Subscripts indicate a differentiation of the parameters :

.c the given parameter is related to a crest

${ }^{\cdot} \mathrm{T}$ the given parameter is related to a trough

. a value between two upwards base-line crossings

d a value between two downwards base-line crossings

Possible base lines are :

- the zero value 
- the ZEROLEVEL value from a SERIES block

- the level described by one of the POLYi functions

In program WAVES the following parameters are computed and sent to output :

- the number of waves between the upward crossings.

- the average wave period Tm

- average of the highest one-third upward crossings :

$\mathrm{H} 1 / 3 \mathrm{u}$ the total height

$\mathrm{aCl} / 3$,u the crest amplitude

aT1/3,u the trough amplitude

- average of the highest one-tenth upward crossings :

$\mathrm{H} 1 / 10 \mathrm{u}$ the total height

$\mathrm{aC} 1 / 10, \mathrm{u}$ the crest amplitude

aT $1 / 10, \mathrm{u}$ the trough amplitude

- correlation values, with the general formula for the linear correlation coefficient

$I(\bar{Z})=\frac{N}{N-1} \frac{\sum_{i=1}^{N-1}\left(z_{i}-\bar{Z}\right)\left(z_{i+1}-\bar{Z}\right)}{\sum_{i=1}^{N}\left(z_{i}-\bar{H}\right)^{2}}$

In WAVES two parameter types are computed with the correlation function :

1. Kappa $\quad \mathrm{K}=\sqrt{I\left(X^{2}\right)}$

2. Gamma $\gamma=r(X)$

For $\mathrm{X} \mathrm{H}_{u}, \mathrm{a}_{\mathrm{C}}$ or $\mathrm{a}_{\mathrm{T}}$ can be selected.

- the gamma value computed from the $\kappa$ value for one of the three possible wave parameters :

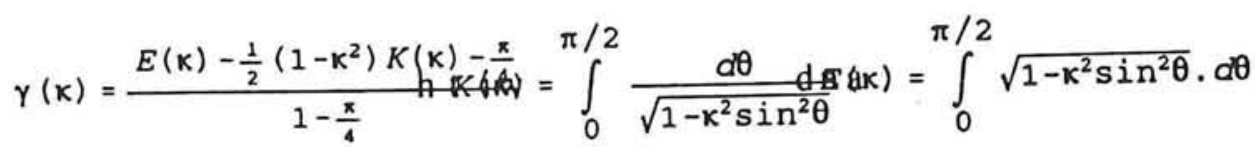


Output in the form of a table presents the minimum and maximum values of $\mathrm{H}_{v}, \mathrm{a}_{\mathrm{c}}$ and $\mathrm{a}_{\mathrm{T}}$ together with the position (time) in the series of the related wave. As each of these parameters may be derived from another location, the values of the other parameters for that wave are also presented together with their position. Also $T_{u}$ of the related wave is presented for each parameter.

The location of $\mathrm{H}_{u}$ is the position where the wave starts, so the upward base-line crossing. The location of $a_{c}$ and $a_{T}$ is the position of the value itself.

The values $H_{v}, a_{c}$ and $a_{T}$ can be presented for various percentage values $Z_{i}$ being the probability of exceedance. This is according to the Rayleigh probability distribution function $P\left(x>Z_{j}\right)=e^{-\left(\frac{z_{i}}{z_{\text {rns }}}\right)^{2}}$ with $\mathrm{P}\left(x>Z_{\mathrm{i}}\right)$ the number of waves $\mathrm{n}$ larger than $\mathrm{Z}_{\mathrm{i}}$ divided by the total number $N$ of waves. So $P$ has the form $n / N$. Also on output is the root mean square of a height $Y_{\text {rms }}=\sqrt{\frac{1}{N} \sum_{j=1}^{N} Y_{j}^{2}}$, where $\mathrm{Y}$ can be $\mathrm{H}_{\mathrm{u}}$,
$\mathrm{a}_{\mathrm{c}}$ and $\mathrm{a}_{\mathrm{T}}$.

Information can be retrieved of maximum 50 highest values of $\mathrm{H}_{v}, \mathrm{a}_{\mathrm{c}}$ and $\mathrm{a}_{\mathrm{T}}$ together with their location. This information is sent to output.

For further use the series of all values of $\mathrm{H}_{u}, \mathrm{a}_{\mathrm{c}}$ and $\mathrm{a}_{\mathrm{T}}$ can be stored together with their location in a new series file. By doing so it is for instance possible to make a H-T correlation graph with AUKEPLOT.

\section{Commands summary}

General commands

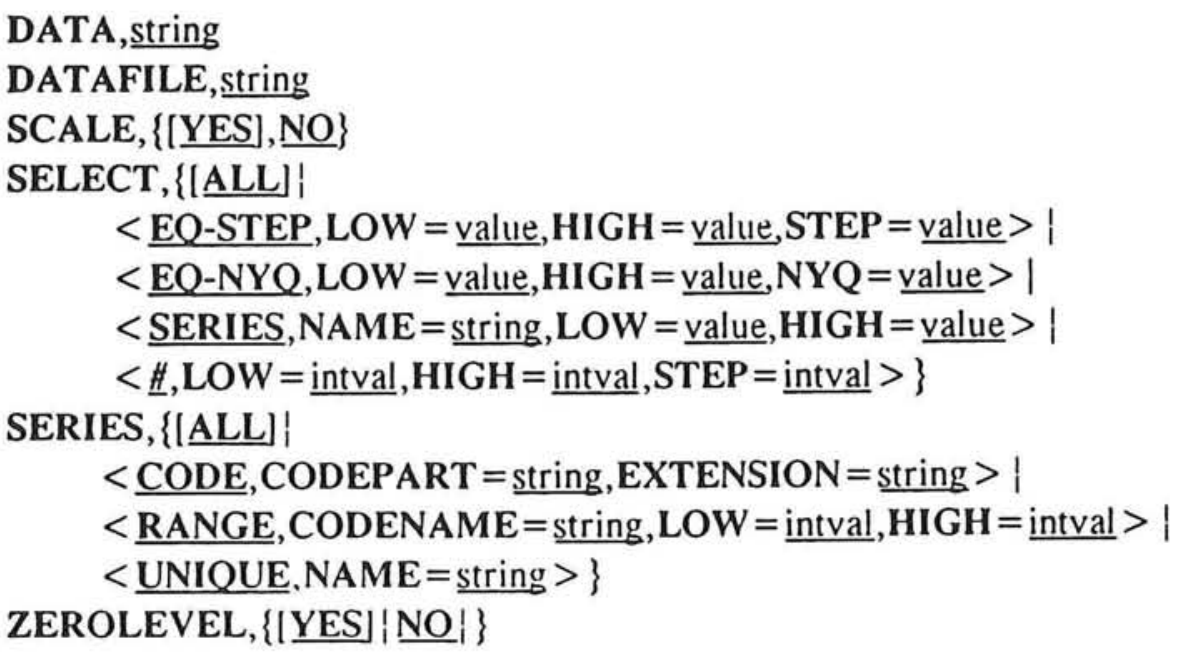


Special USE commands

USE

POLY0,\{CALCULATE $\mid\}$

POLY1, CALCULATE $\mid\}$

POLY2, CALCULATE $\}$

POLY3, CALCULATE $\mid\}$

END:USE

frogran WAVES commands

MAX, $\{$ [WAVE $\mid$ CREST $\mid$ TROUGH $\}$, number[50]

CORRELATION,SELECT $=\{[\underline{\text { WAVE }}|\underline{\text { CREST }}| \underline{T R O U G H}\}$

EXCEEDANCE, PERC $=$ perc $[100$.

STORE, choice[WAVES]

\section{Commands description}

MAX, $\{$ [WAVE $\mid$ CREST $\mid$ TROUGH $\}$, number[50]

In a wave signal a number of waves with height $\mathrm{H}_{u}$ is available. With the MAX command the $n$ highest values can be sent to output. When a user is not interested in the heights but in the crest height $a_{c}$ or the trough height $a_{T}$ only that quantity may be selected.

The number of values at output is default set at 50 , but it may be set at a lower number.

When a quantity is selected some complementary information is presented too.

With WAVE both the period and the starting time of the wave is at output. When either CREST or TROUGH is selected the value of that quantity is at output together with the point in time this value appears in the series.

\section{CORRELATION,SELECT $=\{[$ WAVE $\mid$ CREST $\mid$ TROUGH $\}$}

The $\kappa$ and $\gamma$ values can be computed for the WAVE $(\mathrm{H})$, the CREST $\left(\mathrm{a}_{\mathrm{c}}\right)$ or the TROUGH $\left(a_{T}\right)$ set of values. Default the wave height quantity is selected. With CREST or TROUGH the correlation for that quantity is computed.

EXCEEDANCE,PERC $=$ perc 100.$]$

The exceedance percentage for a wave height is the percentage of wave heights that is higher than a given wave height according to the Rayleigh probability function. In this way the lowest wave height is chained to the percentage 100.

For the required percentages the connected wave height can be printed. These percentages will not always fit an exact available percentage, and for that reason the wave height closest to the asked percentage will be presented, together with the real percentage. 
The number of command lines to be used with EXCEEDANCE is unlimited. The crest and trough value for the selected wave are also presented.

Default the 100,90,50,20,10,2 and 1 are used. When the command is used they are overruled and the exceedance values are in output in the order of the command file.

\section{DATA, filename}

This command lets the program know that special results to the filename series file must be stored. The series are related and the values are stored according to the occurence of the waves.

The series stored are described in the STORE command. When only the DATA command is present and no other STORE commands then all quantities are stored.

\section{STORE, choice}

If the DATA command is in the file and no STORE command then all quantities are stored.

A number of resulting quantities can be stored in a new file. The number of series is the same as the number of STORE commands. A quantity is a series with as much values as the number of waves in the original series. These quantities can be :

$\begin{array}{ll}\text { Waves } & \text { the wave heights found } \\ t \text {-waves } & \text { the starting location of the wave } \\ \text { Period } & \text { the period } \mathrm{T}_{\mathrm{u}} \text { of the wave } \\ \text { Crest } & \text { the highest value } \eta_{\mathrm{u}} \text { in the wave } \\ t \text {-crest } & \text { the location of the crest } \\ \text { Trough } & \text { the lowest value } \eta_{\mathrm{u}} \text { in the wave } \\ t \text {-trough } & \text { the location of the trough }\end{array}$

The names in italics represent the names in choice which may be used. More than one quantity can be selected by repeating the command with another selection name. The new series are not time-based. The resulting quantity series for one original series, however, are related so one series can act as a base for the other series. In this way the series can be plotted as a function of one of the other quantity series that acts as a master series.

Series may also be plotted according to their sequence in file.

Depending on the number of selected original series and the number of used STORE commands, one or more new series files will be created. Four possible combinations of selection are possible :

1. one series, one quantity 1 file

2. one series, more quantities 1 file

3. more series, one quantity 1 tïle

4. more series, more quantities more files, one for each series 
The content of the SEQ information file contains the SERIES blocks with the series name. Each new series name is composed of the original name and the quantity name, linked to each other with a colon.

Example Suppose the name of the series is PRESSURE and the quantity to store CREST. Now the name of the new series is PRESSURE:CREST.

In option 4 the number of created series files equals the number of series selected. Each series file has the name from the DATA command followed by the sequence number of the series as selected in the command file. The number of characters used in the name of the file depends on the number of series selected. When this number is $<10$ only one position is used. From 10 until 100 two positions are used. When the name plus the number exceed 8 characters the series name is cut off.

Example Suppose the series name is TRYNAME and the number of series selected is such that two characters are needed. Then the name of the file for the ninth selected series is TRYNAM09.

This construction is needed to be able to select related series for plotting as the resulting quantity series will have a different number of values for the various original series. 


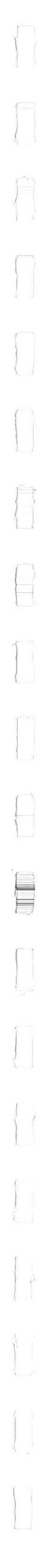


APPENDIX B

RESAMPLE.CPP 
if $(($ outstr $=$ Openoutput $f$ ile (sub_name $))==N U L L)$ exit (1);

// read heading and write to outputfile

//

$/ /$ continue if no error in inputfile

// -....................................

if (fileinfo1->error $=0$ )

// bepaal de te lezen samplenumers

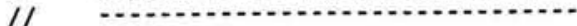

Readnums(fileinfo1);

// read samples in array a

// Readdata (fileinfol, instr, a);

fclose(instr);

// resample the data

// Resampdata(fileinfo1, a, b);

// write the resampled data to the outputfile

// Writedata(fileinfo1, outstr, b); fclose(outstr)

// no error in Readdata

了

FILE * Openinputfile(char sub_name [])

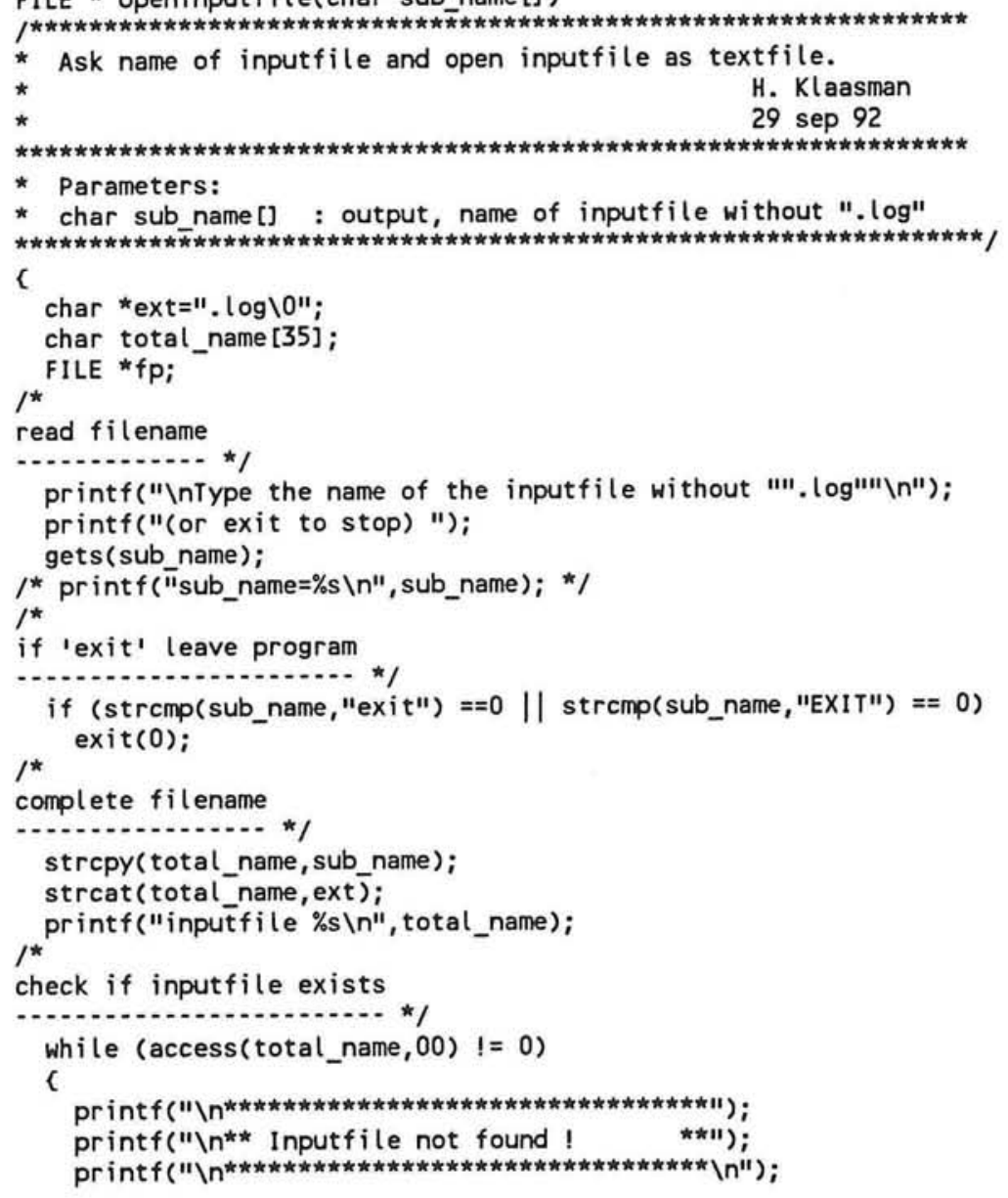




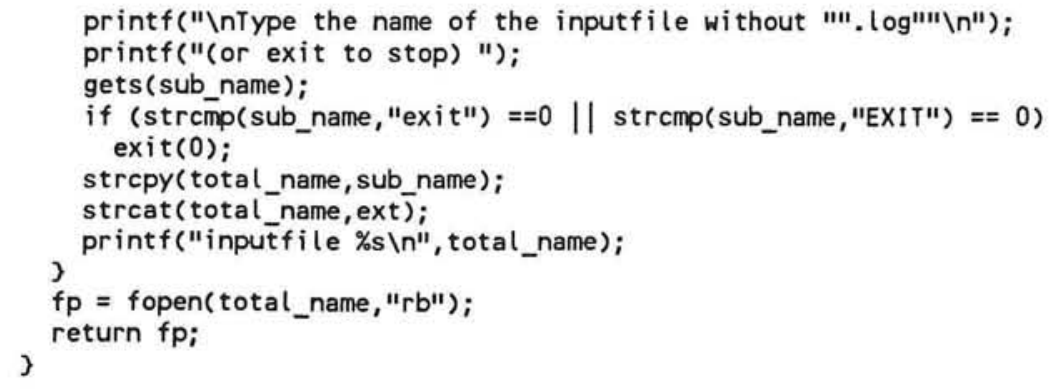


void Init(fileinfo *fifo)

/

* initialise some values to be read later

$\star$

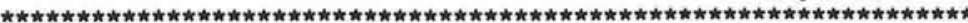

* Parameters:

* fileinfo *fifo: structure with file information, see declaration of fileinfo

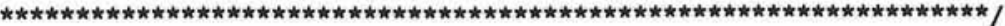

<

// initialisation

//

fifo->n_chan = MAX_CHAN;

fifo->n_samp $\quad=0$;

fifo- $>$ n out $\quad=0$;

fifo->n_sout $\quad=$ OUT_SAMP;

fifo->er̃or $\quad=0$;

return;

void Readhead(fileinfo *fifo, FILE *instr, FILE *outstr)

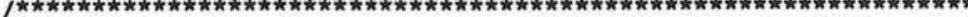

* Read head of DACON data and write to outputfile.

*

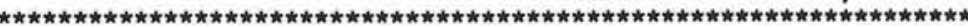

* Parameters:

* fileinfo *fifo: structure with file information, see declaration

*

* FILE *instr : inputfilestream

* FILE *outstr: outputfilestream

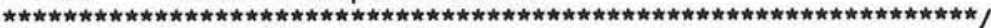

<

int $i, n$;

$/ /$ int ierrs, ind1, ind2, j, lcom, nj;

// double dtime;

// char ident1[MAXLENGTH+1], ident2 [MAXLENGTH+1];

// read heading

ll ..............

fread (fifo->remark, size_t(1), size_t(256), instr);

// print heading

$\mathrm{i}=\mathrm{n}=0$

while $(n<11 \& \& i<256)$

if ( $f$ ifo->remark $[i]==0 X O A$ ) $n++$;

printf("\%c",fifo->remark[i]);

i++

printf("\n");

/I

// lees aantal kanalen

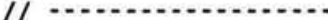

$\mathrm{i}=\mathrm{n}=0$;

while $(n<5$ \&\& $i<256)$

if ( $f$ ifo->remark $[i]==0 X O A$ ) $n++$;

fifo->n_chan = atoi $(\&$ fifo->remark $[i])$;

printf("aantal kanalen \%d\n", fifo->n_chan);

// lees aantal samples 


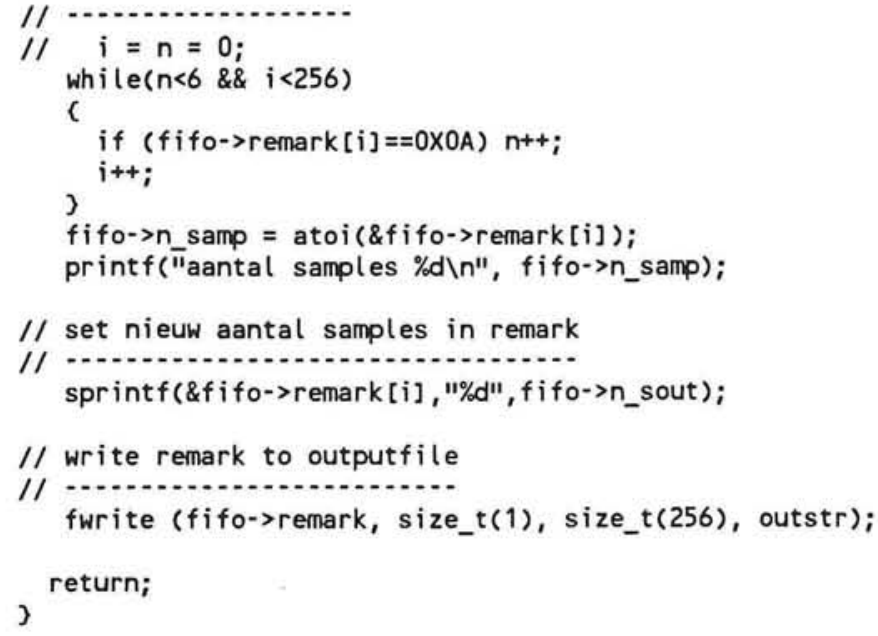




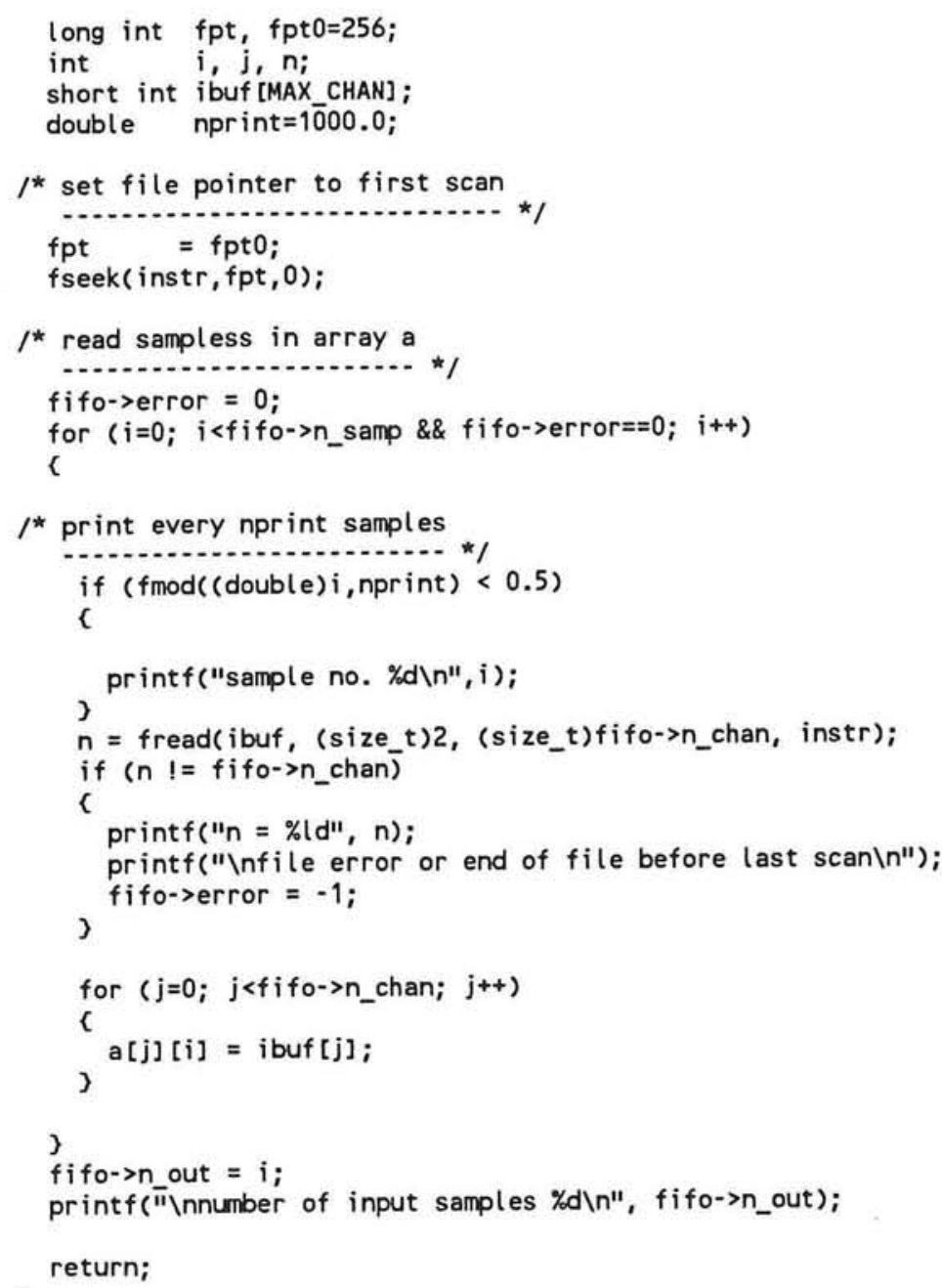
short int b [MAX_CHAN] [OUT_SAMPJ)

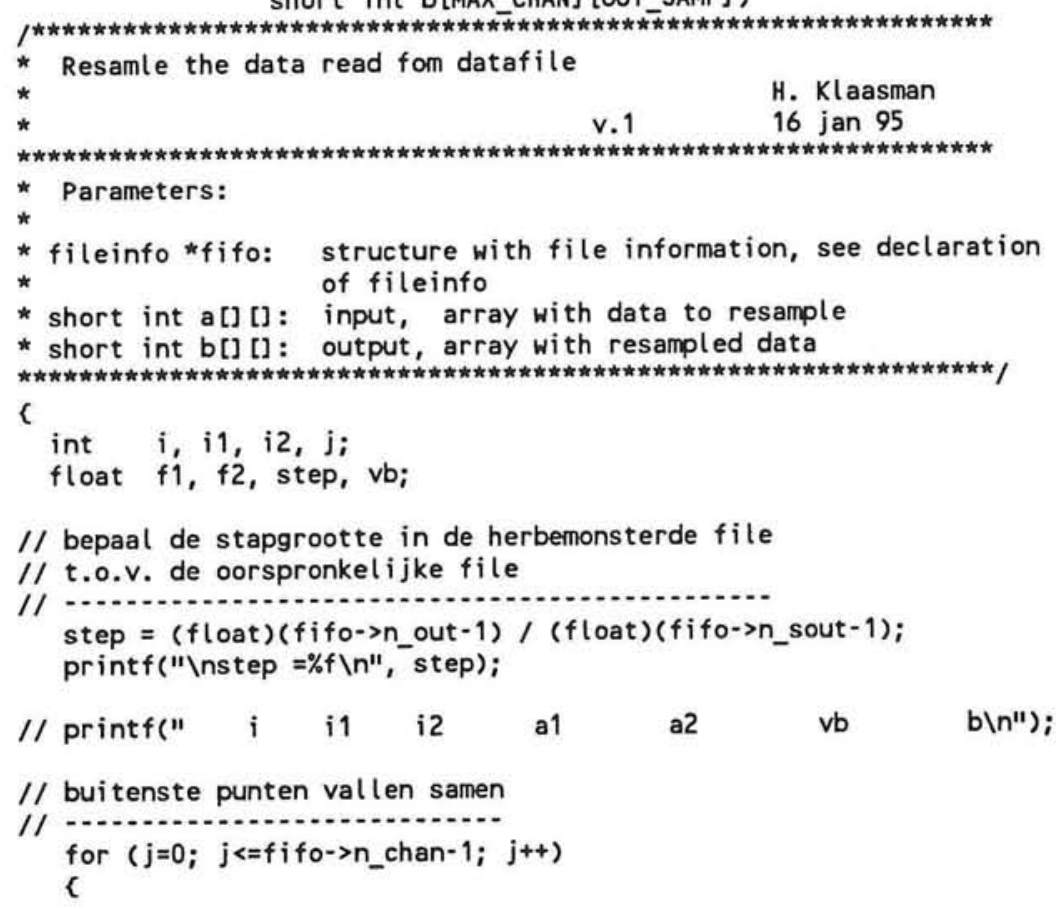




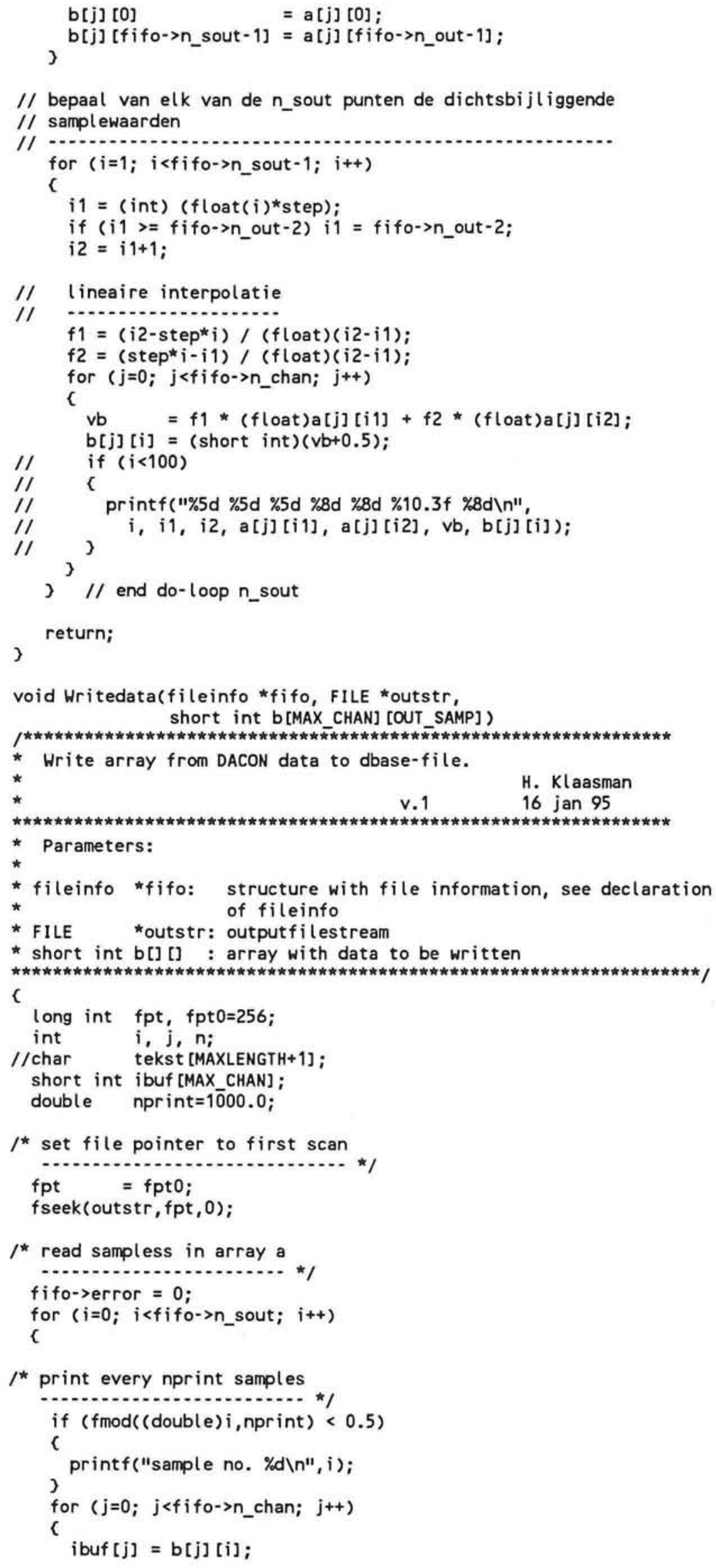


了

$n=$ fwrite(ibuf, $($ size_t $) 2,($ size_t)fifo->n_chan, outstr);

if (n! = fifo->n_chan)

printf("n = \%d", $n)$;

printf("Infile error or end of file before last scan $\backslash n "$ );

)

fifo->error $=-1$;

fifo->n_sout $=i$;

printf("\nnumber of output samples \%d\n", fifo->n_sout);

return; 


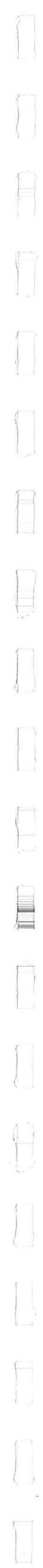




\section{APPENDIX C}

WAVE HEIGHT MEASUREMENTS 
Table C.1 All wave height measurements performed during the experiments

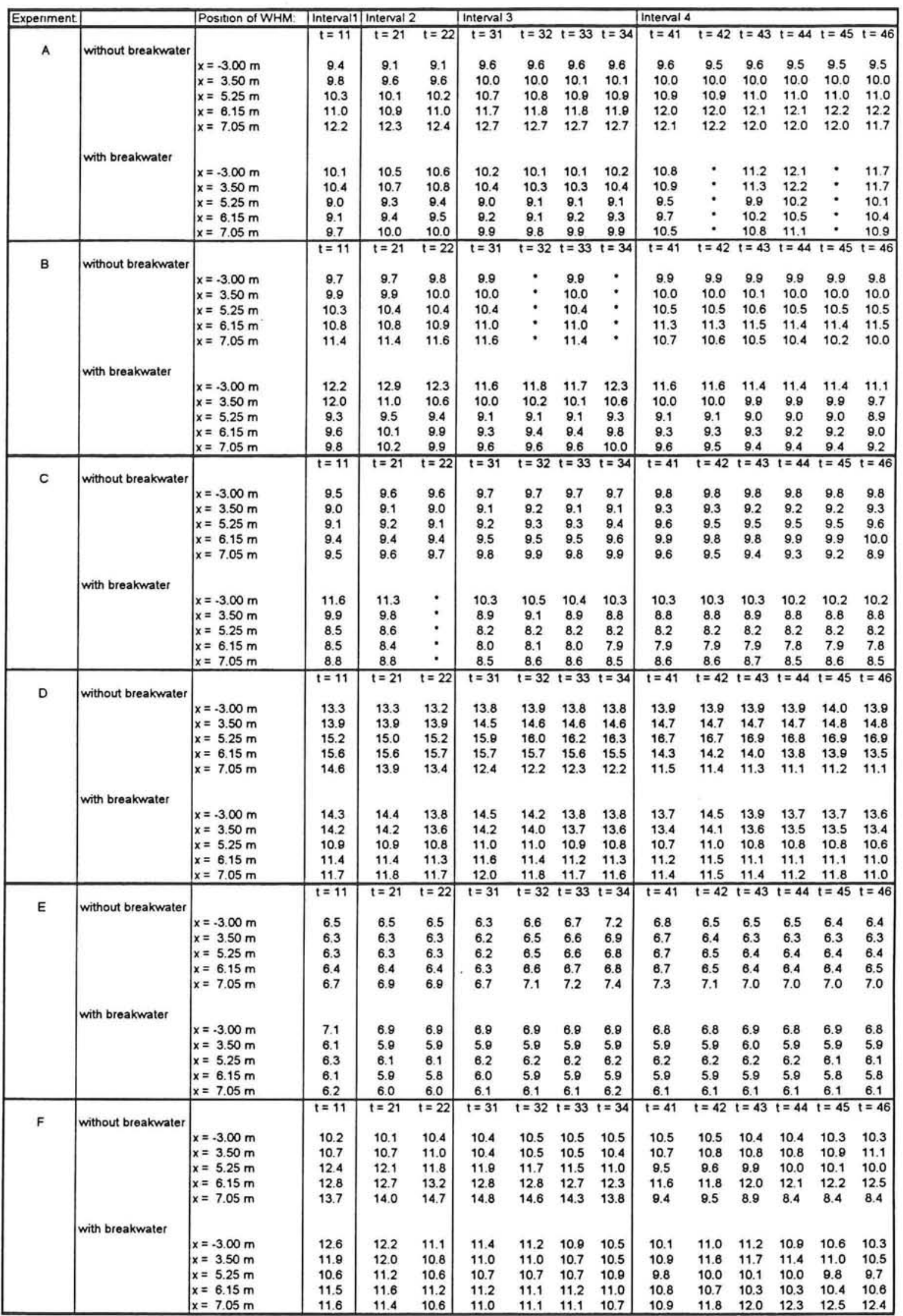

- = measurements failed

all wave heights in [cm] 


\section{$\underline{\text { APPENDIX D }}$}

Mo-PARAMETERS OF INTERVAL 2 


\begin{tabular}{|l|c|c|}
\hline & $\mathbf{m}_{\mathbf{0}}$ at WHM 1 $\left[\mathbf{c m}^{2}\right]$ & $\mathbf{m}_{\mathbf{0}}$ at WHM 3 $\left[\mathbf{c m}^{2}\right]$ \\
\hline experiment A without breakwater & 5.4 & 5.9 \\
experiment B without breakwater & 6.2 & 6.4 \\
experiment C without breakwater & 5.9 & 5.2 \\
experiment D without breakwater & 11.7 & 12.1 \\
experiment E without breakwater & 2.8 & 2.6 \\
& & 4.7 \\
experiment A with breakwater & 7.4 & 5.3 \\
experiment B with breakwater & 10.6 & 4.2 \\
experiment C with breakwater & 8.0 & 7.0 \\
experiment D with breakwater & 13.7 & 2.2 \\
experiment E with breakwater & 3.0 & \\
\hline
\end{tabular}

List of values of first order moments of the spectra of the first series of measurements during Interval 2.

\begin{tabular}{|l|c|c|}
\hline & $\mathbf{m}_{\mathbf{0}}$ at WHM $\mathbf{1}\left[\mathbf{c m}^{2}\right]$ & $\mathbf{m}_{\mathbf{0}}$ at WHM 3 $\left[\mathbf{c m}^{\mathbf{2}}\right]$ \\
\hline experiment A without breakwater & 5.4 & 6.0 \\
experiment B without breakwater & 6.2 & 6.4 \\
experiment C without breakwater & 5.8 & 5.2 \\
experiment D without breakwater & 11.4 & 12.2 \\
experiment E without breakwater & 2.8 & 2.6 \\
& & 4.8 \\
experiment A with breakwater & 7.5 & 5.4 \\
experiment B with breakwater & 10.0 & - \\
experiment C with breakwater & - & 6.7 \\
experiment D with breakwater & 12.6 & 2.2 \\
experiment E with breakwater & 3.1 & \\
\hline
\end{tabular}

List of values of first order moments of the spectra of the second series of measurements during Interval 2. 


\section{APPENDIX E}

CURVE FITTING METHOD OF KLAROS

Partial reprint from the KLAROS user's manual (1994)

Courtesy of Delft University of Technology 


\section{CALCULATION OF THE FIT CURVE}

\subsection{Background of the fit method}

As said, a time-averaged sediment concentration profile can be determined experimentally. The starting point is a series of measurement points at various heights, with distinct values for the sediment concentration. The wanted continuous distribution of the concentration over the total height can be obtained by fitting a curve through these measurement points. This is illustrated in Fig.3.

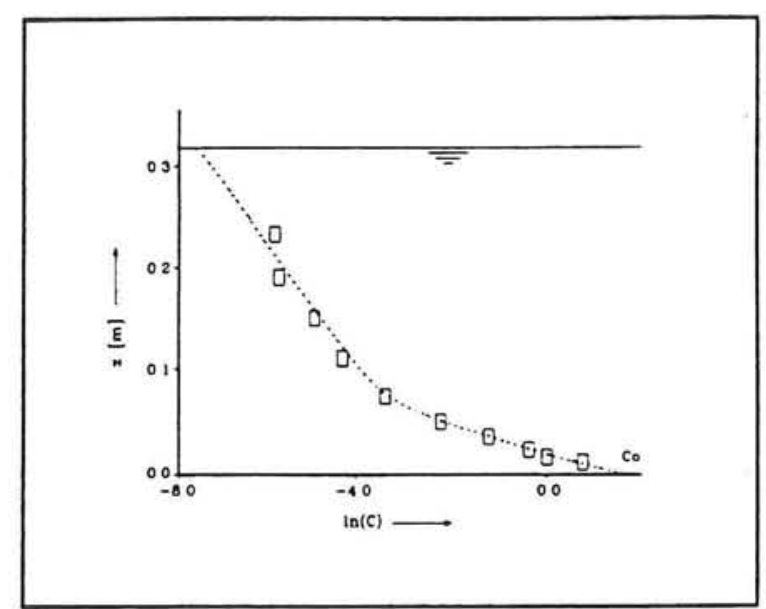

Fig.3 Example of a measured concentration distribution and a calculated fit curve.

In the report "A Curve Fitting Method for Mean Concentration Distributions" (Van Hazendonk, 1991) four restraints are mentioned for a sediment concentration fit curve: 1. The gradient of the concentration over the height is always negative (because the mean concentration decreases with the height).

2. The largest gradient of the concentration distribution is at the bottom (this is observed from many experiments).

3. The gradient of the concentration is constant at the bottom.

4. The number of degrees of freedom is limited, so that the fitting of the curve is still significant (maximum of two bending points).

These restraints concern the gradient of the concentration distribution over the height. The behavior of this curve can therefore quite well be illustrated with help of a figure, where the normalized derivative of $\ln (C)$ to the relative waterheight is represented as a function of the relative height $\left(z^{\circ}\right)$. In this so called standard square, this derivative as well as the relative height $z^{\circ}$ vary from 0 to 1 . To translate the standard square to reality, the concentration gradient has to be scaled by a (negative) factor. See Fig. 4 . 


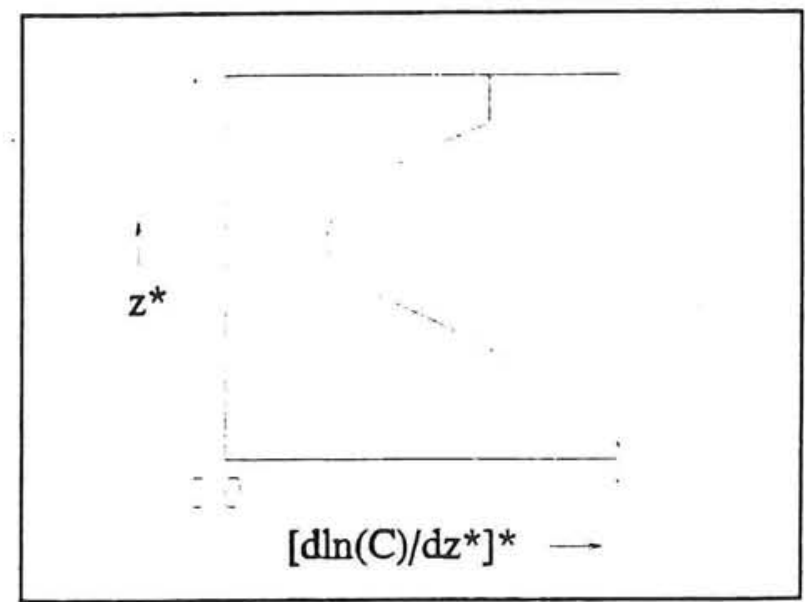

Fig.4 The standard square with an example of the concentration gradient.

A suitable curve that satisfies all criteria mentioned before, is the $n$-degree cosine function. The derivative of this curve is defined by:

$$
\begin{aligned}
& {\left[\frac{\mathrm{d} \ln (C)}{\mathrm{d} z^{*}}\right]^{*}=d+(1-d) \cos ^{n}\left(\frac{z^{*}}{a}\right) ; \quad \frac{z^{*}}{a}<\xi} \\
& {\left[\frac{\mathrm{d} \ln (C)}{\mathrm{d} z^{*}}\right]^{*}=d+(1-d) \cos ^{n}(\xi) ; \quad \frac{z^{*}}{a} \geq \xi}
\end{aligned}
$$

where:

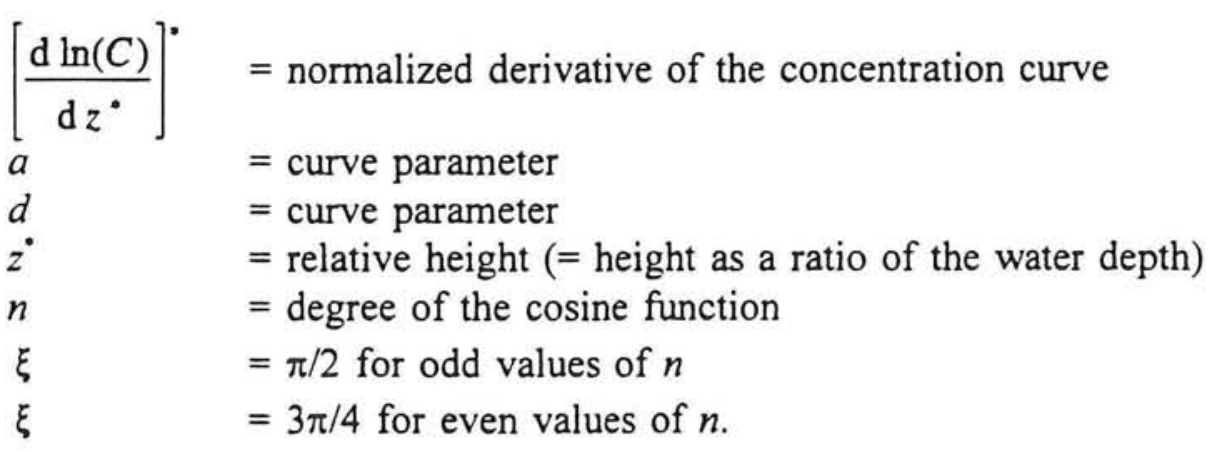

In Fig.5a through Fig.5g, the transformation of a plain cosine curve into a normalized derivative (Eq.6a and Eq.6b) is explained. For example, $n=2, a=1.2 / \pi$ and $d=0.25$ is choosen. In Fig.5d, the complete n-degree cosine curve is shown. The effect of $\xi$ is shown in Fig.5e (for $z^{*} \geq \xi, \operatorname{dln}(C) / \mathrm{d} z^{\circ}$ becomes a straight line). The boundaries of the standard square are indicated in Fig.5f. Note that the $z$-axis is rotated $90^{\circ}$ in the standard square (Fig.5g). To obtain a concentration curve, like Fig.5h, the normalized derivative has to be integrated and scaled (yielding an integration constant $b$ and a scale factor $c$ ). In total, 5 parameters have to be optimized to get a concentration curve that 
fits the measurement points as best as possible (see also Fig.1). The optimization is explained in the next section.

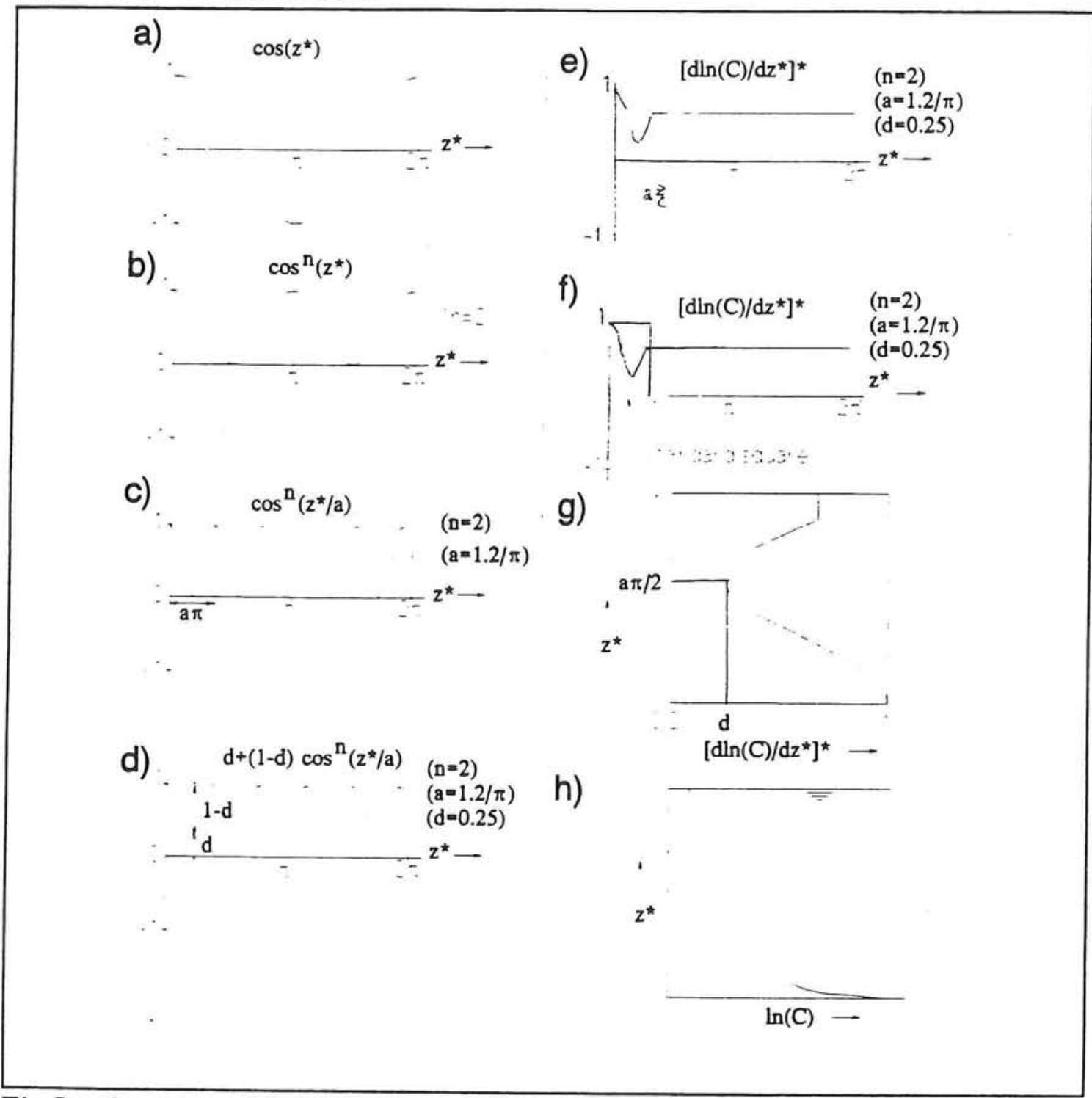

Fig.5 Situation of the cosine curve in the standard square.

\subsection{Optimization of the fitcurve}

In the previous section, a characterization was given of the type of fitcurve that appeared suitable for the description of sediment distributions. The general equation for the concentration distribution contains five variables $(a, b, c, d$, and $n)$, that can vary per experiment. Now it is opportune to choose the values of these variables in such a way, that the curve describes the measured concentrations as good as possible. The principle of this optimizing of the variables is treated in Section 3.2.1. Section 3.2.2 describes the implementation of this procedure for the specific case of the n-degree cosine curve. 


\subsubsection{The principle of the optimization procedure}

The fit curve used by this program is optimized using the method of least squares. The function of this method, and the customization for non-linear curves, is described in this section.

The method of least squares is dealt with in every standard work about regression analysis, e.g., Draper and Smith (1966). The main idea behind the method of least squares is the minimization of the squares of the residues. The residue is the difference between the natural logarithm of the measured concentration and the logarithm of the value of the fit curve at a given measurement point.

The curve that has to be optimized is non-linear. The consequence for the optimization procedure is that the method of least squares does not result directly in optimal parameters. To solve this problem, the parameters are optimized in an iterating process. The curve is linearized with initial values of the parameters. Then the method of least squares results in correction terms for these parameters. The initial values are adjusted and form the input of another optimization using the method of least squares. The correction terms should become smaller and smaller and at the end they should become negligible.

The steps that are followed during this process of optimization are:

1. All variable parameters get an estimated initial value.

2. The curve is linearized around the initial values.

3. The square residue of this approximated curve is minimized. (Method of least squares):

$$
S S=\sum_{i=1}^{m}\left[\ln \left(C_{i, B}\right)-\ln \left(C_{i, M}\right)\right]^{2}
$$

has to become as small as possible, where:

$S S \quad=$ sum of squares of the residues

$C_{i, B}=$ calculated concentration at measurement point no. $i$

$C_{l, M}=$ measured concentration of measurement point no. $i$

$m=$ number of measurement points.

4. This results in correction terms of the estimated values (in stead of fully optimized parameters in a linear case).

5. Steps 2, 3 and 4 are repeated until the corrections are negligible.

NOTE. When optimizing the $n$-degree cosine function, a set of non-linear equations is solved in an iterative way. However, the set of equations can only be convergent if the initial values are estimated quite reasonably. The programmed initial values are chosen in a way that most cases result in a convergent process. In an exceptional case it might occur that those values do not result in a convergent process. That means that the correction terms do not become smaller or will alter between a few constant values. When this happens, the program will stop and display an error message. 


\subsubsection{The implementation of the optimization procedure}

The equations for the n-degree cosine gradient function are described in section 3.1. To find the equation for the natural logarithm of the concentration, it is necessary to integrate and scale Eq.6a and Eq.6b. Integration of these equations results in considerable dissimilar expressions, dependent of the degree of the cosine function. Therefore, every degree had to be programmed separately, and the number of degrees is limited to 6. A choice has been made to program the degrees $1,2,5,8,10$ and 12 .

So, Klaros contains 6 equations for $\ln (C)$. The equations consist for every degree of a more or less curved part (if $z^{*} / a<\xi$ ) and possible of a linear part (if $z^{*} / a \geq \xi$ ). This division in two parts enables the construction of lines that are curved near the bottom but do not oscillate strongly near the water level.

To prevent that the gradient alters in sign, there are different boundary values for $\xi$. If the degree is odd ( 1 or 5 ) the boundary value is: $\xi=\pi / 2$; if the degree is even $(2,8,10$ or 12 ), the boundary value is: $\xi=3 \pi / 4$

The integrated and scaled equations for $\ln (C)$ are:

$1^{\text {st }}$ degree cosine function:

$$
\ln (C)=b+c d z^{*}+a c(1-d) \sin \left(\frac{z^{*}}{a}\right)
$$

For the part where $z^{\circ} / a>\pi / 2$, the equation is:

$$
\ln (C)=b+c d z^{*}+a c(1-d)
$$

where:

$a \quad=$ curve parameter

$b \quad=$ integration constant

c $\quad=$ scale factor

$d \quad=$ curve parameter

$\ln (C) \quad=$ natural logarithm of the sediment concentration at height $z^{*}$

$z^{\circ} \quad=$ relative height

$2^{\text {nd }}$ degree cosine function:

$$
\ln (C)=b+c d z^{*}+\frac{a c(1-d)}{4} \sin \left(\frac{2 z^{\circ}}{a}\right)+\frac{c z^{*}(1-d)}{2}
$$


For the part where $z^{\circ} / a>3 \pi / 4$, the equation is:

$$
\ln (C)=b+\frac{c(1+d) z^{*}}{2}-\frac{a c(1-d)}{4}
$$

$5^{\text {th }}$ degree cosine function:

$$
\begin{aligned}
& \ln (C)=b+c d z^{\circ}+\frac{5 a c(1-d)}{8} \sin \left(\frac{z^{\circ}}{a}\right) \\
& +\frac{5 a c(1-d)}{48} \sin \left(\frac{3 z^{\circ}}{a}\right)+\frac{a c(1-d)}{80} \sin \left(\frac{5 z^{\circ}}{a}\right)
\end{aligned}
$$

For the part where $z^{*} / a>\pi / 2$, the equation is:

$$
\ln (C)=b+c d z^{*}+\frac{128 a c(1-d)}{240}
$$

$8^{\text {th }}$ degree cosine function:

$$
\begin{aligned}
\ln (C)= & b+c d z^{*}+\frac{35 c(1-d) z^{*}}{128}+\frac{7 a c(1-d)}{32} \sin \left(\frac{2 z^{*}}{a}\right) \\
& +\frac{7 a c(1-d)}{128} \sin \left(\frac{4 z^{\circ}}{a}\right)+\frac{a c(1-d)}{96} \sin \left(\frac{6 z^{*}}{a}\right) \\
& +\frac{a c(1-d)}{1024} \sin \left(\frac{8 z^{\circ}}{a}\right)
\end{aligned}
$$

For the part where $z^{\circ} / a>3 \pi / 4$, the equation is:

$$
\ln (C)=b+c d z^{*}+a c(1-d) *\left(\frac{z^{*}}{16 a}+0,288676441\right)
$$


$10^{\text {th }}$ degree cosine function:

$$
\begin{aligned}
\ln (C) & =b+c d z^{*}+\frac{63 c(1-d) z^{*}}{256}+\frac{105 a c(1-d)}{512} \sin \left(\frac{2 z^{*}}{a}\right) \\
& +\frac{15 a c(1-d)}{256} \sin \left(\frac{4 z^{\circ}}{a}\right)+\frac{45 a c(1-d)}{3072} \sin \left(\frac{6 z^{\circ}}{a}\right) \\
& +\frac{5 a c(1-d)}{2048} \sin \left(\frac{8 z^{*}}{a}\right)+\frac{a c(1-d)}{5120} \sin \left(\frac{10 z^{*}}{a}\right)
\end{aligned}
$$

For the part where $z^{*} / a>3 \pi / 4$, the equation is:

$$
\ln (C)=b+c d z^{*}+a c(1-d)\left(\frac{z^{*}}{32 a}+0.315588659\right)
$$

$12^{\text {th }}$ degree cosine function:

$$
\begin{aligned}
\ln (C) & =b+c d z^{*}+\frac{231 c(1-d) z^{*}}{1024}+\frac{99 a c(1-d)}{512} \sin \left(\frac{2 z^{*}}{a}\right) \\
& +\frac{945 a c(1-d)}{8192} \sin \left(\frac{4 z^{\circ}}{a}\right)+\frac{55 a c(1-d)}{3072} \cdot \sin \left(\frac{6 z^{*}}{a}\right) \\
& +\frac{33 a c(1-d)}{8192} \sin \left(\frac{8 z^{*}}{a}\right)+\frac{3 a c(1-d)}{5120} \sin \left(\frac{10 z^{\circ}}{a}\right) \\
& +\frac{a c(1-d)}{24576} \sin \left(\frac{12 z^{\circ}}{a}\right)
\end{aligned}
$$

For the part where $z^{\circ} / a>3 \pi / 4$, the equation is:

$$
\ln (C)=b+c d z^{*}+a c(1-d)\left(\frac{z^{*}}{64 a}+0.318667137\right)
$$

Because of the strong non-linear character of these equations, it is almost impossible to get convergency if all four parameters are optimized at the same time. So it has been decided to optimize the values of $b$ and $c$ first, while $a, d$ and $n$ keep their initial values. Then the value of $a$ increases a little and the values of $b$ and $c$ are optimized again. By repeating the increment of $a$ and optimizing $b$ and $c$ for every value of $a$, it can be found out what is the best combination of $a-, b$ - and $c$-values when $d$ and $n$ still have their initial values. 
If the previous steps are repeated using different values of $d$, the best combination of the $a-, b-, c$ - and $d$-values can be found per $n$-value. (In fact, this optimal combination is still an approach, because the step sizes of $a$ and $d$ are not infinite small. However, the step size is made small enough so that the found parameters do not differ significantly from the really optimized values. The effects of the step size and initial values have been studied profoundly). In the next table, a survey of the initial values of the parameters and step sizes is given.

\begin{tabular}{|c|c|c|c|c|c|c|c|}
\hline \multirow{2}{*}{$\begin{array}{l}\text { para- } \\
\text { meter }\end{array}$} & \multicolumn{6}{|c|}{ initial values for: } & \multirow{2}{*}{$\begin{array}{l}\text { step } \\
\text { size }\end{array}$} \\
\hline & $n=1$ & $n=2$ & $n=5$ & $n=8$ & $n=10$ & $n=12$ & \\
\hline$a$ & $0.6 / \pi$ & $0.6 / \pi$ & $0.6 / \pi$ & $0.6 / \pi$ & $0.6 / \pi$ & $0.6 / \pi$ & $0.2 / \pi$ \\
\hline$b$ & 2 & 2 & 1 & 2 & 2 & 2 & - \\
\hline$c$ & -10 & -10 & -1 & -1 & -1 & -1 & - \\
\hline$d$ & 0 & 0 & 0 & 0 & 0 & 0 & 0.05 \\
\hline
\end{tabular}

The maximum value of $a$ is $4 / \pi$; the maximum value of $d$ is 0.9 .

A flow diagram of the fit procedure is given in Fig.6.

As appears from this diagram, the increase of $a$ is stopped as soon as the result becomes worse or the maximum value is reached. The increase of $d$ stops only if a four times worse result is obtained or if the maximum value is reached (It is very doubtful if results will become better after that it has become a factor four worse). The optimal value of $d$ is the value that belongs to the best fit.

The rate of success of the fit attempt is estimated with help of the calculated sum of squares of the residues $(S S)$. If $S S$ becomes larger, the curve is fitted worse through the measurement points.

When the parameters are optimized for each degree $n$ of the cosine function, the results (SS) of the optimizations of all degrees are compared to each other. The combination with the smallest $S S$ is selected. In this way, the value of $n$ is optimized also.

Fig. 7 shows us that the value of $n$ does not have a very significant influence on the fit curve within the range of the measurement points. (It should be noticed that outside the measurement range, the fitcurves becomes less reliable.) In the given example, $n=5$ leads to the best fit results. 


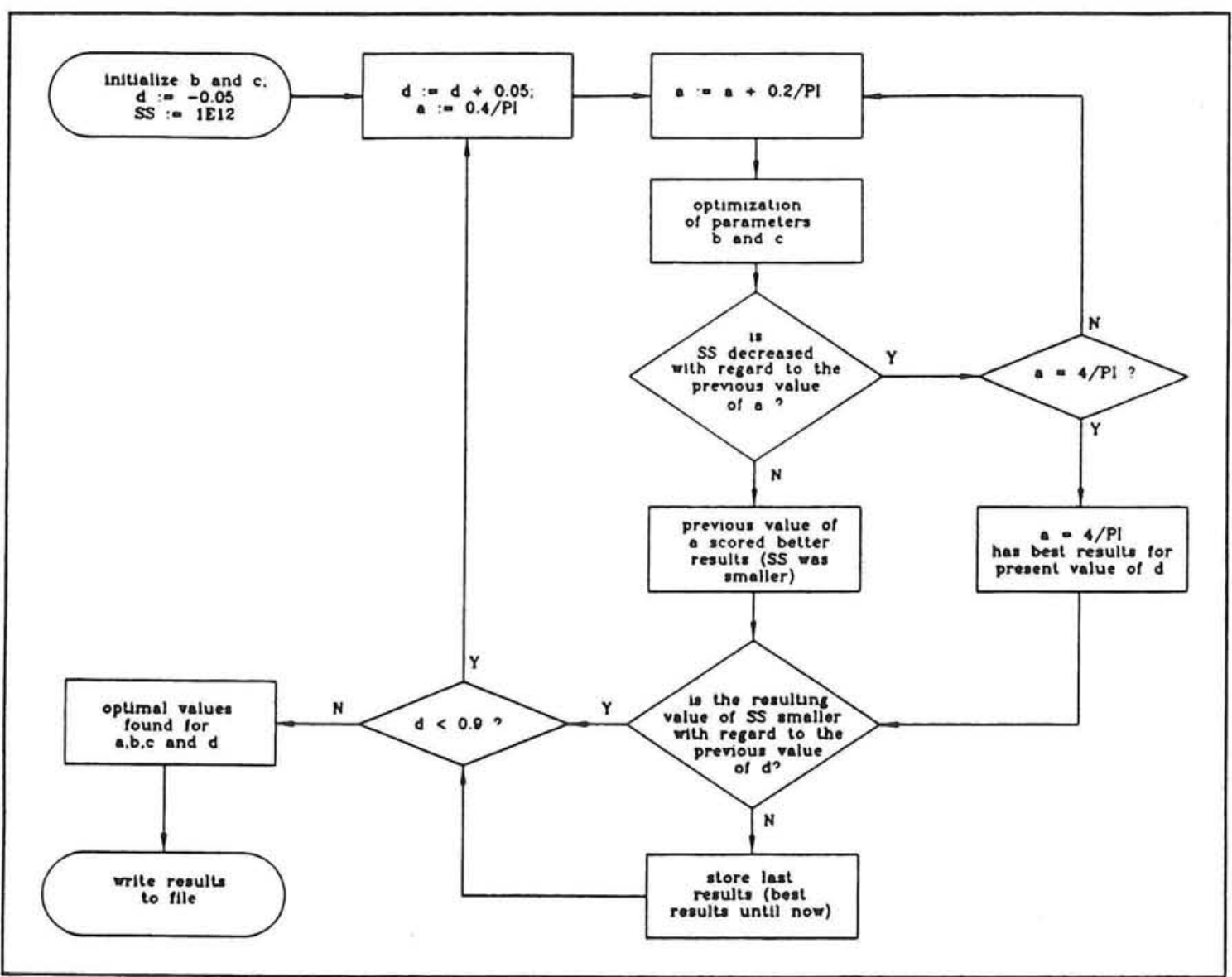

Fig.6 Flow diagram of the optimization of the n-degree cosine function parameters.

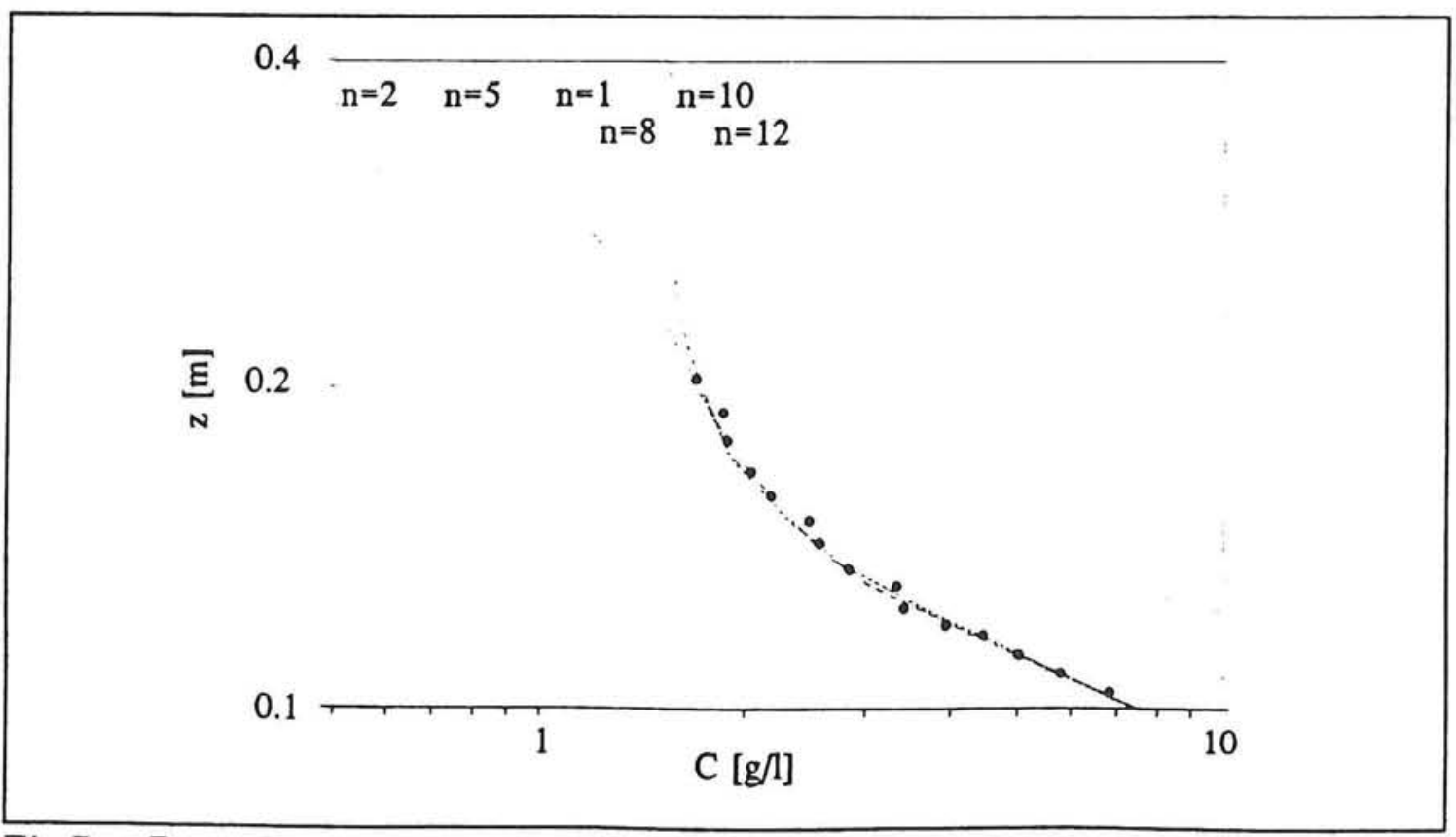

Fig.7 Example of the influence of $n$ on a concentration fitcurve. 
In Fig.8, the influence of parameter $a$ is shown (same measurements as in Fig.7). The curve does not change significantly if $a$ is increased or decreased one step size $(0.2 / \pi)$.

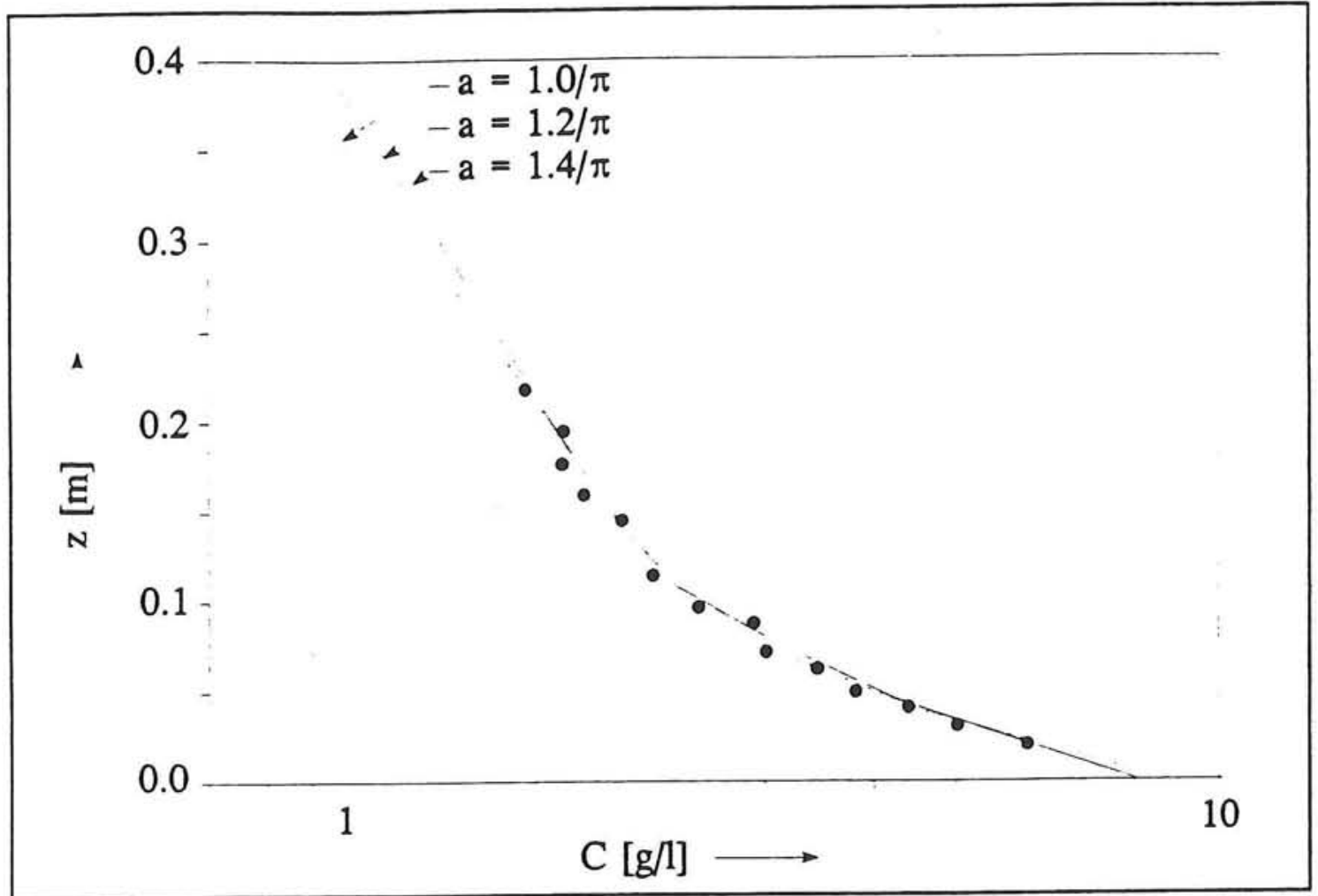

Fig.8 Example of the influence of parameter $a$ on the concentration fitcurve. 


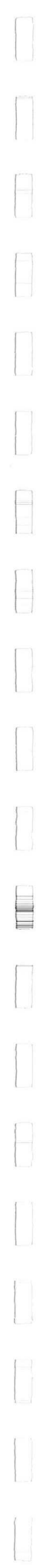




\title{
APPENDIX F
}

\author{
CONVERT
}

EXTRAPOLATION METHOD OF SEDIMENT CONCENTRATION DISTRIBUTION CURVE FROM LOWEST MEASUREMENT POINT TO THE BOTTOM

CALCULATED SEDIMENT CONCENTRATIONS NEAR

THE BED ( $\left.C_{\text {BED }}\right)$ 
The sediment concentrations between the bed and the first measuring point are represented by:

$$
c=e^{A z+B} \quad \text { for } 0<z<z_{1}
$$

with:

$\mathrm{z} \quad=$ height above bed

$\mathrm{A}, \mathrm{B}=$ coefficients

The A and B coefficients are determined by a linear regression method applying the measured concentration of the first three measuring points above the bed, as follows:

$$
\begin{gathered}
A-\frac{3 \sum_{1}^{3}\left(z_{k} \ln c_{k}\right)-\sum_{1}^{3}\left(z_{k}\right) \sum_{1}^{3}\left(\ln c_{k}\right)}{\left.3 \sum_{1}^{3}\left(z_{k} z_{k}\right)-\underset{1}{3} \sum_{k}\right)^{2}} \\
B=\frac{\sum_{1}^{3}\left(z_{k} z_{k}\right) \sum_{1}^{3}\left(\ln c_{k}\right)-\sum_{1}^{3}\left(z_{k}\right) \sum_{1}^{3}\left(z_{k} \ln c_{k}\right)}{3} \\
\frac{3}{3}\left(z_{k} z_{k}\right)-\frac{\left(\sum z_{k}\right)^{2}}{1}
\end{gathered}
$$

Applying Equation 1, the sediment concentration is computed in 50 equidistant points between the bed (defined at $\left.z=2 * D_{50}\right)$ and the first measuring point $\left(z=z_{1}\right)$. The maximum concentration is assumed to be $1590 \mathrm{~kg} / \mathrm{m}^{3}$. 
CALCULATED SEDIMENT CONCENTRATIONS NEAR THE BED ( $\left.\mathrm{C}_{\text {BED }}\right)$

\begin{tabular}{|c|c|c|}
\hline Experiment & Vertical & $\begin{array}{c}\text { Sediment concentration near the } \\
\text { bed }\left(\mathrm{z}=2 * \mathrm{D}_{50}\right) \text { calculated by CONVERT } \\
{[\mathrm{g} /]}\end{array}$ \\
\hline A without breakwater & (a) & 6.2 \\
\hline B without breakwater & (a) & 9.5 \\
\hline C without breakwater & (a) & 0.6 \\
\hline D without breakwater & (a) & 27.8 \\
\hline A with breakwater & (a) & 4.2 \\
\hline B with breakwater & (a) & 0.7 \\
\hline C with breakwater & (a) & 0.9 \\
\hline D with breakwater & (a) & 4.9 \\
\hline A without breakwater & (b) & 7.5 \\
\hline B without breakwater & (b) & 13.1 \\
\hline C without breakwater & (b) & 2.2 \\
\hline D without breakwater & (b) & 17.5 \\
\hline A with breakwater & (b) & 8.3 \\
\hline B with breakwater & (b) & 2.8 \\
\hline C with breakwater & (b) & 32.6 \\
\hline D with breakwater & (b) & 9.2 \\
\hline A without breakwater & (c) & 11.9 \\
\hline B without breakwater & (c) & 8.1 \\
\hline C without breakwater & (c) & 9.5 \\
\hline D without breakwater & (c) & 13.0 \\
\hline A with breakwater & (c) & 12.4 \\
\hline B with breakwater & (c) & 84.5 \\
\hline C with breakwater & (c) & 7.2 \\
\hline D with breakwater & (c) & 8.1 \\
\hline
\end{tabular}

Table F.1 List of calculated sediment concentrations near the bed ( $\left.c_{b e d}\right)$. 


\section{APPENDIX G}

\section{UNIBEST-TC}

- THEORETICAL BACKGROUND

- $\quad$ INPUT PARAMETERS

- $\quad$ LIST OF H $_{\text {RMS }}$ 


\section{MATHEMATICALPPYYSICAL DESCRIPTION}

\section{UST OF SYMBOLS}

Wave propagation

\begin{tabular}{|c|c|c|}
\hline $\mathbf{E}$ & : & wave energy \\
\hline$p$ & $:$ & density of water \\
\hline B & : & gravitational accelaration \\
\hline$H_{n}$ & : & root mean square wave beight \\
\hline$c_{8}$ & : & group velocity \\
\hline$\omega_{\mathrm{x}}$ & : & relative wave frequency \\
\hline $\mathbf{k}$ & : & wavenumber in direction of propagation \\
\hline v & : & alongshore directed depth-averaged velocity \\
\hline$D_{1}$ & : & wave energy dissipation due to bottom friction \\
\hline D. & : & wave energy dissipation due to wave braking \\
\hline Q. & $:$ & fraction of brealing waves \\
\hline $\mathrm{H}_{\mathbf{a}}$ & : & maximum wave height \\
\hline f. & : & friction factor \\
\hline$D_{90}$ & : & $50 \%$ grain diameter \\
\hline
\end{tabular}

Turbulence model
$B_{t} \quad:$ coefficient of order one
$\theta_{d}:$ coefficieat of order one
k. : turbuleat kinetic energy

Cross-shore momenrion equation

$\theta_{4}$ : angle of incidence for waves

$S_{\mathrm{ax}} \quad$ : rediation stress

\section{Longshore momentwon equation}

i) : the longshore water-level gradient due to the tide

A : calibration coefficient

f. : friction factor due to steady current

$\mathrm{O}_{\mathrm{I}_{\mathrm{m}}} \quad$ : amplitude of the orbital velocity for $\mathrm{H}_{\mathrm{n}}$ 


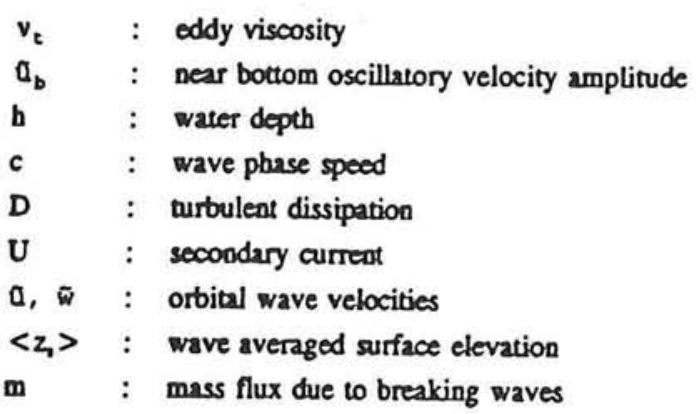

Long waves

G. : transfer function [Sand, 1982]

$2_{0}=\alpha_{2}$ : short wave amplitudes

$\xi_{\text {a }} \quad$ : long bound wave amplitude

$\Delta \omega$ : beat froquency

$\omega_{p} \quad$ : peak frequency

$u_{1} \quad$ : long-wave velocity amplitude

u. : bichromatic velocity

\section{Sedimert transpont}

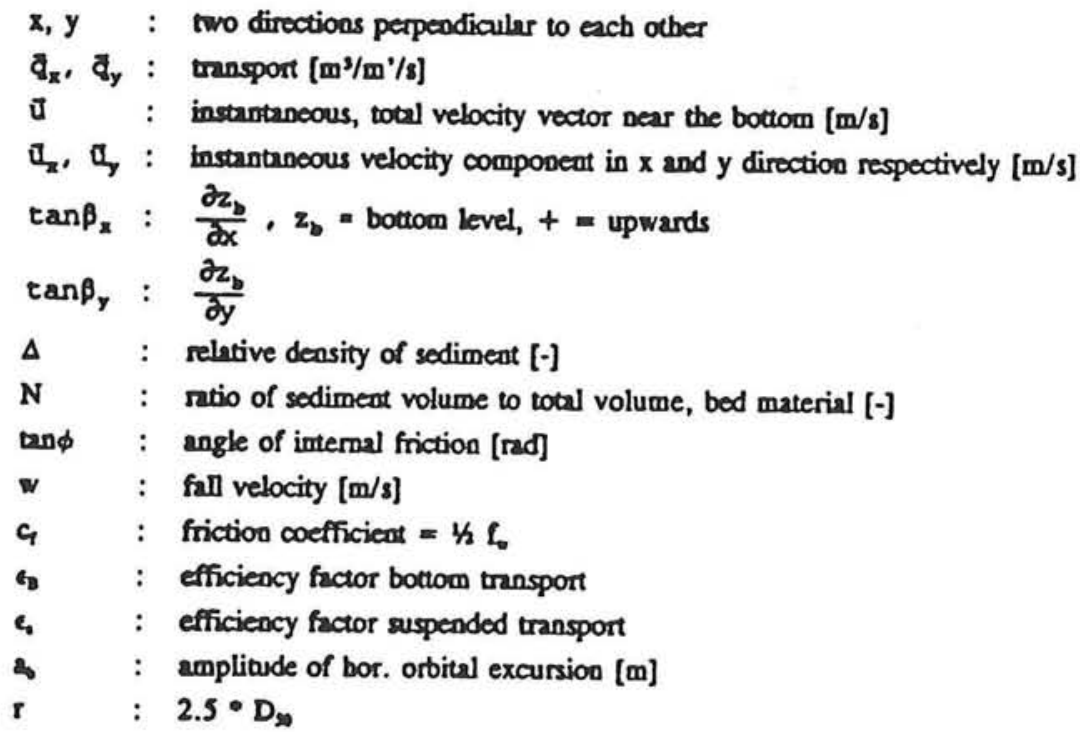




\section{Velocity moments}

$$
\begin{array}{ll}
\overline{\mathrm{u}} & : \text { mean velocity component } \\
\mathrm{a} & : \text { wave velocity component } \\
\mathrm{a}_{\mathrm{a}} & : \text { shon-wave velocity component } \\
\mathrm{a}_{1} & : \text { long-wave velocity component } \\
\mathrm{T}_{\mathrm{p}} & : \text { peak period }
\end{array}
$$

\section{Morphology}

$$
\begin{array}{ll}
\mathbf{z} & : \text { bottom level } \\
\mathrm{S}_{\mathbf{x}} & \text { : cross-shore sediment transport }
\end{array}
$$

\section{Formulations}

The UNIBEST_TC motule is a direct descendant of the models OSTRAN [Stive and Batries, 1984] and CROSTRAN [Stive, 1986]. In Roelvink and Stive [1989], the model is tested agaii wave flume measurements and improved on some points. In this paper a more detailed descripti of the mathematical-physical formulations used in the UNIBEST_TC module is given than presented here.
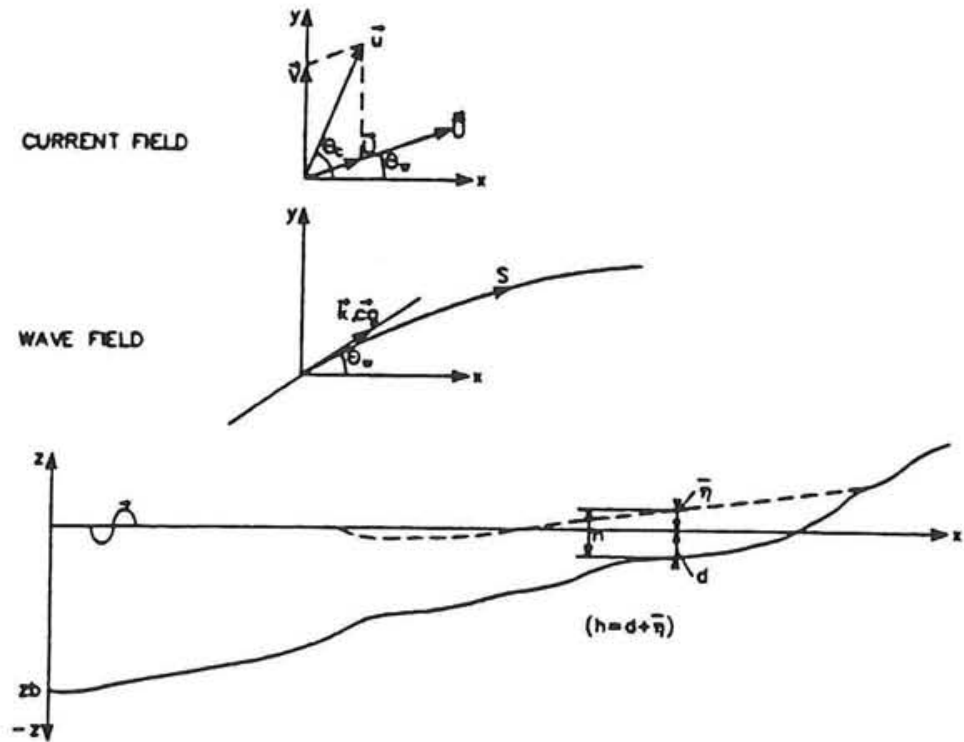

Figure C.I Definition of coordinate system and domain 
Wave propagarion model

The wave energy decay model of Bartjes and Janssen [1978] is used. It includes the wave energy changes due to bottom refraction, shoaling, bottom dissipation and wave breaking. The current refraction due to the longshore velocity component in the short-wave propagation direction is included as well:

$\frac{d}{d x}\left[c_{s} \cos \theta_{v} \frac{E}{\omega_{t}}\right]+\frac{D_{b}}{\omega_{t}}+\frac{D_{f}}{\omega_{t}}=0$

where:

$\mathrm{E}=\frac{1}{8} \rho_{\mathrm{B}} \mathrm{H}^{2}$

$c_{8}=\frac{\partial \omega_{r}}{\partial t}$

$\omega_{t}=\omega-\mathrm{k} \sin \theta_{\sigma} \mathrm{V}, \omega=2 \pi / T$

$\omega_{z}^{2}=\operatorname{gk} \tanh (\mathrm{h})$

$D_{b}=\frac{1}{4} P \& \&_{c} Q_{0}(\omega / 2 x) \mathrm{H}_{\mathrm{c}}^{2}$

and:

$Q_{0}=\exp \left(-\frac{1-Q_{0}}{\left(\frac{\mathrm{H}_{\mathrm{B}_{M}}}{\mathrm{H}_{M}}\right)}\right)$

alibration coefficieat $e_{\epsilon}=O(1)$

H. $=(0.88 / \mathrm{k}) \tanh (\gamma / \mathrm{h} / 0.88)$

$D_{f}=\frac{1}{8} \rho f_{0} x^{-6}\left(\frac{\omega_{1} R}{\sinh (x)}\right)^{3}$

and:

$f_{v}=\exp \left[-5.997+5213\left(\frac{26}{8}\right)^{-.194}\right]$

2 is the amplitude of the hor. orbital excursion near the bottom

$r$ is $2.5 \cdot D_{90}$ 
The urbulent dissipation is derived from the following equation:

$D=D_{b}-\rho \beta_{1} \frac{\partial}{\partial \alpha}\left(\alpha_{\gamma} h \cos \theta_{\nu}\right.$

where:

$D=\rho \beta_{d} K_{d}^{\frac{3}{2}}$

The cross-sbore momentum equation which is used to calculate the wave induced set-up:

$\frac{d}{d x} s_{x}+\rho g b \frac{d \bar{\eta}}{d x}=0$

where:

$S_{z \mathbf{z}}=\mathrm{E}\left(\mathrm{n}\left(1+\cos ^{2} \theta_{\bar{\sigma}}\right)-1 / 2\right)$

$n=\frac{1 \mathrm{~h}}{2 \sinh (2 \mathrm{l} \omega)}$

The wave angle with the shore normal is after Snel's bw:

$\mathbf{k} \sin \theta_{v}=\operatorname{cosstam}$

The longshore momentum equation, which describes the balance between the driving forces of the longshore curreat the to the tide and waves and the bottom friction:

Pghi, $-\frac{D}{\omega} k \sin \theta_{v}=\left.A_{\rho} \frac{\left(1+\sin ^{2} \theta_{w}\right)}{\sqrt{x}} \sqrt{\left(f_{\sigma} f_{0}\right)} \theta\right|_{k} v$

Secondary current

The secondary curreat is due to the vertical noo-uniformity of the driving forces in the nearshore zone. This is modelled according to the formulations given by Stive and de Vriend [1987]. They use a profile function technique in combination with a borizontally two-dimensional current to describe the three-dimensional current system in the coastal zone. The secondary current velocity is estimated using a three-layer concept. 
middle loyer

bottom loyer

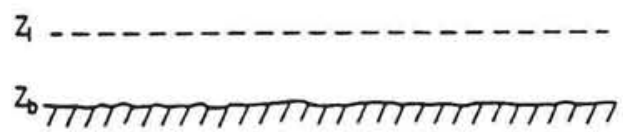

Figure C.2 Three loyer concept

The influence of the surface layer on the underlying layers is acoounted for by an effective shear stress at trough level [Stive and Wind, 1986]. This compensates for the momentum decay in the surface layer due to viscous dissipation and momentum loss due to wave brealing. The effective shear stress is at wave trough level is given by:

$\tau(t)=p v_{1} \frac{q^{2} t}{c} \sinh (2 t h)+\frac{D}{c}$

The use of the turbulent dissipation instead of the wave energy dissipation results in a spatial lag between the wave brealing and the offishore directed secondary current.

The horizoatal wave-average momentum balance for the middle layer in the cross-shore direction reads:

$\frac{\partial}{\partial x}\left(v_{0} \frac{\partial U}{\partial z}\right)=\frac{\partial}{\partial x}\left(\left\langle\theta^{2}\right\rangle-\left\langle w^{2}\right\rangle\right)+g \frac{\partial\left\langle z_{\theta}\right\rangle}{\partial x}$

For the bottom layer the borizontal wave-average momentum equation in the cross-shore direction is given by:

$\left.\frac{\partial}{\partial z}\left(v, \frac{\partial U}{\partial z}\right)=\frac{\partial}{\partial x}\left(\left\langle u^{2}\right\rangle-\left\langle w^{2}\right\rangle\right)+8 \frac{\partial\left\langle z_{0}\right\rangle}{\partial x}+\frac{\partial}{\partial z}(\Delta w w\rangle\right)$

where the last term on the right-hand side is no longer negligible comparod to the Reynolds stress term given on the left-hand side of the equation. With the shear stress condition at the trough level and a no slip condition at the bottom, the solution for the recondary current is obtainod by patching the velocities and shear stresses at the bottom boundary 4 . Using the integral condition of continuity: 
$\int_{4}^{2} U d z=-\frac{m}{p}$

where:

$m=\left(1+Q_{b} \frac{7 \pi h}{2 \pi}\right) \frac{E}{c}$

and $m$ represents the mass flux in the surface layer due to breaking waves yields the final expression for the secondary current.

Long woves

In the case of a random wave field the grouping of the short waves will generate long waves. $\mathrm{I}$ assumption is made that the wave-group related features of a radom wave field may be represented by a bichromatic wave train with accompanying bound long wave. For the amplitud of the bound long wave is used:

$$
\xi_{0}=-G_{0} \frac{a_{a} a_{2}}{b}
$$

The short wave amplitudes are given by:

$$
q_{1}=q_{a}=\frac{1}{8} \mathrm{R}^{2}-\frac{1}{2} \xi_{a}^{2}
$$

where the condition that the schematized wave train tas the same surface variance as the random wave has been used. The individual velocities are obcained using linear wave theory.

For the long wave component is taken:

$a_{1}=\varepsilon_{a} \frac{\sqrt{g}}{b}$

The near bottom time-varying flow due to short and loag waves is given by:

$u_{\mathrm{u}}=\theta_{\mathrm{a}} \cos \left(\omega_{\mathrm{p}}\right)+\theta_{\mathrm{a}} \cos \left(\omega_{\mathrm{p}}+\Delta \omega\right) t+\theta_{1} \cos ((\Delta \omega) t+\phi)$

The beat frequency $\Delta \omega=\frac{1}{s} \omega_{p}$

Shor-wave orbital velociry

The orbital velocities near the bed due to short waves, which determine the strength of the onshore directed wave assymetry transport, have been computed using the model RFWAVE, developed at Delf Hydraulics [G. Klopman, 1989]. It is based on the Fourier approximation of the stram function method as developed by Rienocker and Fenton [1981], using wave coergy as imput. 
The sediment transport is calculated accorting to the formulations given by Bailard [1981], of which only the cross-shore component is used for the time-dependent morphological computations. This formulation includes transport due to the combined actions of steady current, wave orbial mocion and botrom slope effect. The Bailard transport model in 2 horizontal dimensions is given by:

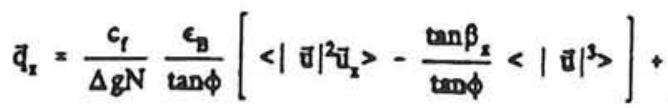

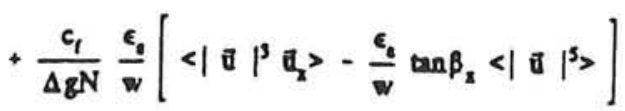

$$
\begin{aligned}
& \left.\left.\Phi_{3}=\frac{c_{f}}{\Delta g N} \frac{c_{B}}{\sin \phi}\left[\langle| \text { ब }\left.\right|^{2} \tilde{z}_{\gamma}\right\rangle-\frac{\tan \beta_{y}}{\tan \phi}\langle| \text { ब }\left.\right|^{3}\right\rangle\right]+
\end{aligned}
$$

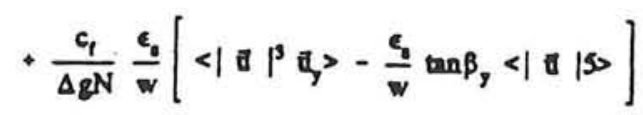

where:

$c_{4}: 0.5 f_{0}$

f. $: \exp \left[-5.97+5.213(2 / \mathrm{r})^{-2 \pi}\right]$

$<>$ indicate averaging over time.

The longsbore transport is defined as the componeat of the total transport vector in longshore direction.

The transport formulation contains several velocity moments. The time-averaged total near bed velocity is split up into a mean and a time varying velocity compooent:

$\mathbf{u}=\overline{\mathrm{u}}+\mathbb{Q}(t)$

where $t(t)$ stands for the velocity variation on the time scale of the wave groups and that of the individual waves. With these separate terms for the wave-orbital motion and the steady curreat the terms $\left\langle|\vec{u}|^{*} a_{1}\right\rangle$, with $m=2,3,5$ and $n=0,1$ can be approximated by a Taylor series. In these series the angle between waves and curreats has also been included.

Two Taylor expansions are possible: one with the orbital motion small compared to the steady curreat and vice versa. 


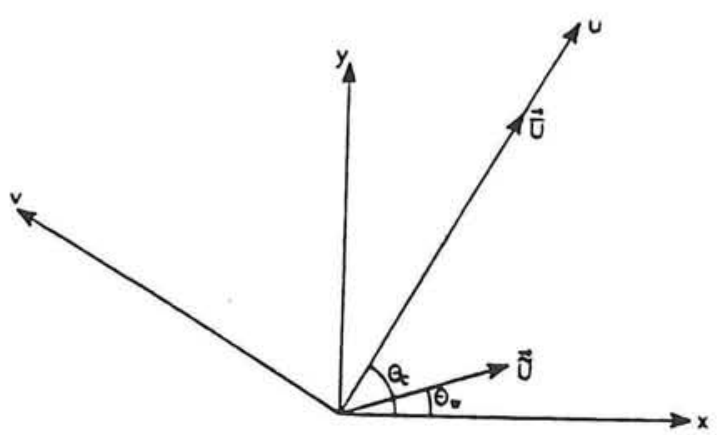

Figure C.3 Velocity directions

The first yields the following expressions for the odd velocity moments in u-direction:

$\left\langle\left|u_{1}\right|^{2} u\right\rangle=\vec{u}+\vec{u}\left\langle\vec{u}^{2}\right\rangle\left(1+\cos ^{2} \varphi\right)+\left\langle t^{3}\right\rangle \cos \varphi$

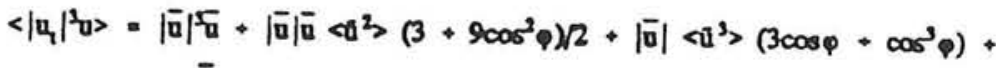
$+\frac{\overline{\mathrm{n}}}{|\overline{\mathrm{u}}|} \mathrm{Qr}^{\mathrm{s}}\left(3+6 \cos ^{2} \varphi-\cos ^{4} \varphi\right) / 8$

the odd velocity moments in v-direction:

$\left.\left\langle|\mathrm{u}|^{2} v\right\rangle=\overline{\mathrm{a}}\left\langle\mathrm{x}^{2}\right\rangle(2 \sin \varphi \cos \varphi)+\mathrm{s}^{2}\right\rangle \sin \varphi$

$\left.\left.\left\langle\left|\mathrm{u}_{1}\right|^{2} v\right\rangle=|\overline{\mathrm{u}}| \overline{\mathrm{u}}<\mathrm{e}^{2}\right\rangle(3 \sin \varphi \cos \varphi)+|\overline{\mathrm{v}}| \cos ^{2}\right\rangle\left(3 \sin \varphi+3 \sin \varphi \cos ^{2} \varphi\right) / 2$

$+\frac{\overline{\mathrm{a}}}{|\overline{\mathrm{a}}|}<\mathrm{s}>\left(3 \sin \varphi \cos \varphi-\sin \varphi \cos ^{3} \varphi\right) / 2$

and the even velocity moments:

$\left.\left.\left\langle\left|u_{1}\right|^{3}\right\rangle=|\bar{a}|^{3}+|\bar{a}| \cos ^{2}\right\rangle\left(3+3 \cos ^{2} \varphi\right) / 2+\frac{\bar{a}}{|\bar{a}|} \alpha^{3}\right\rangle\left(3 \cos \varphi-\cos ^{3} \varphi\right) / 2$

$\left\langle|u|^{3}=|\overline{\mathrm{u}}|^{3}+|\overline{\mathrm{v}}|^{3} \overline{\mathrm{u}}^{-2}\right\rangle\left(\mathrm{s}+15 \cos ^{2} \varphi\right) / 2+|\overline{\mathrm{u}}| \overline{\mathrm{u}}\left\langle\mathrm{u}^{3}\right\rangle\left(15 \cos \varphi+5 \cos ^{3} \phi\right) / 2$

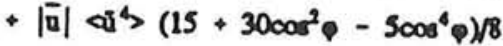

$+\frac{\overline{\mathrm{v}}}{|\overline{\mathrm{v}}|}<\mathrm{e}^{3}>\left(15 \cos \varphi-10 \cos ^{3} \varphi+3 \cos ^{3} \varphi\right) / 8$ 
Where:

$\varphi=\theta_{v}-\theta_{c}$.

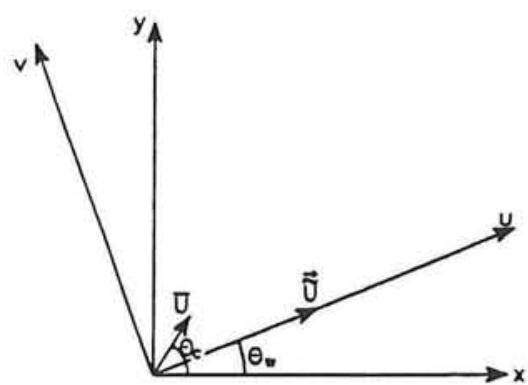

Figure C.4 Velociry directions

If the mean velocity component is small compared to the orbital velocity the Taylor expansion yields for the odd velocity moments:

$$
\begin{aligned}
& \left\langle\left|u_{1}\right|^{2} u\right\rangle=\left\langle\vec{u}^{3}\right\rangle+\bar{u}\left\langle\tilde{u}^{2}\right\rangle(3 \cos \varphi)+\vec{u}^{3}(\cos \varphi)
\end{aligned}
$$

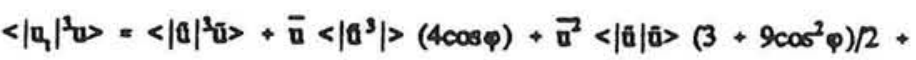

$$
\begin{aligned}
& +\overrightarrow{\mathrm{a}}<|\overline{\mathrm{u}}|>\left(3 \cos \varphi+\cos ^{3} \varphi\right)
\end{aligned}
$$

the odd velocity moments in v-direction:

$$
\begin{aligned}
& \left\langle\left|u_{1}\right|^{2} v\right\rangle=\overline{\mathrm{u}}\left\langle\overline{\mathrm{u}}^{2}\right\rangle(\sin \varphi)+\overrightarrow{\mathrm{u}}(\sin \varphi) \\
& \left.\left\langle\left|\mathrm{u}_{1}\right|^{3} v\right\rangle=\overline{\mathrm{u}}<\left|\mathrm{a}^{3}\right|>(\sin \varphi)+\overline{\mathrm{u}}^{2}<|\hat{\sigma}| \omega\right\rangle(3 \sin \varphi \cos \varphi) \\
& +\overrightarrow{\mathrm{a}}<|\hat{0}|>\left(3 \sin \varphi+3 \sin \varphi \cos ^{2} \varphi\right) / 2
\end{aligned}
$$

and the even velocity moments:

$$
\begin{aligned}
& \left\langle|\mathrm{u}|^{3}\right\rangle=\left\langle|\tilde{u}|^{3}\right\rangle+\overline{\mathrm{u}}\langle|\hat{\mathrm{u}}| \hat{\mathrm{u}}\rangle(3 \cos \varphi)+\overrightarrow{\mathrm{u}}^{2}\langle|\hat{\mathrm{a}}|\rangle\left(3+3 \cos ^{2} \varphi\right) / 2 \\
& \left\langle|\bar{u}|^{3}\right\rangle=\left\langle|\bar{u}|^{3}\right\rangle+\bar{u}\left\langle|\bar{u}|^{3} \bar{u}\right\rangle(5 \cos \varphi)+\overrightarrow{u^{2}}\left\langle|\bar{u}|^{3}\right\rangle\left(5+15 \cos ^{2} \varphi\right) / 2 \\
& +\overrightarrow{\mathrm{u}}<|\tilde{u}| \tilde{u}>\left(15 \cos \varphi+5 \cos ^{3} \varphi\right) / 2 \\
& +\overline{\mathrm{u}}^{4}<|\mathrm{u}|>\left(15+30 \cos ^{2} \varphi-5 \cos ^{4} \varphi\right) / 8
\end{aligned}
$$


where:

$\theta=\theta_{c}-\theta_{v}$.

A smooth transition from one formulation to the other is aken care of.

The orbital velocity component is split up into a short-wave and a long-wave component:

$6=6+\frac{6}{4}$

The long-wave velocity is assumed to be significantly smaller than the sbort-wave component. A second assumption is that there is no correlation between u, and $\left|u_{1}\right|^{n}$. With these assumptions " can write for the following odd velocity momeat:

$\left\langle\bar{u}|\bar{u}|^{2}\right\rangle=\left\langle u_{3}\left|u_{s}\right|^{2}\right\rangle+3\left\langle u_{1}\left|u_{3}\right|^{2}\right\rangle$

Where the first term on the right is non-zero in the case of an assymmetry about the borizontal plane caused by the non-linearity of the shor waves. As mentioned before, this part is calculate" with RFWAVE.

The second term on the right is nonzero if there is a correlation between $\mathrm{q}_{1}$ and $\mathrm{q}_{4}{ }^{2}$. Thi correlation is present in the case of long bound waves accompanying a short-wave group, resultin in a negative correlation. In that case the velocity moment is approximated by:

$\left.3<0_{1}\left|u_{1}\right|^{2}\right\rangle=\left\langle 0_{1}{ }_{1}^{3}\right.$

where the bi-chromatic velocity component is alculated as described previously. The other velocity moments are expanded in a similar way. In order to reduce computing time, the results have beea tabulated as function of two dimensionless variables:

$f\left(\frac{\mathrm{H}}{\mathrm{b}}, \mathrm{T}, \sqrt{\frac{\mathrm{g}}{\mathrm{b}}}\right)$

Morphology

The bottom level changes are computed from the mass balance:

$\frac{\partial z}{\partial t}+\frac{w_{2}}{\partial x}=0$ 


\section{INPUT PARAMETERS}

Run-constant parameters:

- wavebreaking parameter

$\begin{array}{llll}\alpha_{c} & = & 1.00 & {[-]} \\ \gamma & = & 0.75 & {[-]} \\ f_{w} & = & 0.01 & {[-]} \\ \text { rkls } & = & 0.05 & {[\mathrm{~m}]}\end{array}$

Sediment parameters:

- coefficient bottom transport:

$\begin{array}{llll}\text { epsb } & = & 0.15 & {[-]} \\ \text { epss } & =0.03 & {[-]} \\ \text { wee } & = & 0.01 & {[\mathrm{~m} / \mathrm{s}]} \\ \text { tant } & =0.63 & {[-]} \\ \text { dsed } & = & 95 & {[\mu \mathrm{m}]} \\ \text { rhos } & =2650 & {\left[\mathrm{~kg} / \mathrm{m}^{3}\right]} \\ \text { por } & = & 0.4 & {[-]}\end{array}$

Other parameters:

- definition of 'dry'

$\mathrm{T}_{\mathrm{dry}}=21$

( $\mathrm{T}_{\text {dry }}$ : computations carried out up to the point where the relative wave length becomes to great: depth $\left.<\mathrm{g}^{*}\left(\mathrm{~T}_{\mathrm{p}} / \mathrm{T}_{\mathrm{dry}}\right)^{\wedge} 2\right) \Rightarrow$ depth $\left.\approx 0.05 \mathrm{~m}\right)$

Definition of grid (from $\mathrm{x}=-3.0 \mathrm{~m}$ till $\mathrm{x}=12.0 \mathrm{~m}$ ):

\begin{tabular}{|c|c|c|}
\hline$\Delta \mathrm{x}[\mathrm{m}]$ & Number of $\Delta \mathrm{x}$ & From/to \\
\hline 0.50 & 13 & $-3.00 \mathrm{~m}-3.50 \mathrm{~m}$ \\
\hline 0.05 & 14 & $3.50 \mathrm{~m}-4.20 \mathrm{~m}$ \\
\hline 0.04 & 4 & $4.20 \mathrm{~m}-4.36 \mathrm{~m}$ \\
\hline 0.05 & 7 & $4.36 \mathrm{~m}-4.71 \mathrm{~m}$ \\
\hline 0.09 & 1 & $4.71 \mathrm{~m}-4.80 \mathrm{~m}$ \\
\hline 0.10 & 32 & $4.80 \mathrm{~m}-8.00 \mathrm{~m}$ \\
\hline 0.25 & 16 & $8.00 \mathrm{~m}-12.00 \mathrm{~m}$ \\
\hline
\end{tabular}

Table G.1 Definition of gridpoints in Unibest-TC.

The grid of the first part of the slope (till $\mathrm{x}=3.50 \mathrm{~m}$ ) is quite wide. From the position of the toe of the breakwater $(x=3.75 \mathrm{~m})$ the grid is much smaller. Near the breakwater the water motion changes strongly so a small grid is necessary. In the area of the waterline the gridsize was slightly increased again because with a small gridsize sometimes divergence in the calculations occured. 
MEASURED $\mathrm{H}_{\mathrm{RMS}}$ AT WHM 1

\begin{tabular}{|l|c|c|c|c|c|c|c|c|c|c|c|c|c|}
\hline \multicolumn{1}{|c|}{ time-point* } & $\mathbf{1 . 1}$ & $\mathbf{2 . 1}$ & $\mathbf{2 . 2}$ & $\mathbf{3 . 1}$ & $\mathbf{3 . 2}$ & $\mathbf{3 . 3}$ & $\mathbf{3 . 4}$ & $\mathbf{4 . 1}$ & $\mathbf{4 . 2}$ & $\mathbf{4 . 3}$ & $\mathbf{4 . 4}$ & $\mathbf{4 . 5}$ & $\mathbf{4 . 6}$ \\
\hline $\begin{array}{l}\text { exp A without } \\
\text { breakwater }\end{array}$ & 6.8 & 6.6 & 6.6 & 6.9 & 6.9 & 7.0 & 7.0 & 6.9 & 6.9 & 7.0 & 6.9 & 6.9 & 6.9 \\
\hline $\begin{array}{l}\text { exp B without } \\
\text { breakwater }\end{array}$ & 7.0 & 7.0 & 7.0 & 7.1 & & 7.1 & & 7.1 & 7.1 & 7.2 & 7.1 & 7.1 & 7.1 \\
\hline $\begin{array}{l}\text { exp C without } \\
\text { breakwater }\end{array}$ & 6.8 & 6.8 & 6.8 & 7.0 & 7.0 & 7.0 & 6.9 & 7.0 & 7.0 & 7.0 & 7.0 & 7.0 & 7.0 \\
\hline $\begin{array}{l}\text { exp D without } \\
\text { breakwater }\end{array}$ & 9.6 & 9.7 & 9.5 & 10.0 & 10.0 & 10.0 & 10.0 & 10.1 & 10.1 & 10.0 & 10.1 & 10.1 & 10.0 \\
\hline $\begin{array}{l}\text { exp E without } \\
\text { breakwater }\end{array}$ & 4.7 & 4.7 & 4.7 & 4.6 & 4.8 & 4.9 & 5.2 & 4.9 & 4.7 & 4.7 & 4.7 & 4.7 & 4.6 \\
\hline $\begin{array}{l}\text { exp A with } \\
\text { breakwater }\end{array}$ & 7.4 & 7.7 & 7.7 & 7.5 & 7.5 & 7.5 & 7.5 & 7.9 & & 8.3 & 8.8 & & 8.6 \\
\hline $\begin{array}{l}\text { exp B with } \\
\text { breakwater }\end{array}$ & 8.7 & 9.2 & 9.0 & 8.3 & 8.4 & 8.4 & 8.8 & 8.3 & 8.3 & 8.2 & 8.2 & 8.2 & 8.0 \\
\hline $\begin{array}{l}\text { exp C with } \\
\text { breakwater }\end{array}$ & 8.1 & 8.0 & & 7.3 & 7.5 & 7.4 & 7.3 & 7.3 & 7.3 & 7.3 & 7.3 & 7.3 & 7.3 \\
\hline $\begin{array}{l}\text { exp D with } \\
\text { breakwater }\end{array}$ & 10.3 & 10.5 & 10.0 & 10.5 & 10.3 & 10.1 & 10.0 & 9.9 & 10.4 & 10.0 & 9.9 & 9.9 & 9.8 \\
\hline $\begin{array}{l}\text { exp E with } \\
\text { breakwater }\end{array}$ & 5.1 & 4.9 & 4.9 & 5.0 & 4.9 & 4.9 & 4.9 & 4.9 & 4.9 & 4.9 & 4.9 & 4.9 & 4.9 \\
\hline
\end{tabular}

* The first number indicates the interval number, the second number indicates the number of the measurement during that interval (e.g., 4.2 means: Interval 4, measurement 2).

Table G.2 Measured Hrms [cm] at WHM 1. 


\section{Effect of submerged breakwater on profile development}

Volume 2: Graphics

August 1995

E.W.M. Claessen

M.D. Groenewoud
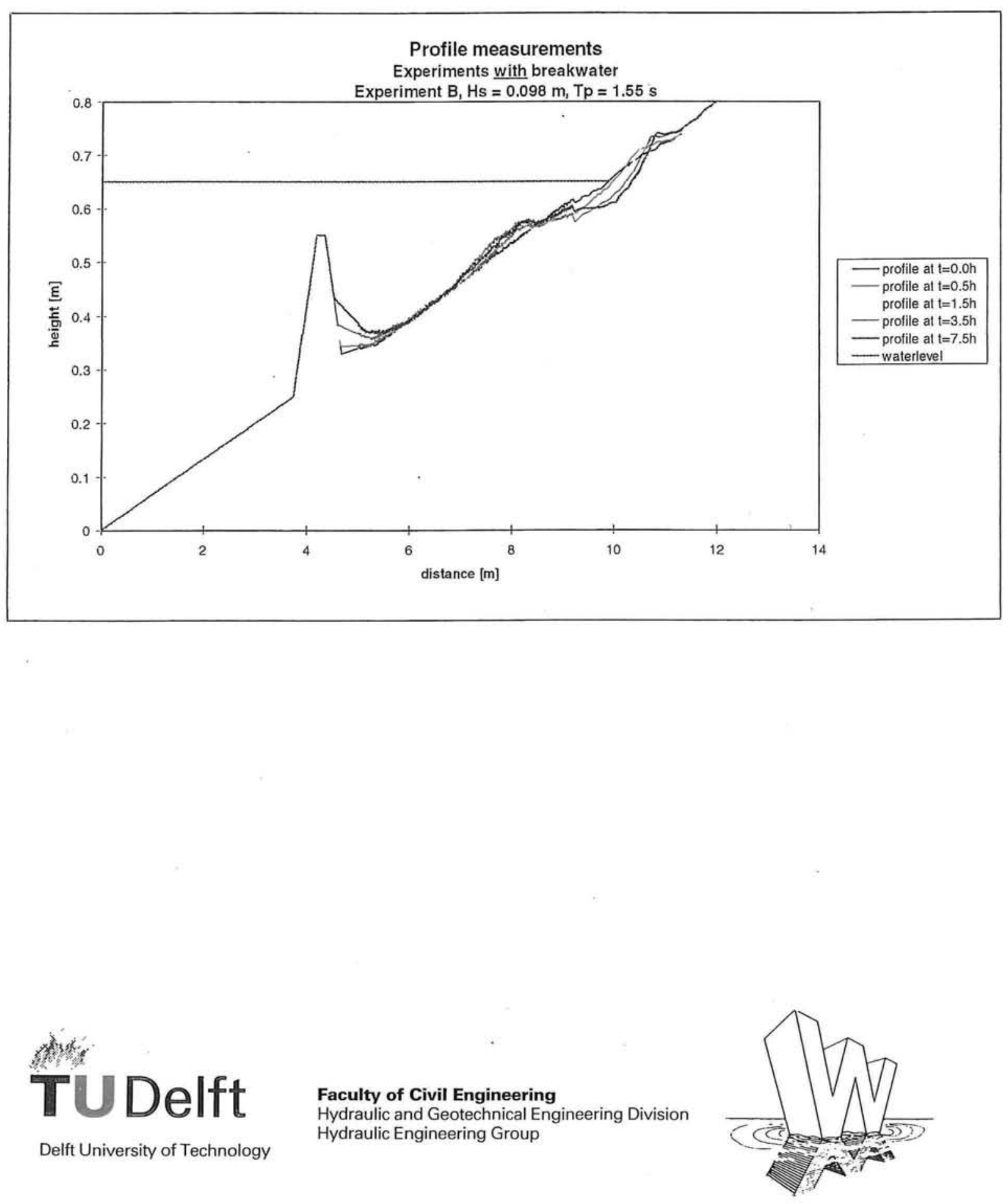


\section{TABLE OF CONTENTS}

\section{Part 1: Profile graphs and sediment transport volume graphs}

\section{Profile graphs}

\section{Graph no.: description:}

1.1

Experiment A without breakwater prof.no. 0-4

Experiment B without breakwater prof.no. 0-4

Experiment $C$ without breakwater prof.no. 0-4

Experiment D without breakwater prof.no. 0-4

Experiment E without breakwater prof.no. 0-4

Experiment $\mathrm{F}$ without breakwater prof.no. 0-4

Experiment A with breakwater prof.no. 0-4

Experiment B with breakwater prof.no. 0-4

Experiment $C$ with breakwater prof.no. 0-4

Experiment D with breakwater prof.no. 0-4

Experiment E with breakwater prof.no. 0-4

Experiment $\mathrm{F}$ with breakwater prof.no. 0-4

Experiment A with and without breakwater prof.no.4 Experiment B with and without breakwater prof.no. 4

Experiment $\mathrm{C}$ with and without breakwater prof.no. 4

Experiment D with and without breakwater prof.no. 4

Experiment E with and without breakwater prof.no. 4

Experiment $\mathrm{F}$ with and without breakwater prof.no. 4 
1.19 Experiments A, B and C without breakwater prof.no.2

1.20 Experiments A, B and C without breakwater prof.no. 4

1.21 Experiments A, B and C with breakwater prof.no.2

1.22 Experiments A, B and C with breakwater prof.no. 4

1.23 Experiments B, D and E without breakwater prof.no.2

1.24 Experiments B, D and E without breakwater prof.no.4

1.25 Experiments B, D and E with breakwater prof.no.2

1.26 Experiments B, D and E with breakwater prof.no.4

\section{Sediment transport volume graphs}

1.27 Experiment A with and without breakwater through Vertical (a): $x=5.25 \mathrm{~m}$

Experiment A with and without breakwater through horizontal (b): $y=0.55 \mathrm{~m}$ Experiment A with and without breakwater through horizontal (c): $y=0.65 \mathrm{~m}$ Experiment B with and without breakwater through Vertical (a): $x=5.25 \mathrm{~m}$ Experiment B with and without breakwater through Vertical (b): $x=6.15 \mathrm{~m}$ Experiment B with and without breakwater through Vertical (c): $x=7.05 \mathrm{~m}$ Experiment B with and without breakwater through horizontal (a): $y=0.45 \mathrm{~m}$ Experiment B with and without breakwater through horizontal (b): $y=0.55 \mathrm{~m}$ Experiment B with and without breakwater through horizontal (c): $y=0.65 \mathrm{~m}$ Experiment $C$ with and without breakwater through Vertical (a): $x=5.25 \mathrm{~m}$ Experiment $C$ with and without breakwater through Vertical (b): $x=6.15 \mathrm{~m}$ Experiment $\mathrm{C}$ with and without breakwater through Vertical (c): $\mathrm{x}=7.05 \mathrm{~m}$ Experiment $C$ with and without breakwater through horizontal (a): $y=0.45 \mathrm{~m}$ Experiment $C$ with and without breakwater through horizontal (b): $y=0.55 \mathrm{~m}$ 
Experiment $\mathrm{C}$ with and without breakwater through horizontal (c): $y=0.65 \mathrm{~m}$ Experiment D with and without breakwater through Vertical (a): $x=5.25 \mathrm{~m}$ Experiment D with and without breakwater through Vertical (b): $x=6.15 \mathrm{~m}$ Experiment D with and without breakwater through Vertical (c): $x=7.05 \mathrm{~m}$ Experiment D with and without breakwater through horizontal (a): $y=0.45 \mathrm{~m}$ Experiment D with and without breakwater through horizontal (b): $y=0.55 \mathrm{~m}$ Experiment D with and without breakwater through horizontal (c): $y=0.65 \mathrm{~m}$ Experiment $E$ with and without breakwater through Vertical (a): $x=5.25 \mathrm{~m}$ Experiment $E$ with and without breakwater through Vertical (b): $x=6.15 \mathrm{~m}$ Experiment $E$ with and without breakwater through Vertical (c): $x=7.05 \mathrm{~m}$ Experiment $E$ with and without breakwater through horizontal (a): $y=0.45 \mathrm{~m}$ Experiment E with and without breakwater through horizontal (b): $y=0.55 \mathrm{~m}$ Experiment E with and without breakwater through horizontal (c): $y=0.65 \mathrm{~m}$ Experiment $F$ with and without breakwater through Vertical (a): $x=5.25 \mathrm{~m}$ Experiment $F$ with and without breakwater through Vertical (b): $x=6.15 \mathrm{~m}$ Experiment $F$ with and without breakwater through Vertical (c): $x=7.05 \mathrm{~m}$ Experiment $F$ with and without breakwater through horizontal (a): $y=0.45 \mathrm{~m}$ Experiment F with and without breakwater through horizontal (b): $y=0.55 \mathrm{~m}$ Experiment $F$ with and without breakwater through horizontal (c): $y=0.65 \mathrm{~m}$

Experiments A, B and C without breakwater through Vertical (a): $x=5.25 \mathrm{~m}$ Experiments A, B and C without breakwater through Vertical (b): $x=6.15 \mathrm{~m}$ Experiments A, B and C without breakwater through Vertical (c): $x=7.05 \mathrm{~m}$ Experiments A, B and C without breakwater through horizontal (a): $y=0.45 \mathrm{~m}$ Experiments A, B and C without breakwater through horizontal (b): $y=0.55 \mathrm{~m}$ Experiments A, B and C without breakwater through horizontal (c): $y=0.65 \mathrm{~m}$

Experiments A, B and C with breakwater through Vertical (a): $\mathrm{x}=5.25 \mathrm{~m}$ Experiments A, B and C with breakwater through Vertical (b): $x=6.15 \mathrm{~m}$. 

Experiments A, B and C with breakwater through horizontal (a): $y=0.45 \mathrm{~m}$ Experiments A, B and C with breakwater through horizontal (b): $y=0.55 \mathrm{~m}$ Experiments A, B and C with breakwater through horizontal (c): $y=0.65 \mathrm{~m}$

Experiments B, D and E without breakwater through Vertical (a): $x=5.25 \mathrm{~m}$ Experiments B, D and E without breakwater through Vertical (b): $x=6.15 \mathrm{~m}$ Experiments B, D and E without breakwater through Vertical (c): $x=7.05 \mathrm{~m}$ Experiments B, D and E without breakwater through horizontal (a): $y=0.45 \mathrm{~m}$ Experiments B, D and E without breakwater through horizontal (b): $y=0.55 \mathrm{~m}$ Experiments B, D and E without breakwater through horizontal (c): $y=0.65 \mathrm{~m}$

1.81 Experiments B, D and E with breakwater through Vertical (a): $x=5.25 \mathrm{~m}$

\section{Part 2: Wave height measurement and spectra graphs}

\section{Wave height measurements graphs}

\section{Graph no.: description:}

$2.1 \quad$ Experiment A without breakwater

2.2 Experiment B without breakwater

2.3 Experiment $\mathrm{C}$ without breakwater 
Experiment D without breakwater

Experiment $\mathrm{F}$ without breakwater

Experiment A with breakwater

Experiment B with breakwater

Experiment $\mathrm{C}$ with breakwater

Experiment D with breakwater

Experiment E with breakwater

2.13 Experiment A with and without breakwater

2.14 Experiment B with and without breakwater

2.15 Experiment $C$ with and without breakwater

2.16 Experiment D with and without breakwater

2.17 Experiment $\mathrm{E}$ with and without breakwater

2.18 Experiment $\mathrm{F}$ with and without breakwater

2.19 Experiments without breakwater at WHM 1

2.20 Experiments with breakwater at WHM 1

\section{Spectra graphs}

2.21 Experiment A without breakwater

2.22 Experiment B without breakwater

2.23 Experiment $\mathrm{C}$ without breakwater

2.24 Experiment D without breakwater

2.25 Experiment E without breakwater 
Experiment A with breakwater Experiment B with breakwater

\section{Part 3: Velocity measurement graphs}

\section{Graph no.: description:}

$3.1 \quad$ Experiment A without breakwater

3.2

Experiment B without breakwater

Experiment $\mathrm{C}$ without breakwater

Experiment D without breakwater

Experiment E without breakwater

\section{Experiment A with breakwater \\ Experiment B with breakwater \\ Experiment $\mathrm{C}$ with breakwater \\ Experiment D with breakwater \\ Experiment E with breakwater}

Experiment A with and without breakwater: Vertical (a): $x=5.25 \mathrm{~m}$

Experiment A with and without breakwater: Vertical (b): $x=6.15 \mathrm{~m}$

Experiment A with and without breakwater: Vertical (c): $x=7.05 \mathrm{~m}$

Experiment B with and without breakwater: Vertical (a): $x=5.25 \mathrm{~m}$

Experiment B with and without breakwater: Vertical (b): $x=6.15 \mathrm{~m}$ 
Experiment B with and without breakwater: Vertical (c): $x=7.05 \mathrm{~m}$ Experiment $C$ with and without breakwater: Vertical (a): $x=5.25 \mathrm{~m}$

Experiment $C$ with and without breakwater: Vertical (c): $x=7.05 \mathrm{~m}$

Experiment D with and without breakwater: Vertical (a): $x=5.25 \mathrm{~m}$

Experiment D with and without breakwater: Vertical (c): $x=7.05 \mathrm{~m}$

Experiment E with and without breakwater: Vertical (a): $x=5.25 \mathrm{~m}$

\section{Part 4: Sediment concentration graphs}

\section{Section 1 Sediment concentrations during Interval 1}

\section{Graph no.: description:}

$\begin{array}{lll}4.1 .1 & \text { Experiment A without breakwater: } & \text { Vertical (a), (b) and (c) } \\ 4.1 .2 & \text { Experiment B without breakwater: } & \text { Vertical (a), (b) and (c) } \\ 4.1 .3 & \text { Experiment C without breakwater: } & \text { Vertical (a), (b) and (c) } \\ 4.1 .4 & \text { Experiment D without breakwater: } & \text { Vertical (a), (b) and (c) } \\ 4.1 .5 & \text { Experiment A with breakwater: } & \text { Vertical (a), (b) and (c) } \\ 4.1 .6 & \text { Experiment B with breakwater: } & \text { Vertical (a), (b) and (c) } \\ 4.1 .7 & \text { Experiment C with breakwater: } & \text { Vertical (a), (b) and (c) } \\ 4.1 .8 & \text { Experiment D with breakwater: } & \text { Vertical (a), (b) and (c) }\end{array}$

4.1.9 Experiment A with and without breakwater: Vertical (a): $x=5.25 \mathrm{~m}$ 4.1.10 Experiment A with and without breakwater: Vertical (b): $x=6.15 \mathrm{~m}$ 4.1.11 Experiment A with and without breakwater: Vertical (c): $x=7.05 \mathrm{~m}$ 4.1.12 Experiment B with and without breakwater: Vertical (a): $x=5.25 \mathrm{~m}$ 
4.1.13 Experiment B with and without breakwater: Vertical (b): $x=6.15 \mathrm{~m}$

4.1.14 Experiment B with and without breakwater: Vertical (c): $x=7.05 \mathrm{~m}$

4.1.15 Experiment $C$ with and without breakwater: Vertical (a): $x=5.25 \mathrm{~m}$

4.1.16 Experiment $C$ with and without breakwater: Vertical (b): $x=6.15 \mathrm{~m}$

4.1.17 Experiment $C$ with and without breakwater: Vertical (c): $x=7.05 \mathrm{~m}$

4.1.18 Experiment D with and without breakwater: Vertical (a): $x=5.25 \mathrm{~m}$

4.1.19 Experiment D with and without breakwater: Vertical (b): $x=6.15 \mathrm{~m}$

4.1.20 Experiment D with and without breakwater: Vertical (c): $x=7.05 \mathrm{~m}$

4.1.21 Experiments A, B, C and D without breakwater: Vertical (a): $x=5.25 \mathrm{~m}$

4.1.22 Experiments A, B, C and D without breakwater: Vertical (b): $x=6.15 \mathrm{~m}$

4.1.23 Experiments A, B, C and D without breakwater: Vertical (c): $x=7.05 \mathrm{~m}$

4.1.24 Experiments A, B, C and D with breakwater: Vertical (a): $x=5.25 \mathrm{~m}$

4.1.25 Experiments A, B, C and D with breakwater: Vertical (b): $x=6.15 \mathrm{~m}$

4.1.26 Experiments A, B, C and D with breakwater: Vertical (c): $x=7.05 \mathrm{~m}$

\section{Section 2 Sediment concentrations during Interval 4}

\section{Graph no.: description:}

4.2.1 Experiment A without breakwater:

Experiment B without breakwater:

4.2 .3

4.2 .4

Experiment $\mathrm{C}$ without breakwater:

Experiment D without breakwater:

Experiment A with breakwater:

Experiment B with breakwater:

Experiment $\mathrm{C}$ with breakwater:

4.2 .8

Experiment D with breakwater:

Vertical (a), (b) and (c)

Vertical (a), (b) and (c)

Vertical (a), (b) and (c)

Vertical (a), (b) and (c)

Vertical (a), (b) and (c)

Vertical (a), (b) and (c)

Vertical (a), (b) and (c)

Vertical (a), (b) and (c) 
Experiment A with and without breakwater: Vertical (a): $x=5.25 \mathrm{~m}$ Experiment A with and without breakwater: Vertical (b): $x=6.15 \mathrm{~m}$ Experiment A with and without breakwater: Vertical (c): $x=7.05 \mathrm{~m}$ Experiment B with and without breakwater: Vertical (a): $x=5.25 \mathrm{~m}$ Experiment B with and without breakwater: Vertical (b): $x=6.15 \mathrm{~m}$ Experiment B with and without breakwater: Vertical (c): $x=7.05 \mathrm{~m}$ Experiment $C$ with and without breakwater: Vertical (a): $x=5.25 \mathrm{~m}$ Experiment $C$ with and without breakwater: Vertical (b): $x=6.15 \mathrm{~m}$ Experiment $C$ with and without breakwater: Vertical (c): $x=7.05 \mathrm{~m}$ Experiment D with and without breakwater: Vertical (a): $x=5.25 \mathrm{~m}$ Experiment D with and without breakwater: Vertical (b): $x=6.15 \mathrm{~m}$ Experiment D with and without breakwater: Vertical (c): $x=7.05 \mathrm{~m}$

\section{Part 5: Unibest-TC graphs}

\section{Graph' no.: description:}

5.1 Measured $\mathrm{H}_{\text {ms }}$ at WHM 1: Experiments without breakwater

5.2 Measured $\mathrm{H}_{\text {ms }}$ at WHM 1: Experiments with breakwater

Vertical (a): $x=5.25 \mathrm{~m}$
Vertical (b): $x=6.15 \mathrm{~m}$
Vertical (c): $x=7.05 \mathrm{~m}$
Vertical (a): $x=5.25 \mathrm{~m}$
Vertical (b): $x=6.15 \mathrm{~m}$
Vertical (c): $x=7.05 \mathrm{~m}$
Experiments $\mathrm{A}, \mathrm{B}, \mathrm{C}$ ar

Experiments A, B, C and D with breakwater: 
Measured $\mathrm{H}_{\mathrm{rms}}$ versus $\mathrm{H}_{\mathrm{ms}}$ calculated by Unibest-TC : Measured $\mathrm{H}_{\mathrm{ms}}$ versus $\mathrm{H}_{\mathrm{ms}}$ calculated by Unibest-TC : Measured $\mathrm{H}_{\mathrm{ms}}$ versus $\mathrm{H}_{\mathrm{ms}}$ calculated by Unibest-TC : Measured $\mathrm{H}_{\mathrm{ms}}$ versus $\mathrm{H}_{\mathrm{ms}}$ calculated by Unibest-TC : Measured $\mathrm{H}_{\mathrm{ms}}$ versus $\mathrm{H}_{\mathrm{ms}}$ calculated by Unibest-TC : Measured $\mathrm{H}_{\mathrm{rms}}$ versus $\mathrm{H}_{\mathrm{rms}}$ calculated by Unibest-TC : Measured $\mathrm{H}_{\mathrm{rms}}$ versus $\mathrm{H}_{\mathrm{rms}}$ calculated by Unibest-TC : Measured $\mathrm{H}_{\mathrm{ms}}$ versus $\mathrm{H}_{\mathrm{ms}}$ calculated by Unibest-TC : Measured $\mathrm{H}_{\mathrm{ms}}$ versus $\mathrm{H}_{\mathrm{ms}}$ calculated by Unibest-TC :

Measured profile versus profile calculated by Unibest-TC: Experiment A without breakwater Measured profile versus profile calculated by Unibest-TC: Experiment B without breakwater Measured profile versus profile calculated by Unibest-TC: Experiment $\mathrm{C}$ without breakwater Measured profile versus profile calculated by Unibest-TC: Experiment D without breakwater Measured profile versus profile calculated by Unibest-TC: Experiment E without breakwater

Measured profile versus profile calculated by Unibest-TC: Experiment A with breakwater Measured profile versus profile calculated by Unibest-TC: Experiment B with breakwater Measured profile versus profile calculated by Unibest-TC: Experiment $\mathrm{C}$ with breakwater Measured profile versus profile calculated by Unibest-TC: Experiment D with breakwater

Measured profile versus profile calculated by Unibest-TC: Experiment E with breakwater
Experiment B without breakwater Experiment $\mathrm{C}$ without breakwater Experiment D without breakwater Experiment E without breakwater (x) Experiment $C$ with breakwater Experiment D with breakwater Experiment E with breakwater 


\section{Profile measurements}

Experiments without breakwater

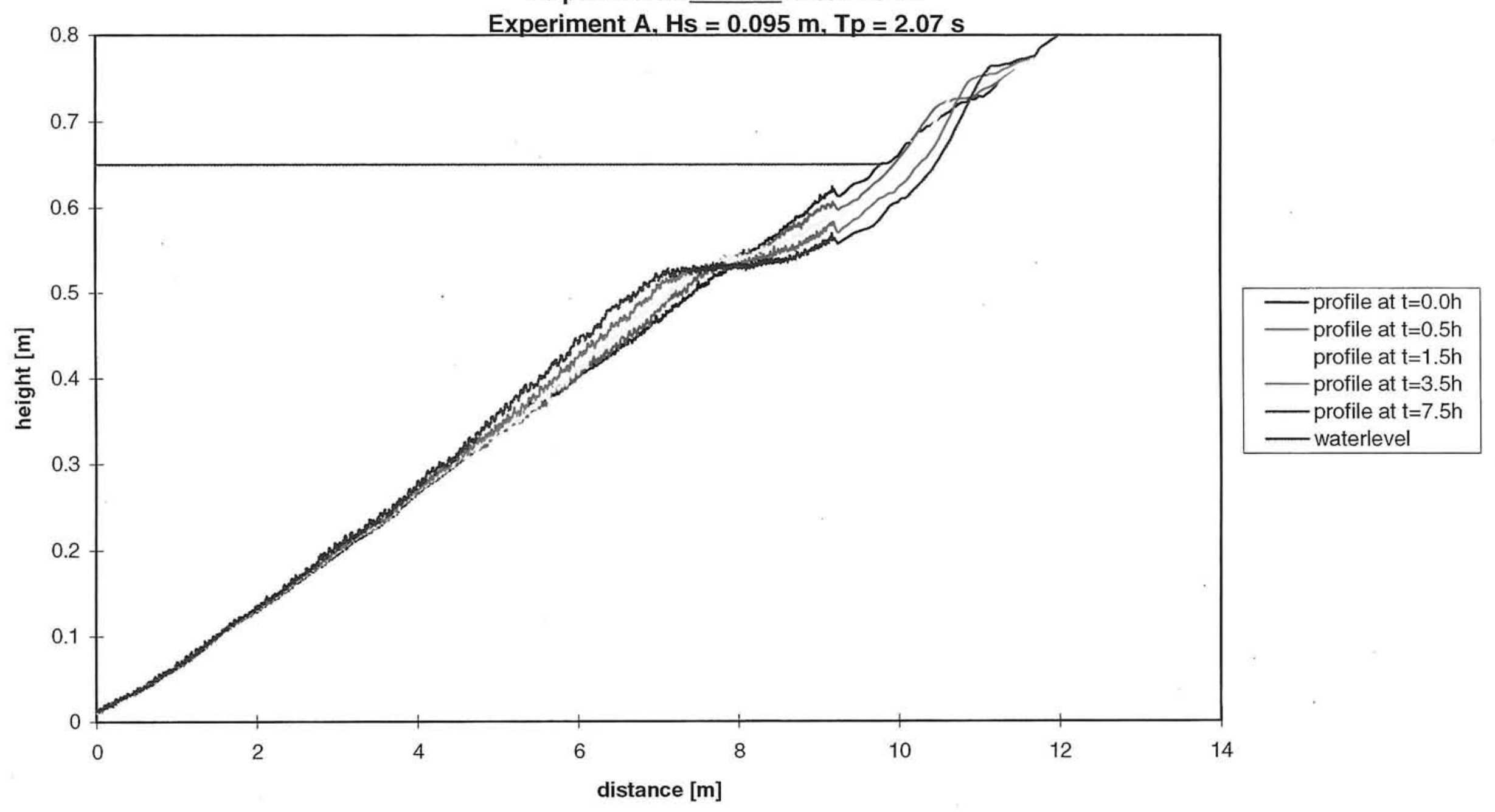

Graph 1.1 
Profile measurements

Experiments without breakwater

Experiment $\mathrm{B}, \mathrm{Hs}=0.098 \mathrm{~m}, \mathrm{Tp}=1.55 \mathrm{~s}$

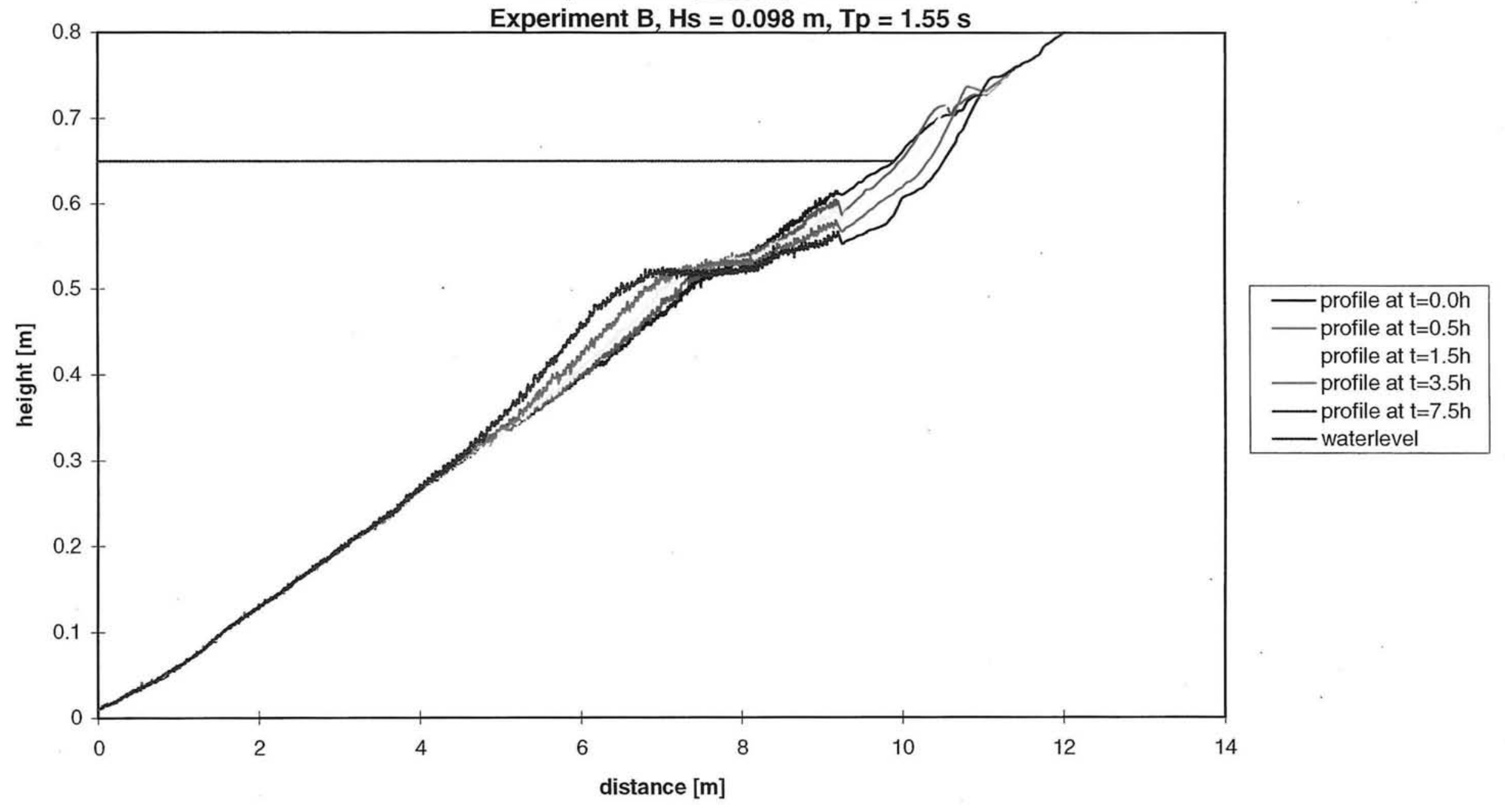

Graph 1.2 


\section{Profile measurements}

Experiments without breakwater

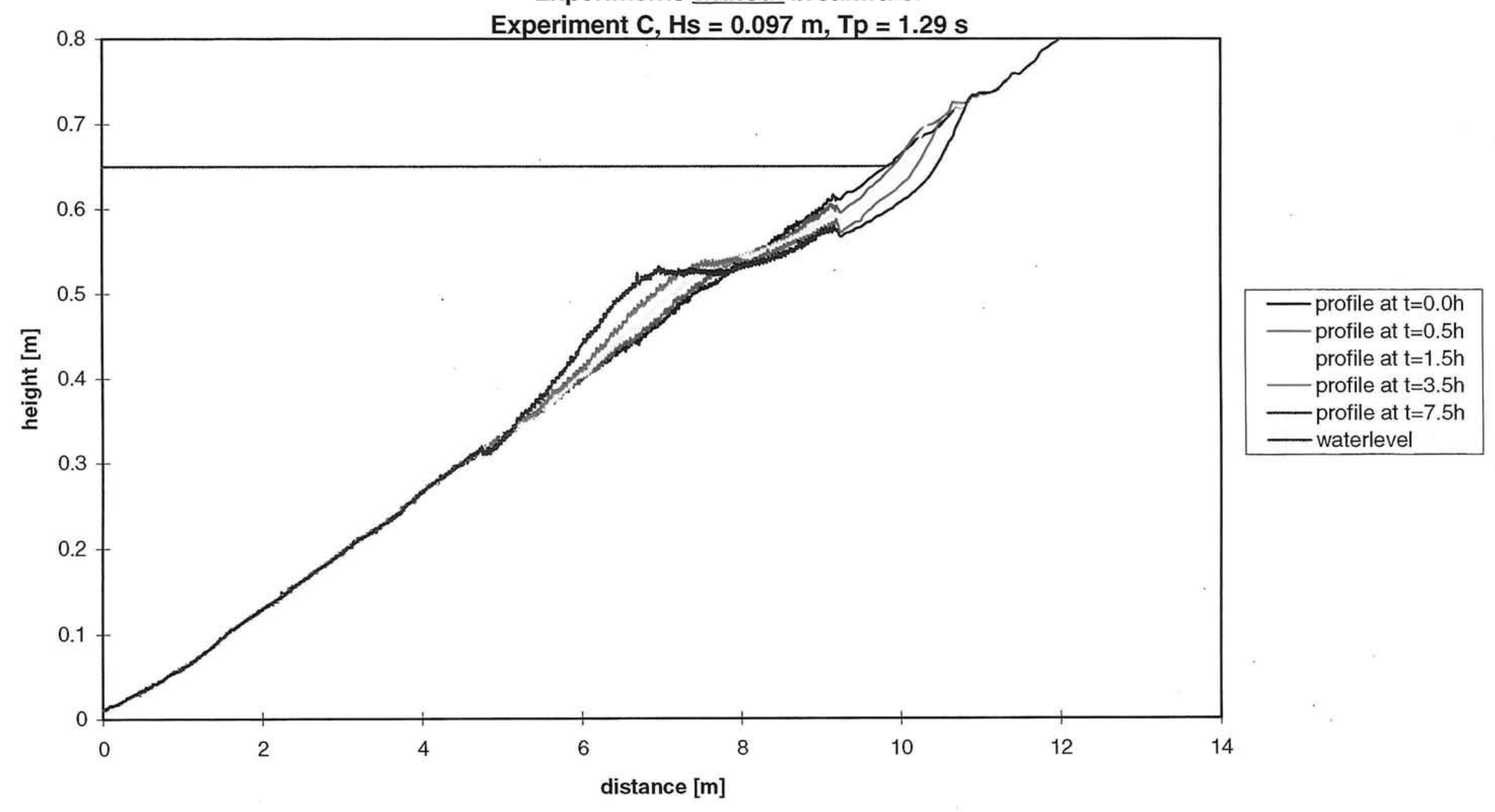

Graph 1.3 


\section{Profile measurements}

Experiments without breakwater

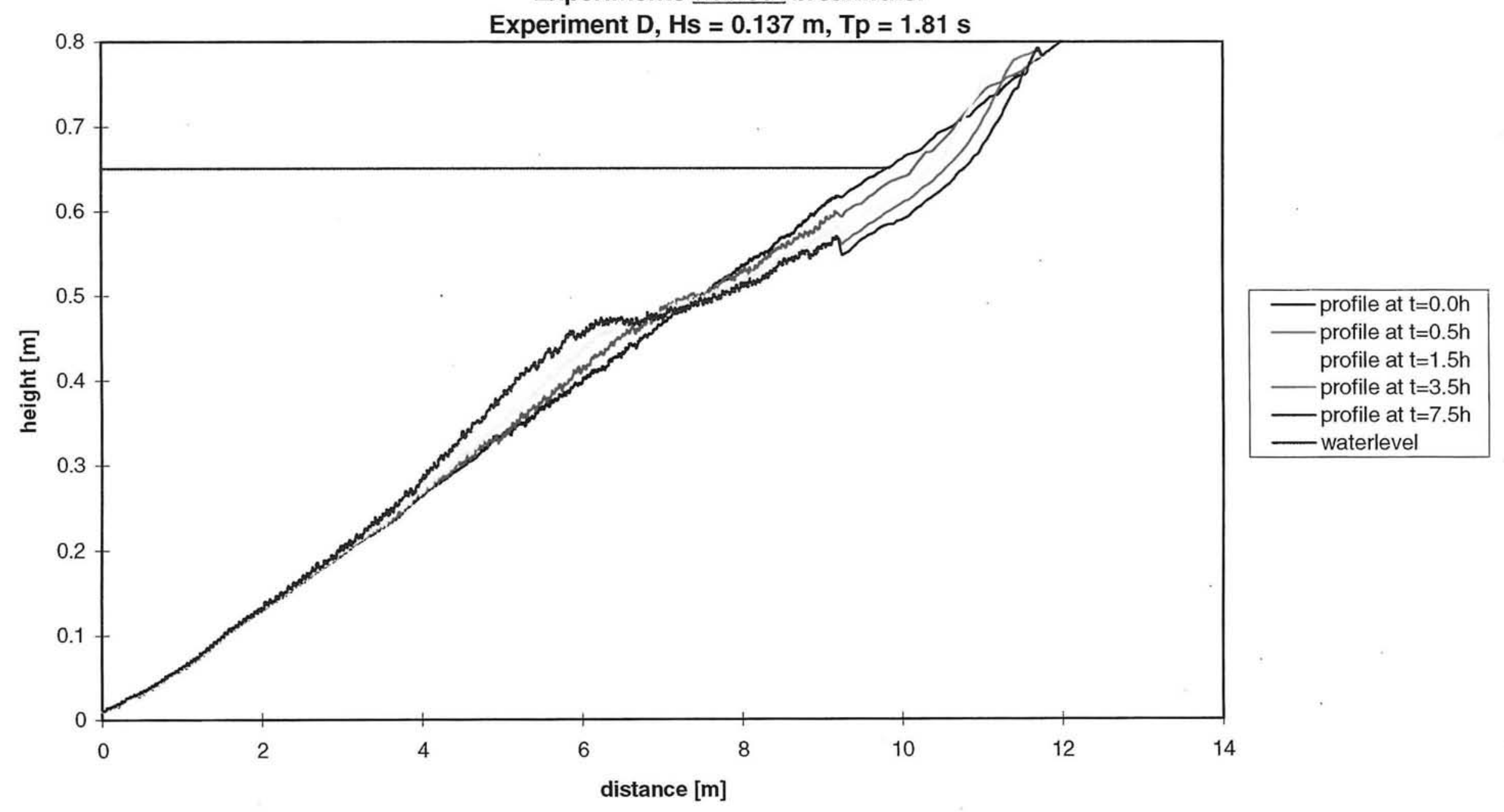

Graph 1.4 


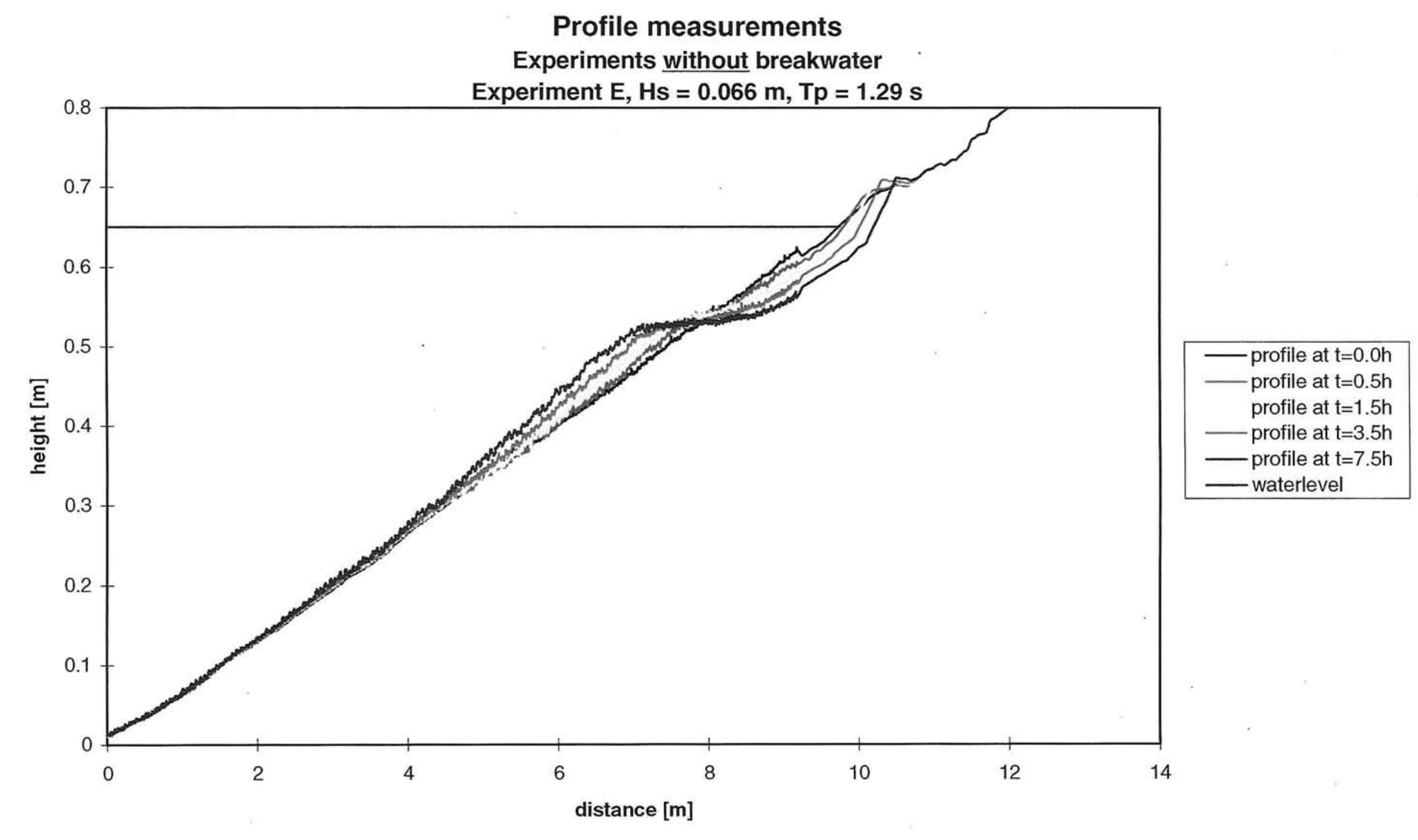

Graph 1.5 


\section{Profile measurements}

Experiments without breakwater

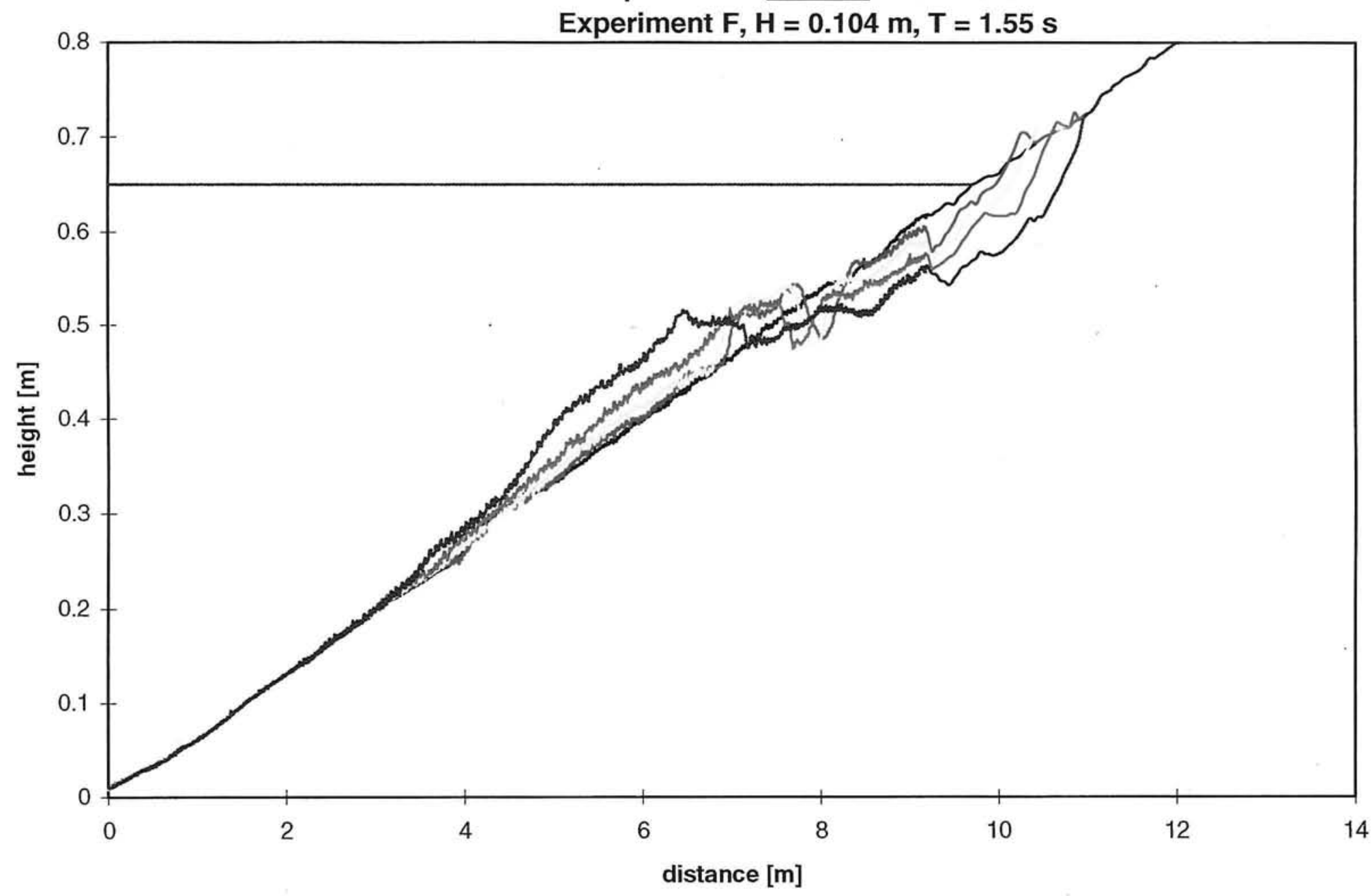

- profile at $\mathrm{t}=0.0 \mathrm{~h}$

- profile at $\mathrm{t}=0.5 \mathrm{~h}$ profile at $t=1.5 \mathrm{~h}$

- profile at $\mathrm{t}=3.5 \mathrm{~h}$

- profile at $\mathrm{t}=7.5 \mathrm{~h}$

- waterlevel

Graph 1.6 


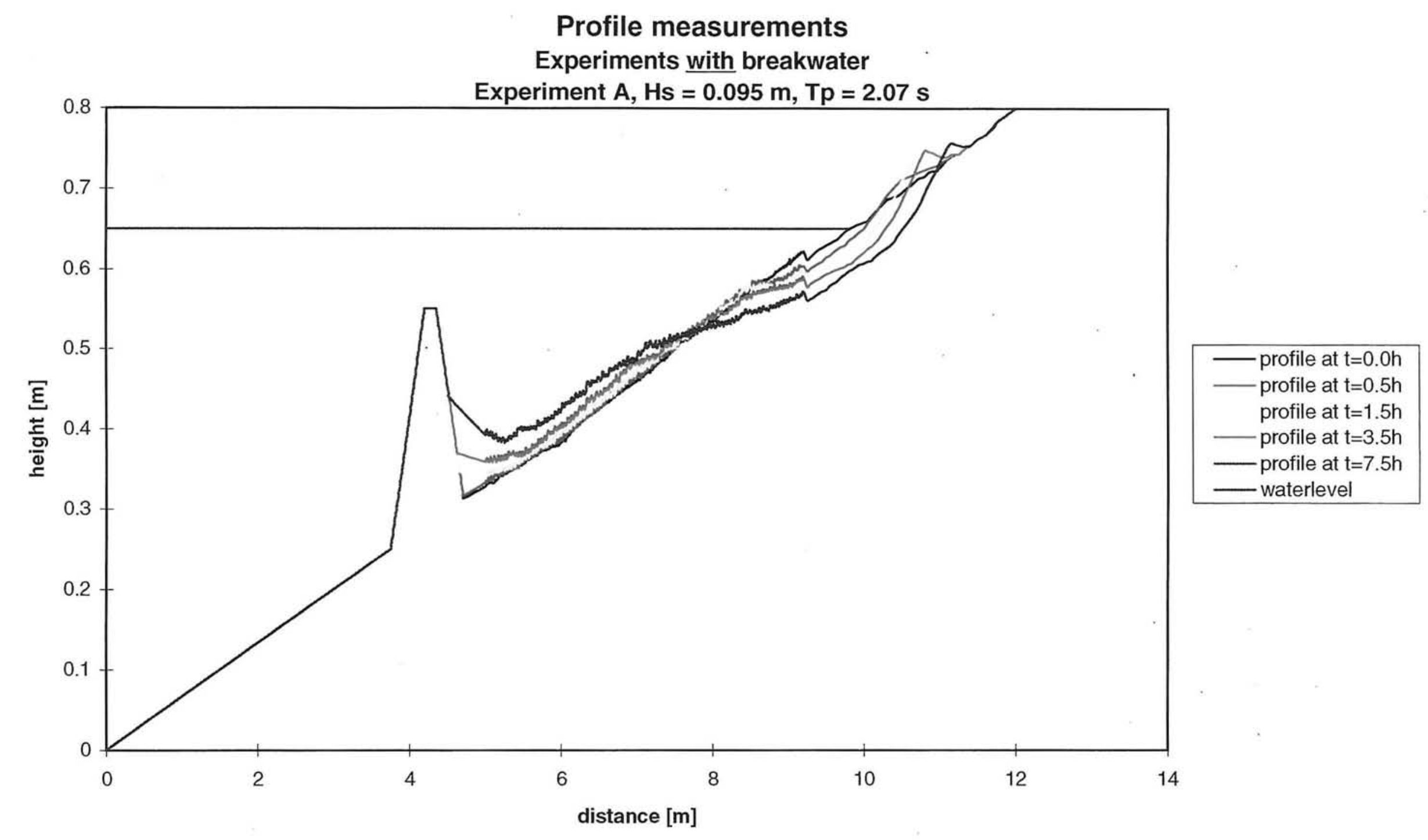

Graph 1.7 


\section{Profile measurements}

Experiments with breakwater

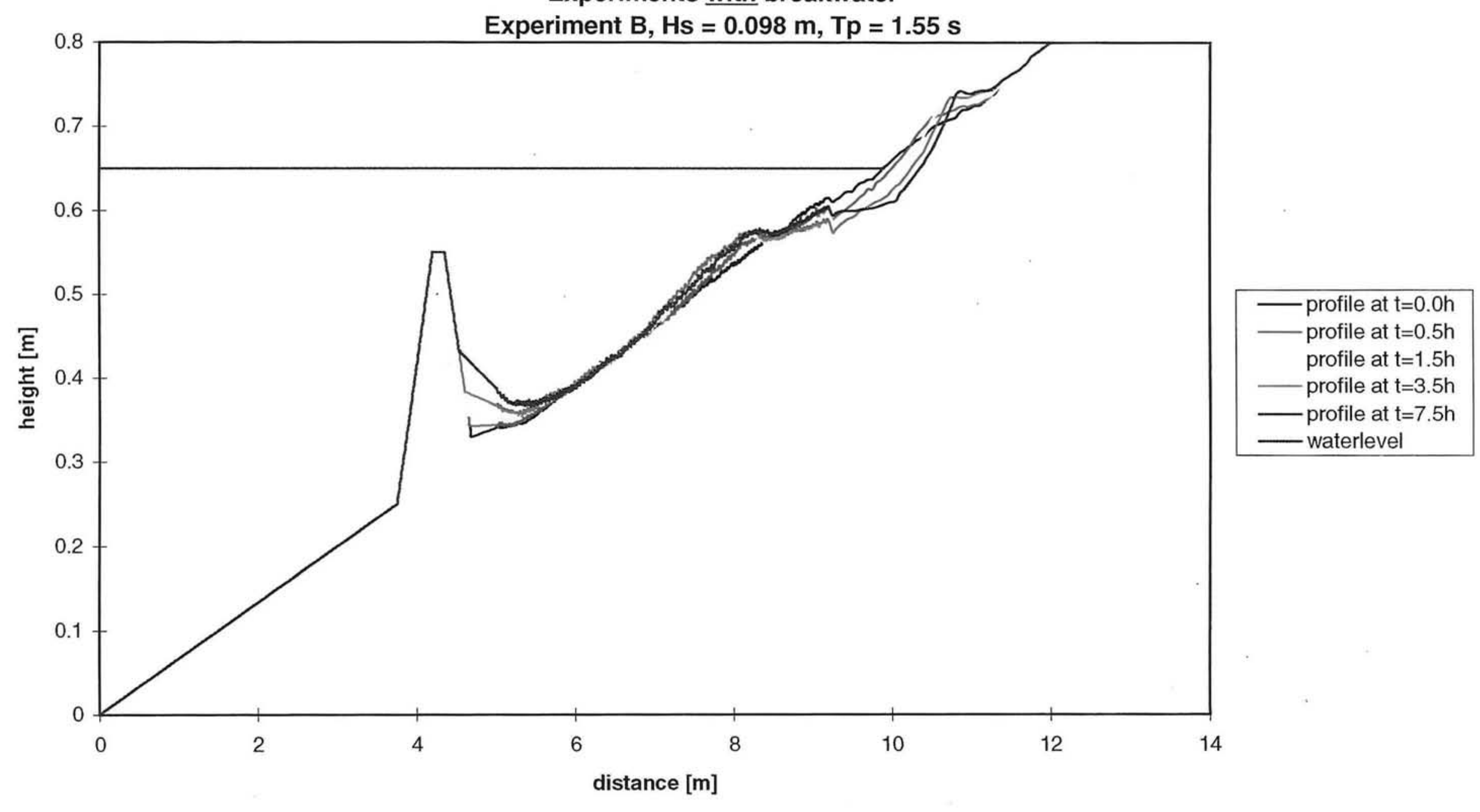

Graph 1.8 


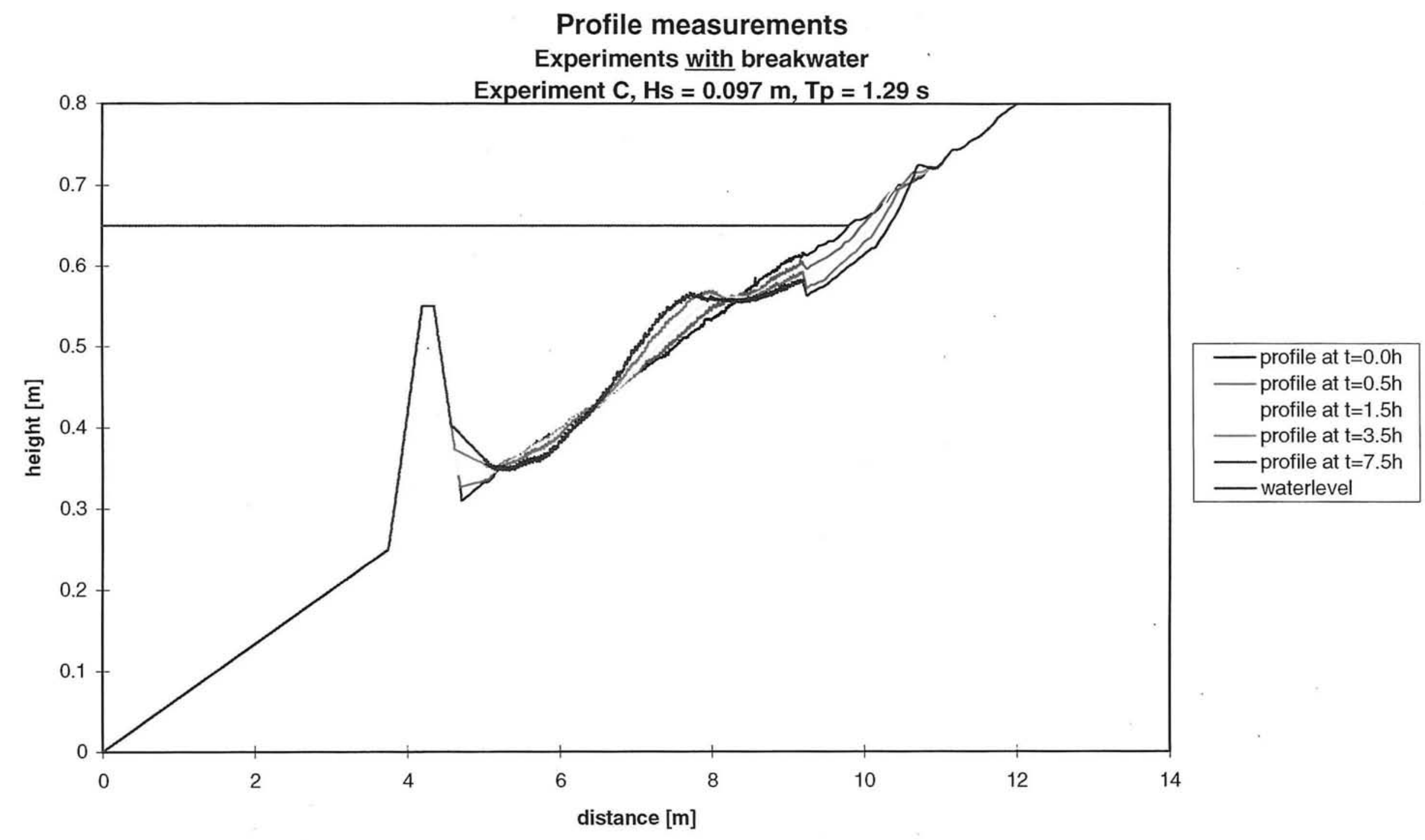

Graph 1.9 


\section{Profile measurements}

Experiments with breakwater

Experiment $\mathrm{D}, \mathrm{Hs}=0.137 \mathrm{~m}, \mathrm{Tp}=1.81 \mathrm{~s}$

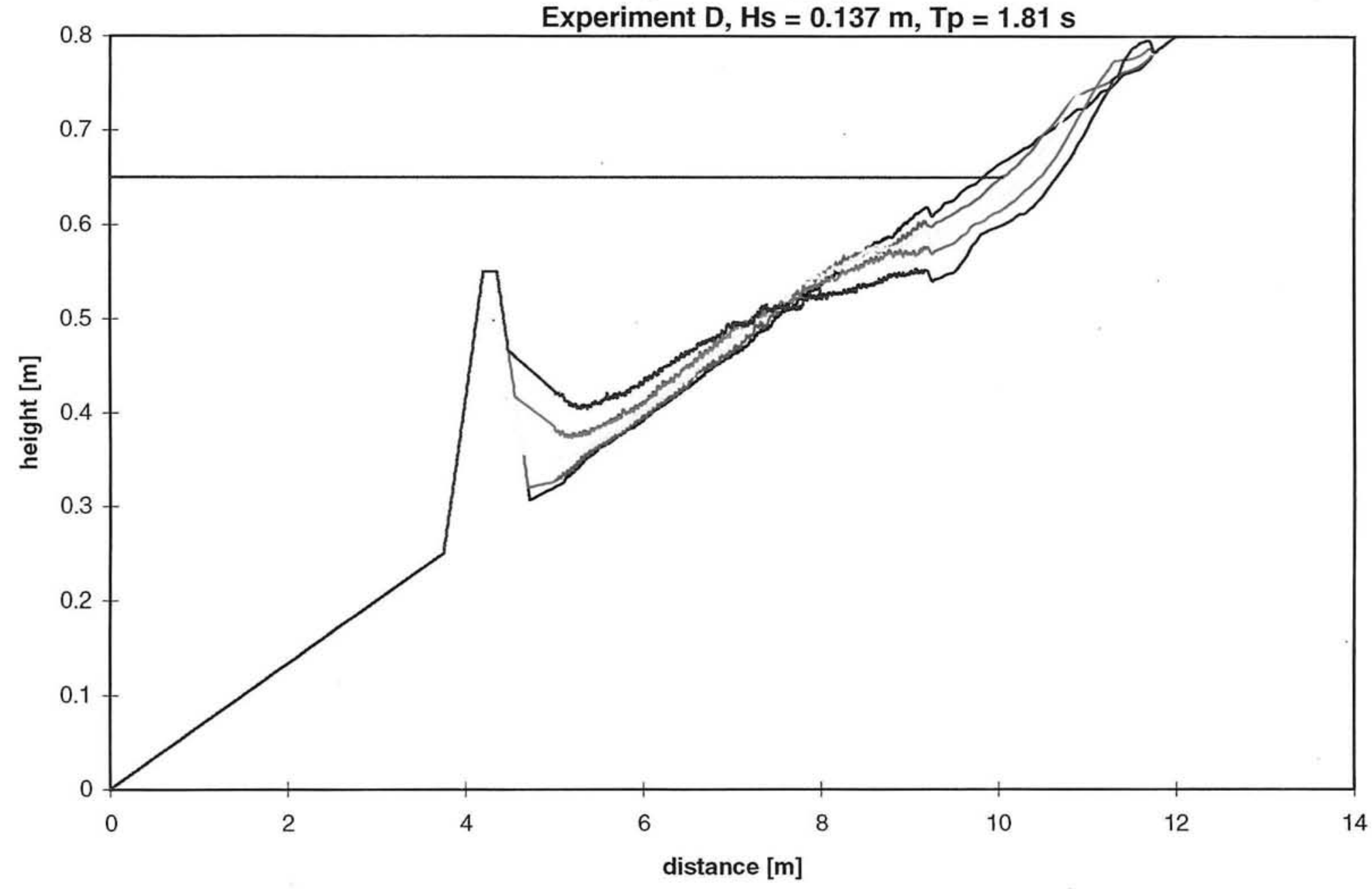
- profile at $\mathrm{t}=0.0 \mathrm{~h}$
- profile at $\mathrm{t}=0.5 \mathrm{~h}$ profile at $t=1.5 \mathrm{~h}$
- profile at $\mathrm{t}=3.5 \mathrm{~h}$
- profile at $\mathrm{t}=7.5 \mathrm{~h}$
- waterlevel

Graph 1.10 
Profile measurements

Experiments with breakwater

Experiment $\mathrm{E}, \mathrm{Hs}=0.066 \mathrm{~m}, \mathrm{Tp}=1.29 \mathrm{~s}$

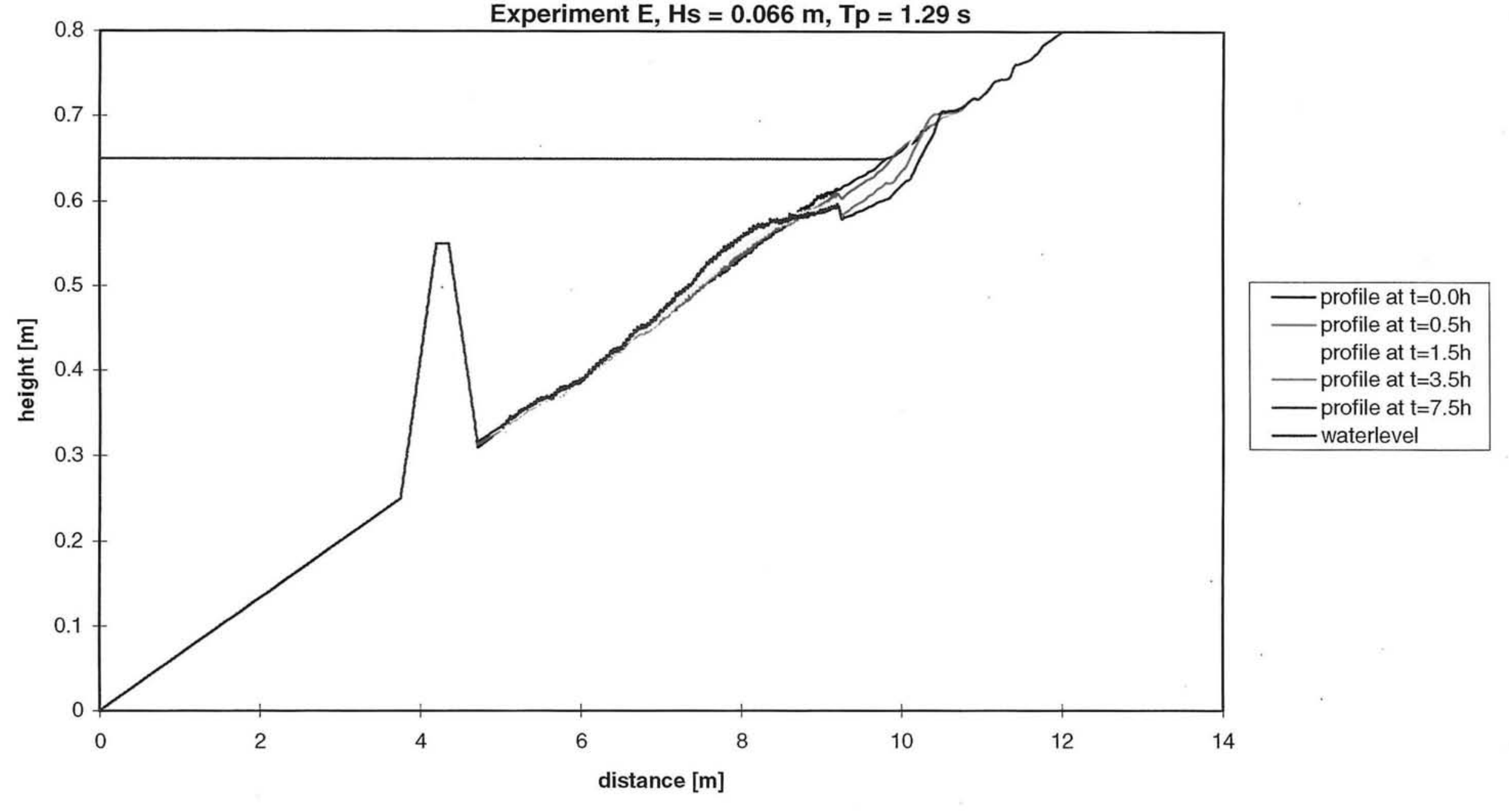

Graph 1.11 
Profile measurements

Experiments with breakwater

Experiment $\mathrm{F}, \mathrm{H}=\mathbf{0 . 1 0 4} \mathrm{m}, \mathrm{T}=1.55 \mathrm{~s}$

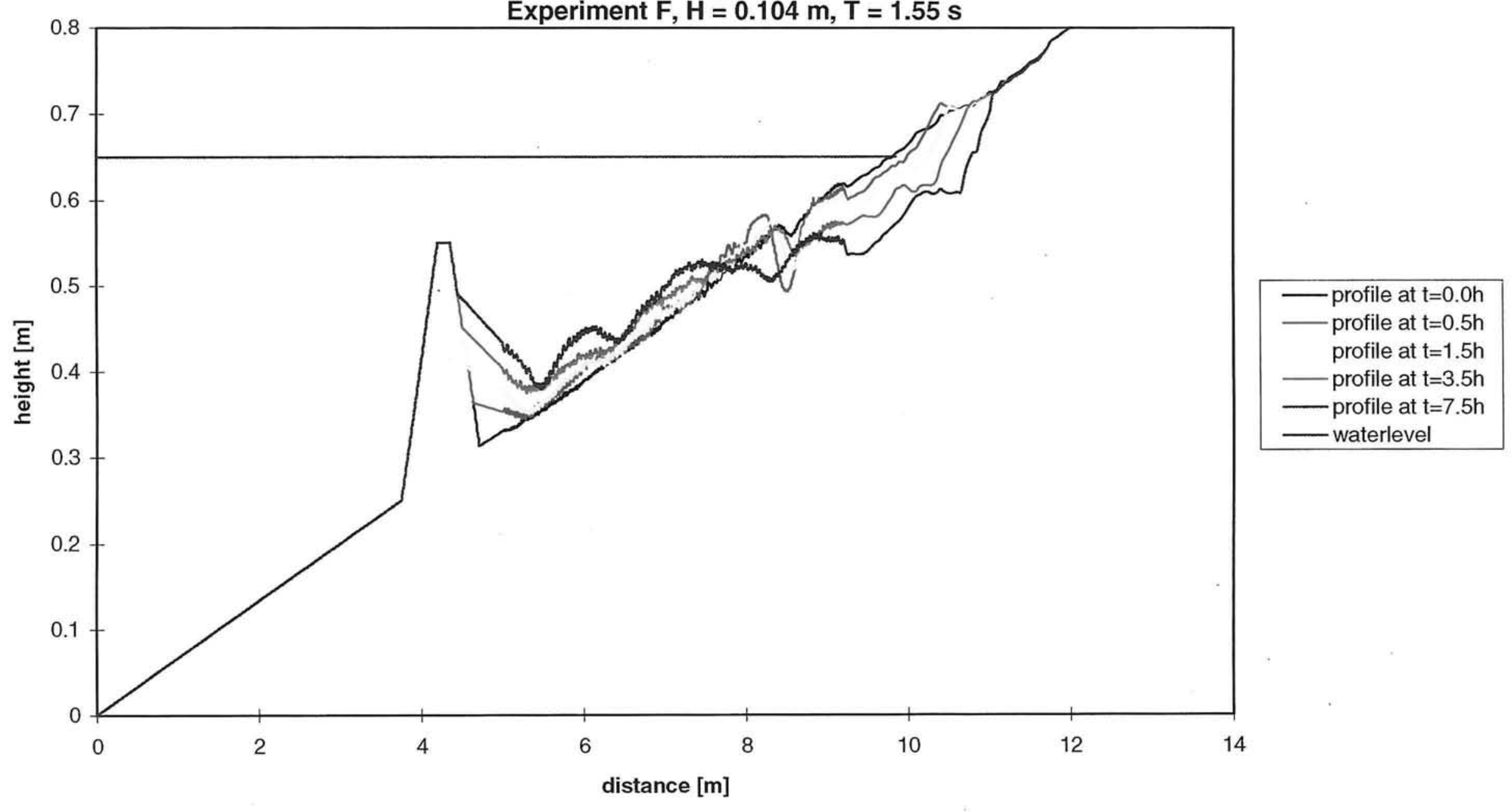

Graph 1.12 


\section{Profile measurements}

Experiments with and without breakwater

Experiment $\mathrm{A}$ at $\mathrm{t}=7.5 \mathrm{~h}, \mathrm{Hs}=0.095 \mathrm{~m}, \mathrm{Tp}=2.07 \mathrm{~s}$

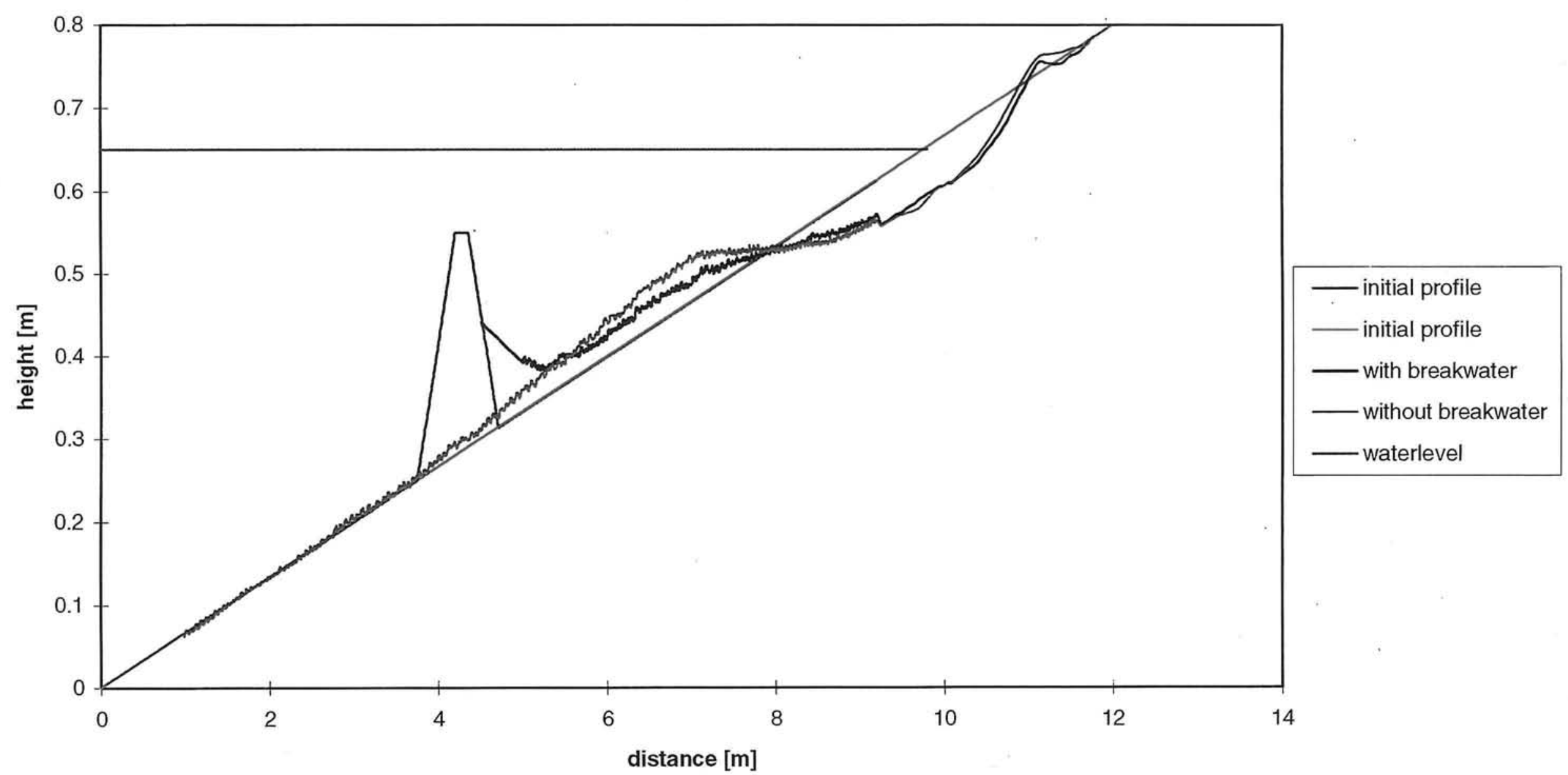

Graph 1.13 
Profile measurements

Experiments with and without breakwater

Experiment $\mathrm{B}$ at $\mathrm{t}=7.5 \mathrm{~h}, \mathrm{Hs}=0.098 \mathrm{~m}, \mathrm{Tp}=1.55 \mathrm{~s}$

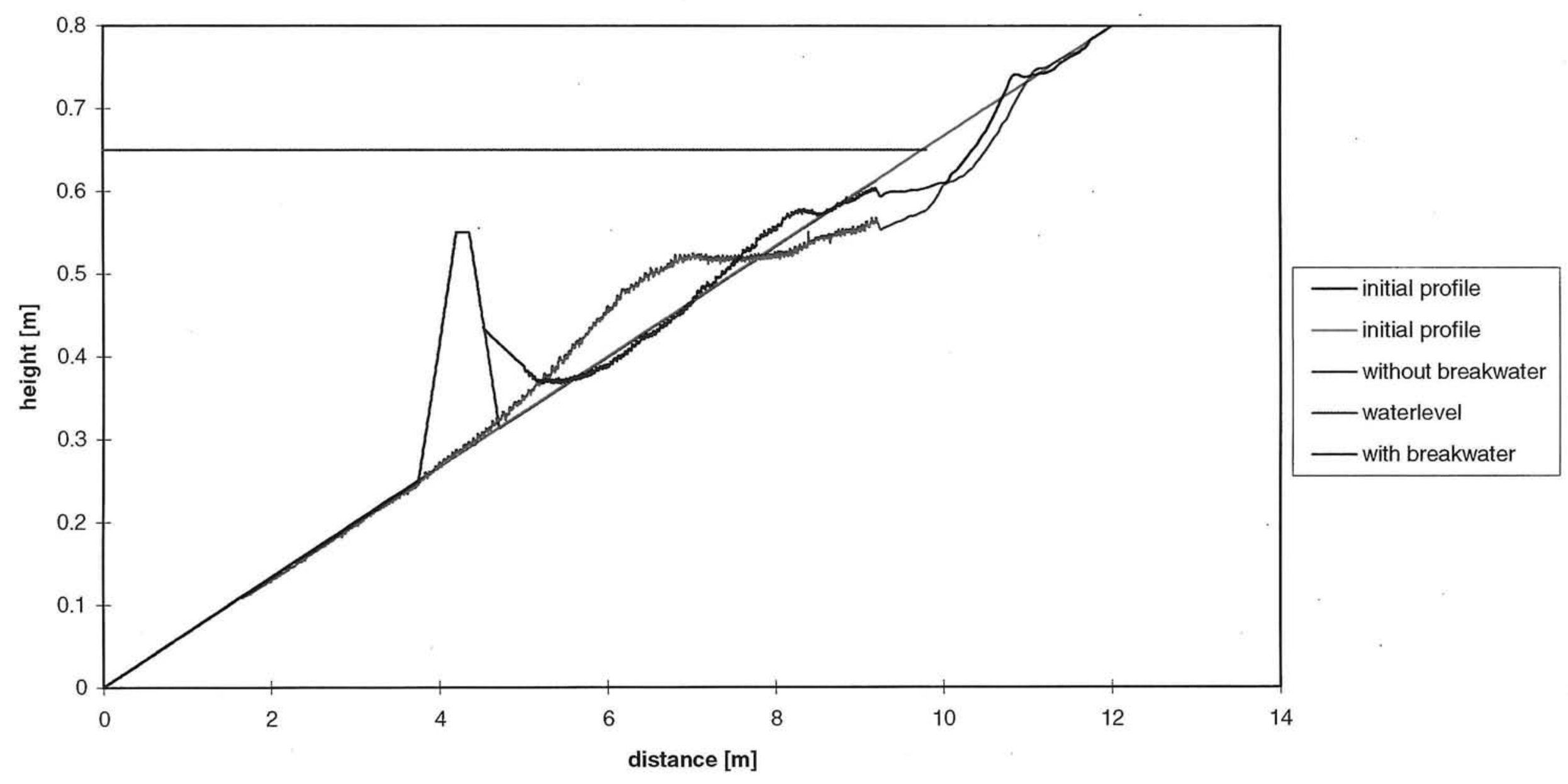

Graph 1.14 
Profile measurements

Experiments with and without breakwater

Experiment $\mathrm{C}$ at $\mathrm{t}=7.5 \mathrm{~h}, \mathrm{Hs}=0.097 \mathrm{~m}, \mathrm{Tp}=1.29 \mathrm{~s}$

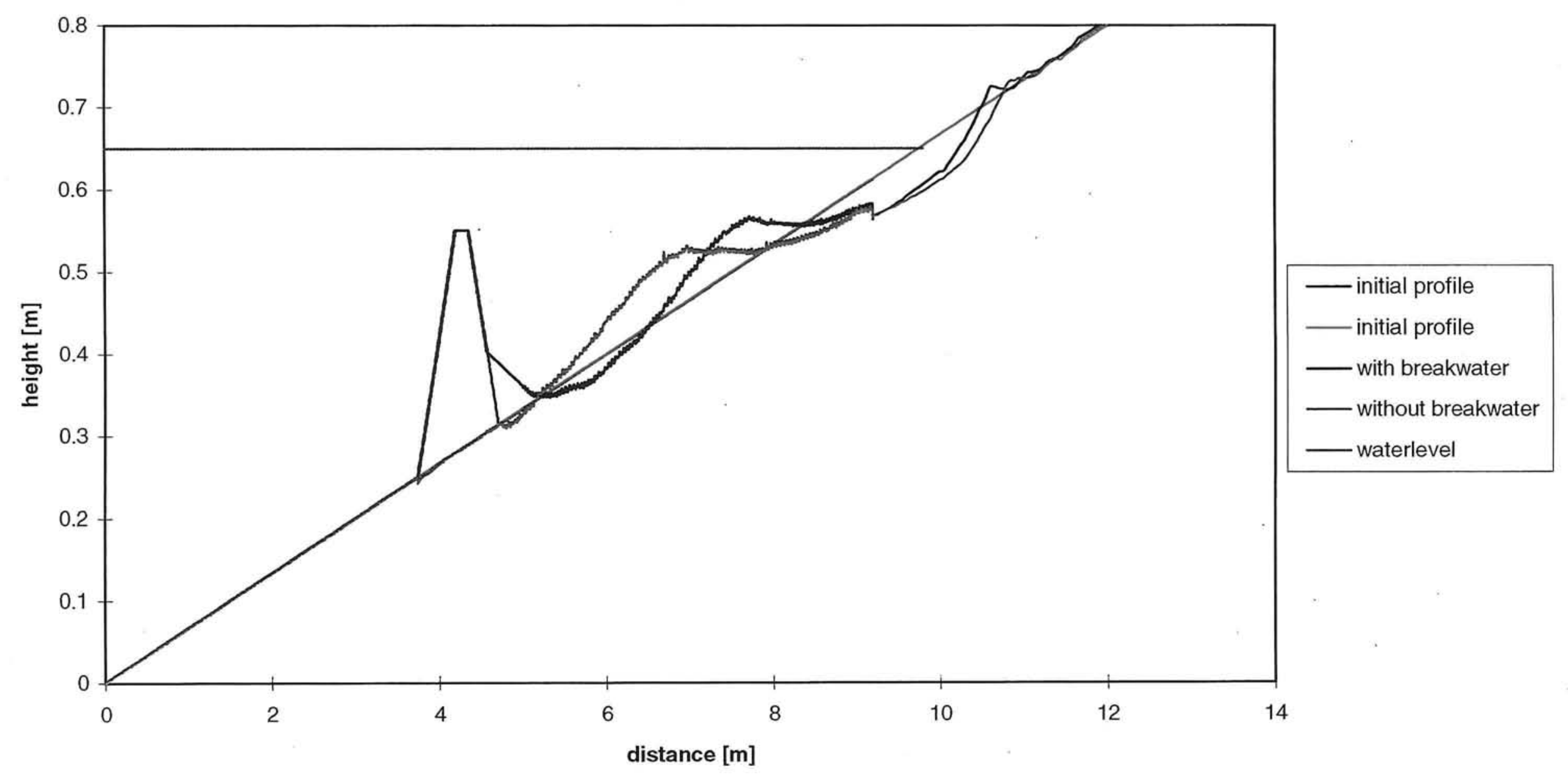

Graph 1.15 
Profile measurements

Experiments with and without breakwater

Experiment $\mathrm{D}$ at $\mathrm{t}=7.5 \mathrm{~h}, \mathrm{Hs}=0.137 \mathrm{~m}, \mathrm{Tp}=1.81 \mathrm{~s}$

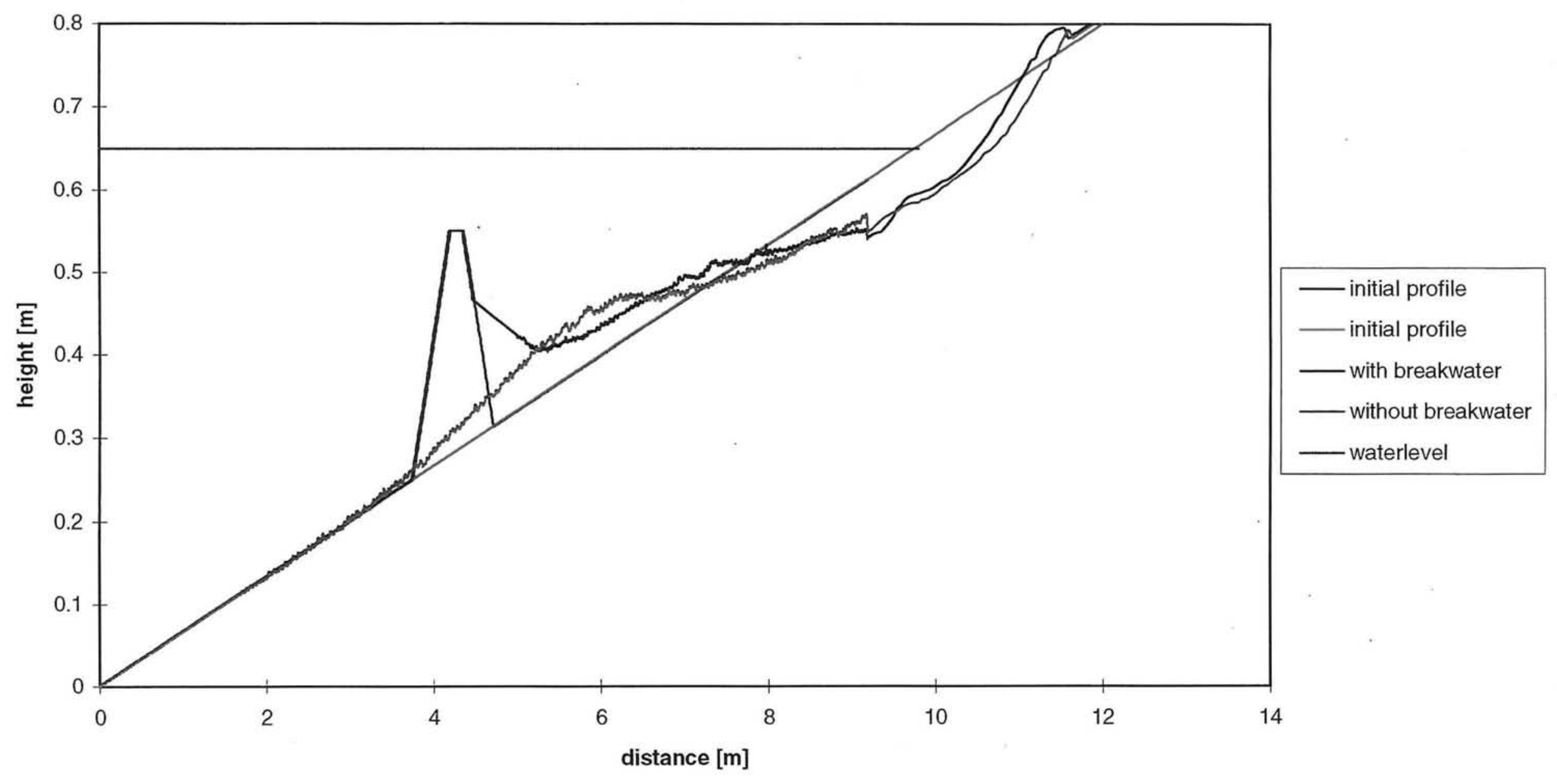

Graph 1.16 


\section{Profile measurements}

Experiments with and without breakwater

Experiment $\mathrm{E}$ at $\mathrm{t}=7.5 \mathrm{~h}, \mathrm{Hs}=0.066 \mathrm{~m}, \mathrm{Tp}=1.29 \mathrm{~s}$

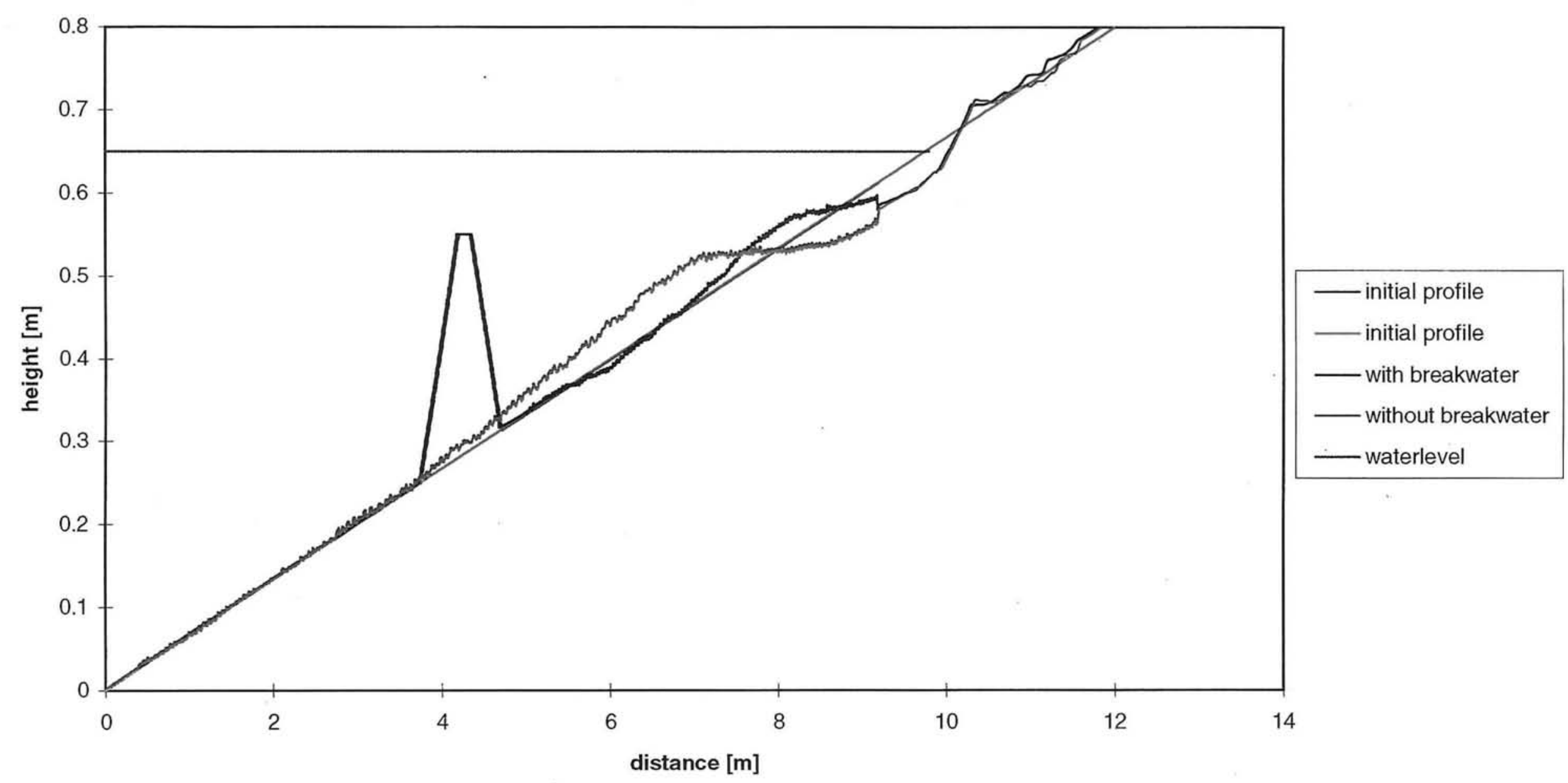

Graph 1.17 


\section{Profile measurements}

Experiments with and without breakwater

Experiment $\mathrm{F}$ at $\mathrm{t}=7.5 \mathrm{~h}, \mathrm{H}=0.104 \mathrm{~m}, \mathrm{~T}=1.55 \mathrm{~s}$

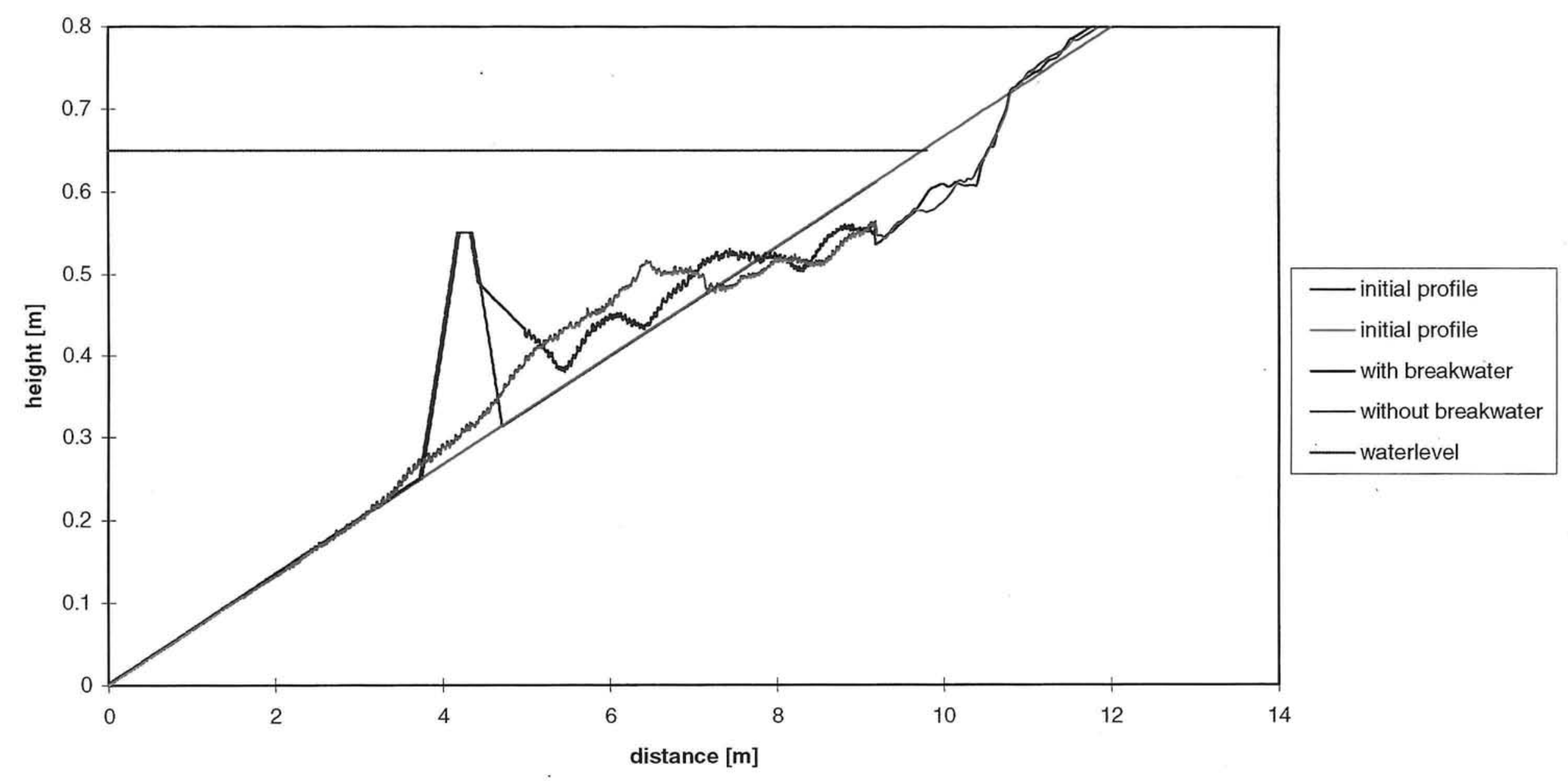

Graph 1.18 


\section{Profile measurements}

Experiments without breakwater

Experiments $\mathrm{A}, \mathrm{B}$ and $\mathrm{C}$ at $\mathrm{t}=1.5 \mathrm{~h}$

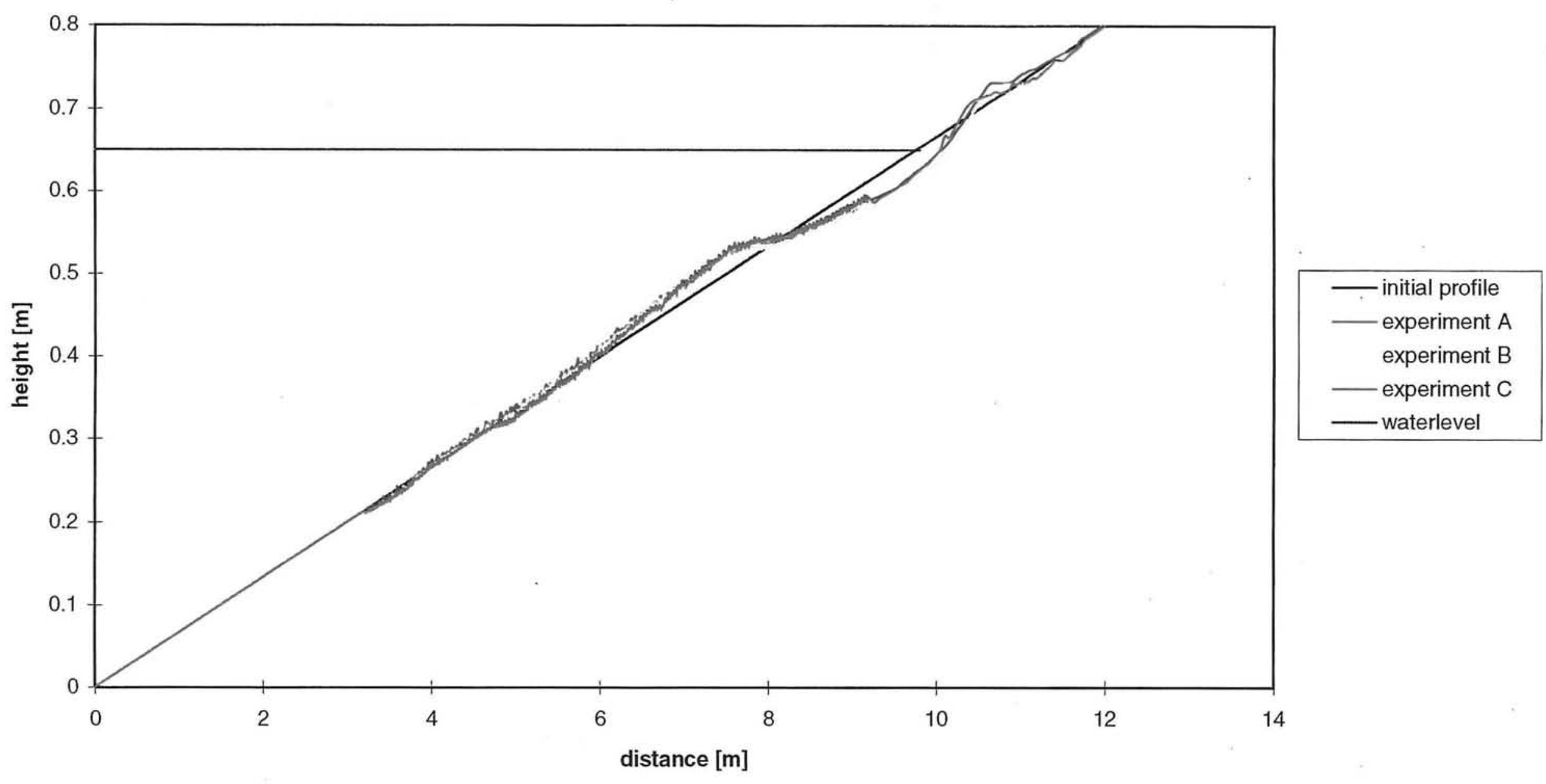

Graph 1.19 
Profile measurements

Experiments without breakwater

Experiments A, B and $\mathrm{C}$ at $\mathrm{t}=7.5 \mathrm{~h}$

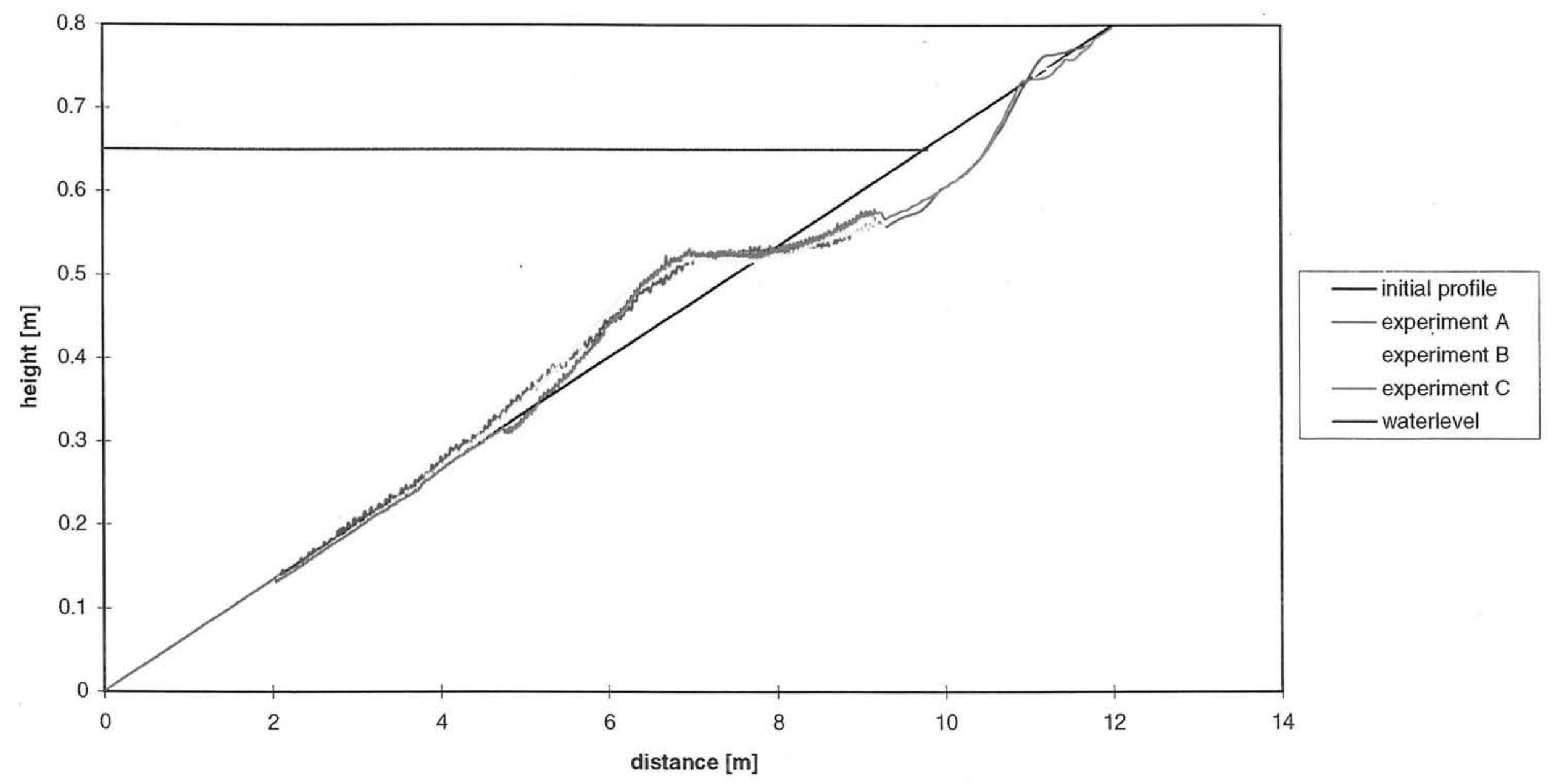

Graph 1.20 


\section{Profile measurements}

Experiments with breakwater

Experiments $\mathrm{A}, \mathrm{B}$ and $\mathrm{C}$ at $\mathrm{t}=1.5 \mathrm{~h}$

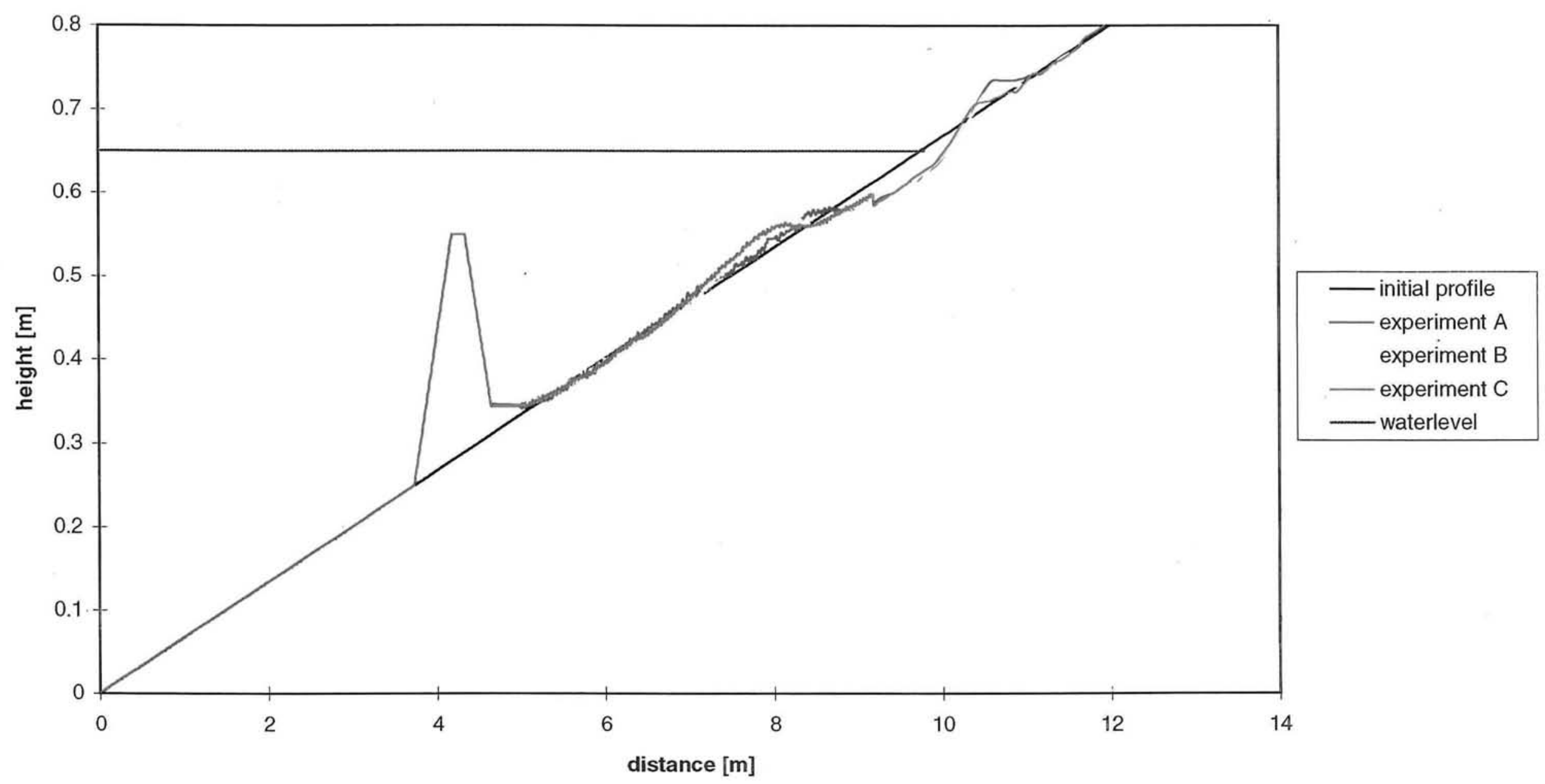

Graph 1.21 
Profile measurements

Experiments with breakwater

Experiments $\mathrm{A}, \mathrm{B}$ and $\mathrm{C}$ at $\mathrm{t}=7.5 \mathrm{~h}$

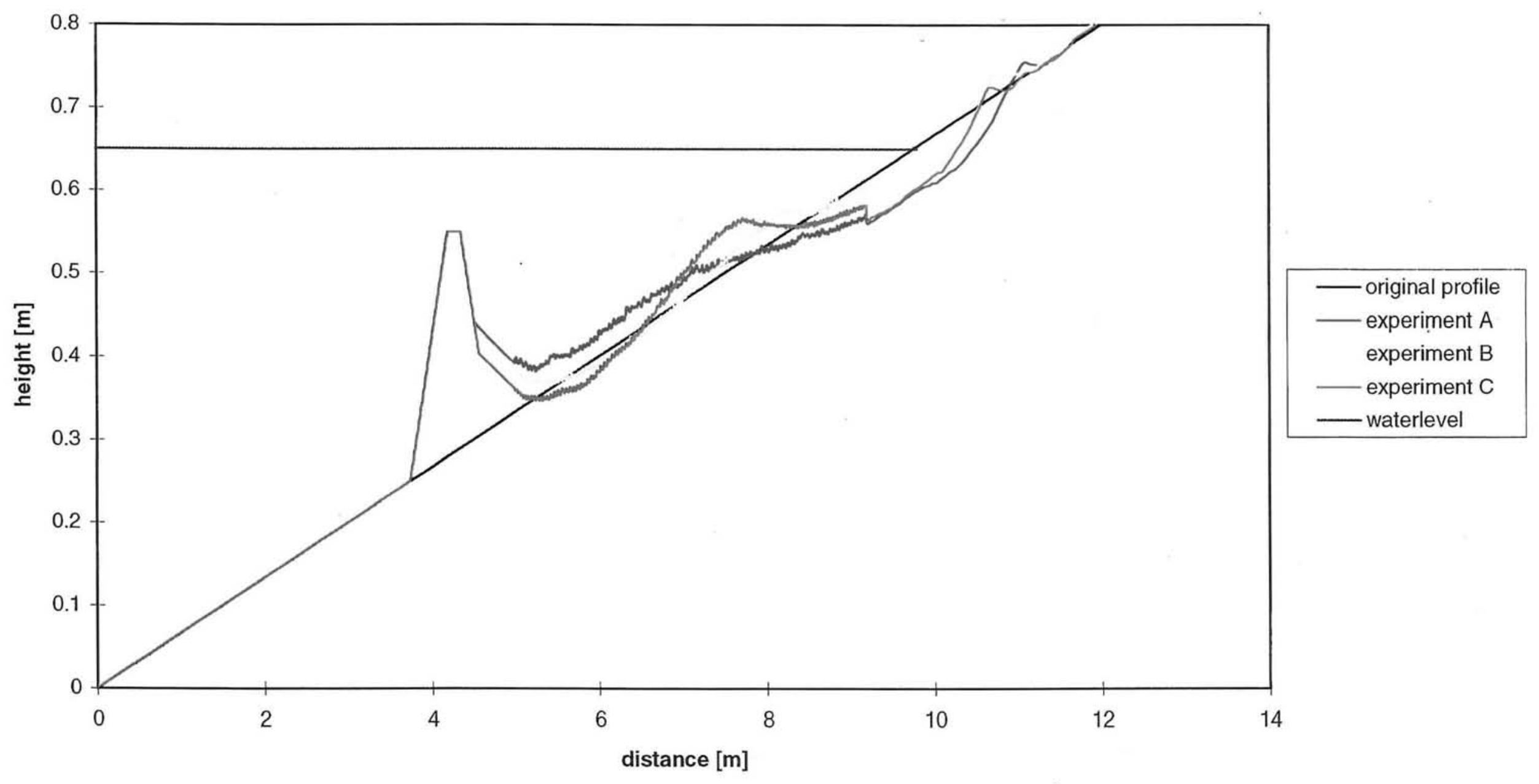

Graph 1.22 


\section{Profile measurements}

Experiments without breakwater

Experiments $\mathrm{B}, \mathrm{D}$ and $\mathrm{E}$ at $\mathrm{t}=1.5 \mathrm{~h}$

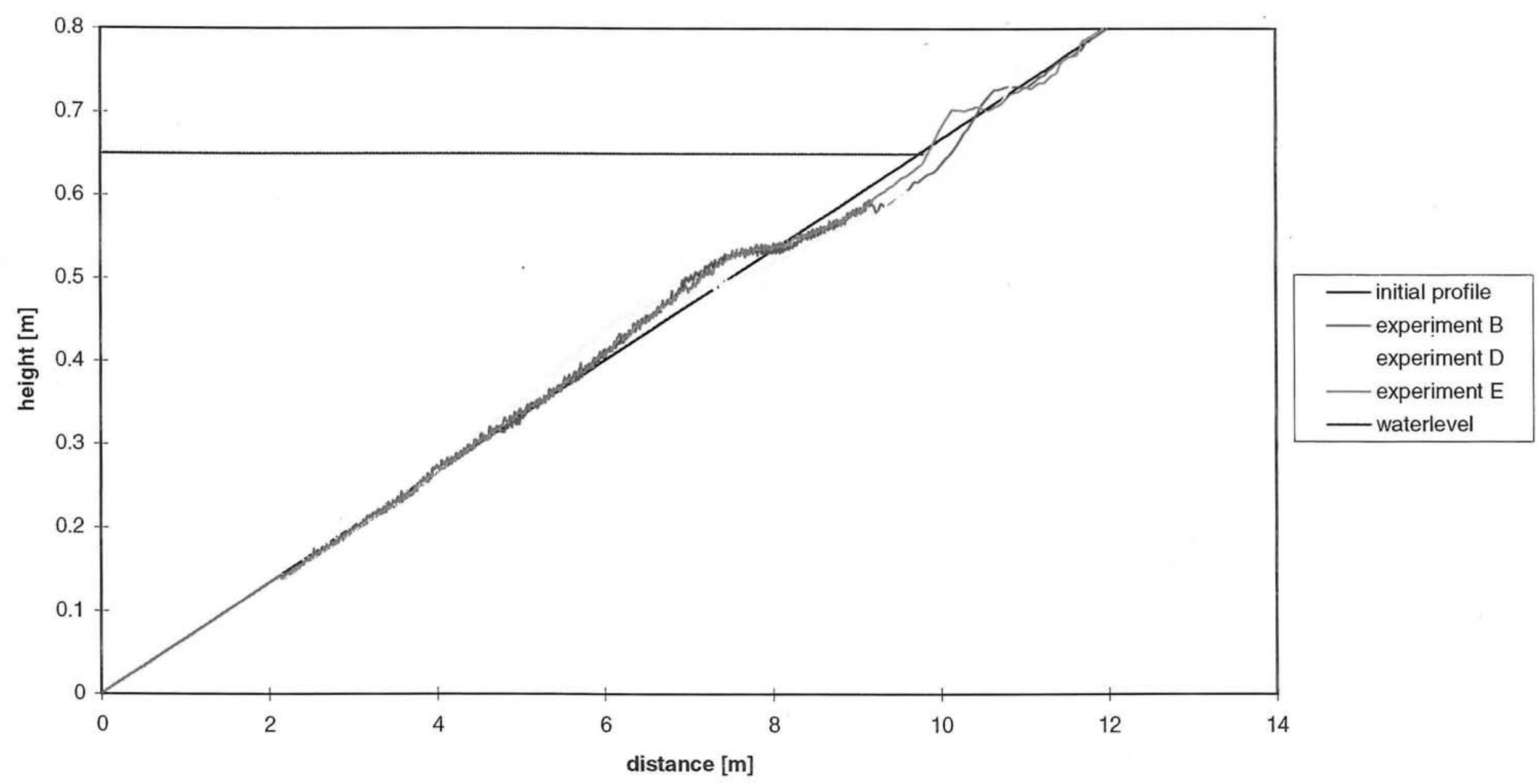

Graph 1.23 
Profile measurements

Experiments without breakwater

Experiments $\mathrm{B}, \mathrm{D}$ and $\mathrm{E}$ at $\mathrm{t}=7.5 \mathrm{~h}$

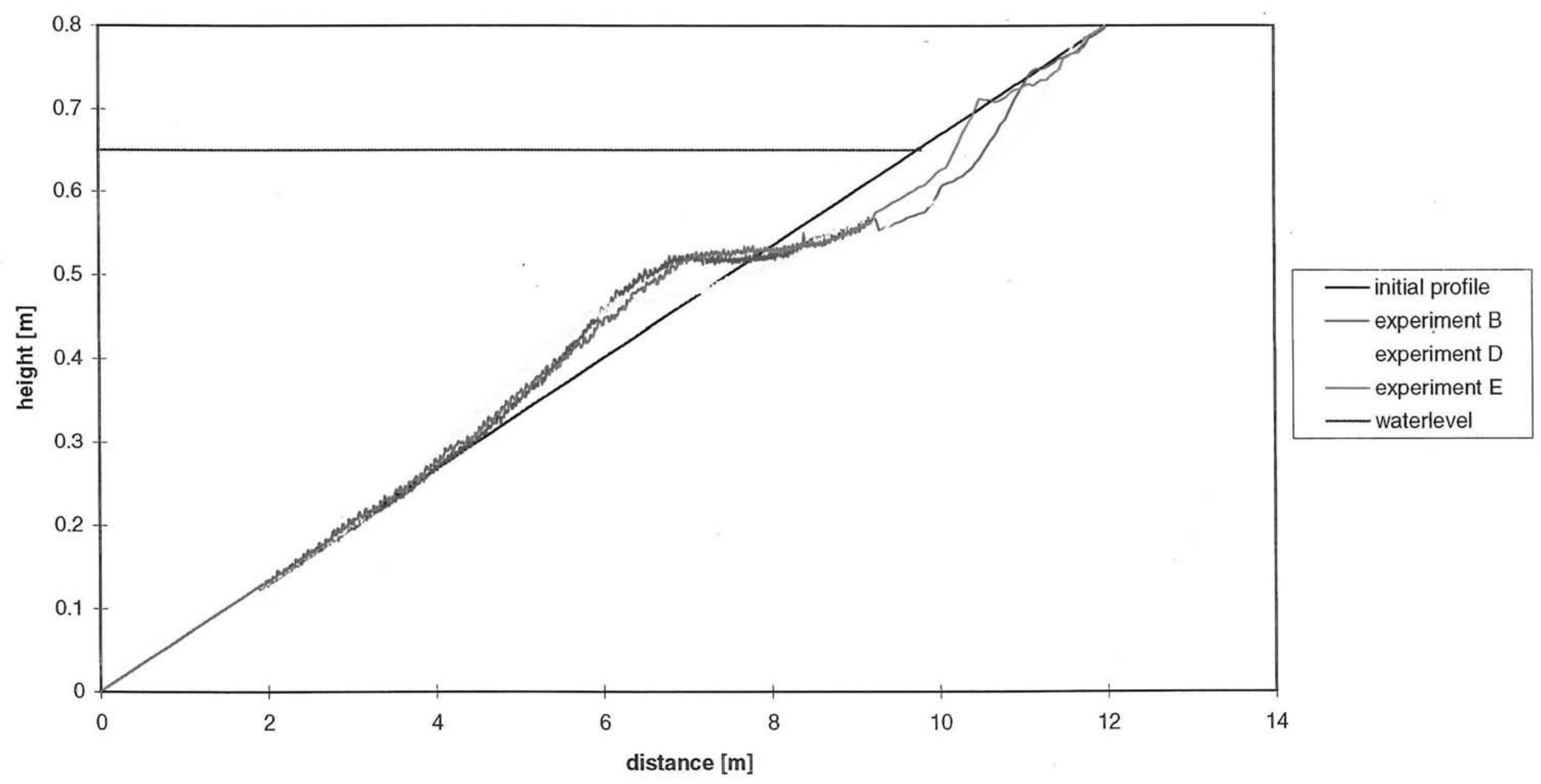

Graph 1.24 


\section{Profile measurements}

Experiments with breakwater

Experiments $\mathrm{B}, \mathrm{D}$ and $\mathrm{E}$ at $\mathrm{t}=1.5 \mathrm{~h}$

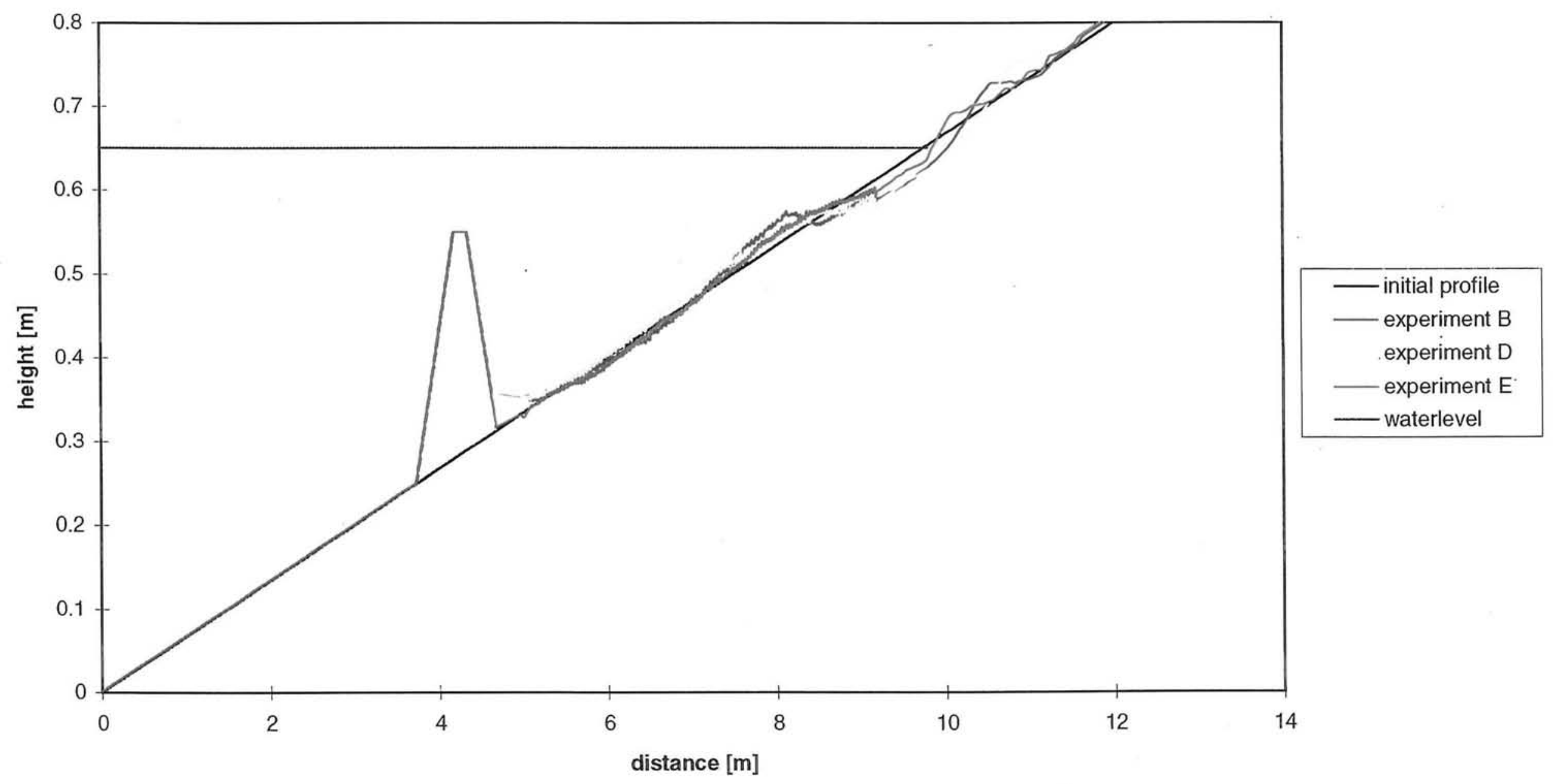

Graph 1.25 
Profile measurements

Experiments with breakwater

Experiments B, D and $\mathrm{E}$ at $\mathrm{t}=7.5 \mathrm{~h}$

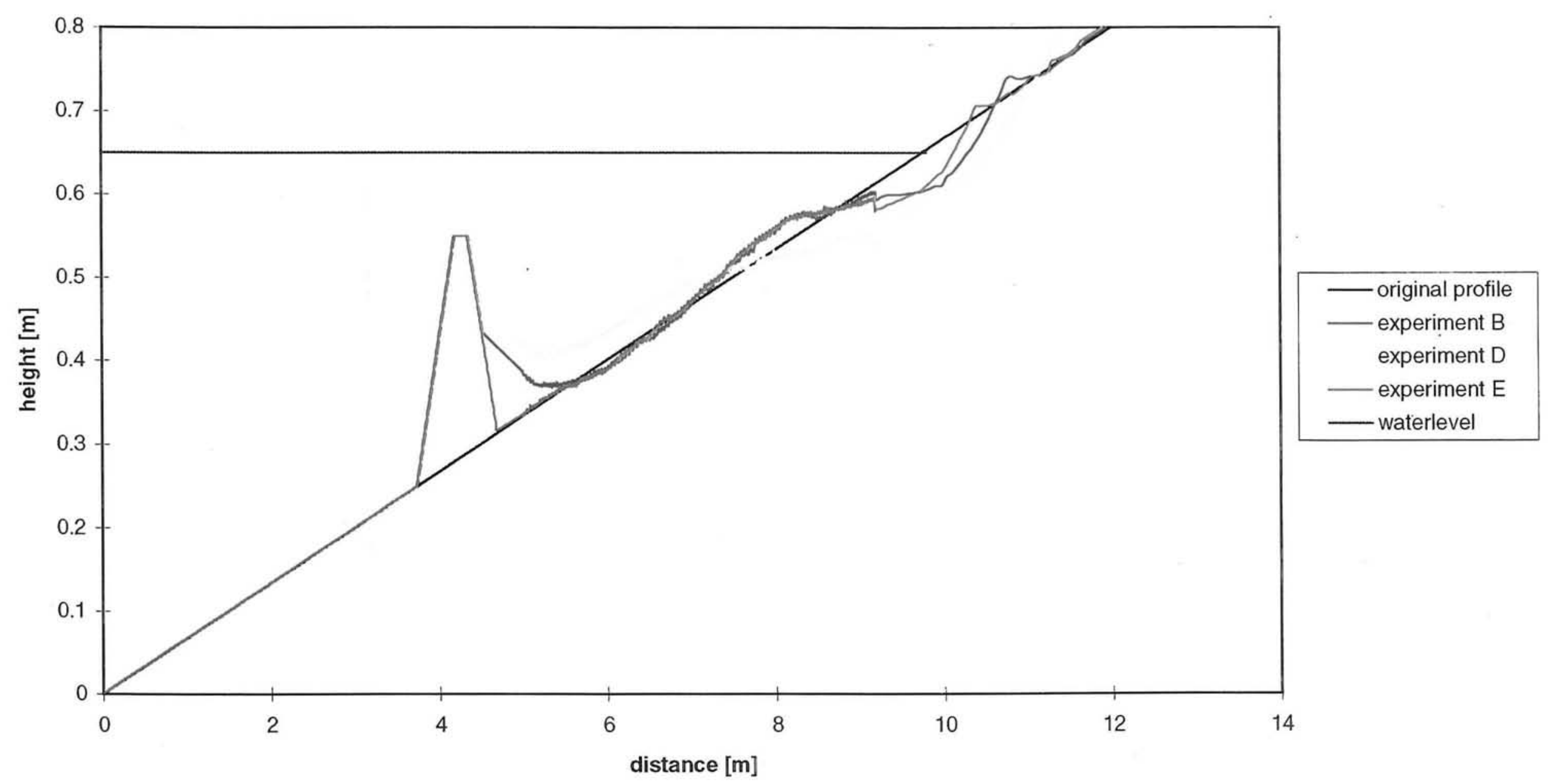

Graph 1.26 


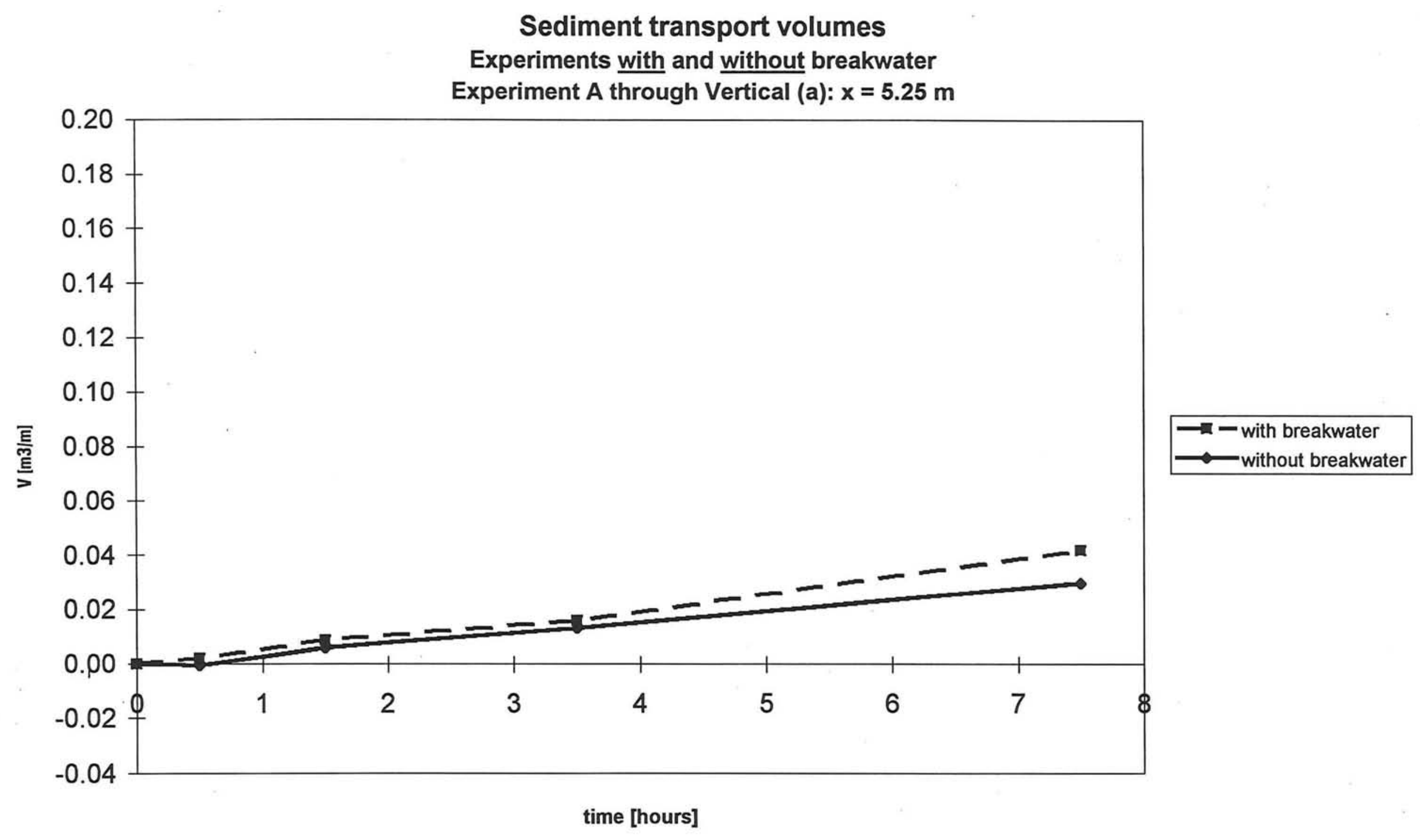

Graph 1.27 


\section{Sediment transport volumes}

Experiments with and without breakwater

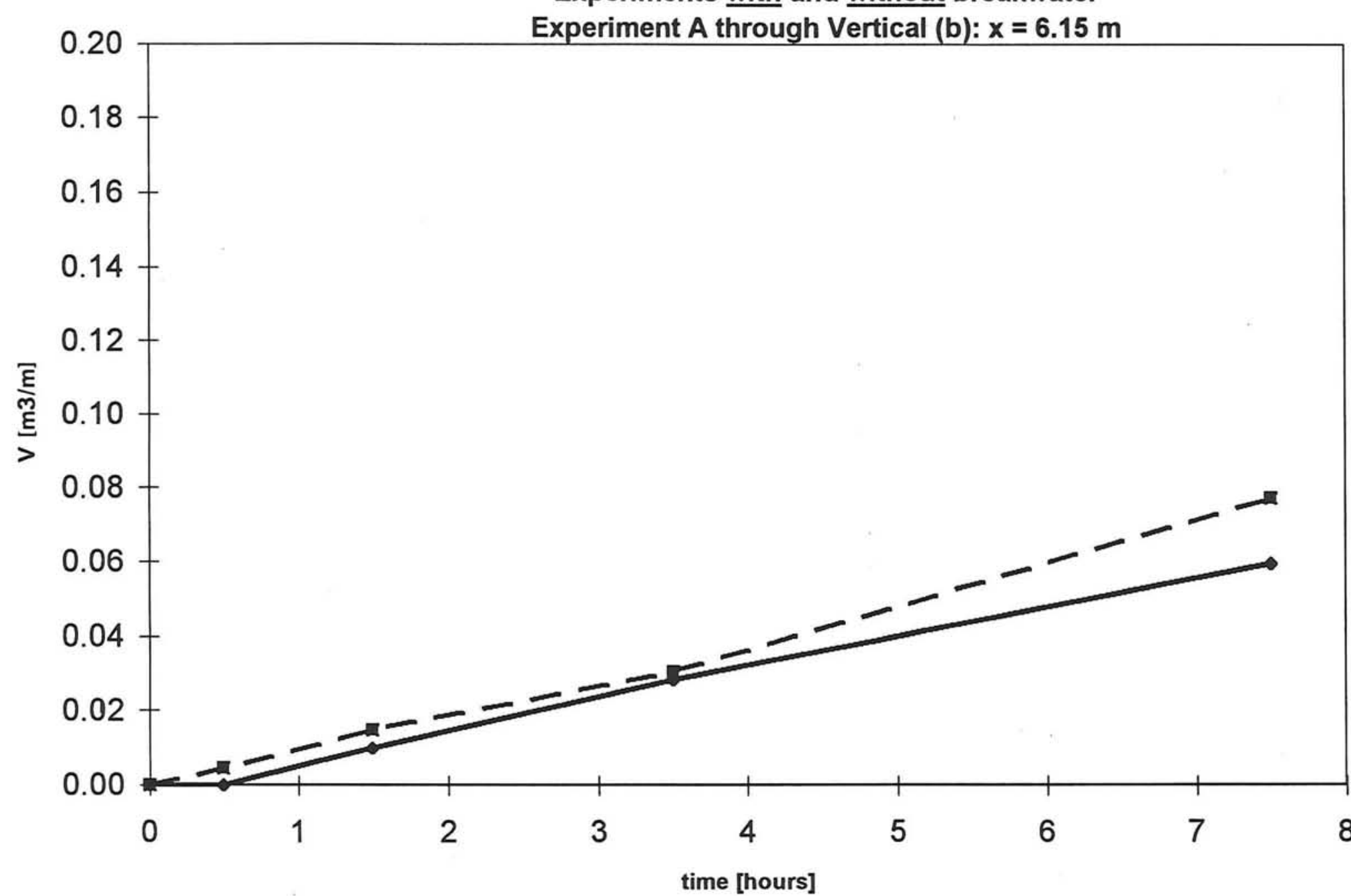




\section{Sediment transport volumes}

Experiments with and without breakwater

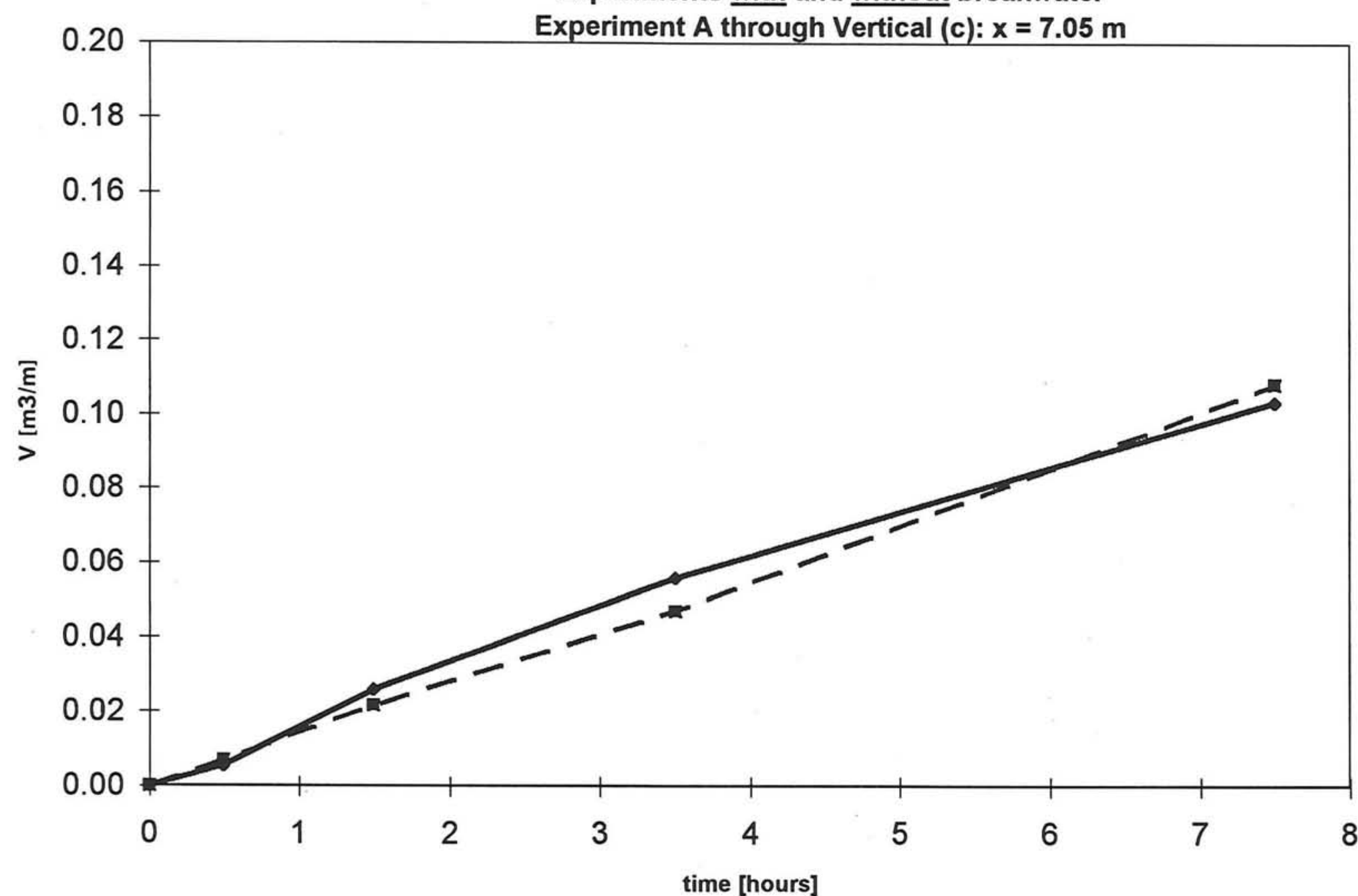

Graph 1.29 


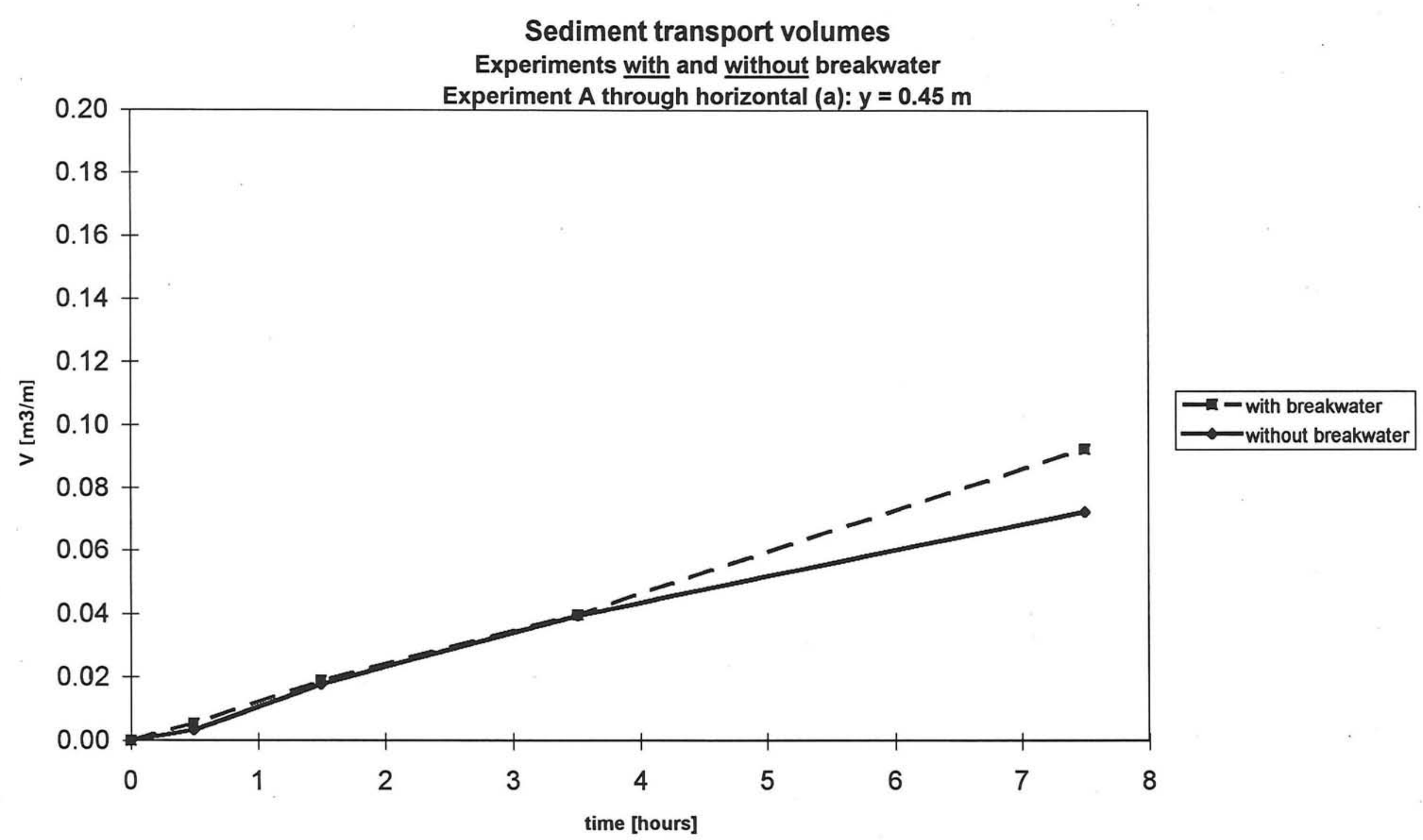

Graph 1.30 


\section{Sediment transport volumes}

Experiments with and without breakwater

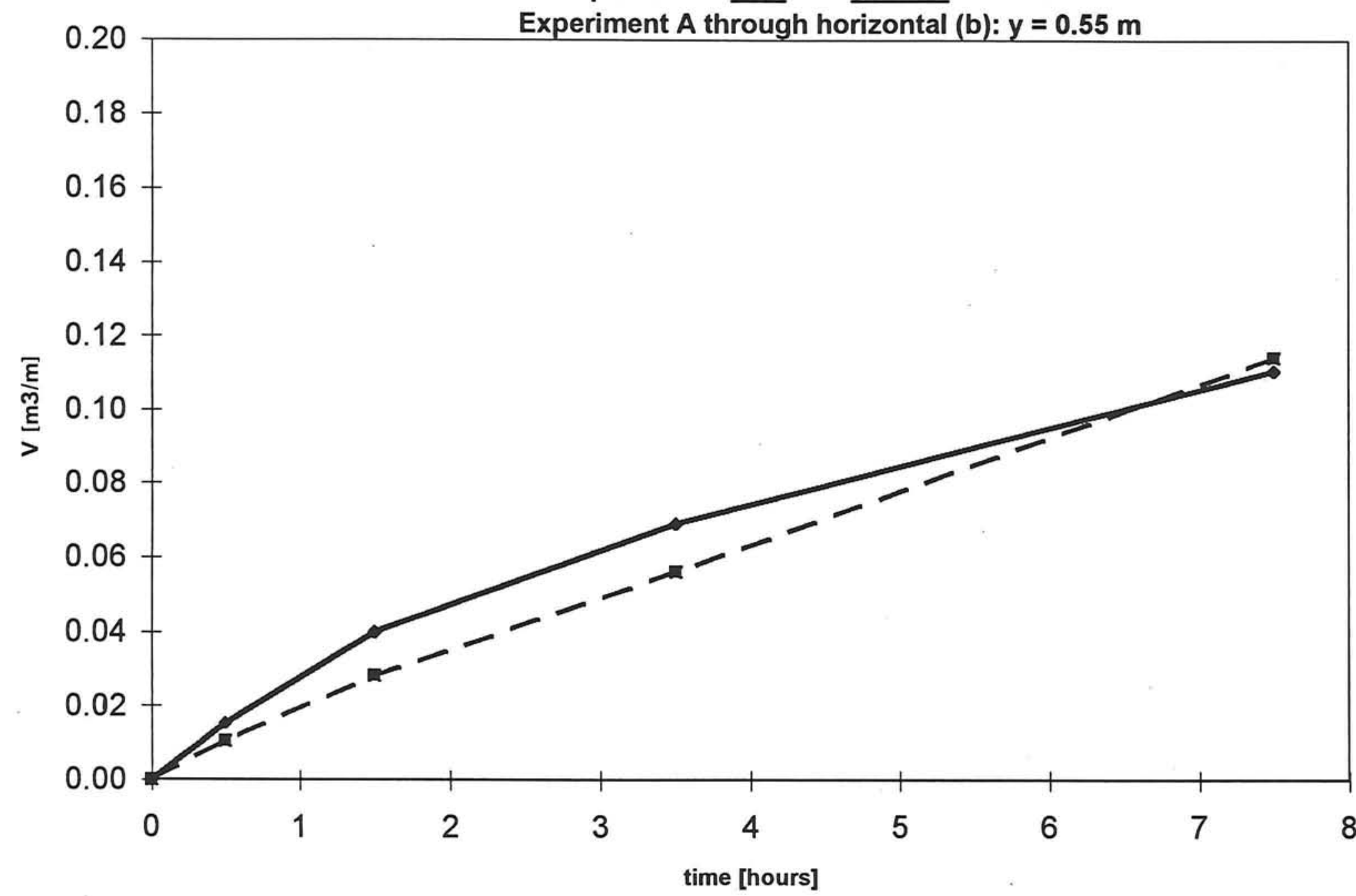




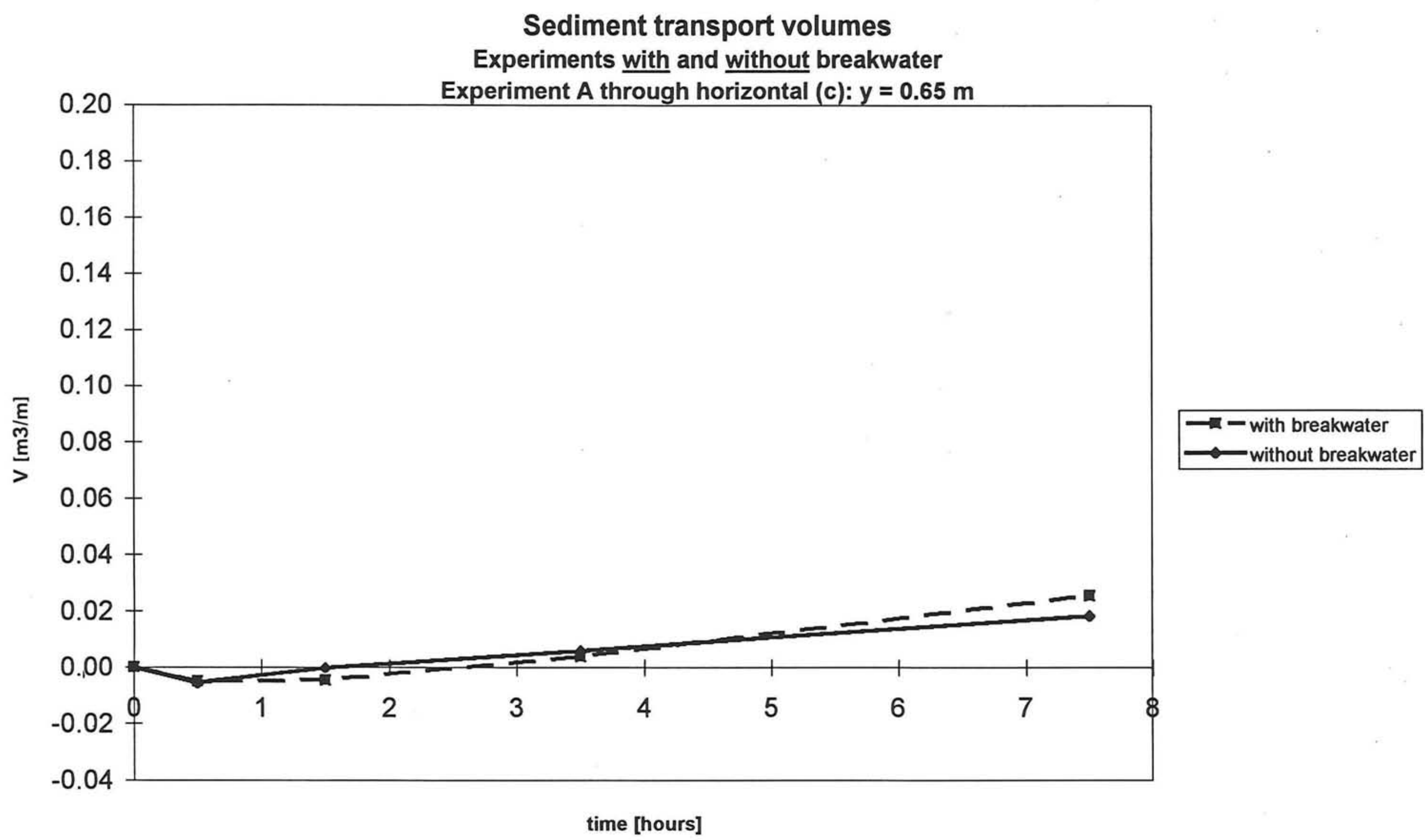

Graph 1.32 
Sediment transport volumes

Experiments with and without breakwater

Experiment $B$ through Vertical (a): $x=5.25 \mathrm{~m}$

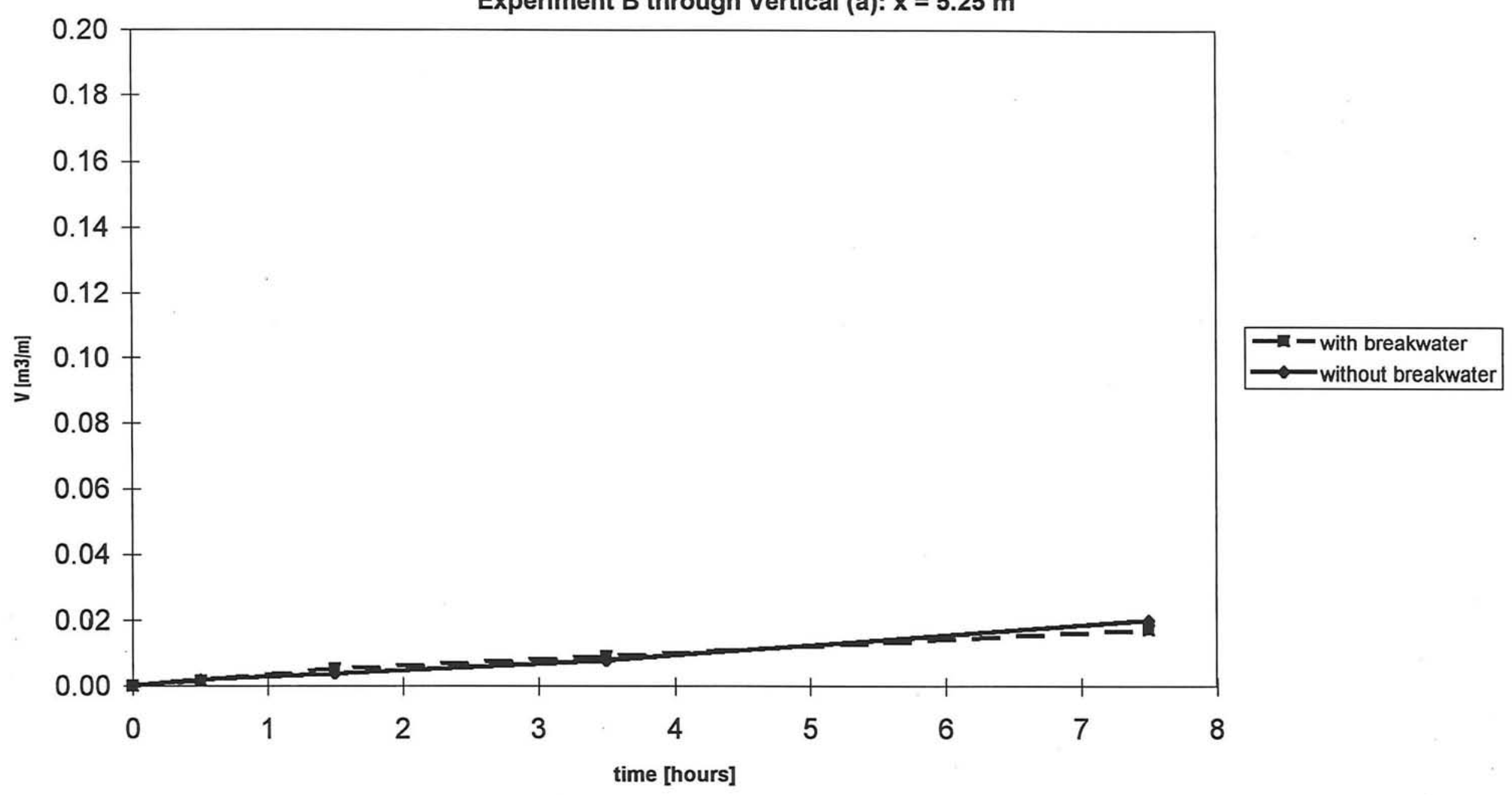

Graph 1.33 


\section{Sediment transport volumes}

Experiments with and without breakwater

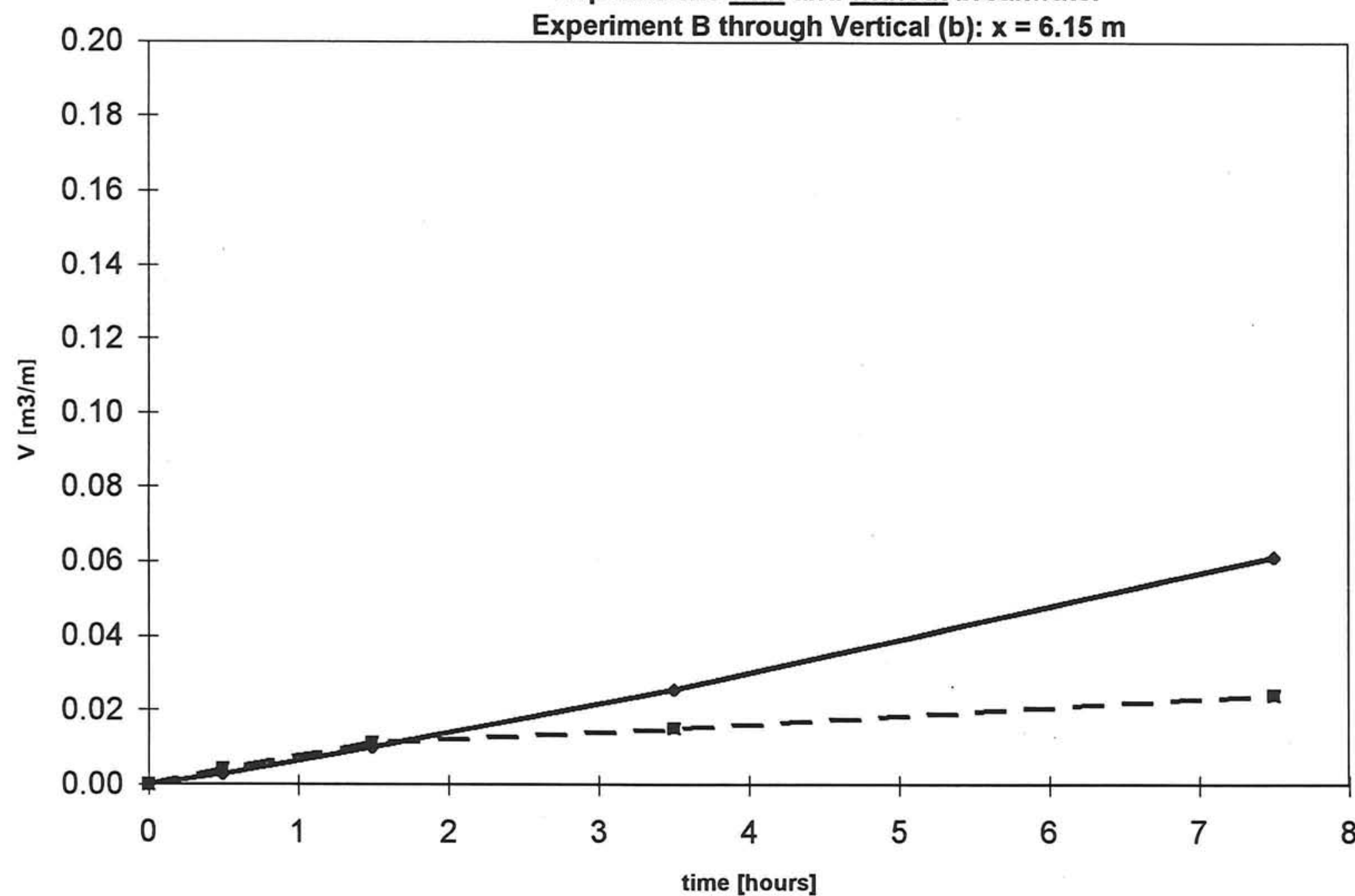

Graph 1.34 


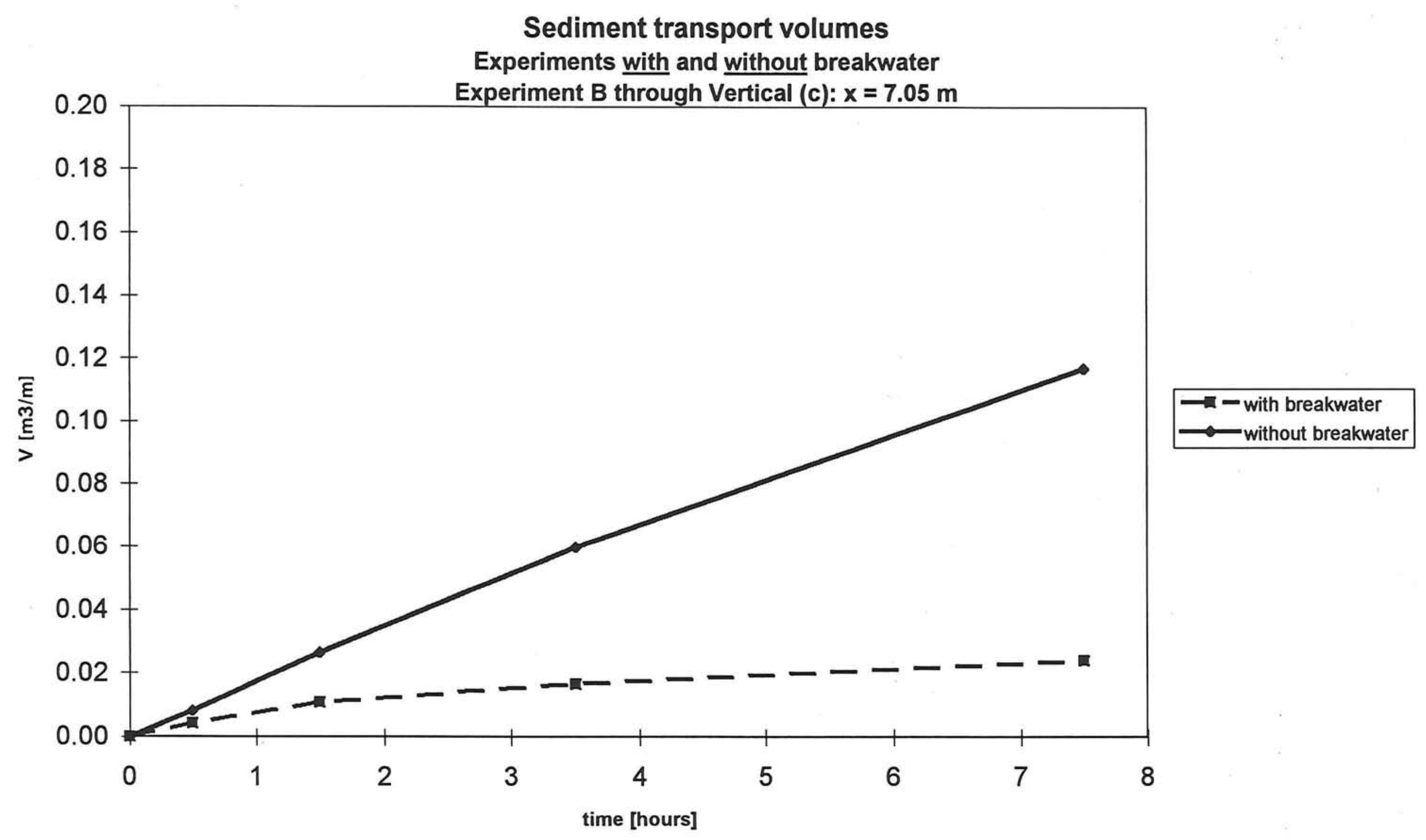

Graph 1.35 


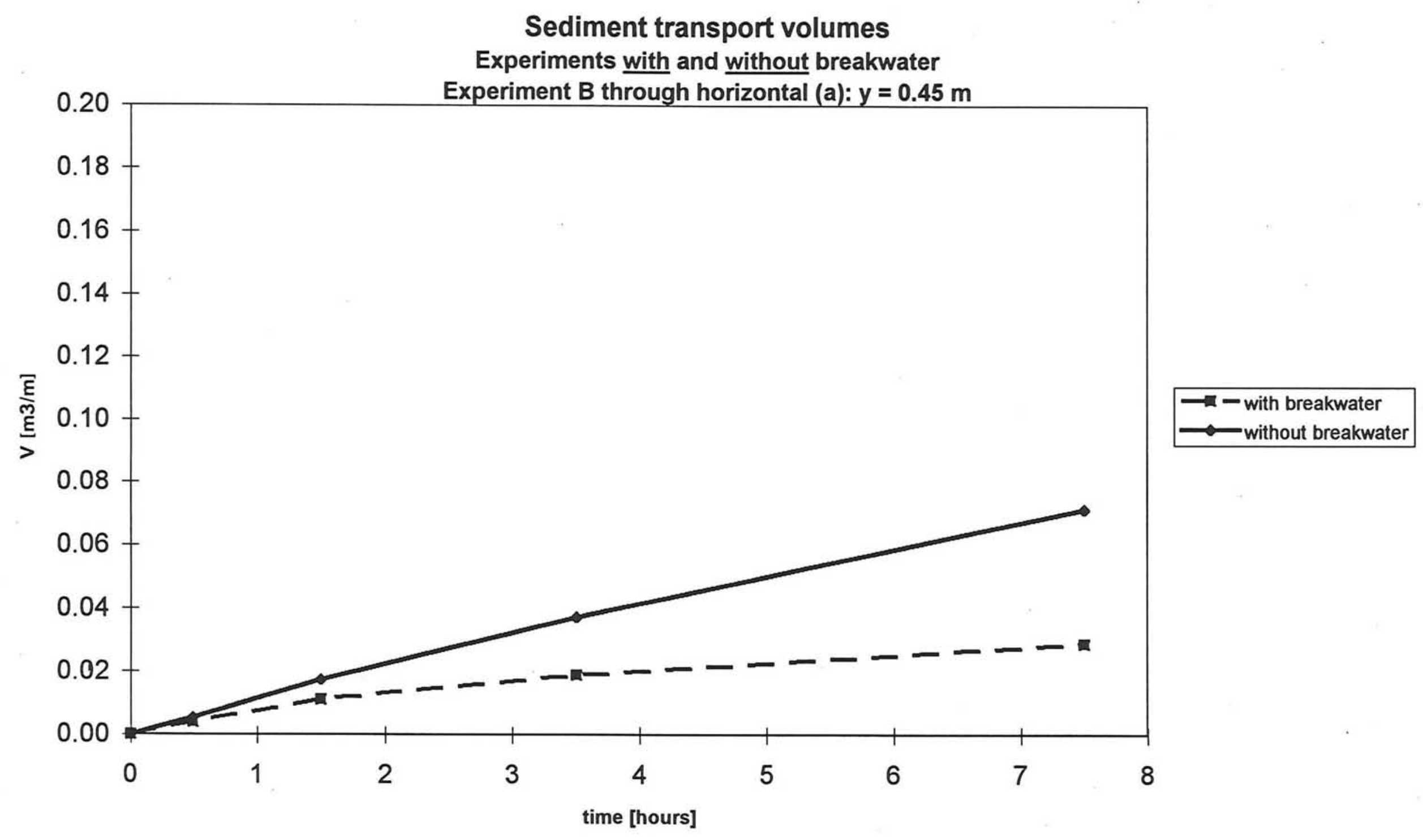

Graph 1.36 


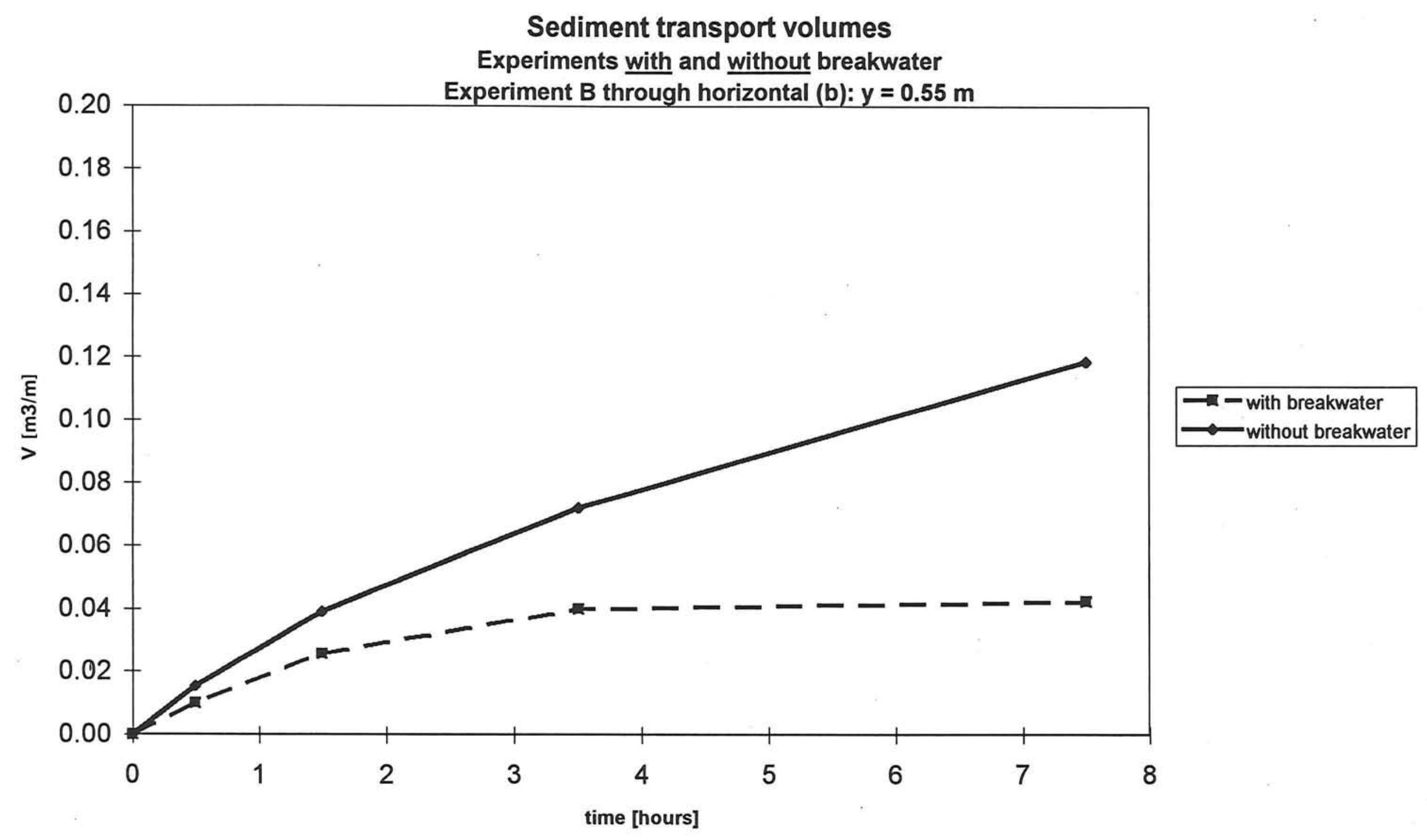

Graph 1.37 


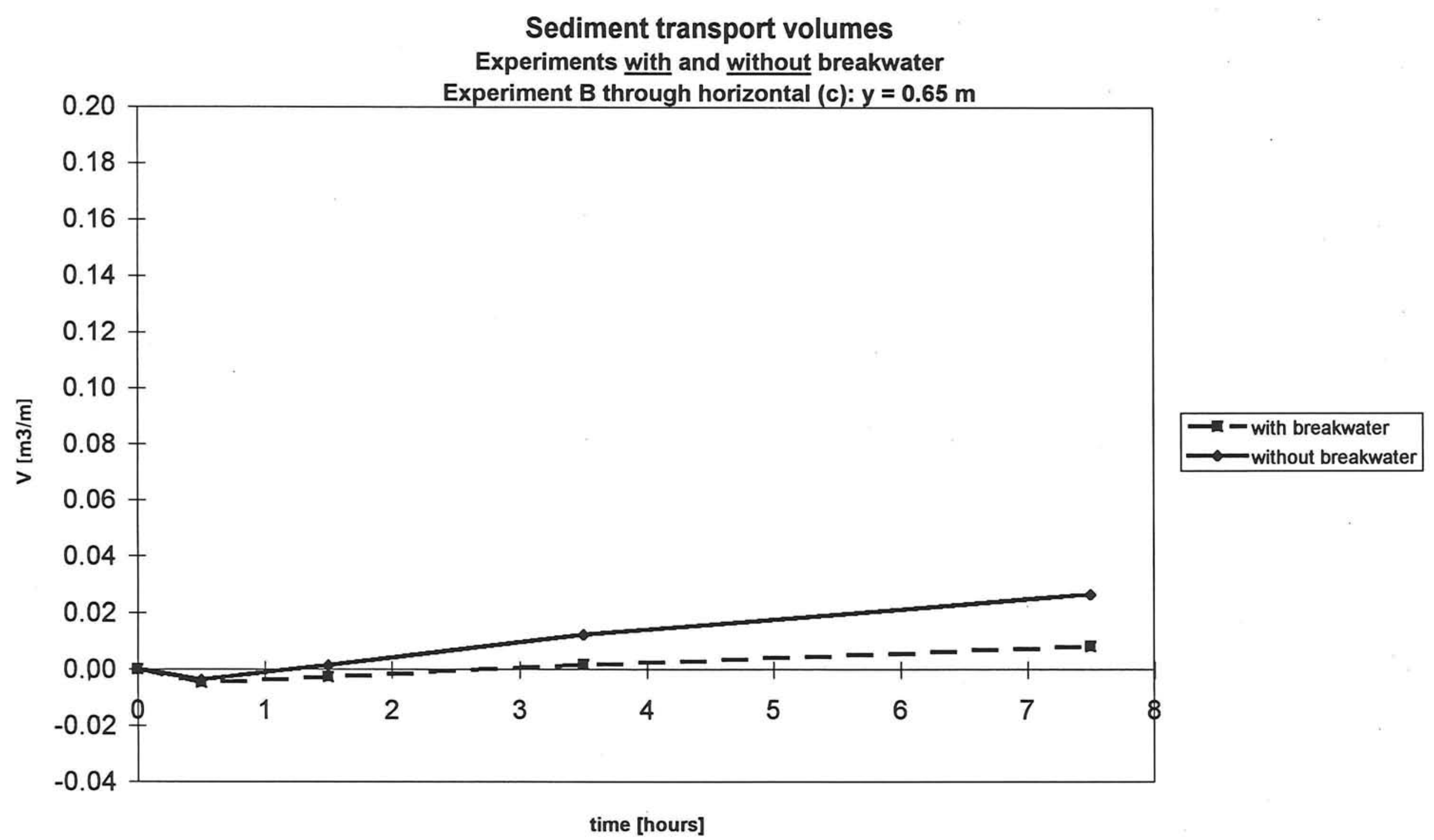

Graph 1.38 
Sediment transport volumes

Experiments with and without breakwater

Experiment $C$ through Vertical (a): $x=5.25 \mathrm{~m}$

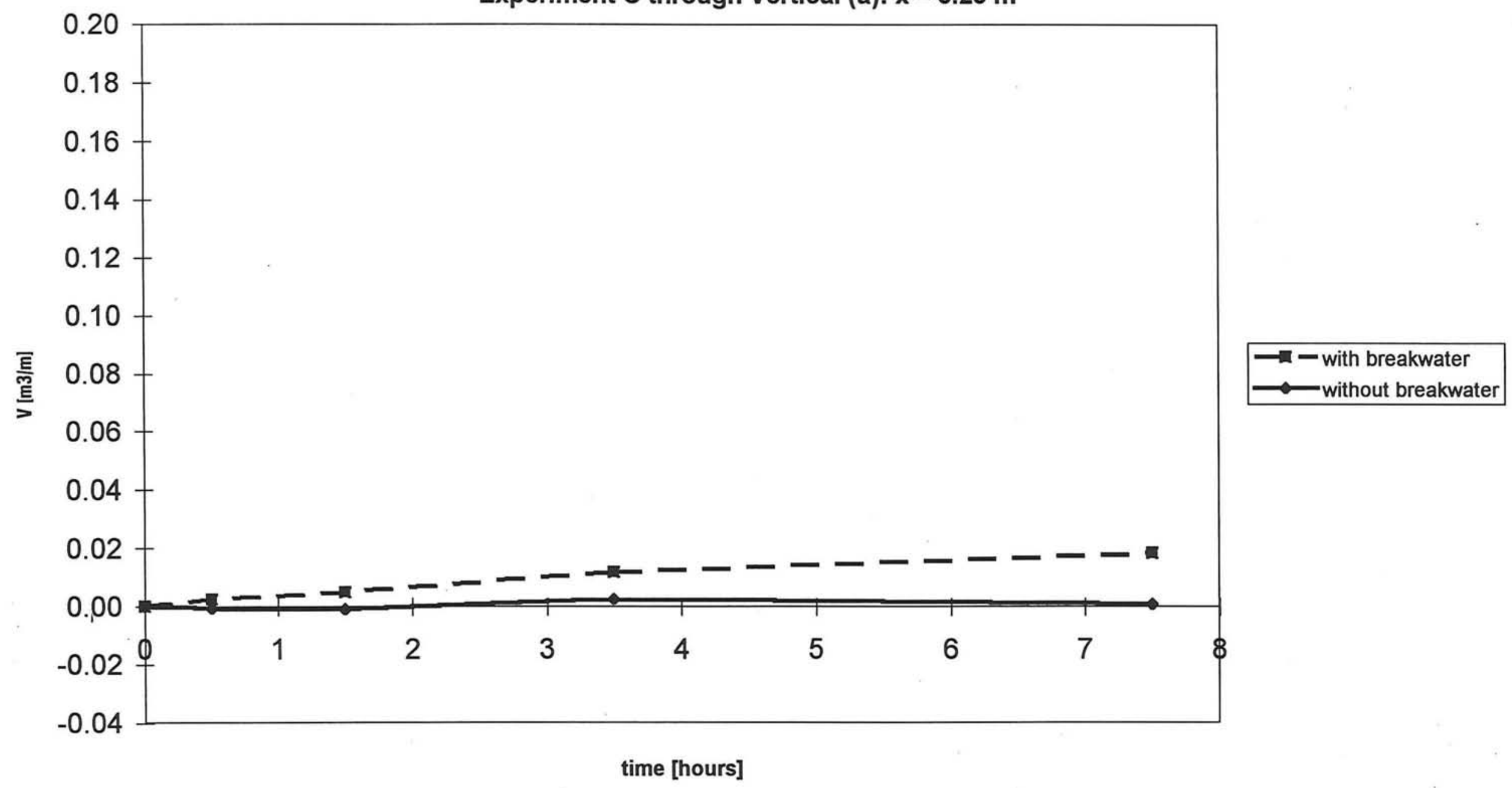

Graph 1.39 


\section{Sediment transport volumes}

Experiments with and without breakwater

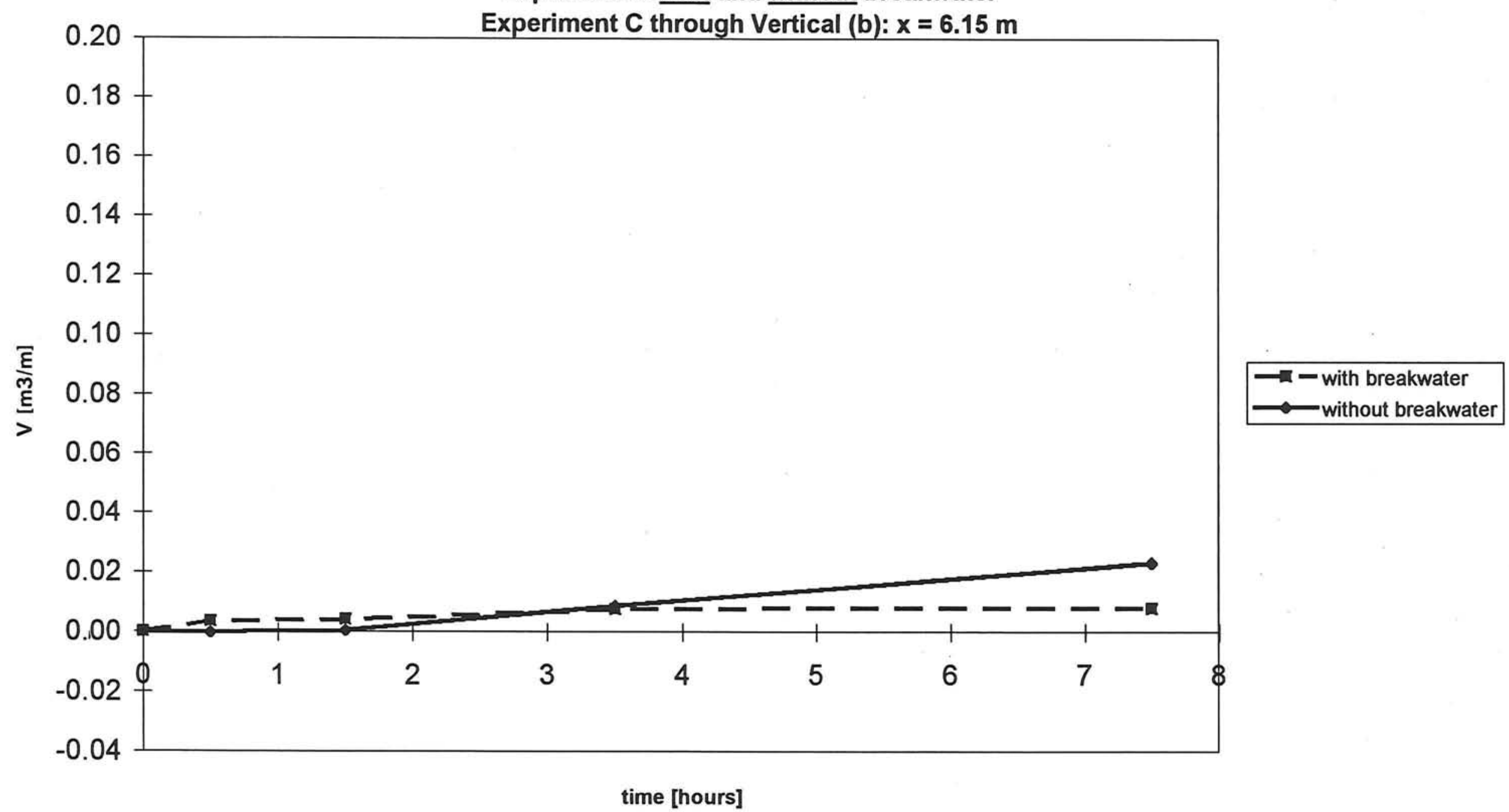

Graph 1.40 


\section{Sediment transport volumes}

Experiments with and without breakwater

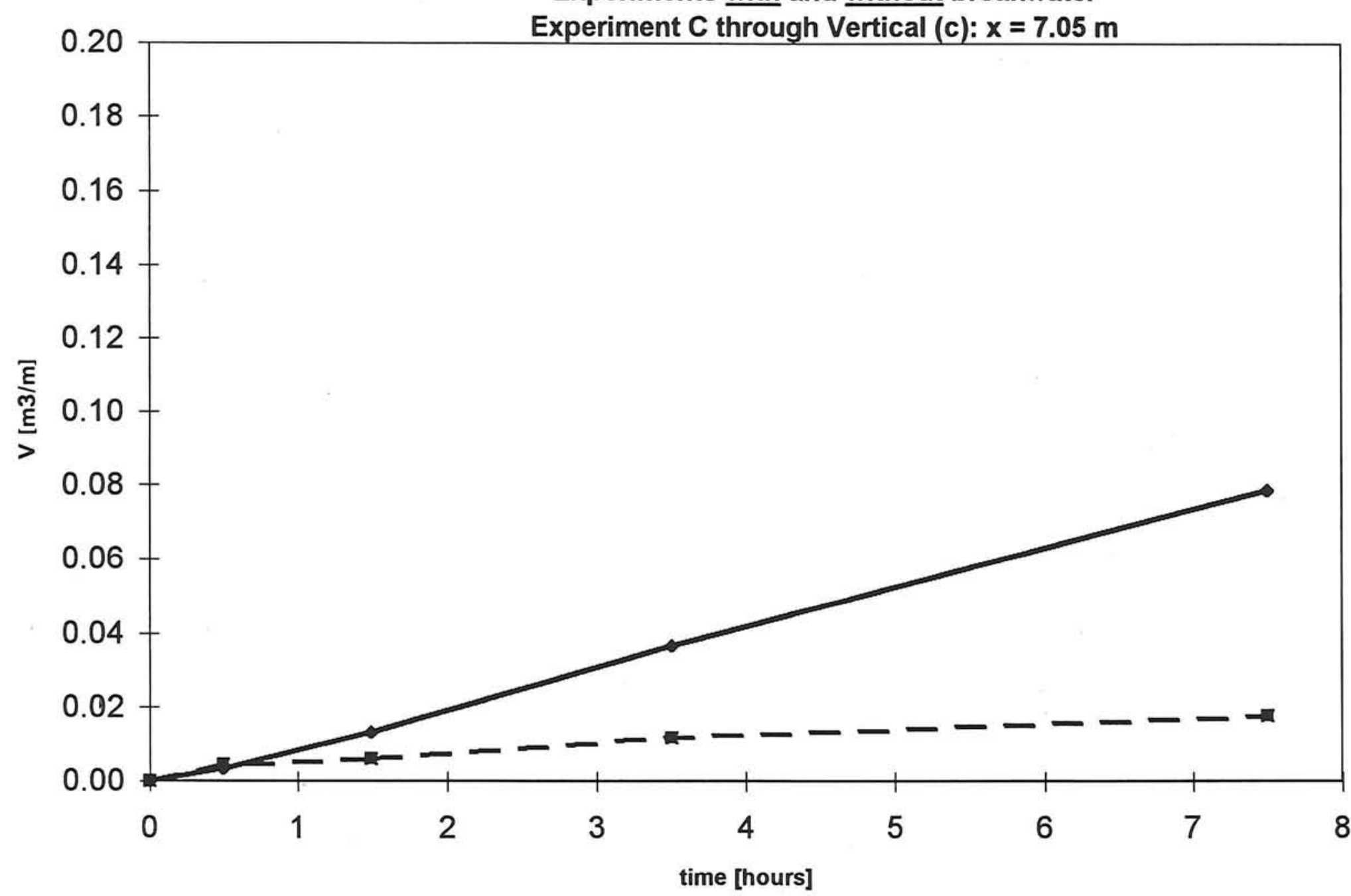




\section{Sediment transport volumes}

Experiments with and without breakwater

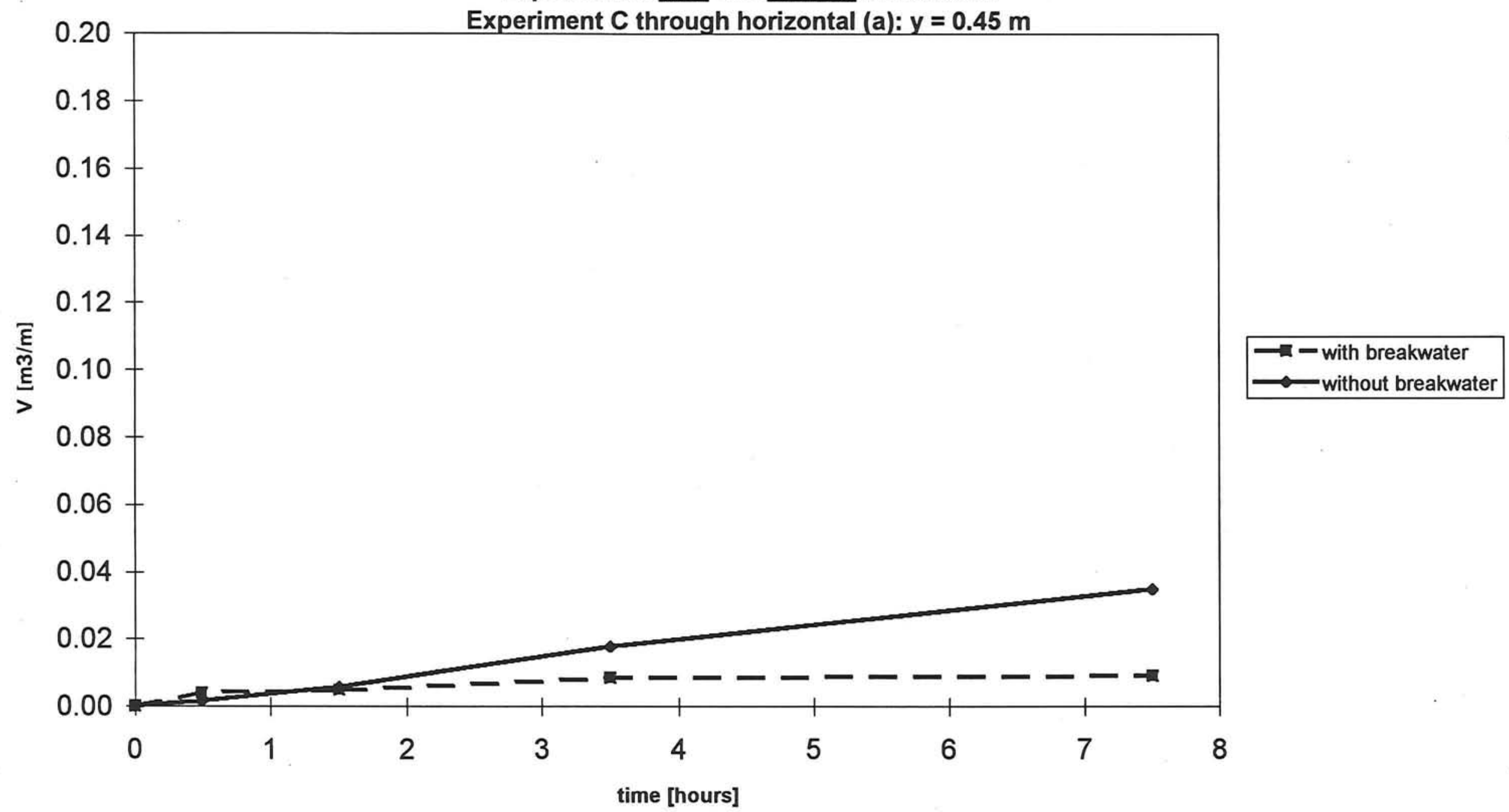

Graph 1.42 


\section{Sediment transport volumes}

Experiments with and without breakwater

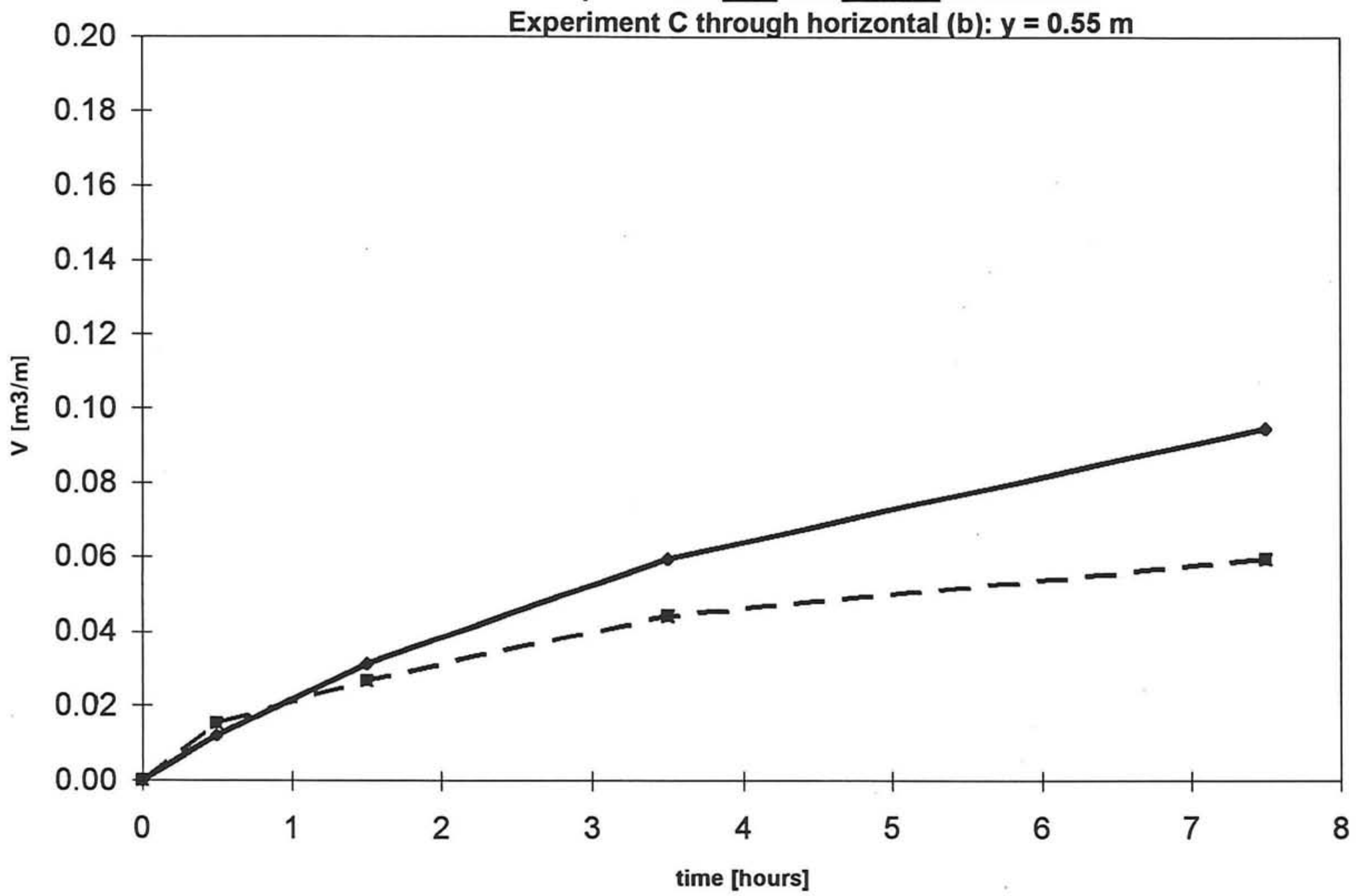

Graph 1.43 


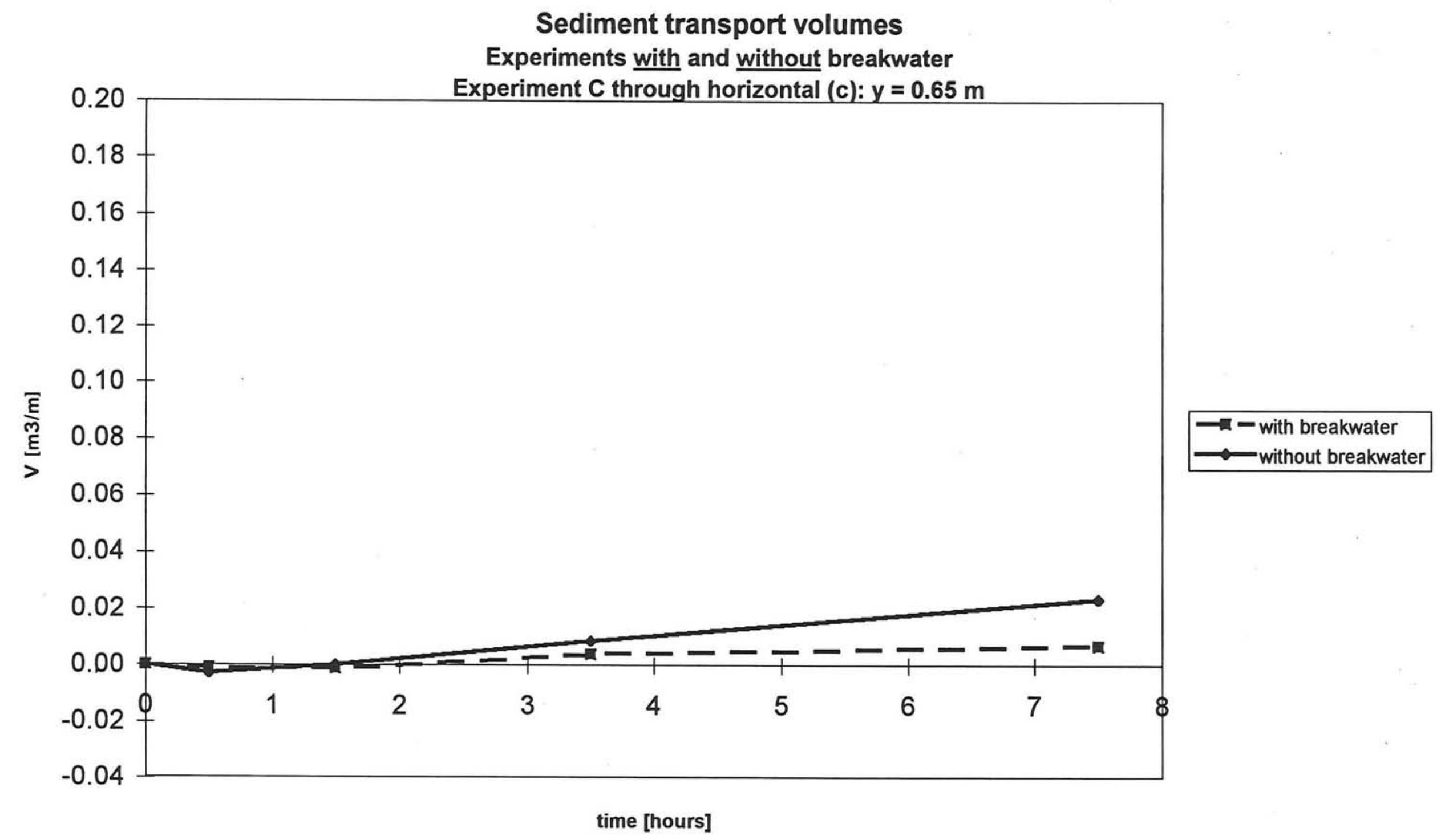

Graph 1.44 


\section{Sediment transport volumes}

Experiments with and without breakwater

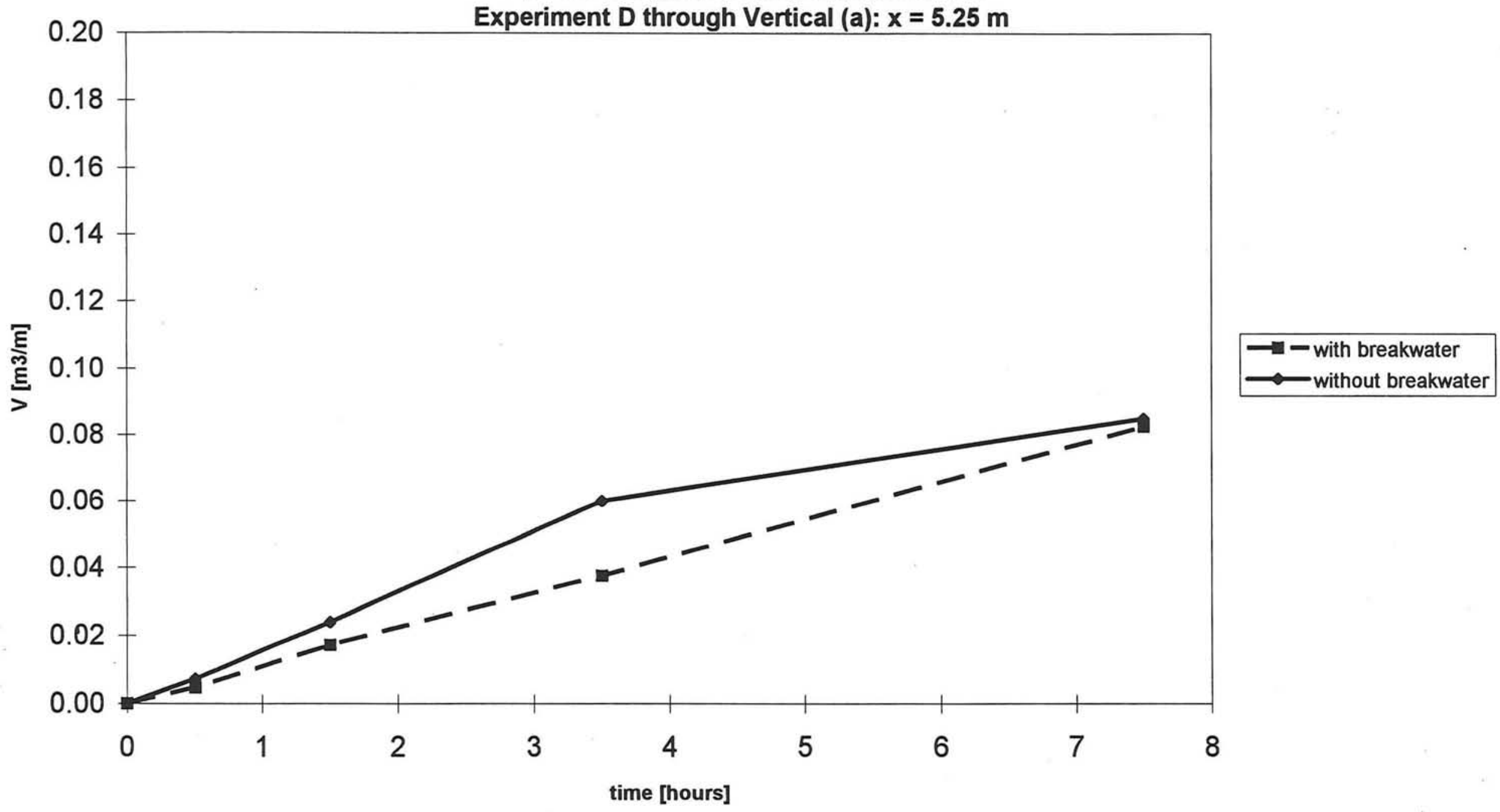

Graph 1.45 
Sediment transport volumes

Experiments with and without breakwater

Experiment $D$ through Vertical (b): $x=6.15 \mathrm{~m}$

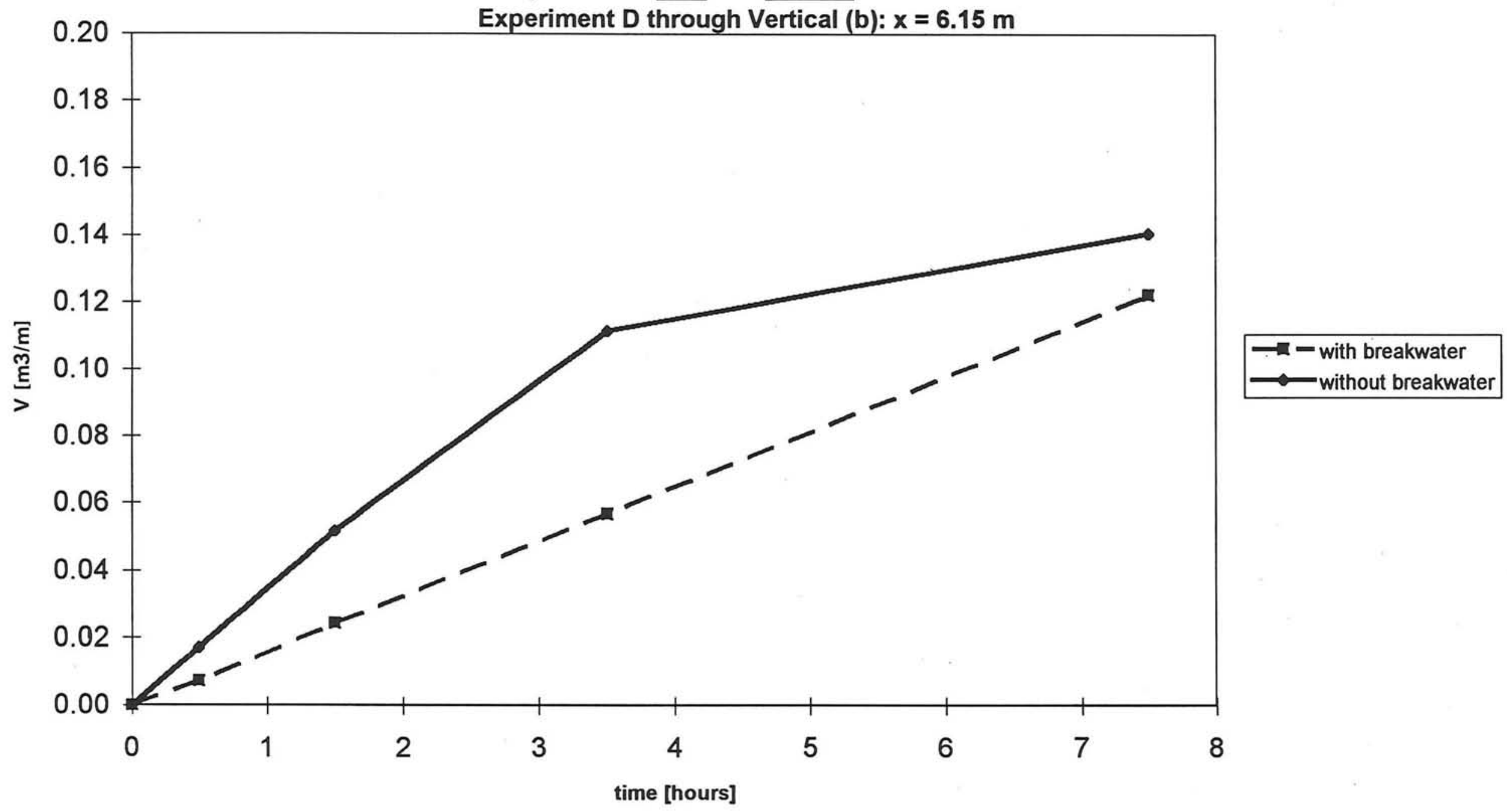

Graph 1.46 
Sediment transport volumes

Experiments with and without breakwater Experiment $D$ through Vertical (c): $x=7.05 \mathrm{~m}$

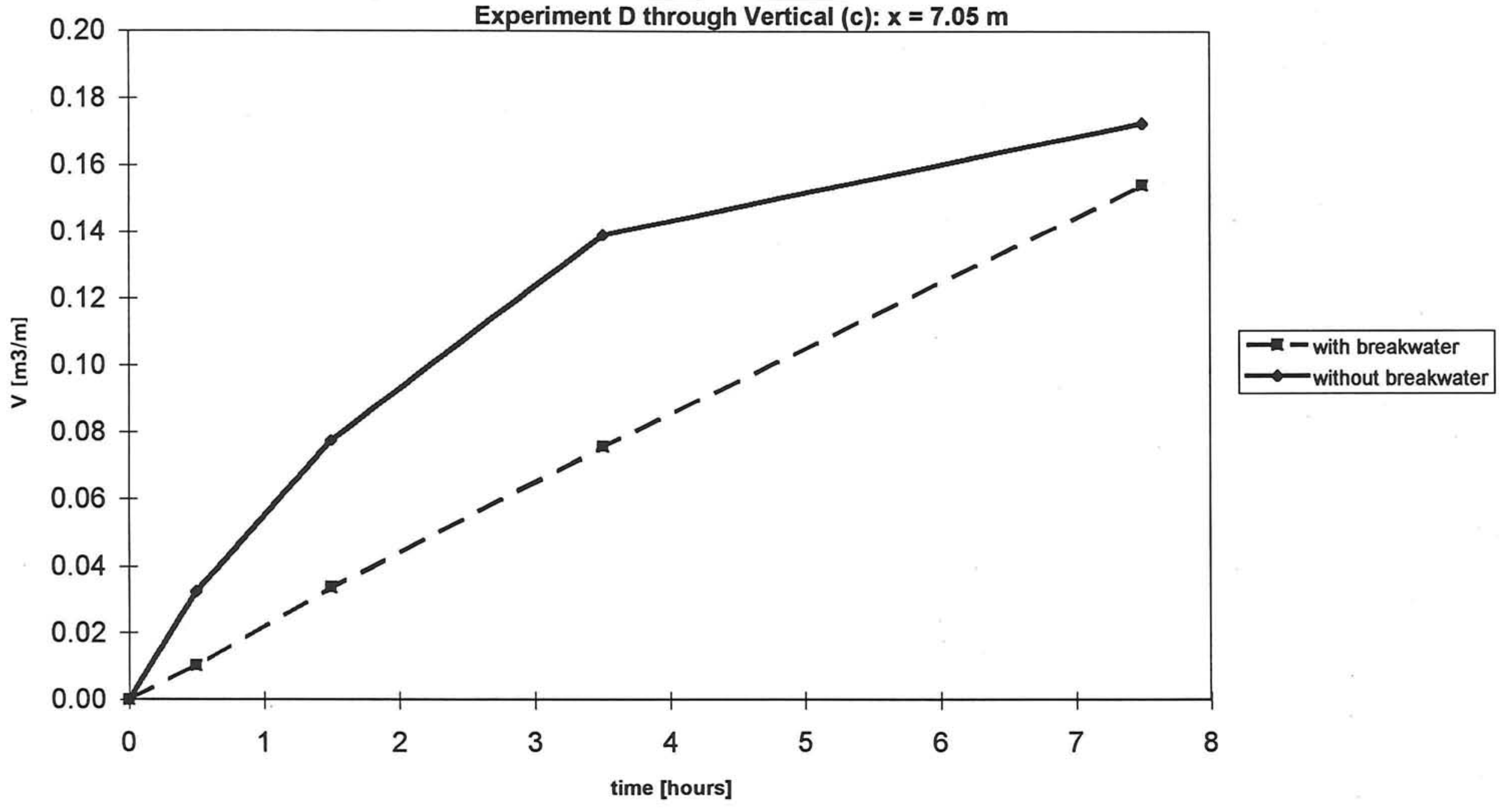

Graph 1.47 
Sediment transport volumes

Experiments with and without breakwater

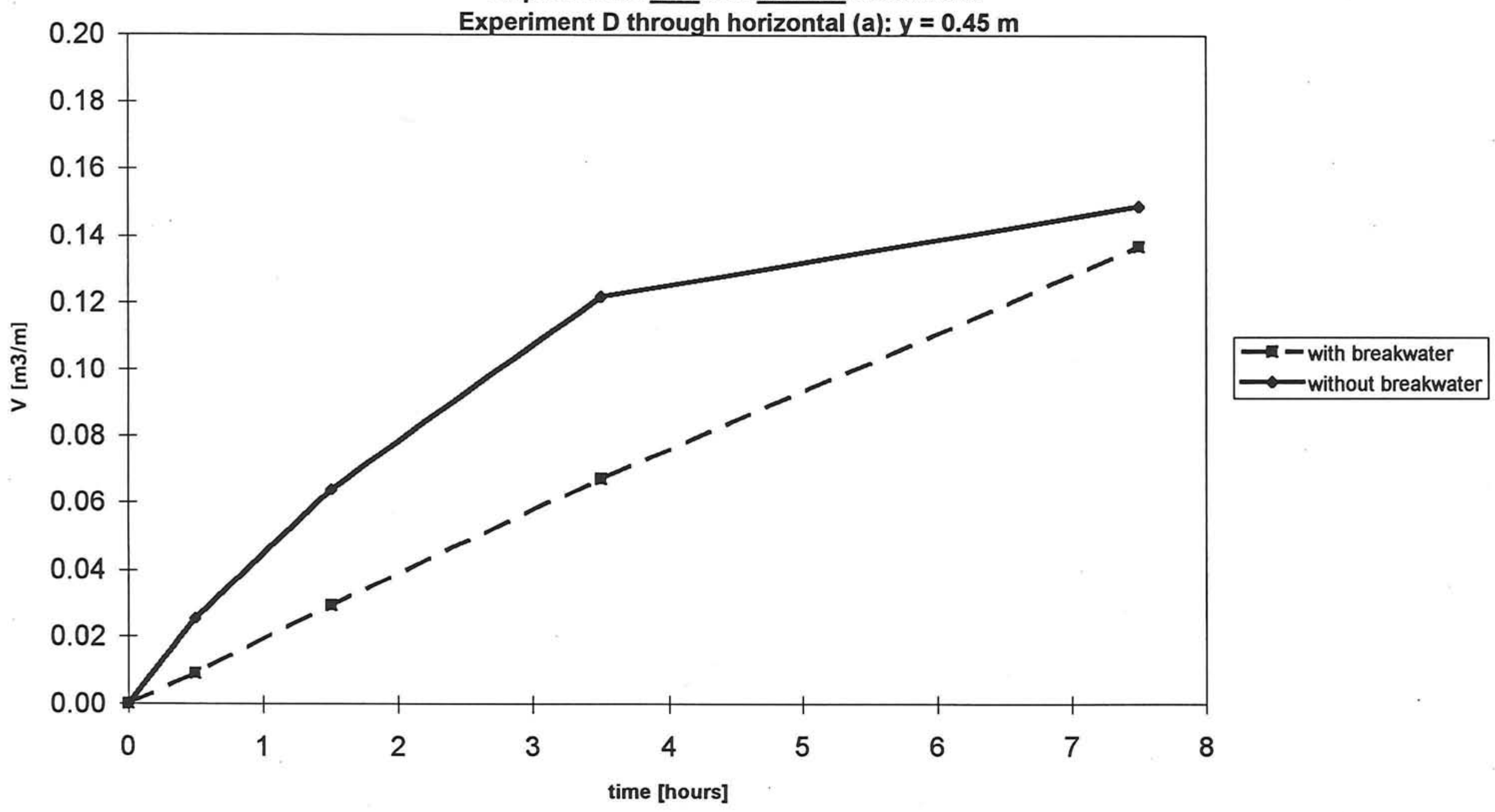

Graph 1.48 
Sediment transport volumes

Experiments with and without breakwater

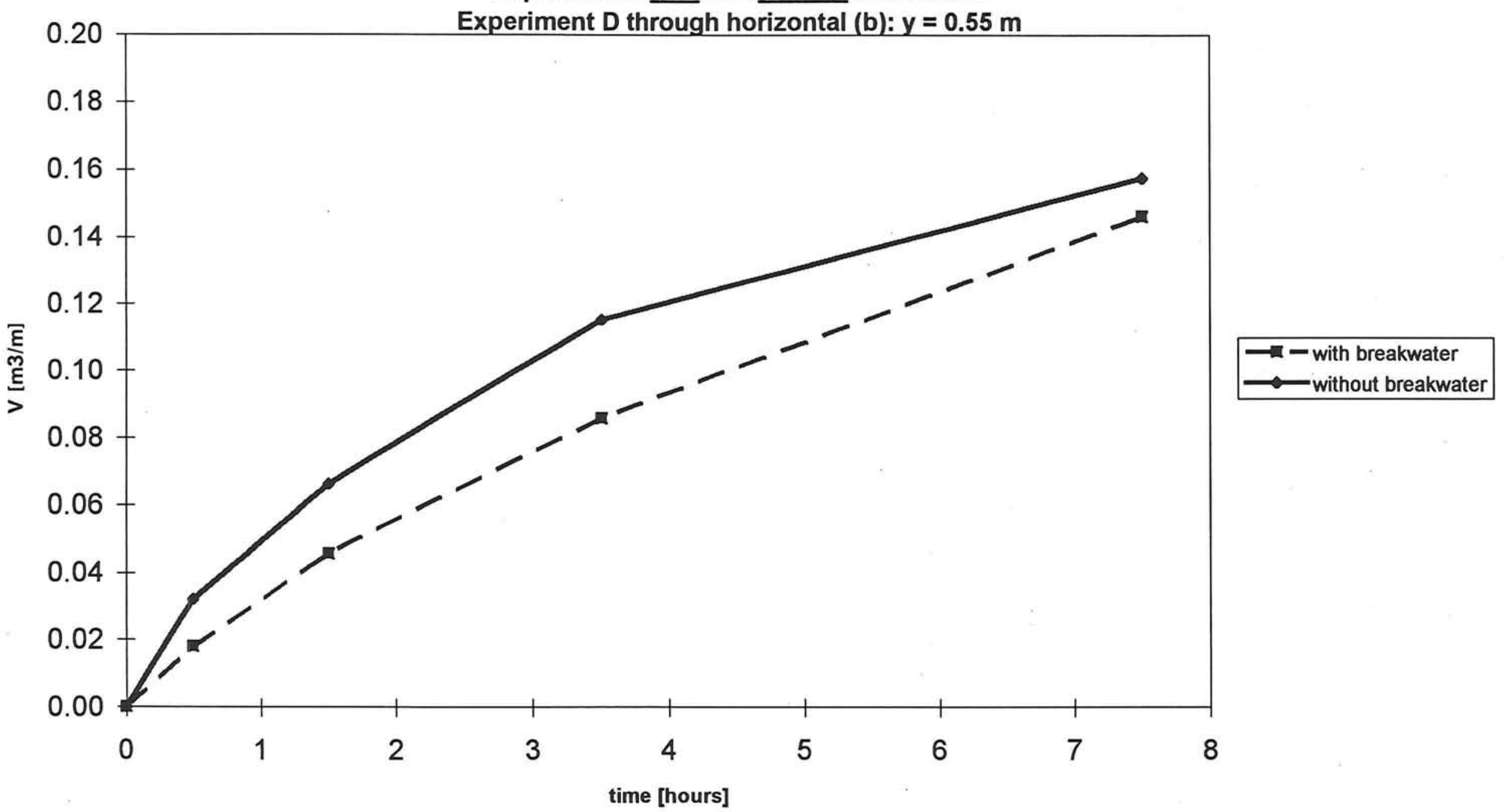

Graph 1.49 


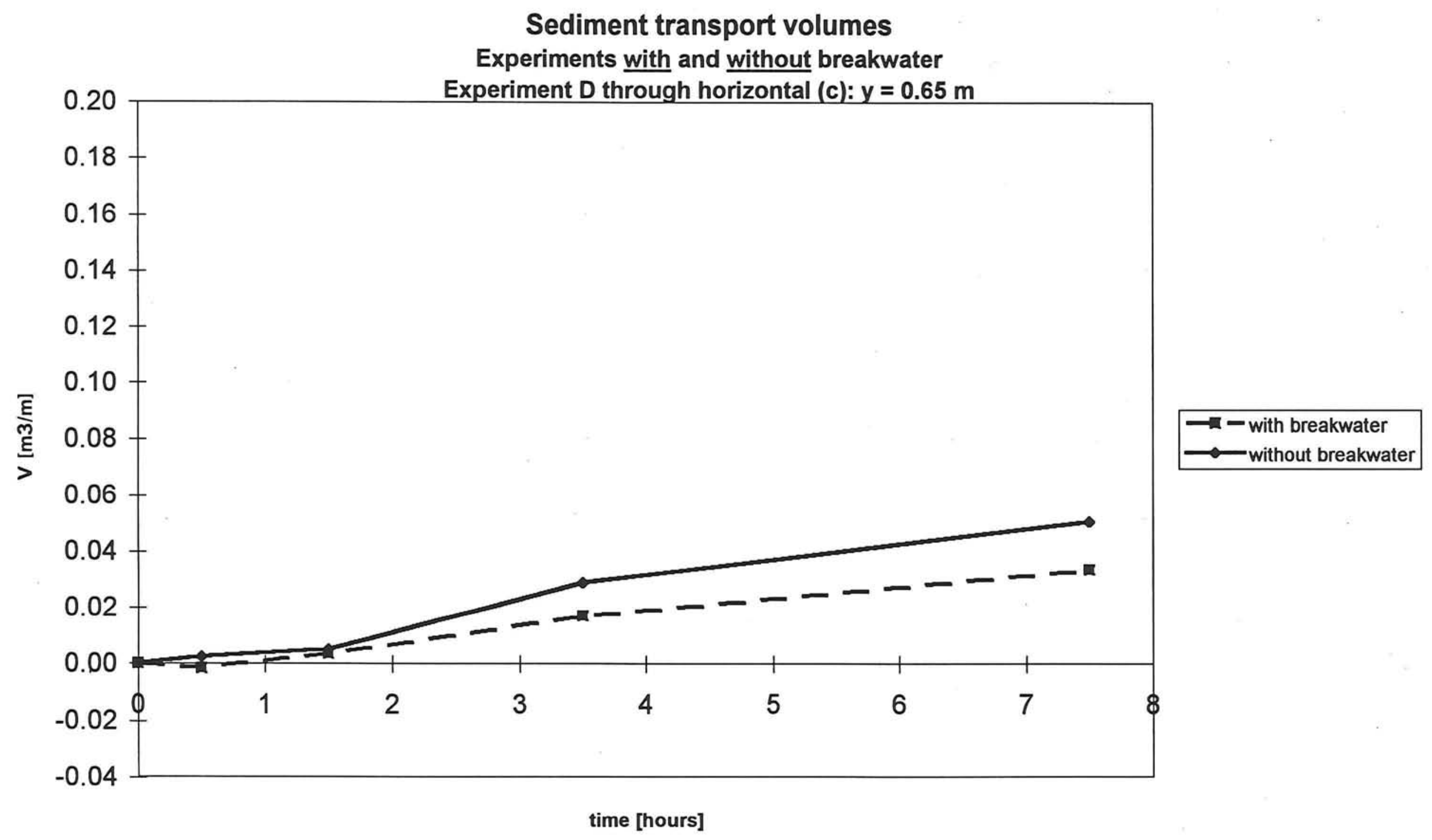

Graph 1.50 


\section{Sediment transport volumes}

Experiments with and without breakwater

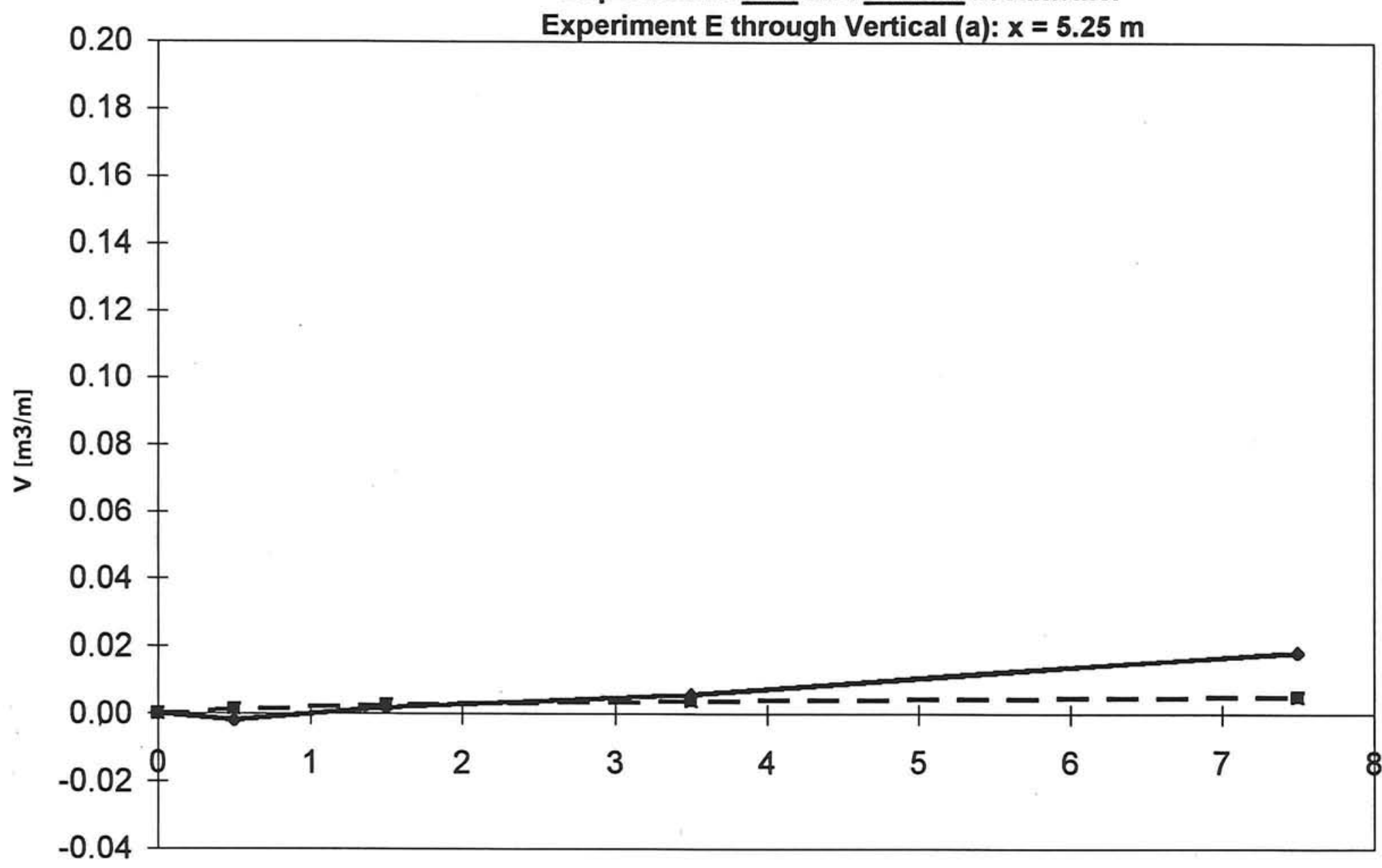

- with breakwater

without breakwater

time [hours]

Graph 1.51 


\section{Sediment transport volumes}

Experiments with and without breakwater

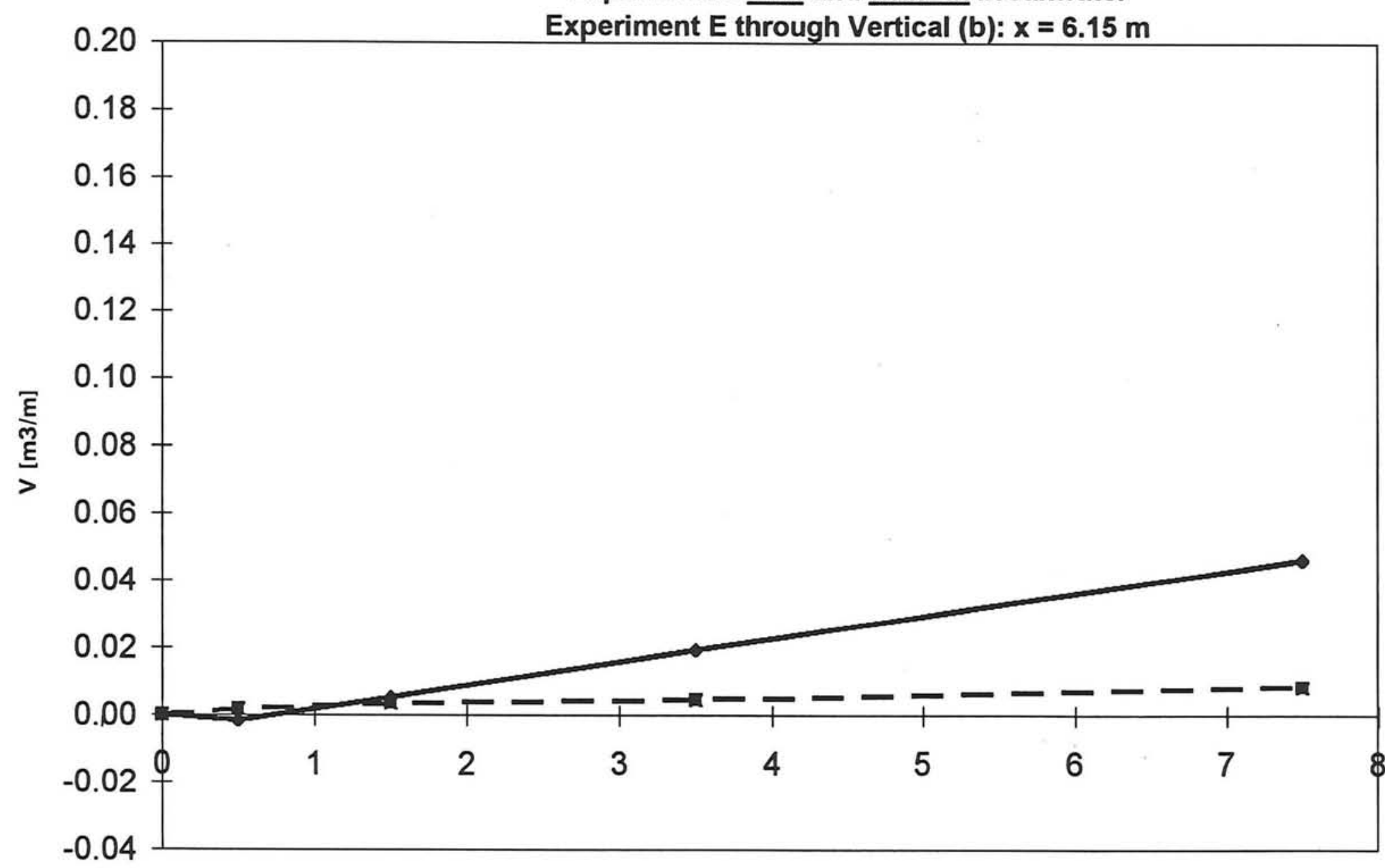

$\longrightarrow$ - with breakwater

$\sim$ without breakwater

time [hours]

Graph 1.52 


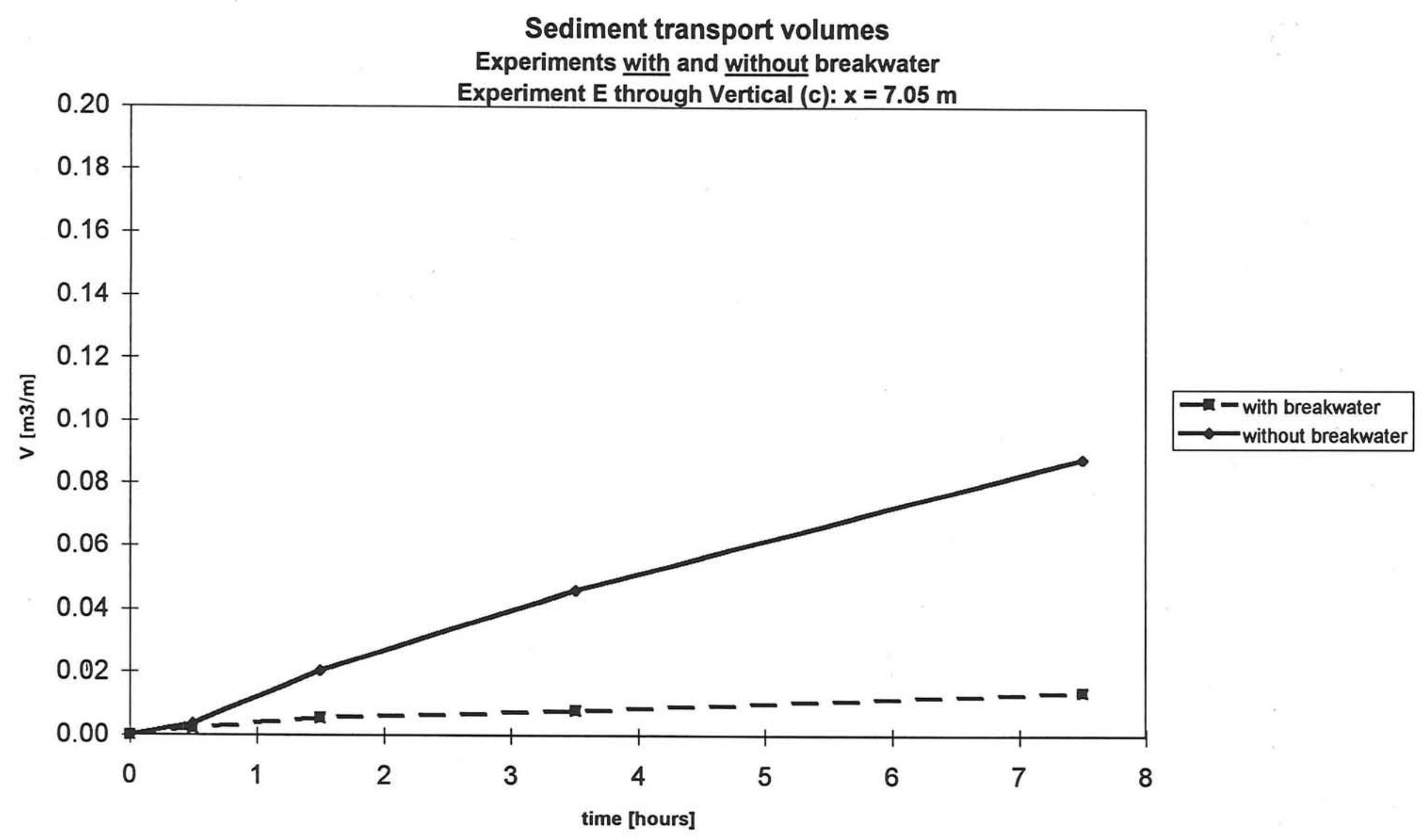

Graph 1.53 


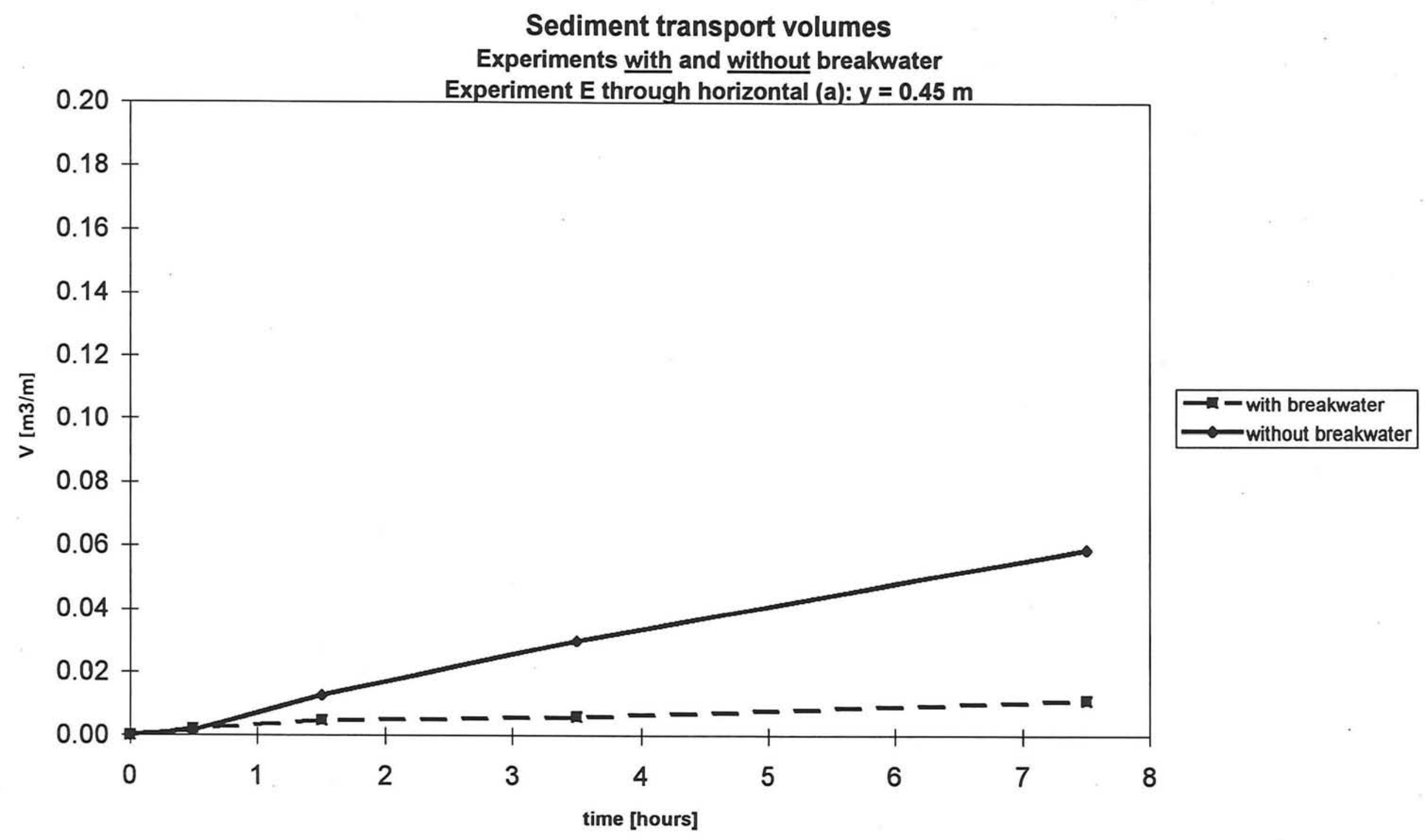

Graph 1.54 


\section{Sediment transport volumes}

Experiments with and without breakwater

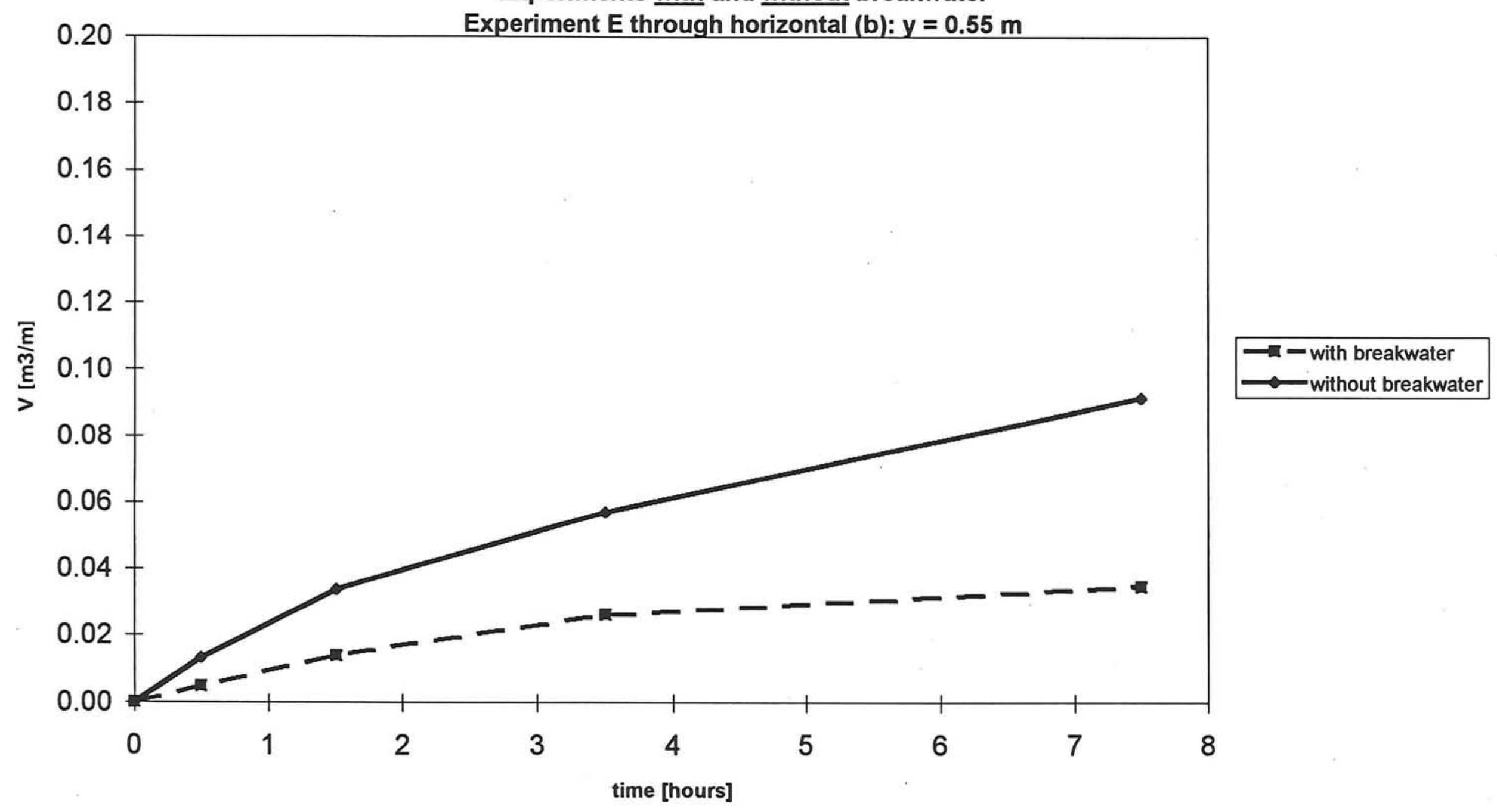

Graph 1.55 


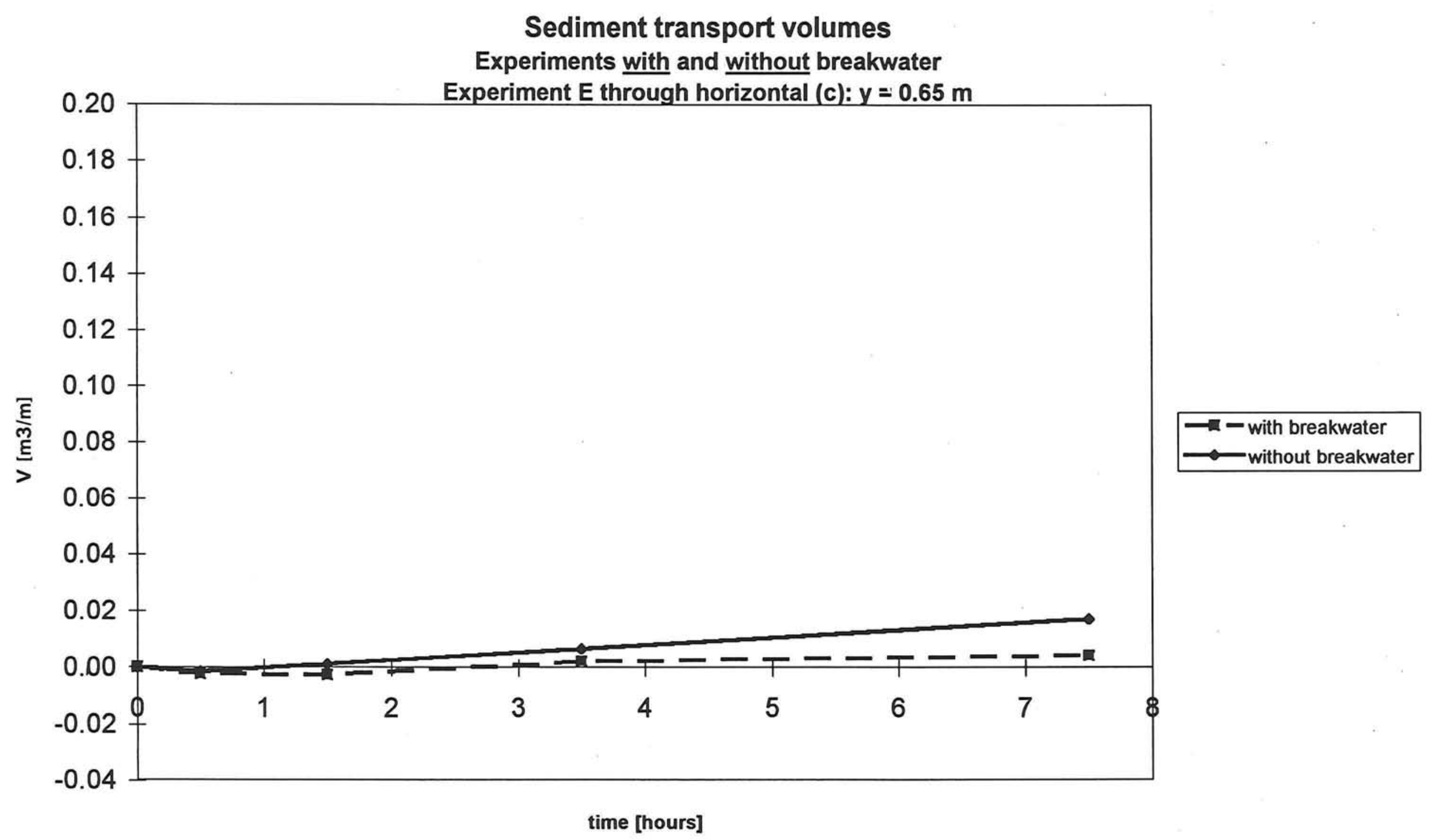

Graph 1.56 


\section{Sediment transport volumes}

Experiments with and without breakwater

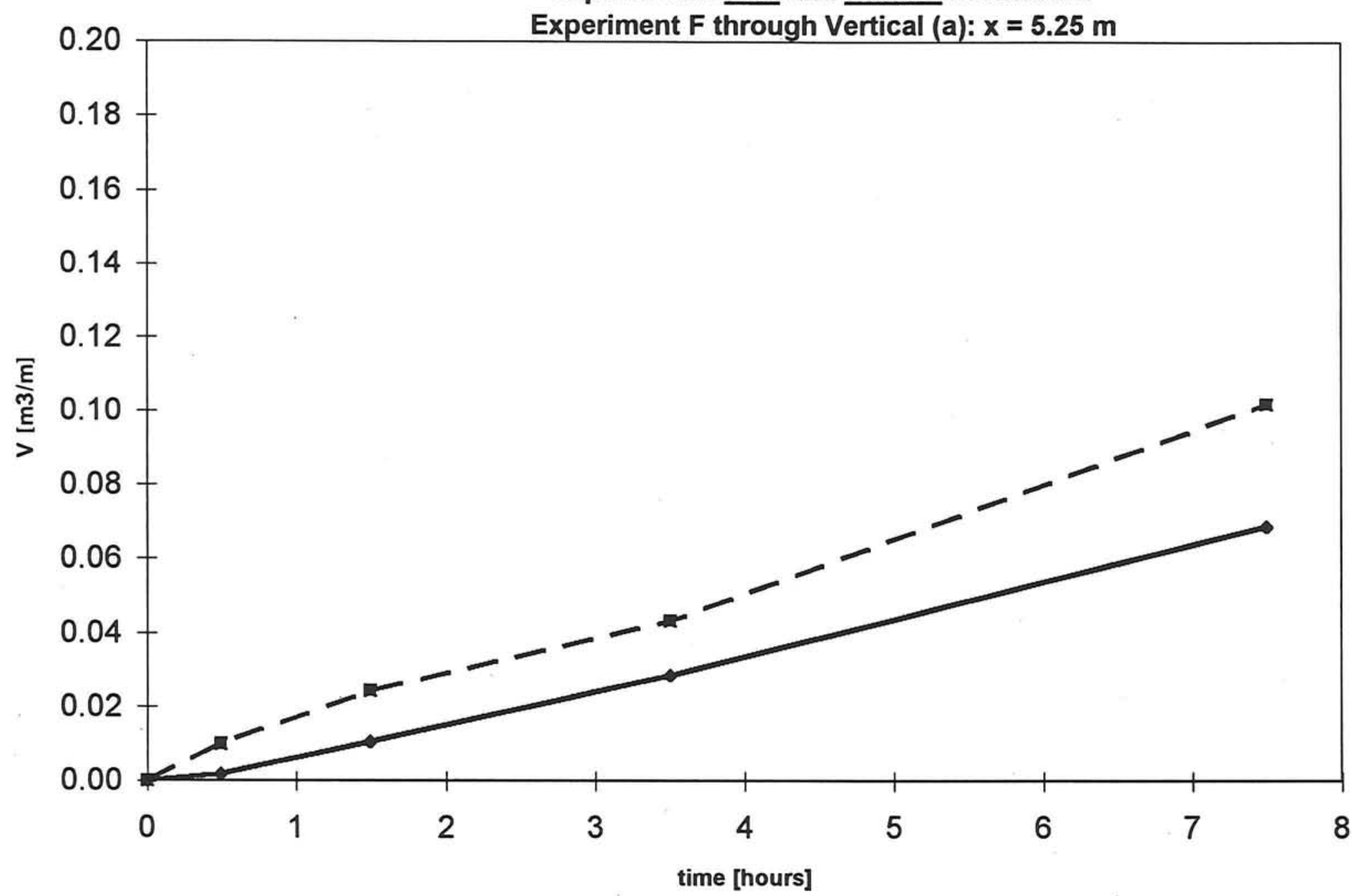

Graph 1.57 


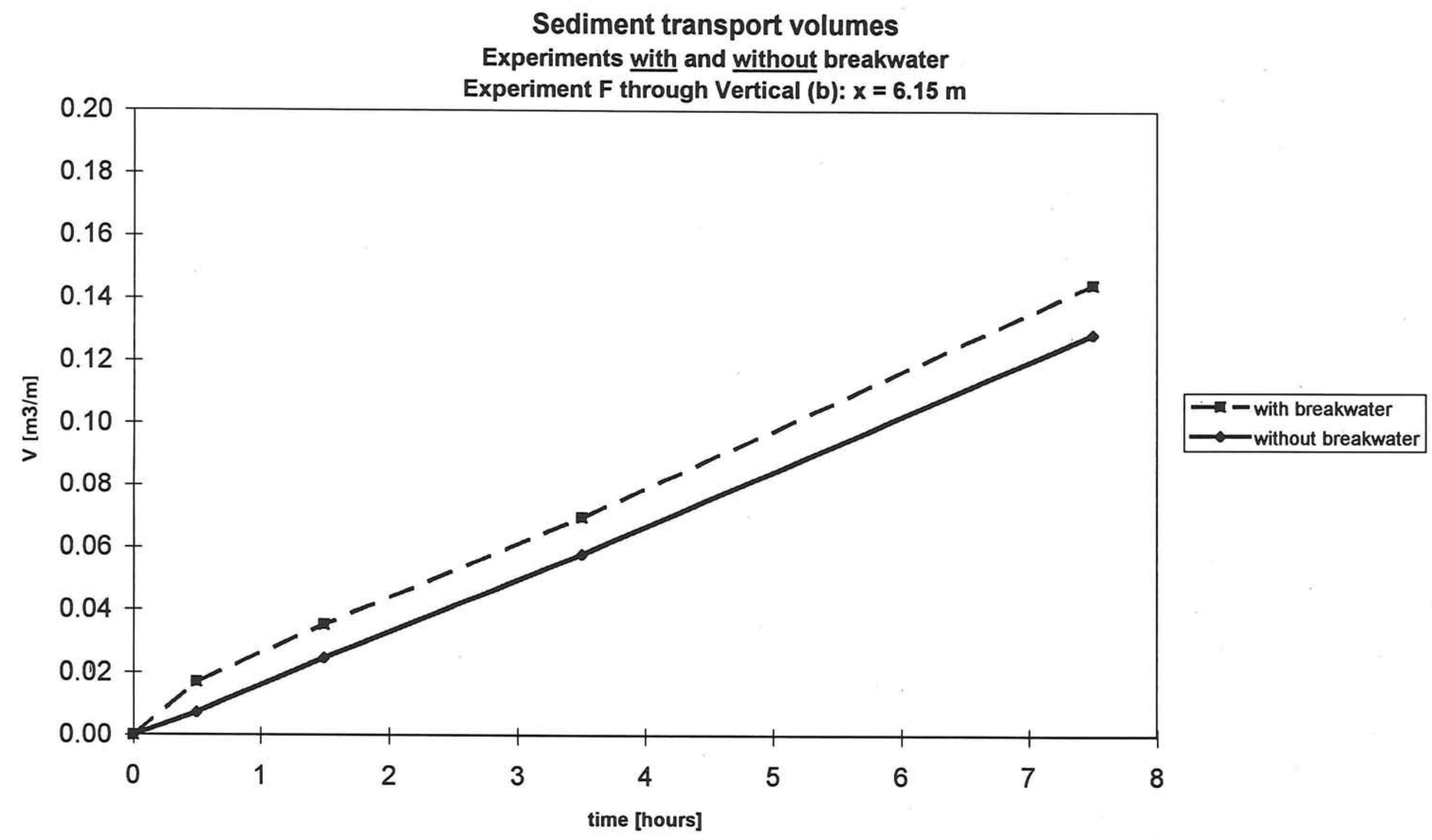

Graph 1.58 


\section{Sediment transport volumes}

Experiments with and without breakwater

Experiment $F$ through Vertical (c): $x=7.05 \mathrm{~m}$

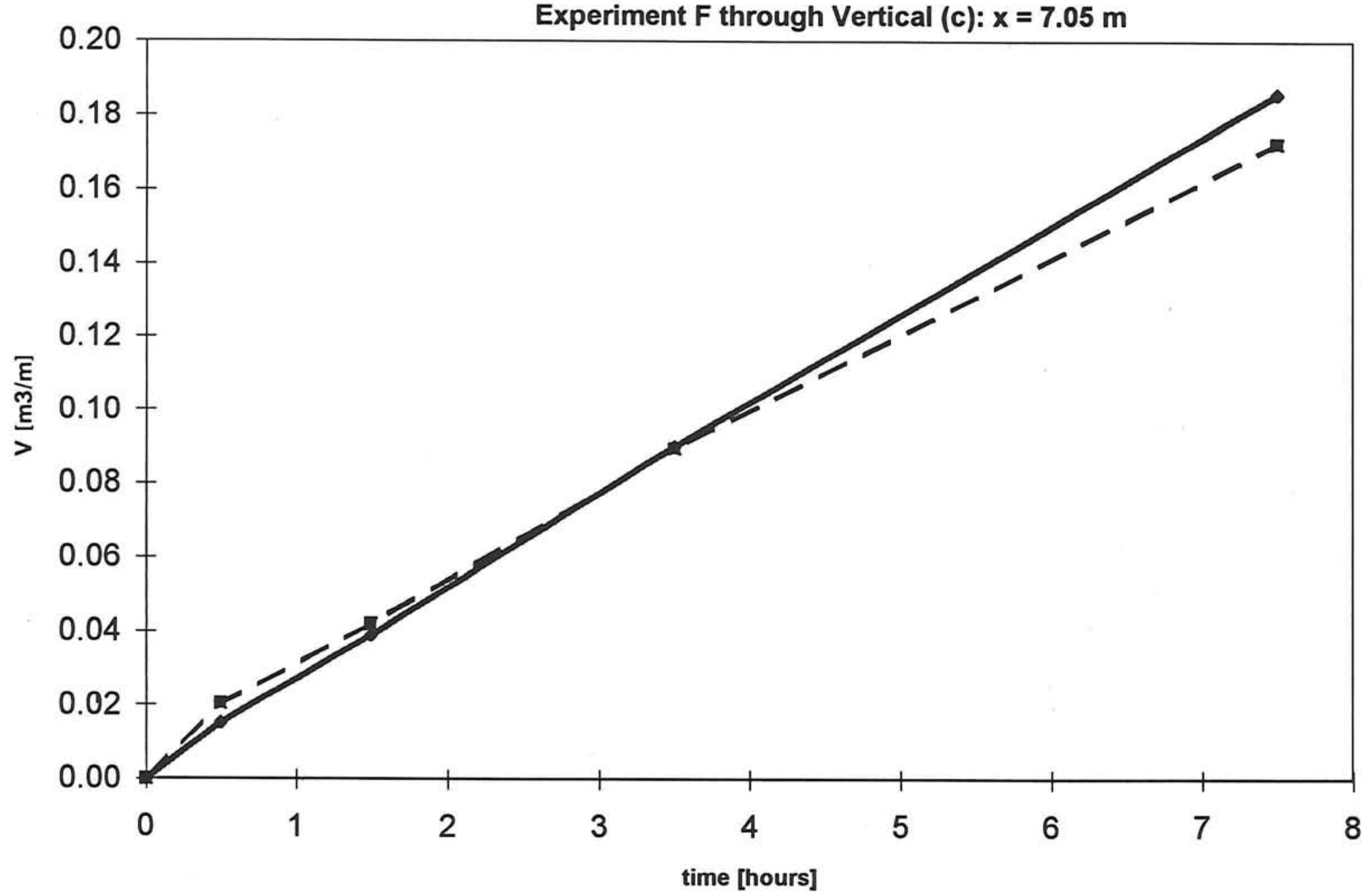

$\longrightarrow$ - with breakwater

$\longrightarrow$ without breakwater

Graph 1.59 


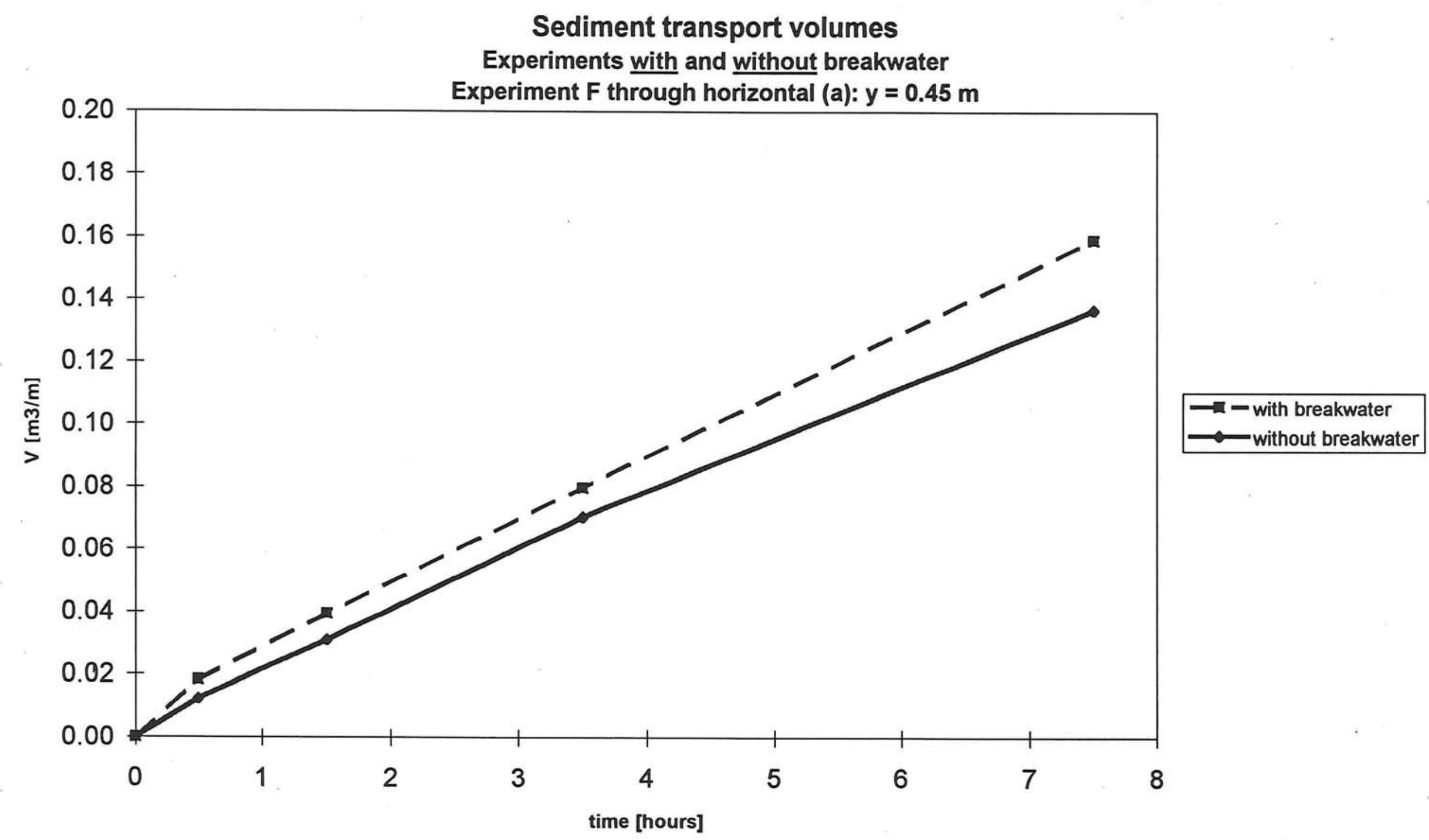

Graph 1.60 


\section{Sediment transport volumes}

Experiments with and without breakwater

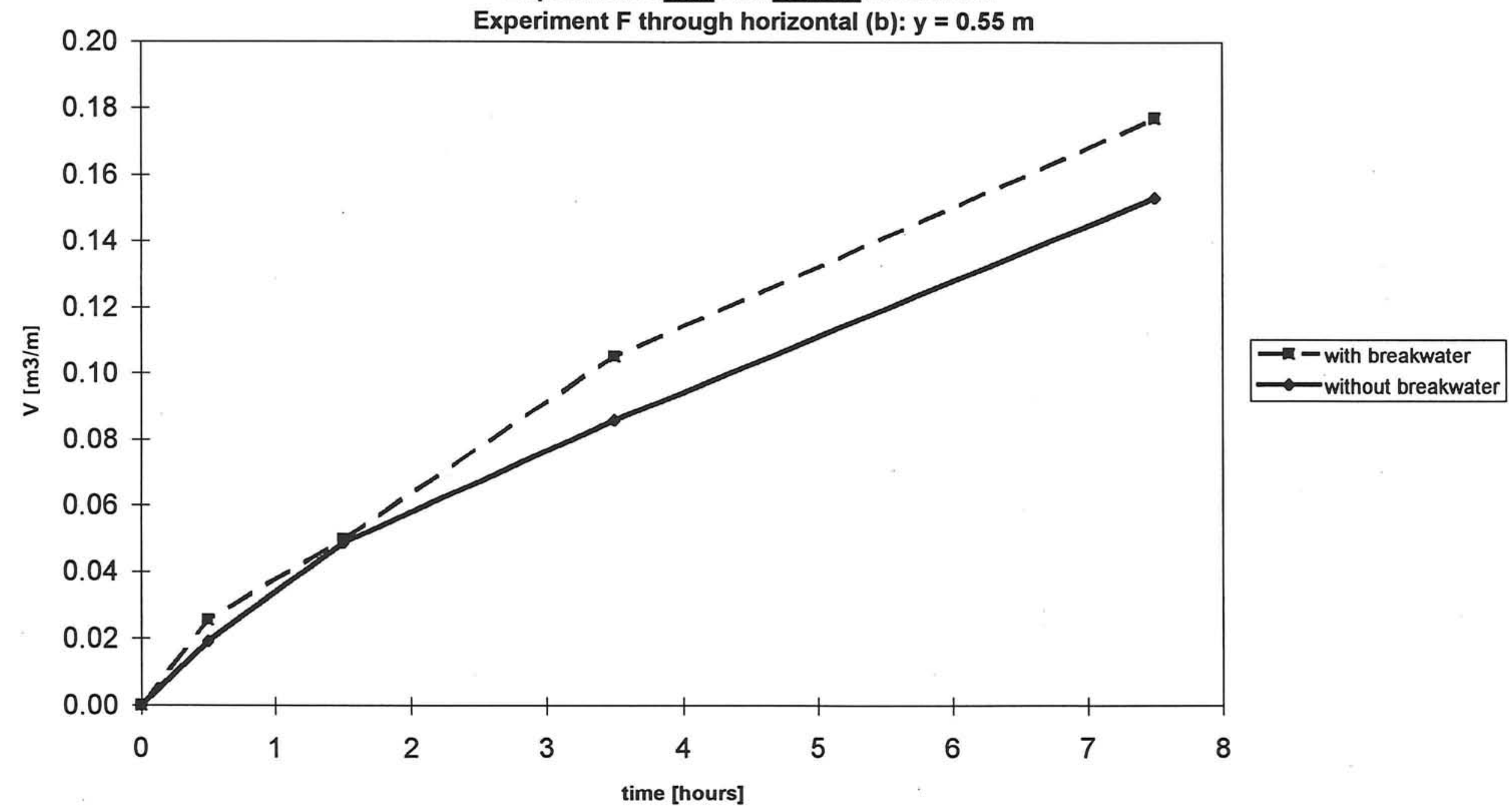

Graph 1.61 


\section{Sediment transport volumes}

Experiments with and without breakwater

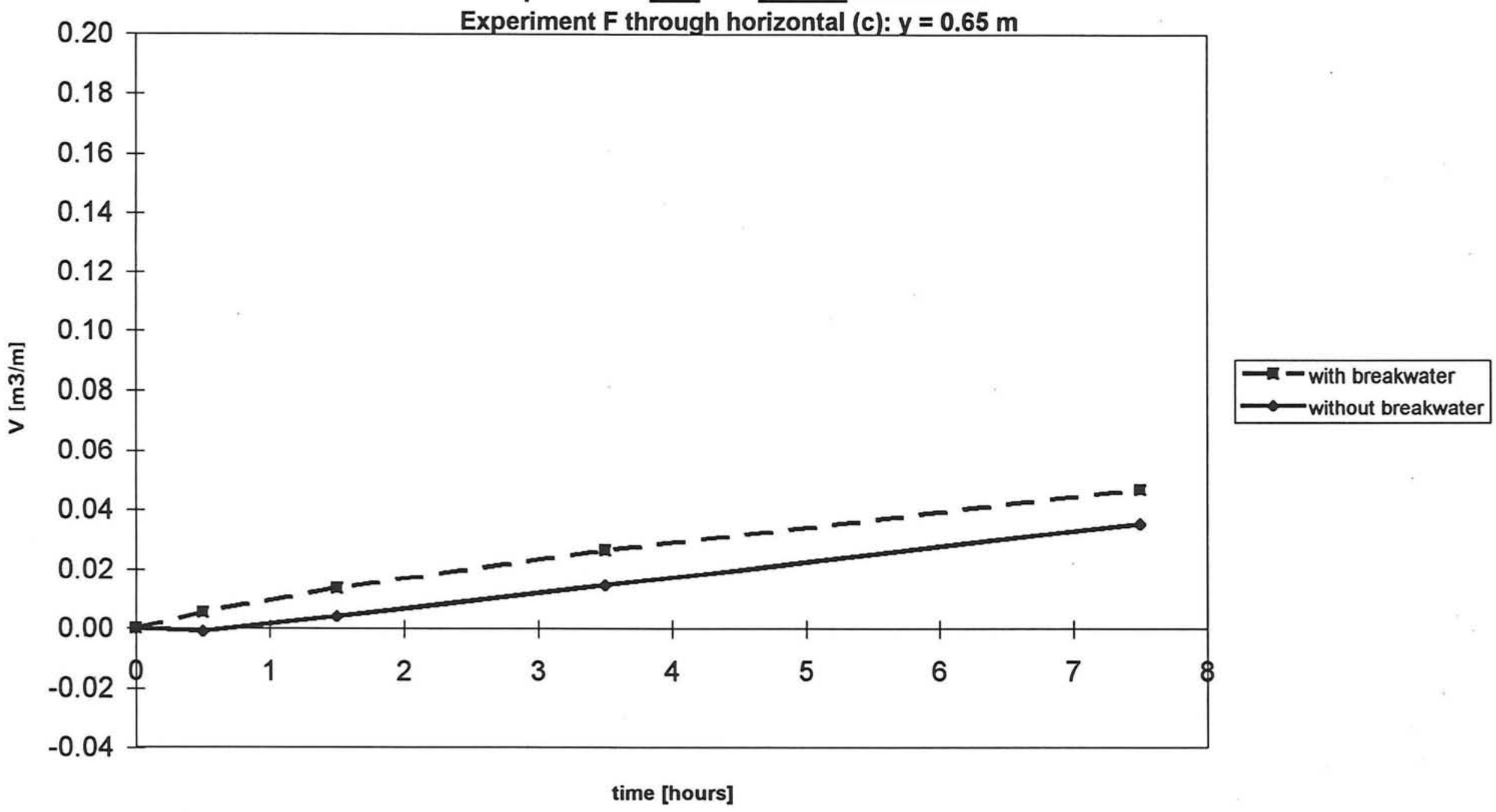

Graph 1.62 


\section{Sediment transport volumes}

Experiments without breakwater

Experiments A, B and C through Vertical (a): $x=5.25 \mathrm{~m}$

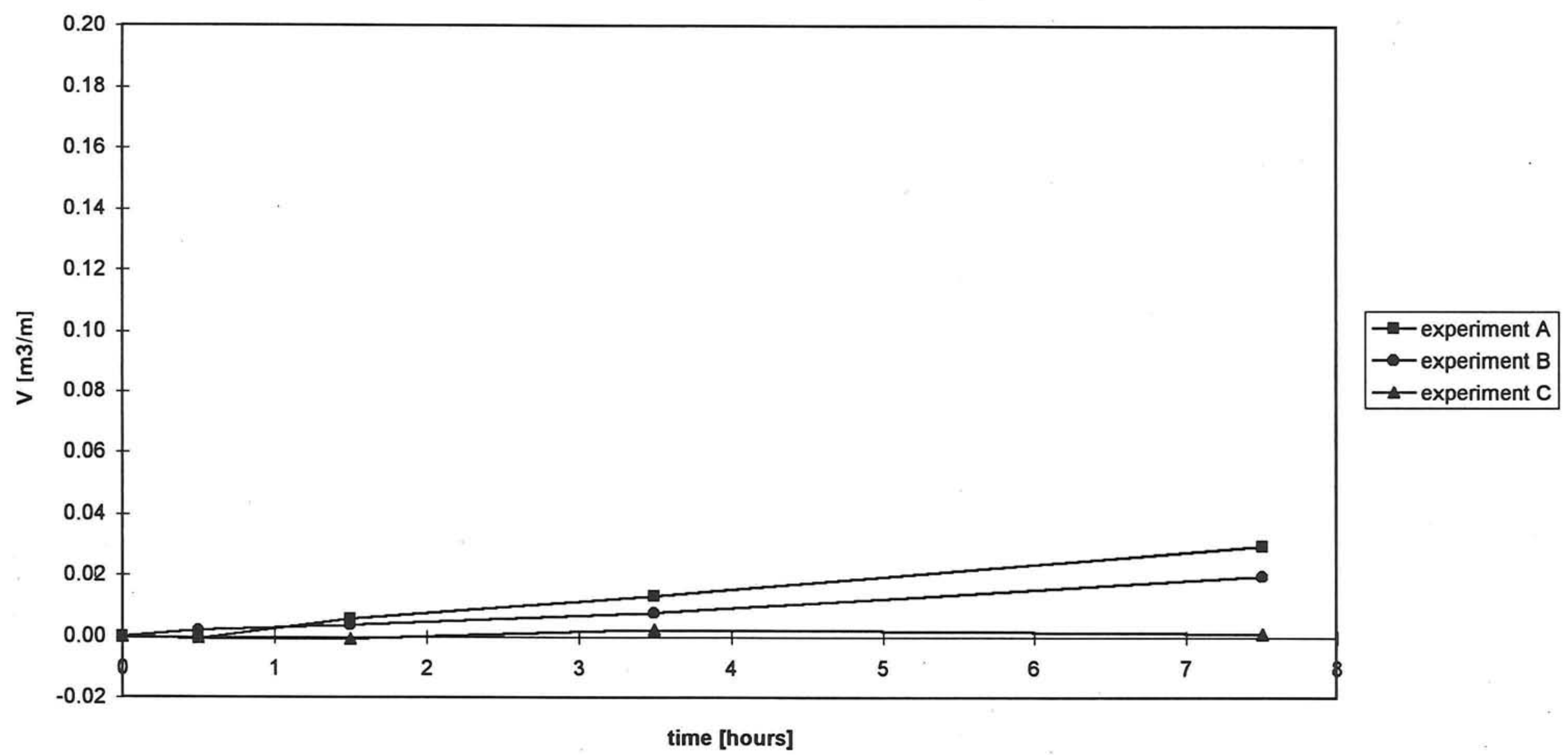

Graph 1.63 


\section{Sediment transport volumes}

Experiments without breakwater

Experiments A, B and C through Vertical (b): $x=6.15 \mathrm{~m}$

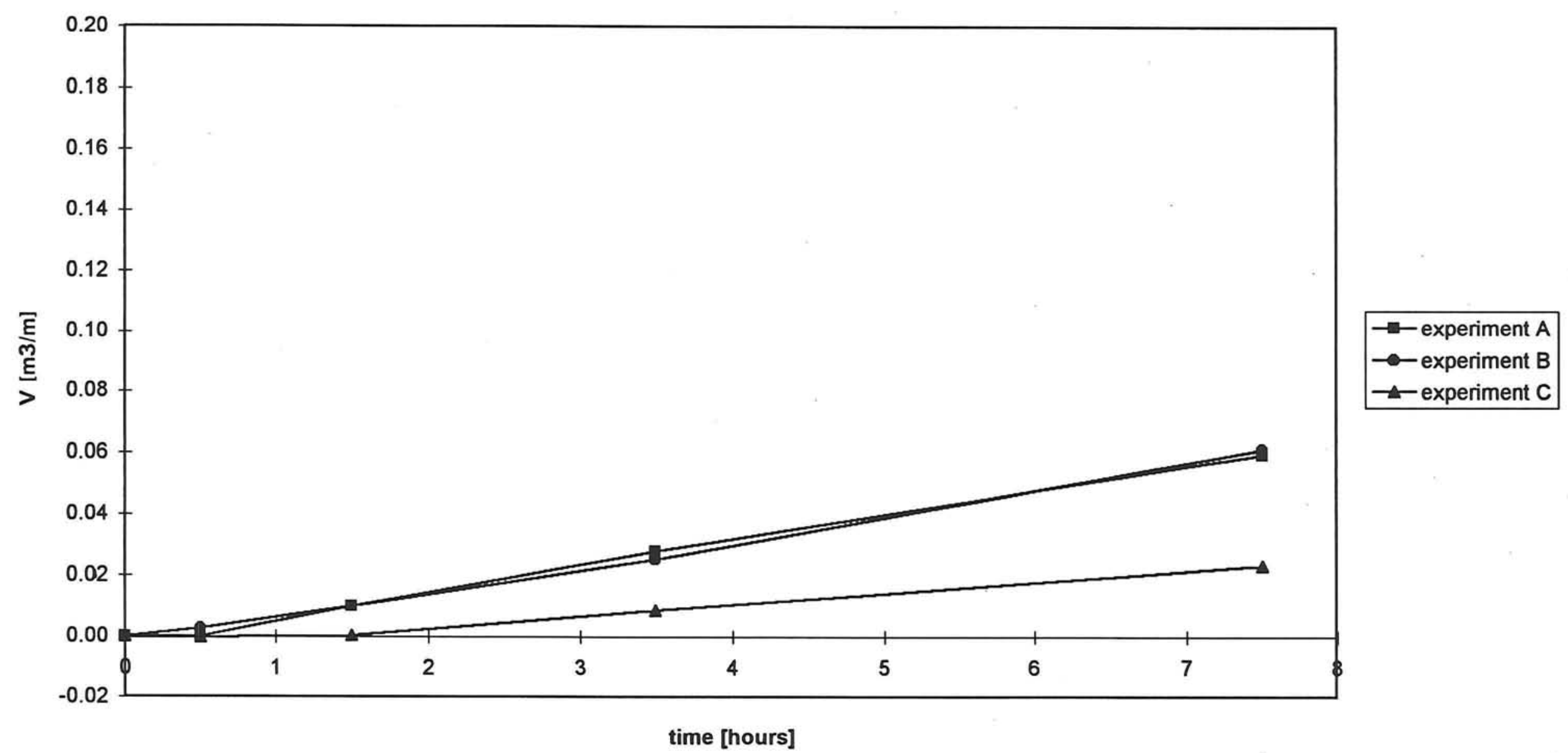

Graph 1.64 


\section{Sediment transport volumes}

Experiments without breakwater

Experiments A, B and C through Vertical (c): $x=7.05 \mathrm{~m}$

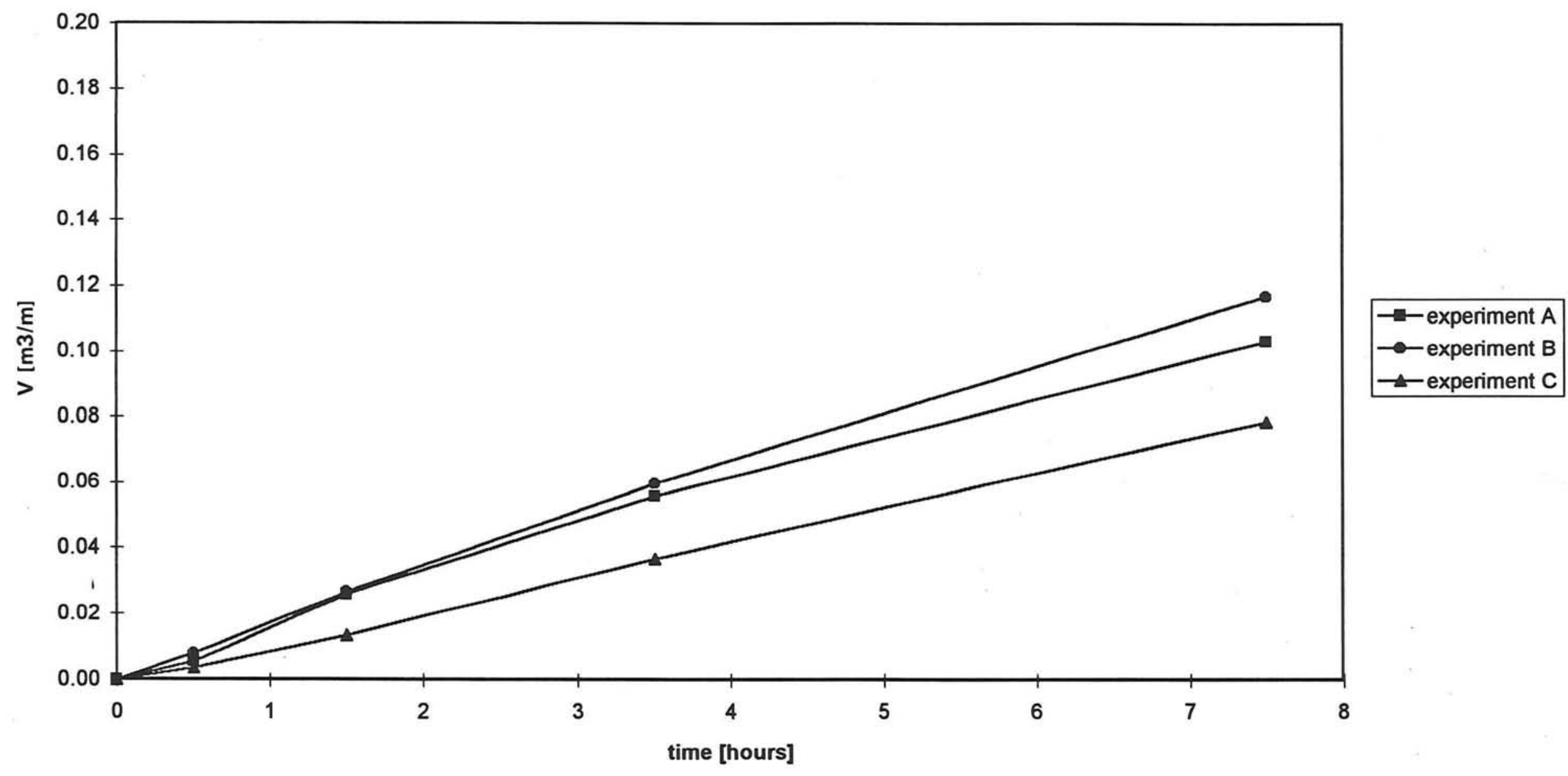

Graph 1.65 


\section{Sediment transport volumes}

Experiments without breakwater

Experiments A, B and C through horizontal (a): $y=0.45 \mathrm{~m}$

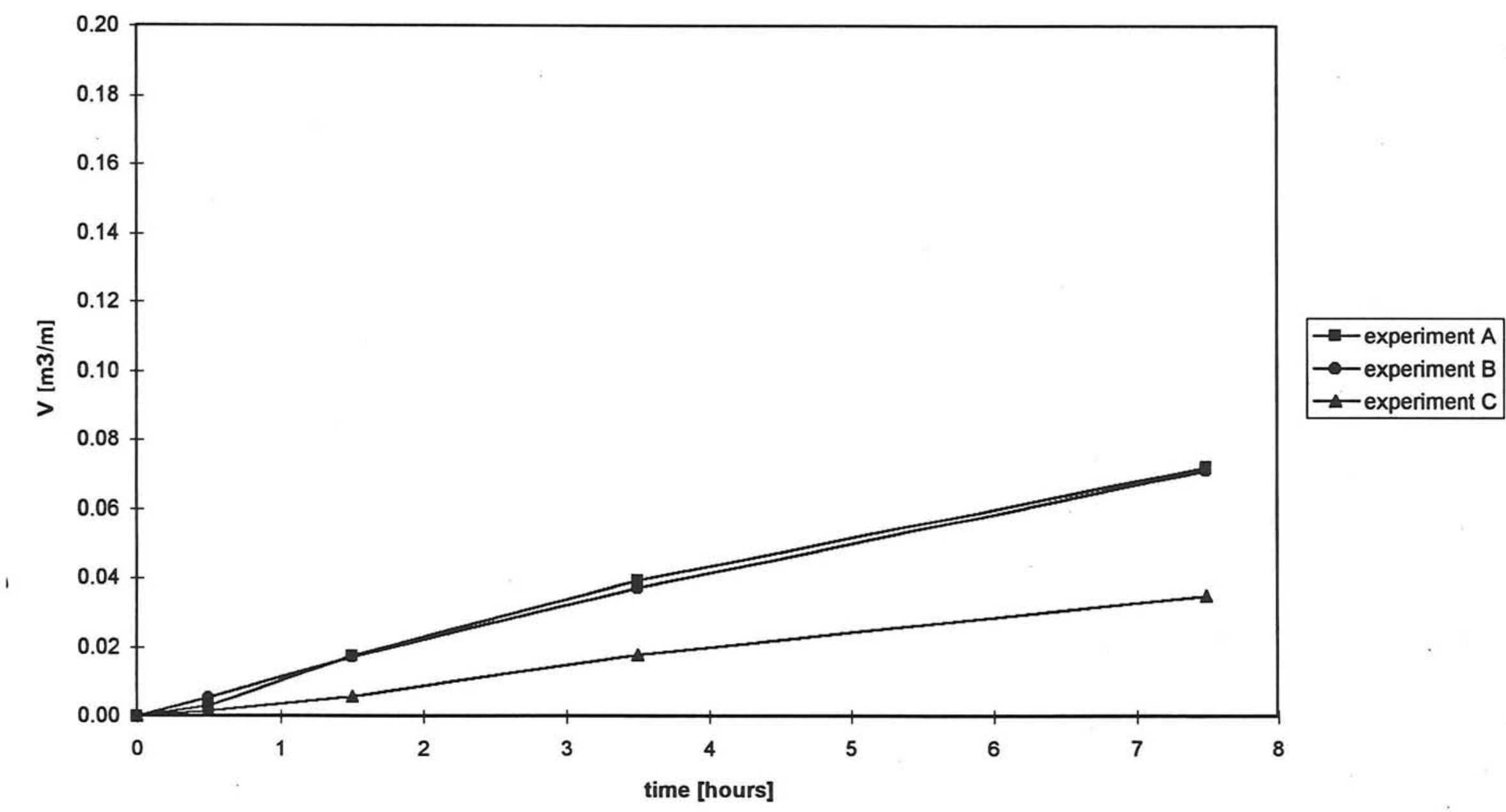

Graph 1.66 


\section{Sediment transport volumes}

Experiments without breakwater

Experiments A, B and C through horizontal (b): $y=0.55 \mathrm{~m}$

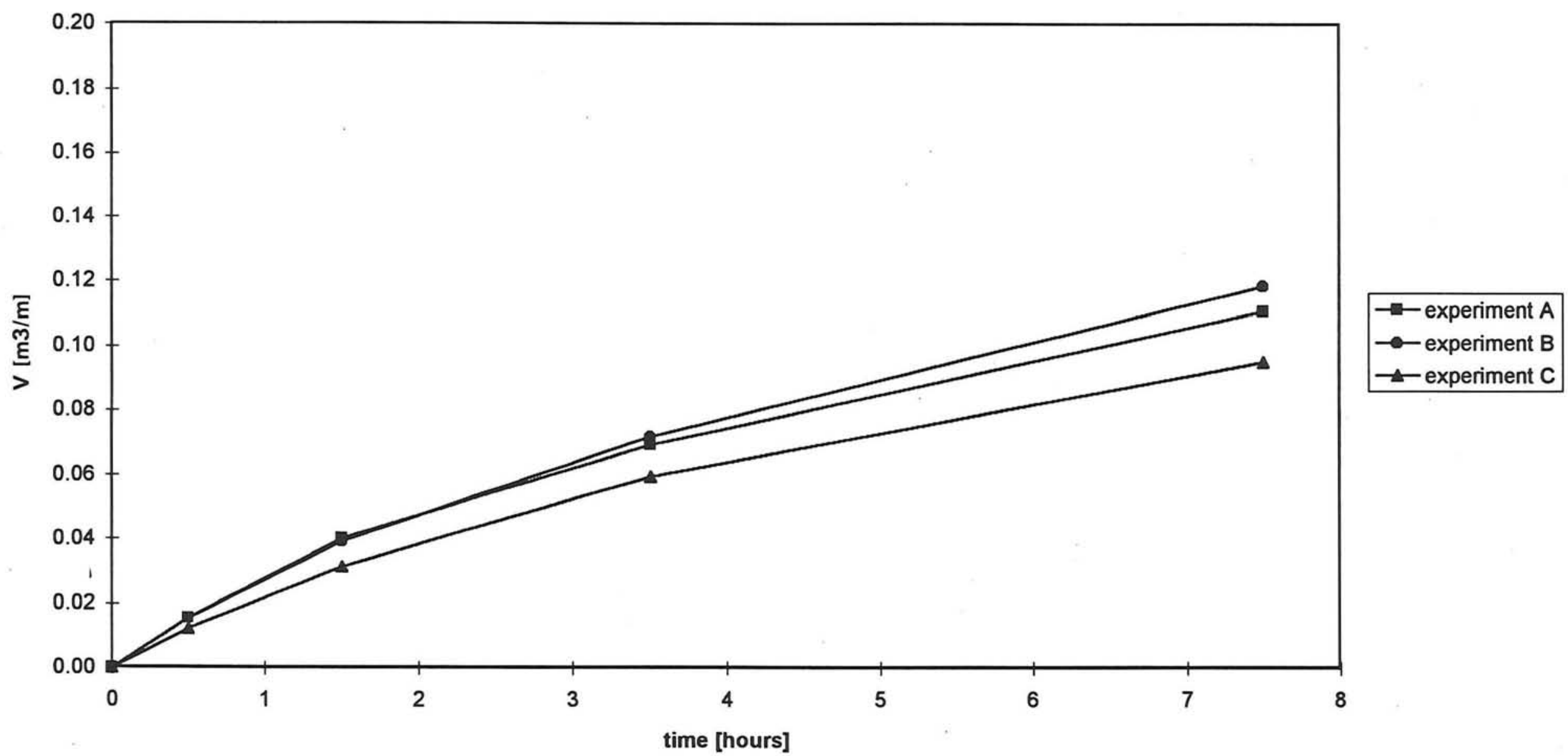

Graph 1.67 


\section{Sediment transport volumes}

Experiments without breakwater

Experiments A, B and C through horizontal (c): $y=0.65 \mathrm{~m}$

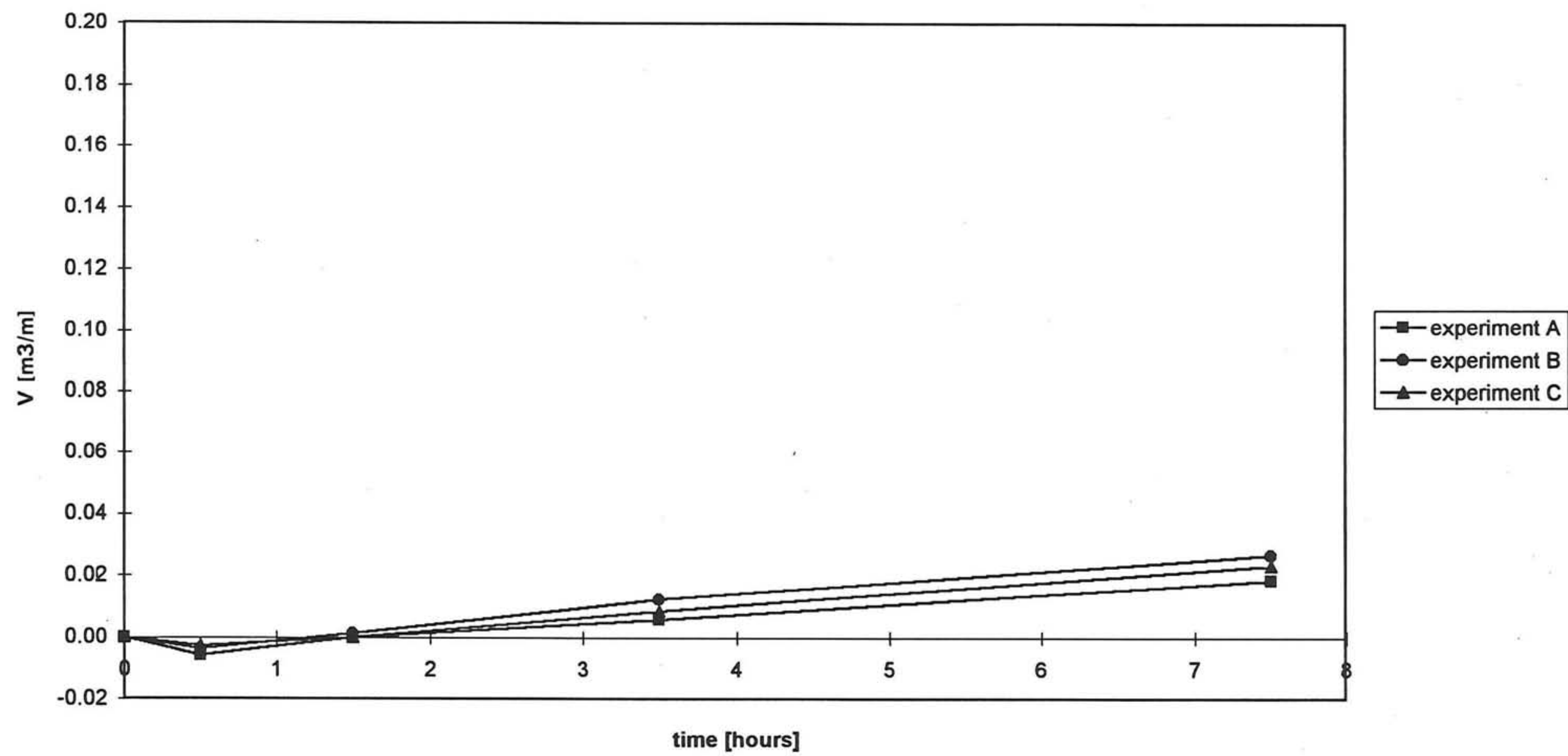

Graph 1.68 


\section{Sediment transport volumes}

Experiments with breakwater

Experiments A, B and C through Vertical (a): $x=5.25 \mathrm{~m}$

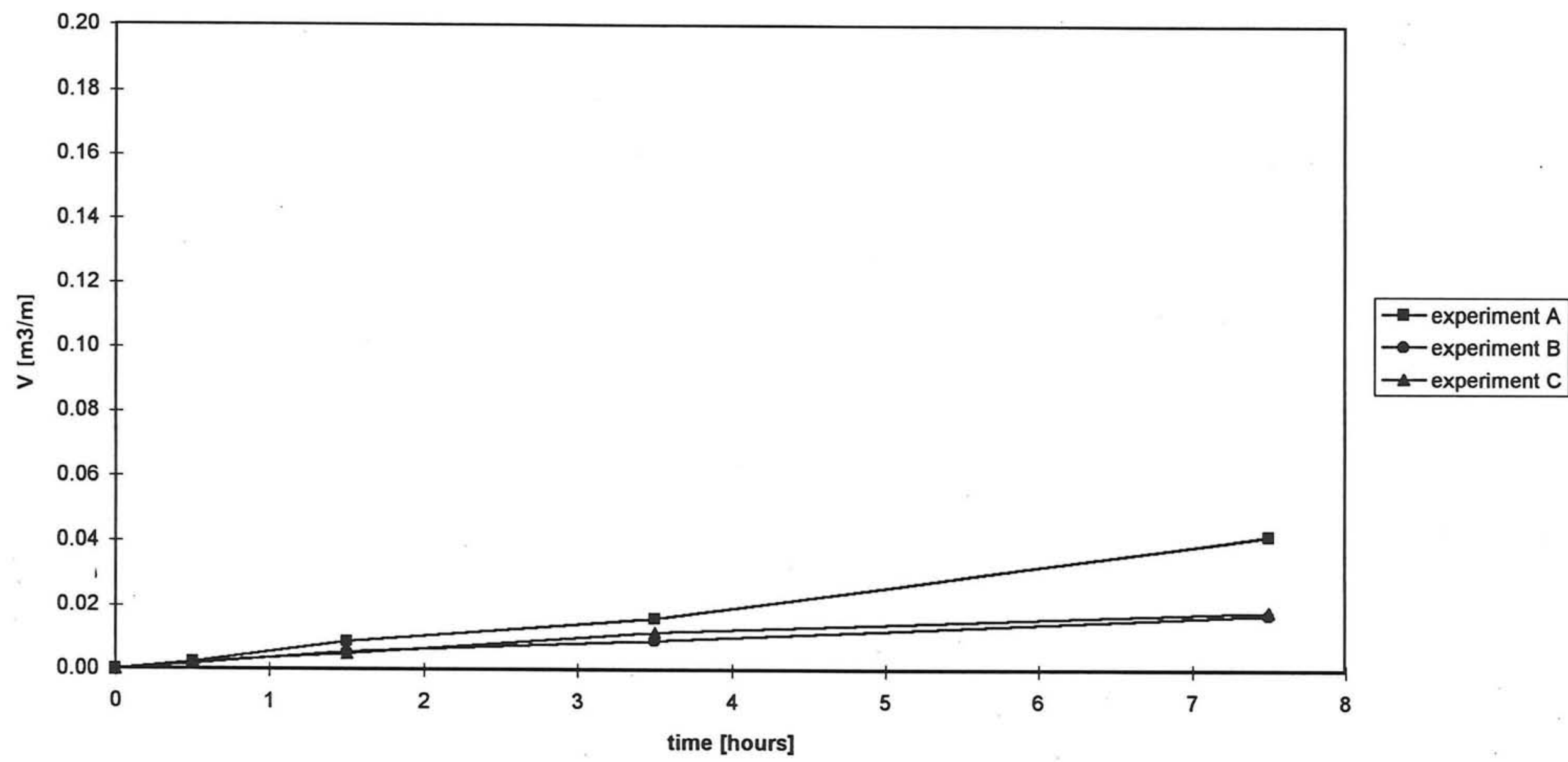

Graph 1.69 


\section{Sediment transport volumes}

Experiments with breakwater

Experiments A, B and C through Vertical (b): $x=6.15 \mathrm{~m}$

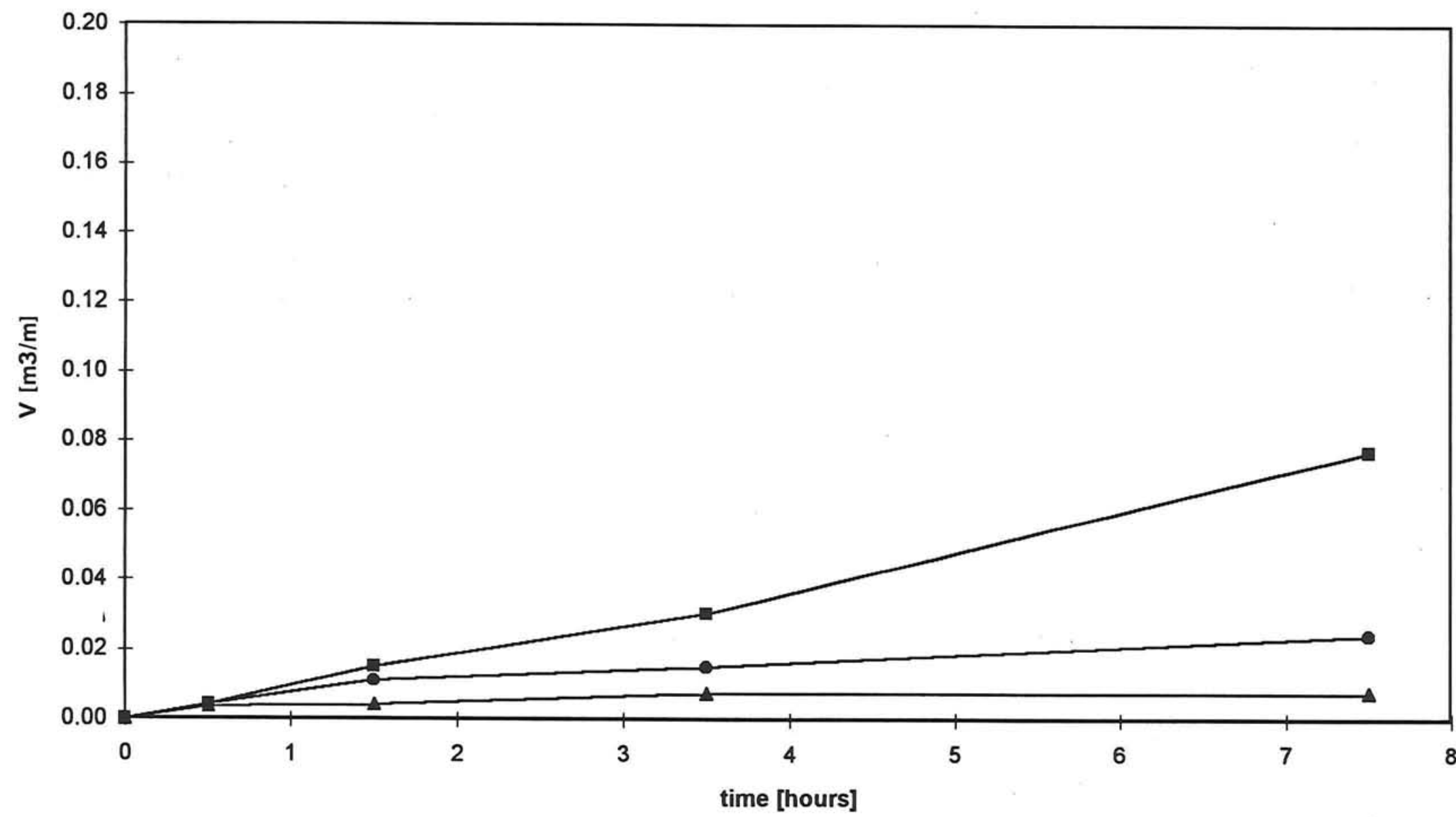

Graph 1.70 


\section{Sediment transport volumes}

Experiments with breakwater

Experiments A, B and C through Vertical (c): $x=7.05 \mathrm{~m}$

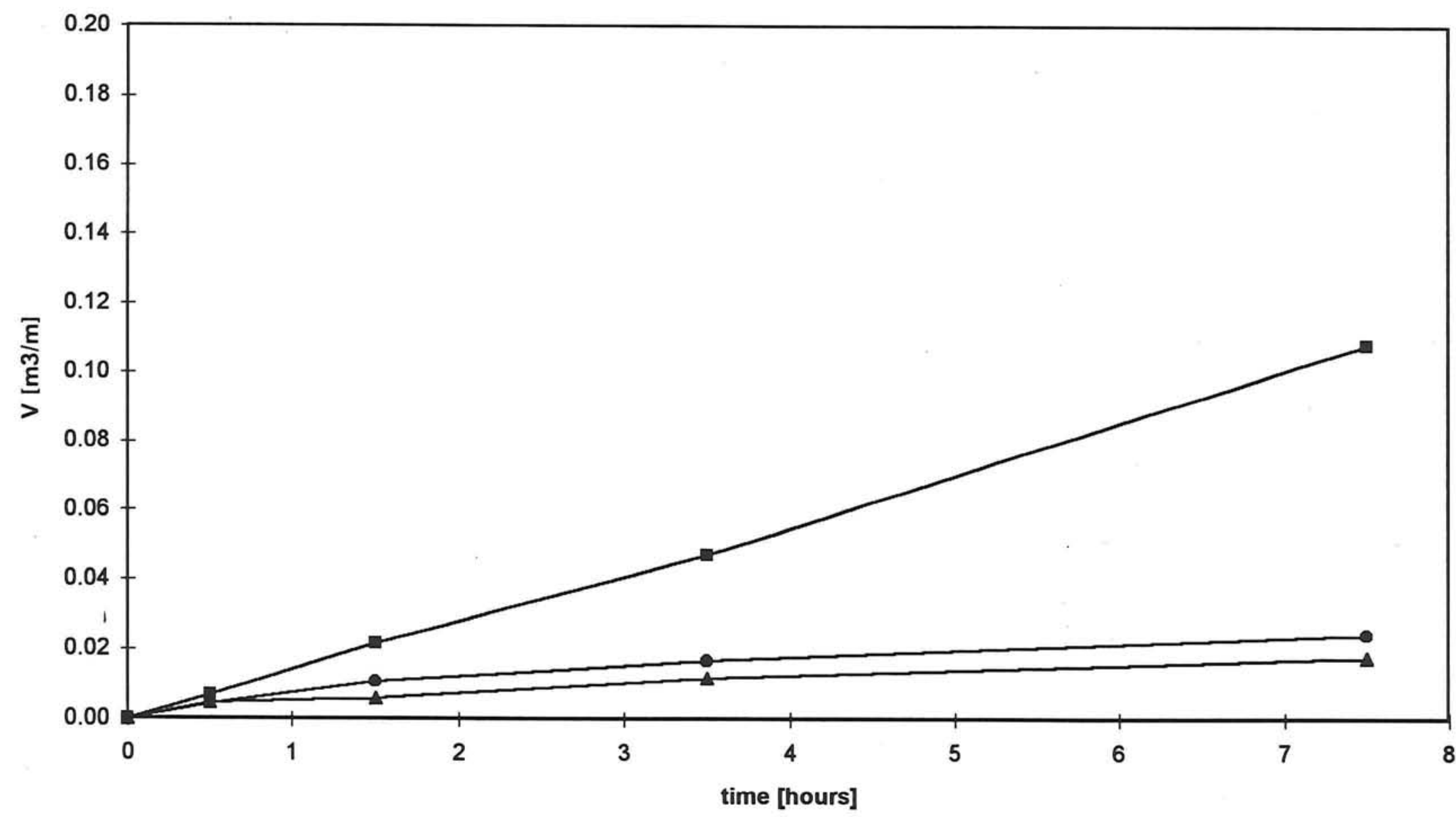




\section{Sediment transport volumes}

Experiments with breakwater

Experiments A, B and C through horizontal (a): $y=0.45 \mathrm{~m}$

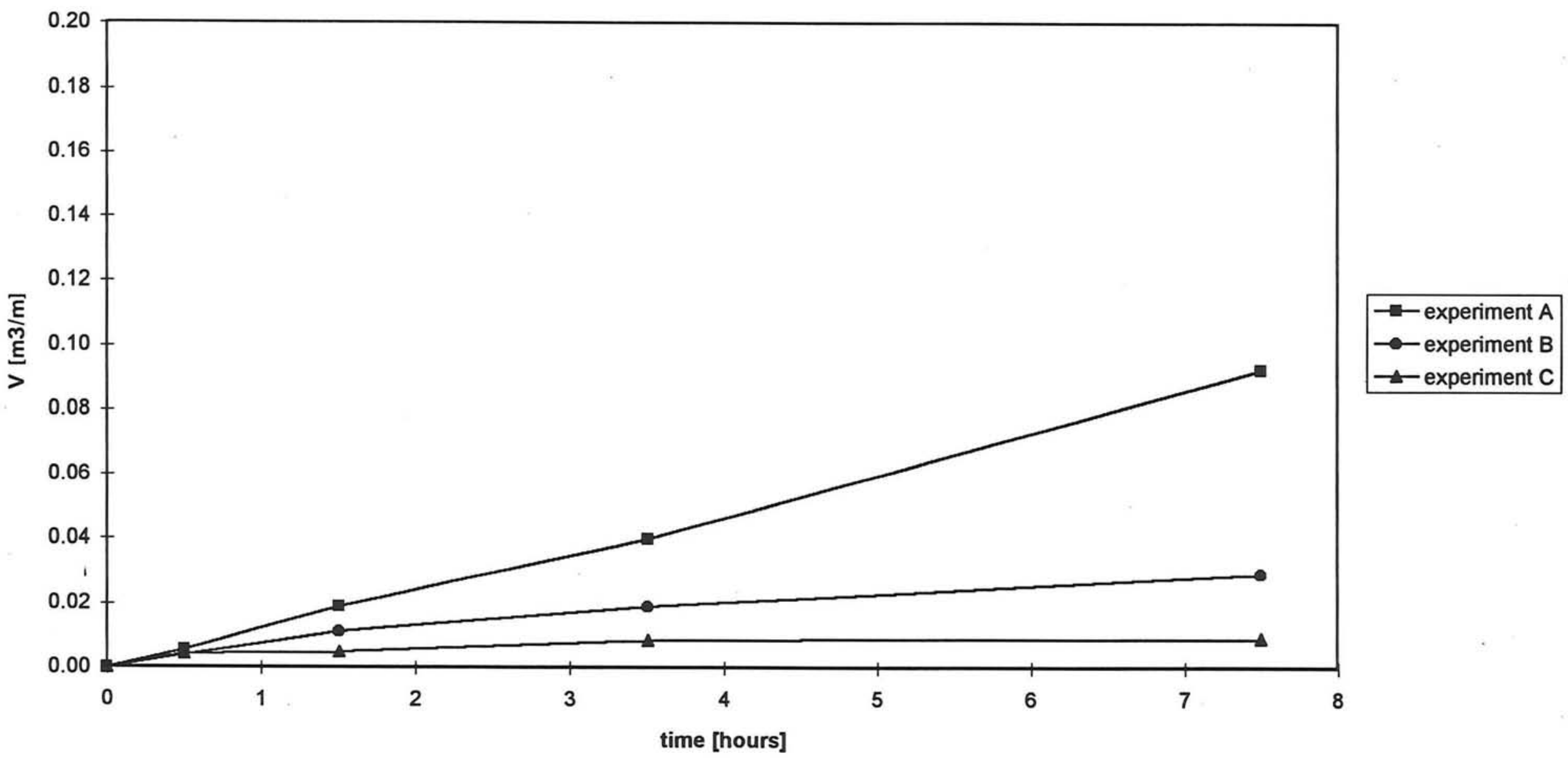

Graph 1.72 


\section{Sediment transport volumes}

Experiments with breakwater

Experiments A, B and C through horizontal (b): $y=0.55 \mathrm{~m}$

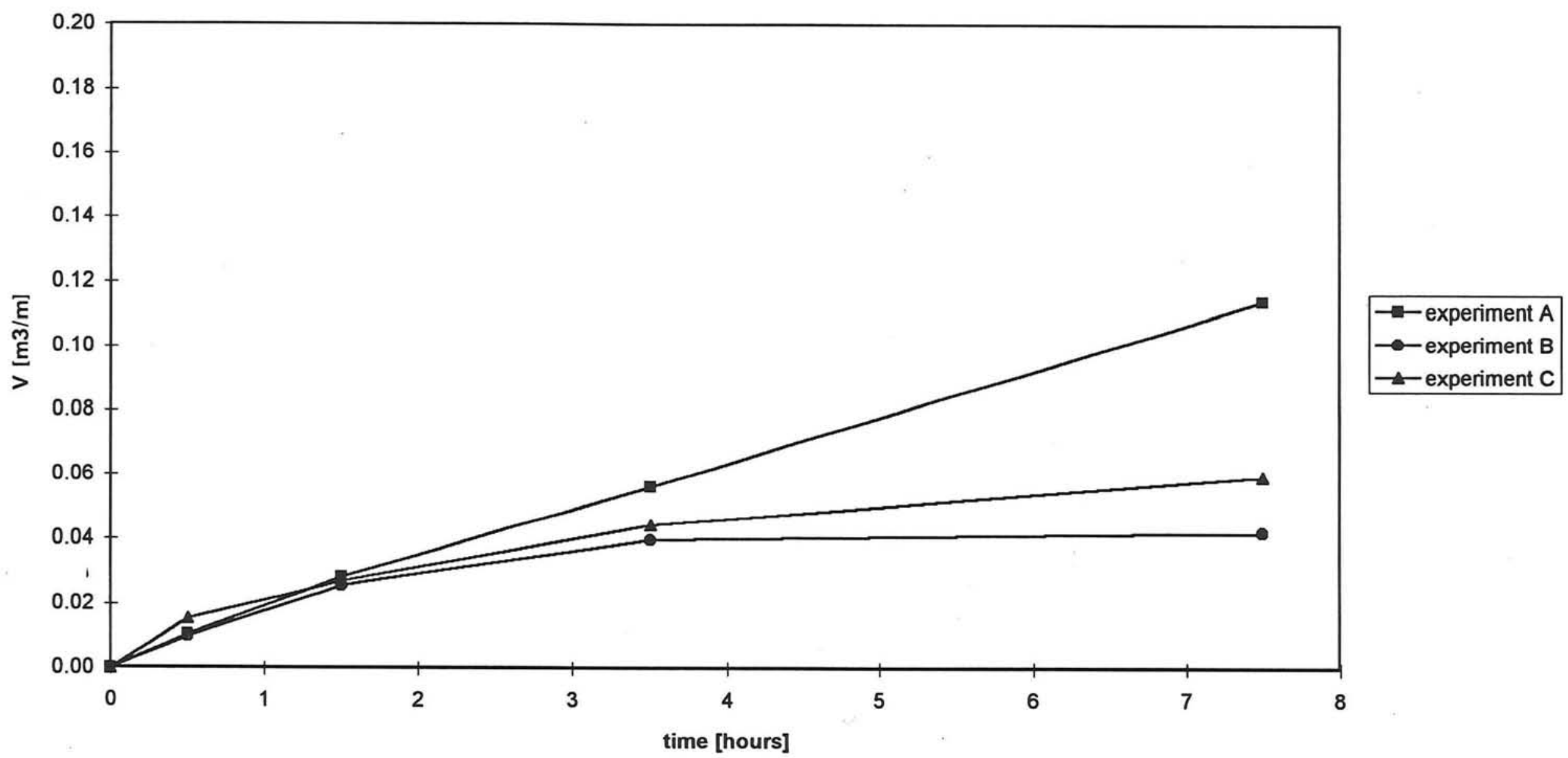

Graph 1.73 


\section{Sediment transport volumes}

Experiments with breakwater

Experiments A, B and C through horizontal (c): $y=0.65 \mathrm{~m}$

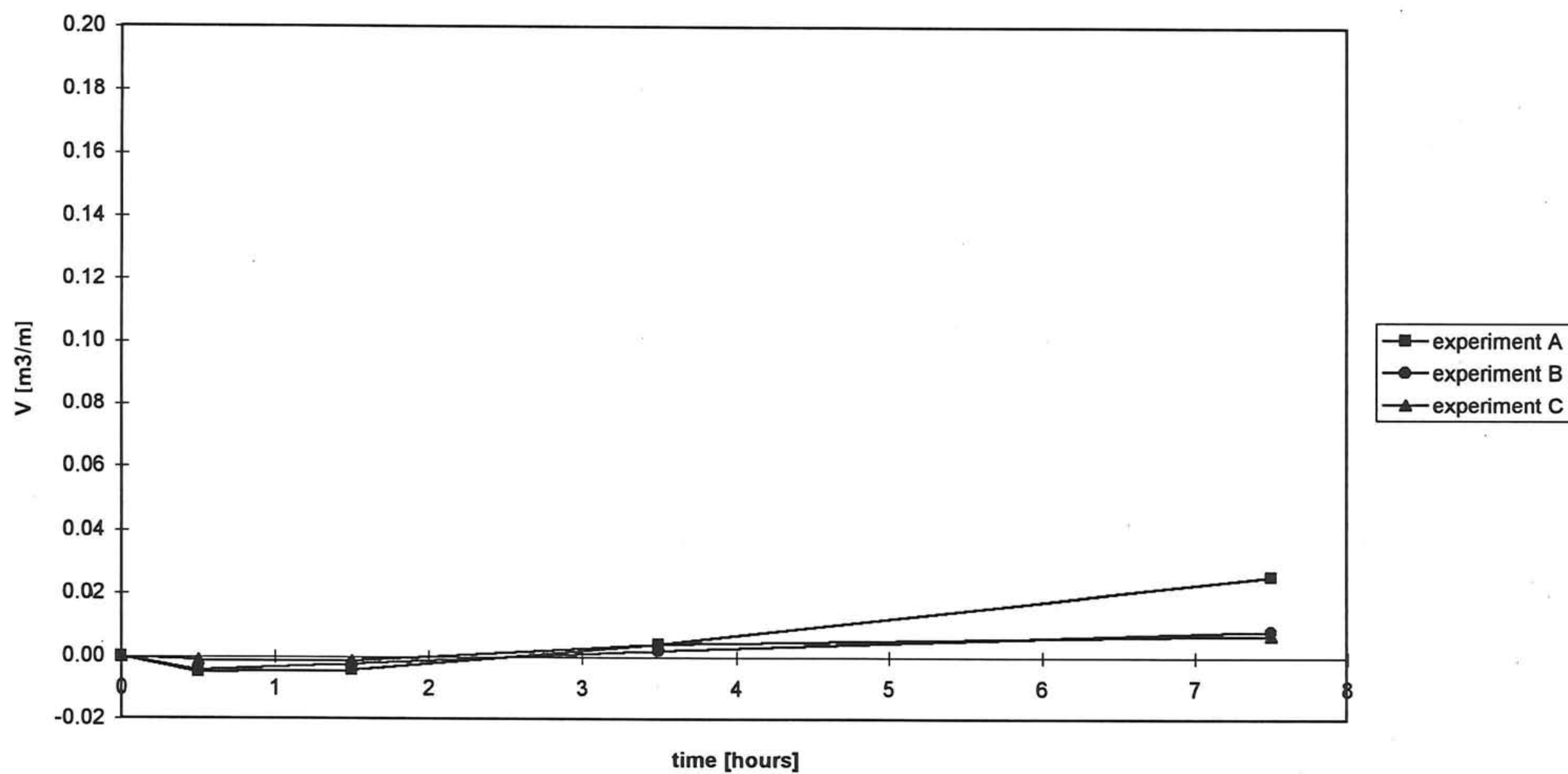

Graph 1.74 


\section{Sediment transport volumes}

Experiments without breakwater

Experiments B, D and E through Vertical (a): $x=5.25 \mathrm{~m}$

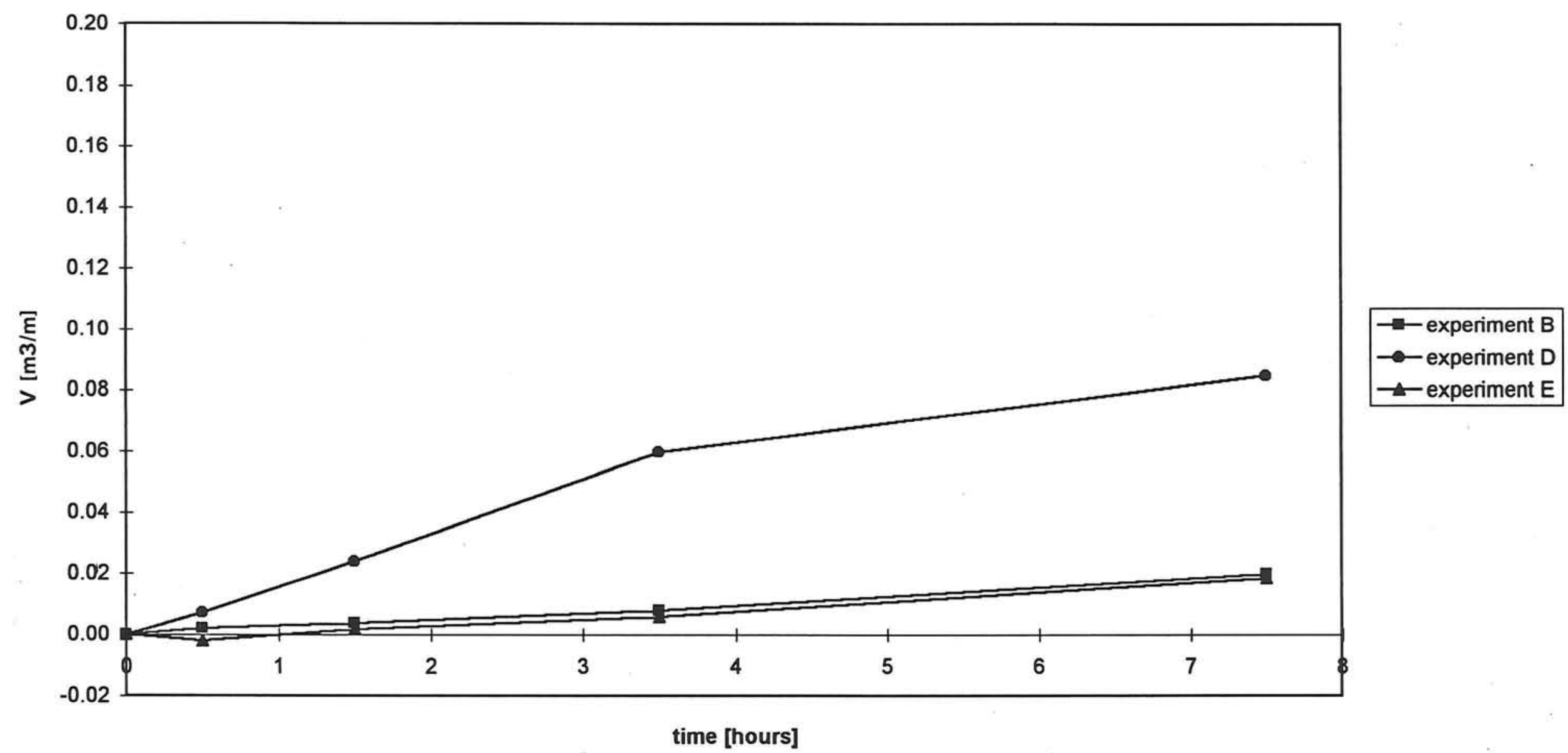

Graph 1.75 


\section{Sediment transport volumes}

Experiments without breakwater

Experiments $B, D$ and $E$ through Vertical (b): $x=6.15 \mathrm{~m}$

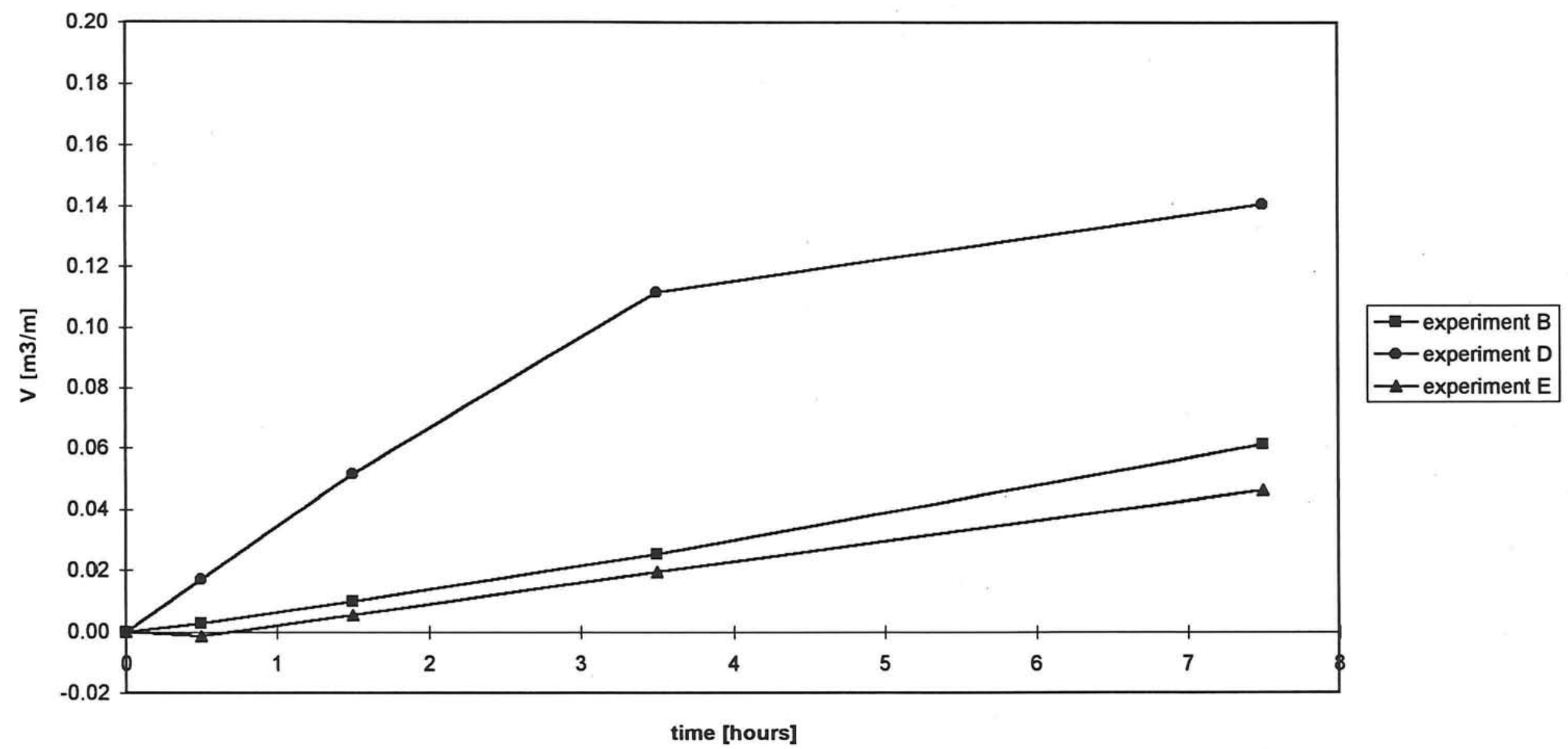

Graph 1.76 
Sediment transpert volumes

Experiments without breakwater

Experiments $B, D$ and $E$ through horizontal (a): $y=0.45 \mathrm{~m}$

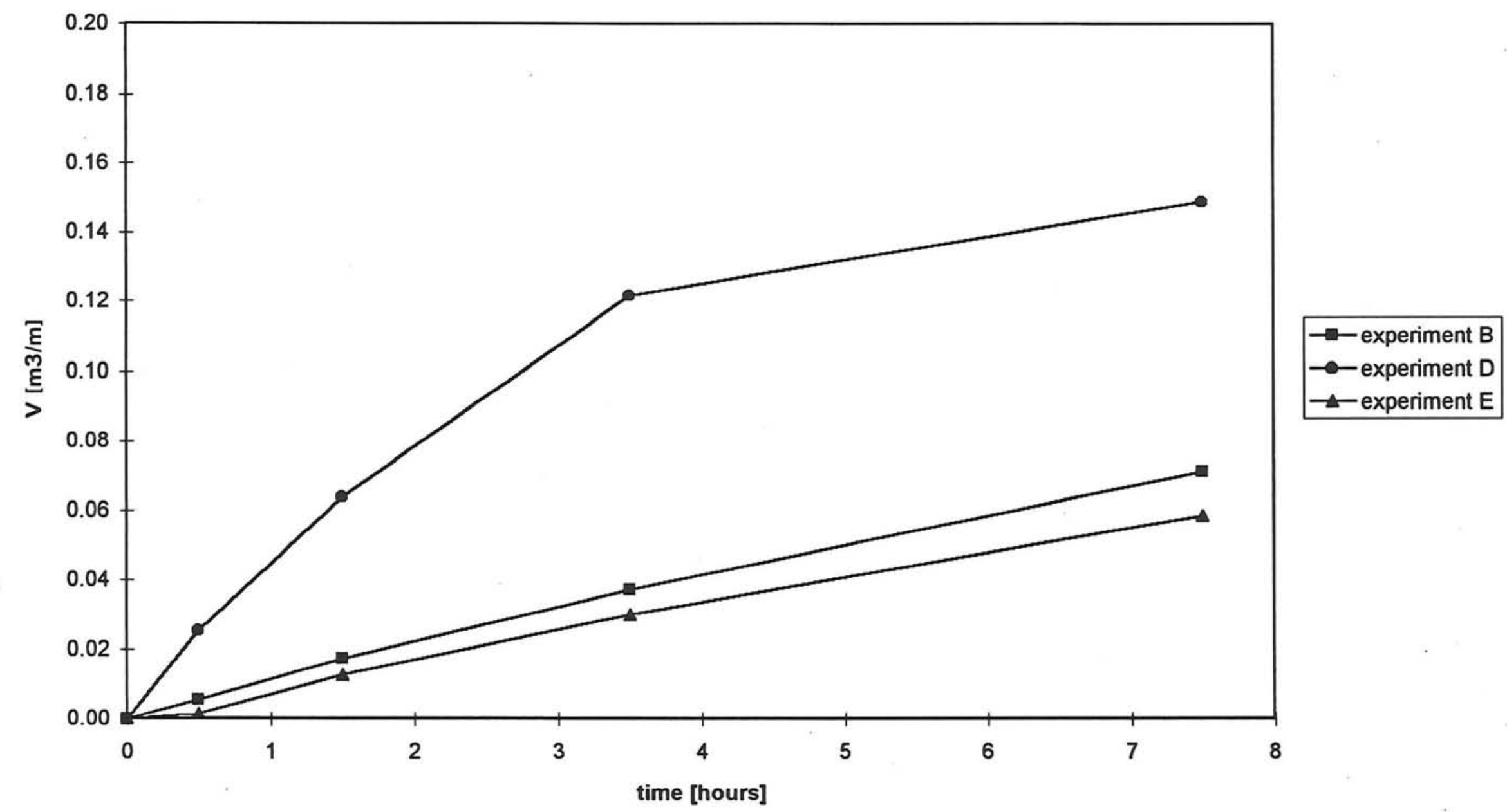

Graph 1.78 


\section{Sediment transport volumes}

Experiments without breakwater

Experiments $B, D$ and $E$ through horizontal (b): $y=0.55 \mathrm{~m}$

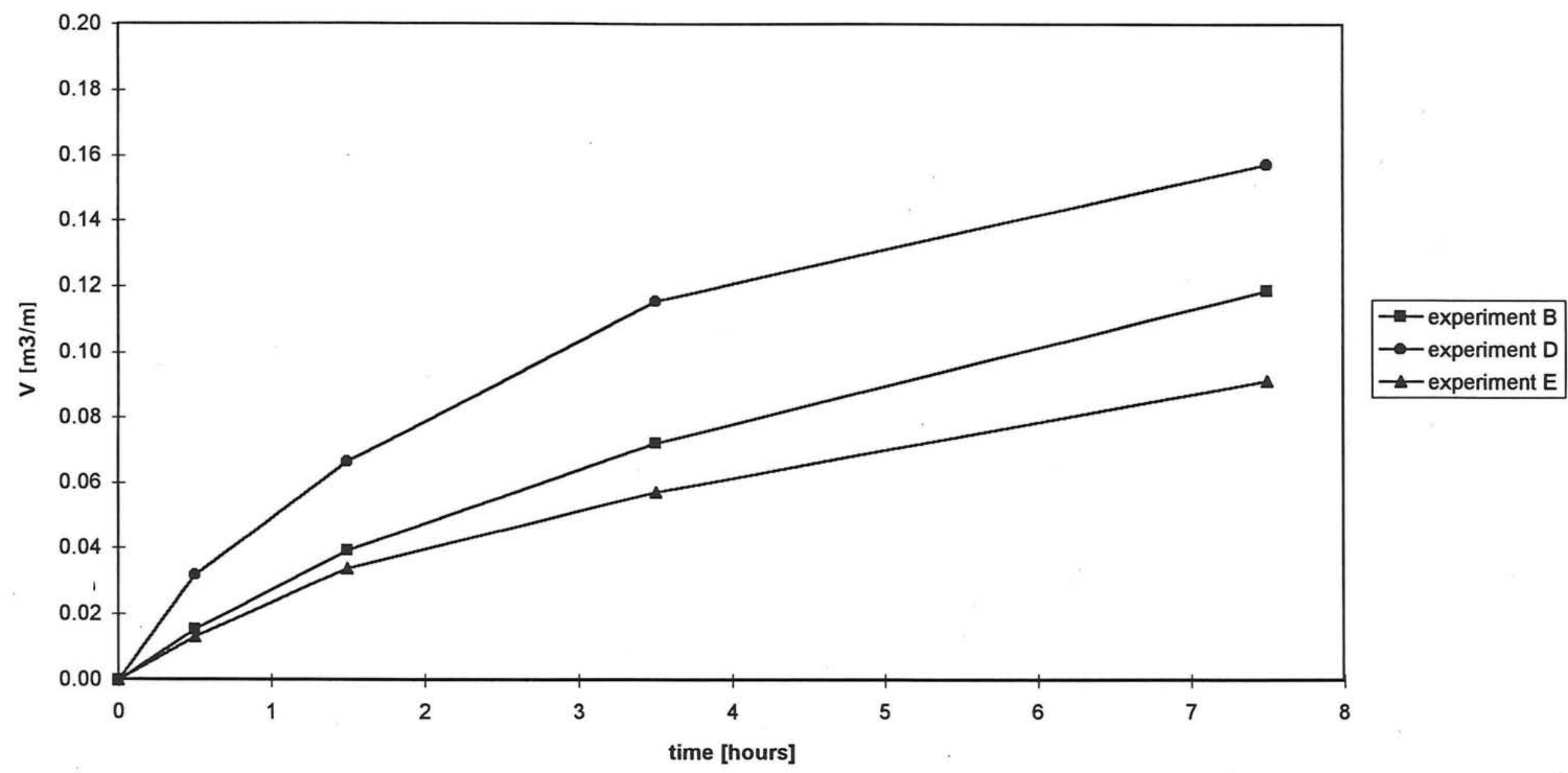

Graph 1.79 


\section{Sediment transport volumes}

Experiments without breakwater

Experiments $B, D$ and $E$ through horizontal (c): $y=0.65 \mathrm{~m}$

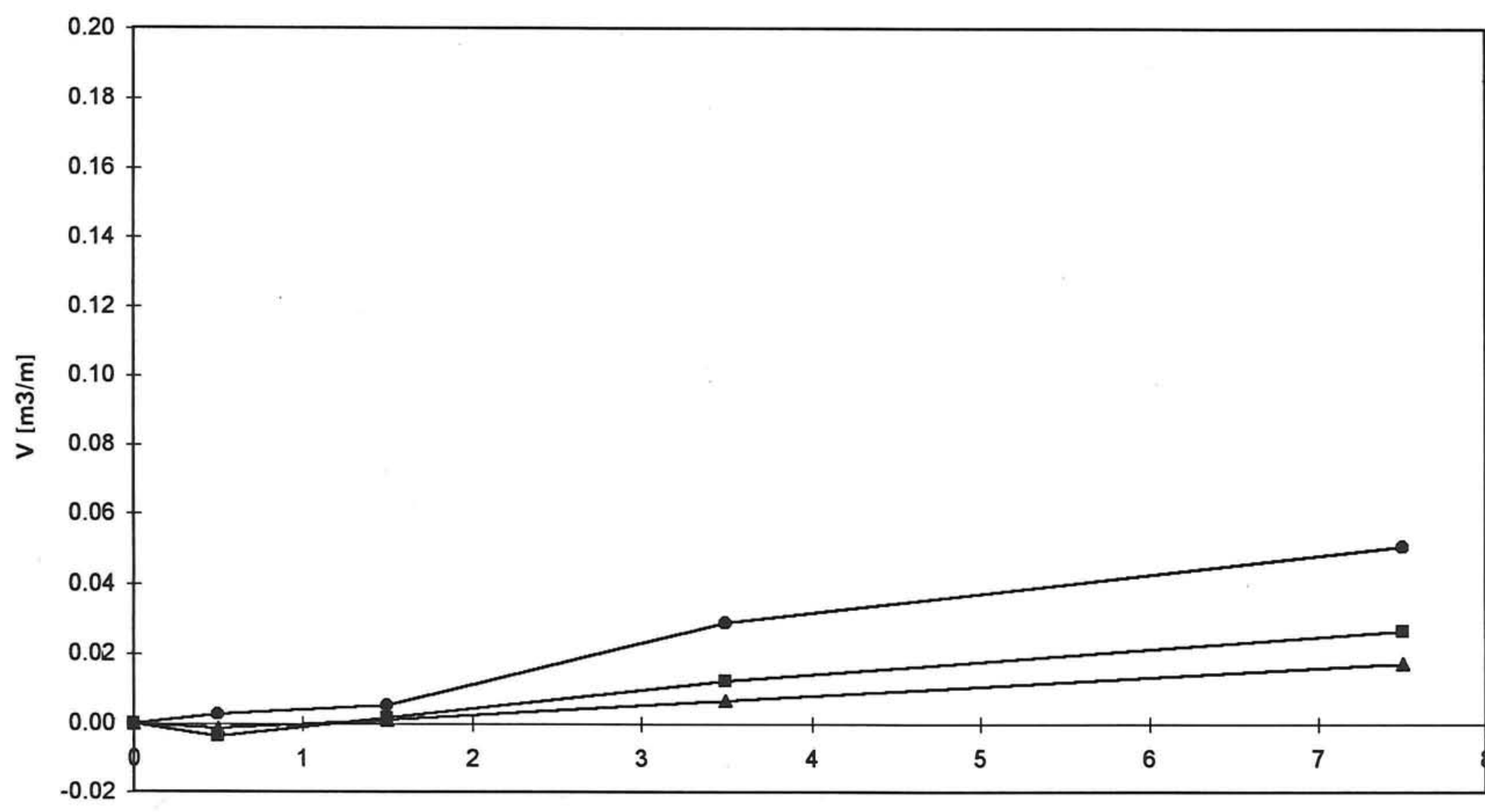

time [hours] 


\section{Sediment transport volumes}

Experiments with breakwater

Experiments B, D and E through Vertical (a): $x=5.25 \mathrm{~m}$

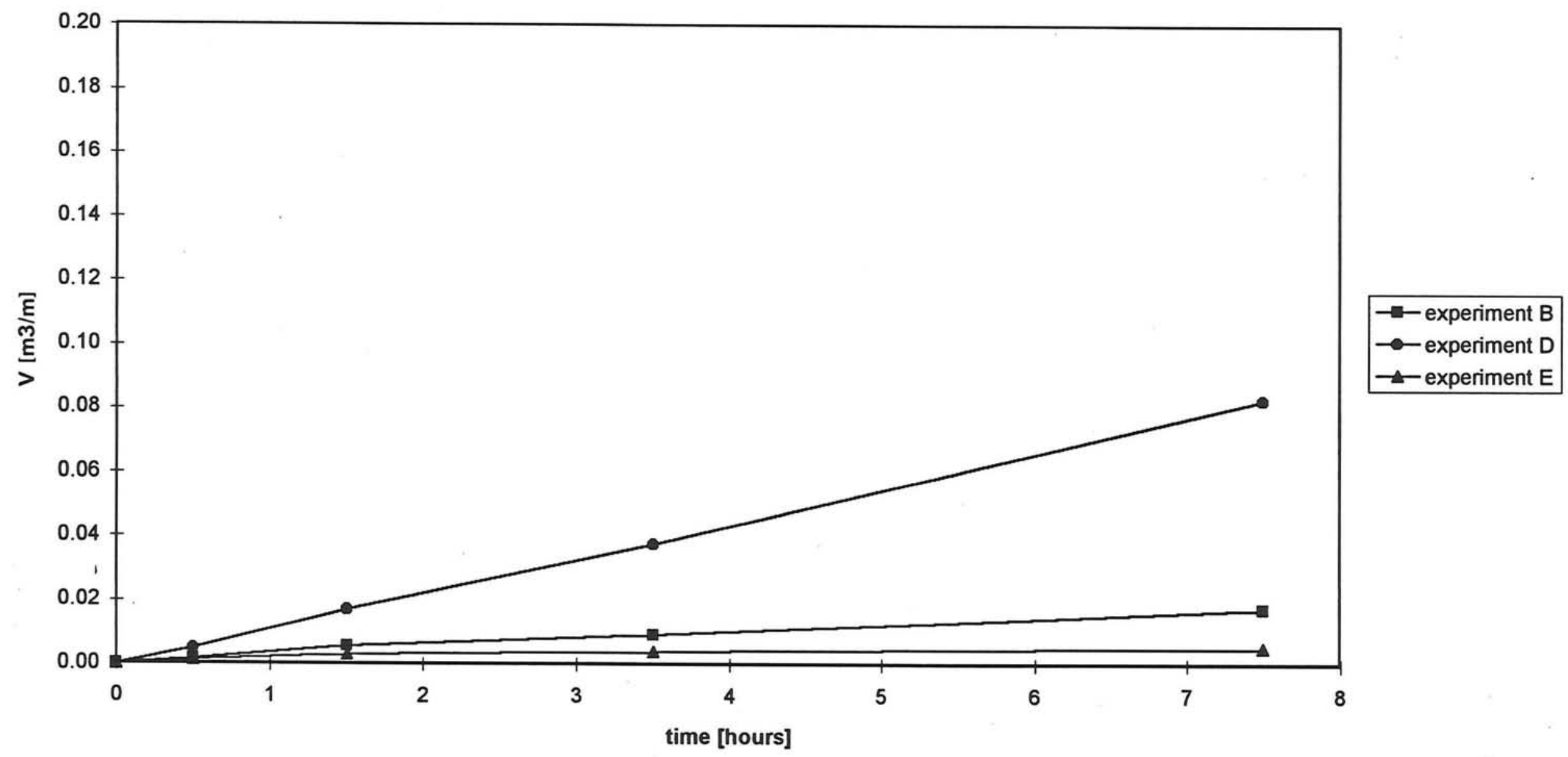

Graph 1.81 


\section{Sediment transport volumes}

Experiments with breakwater

Experiments B, D and E through Vertical (b): $x=6.15 \mathrm{~m}$

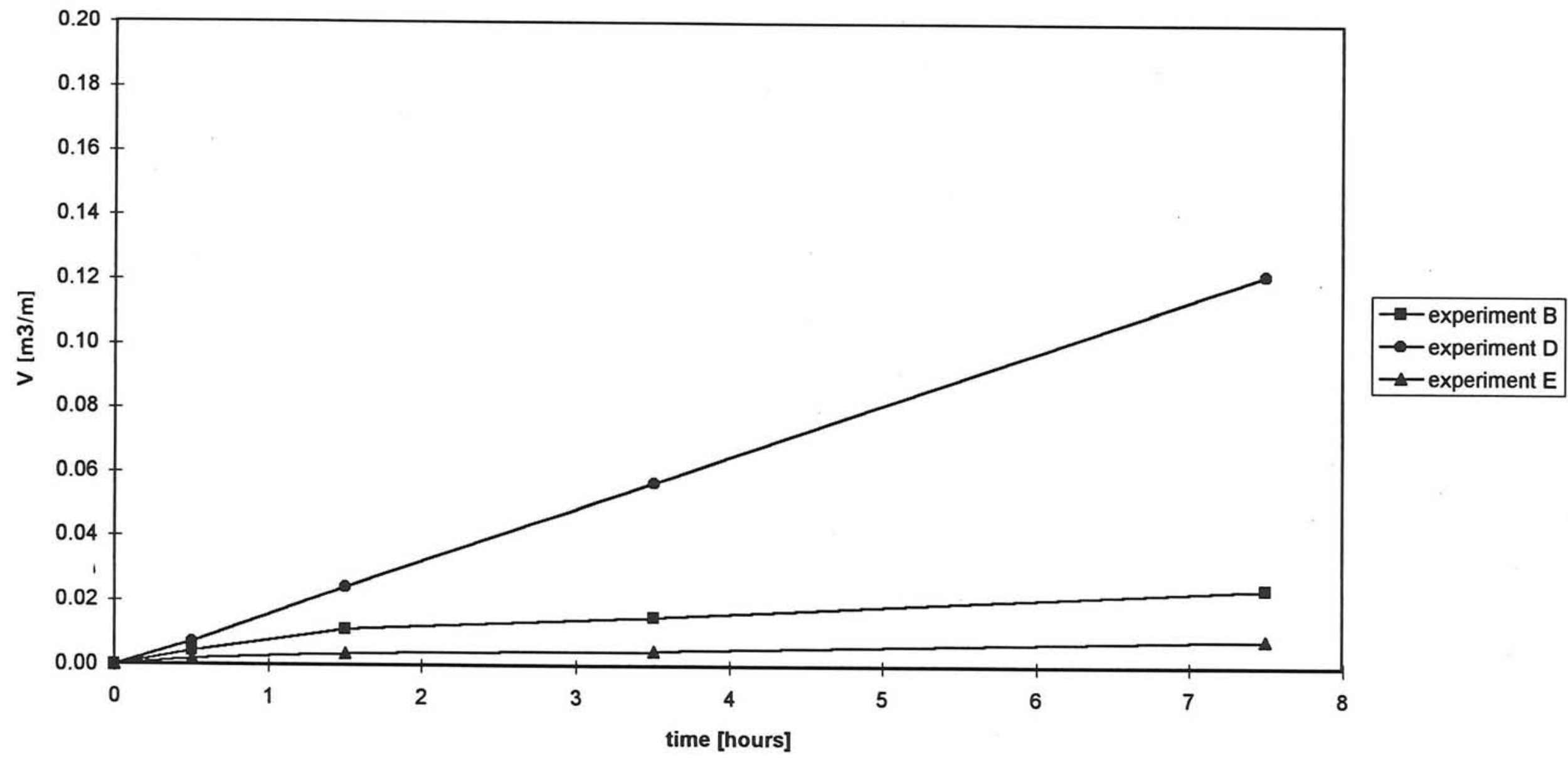

Graph 1.82 


\section{Sediment transport volumes}

Experiments with breakwater

Experiments B, D and E through Vertical (c): $x=7.05 \mathrm{~m}$

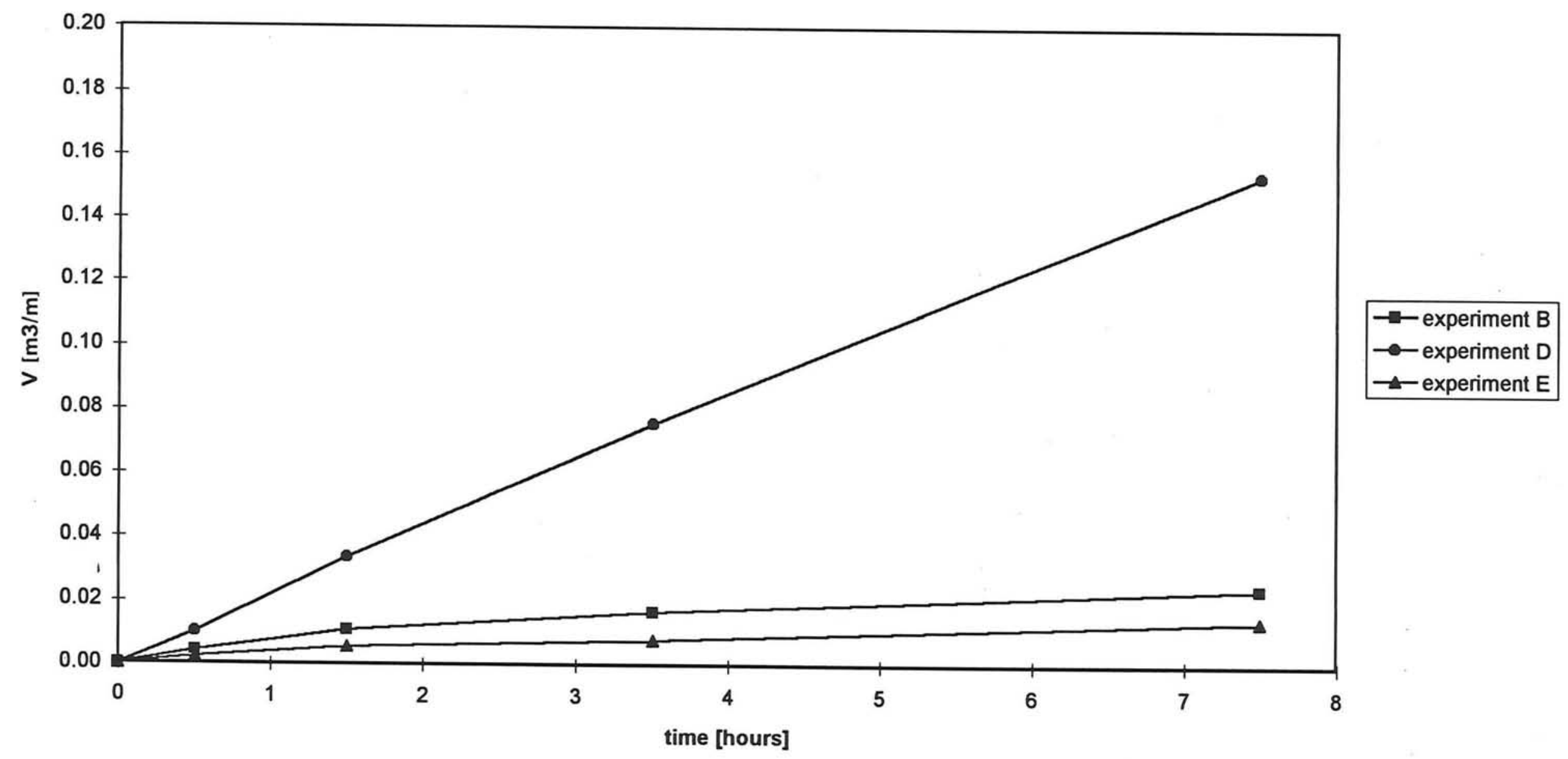

Graph 1.83 


\section{Sediment transport volumes}

Experiments with breakwater

Experiments $B, D$ and $E$ through horizontal (a): $y=0.45 \mathrm{~m}$

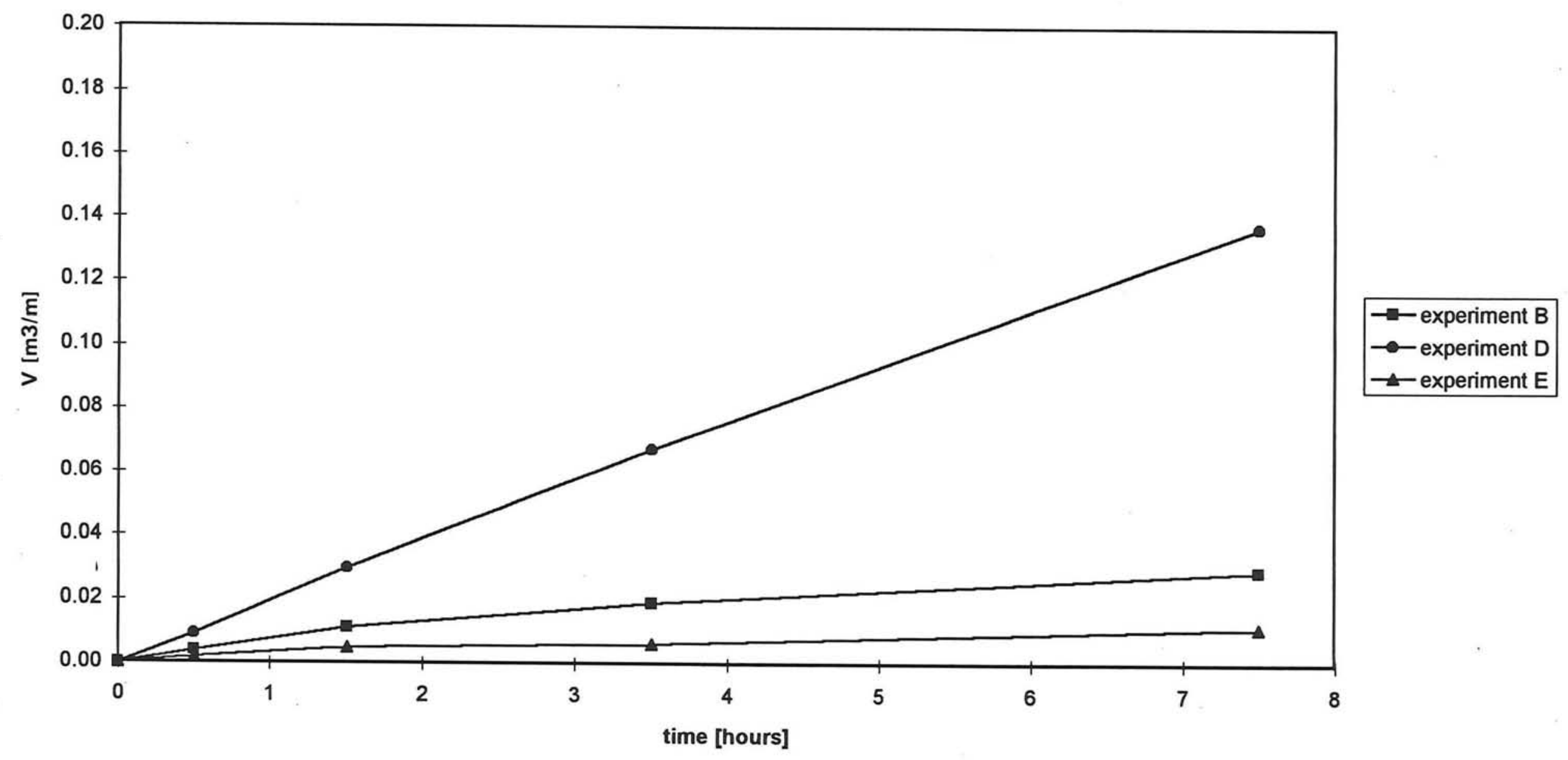

Graph 1.84 


\section{Sediment transport volumes}

Experiments with breakwater

Experiments $B, D$ and $E$ through horizontal $(b): y=0.55 \mathrm{~m}$

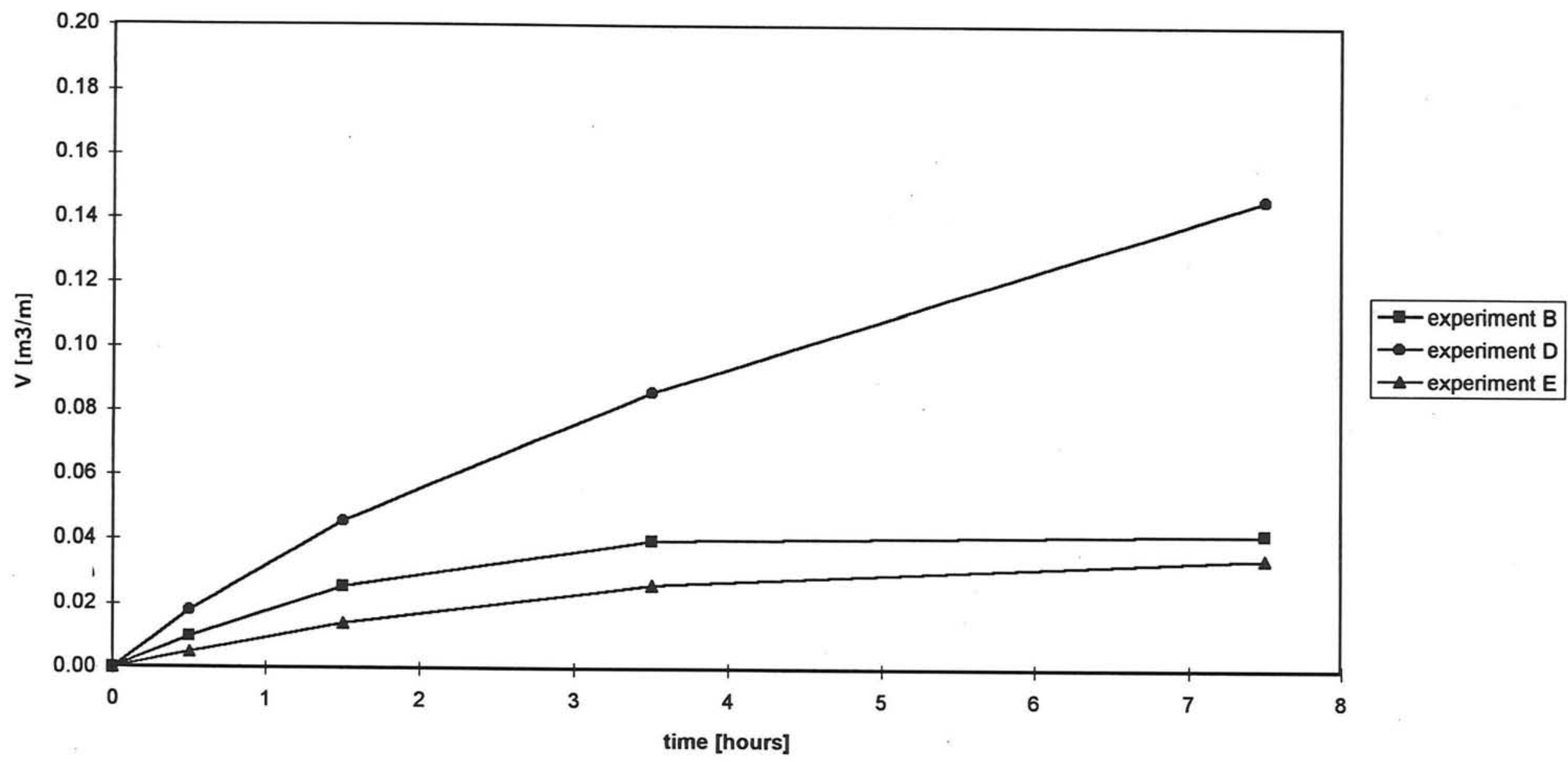

Graph 1.85 


\section{Sediment transport volumes}

Experiments with breakwater

Experiments $B, D$ and $E$ through horizontal (c): $y=0.65 \mathrm{~m}$

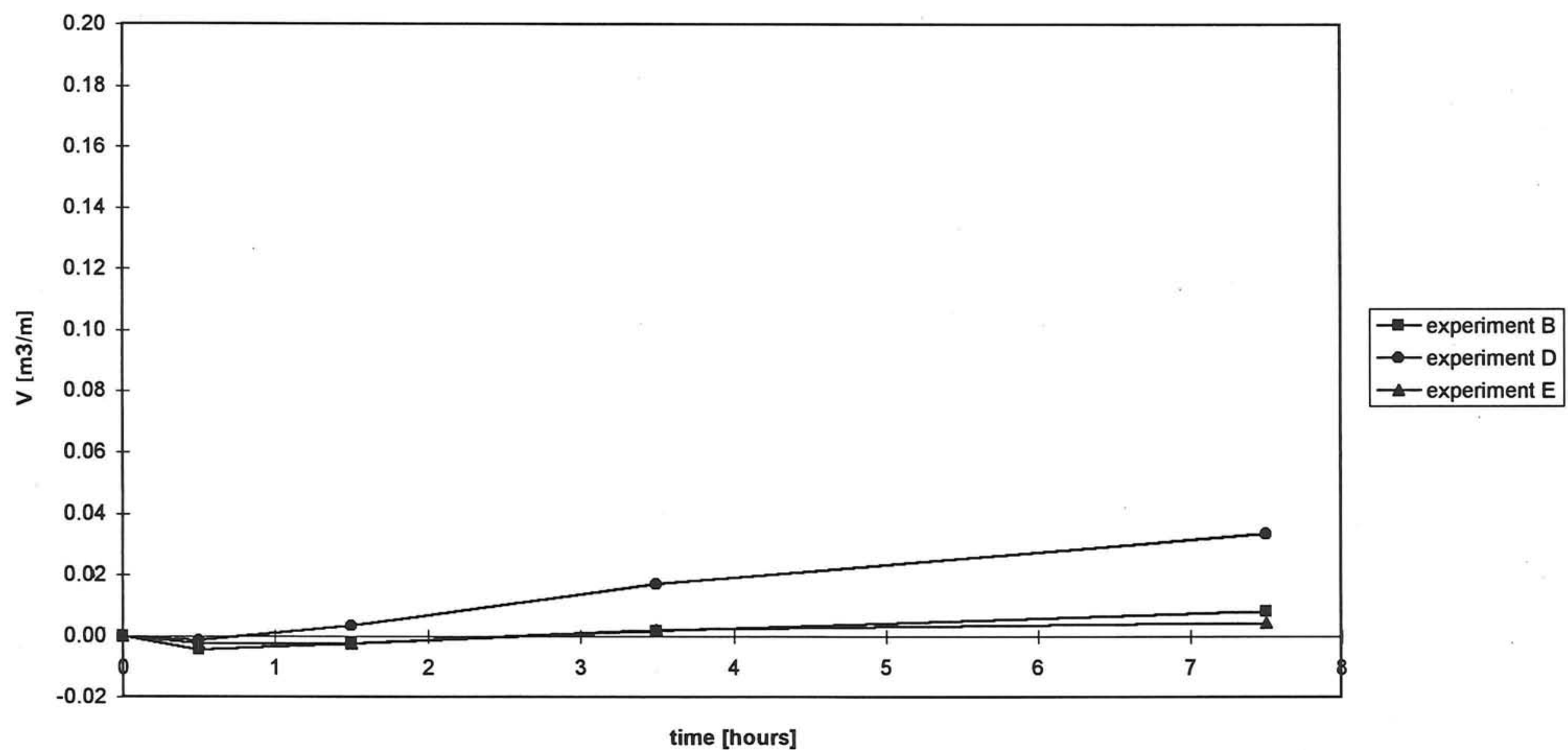

Graph 1.86 
Wave height measurements

Experiments without breakwater

Experiment $\mathbf{A}$

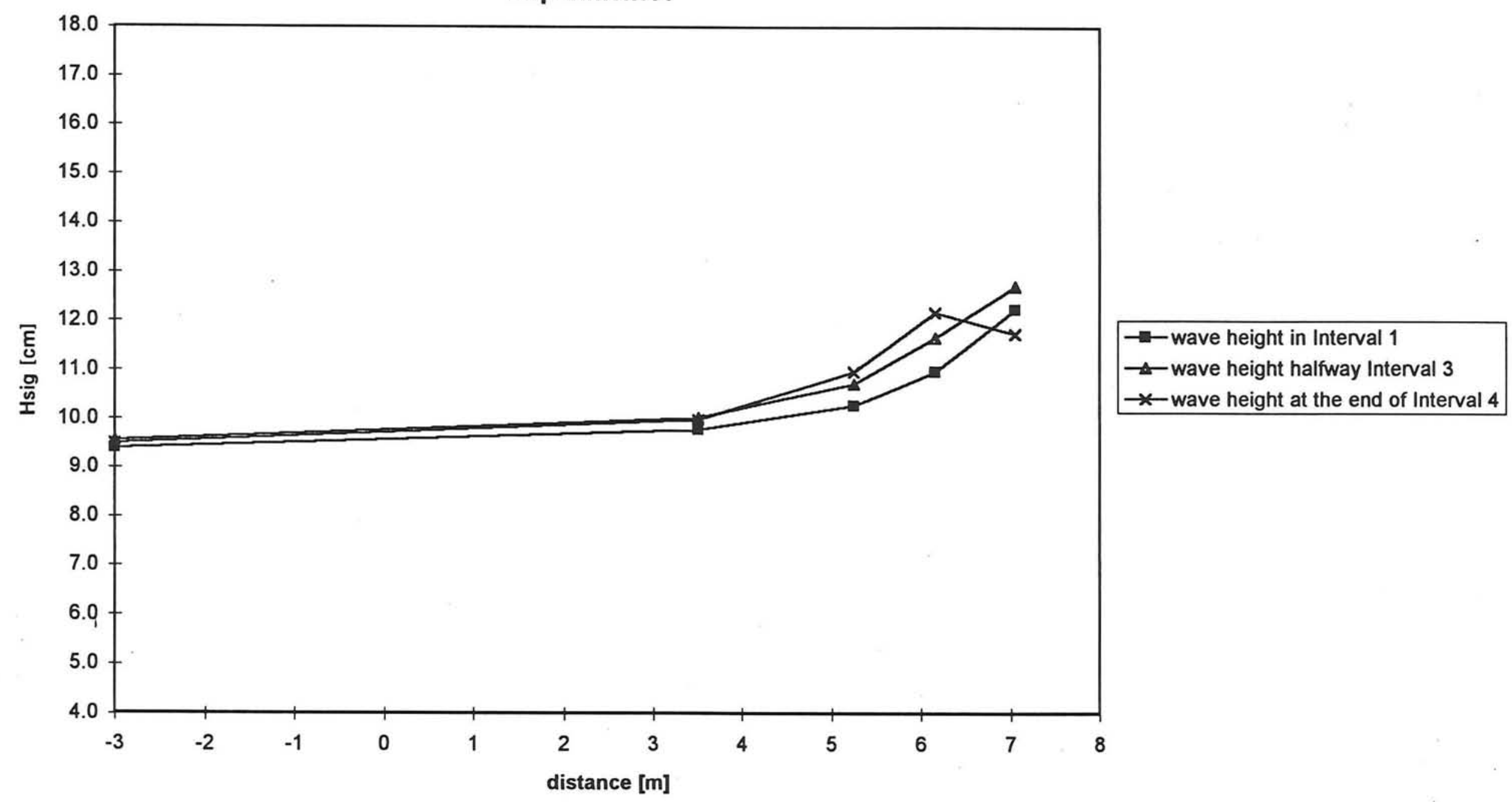

Graph 2.1 
Wave height measurements

Experiments without breakwater

Experiment B

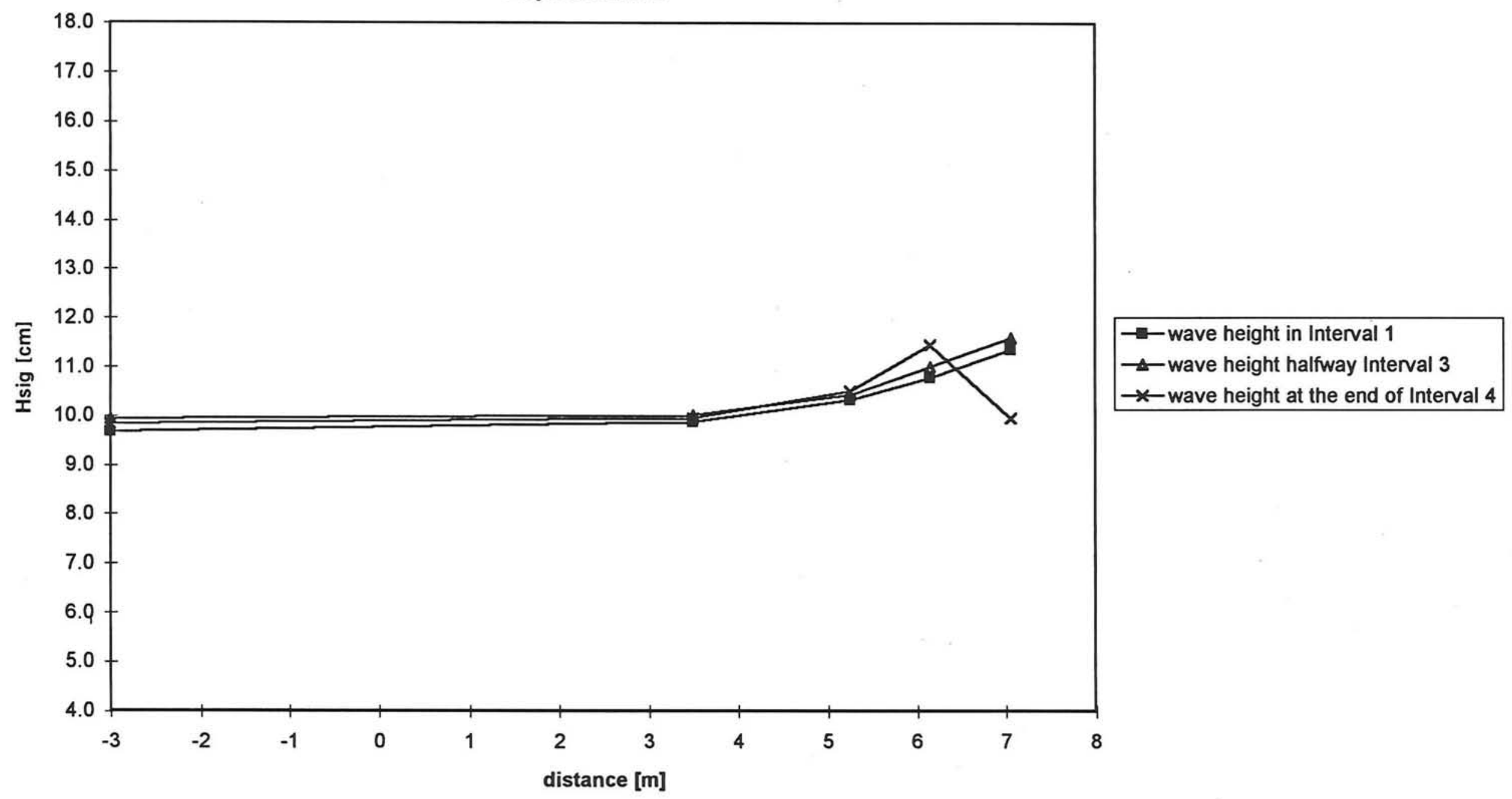

Graph 2.2 
Wave height measurements

Experiments without breakwater

Experiment $\mathbf{C}$

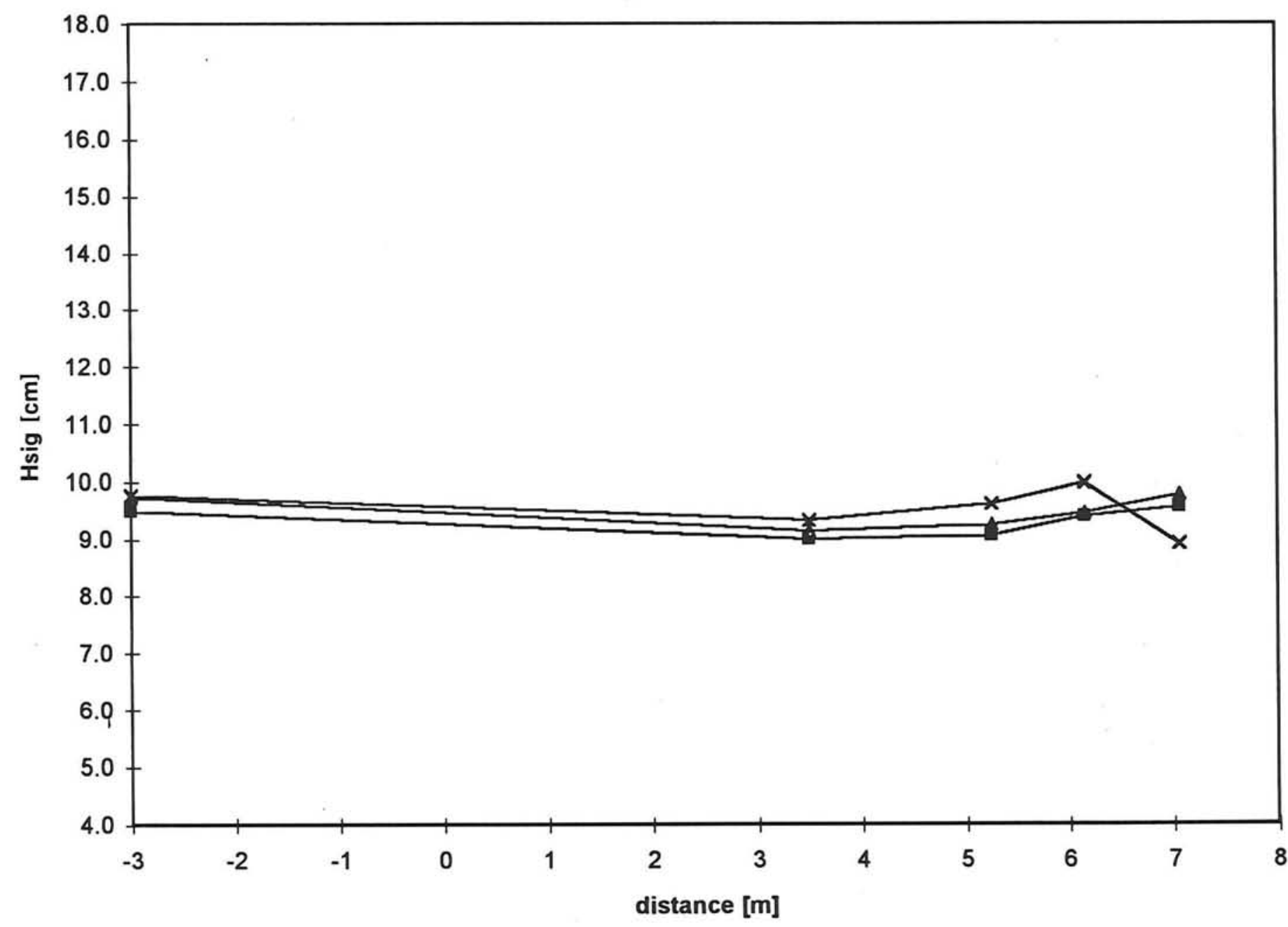

-n-wave height in Interval 1

- - wave height halfway Interval 3

- -wave height at the end of Interval 4

Graph 2.3 
Wave height measurements

Experiments without breakwater

Experiment $D$

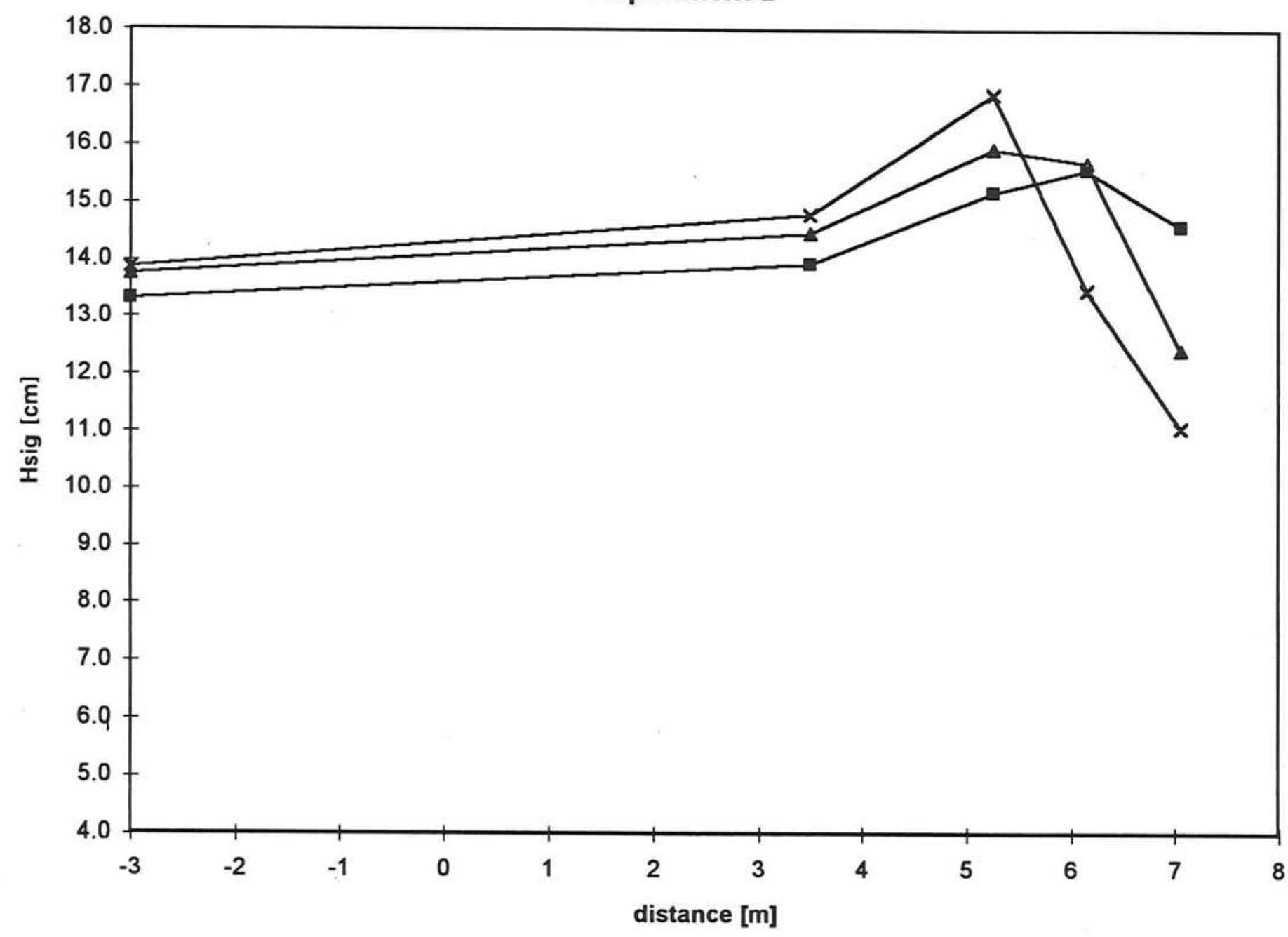

$\rightarrow-$ wave height in Interval 1

$\rightarrow-$ wave height halfway Interval 3

$\rightarrow$ - wave height at the end of Interval 4

Graph 2.4 
Wave height measurements

Experiments without breakwater

Experiment $E$

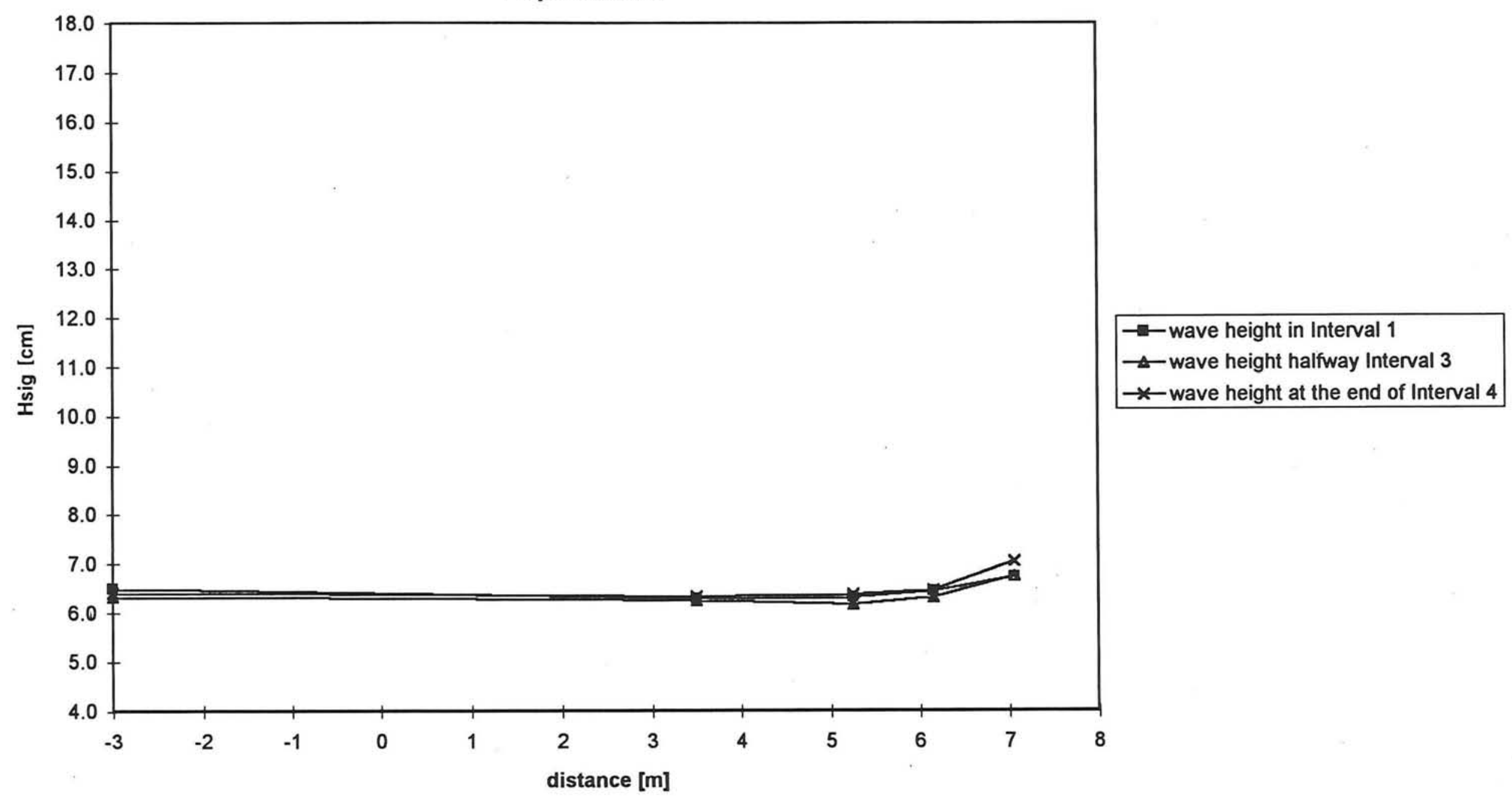

Graph 2.5 
Wave height measurements

Experiments without breakwater

Experiment $F$

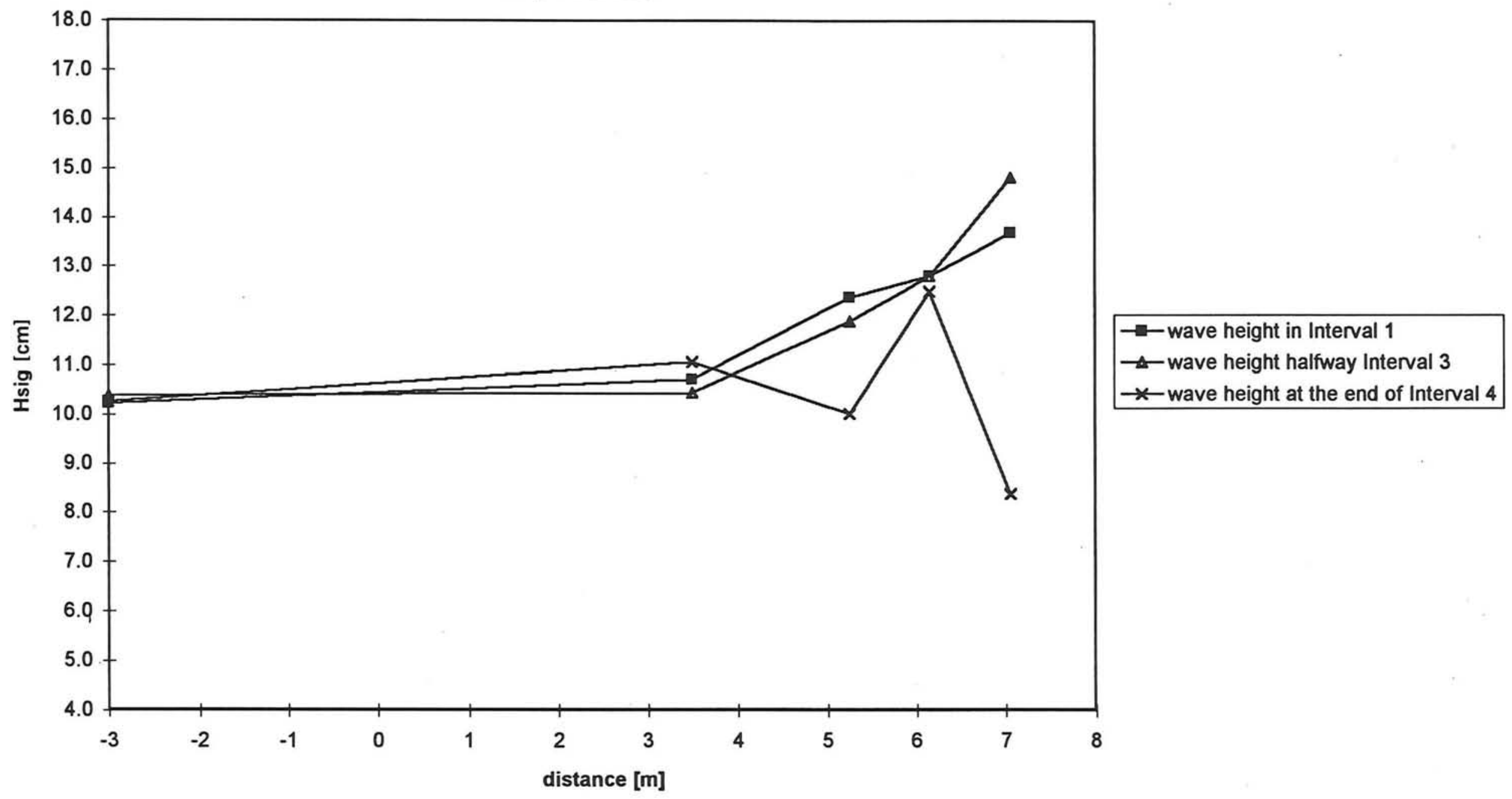

Graph 2.6 


\section{Wave height measurements}

Experiments with breakwater

Experiment $\mathrm{A}$

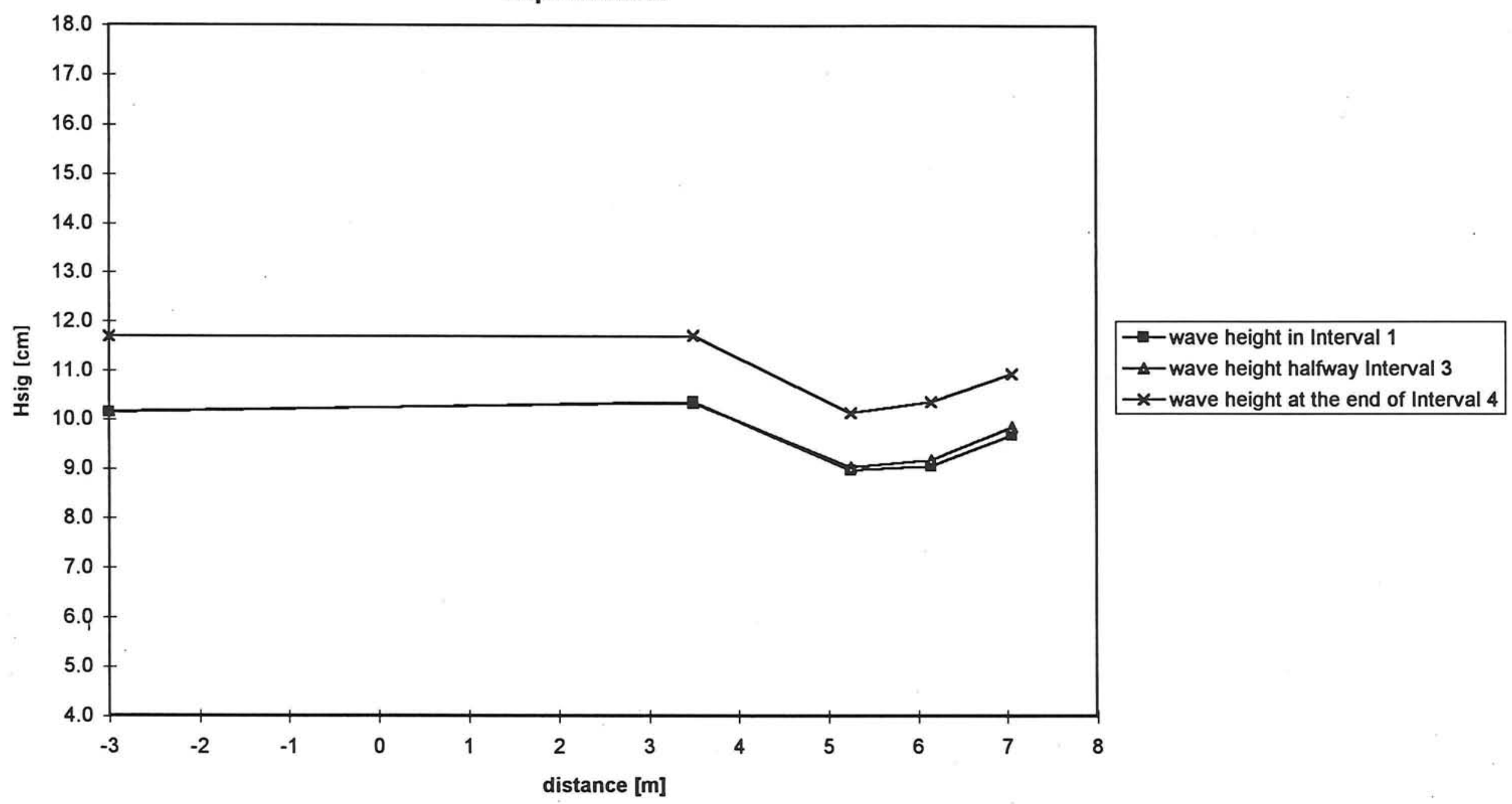

Graph 2.7 


\section{Wave height measurements}

Experiments with breakwater

Experiment B

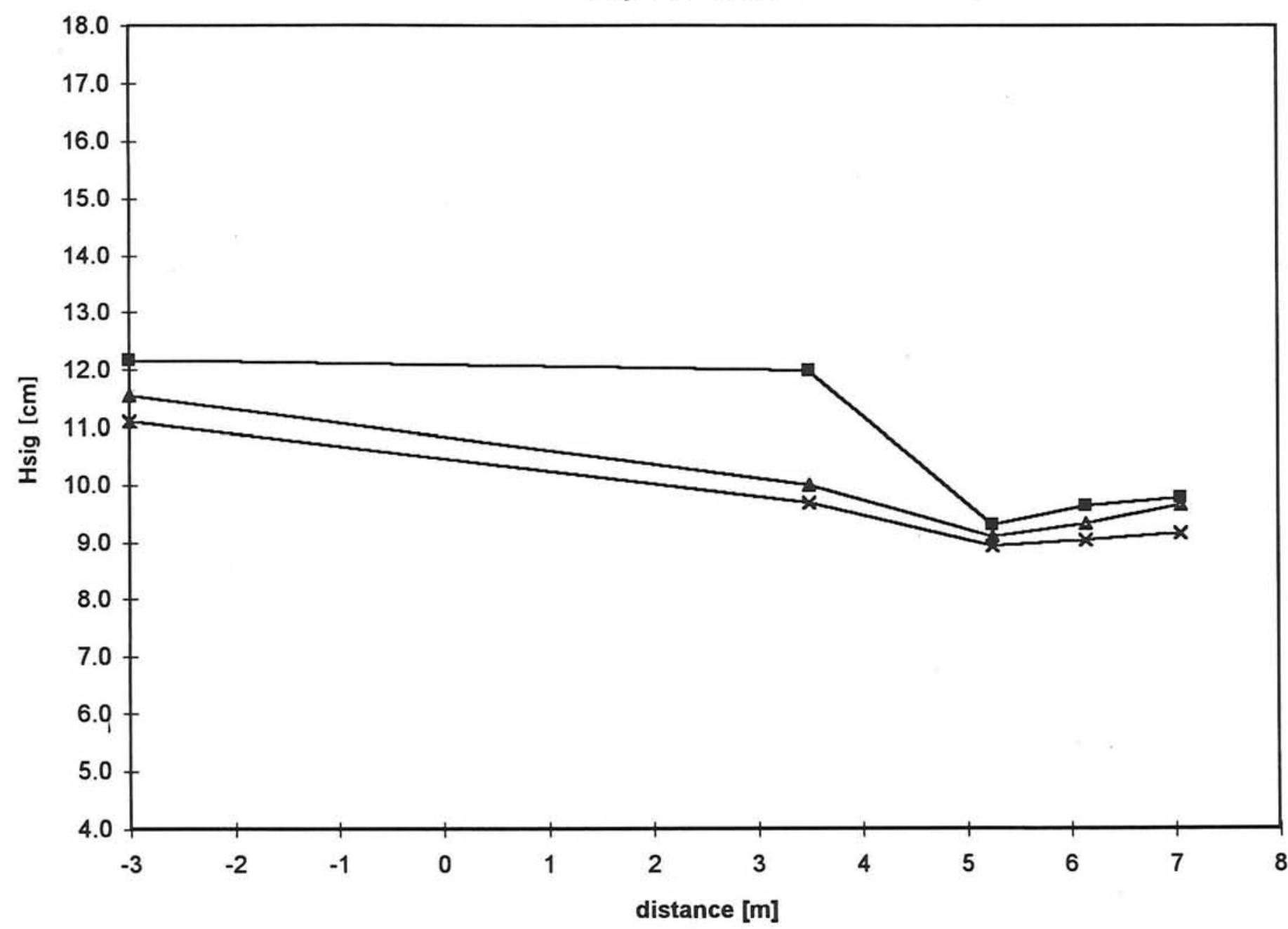

- - wave height in Interval 1

- - wave height halfway Interval 3

$\rightarrow$ - wave height at the end of Interval 4

Graph 2.8 
Wave height measurements

Experiments with breakwater

Experiment $\mathrm{C}$

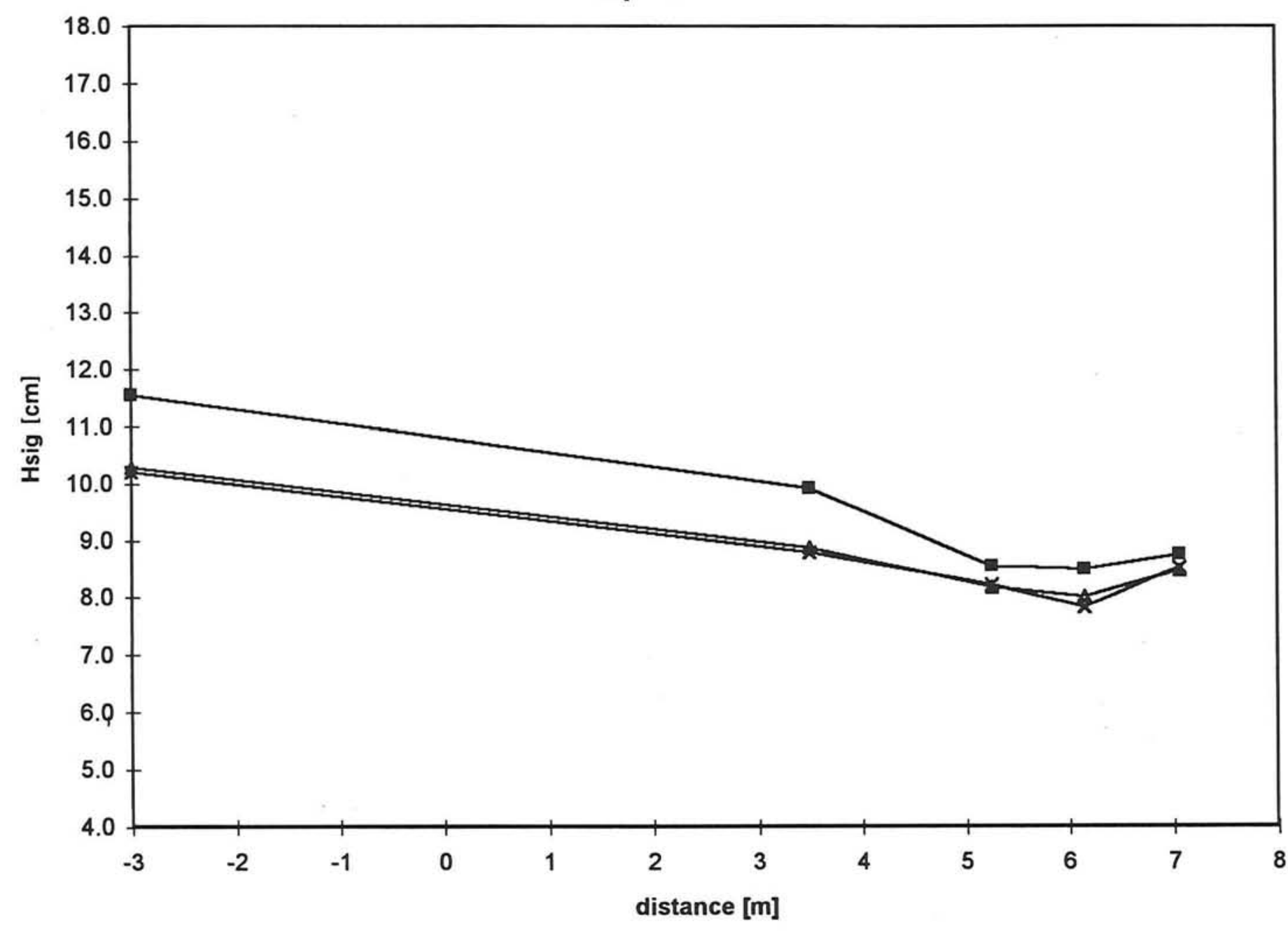

$\rightarrow-$ wave height in Interval 1

$₫$ wave height halfway Interval 3

$\rightarrow$-wave height at the end of Interval 4

Graph 2.9 
Wave height measurements

Experiments with breakwater

Experiment D

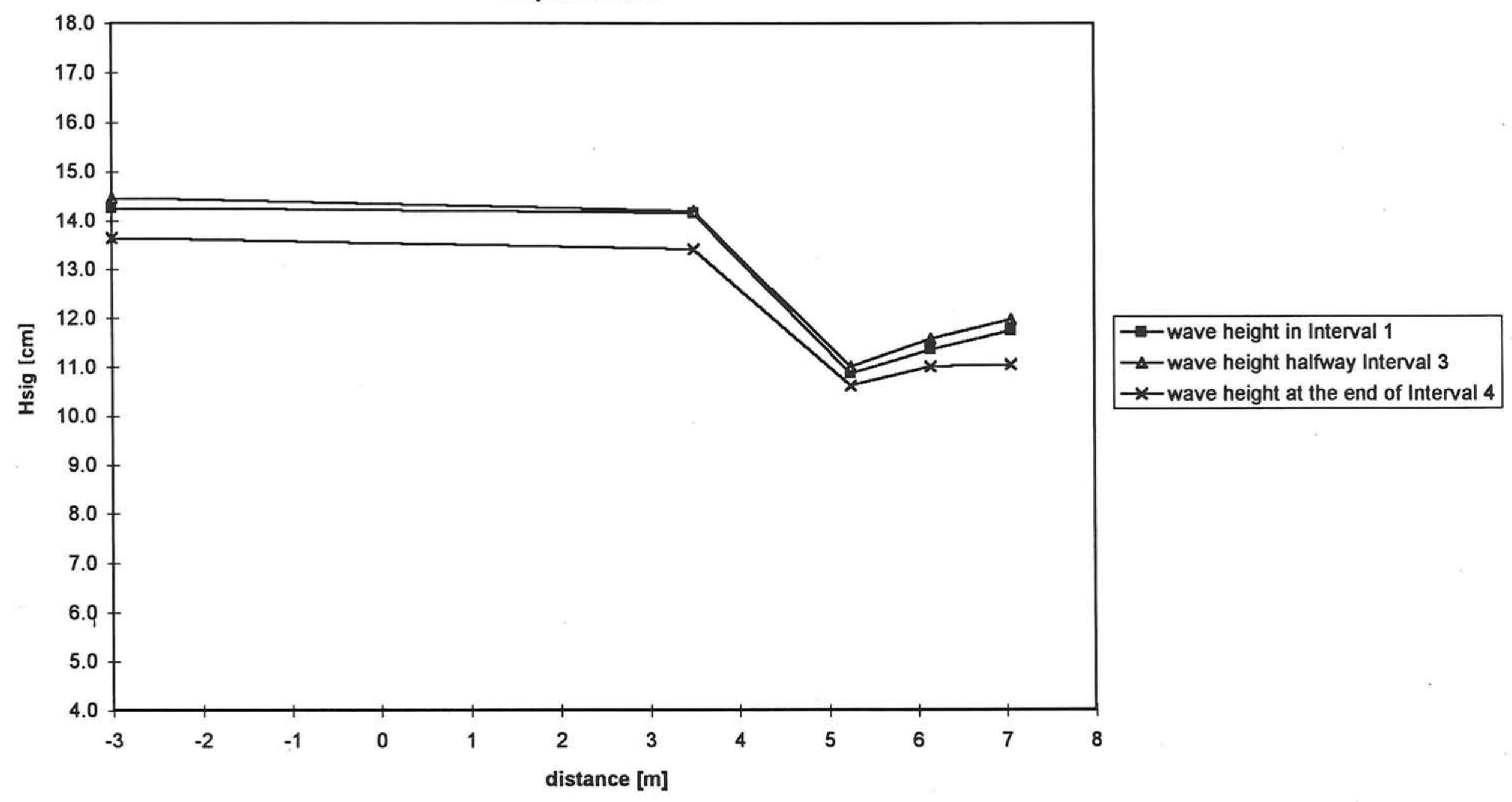

Graph 2.10 
Wave height measurements

Experiments with breakwater

Experiment $\mathrm{E}$

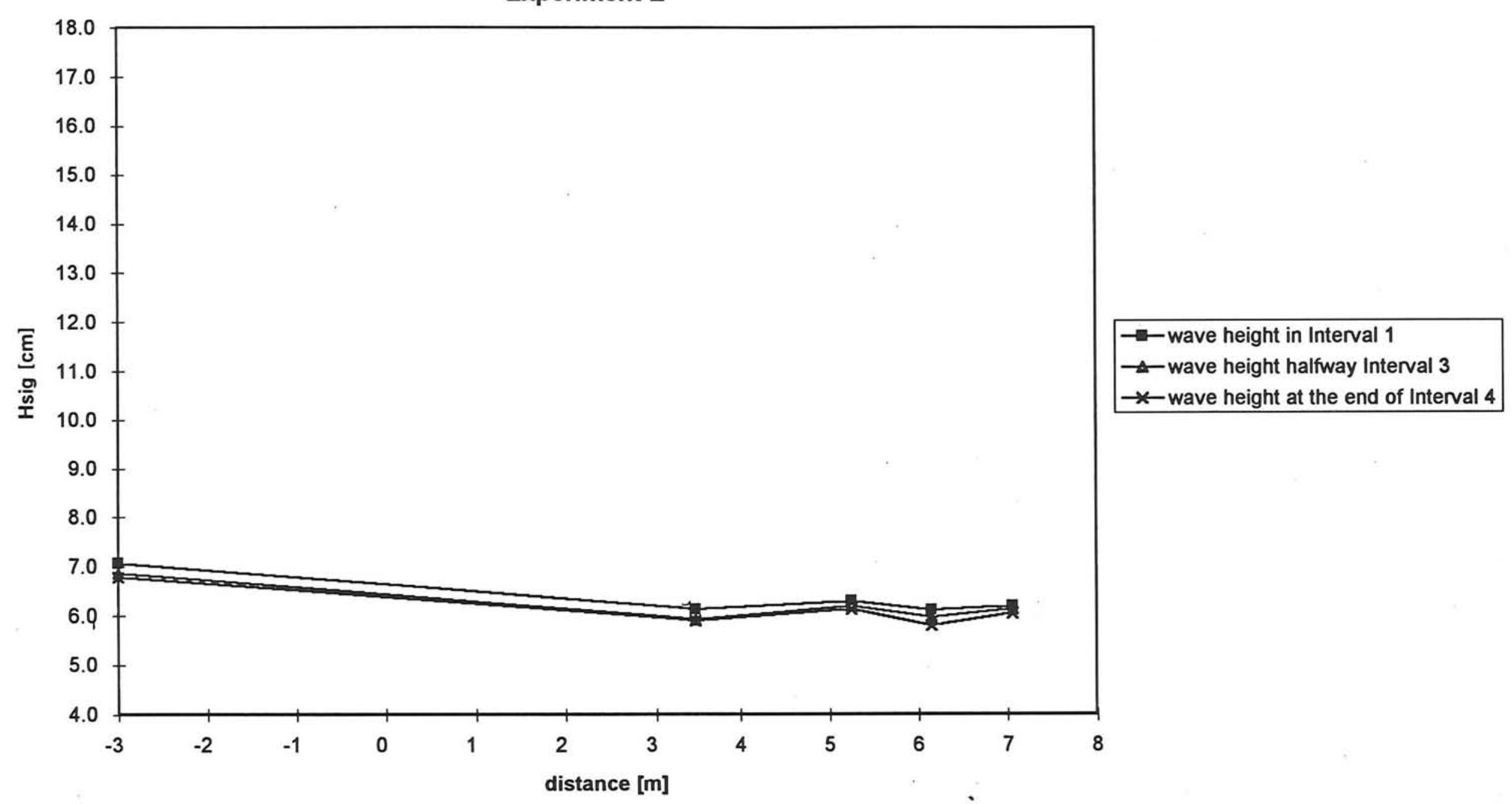

Graph 2.11 


\section{Wave height measurements}

Experiments with breakwater

Experiment $F$

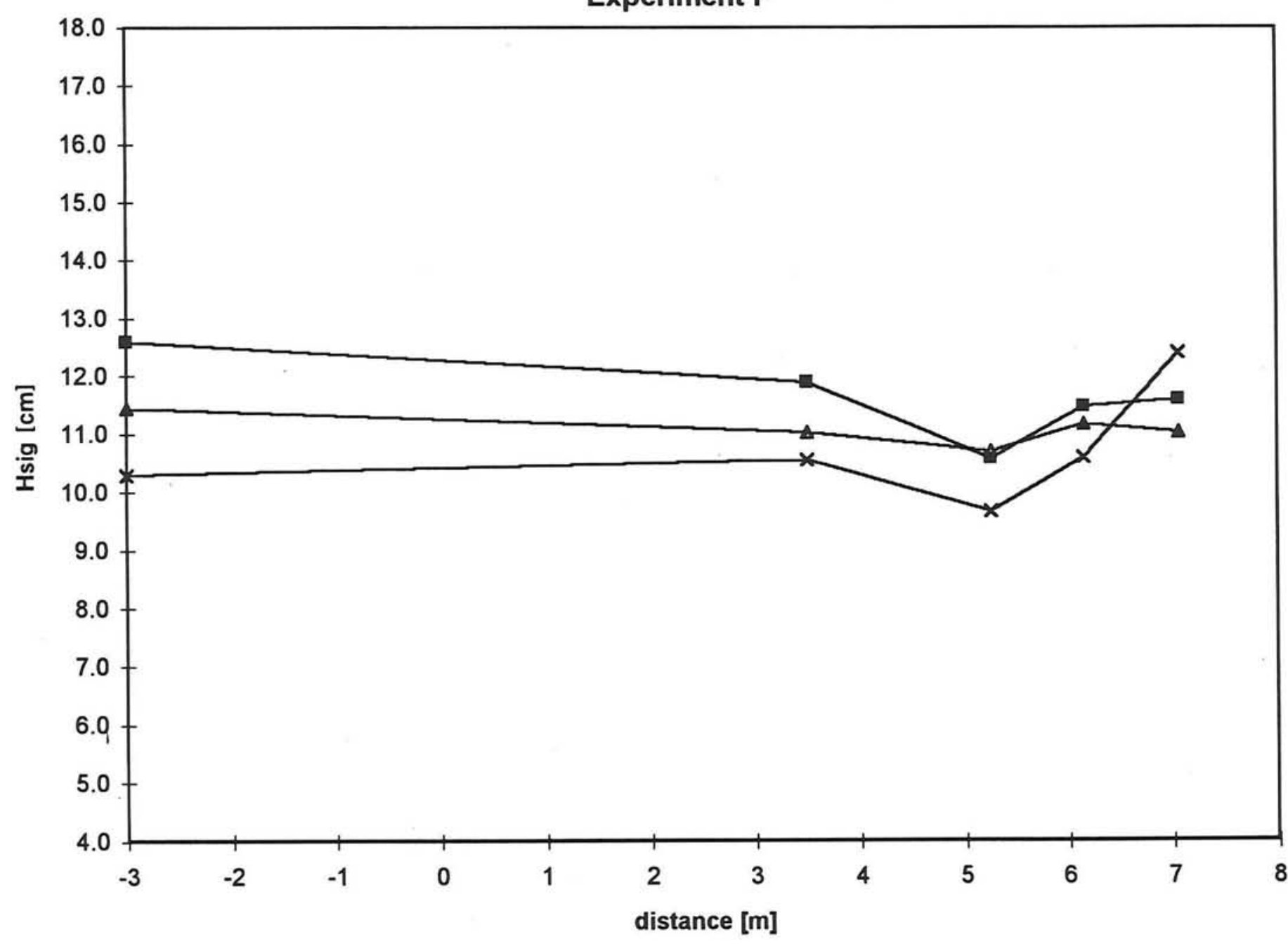


Wave height measurements

Experiments with and without breakwater

Experiment A, at Interval 1

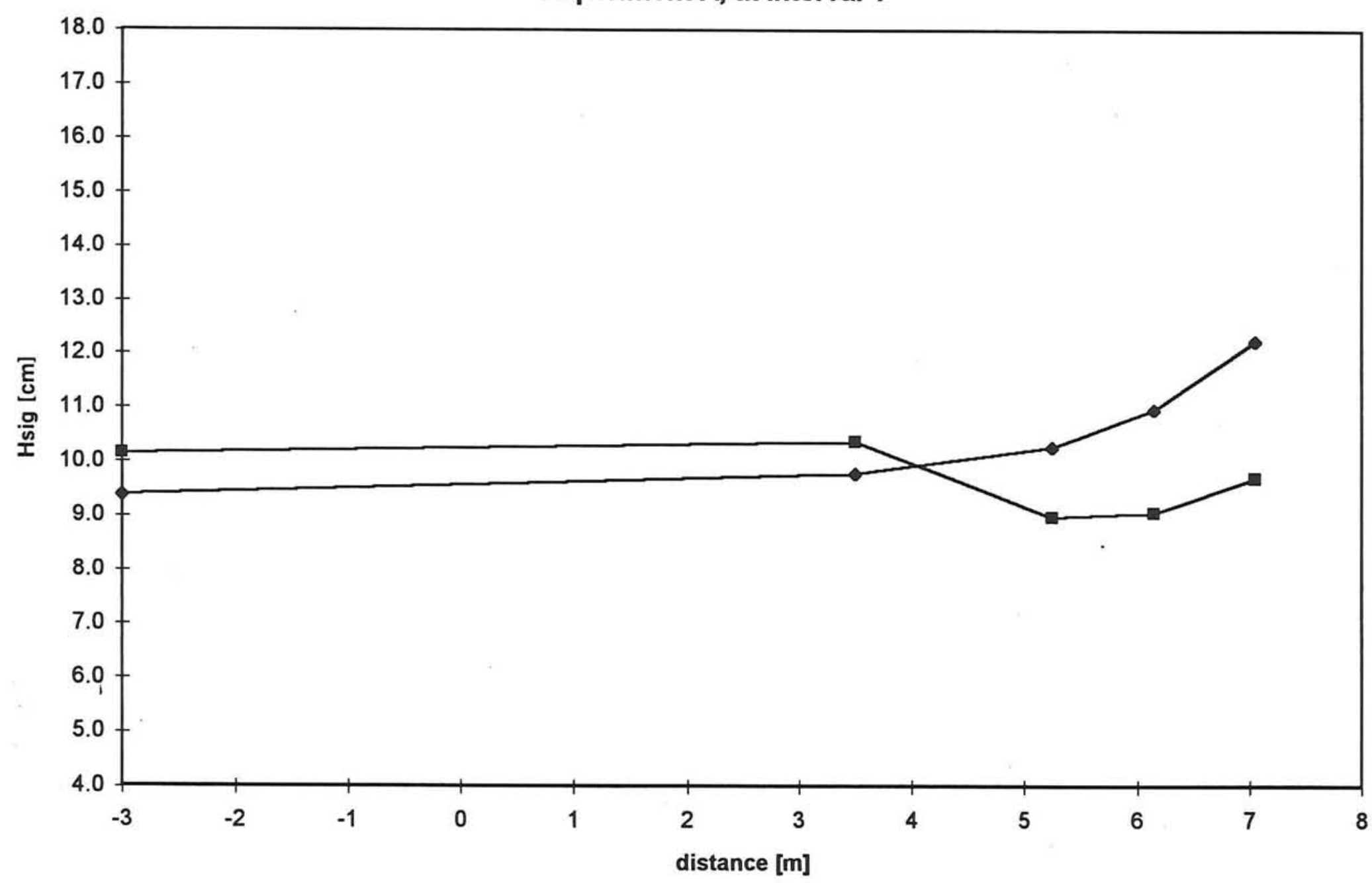

$\sim$ without breakwater

$-\because$ with breakwater

Graph 2.13 
Wave height measurements

Experiments with and without breakwater

Experiment B, at Interval 1

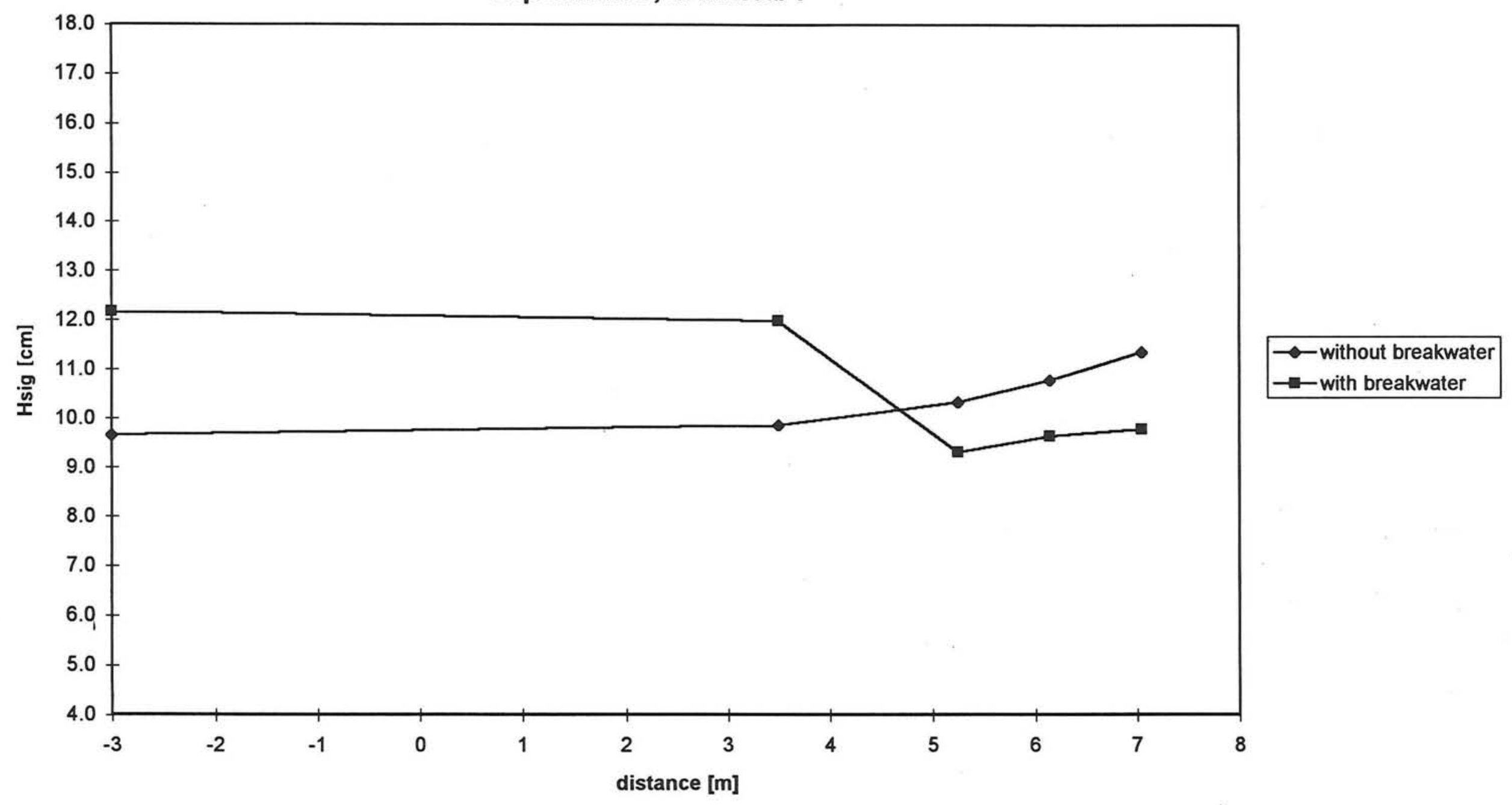

Graph 2.14 
Wave height measurements

Experiments with and without breakwater

Experiment C, at Interval 1

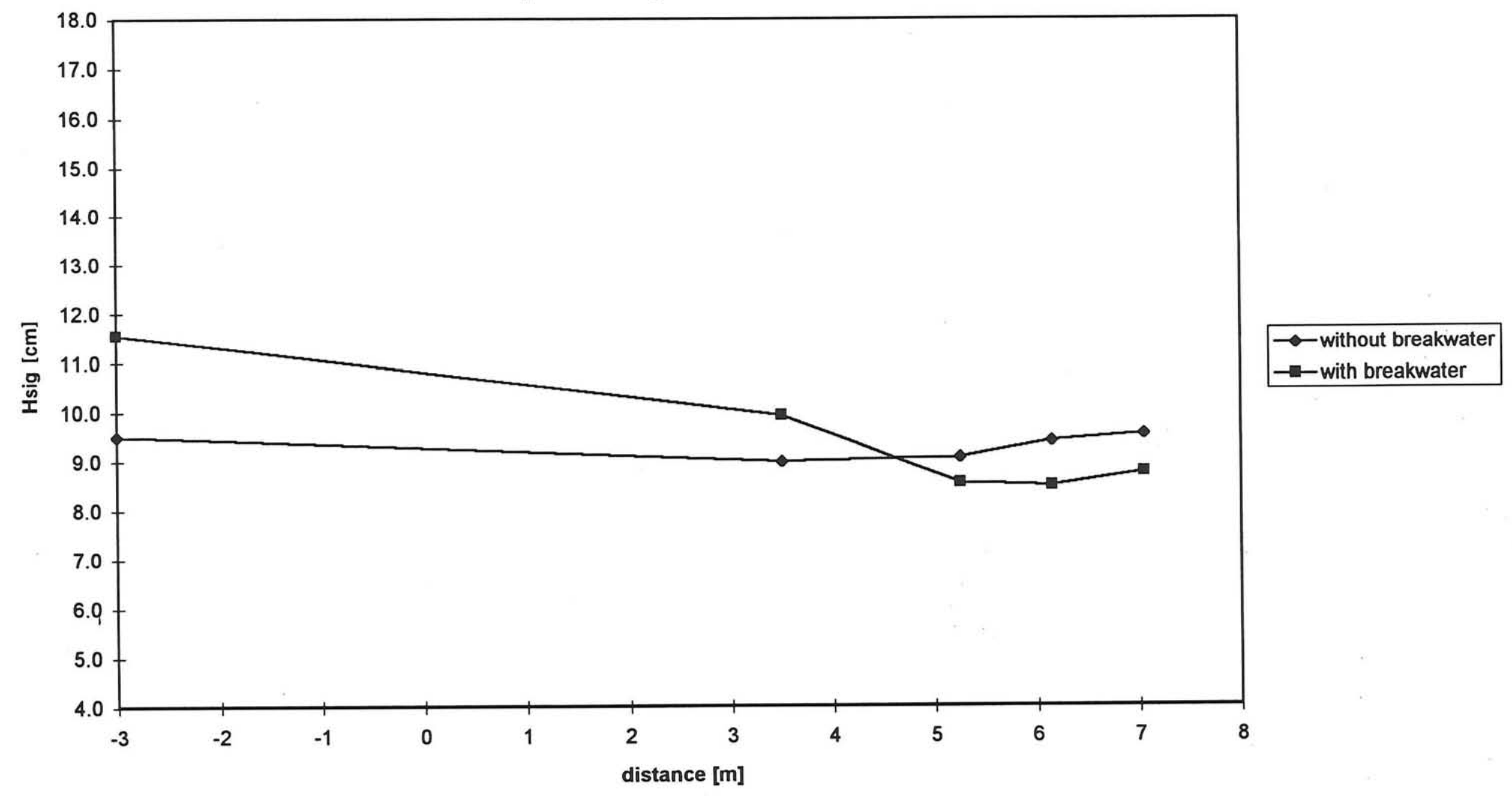

Graph 2.15 
Wave height measurements

Experiments with and without breakwater

Experiment $D$, at Interval 1

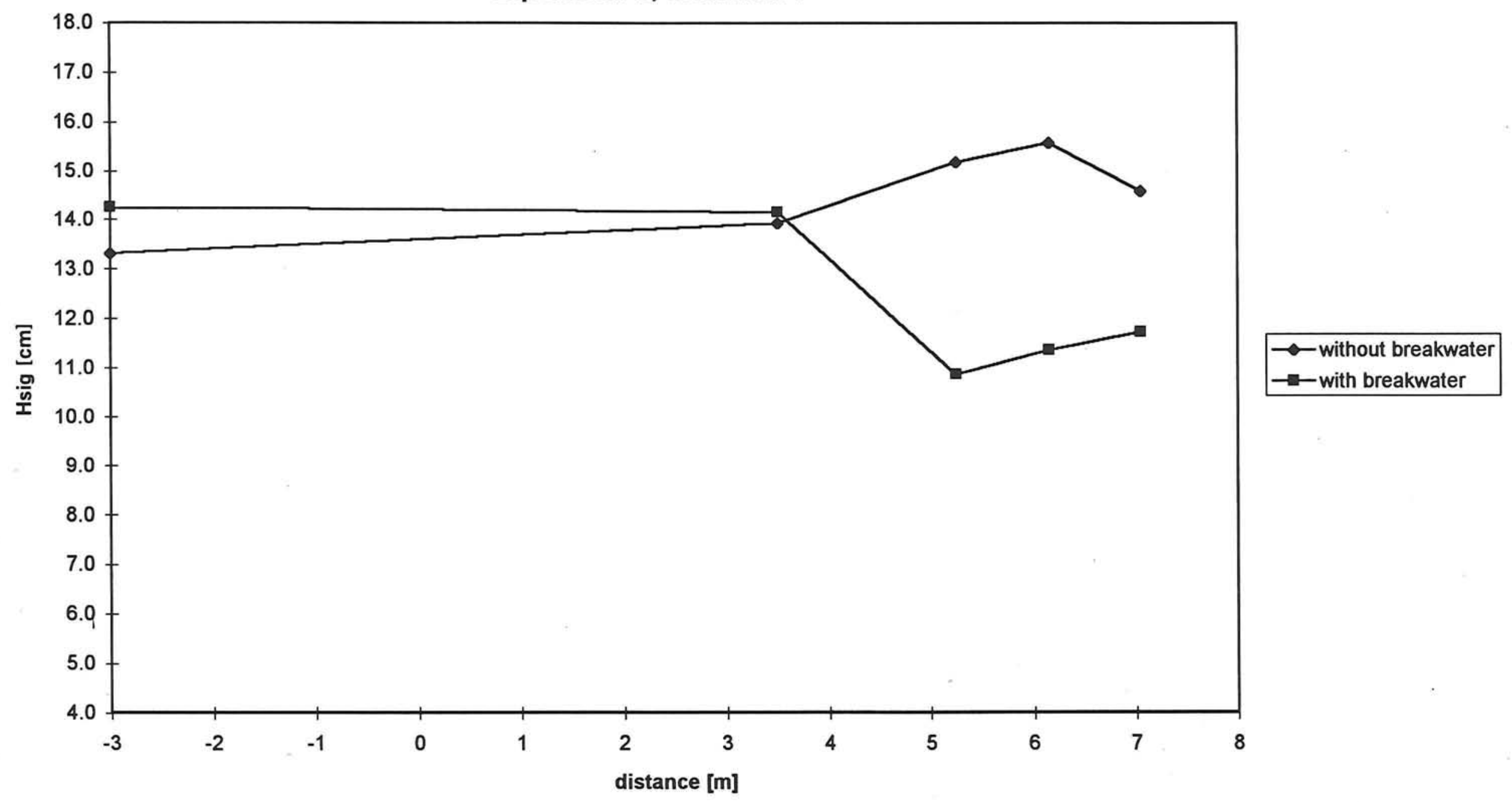

Graph 2.16 
Wave height measurements

Experiments with and without breakwater

Experiment $E$, at Interval 1

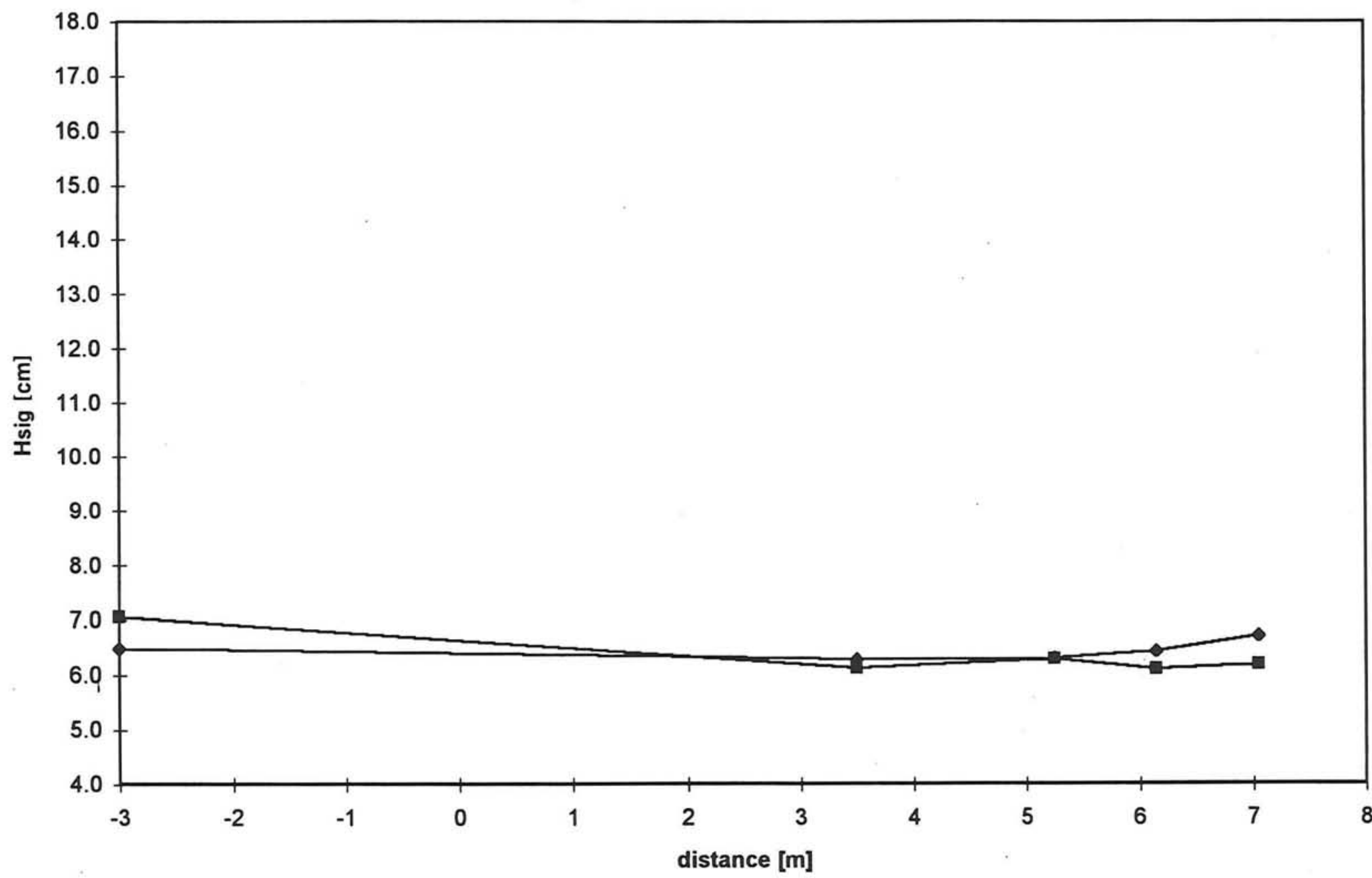

Graph 2.17 
Wave height measurements

Experiments with and without breakwater

Experiment $F$, at Interval 1

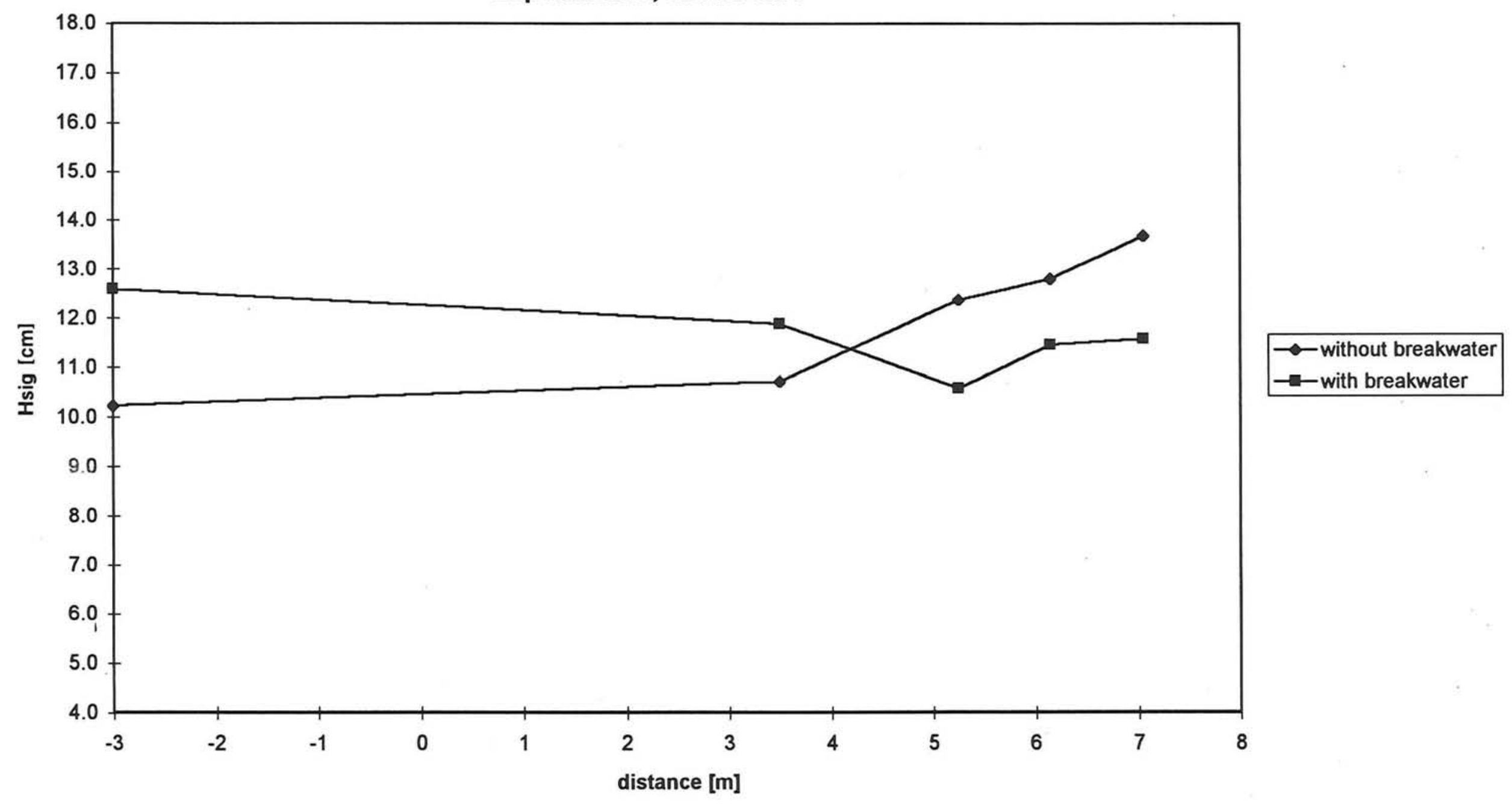

Graph 2.18 


\section{Wave height measurements}

Experiments without breakwater

Wave heights at WHM 1

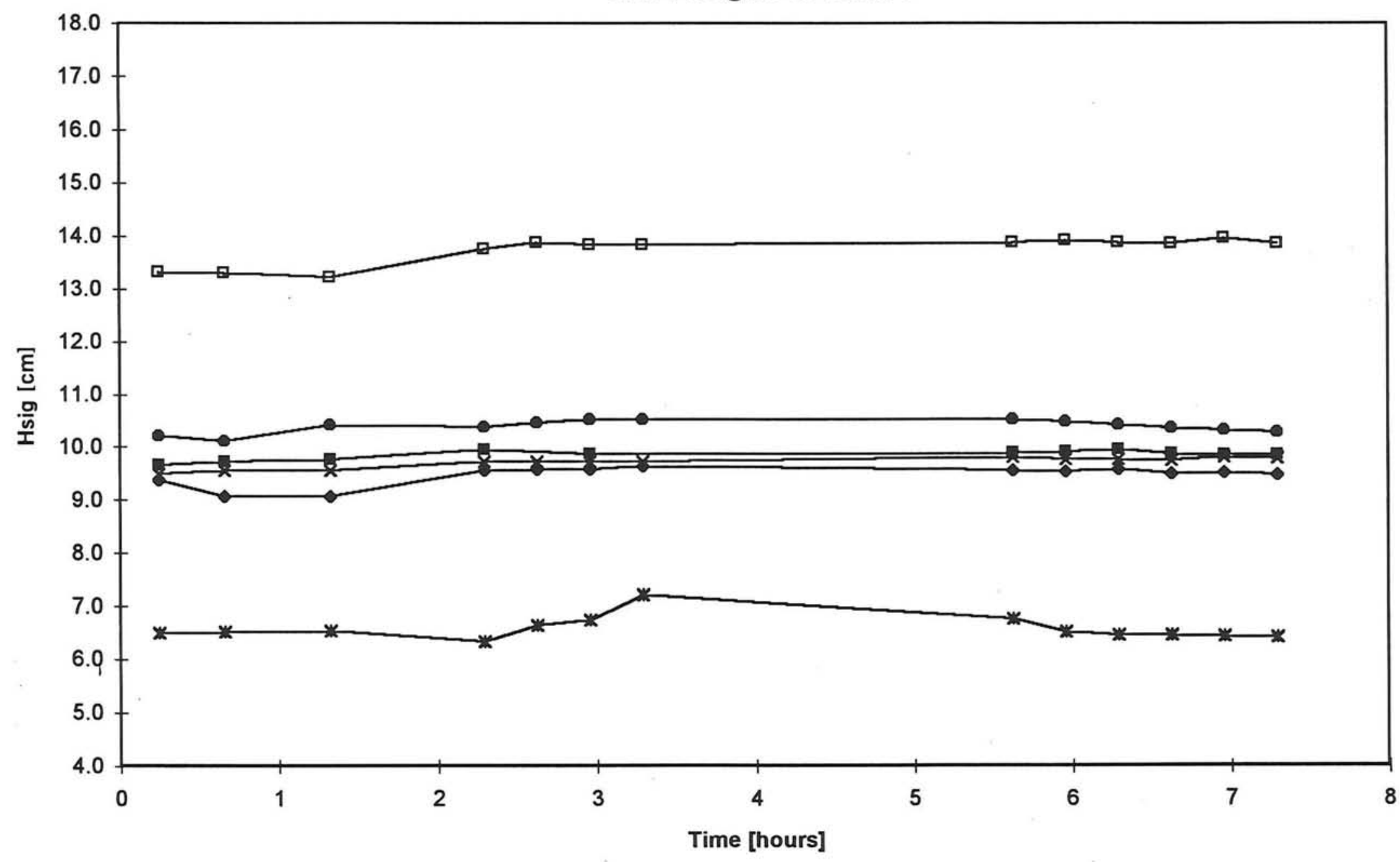




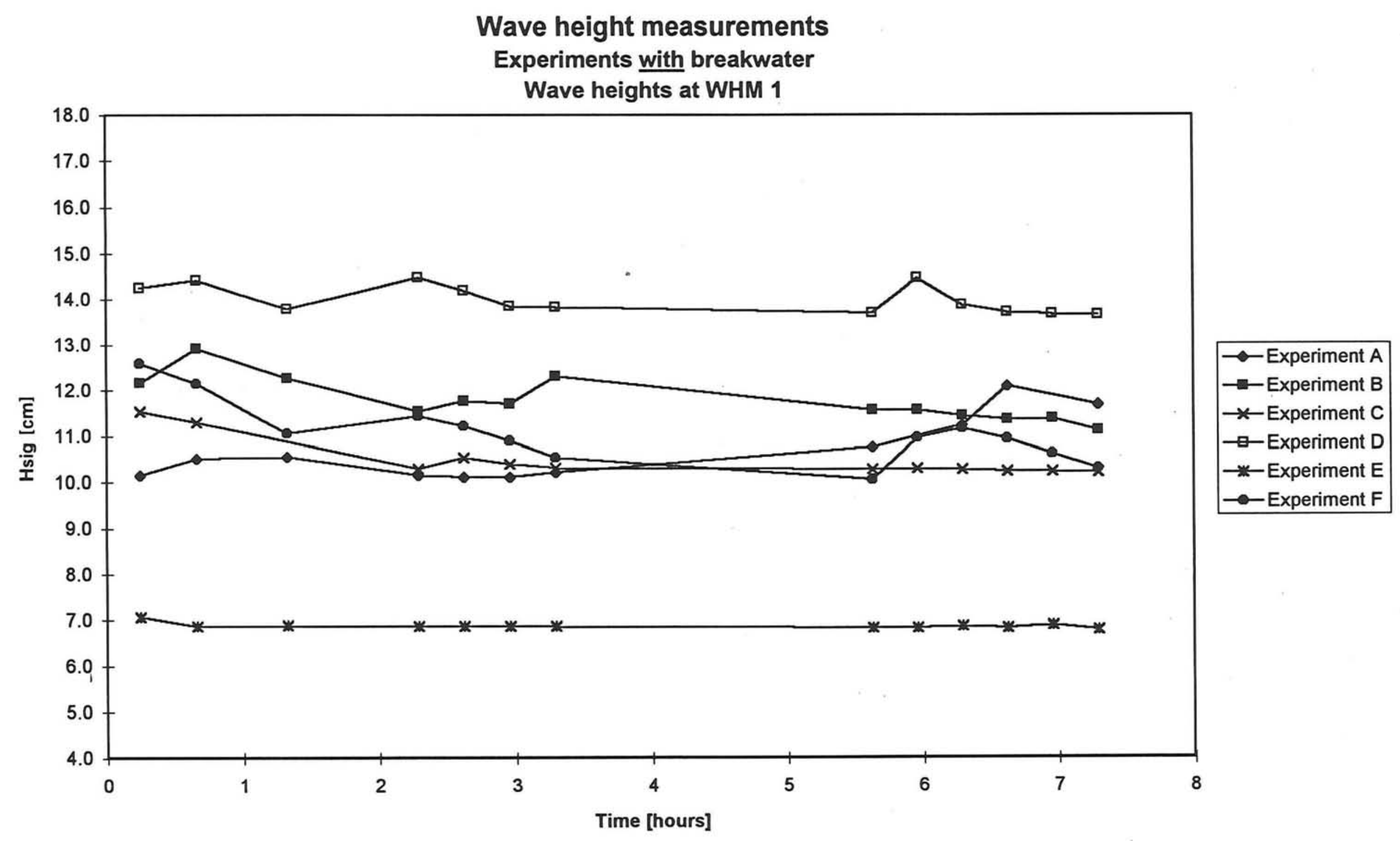

Graph 2.20 


\section{Spectra}

Experiment $\mathrm{A}$ without breakwater Measured during Interval 1

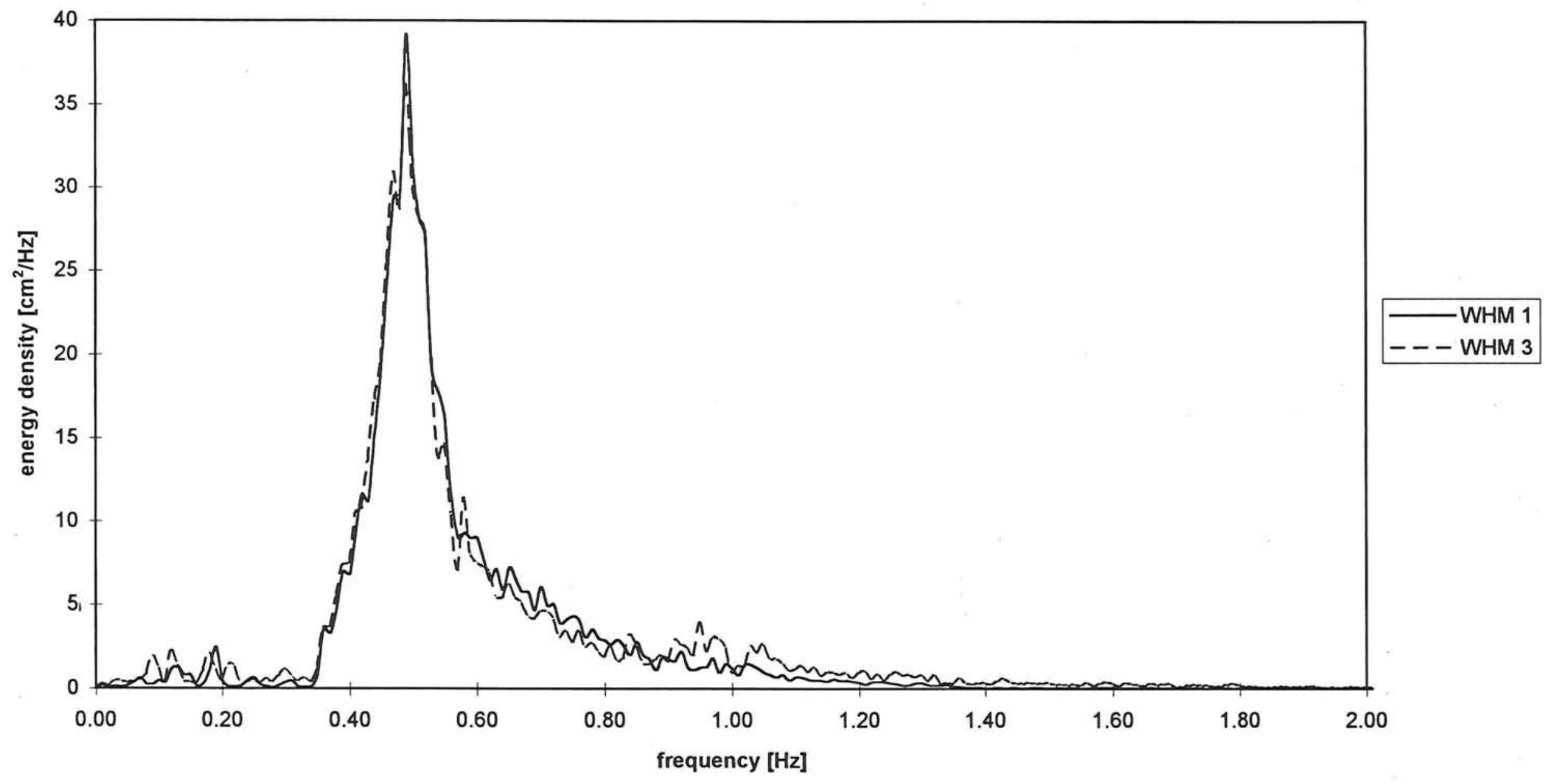

Graph 2.21 
Spectra

Experiment $B$ without breakwater Measured during Interval 1

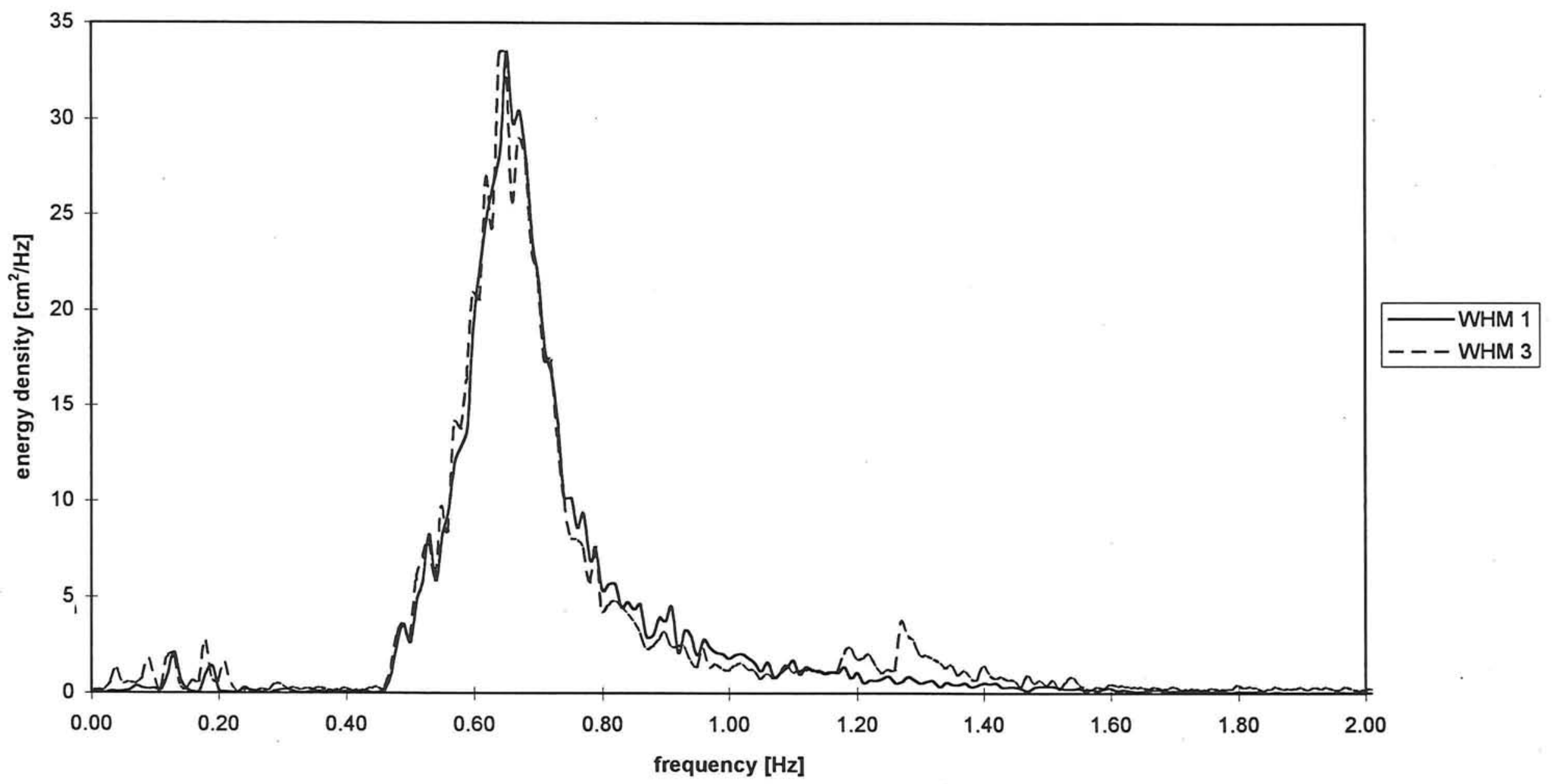

Graph 2.22 


\section{Spectra}

Experiment $\mathrm{C}$ without breakwater

Measured during Interval 1

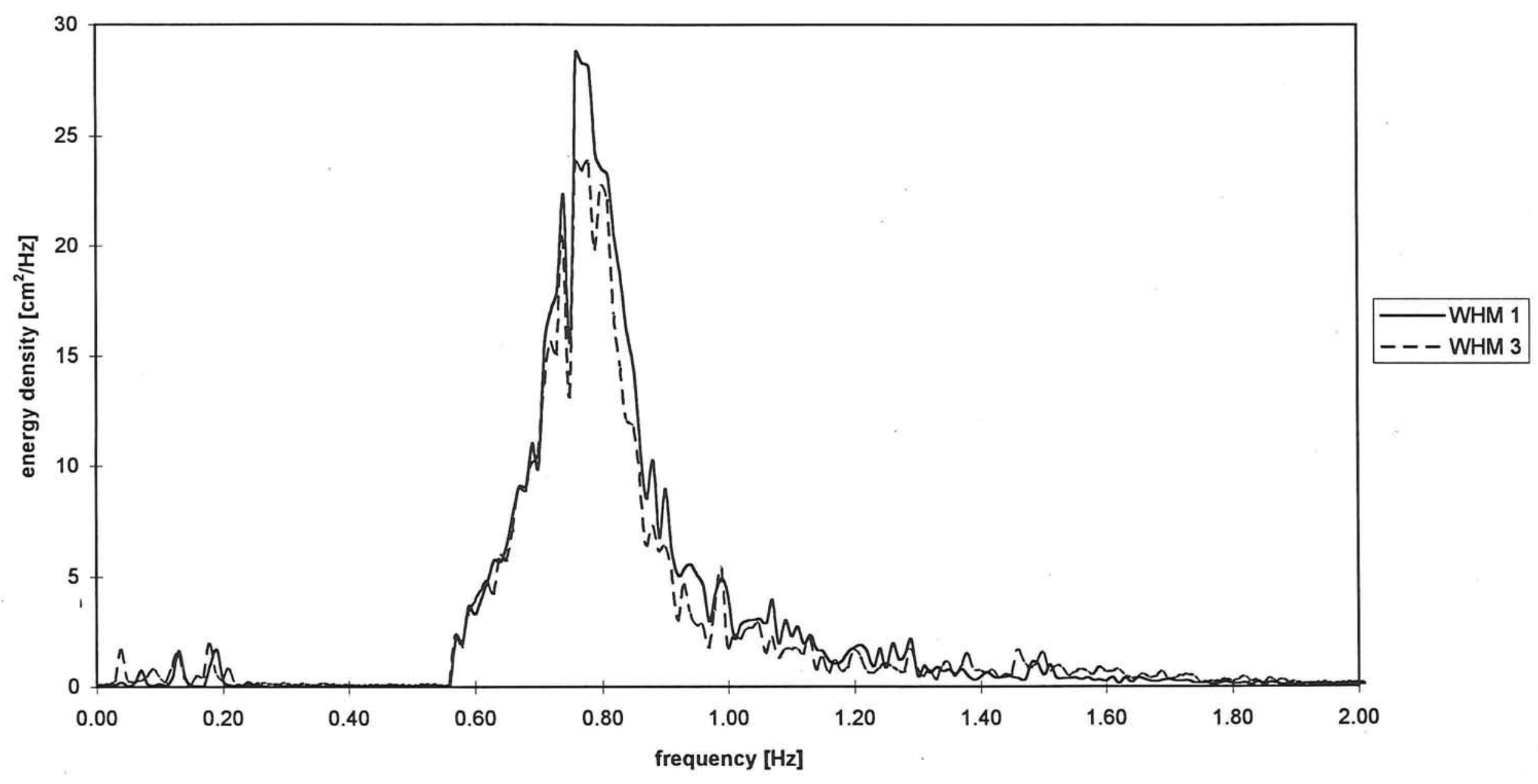

Graph 2.23 


\section{Spectra}

Experiment $\mathrm{E}$ without breakwater

Measured during Interval 1

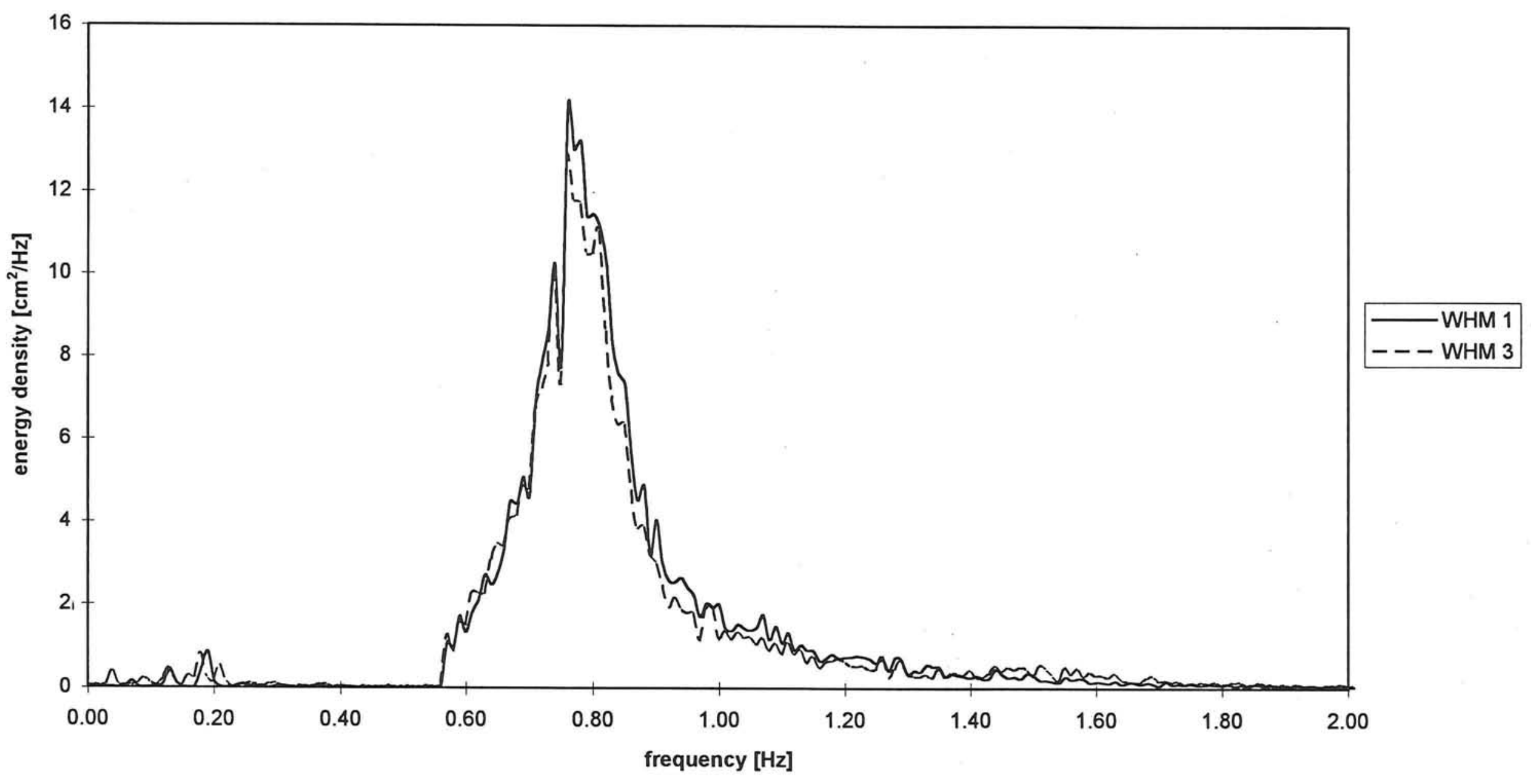

Graph 2.25 


\section{Spectra}

Experiment $A$ with breakwater

Measured during Interval 1

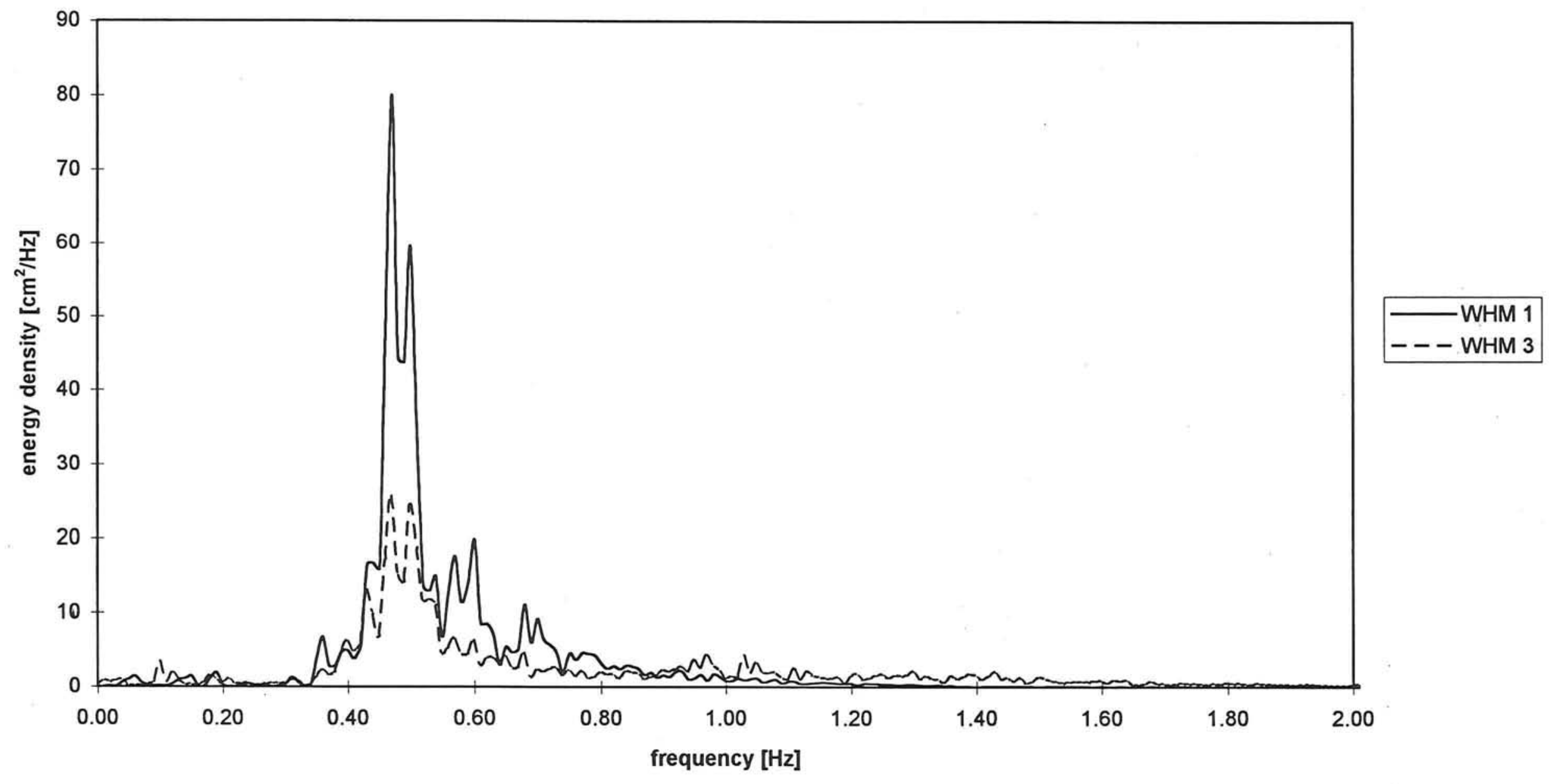

Graph 2.26 


\section{Spectra}

Experiment B with breakwater

Measured during Interval 1

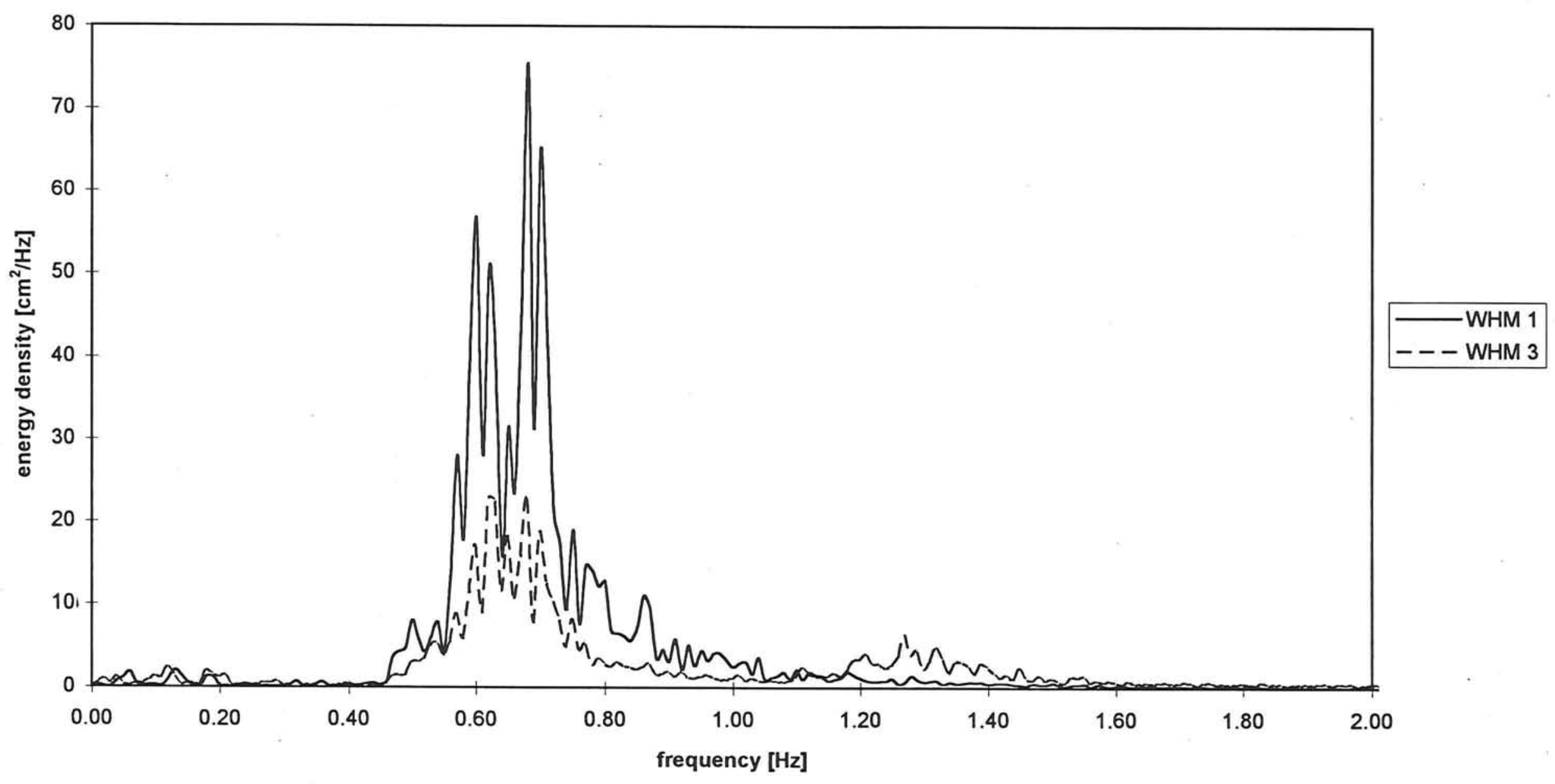

Graph 2.27 


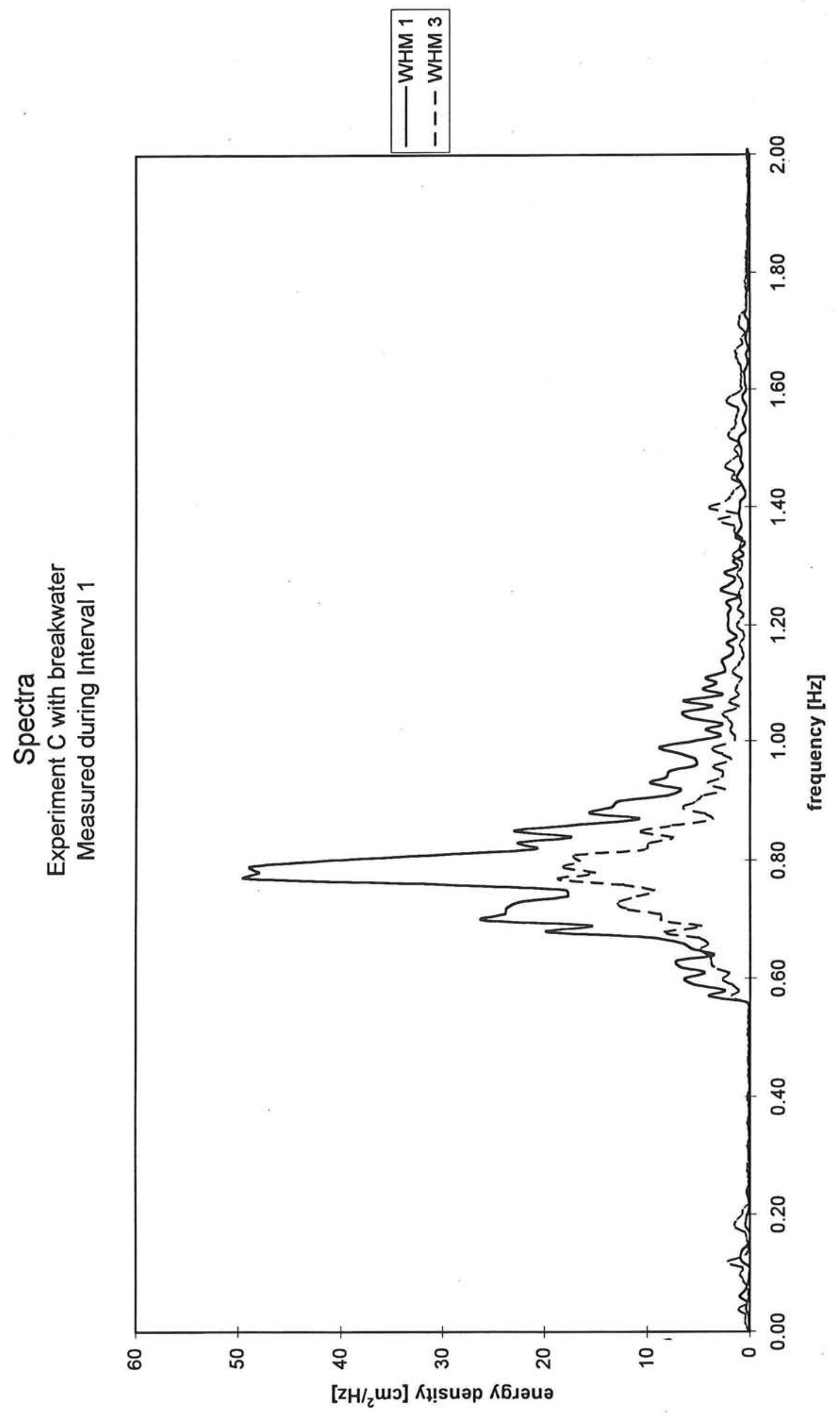

N
ํ.
등
인 


\section{Spectra}

Experiment $\mathrm{D}$ with breakwater

Measured during Interval 1

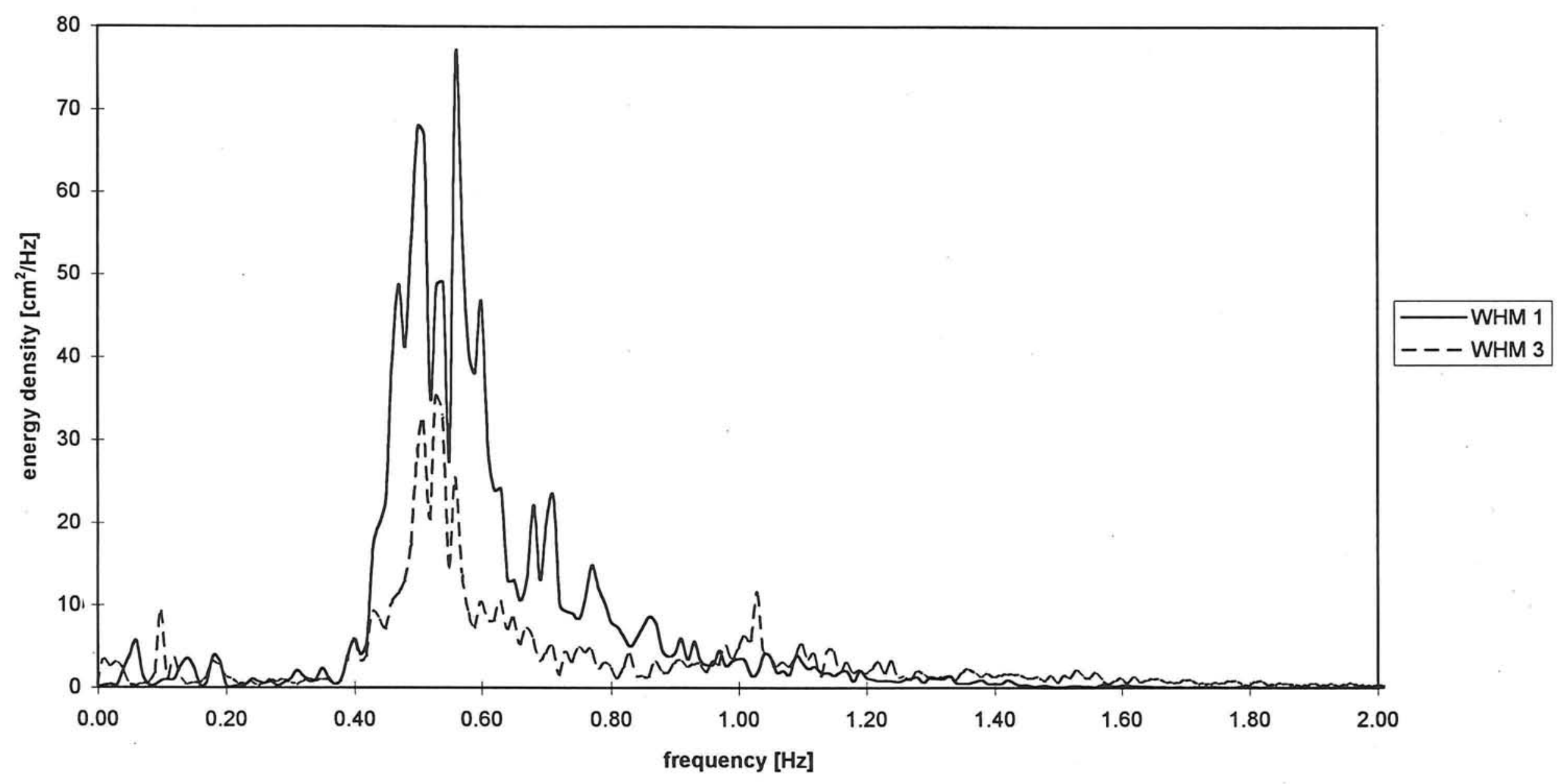

Graph 2.29 


\section{Spectra}

Experiment $E$ with breakwater

Measured during Interval 1

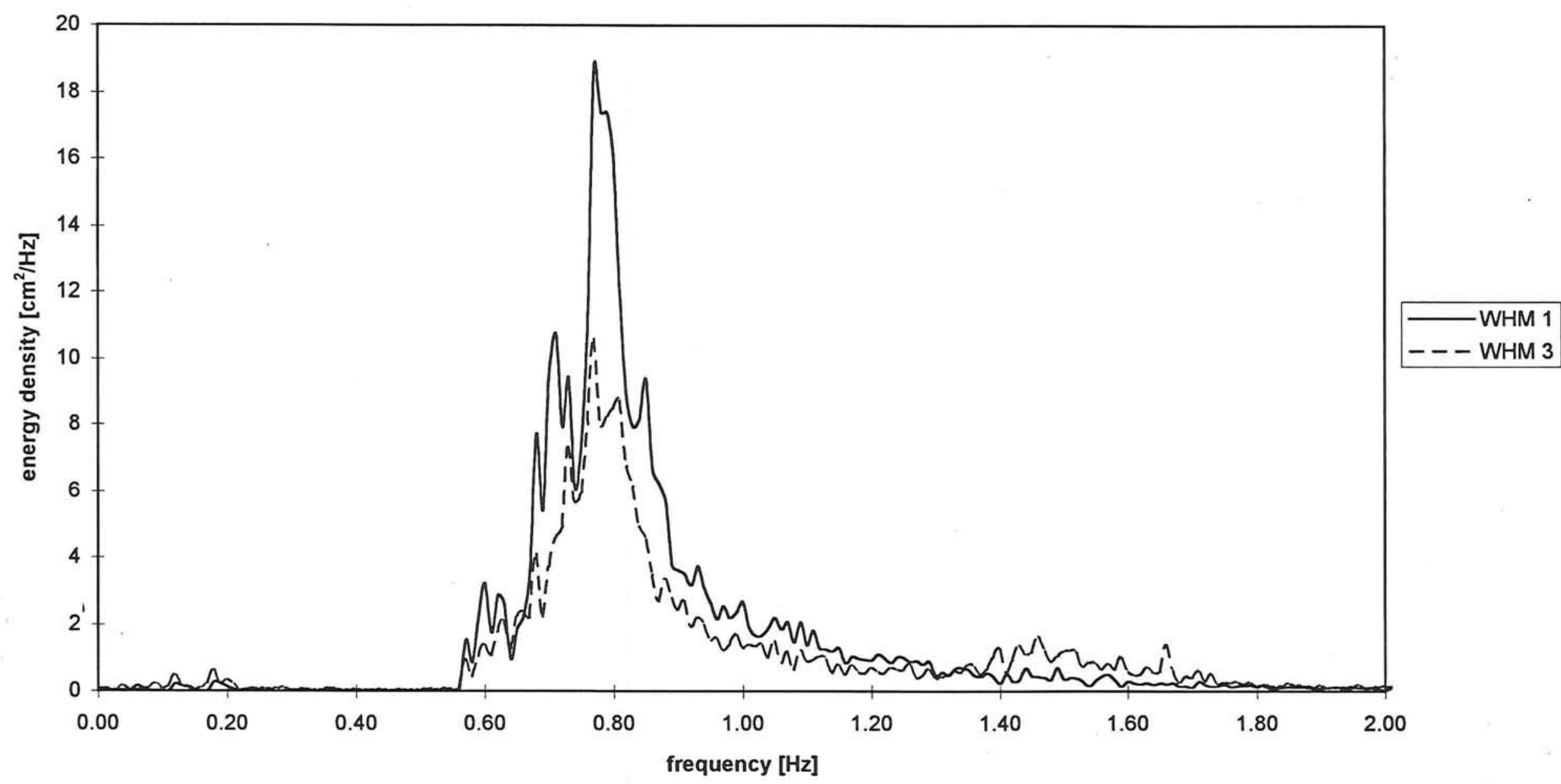

Graph 2.30 


\section{Velocity distributions}

\section{Experiments without breakwater}

Experiment A, Hs $=0.095 \mathrm{~m}, \mathrm{Tp}=2.07 \mathrm{~s}$, during Interval 4

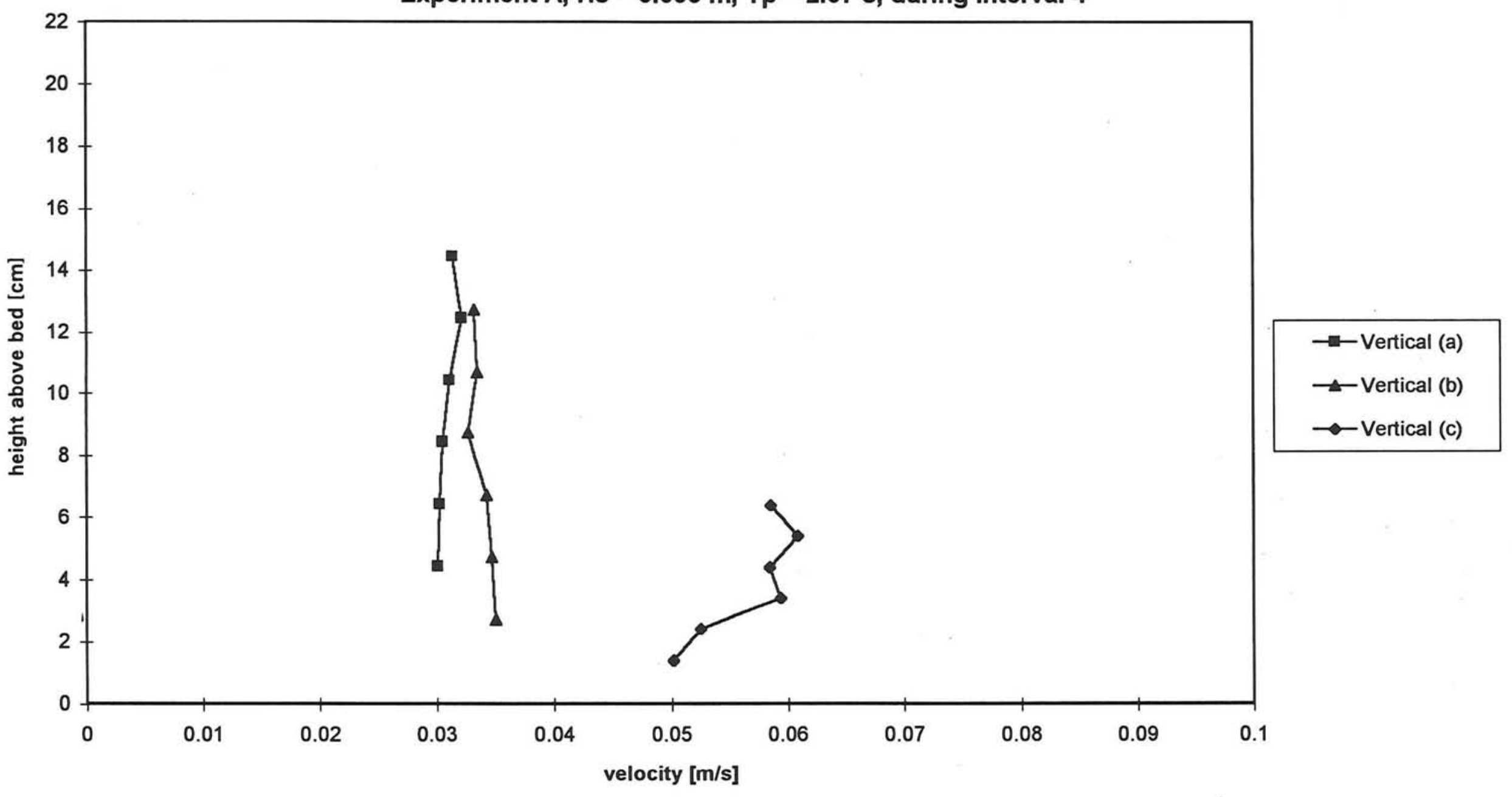

Graph 3.1 
Velocity distributions

Experiments without breakwater

Experiment $B, H s=0.098 m, T p=1.55 \mathrm{~s}$, during Interval 4

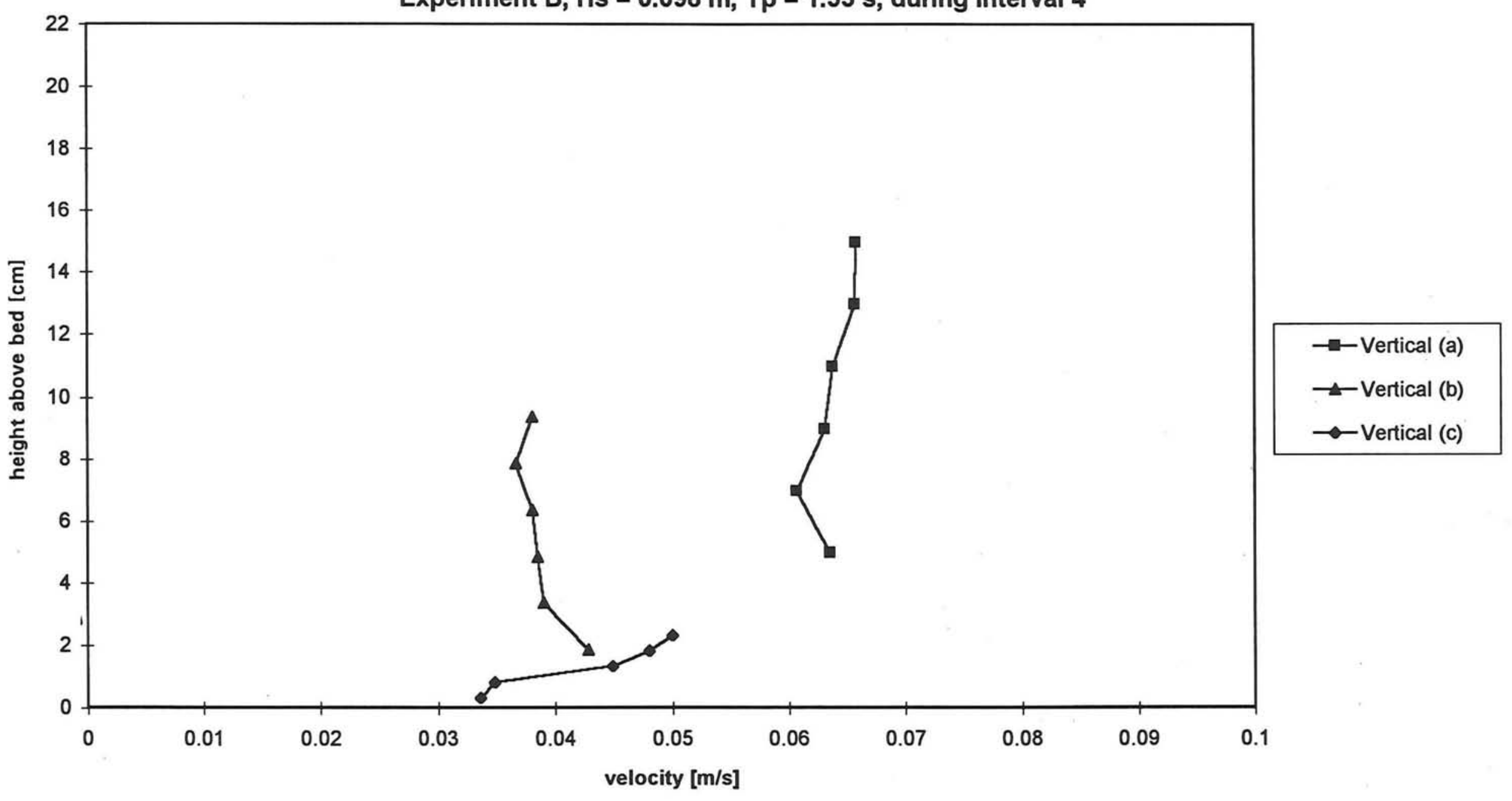

Graph 3.2 


\section{Velocity distributions}

Experiments without breakwater

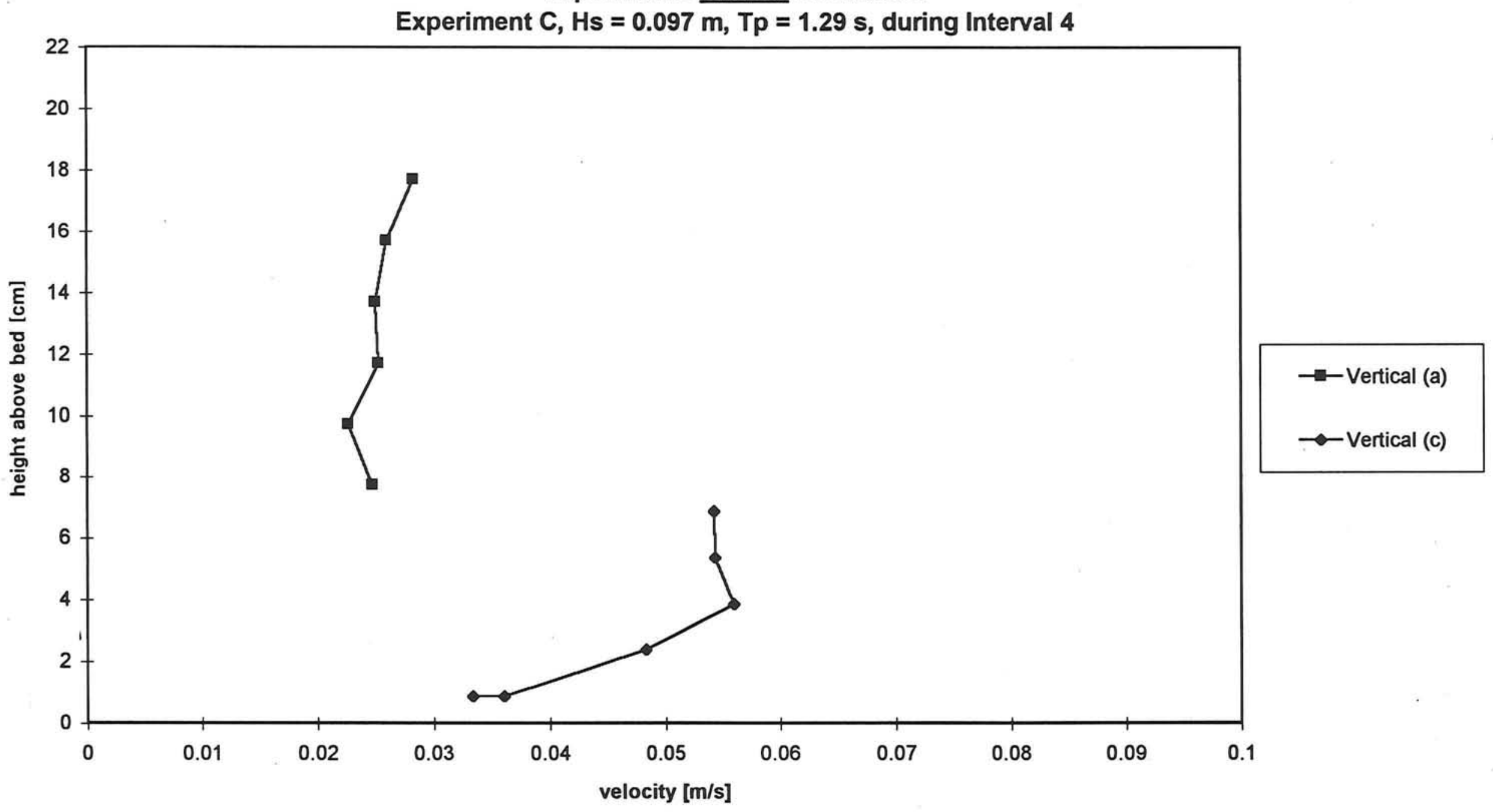

Graph 3.3 


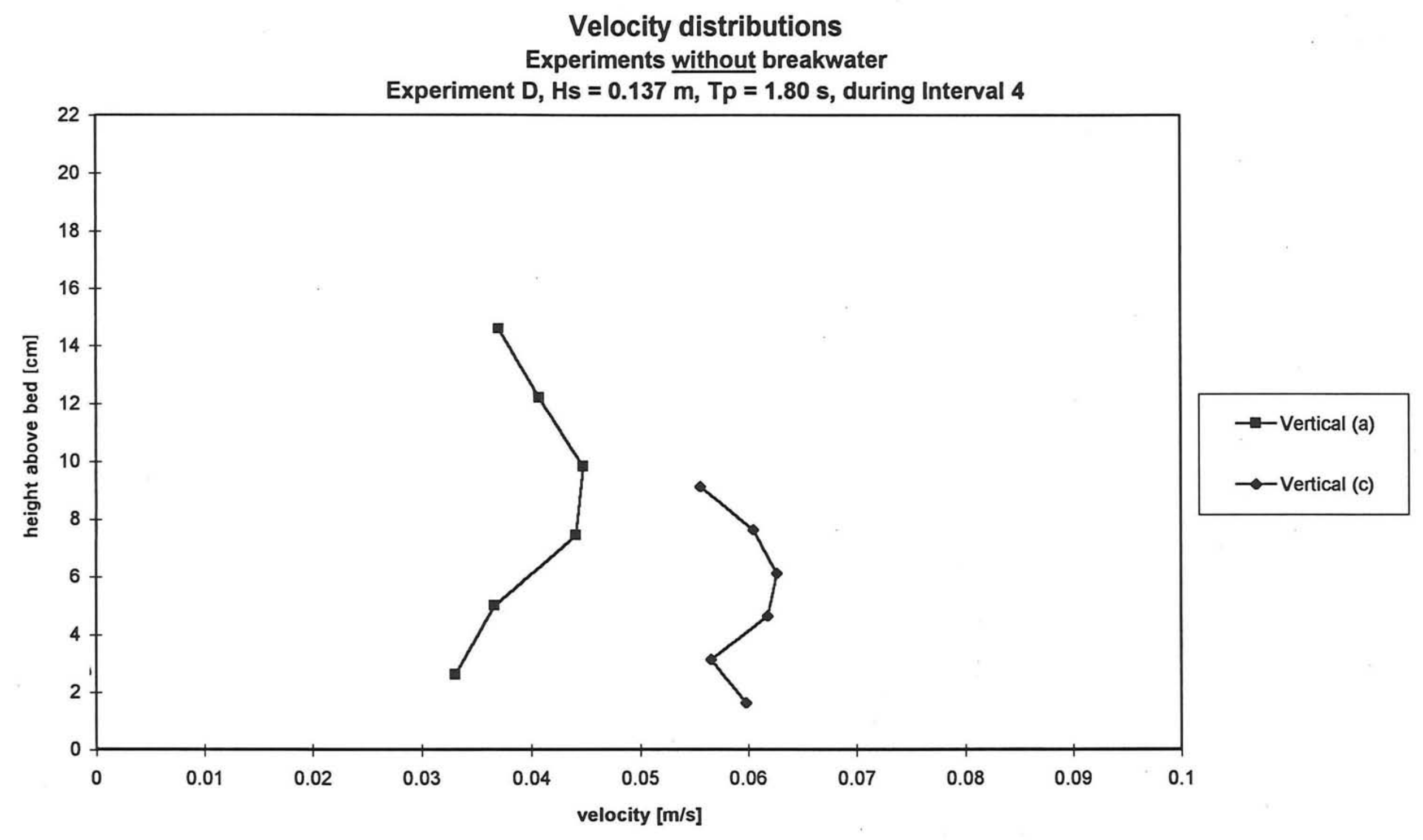

Graph 3.4 
Velocity distributions

Experiments without breakwater

Experiment $\mathrm{E}, \mathrm{Hs}=0.066 \mathrm{~m}, \mathrm{Tp}=1.29 \mathrm{~s}$, during Interval 4

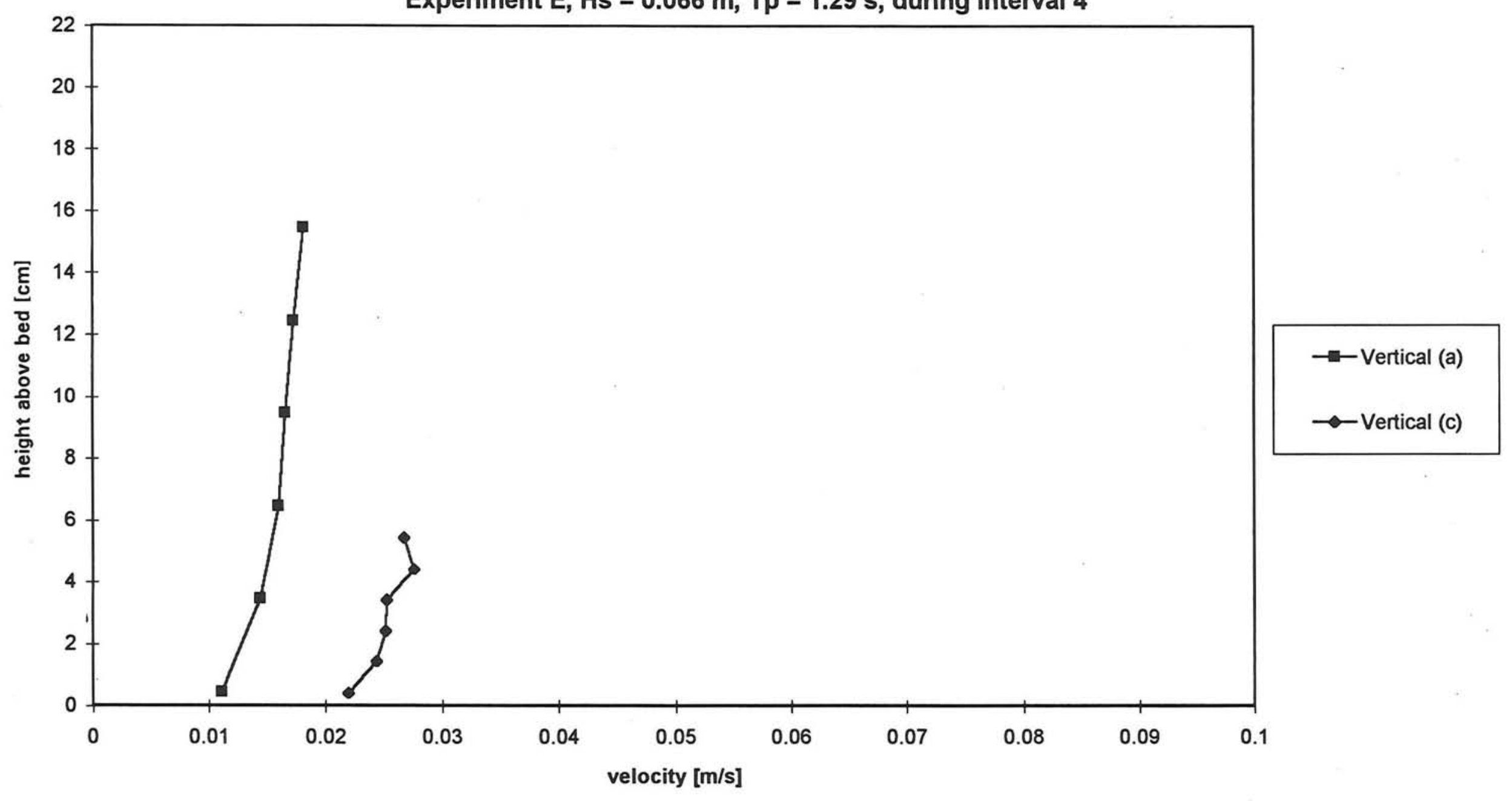

Graph 3.5 


\section{Velocity distributions}

Experiments with breakwater

Experiment $\mathrm{A}, \mathrm{Hs}=\mathbf{0 . 0 9 5} \mathrm{m}, \mathrm{Tp}=\mathbf{2 . 0 7} \mathrm{s}$, during Interval 4

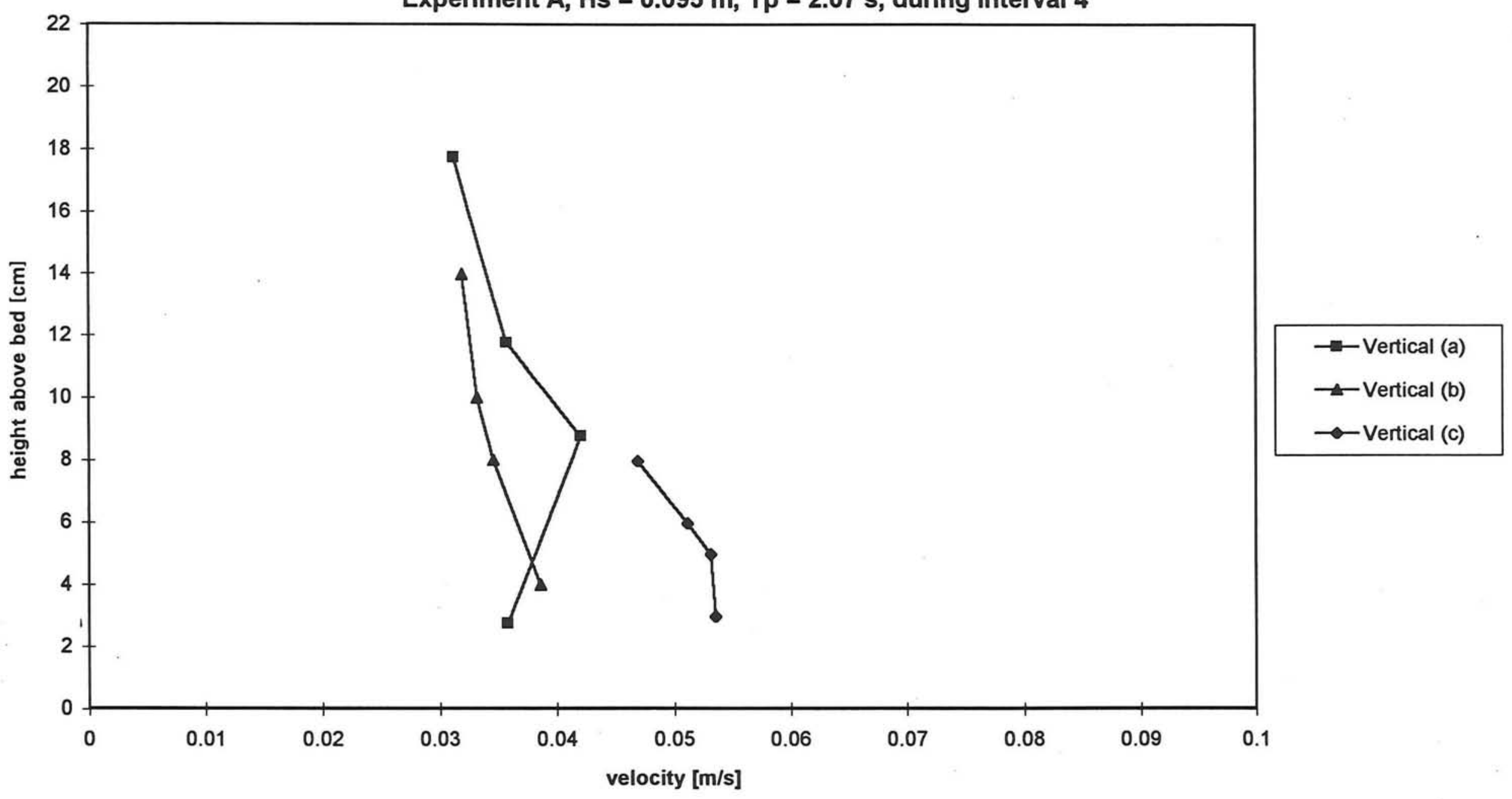

Graph 3.6 


\section{Velocity distributions}

\section{Experiments with breakwater}

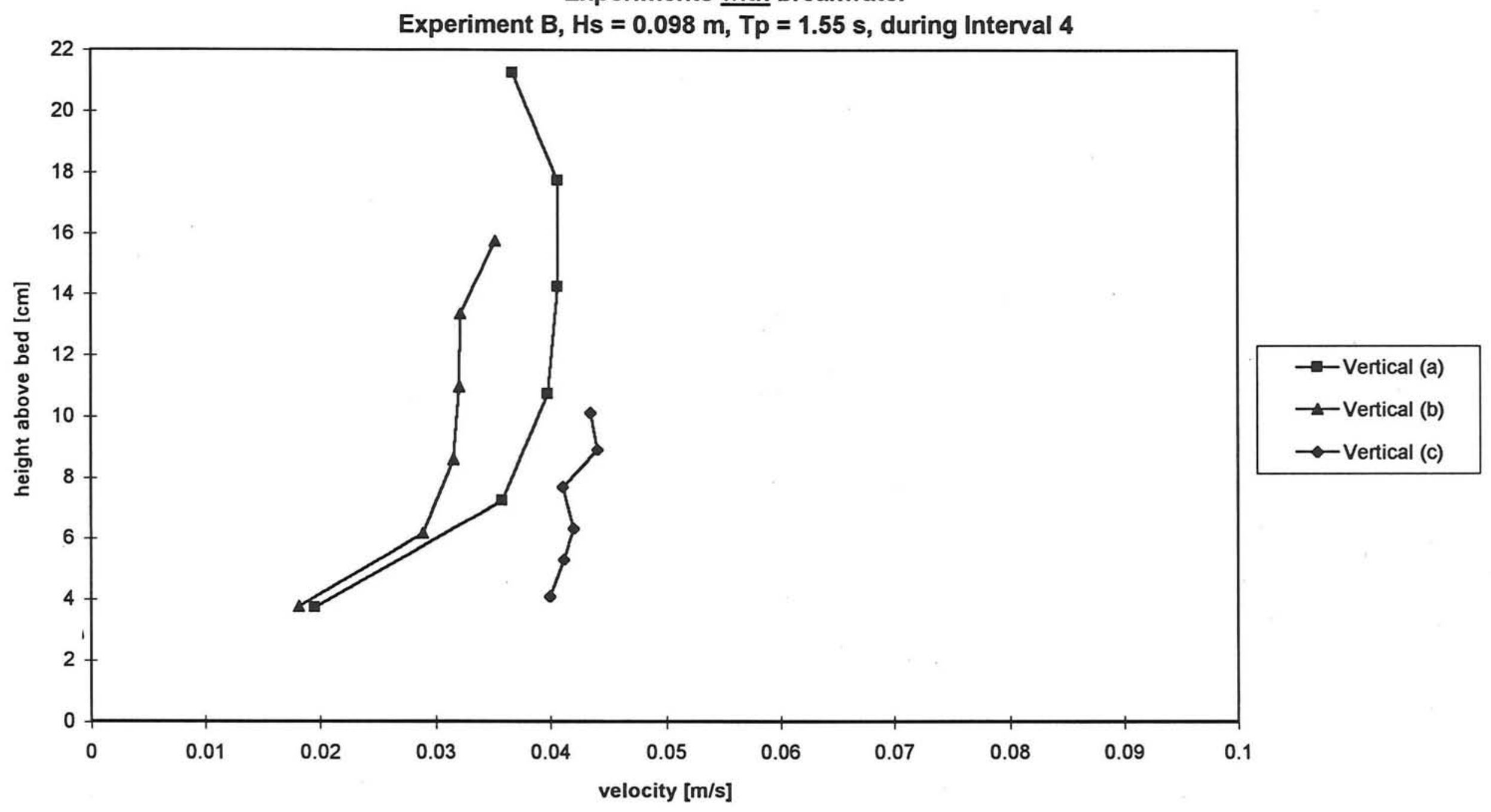

Graph 3.7 


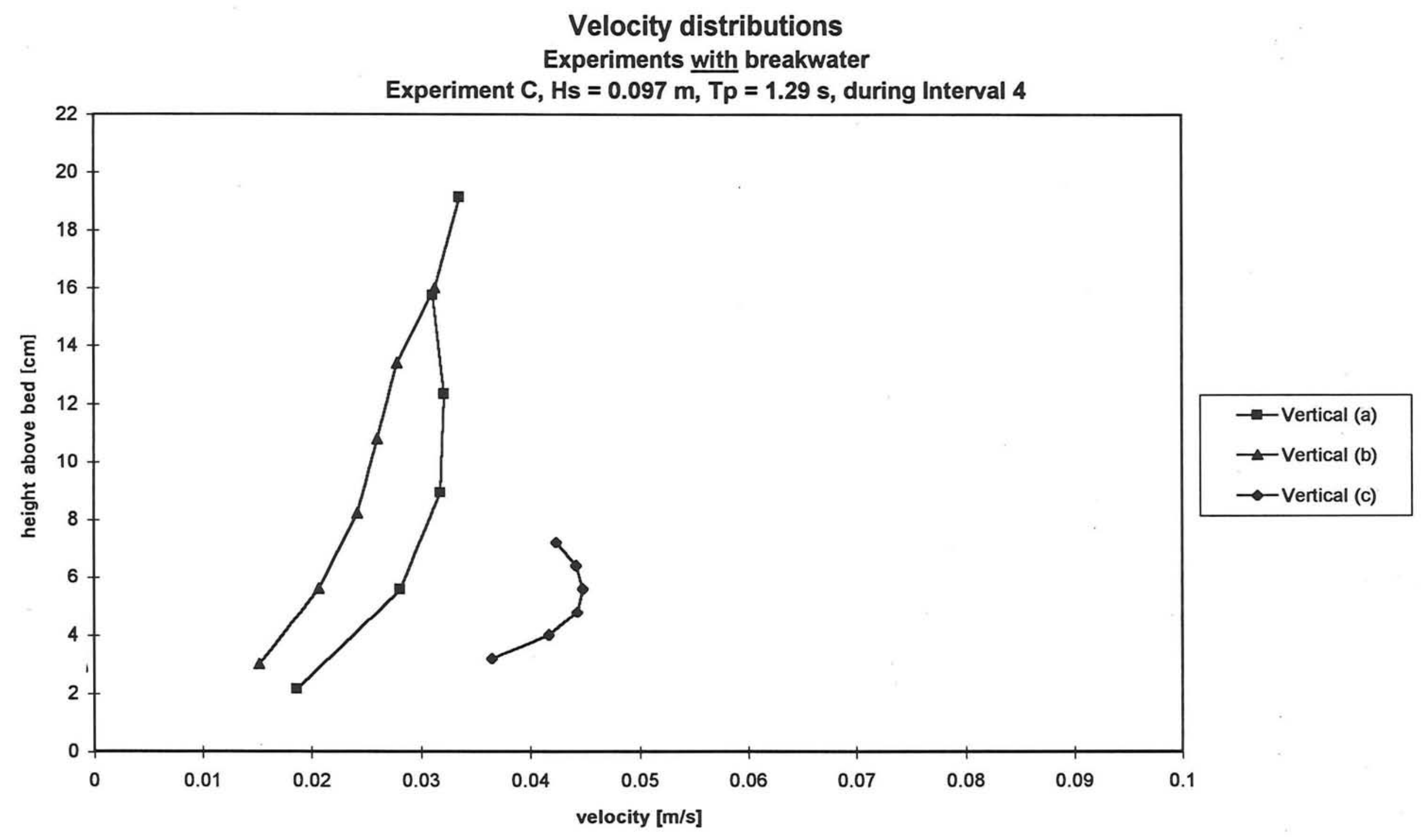

Graph 3.8 


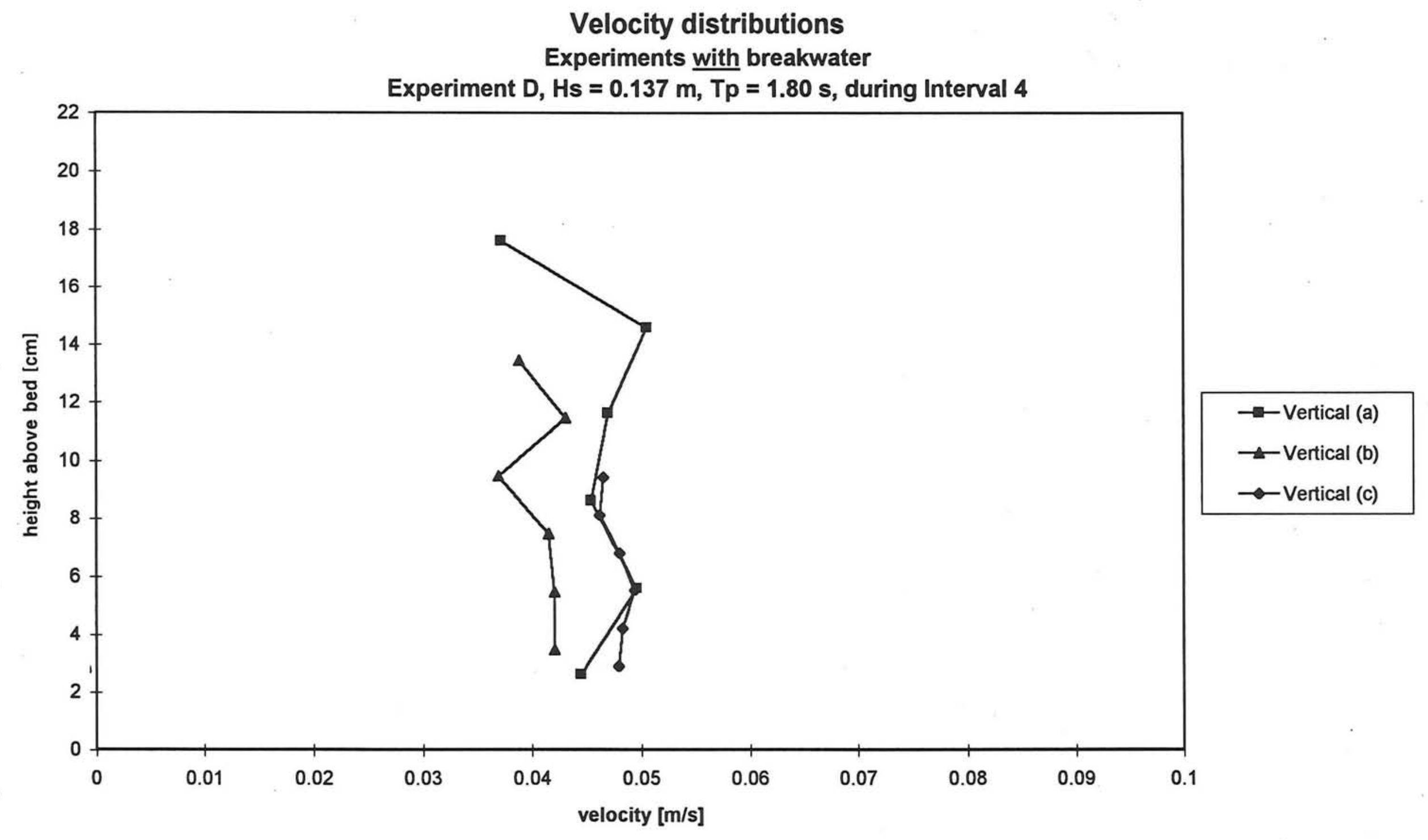

Graph 3.9 


\section{Velocity distributions}

Experiments with breakwater

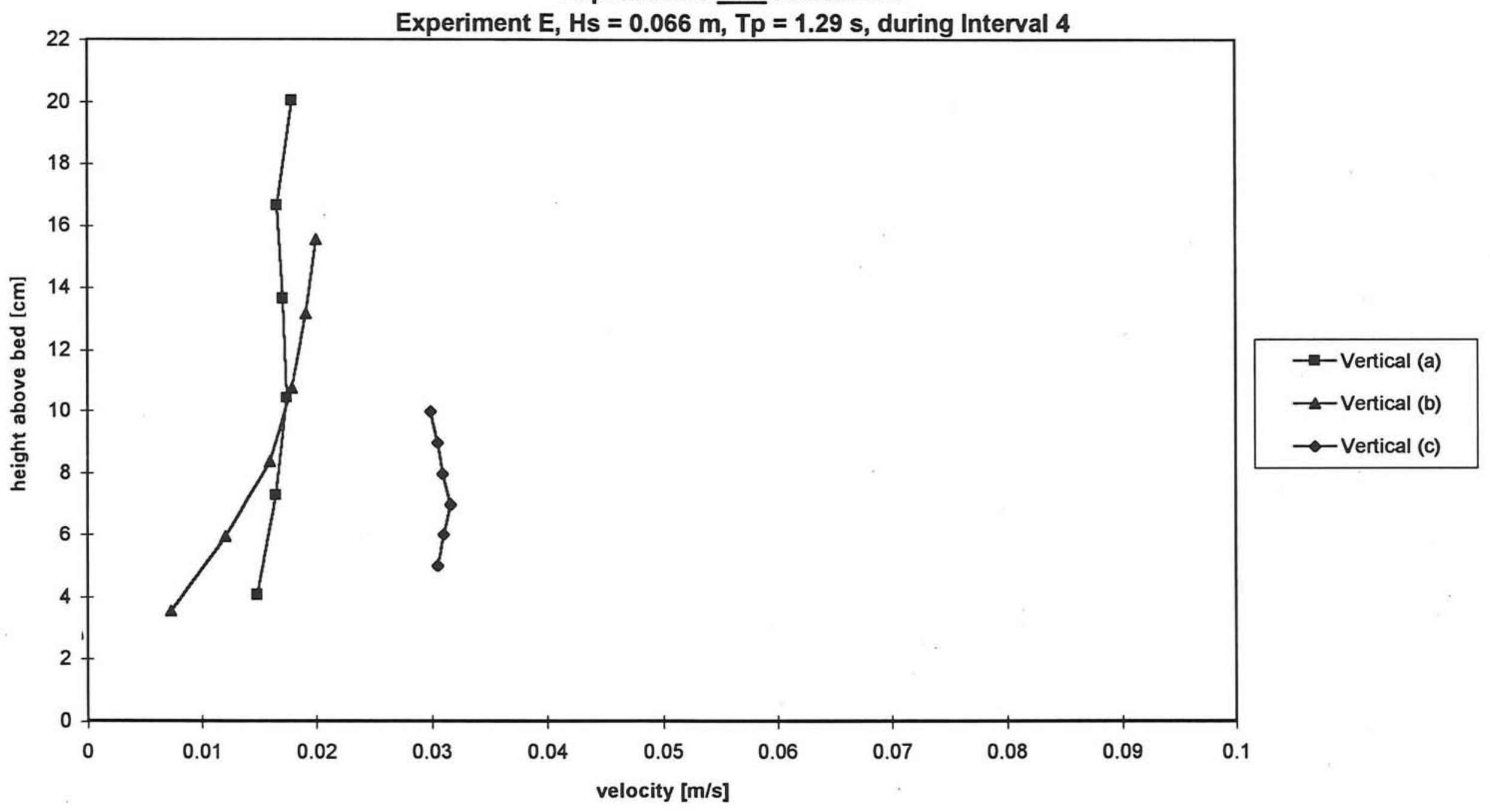

Graph 3.10 
Velocity distributions

Experiments with and without breakwater

Experiment A during Interval 4 at Vertical (a): $x=5.25 \mathrm{~m}$

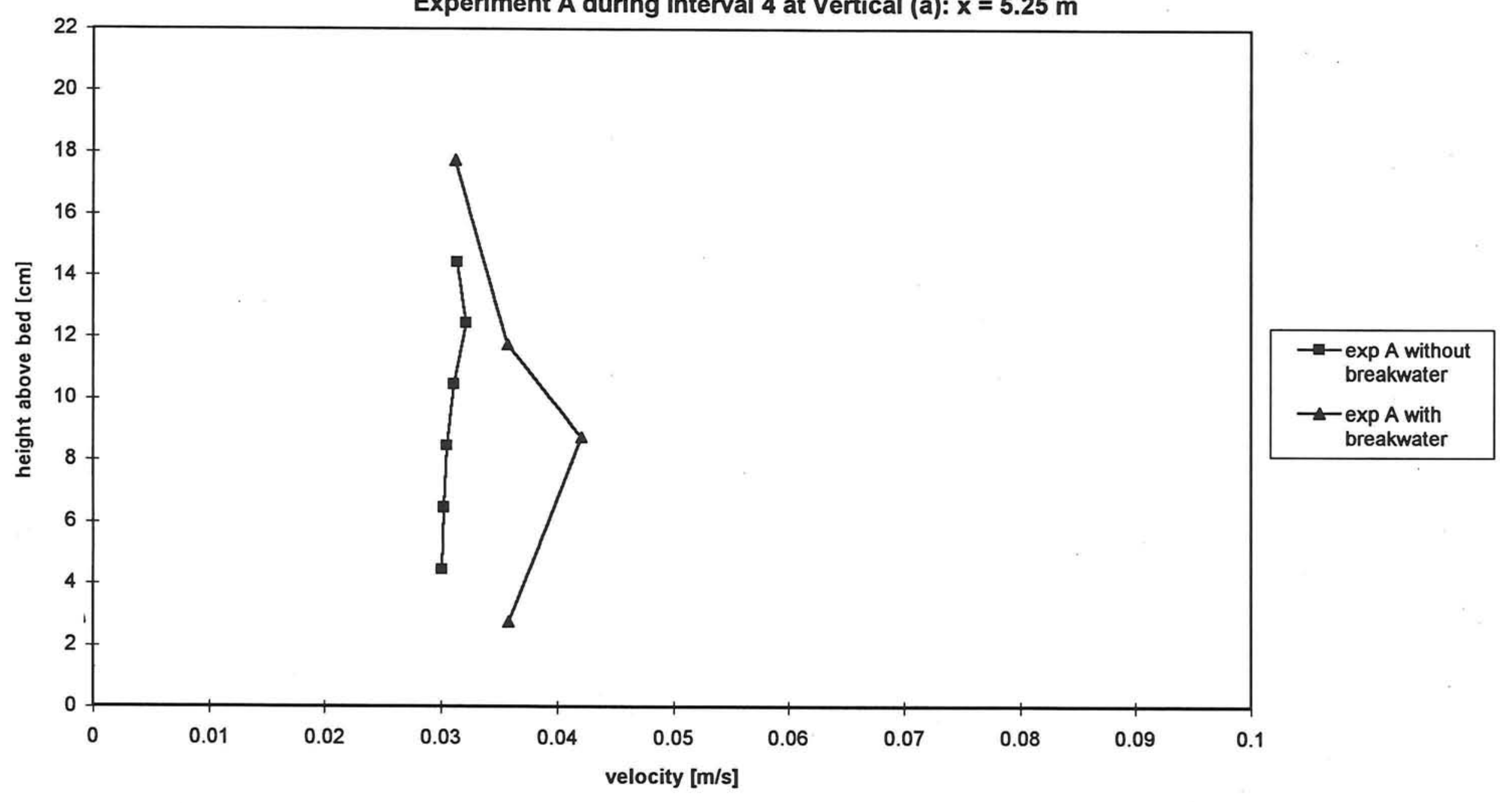

Graph 3.11 
Velocity distributions

Experiments with and without breakwater

Experiment A during Interval 4 at Vertical (b): $x=6.15 \mathrm{~m}$

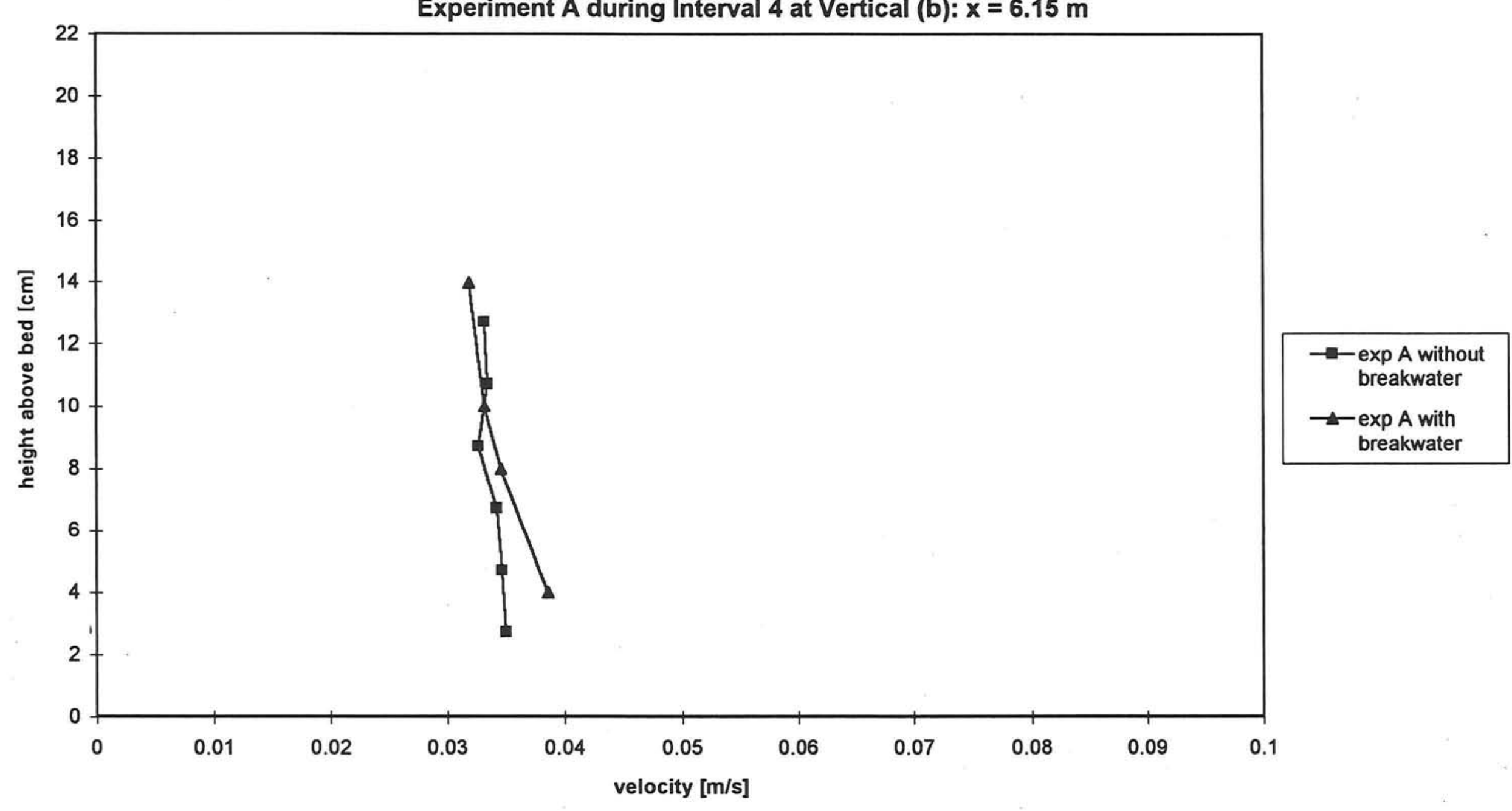

Graph 3.12 


\section{Velocity distributions}

Experiments with and without breakwater

Experiment A during Interval 4 at Vertical (c): $x=7.05 \mathrm{~m}$

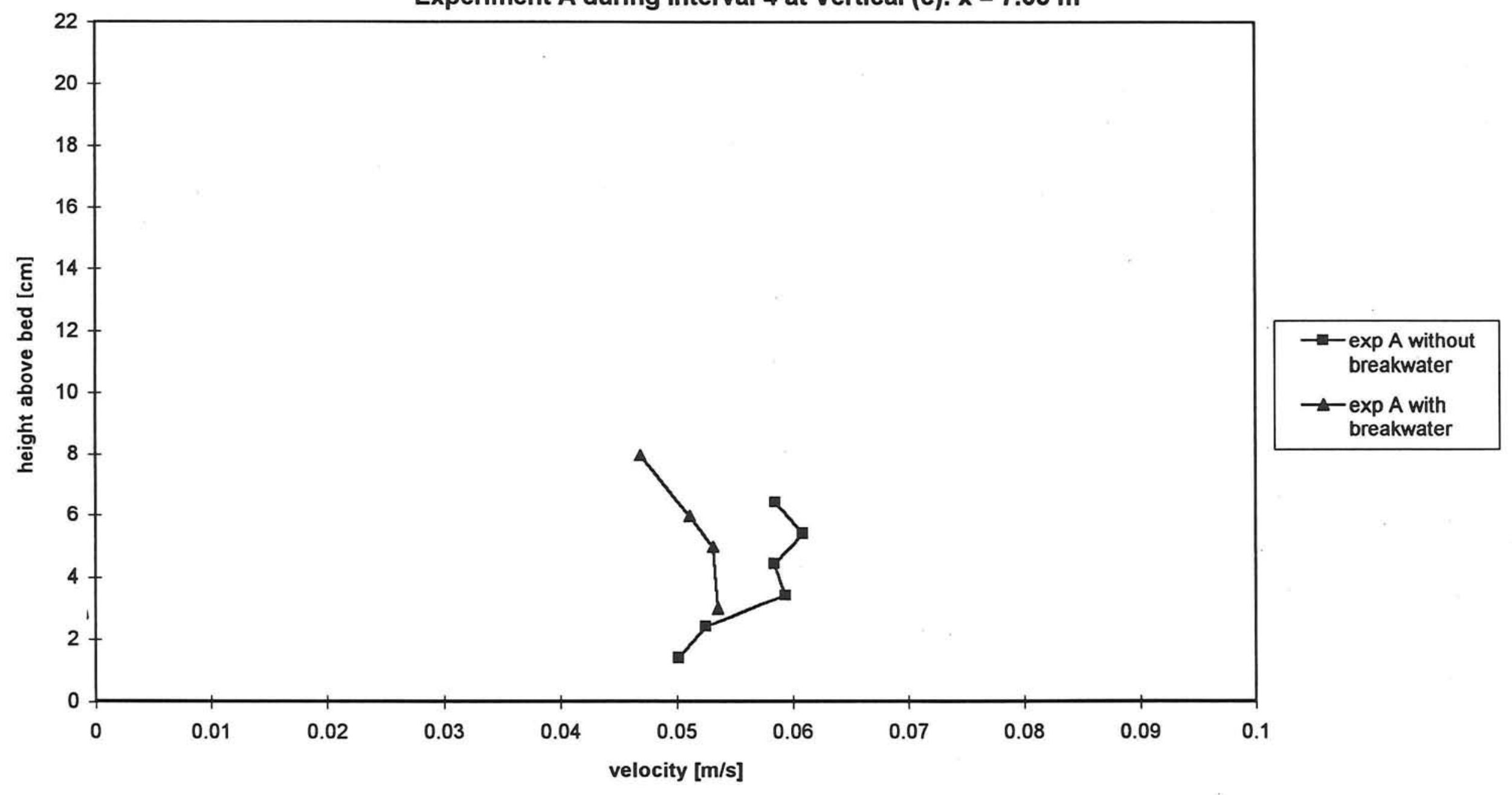

Graph 3.13 
Velocity distributions

Experiments with and without breakwater

Experiment $B$ during Interval 4 at Vertical (a): $x=5.25 \mathrm{~m}$

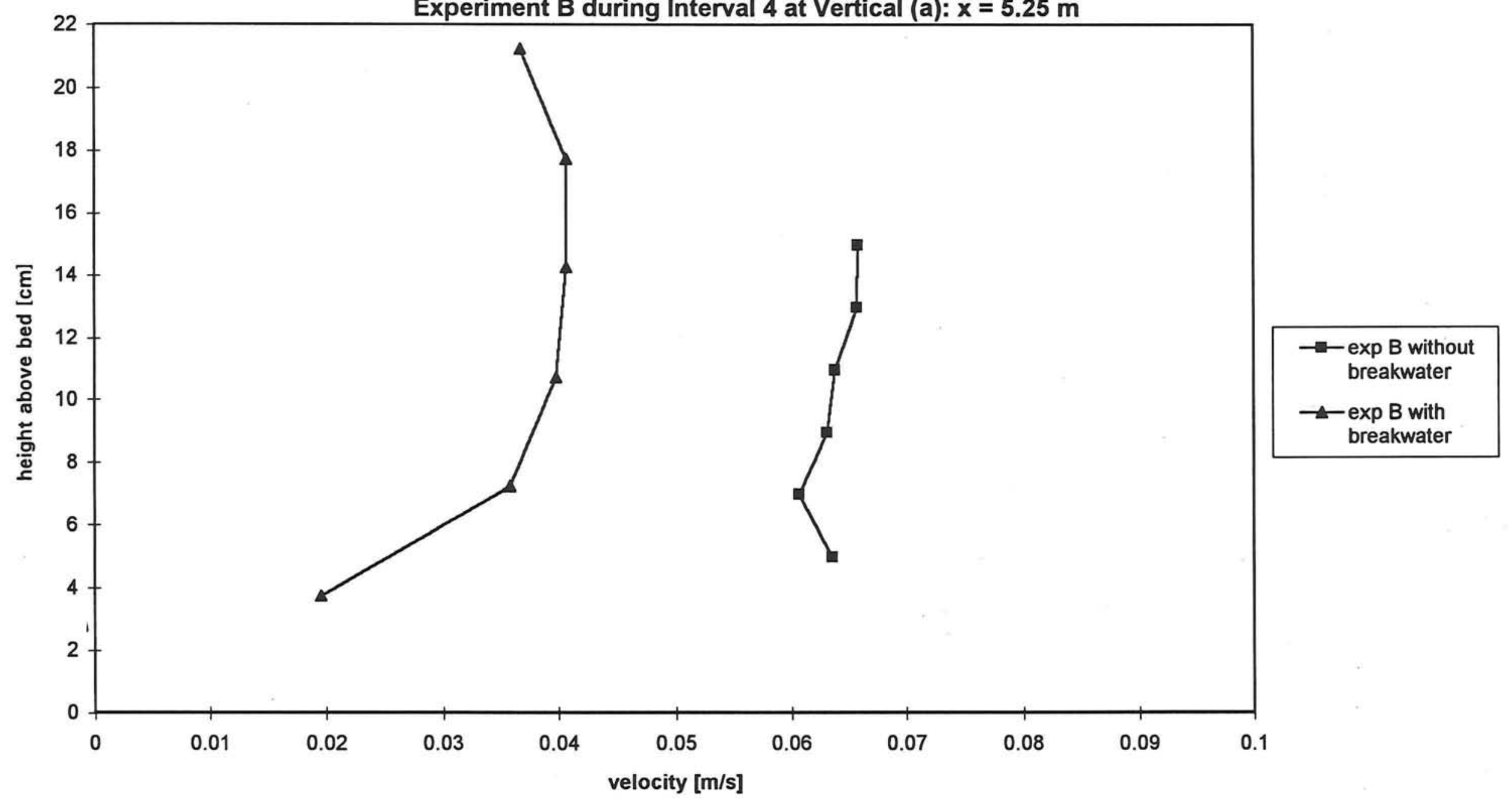

Graph 3.14 
Velocity distributions

Experiments with and without breakwater

Experiment $B$ during Interval 4 at Vertical (b): $x=6.15 \mathrm{~m}$

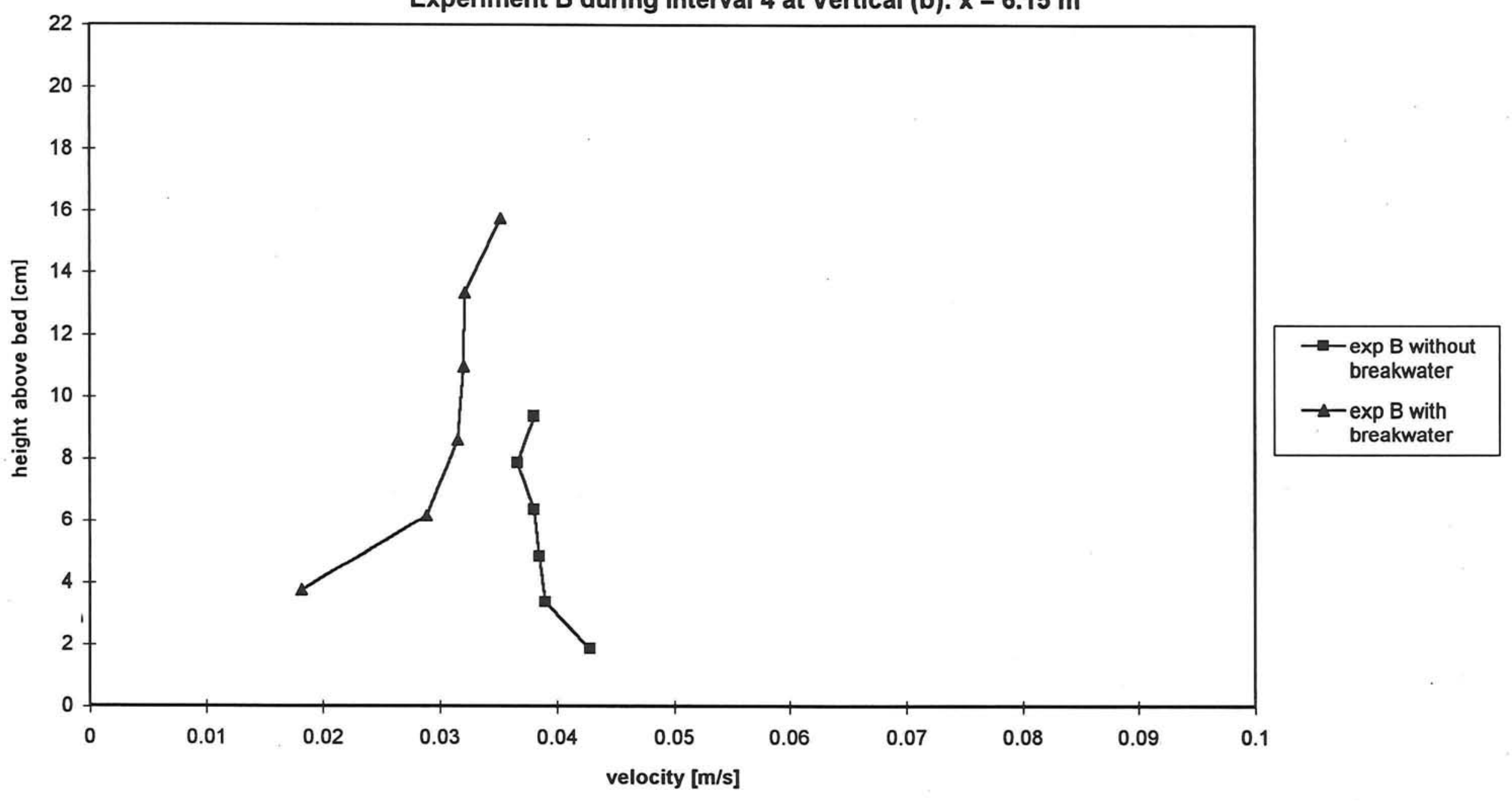

Graph 3.15 


\section{Velocity distributions}

Experiments with and without breakwater

Experiment $B$ during Interval 4 at Vertical (c): $x=7.05 \mathrm{~m}$

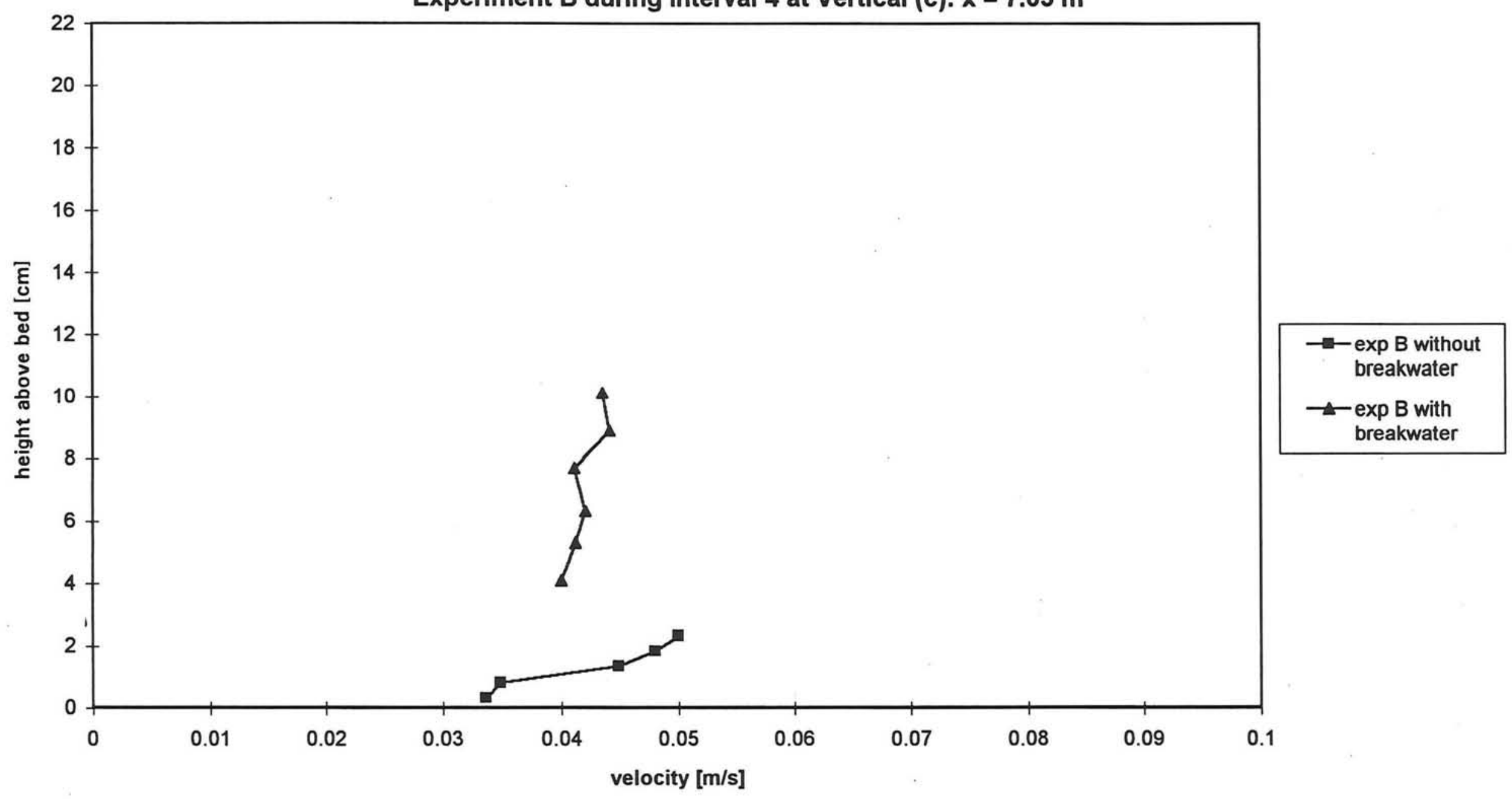

Graph 3.16 
Velocity distributions

Experiments with and without breakwater

Experiment $C$ during Interval 4 at Vertical (a): $x=5.25 \mathrm{~m}$

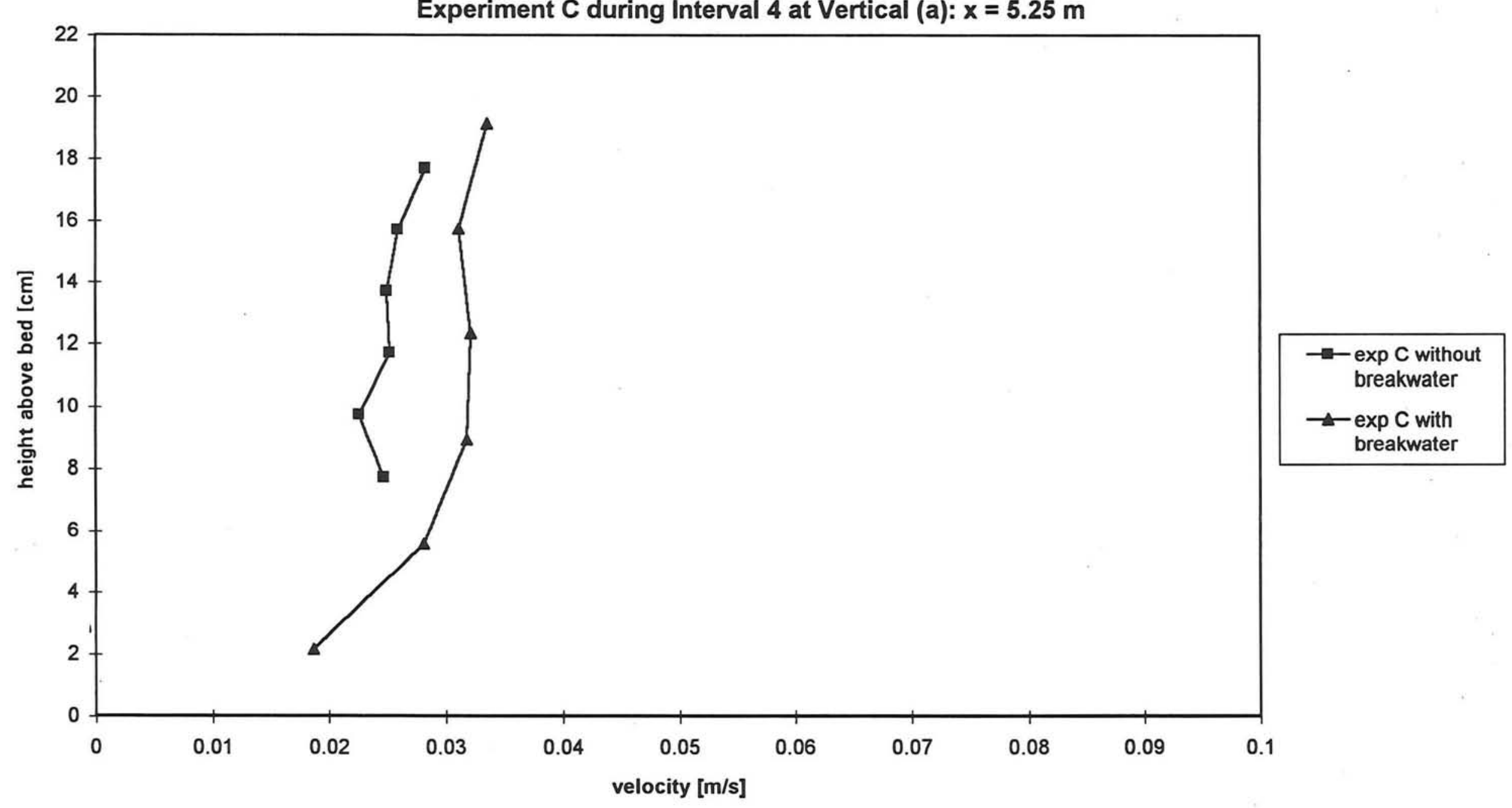

Graph 3.17 


\section{Velocity distributions}

Experiments with and without breakwater

Experiment $C$ during Interval 4 at Vertical (c): $x=7.05 \mathrm{~m}$

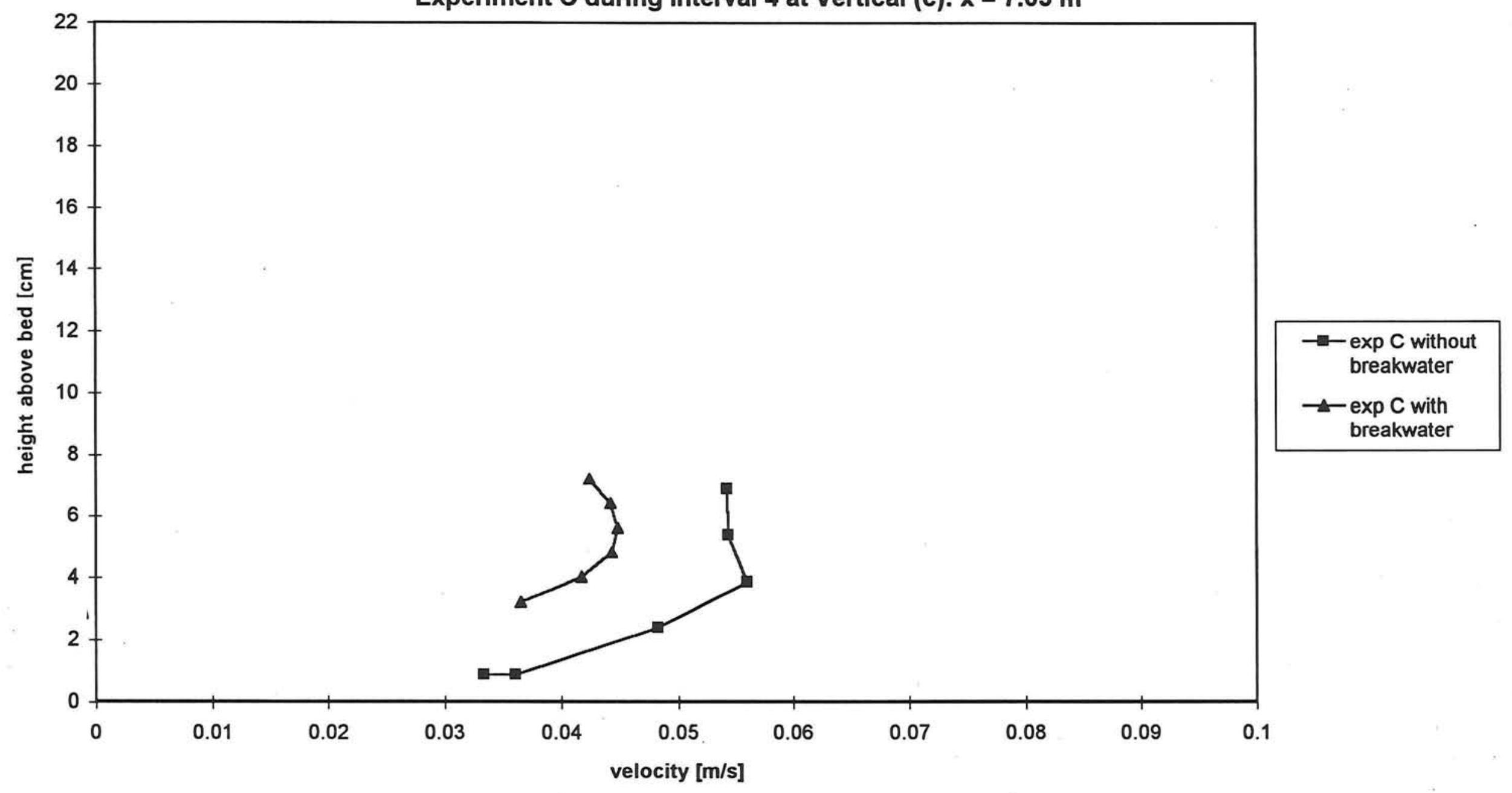

Graph 3.18 


\section{Velocity distributions}

Experiments with and without breakwater

Experiment $D$ during Interval 4 at Vertical (a): $x=5.25 \mathrm{~m}$

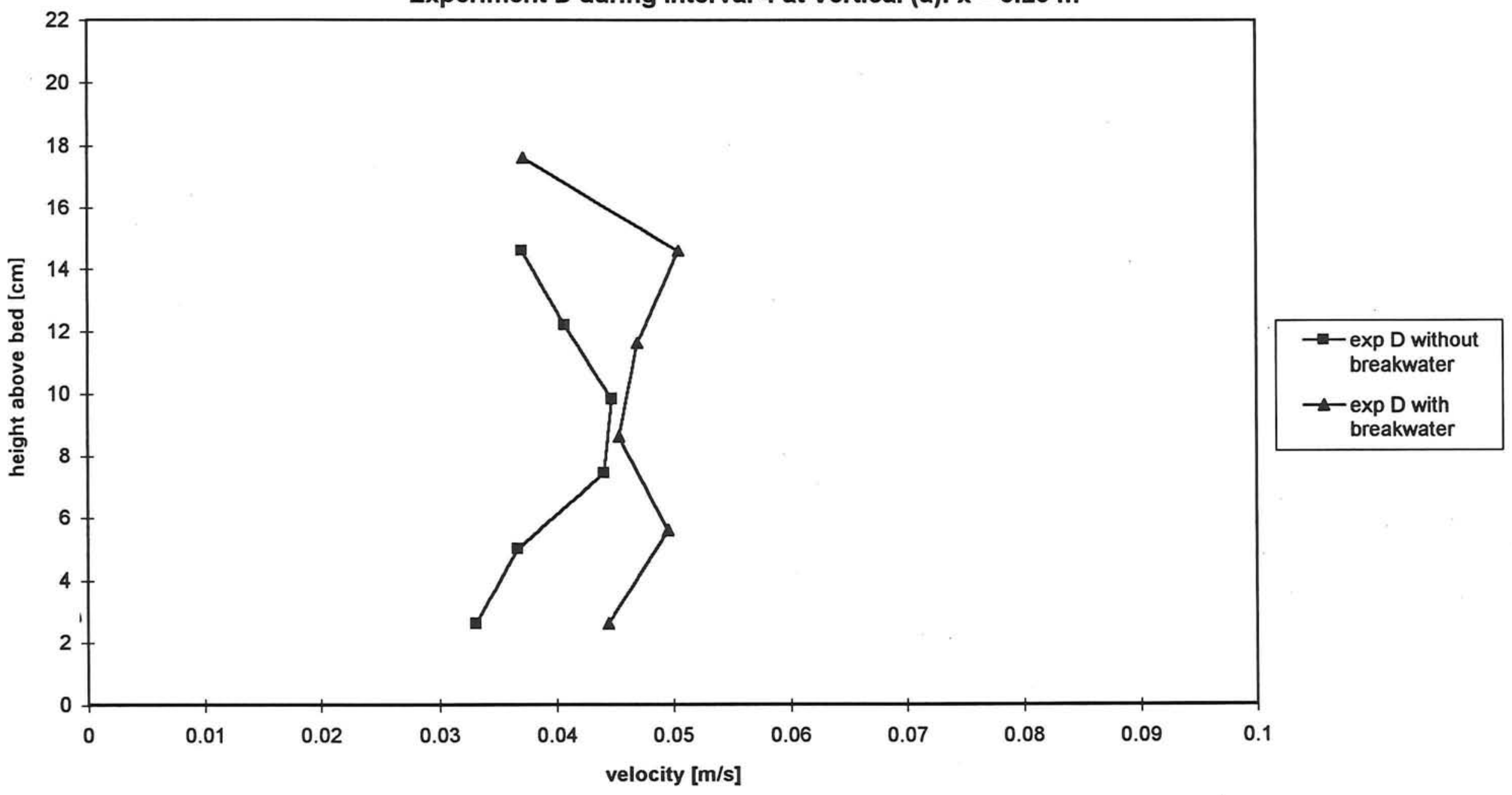

Graph 3.19 
Velocity distributions

Experiments with and without breakwater

Experiment E during Interval 4 at Vertical (a): $x=5.25 \mathrm{~m}$

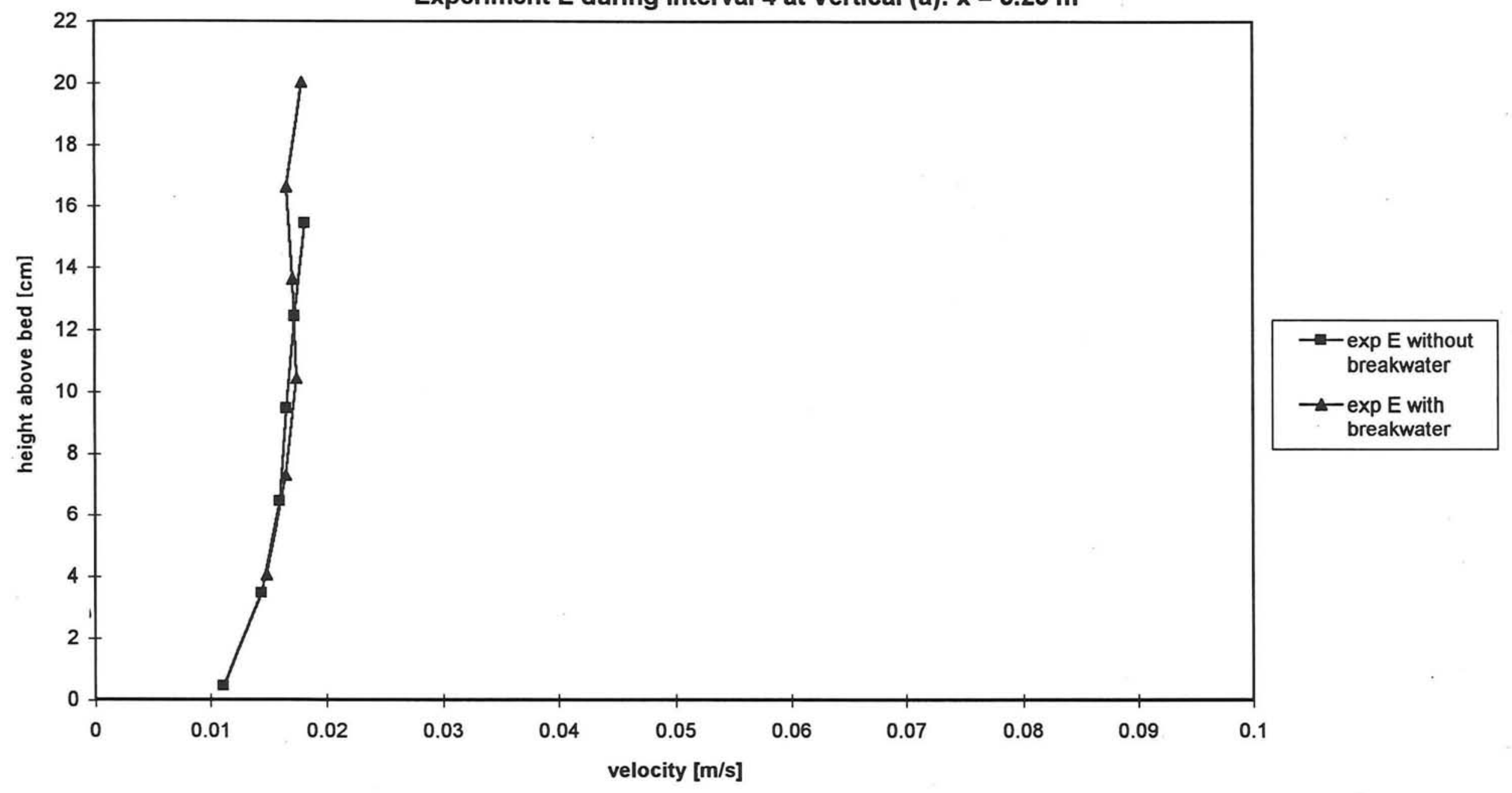

Graph 3.21 
Velocity distributions

Experiments with and without breakwater

Experiment $E$ during Interval 4 at Vertical (c): $x=7.05 \mathrm{~m}$

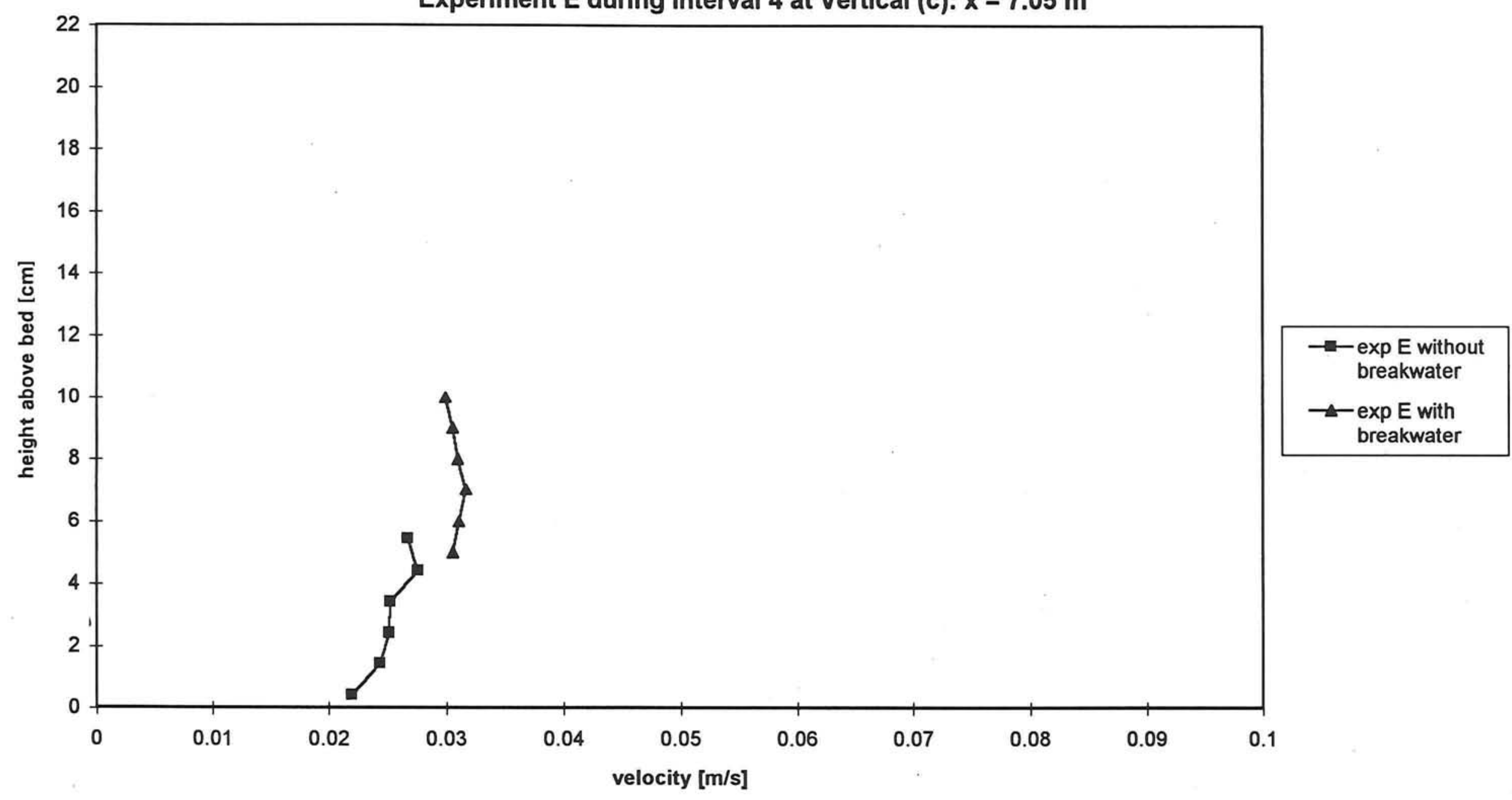

Graph 3.22 


\section{Sediment concentration distributions}

Experiment A without breakwater Measurement during Interval 1

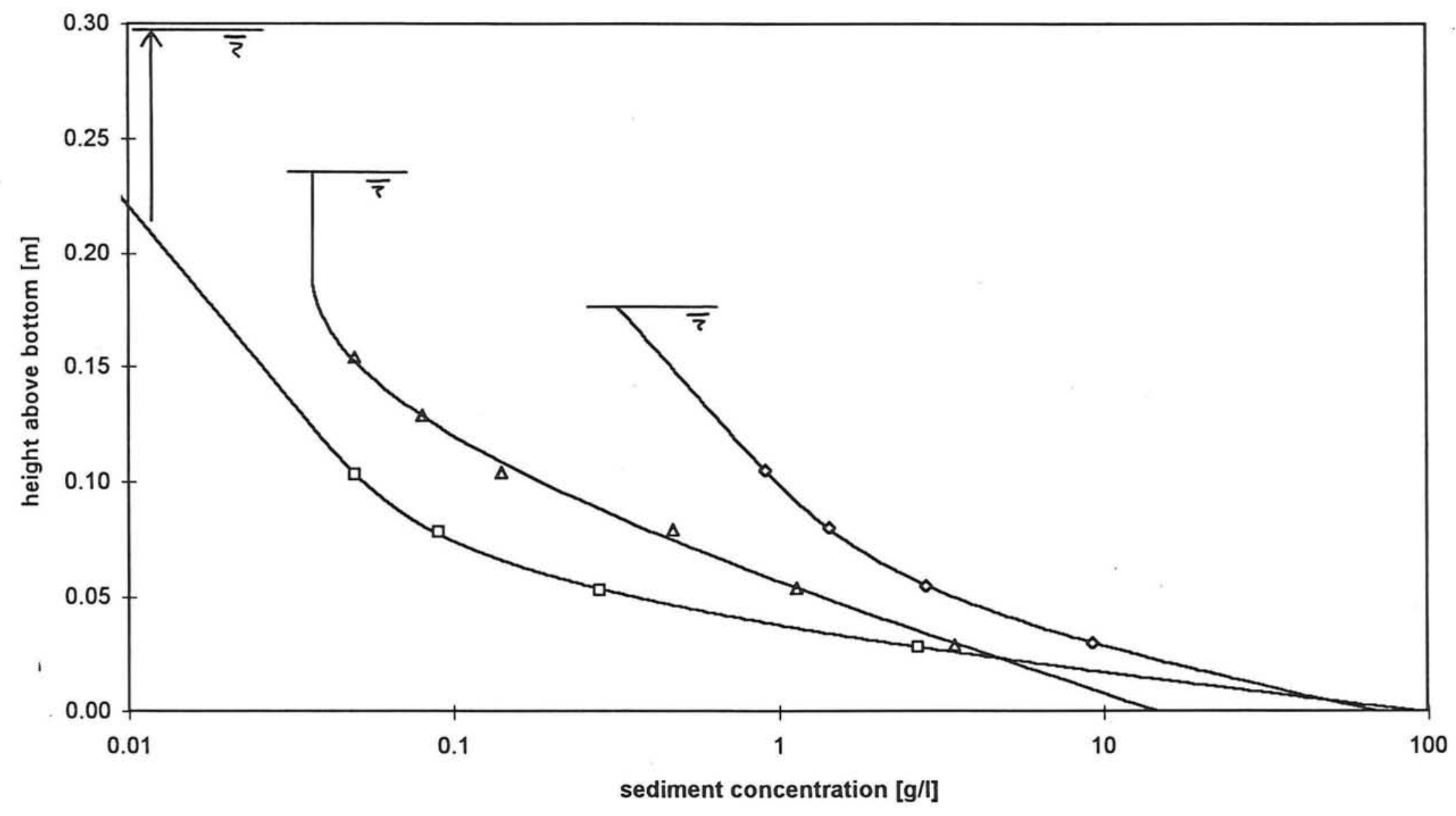




\section{Sediment concentration distributions}

Experiment B without breakwater

Measurement during Interval 1

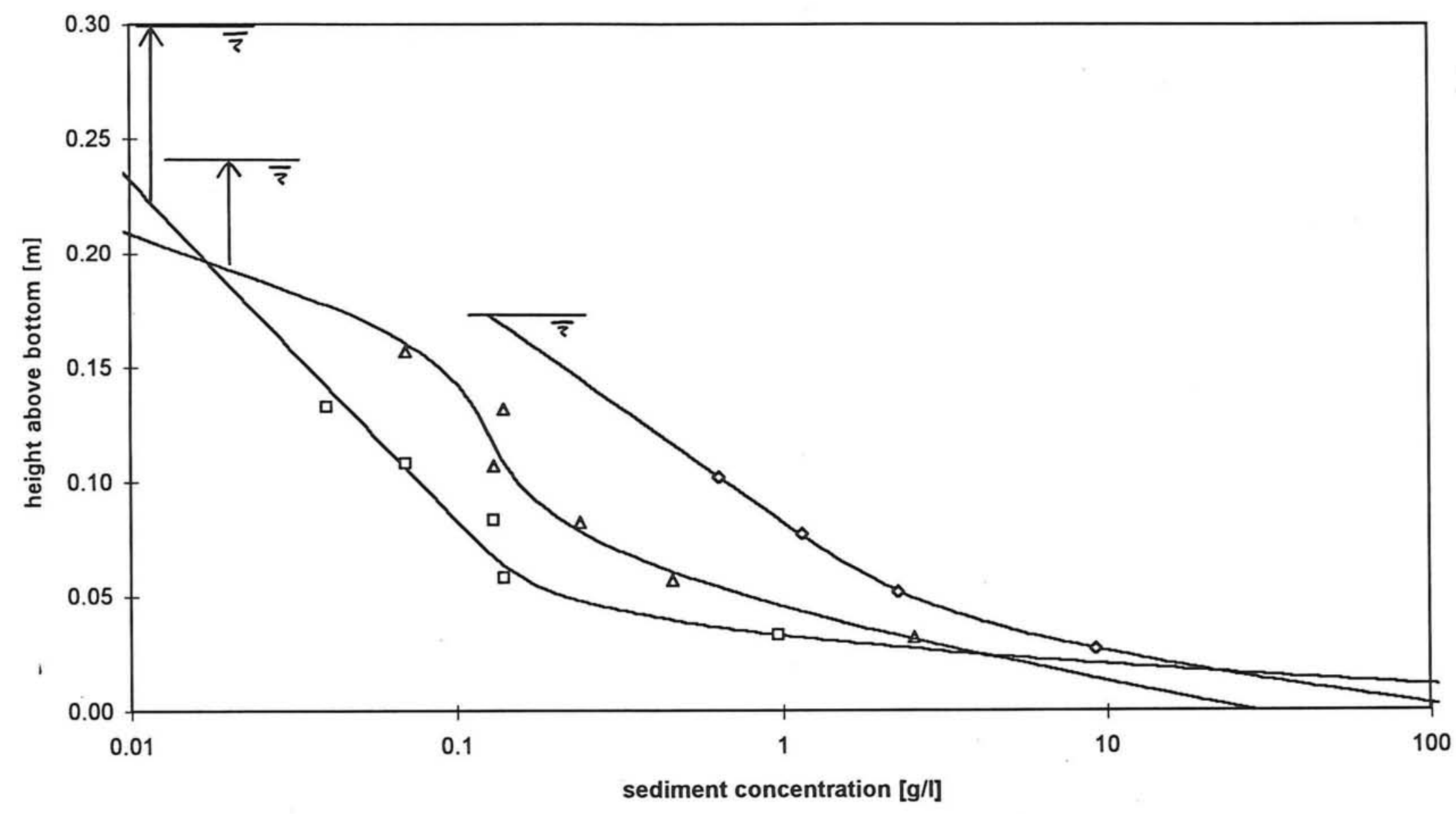




\section{Sediment concentration distributions}

Experiment $D$ without breakwater

Measurement during Interval 1

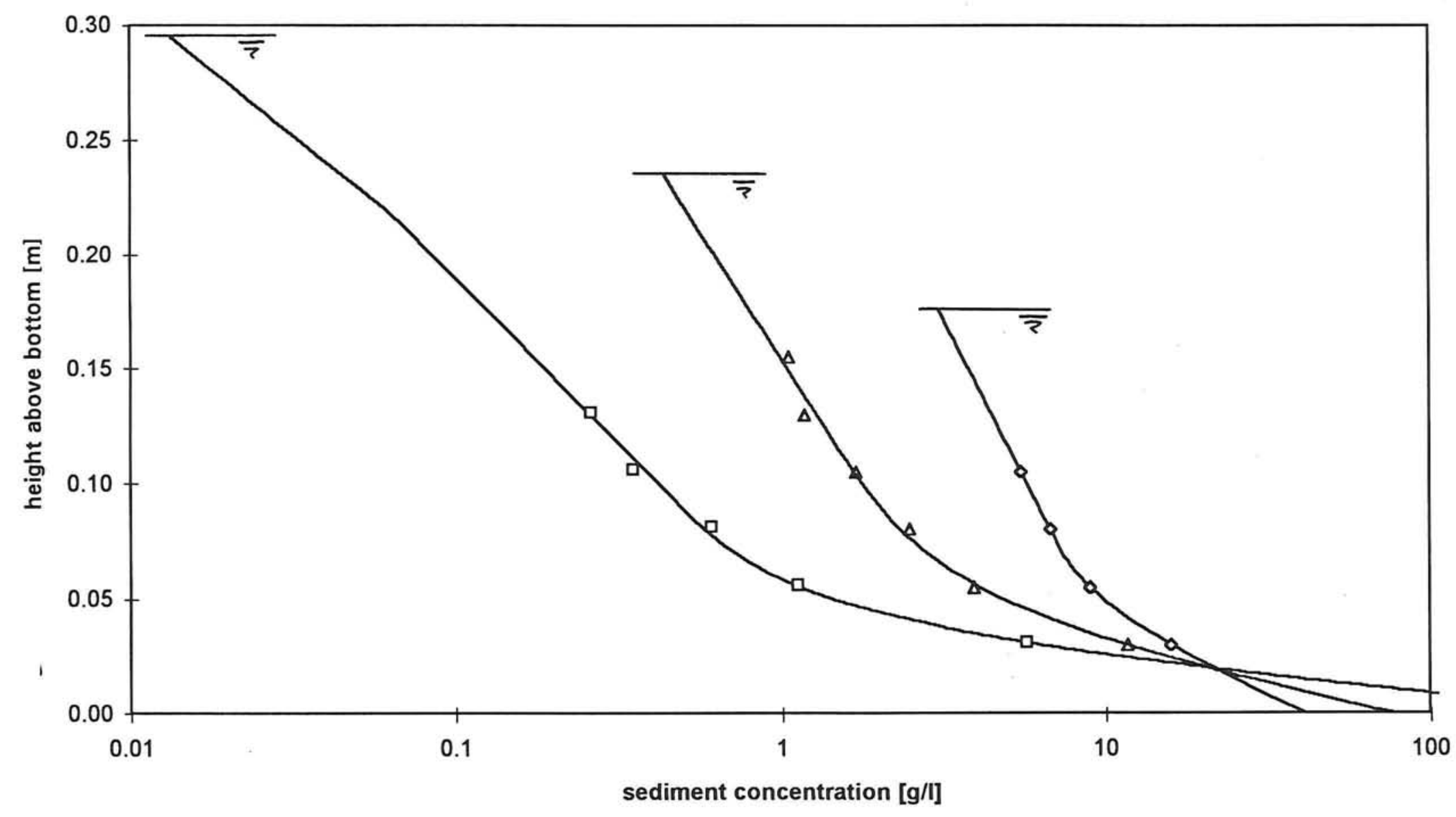




\section{Sediment concentration distributions}

Experiment $\mathrm{A}$ with breakwater

Measurement during Interval 1

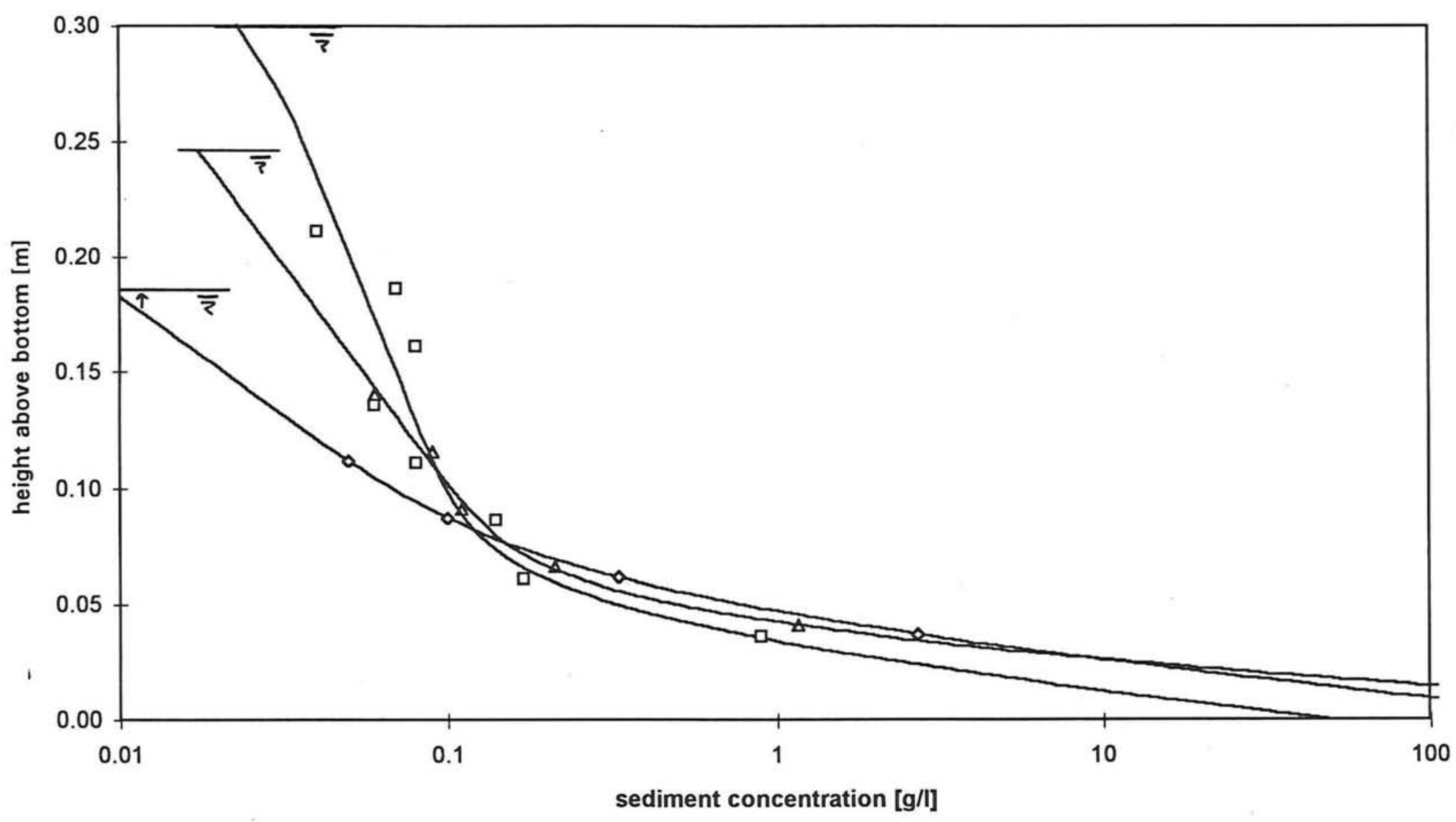




\section{Sediment concentration distributions}

Experiment $B$ with breakwater

Measurement during Interval 1

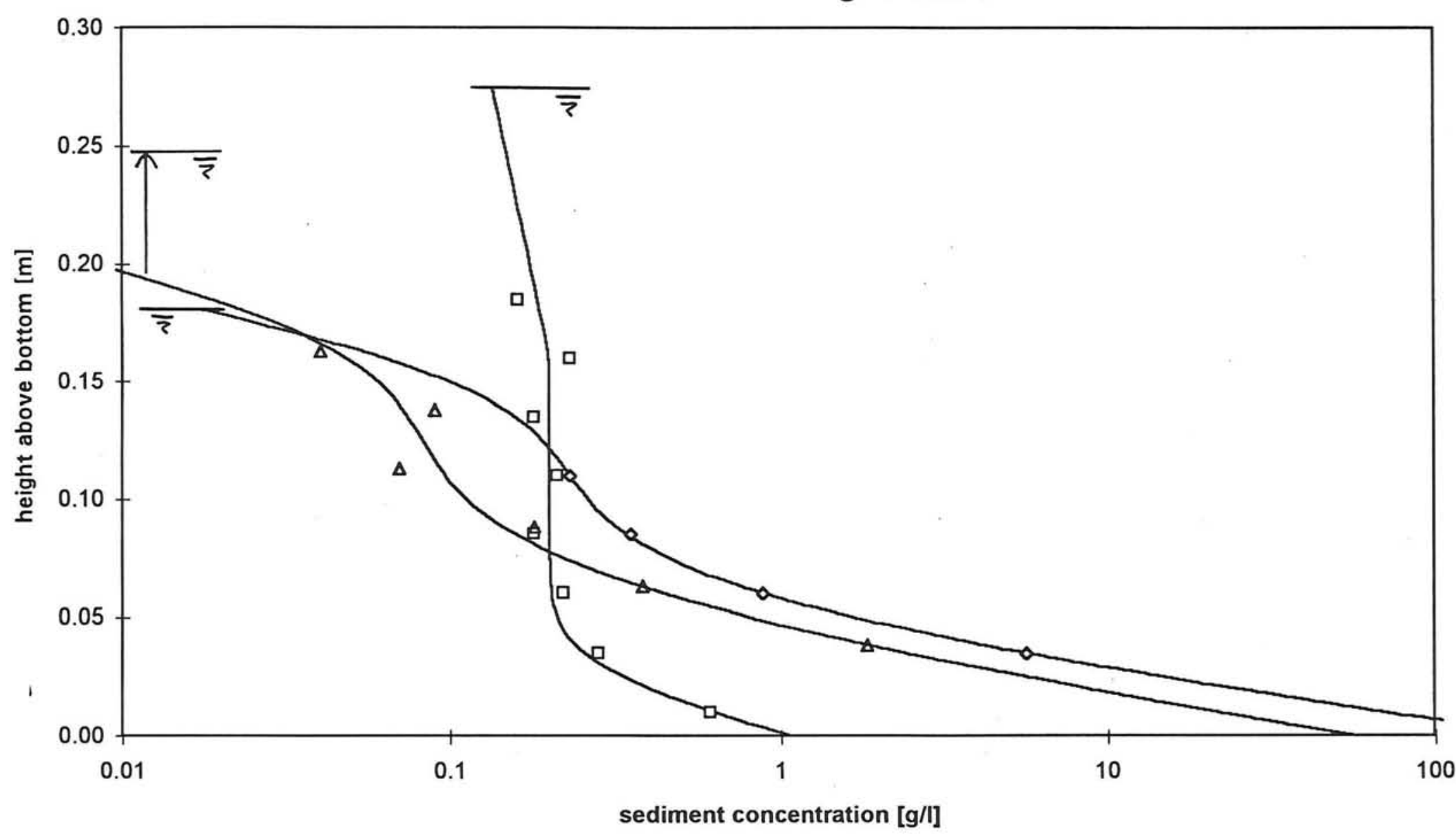




\section{Sediment concentration distributions}

Experiment $\mathbf{C}$ with breakwater

Measurement during Interval 1

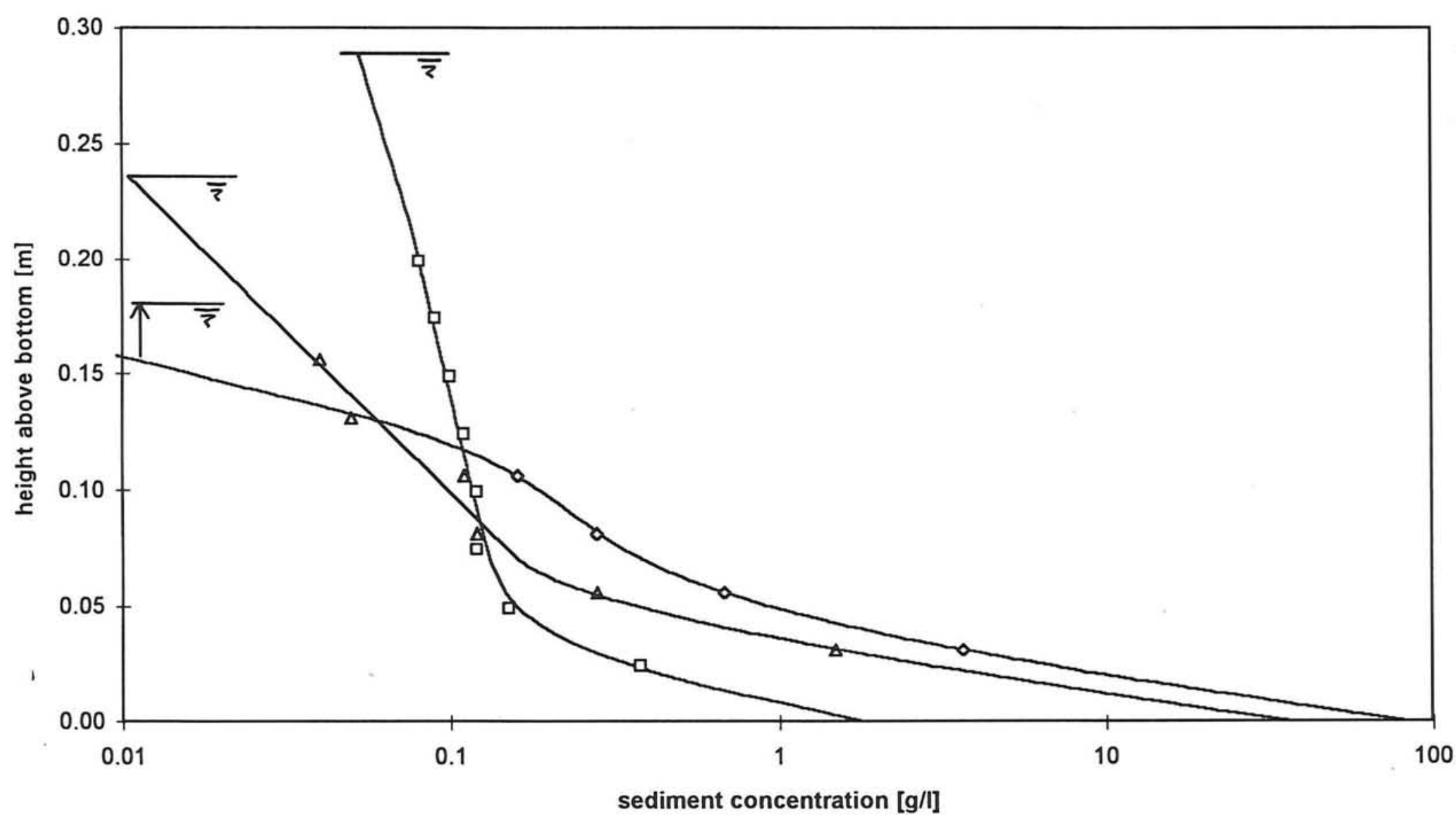




\section{Sediment concentration distributions}

Experiment $\mathrm{D}$ with breakwater

Measurement during Interval 1

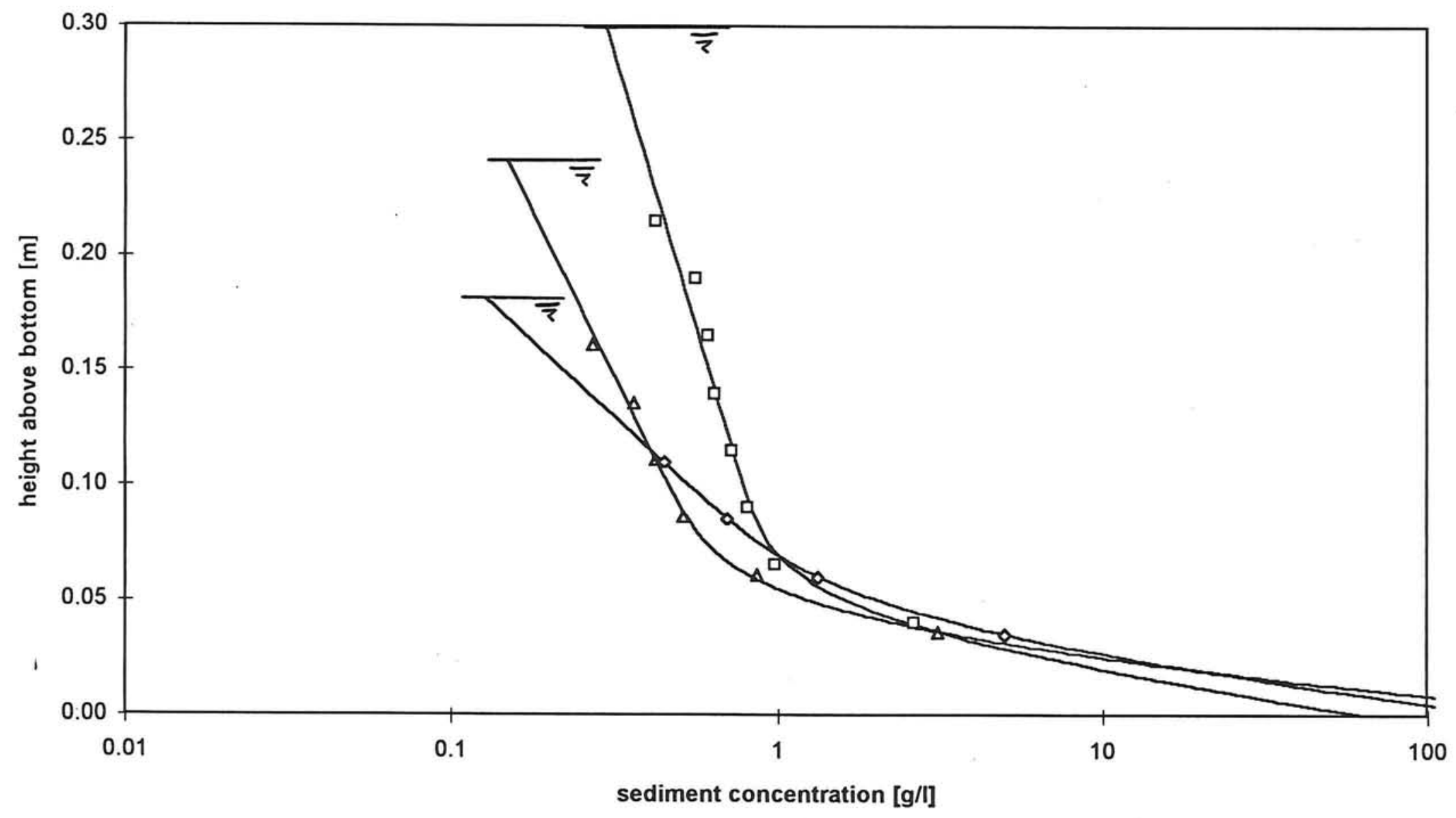




\section{Sediment concentration distributions}

Experiment $A$ with and without breakwater

Measurement during Interval 1 at Vertical (a): $X=5.25 \mathrm{~m}$

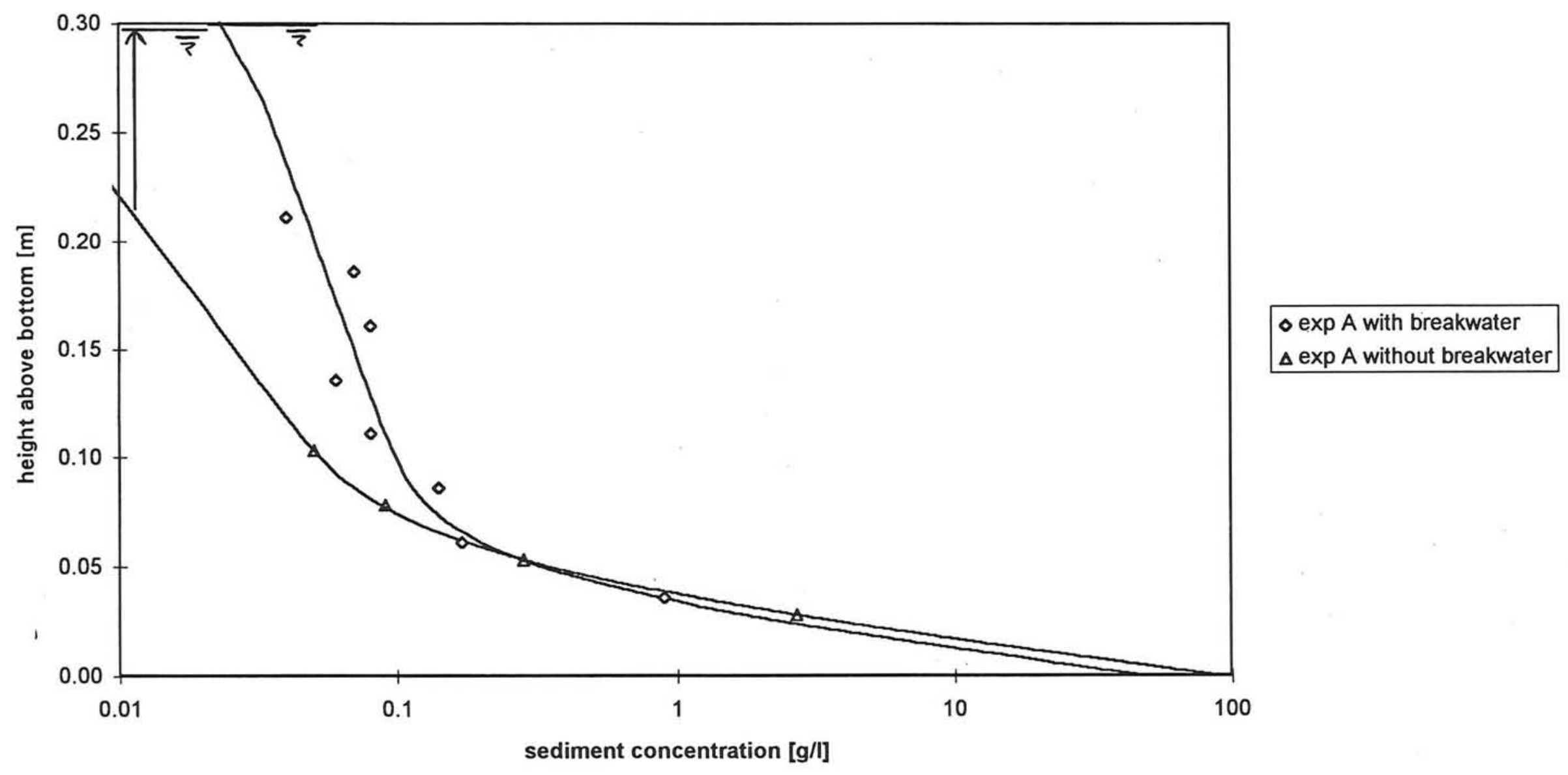

Graph 4.1.9 
Sediment concentration distributions

Experiment $A$ with and without breakwater

Measurement during Interval 1 at Vertical (b): $X=6.15 \mathrm{~m}$

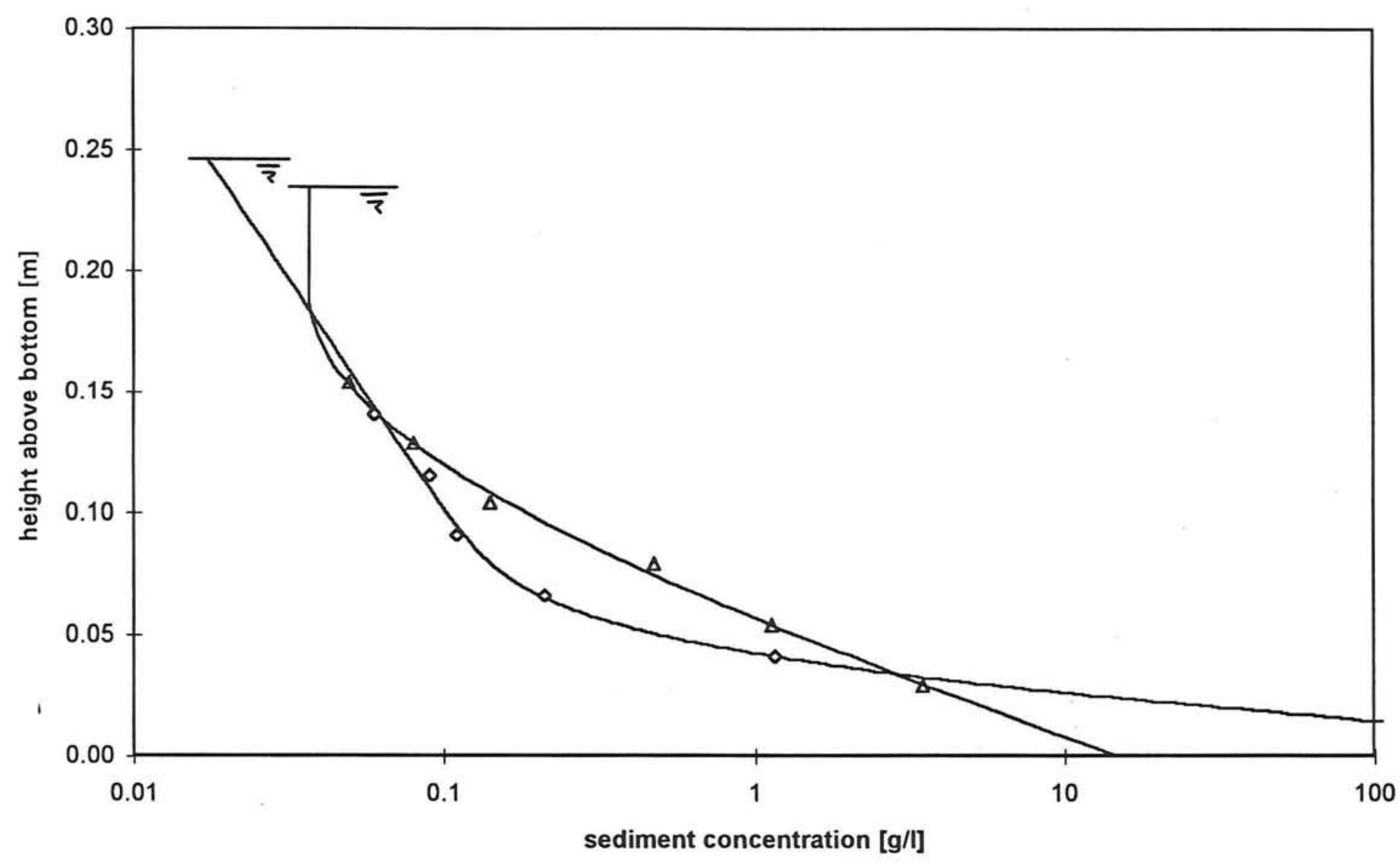

$\triangle \exp A$ with breakwater

$\triangle \exp A$ without breakwater

Graph 4.1.10 


\section{Sediment concentration distributions}

Experiment $A$ with and without breakwater

Measurement during Interval 1 at Vertical (c): $X=7.05 \mathrm{~m}$

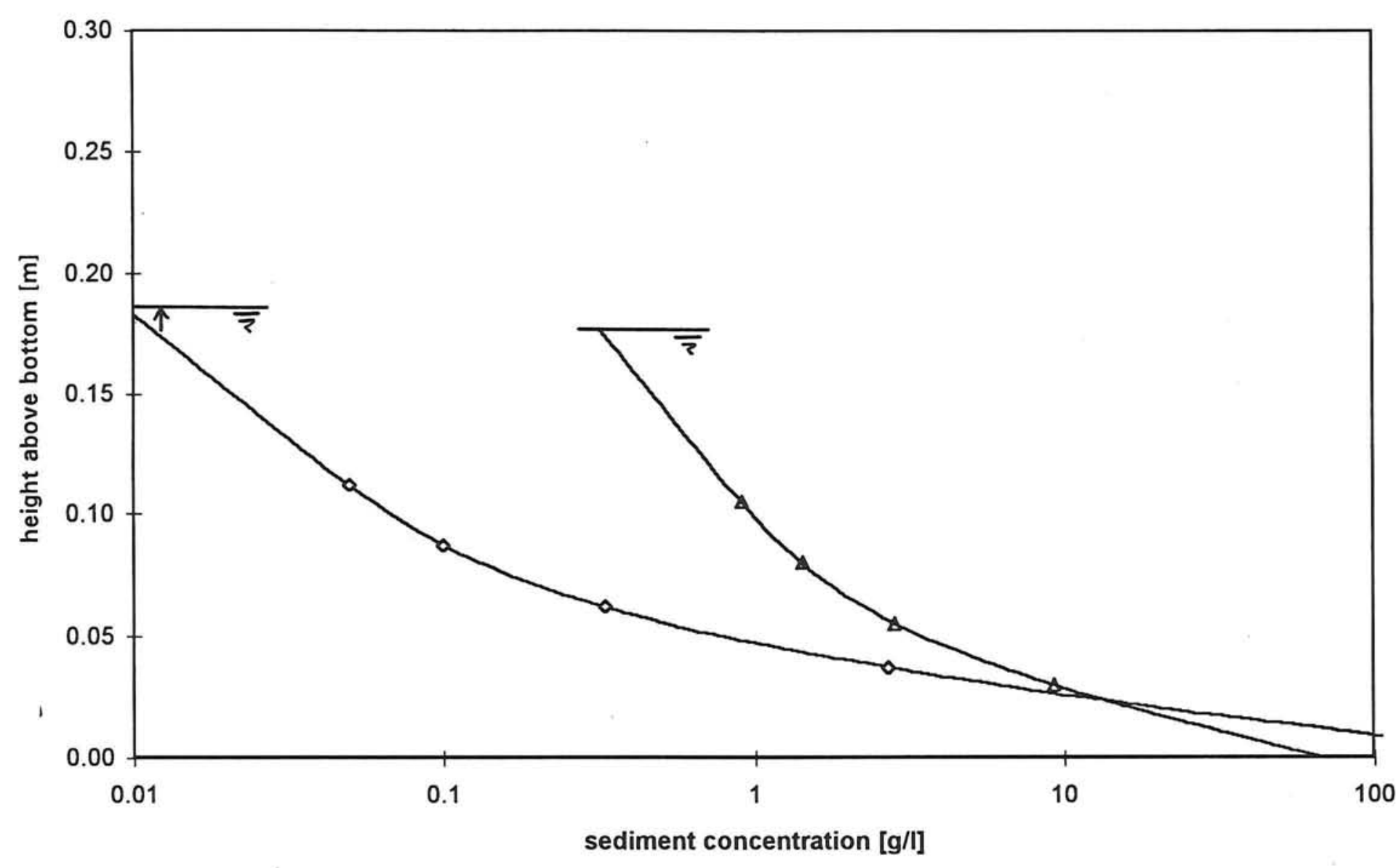




\section{Sediment concentration distributions}

Experiment $B$ with and without breakwater

Measurement during Interval 1 at Vertical (a): $X=5.25 \mathrm{~m}$

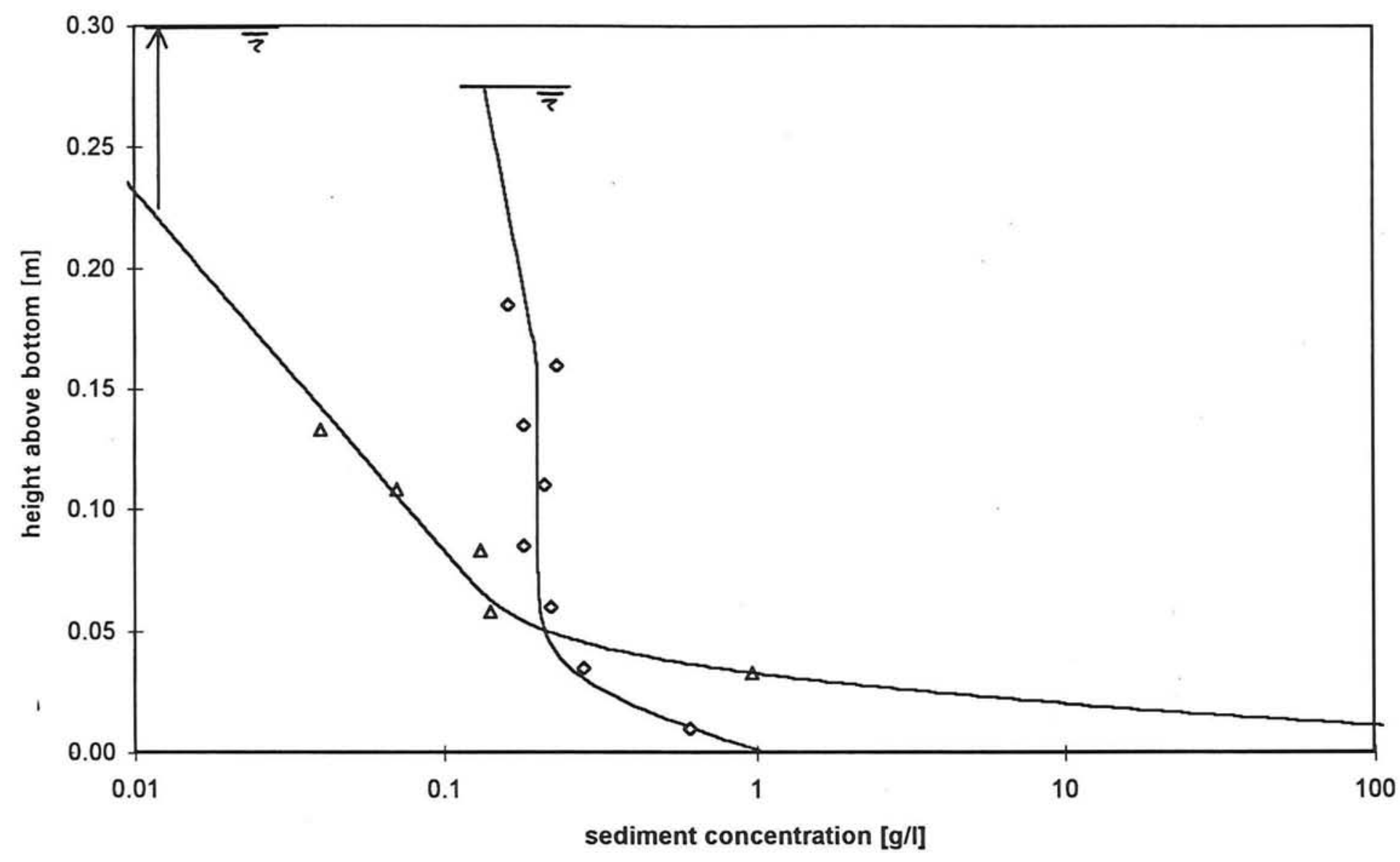




\section{Sediment concentration distributions}

Experiment $B$ with and without breakwater

Measurement during Interval 1 at Vertical (b): $X=6.15 \mathrm{~m}$

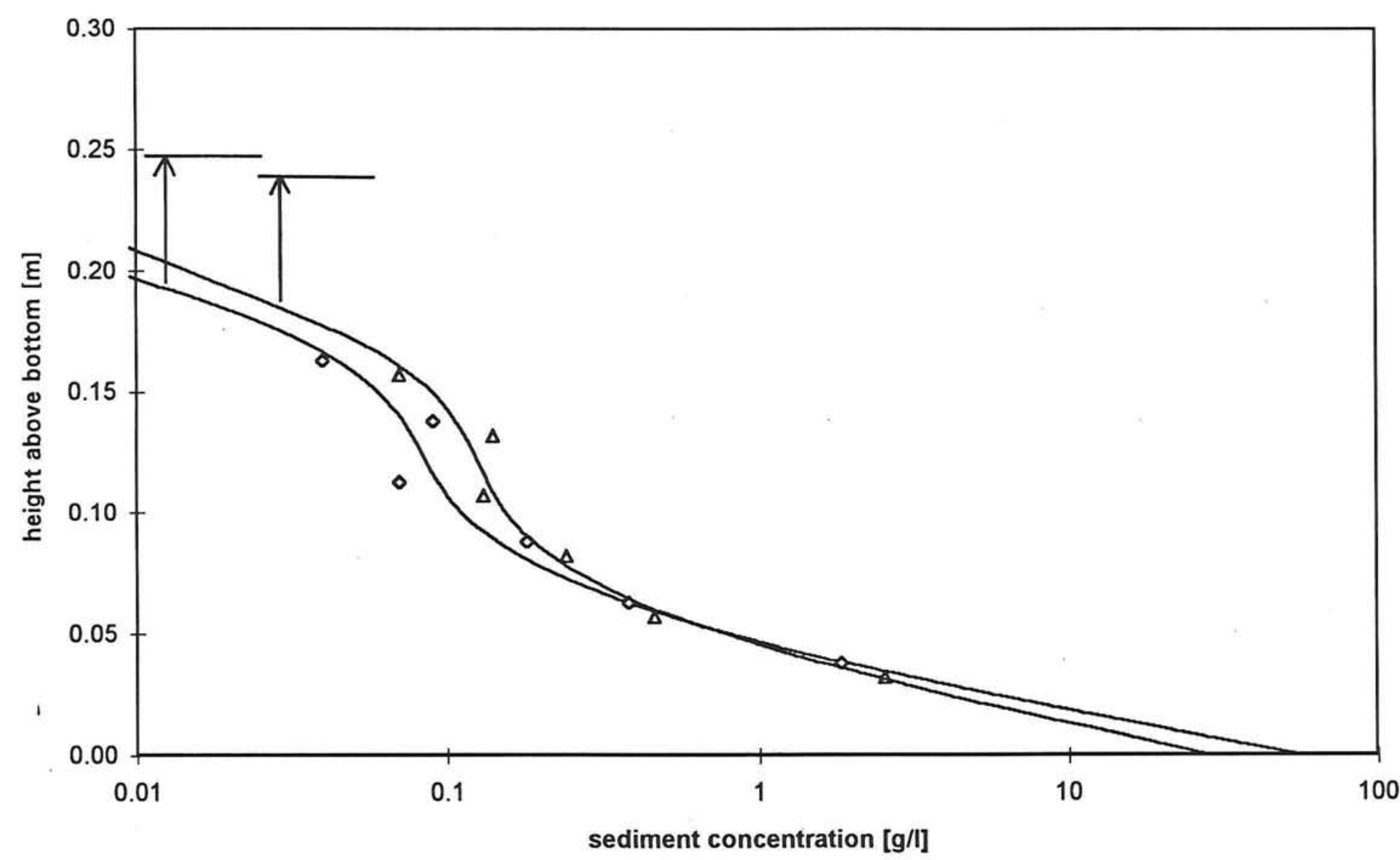




\section{Sediment concentration distributions}

Experiment $B$ with and without breakwater

Measurement during Interval 1 at Vertical (c): $X=7.05 \mathrm{~m}$

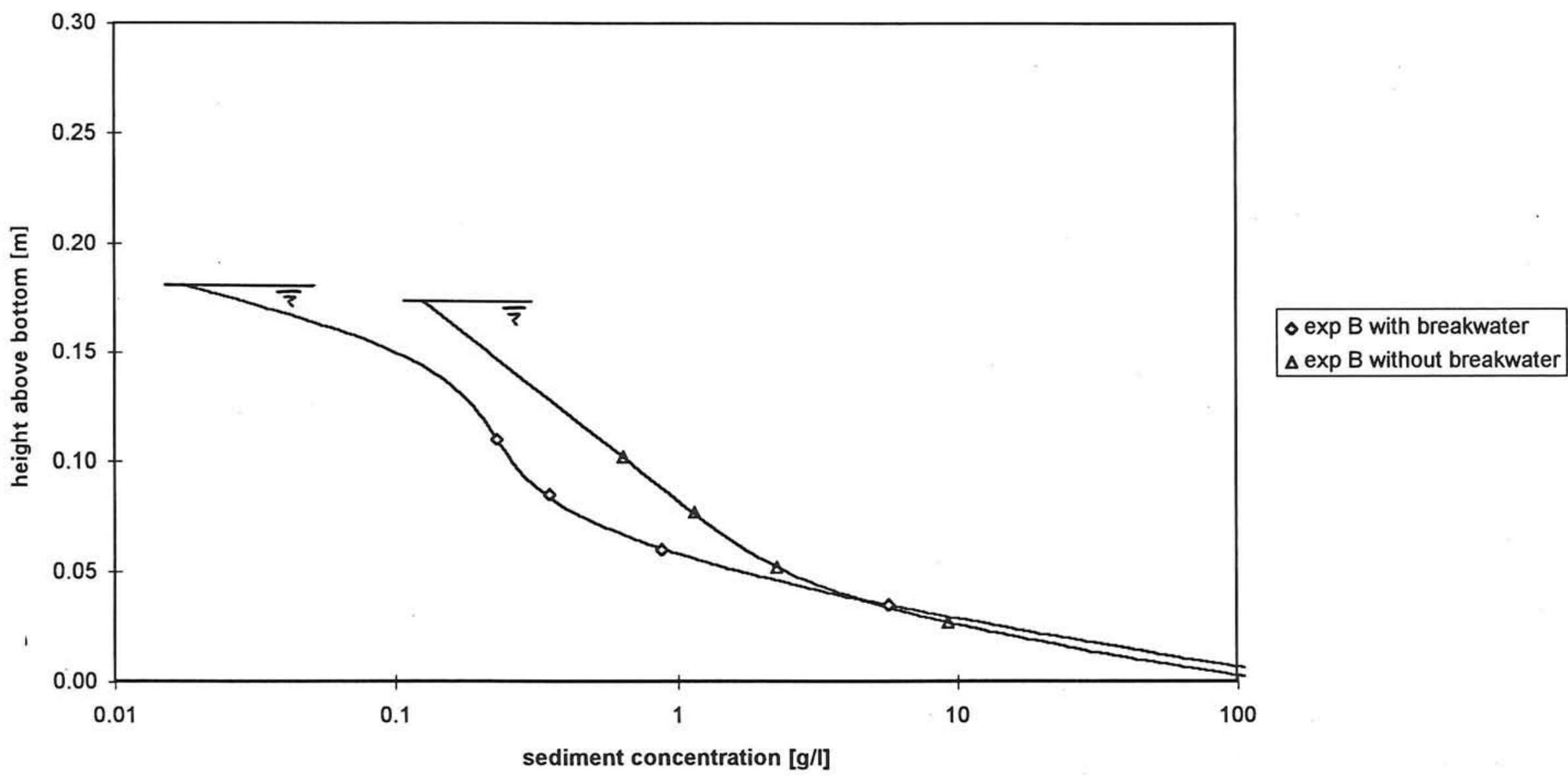

Graph 4.1.14 
Sediment concentration distributions

Experiment $C$ with and without breakwater

Measurement during Interval 1 at Vertical (a): $X=5.25 \mathrm{~m}$

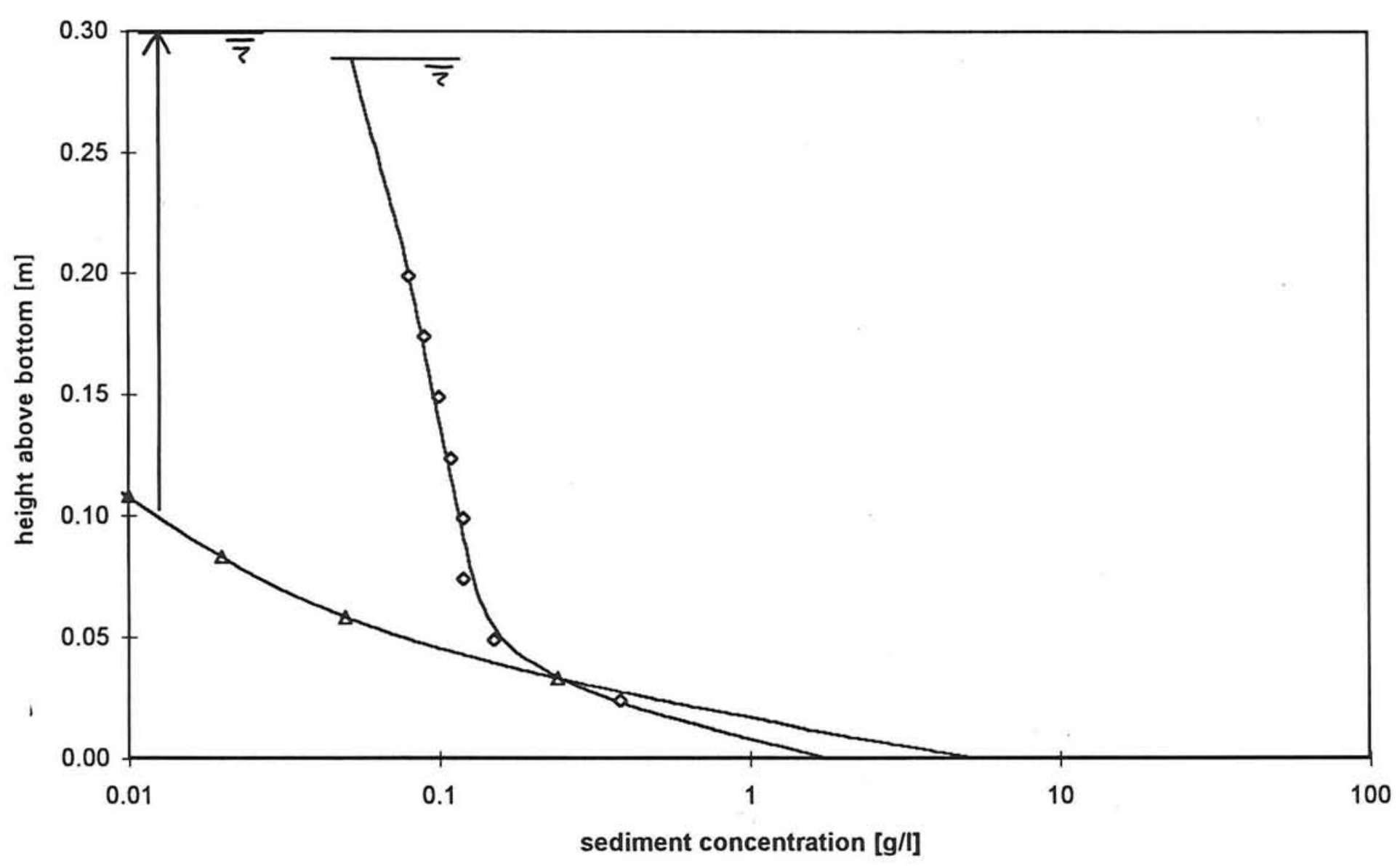




\section{Sediment concentration distributions}

Experiment $C$ with and without breakwater

Measurement during Interval 1 at Vertical (b): $X=6.15 \mathrm{~m}$

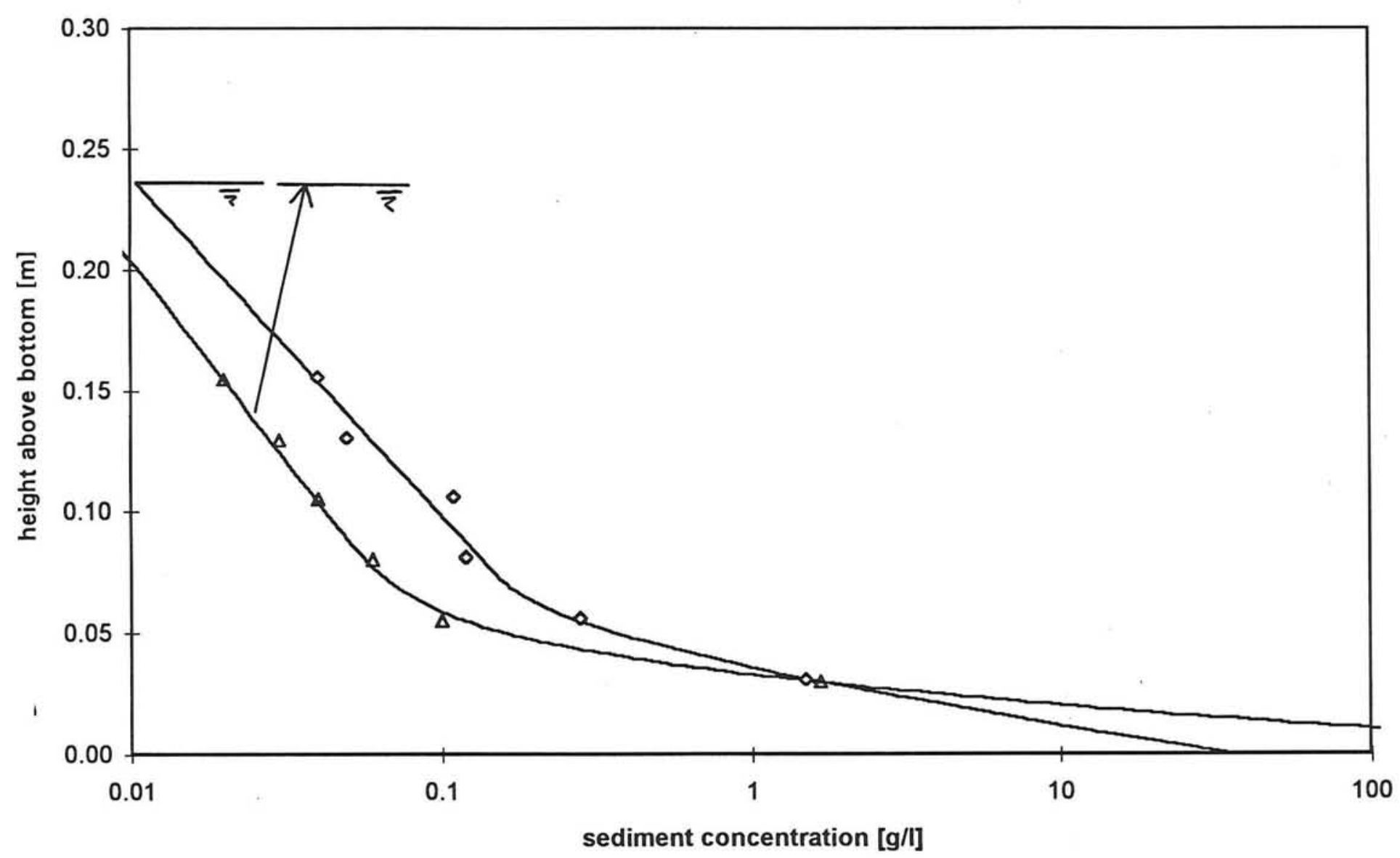




\section{Sediment concentration distributions}

Experiment $\mathbf{C}$ with and without breakwater

Measurement during Interval 1 at Vertical (c): $X=7.05 \mathrm{~m}$

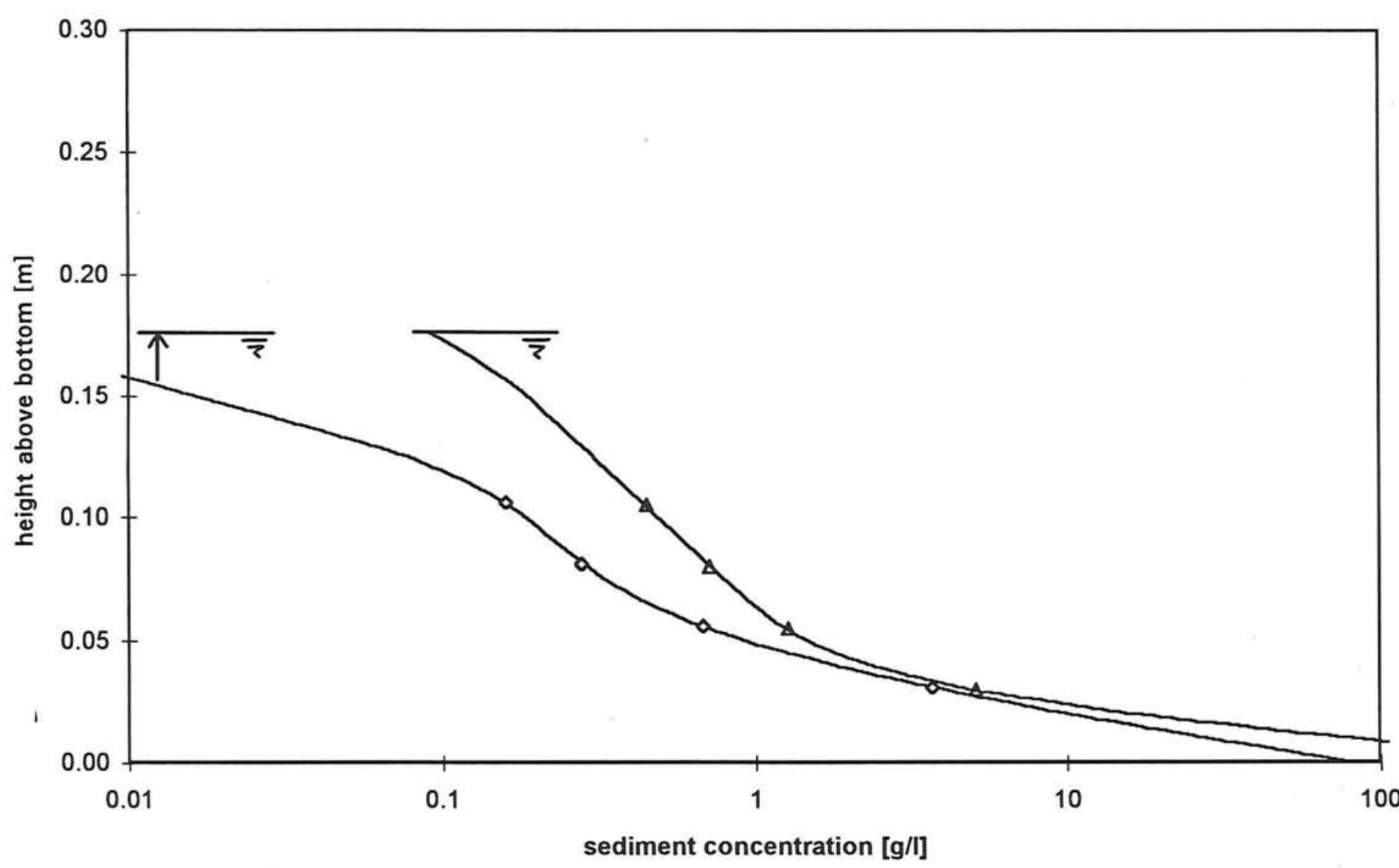

$\triangle \exp C$ with breakwater

$\triangle \exp C$ without breakwater

Graph 4.1.17 


\section{Sediment concentration distributions}

Experiment $D$ with and without breakwater

Measurement during Interval 1 at Vertical (a): $X=\mathbf{5 . 2 5} \mathrm{m}$

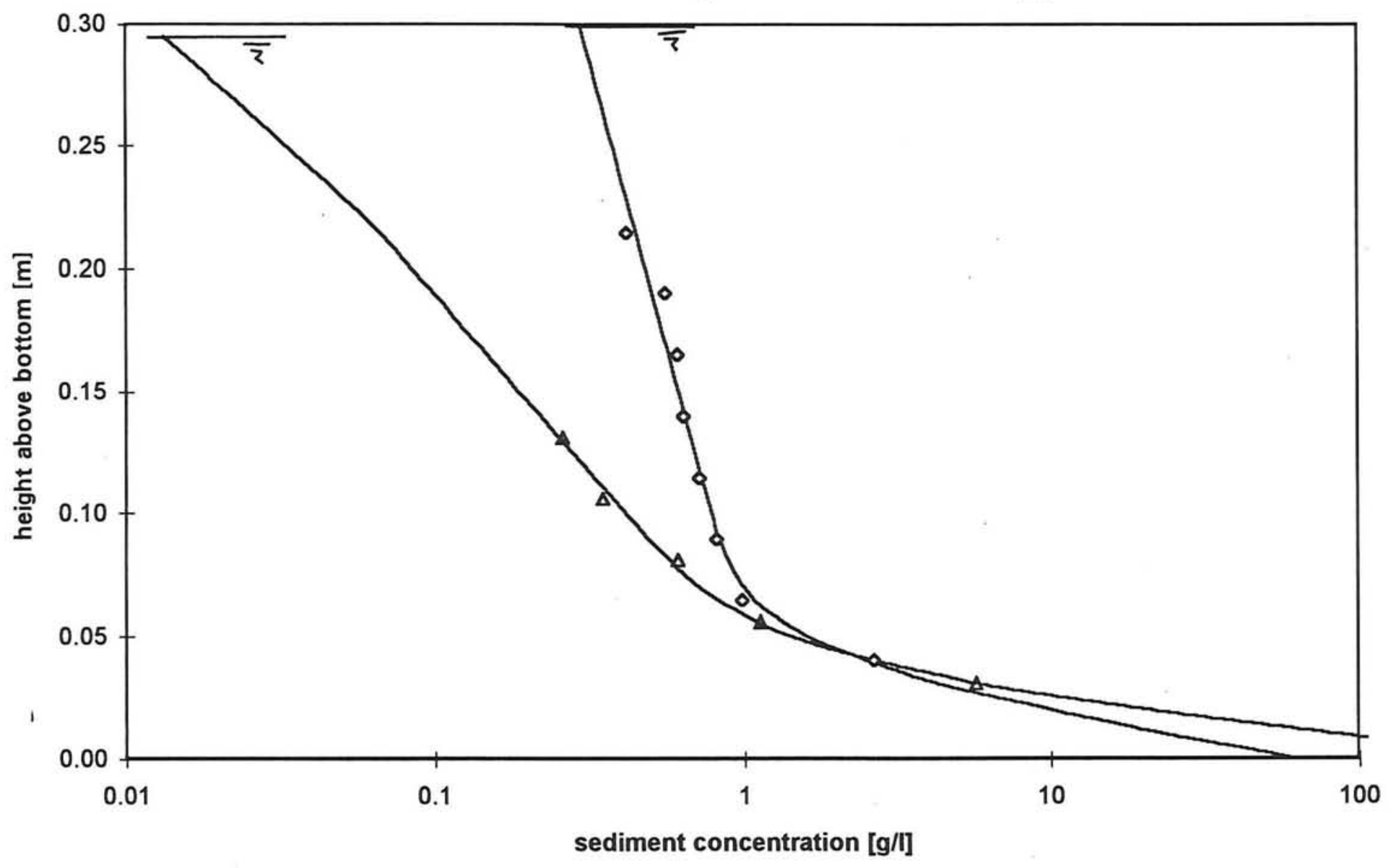




\section{Sediment concentration distributions}

Experiment $D$ with and without breakwater

Measurement during Interval 1 at Vertical (b): $X=6.15 \mathrm{~m}$

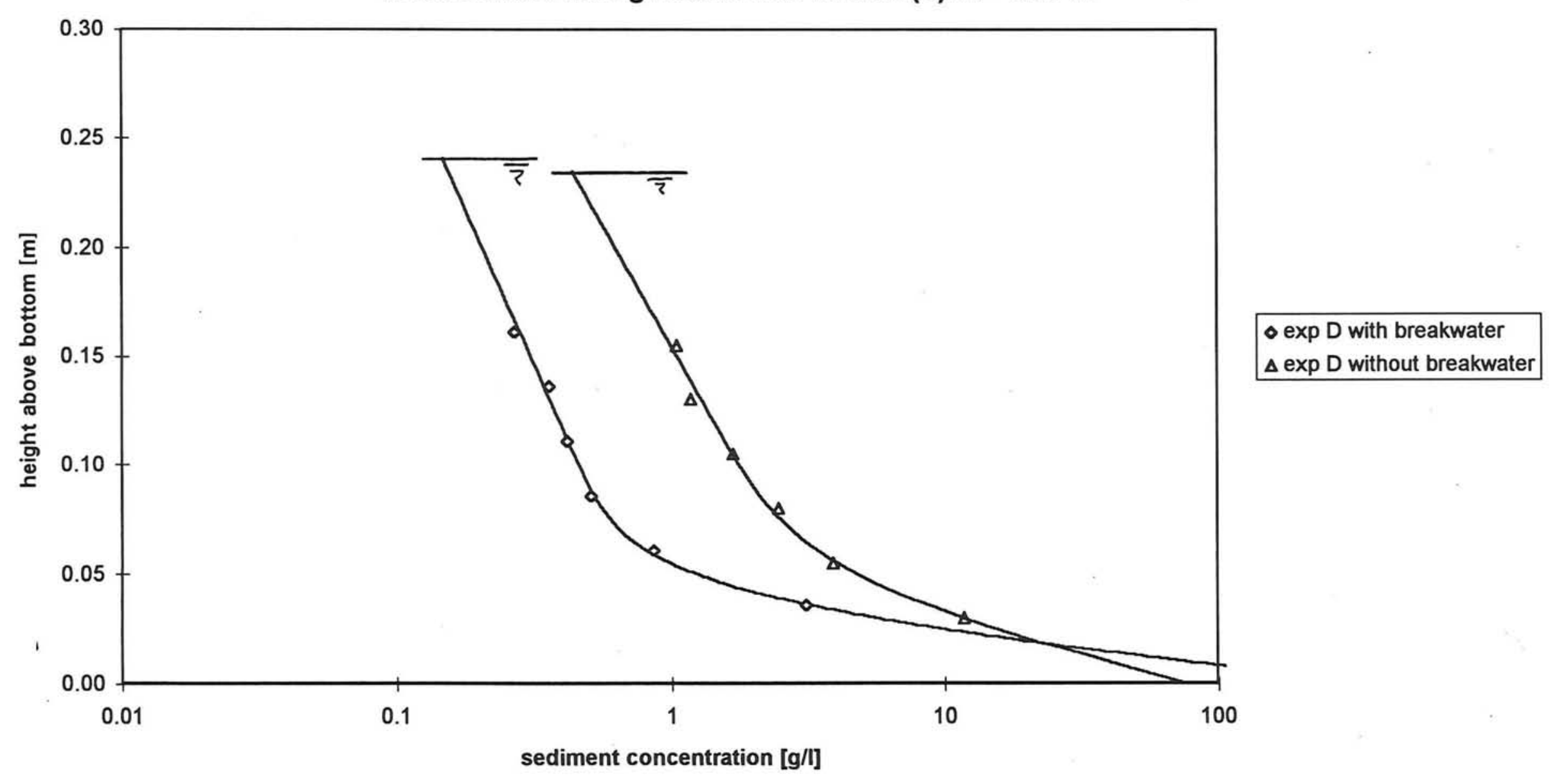

Graph 4.1.19 


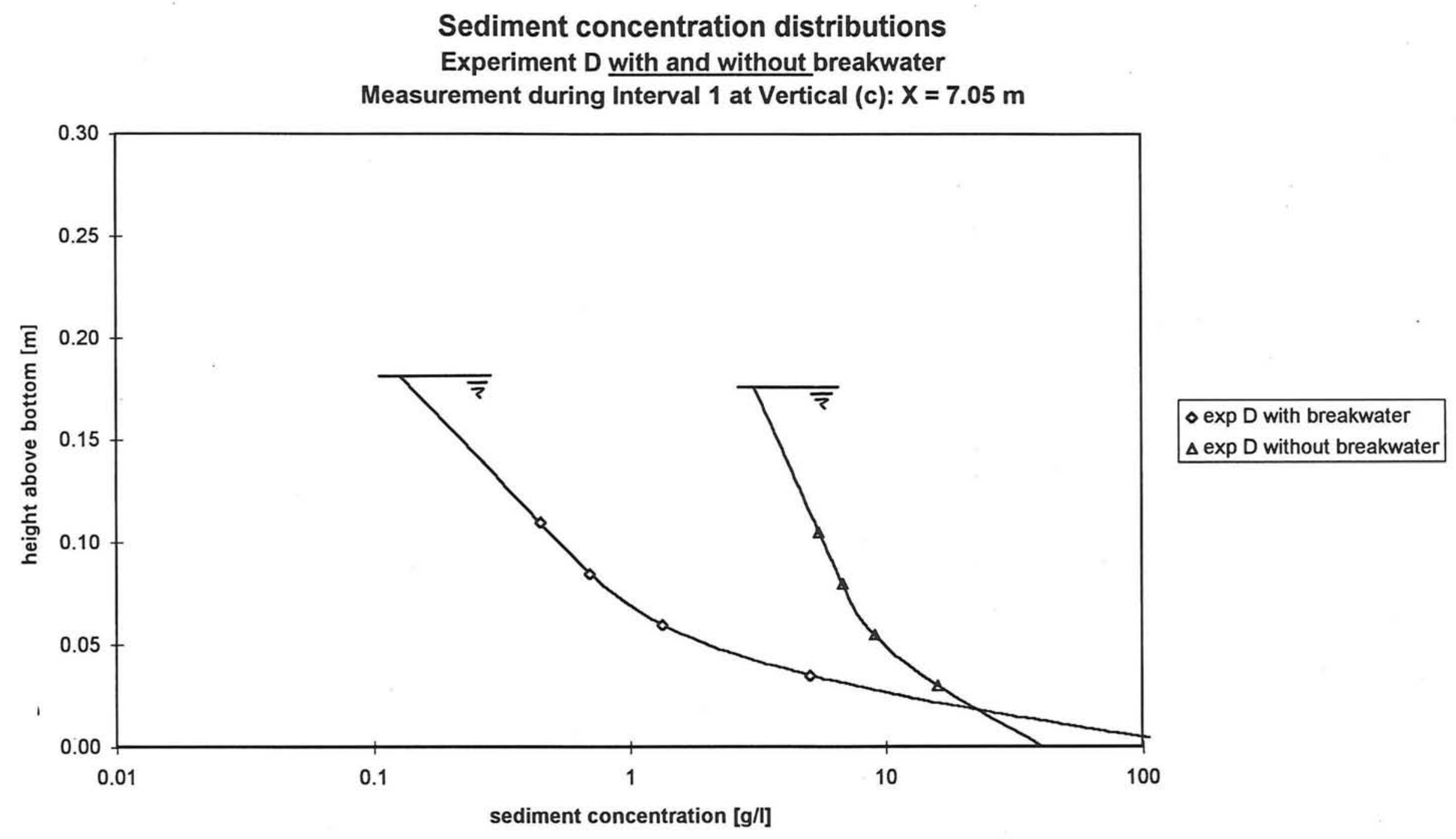

Graph 4.1.20 


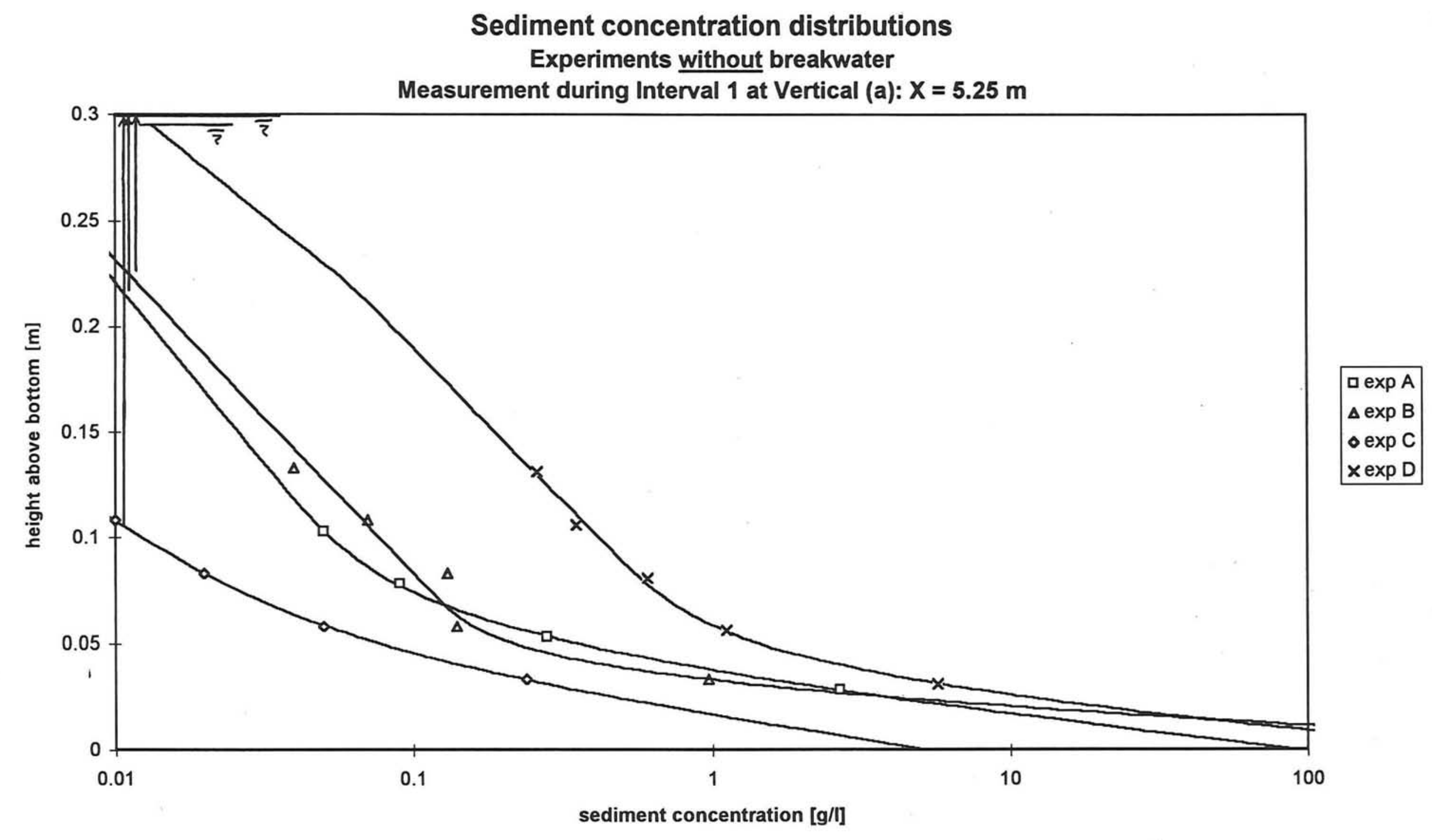

Graph 4.1.21 


\section{Sediment concentration distributions}

Experiments without breakwater

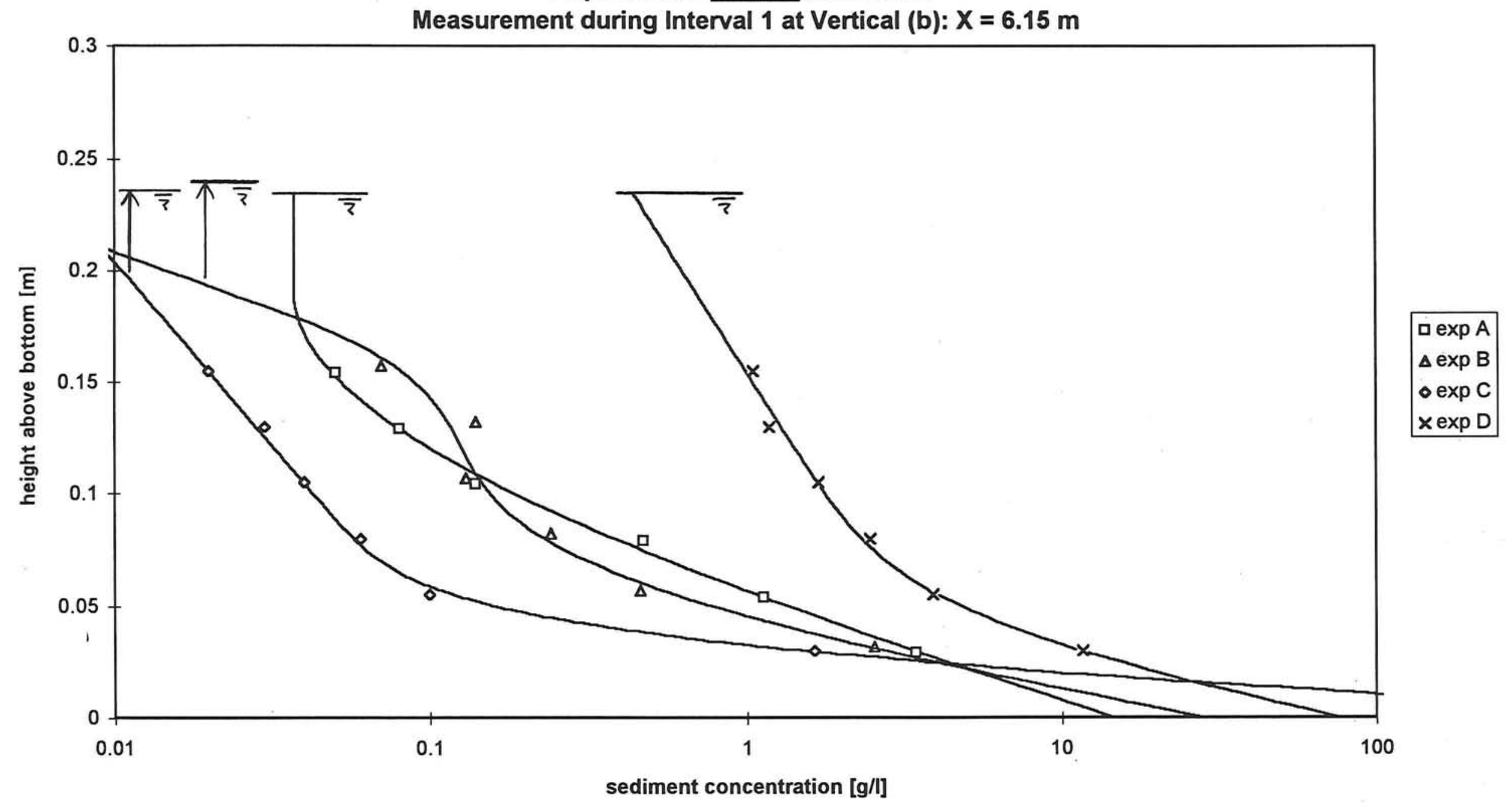

Graph 4.1.22 


\section{Sediment concentration distributions}

Experiments without breakwater

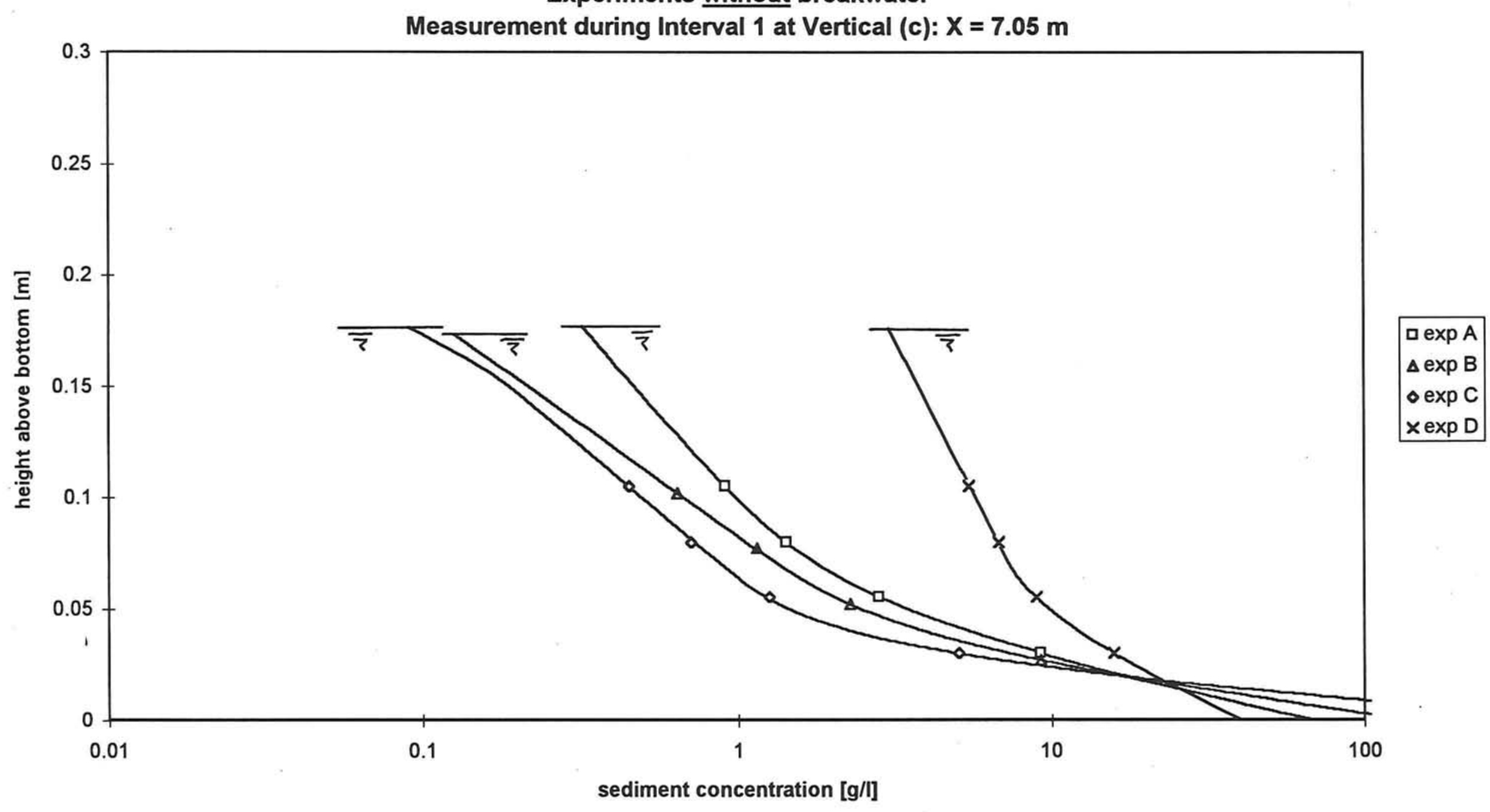

Graph 4.1.23 


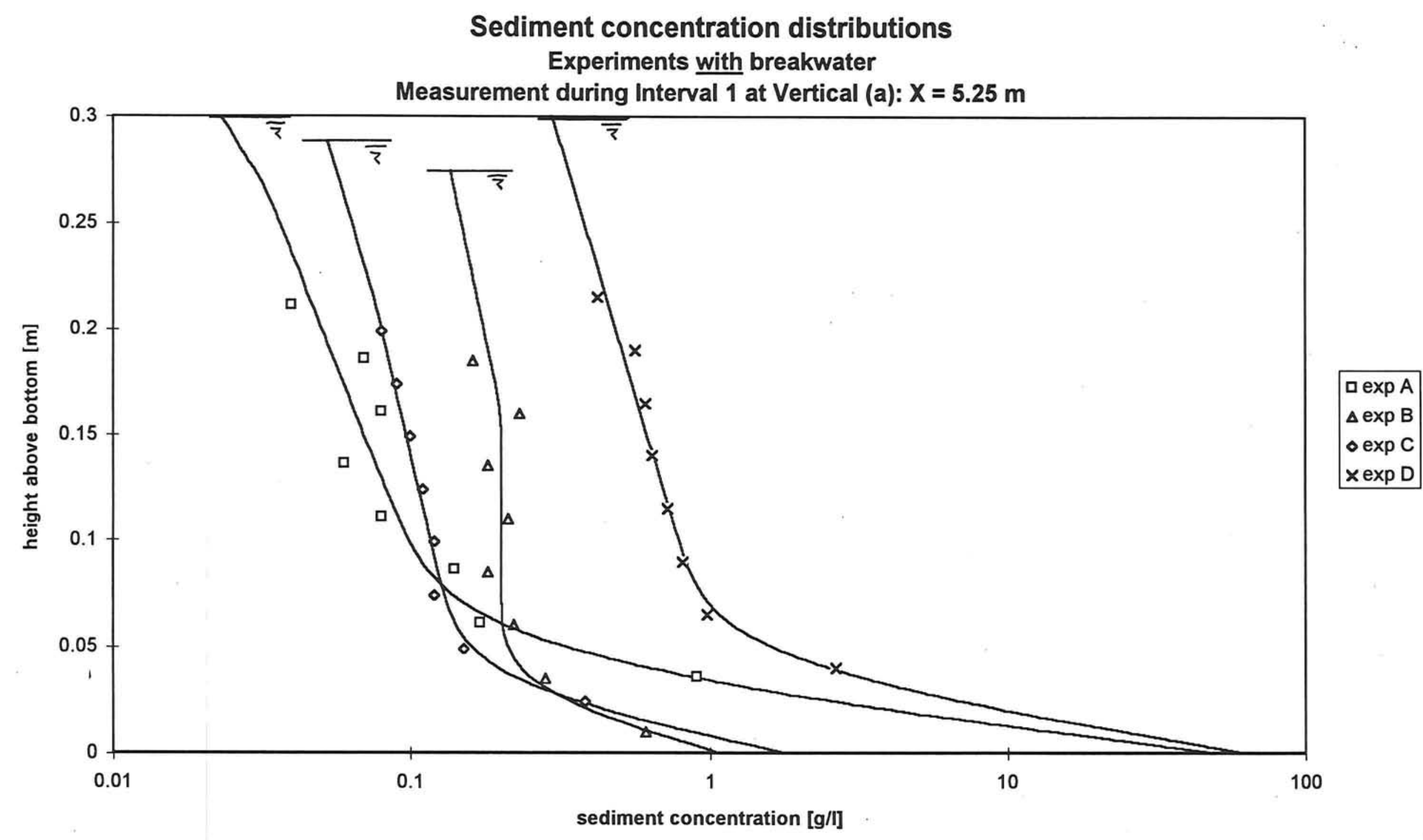

Graph 4.1.24 


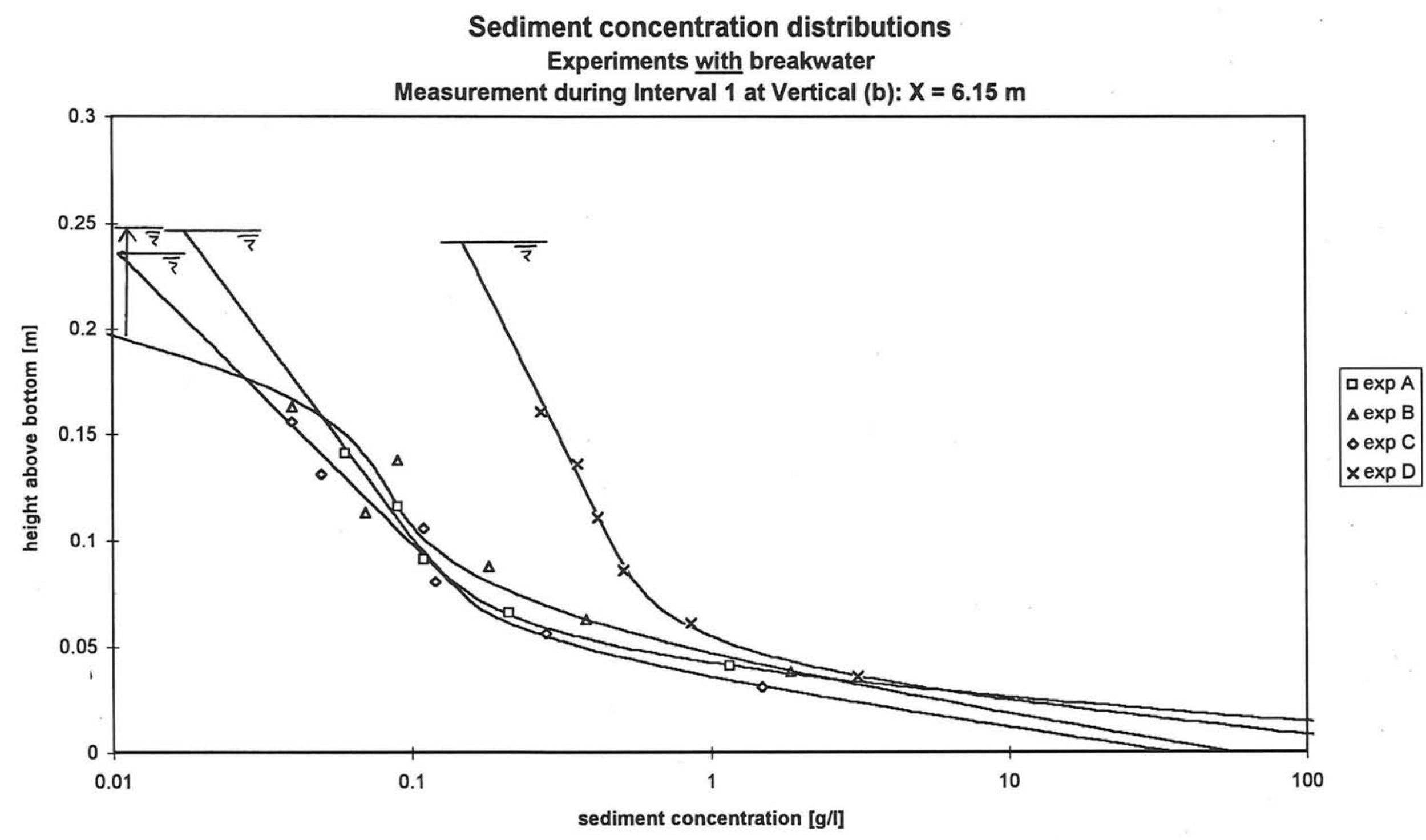

Graph 4.1.25 


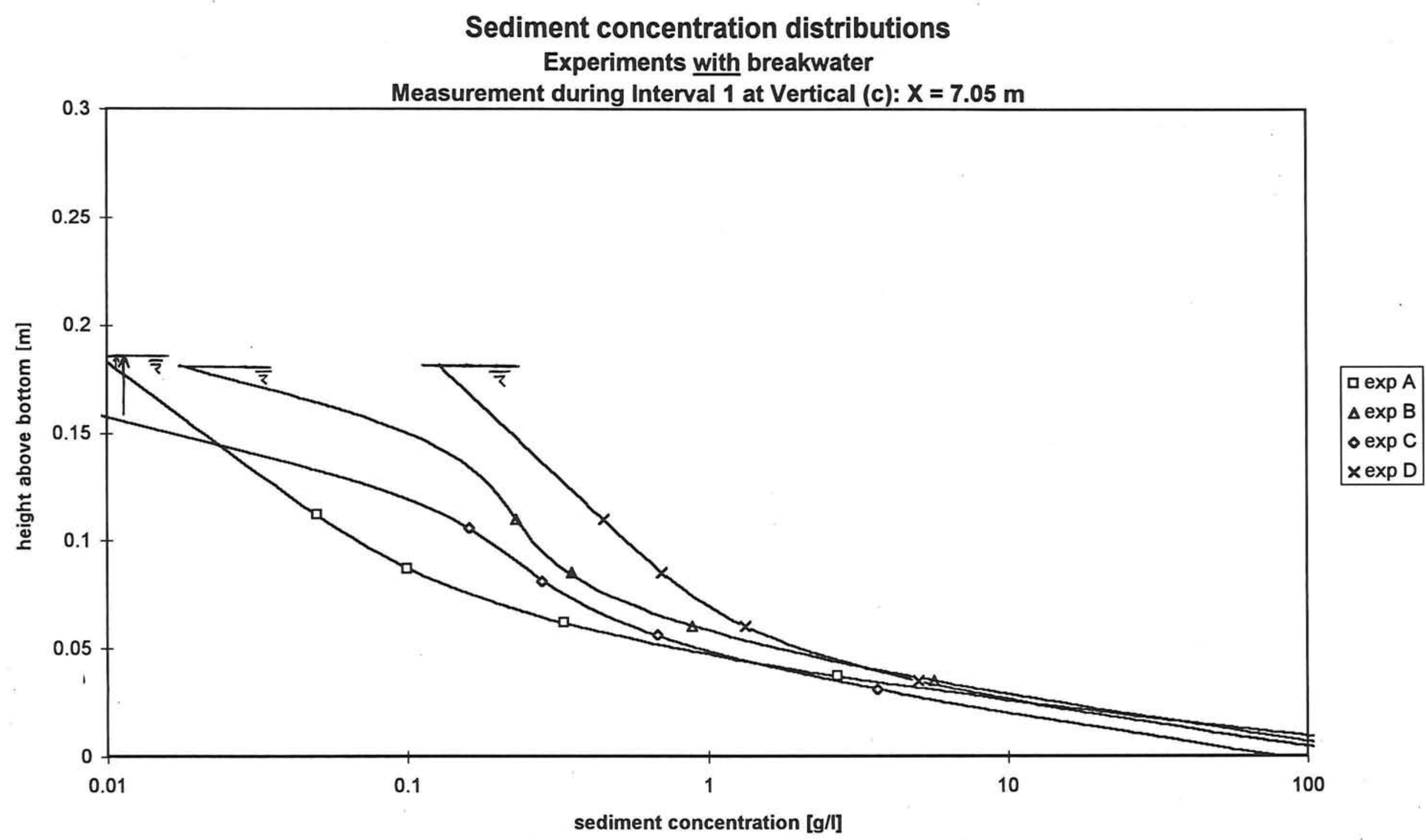

Graph 4.1.26 


\section{Sediment concentration distributions}

Experiment $B$ without breakwater

Measurement during Interval 4

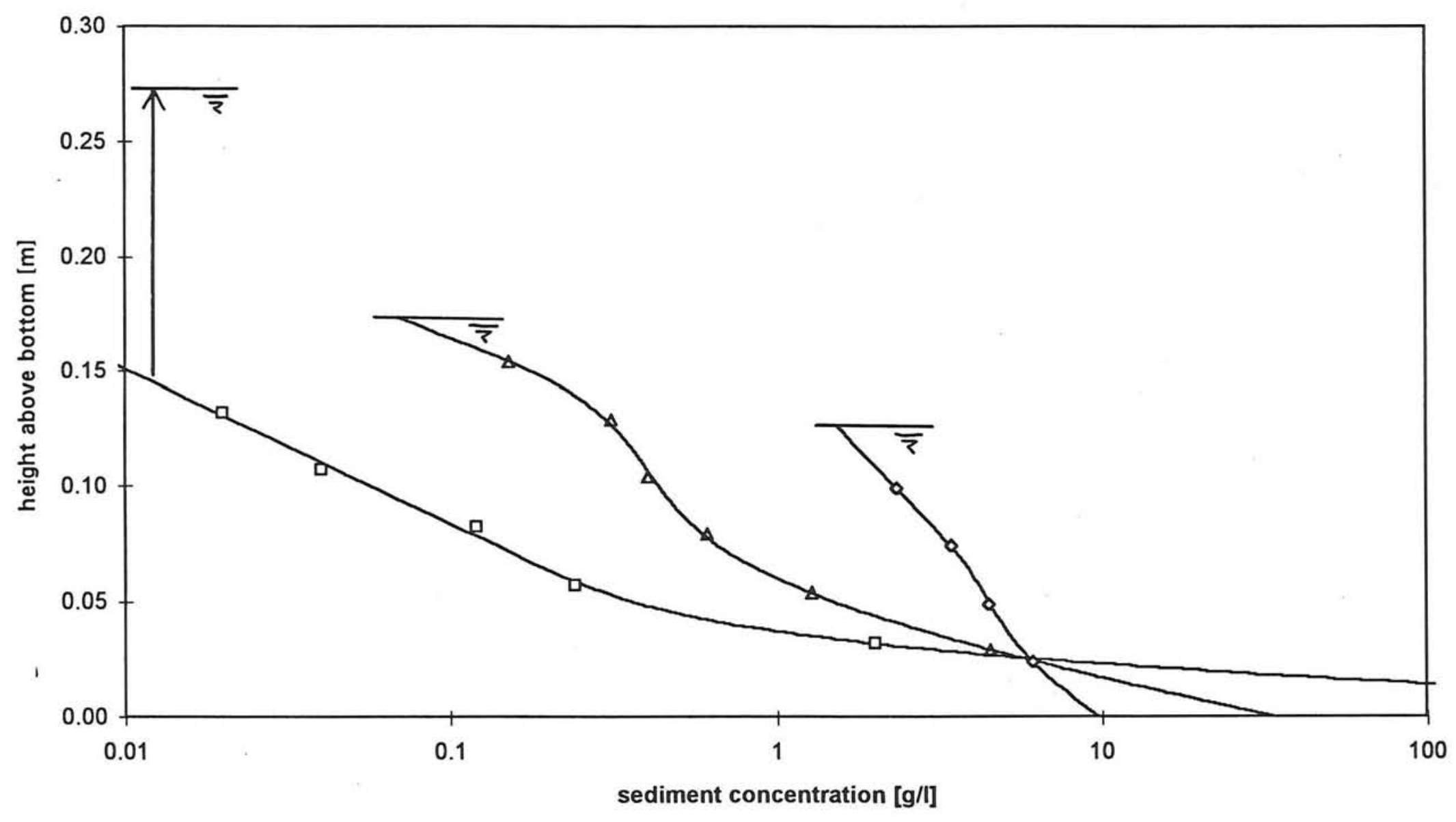




\section{Sediment concentration distributions}

Experiment $\mathbf{C}$ without breakwater

Measurement during Interval 4

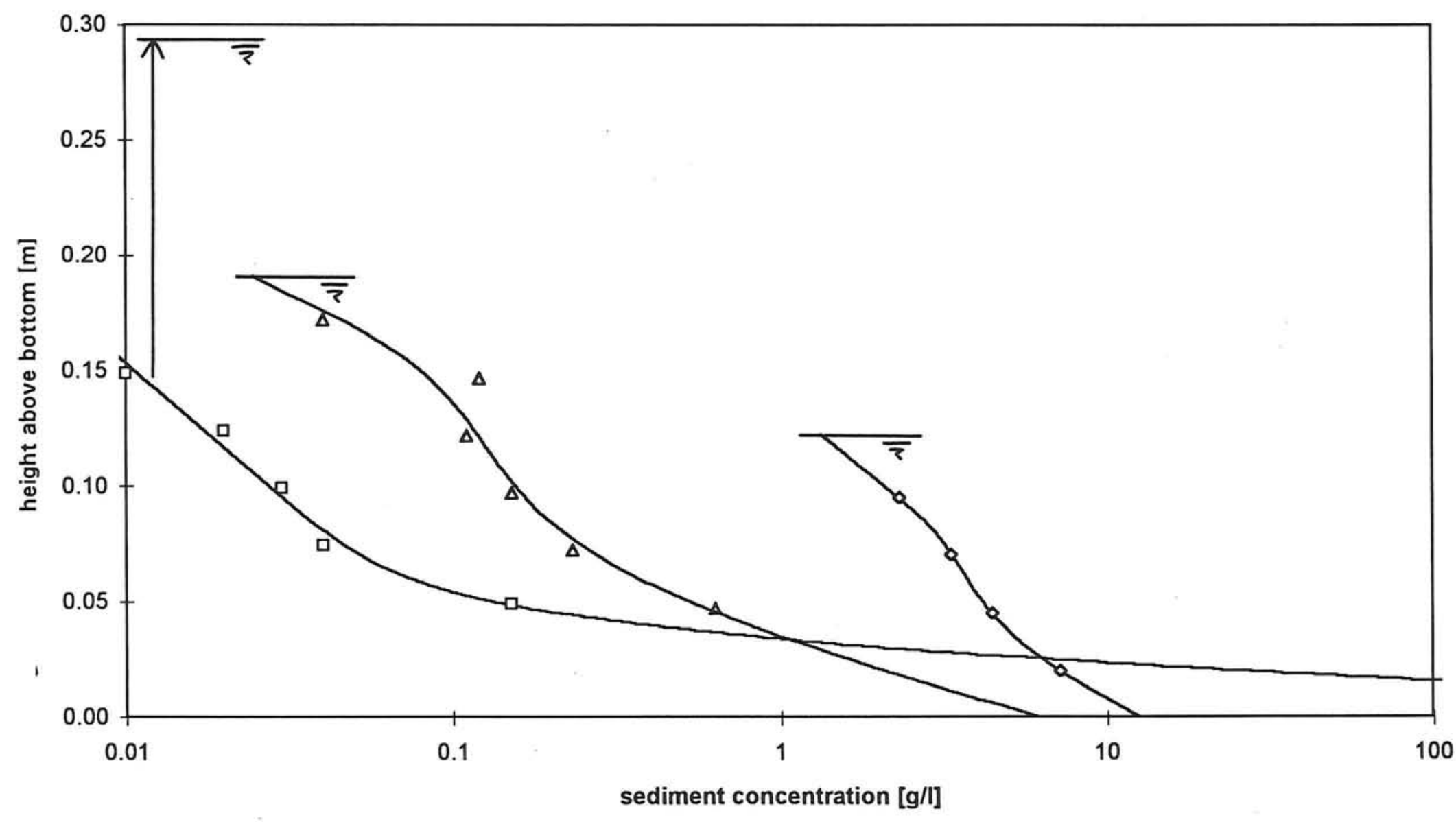




\section{Sediment concentration distributions}

Experiment $D$ without breakwater

Measurement during Interval 4

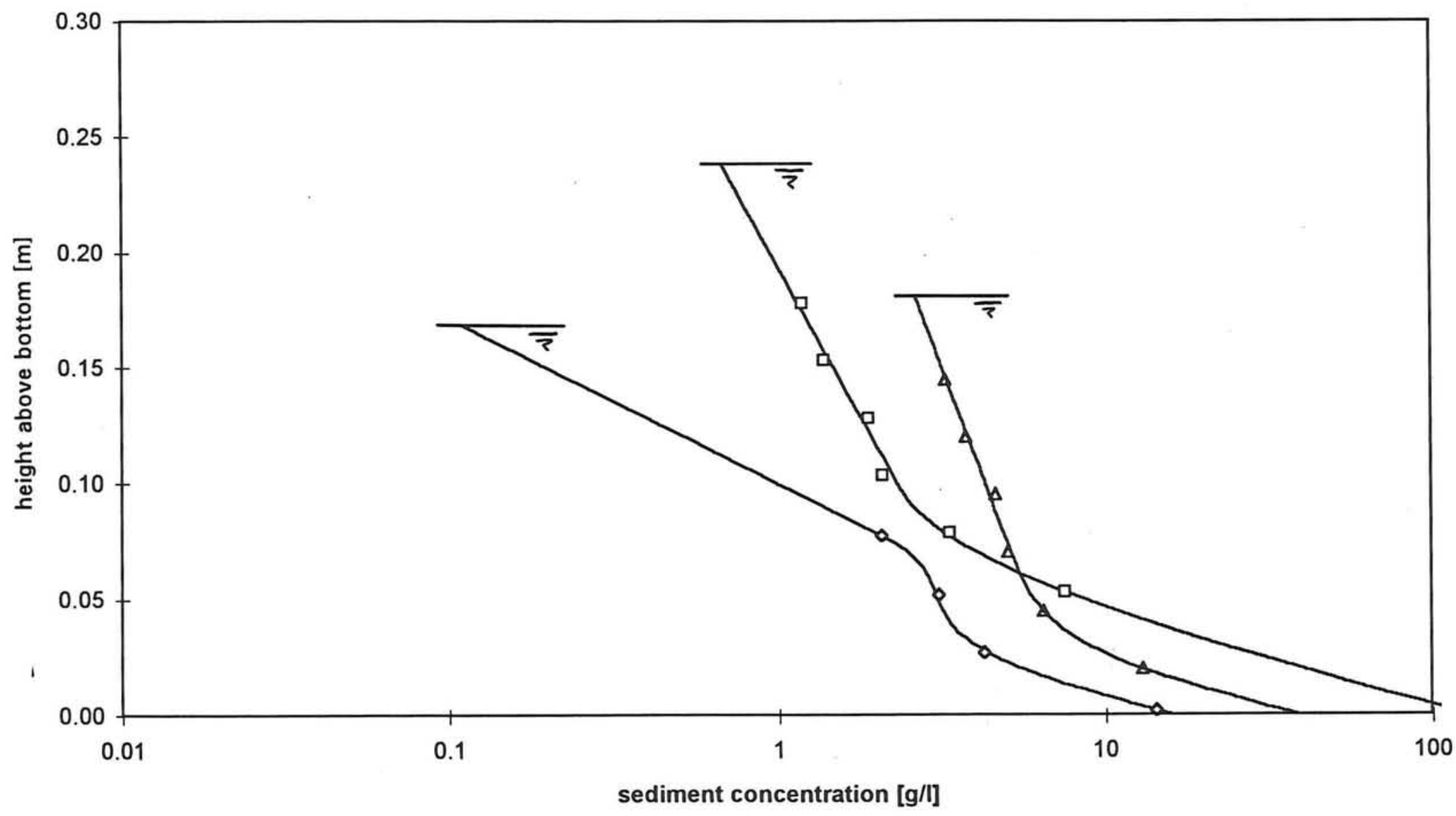




\section{Sediment concentration distributions}

Experiment A with breakwater

Measurement during Interval 4

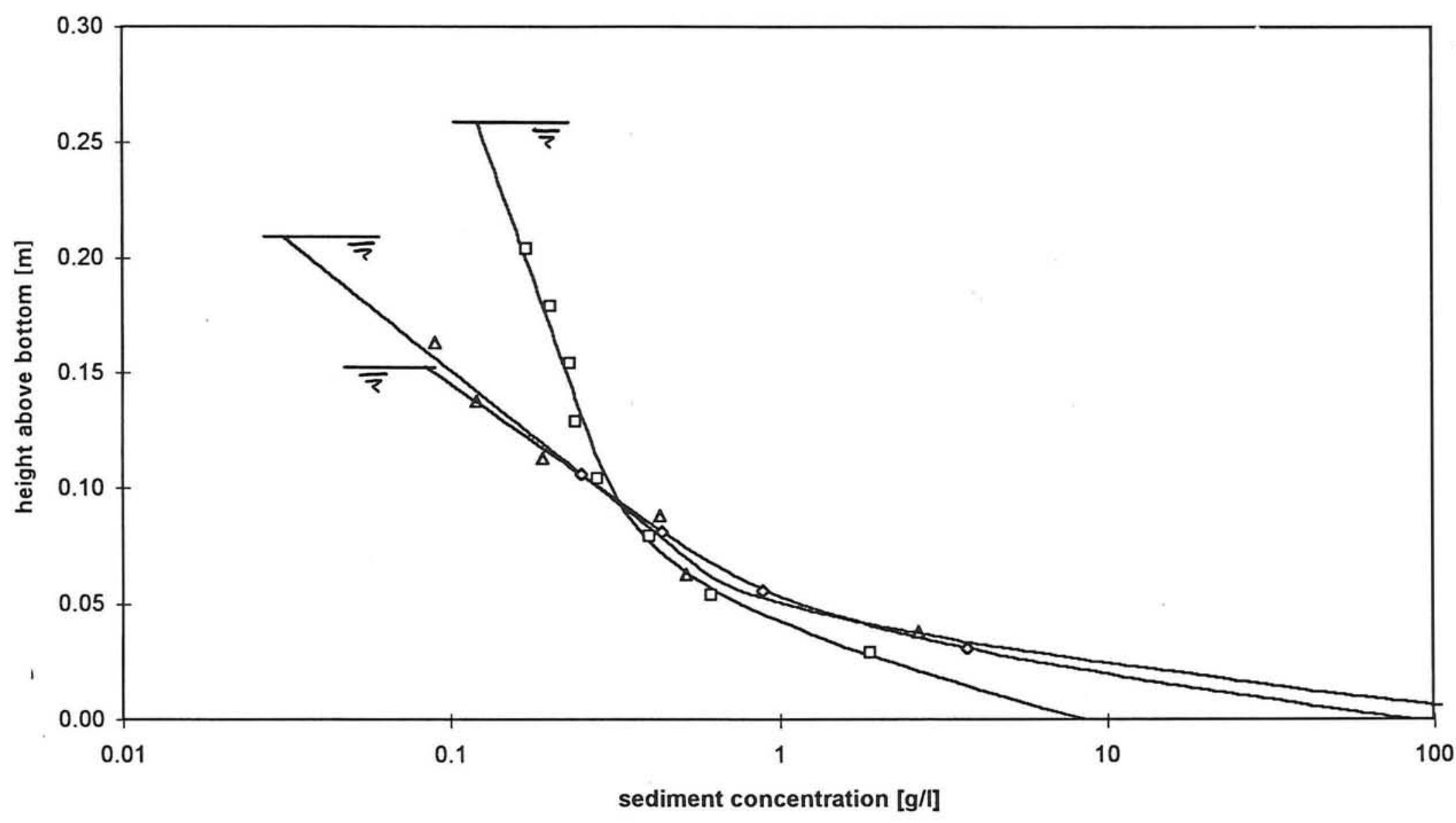




\section{Sediment concentration distributions}

Experiment $B$ with breakwater

Measurement during Interval 4

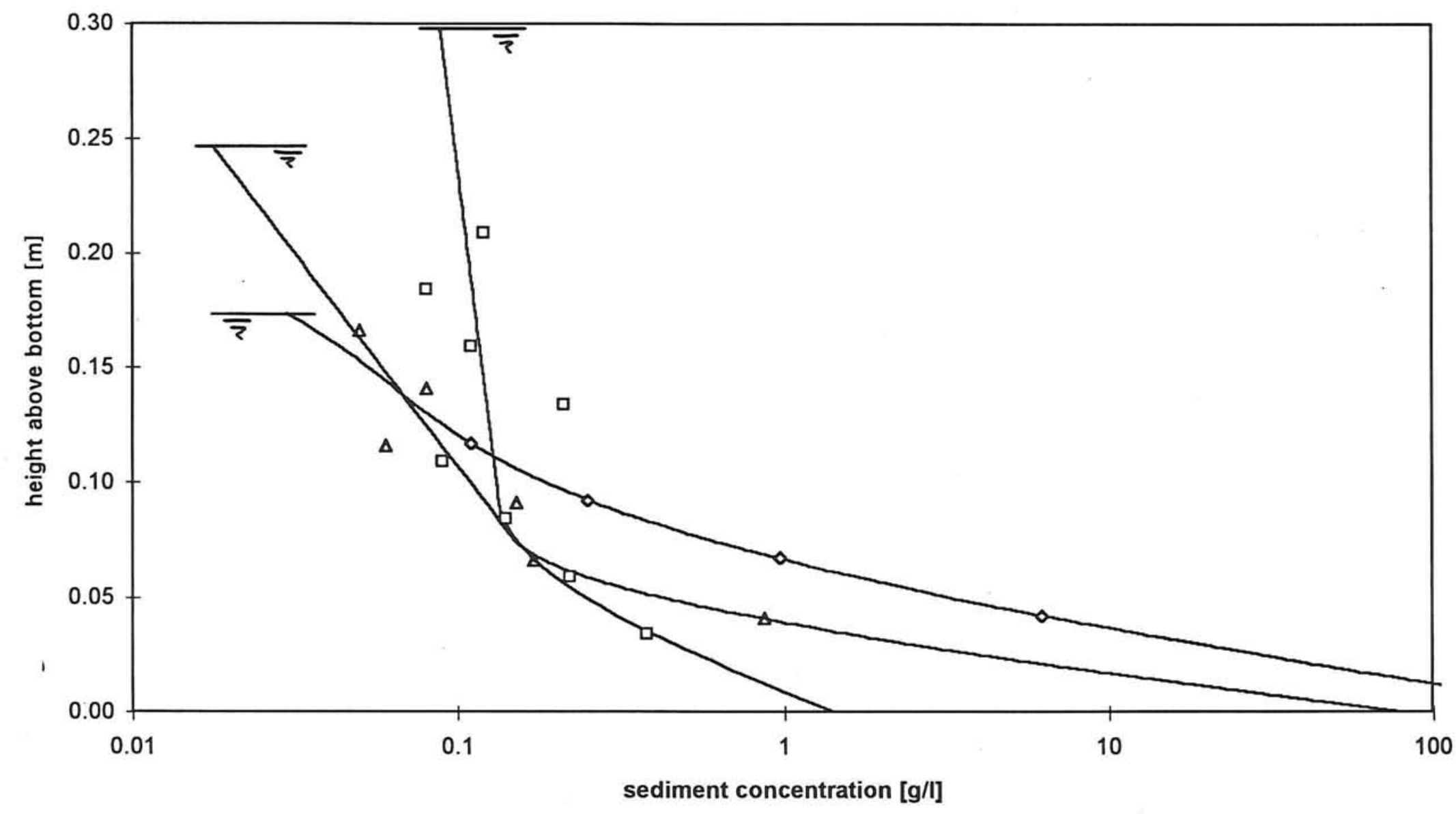




\section{Sediment concentration distributions}

Experiment A with breakwater

Measurement during Interval 4

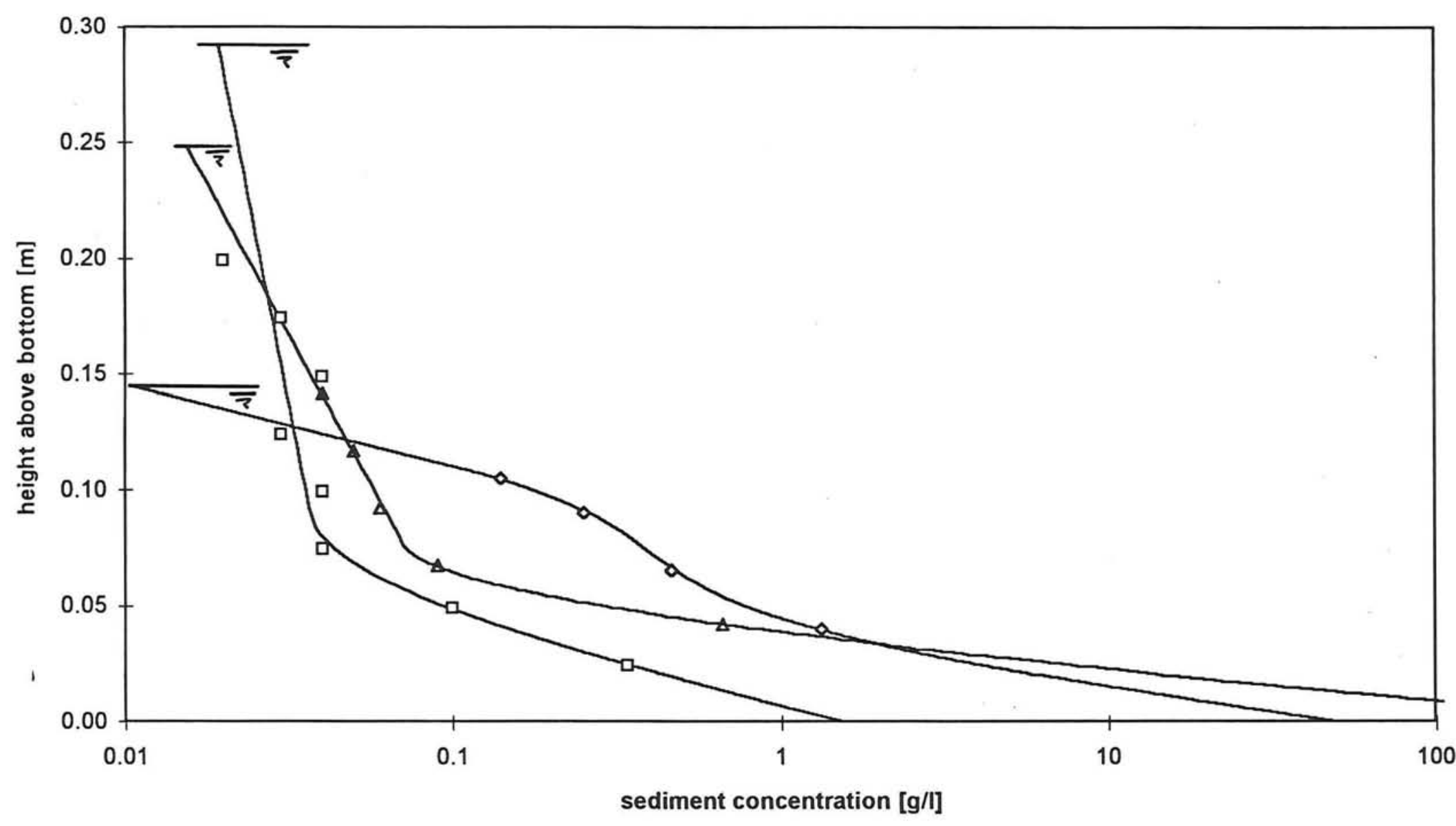




\section{Sediment concentration distributions}

Experiment $D$ with breakwater

Measurement during Interval 4

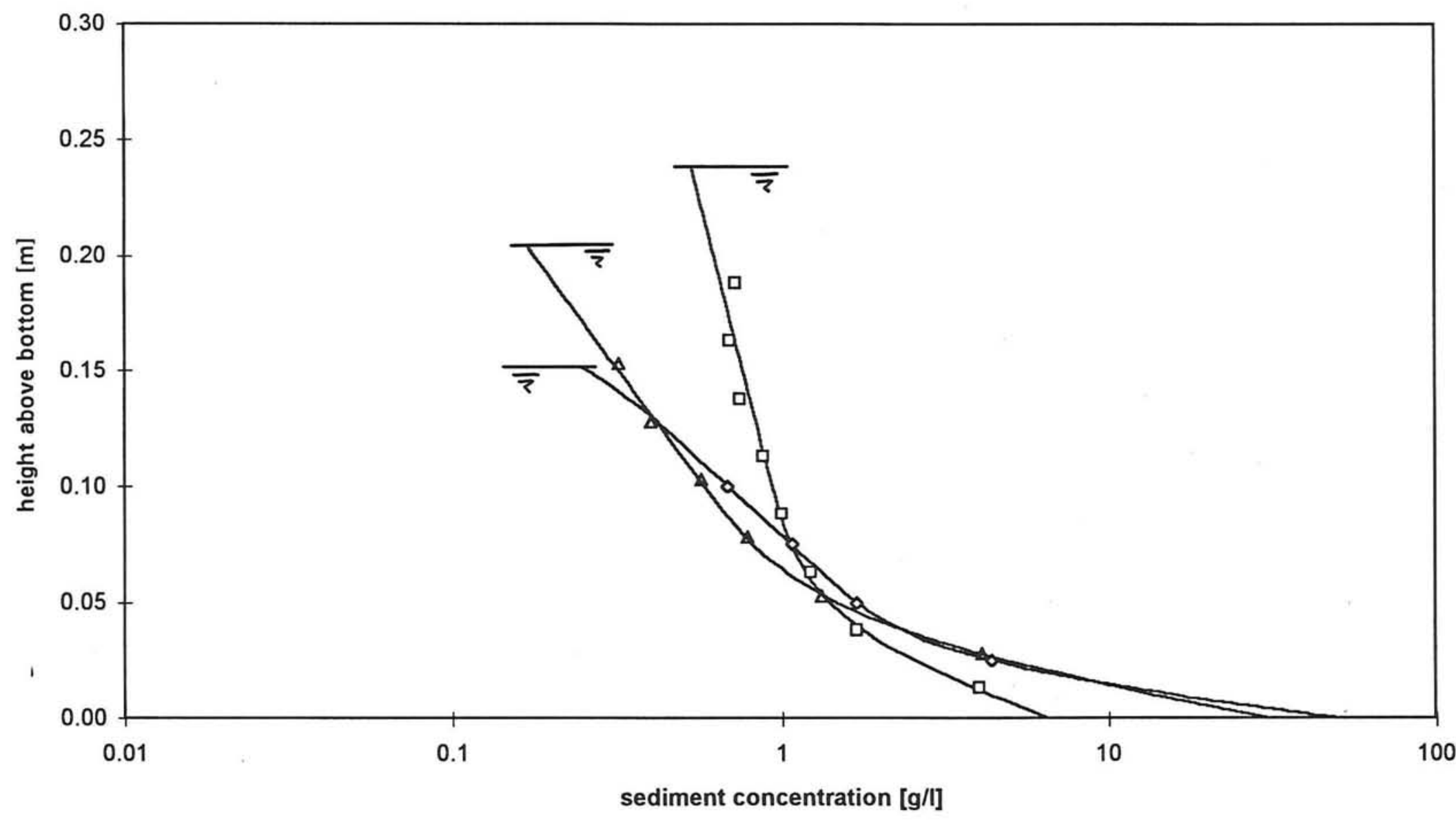




\section{Sediment concentration distributions}

Experiment $A$ with and without breakwater

Measurement during Interval 4 at Vertical (a): $X=5.25 \mathrm{~m}$

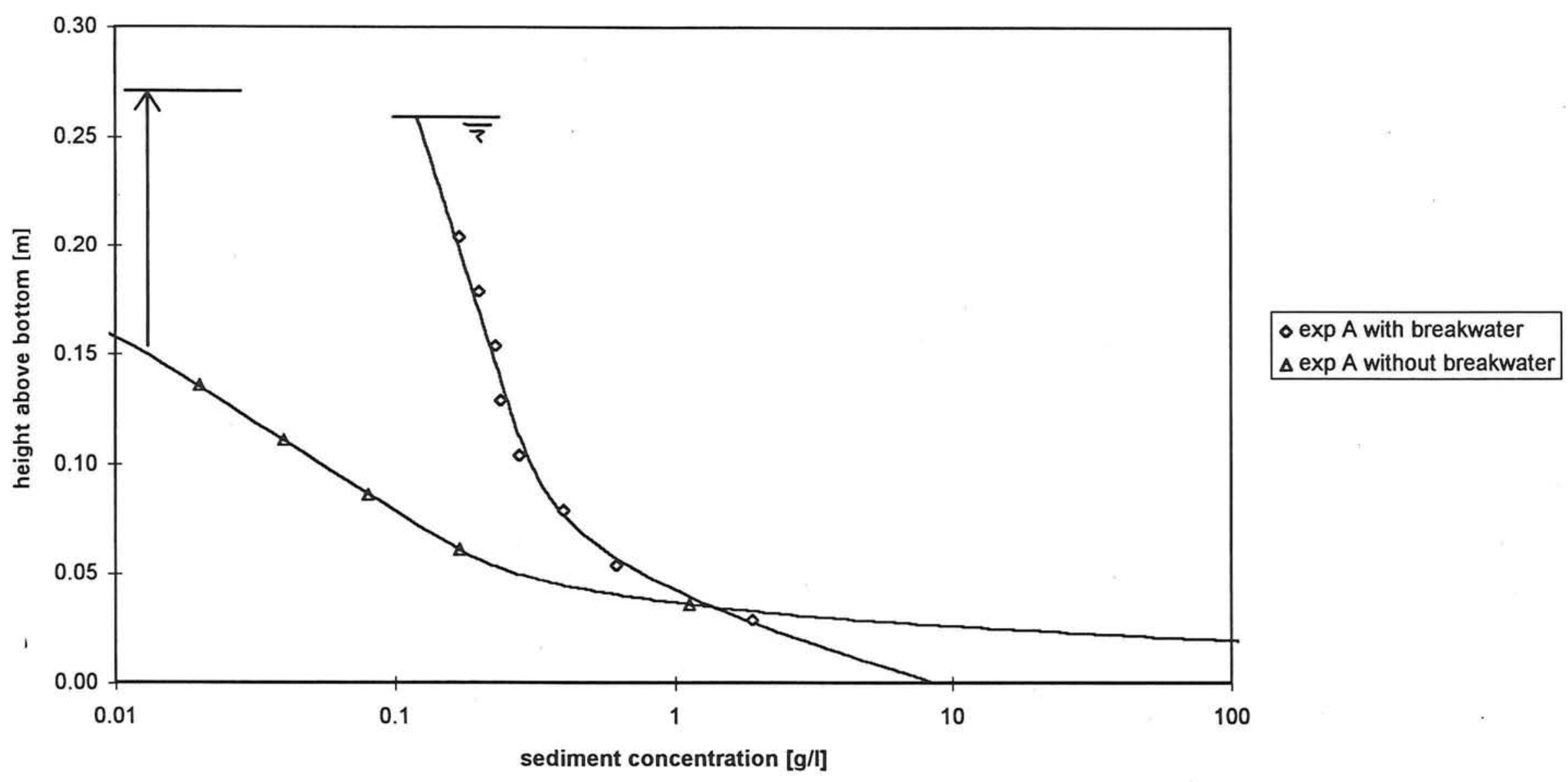

Graph 4.2.9 


\section{Sediment concentration distributions}

Experiment $A$ with and without breakwater

Measurement during Interval 4 at Vertical (b): $X=6.15 \mathrm{~m}$

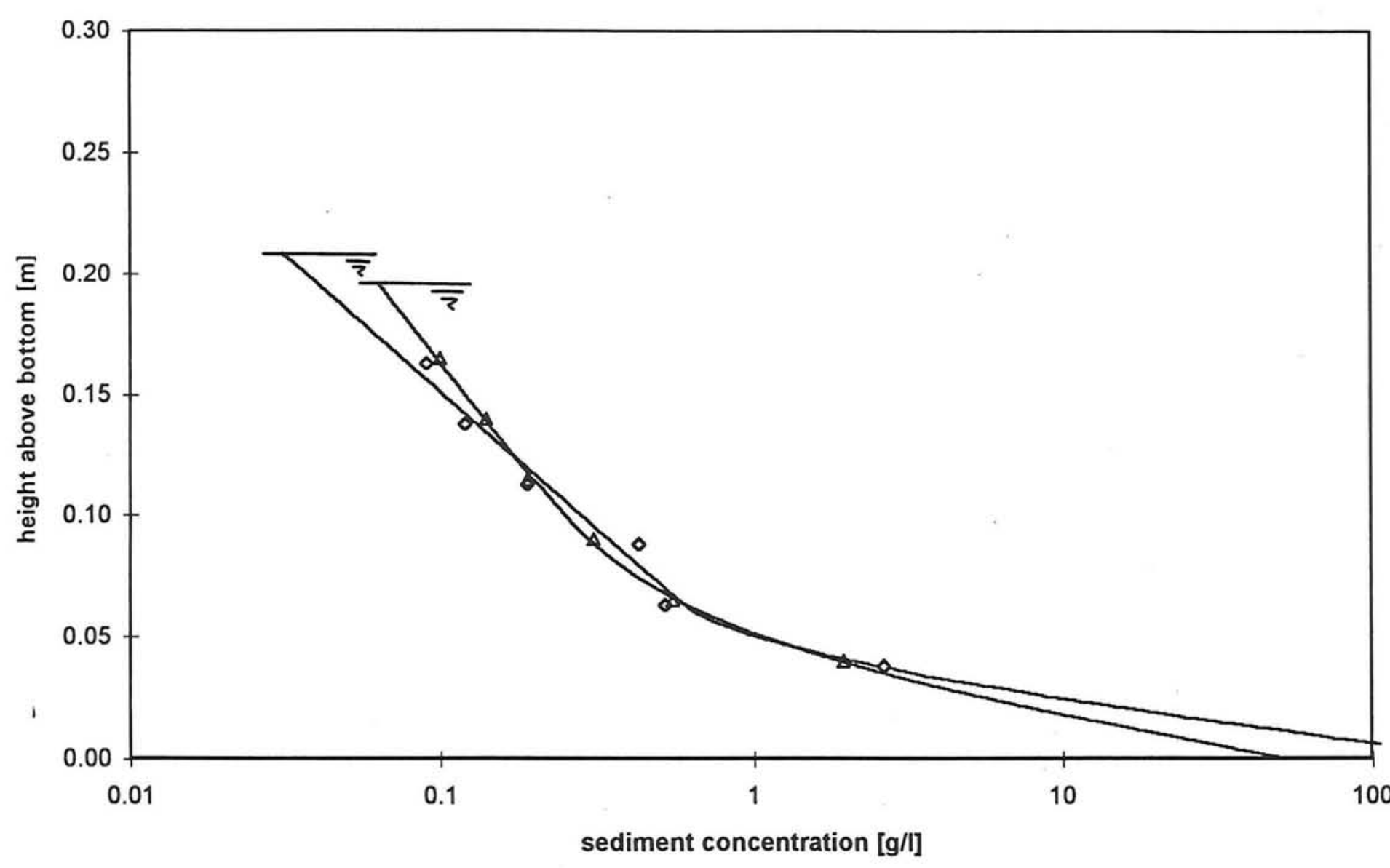

$\triangle \exp A$ with breakwater

$\Delta \exp A$ without breakwater

Graph 4.2.10 


\section{Sediment concentration distributions}

Experiment $A$ with and without breakwater

Measurement during Interval 4 at Vertical (c): $X=7.05 \mathrm{~m}$

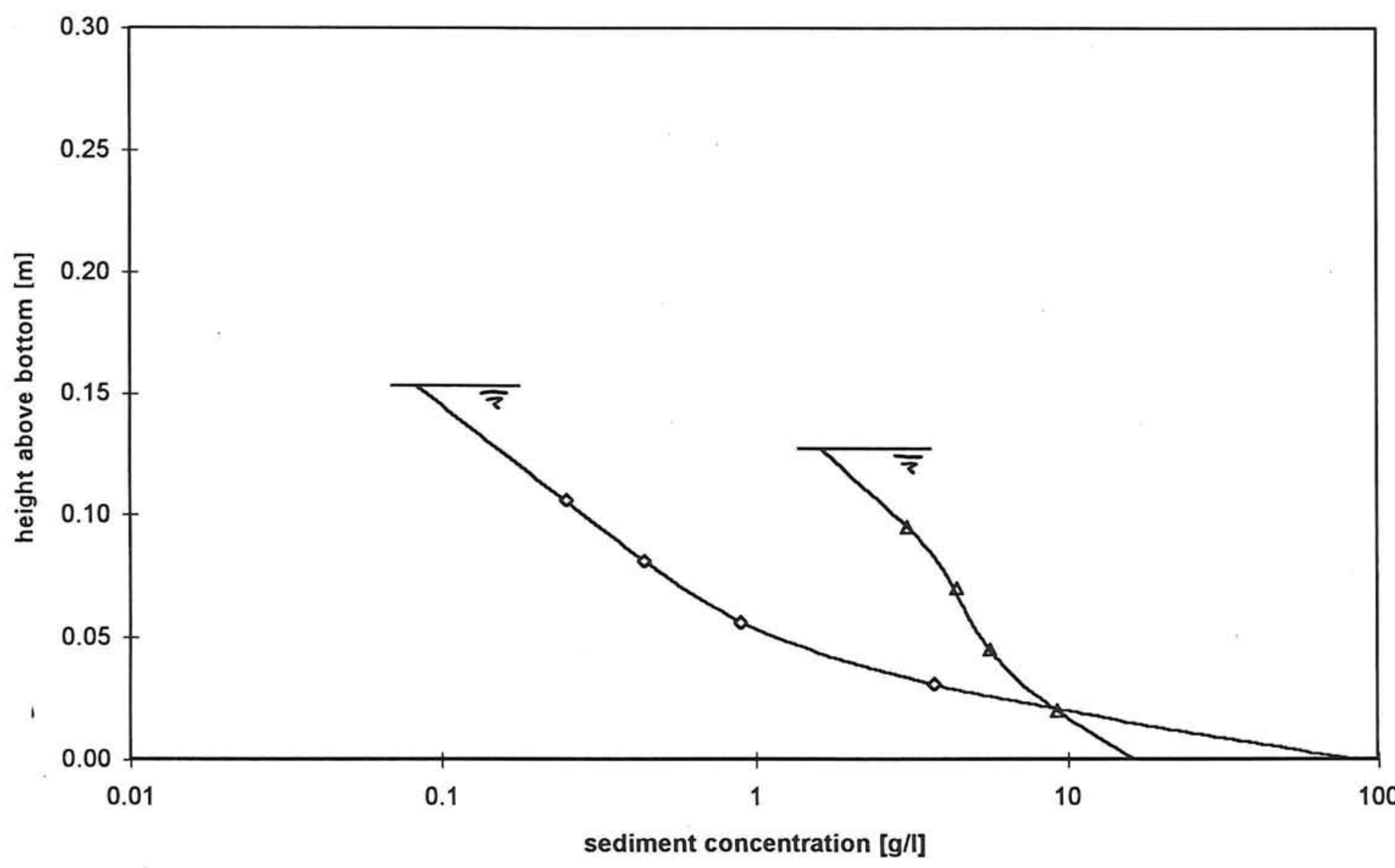

$\checkmark \exp A$ with breakwater

$\triangle \exp A$ without breakwater

Graph 4.2.11 


\section{Sediment concentration distributions}

Experiment $B$ with and without breakwater

Measurement during Interval 4 at Vertical (a): $X=5.25 \mathrm{~m}$

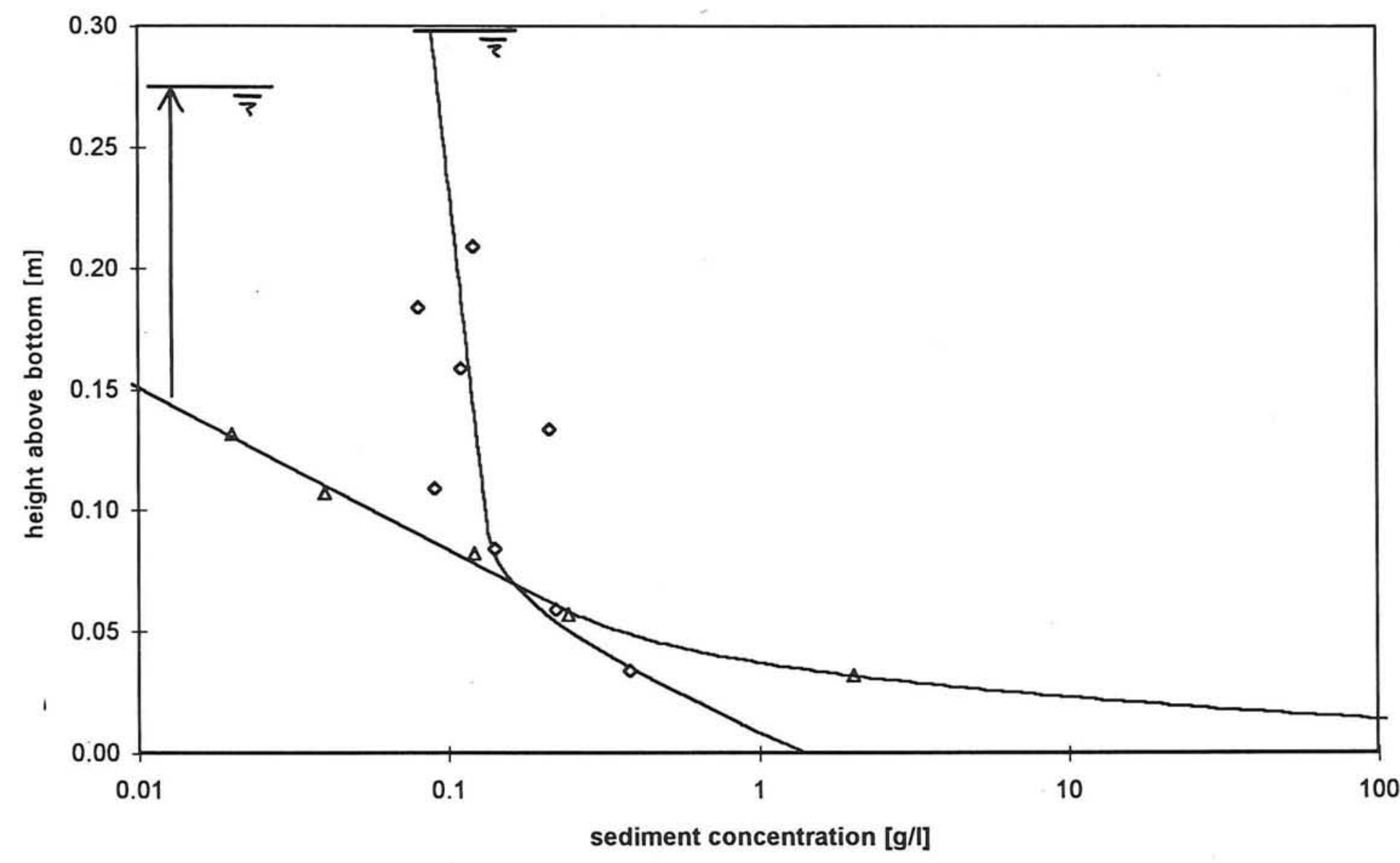




\section{Sediment concentration distributions}

Experiment $B$ with and without breakwater

Measurement during Interval 4 at Vertical (b): $X=6.15 \mathrm{~m}$

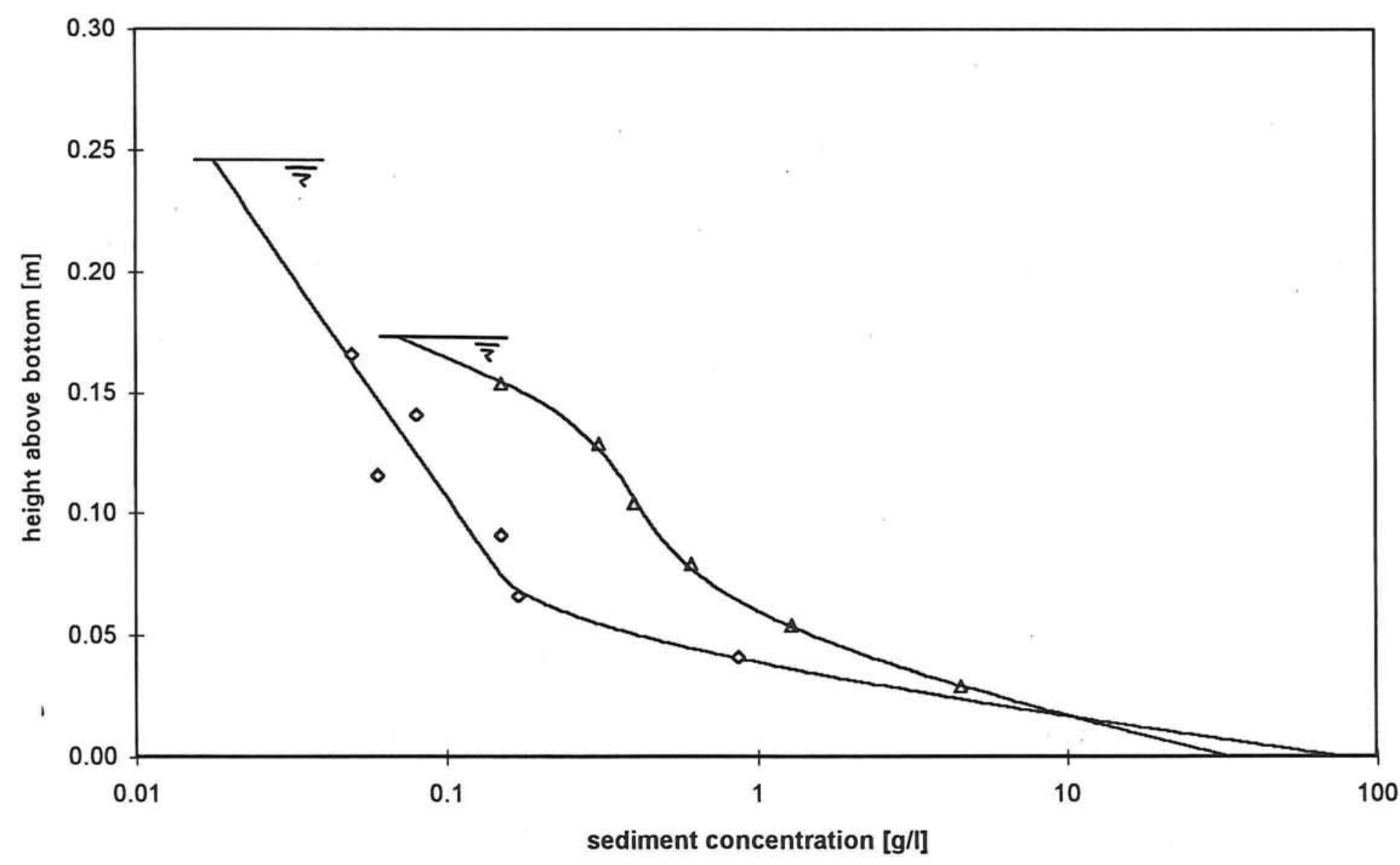




\section{Sediment concentration distributions}

Experiment $B$ with and without breakwater

Measurement during Interval 4 at Vertical (c): $X=7.05 \mathrm{~m}$

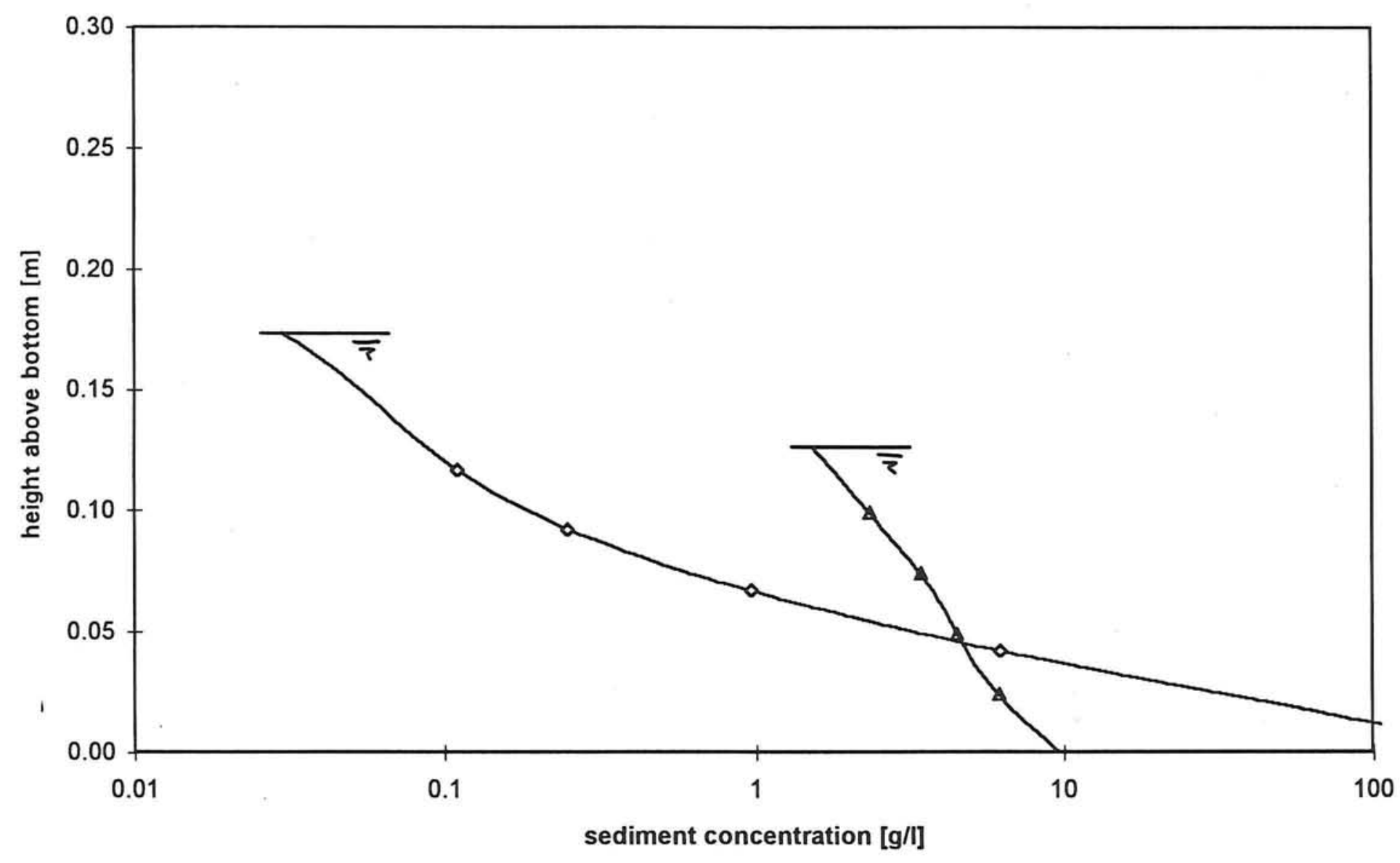




\section{Sediment concentration distributions}

Experiment $C$ with and without breakwater

Measurement during Interval 4 at Vertical (a): $X=5.25 \mathrm{~m}$

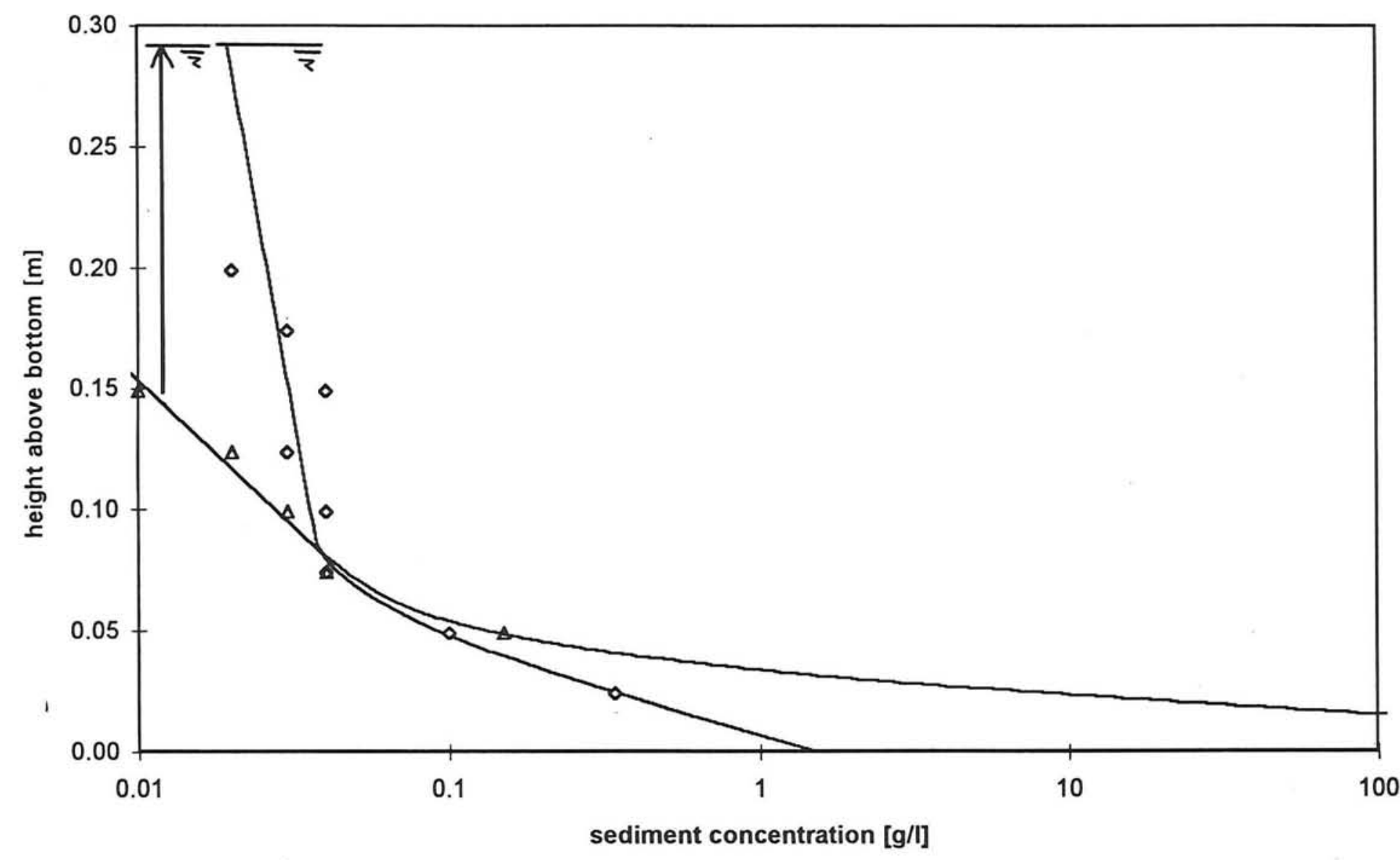


Sediment concentration distributions

Experiment $C$ with and without breakwater

Measurement during Interval 4 at Vertical (b): $X=6.15 \mathrm{~m}$

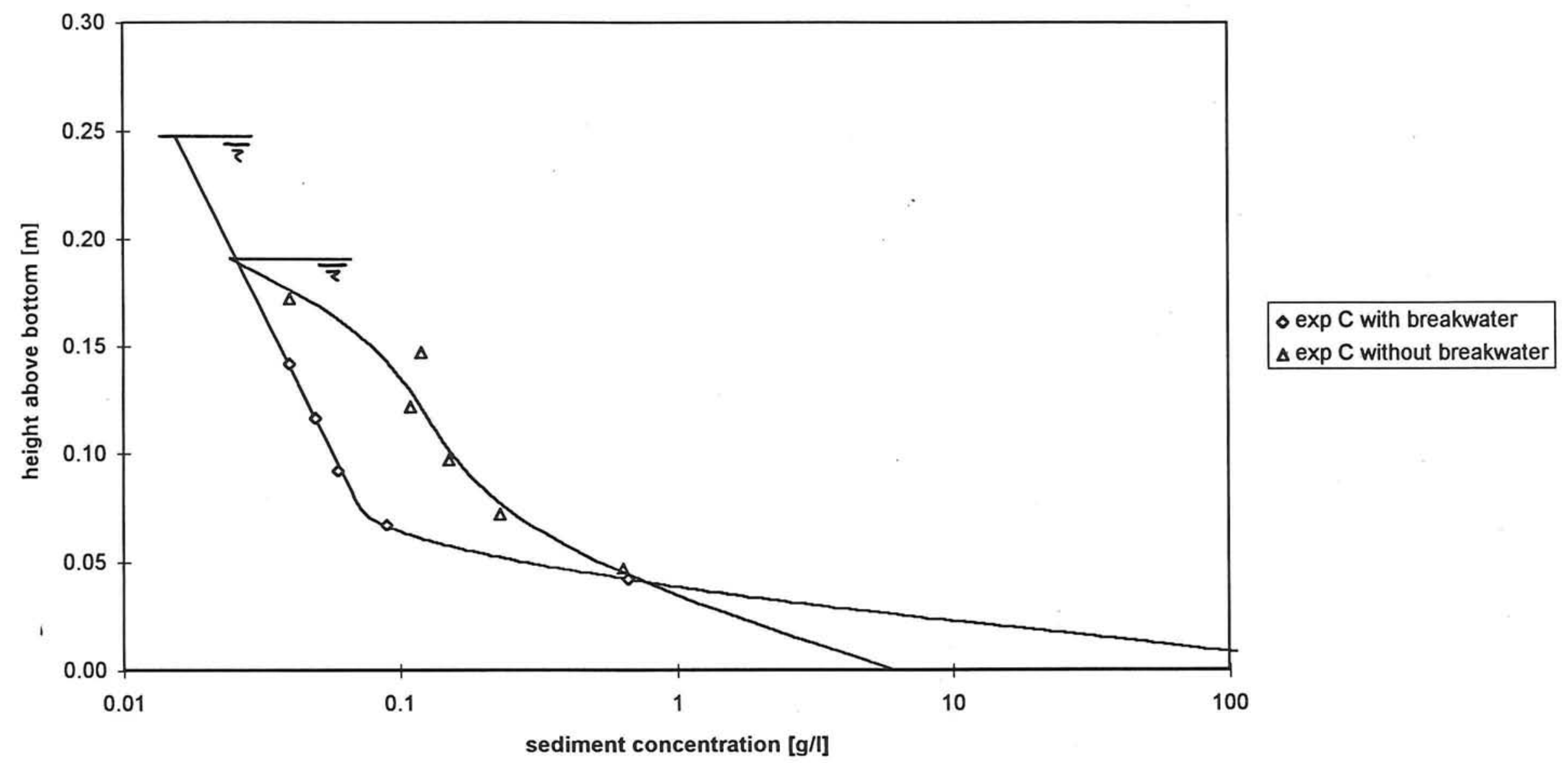

Graph 4.2.16 


\section{Sediment concentration distributions}

Experiment $\mathrm{C}$ with and without breakwater

Measurement during Interval 4 at Vertical (c): $X=7.05 \mathrm{~m}$

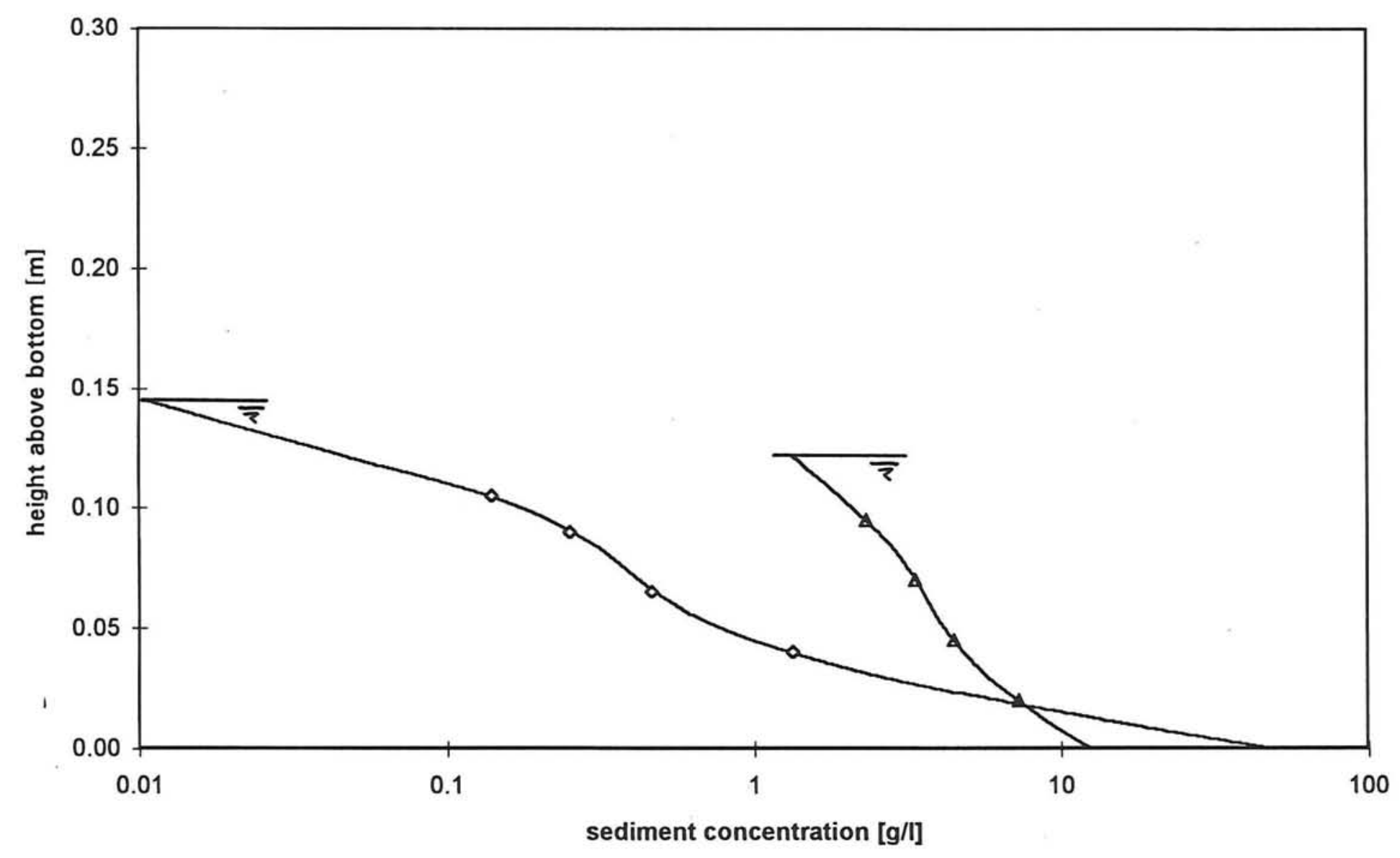

- $\exp C$ with breakwater

$\triangle \exp C$ without breakwater

Graph 4.2.17 
Sediment concentration distributions

Experiment $D$ with and without breakwater

Measurement during Interval 4 at Vertical (a): $X=5.25 \mathrm{~m}$

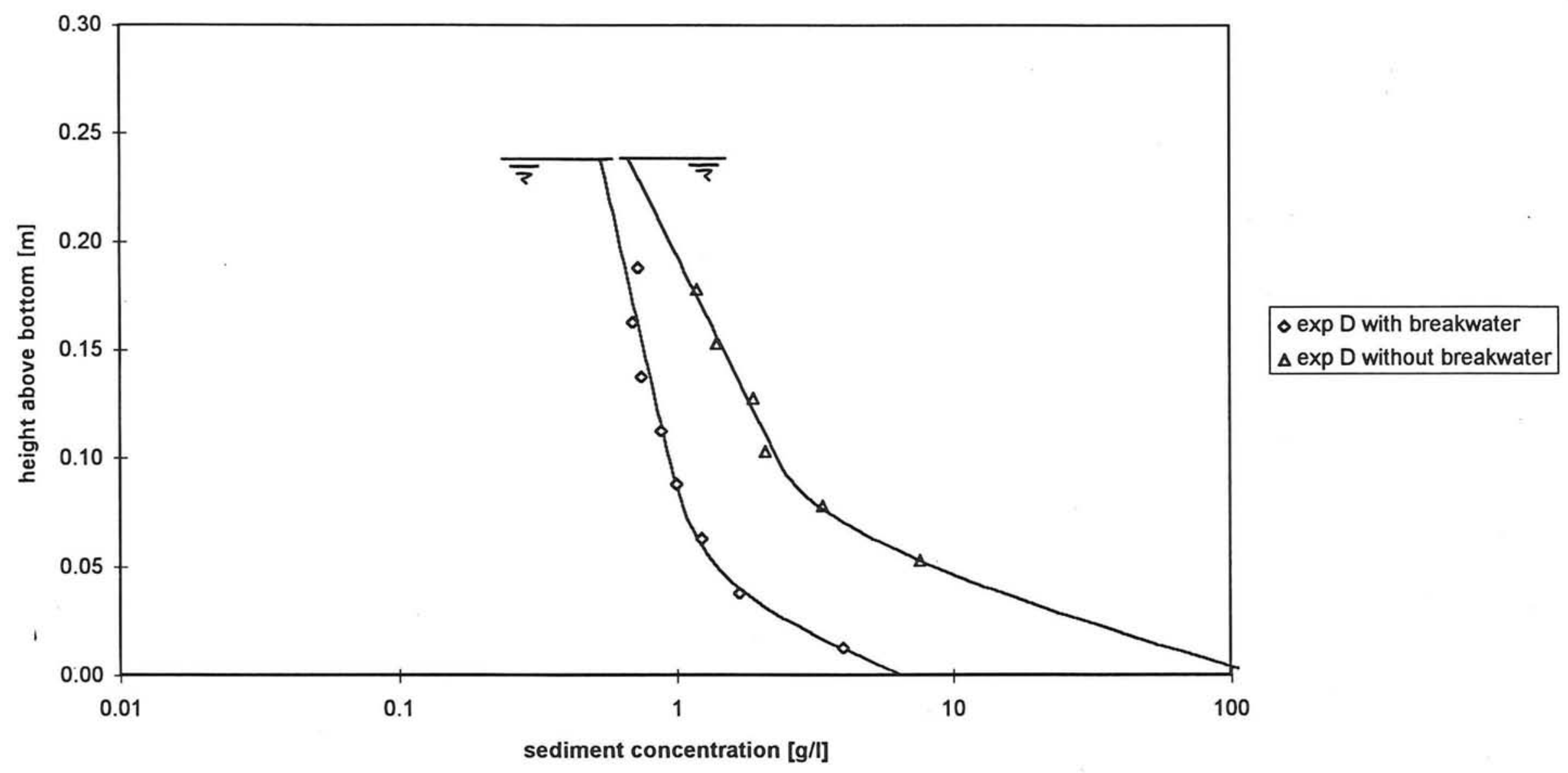

Graph 4.2.18 


\section{Sediment concentration distributions}

Experiment $D$ with and without breakwater

Measurement during Interval 4 at Vertical (b): $X=6.15 \mathrm{~m}$

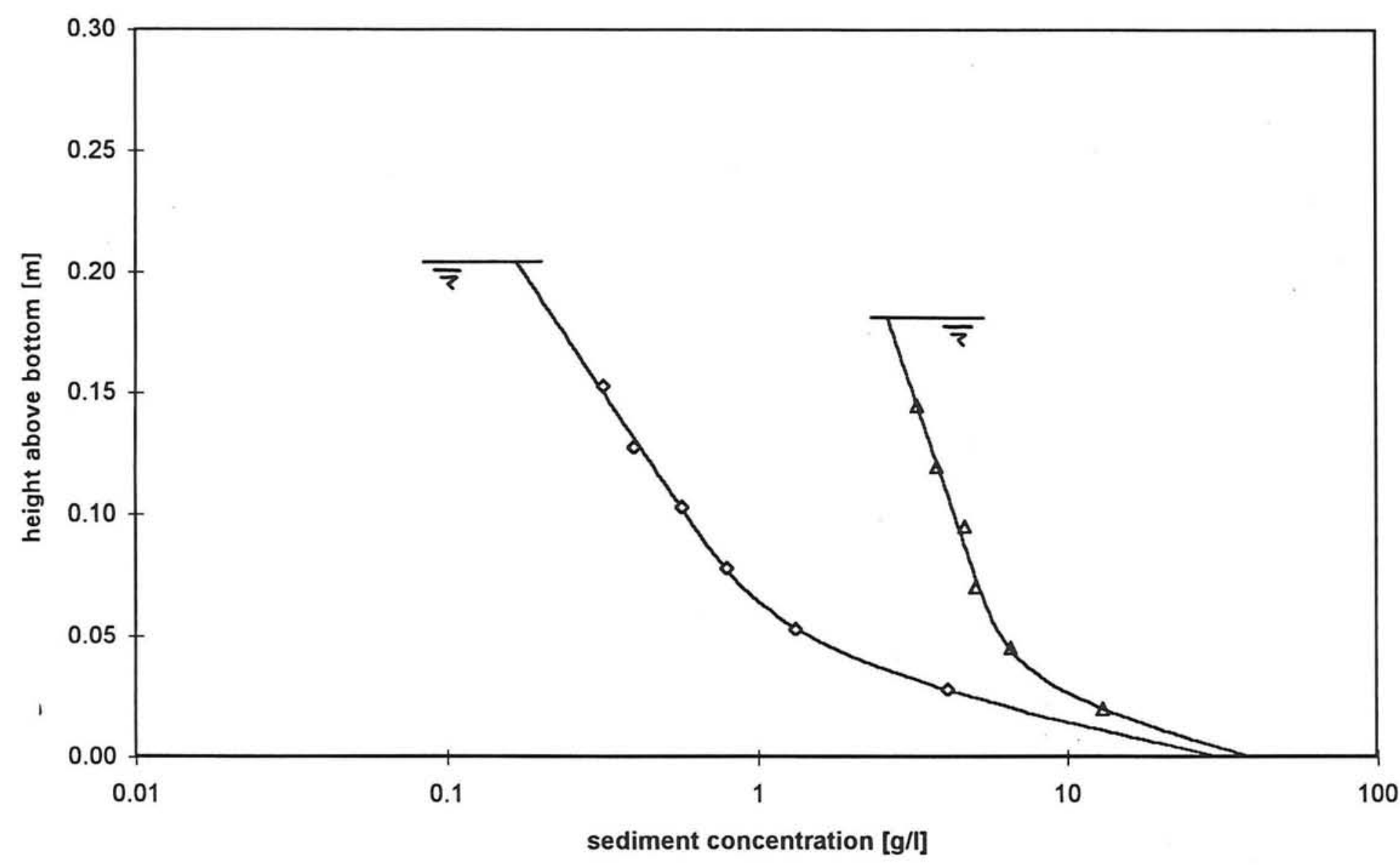




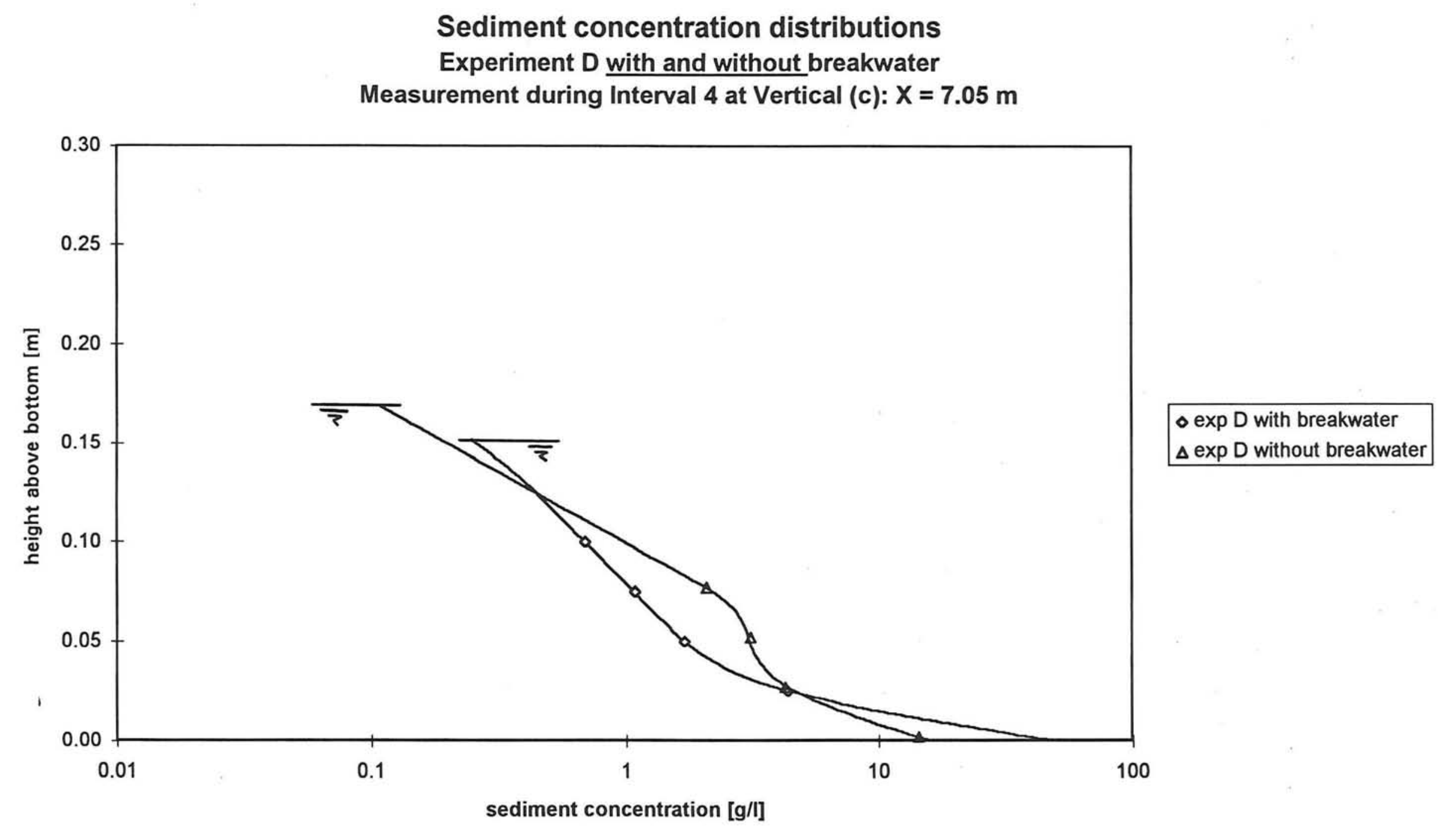

Graph 4.2.20 


\section{Sediment concentration distributions}

Experiments without breakwater

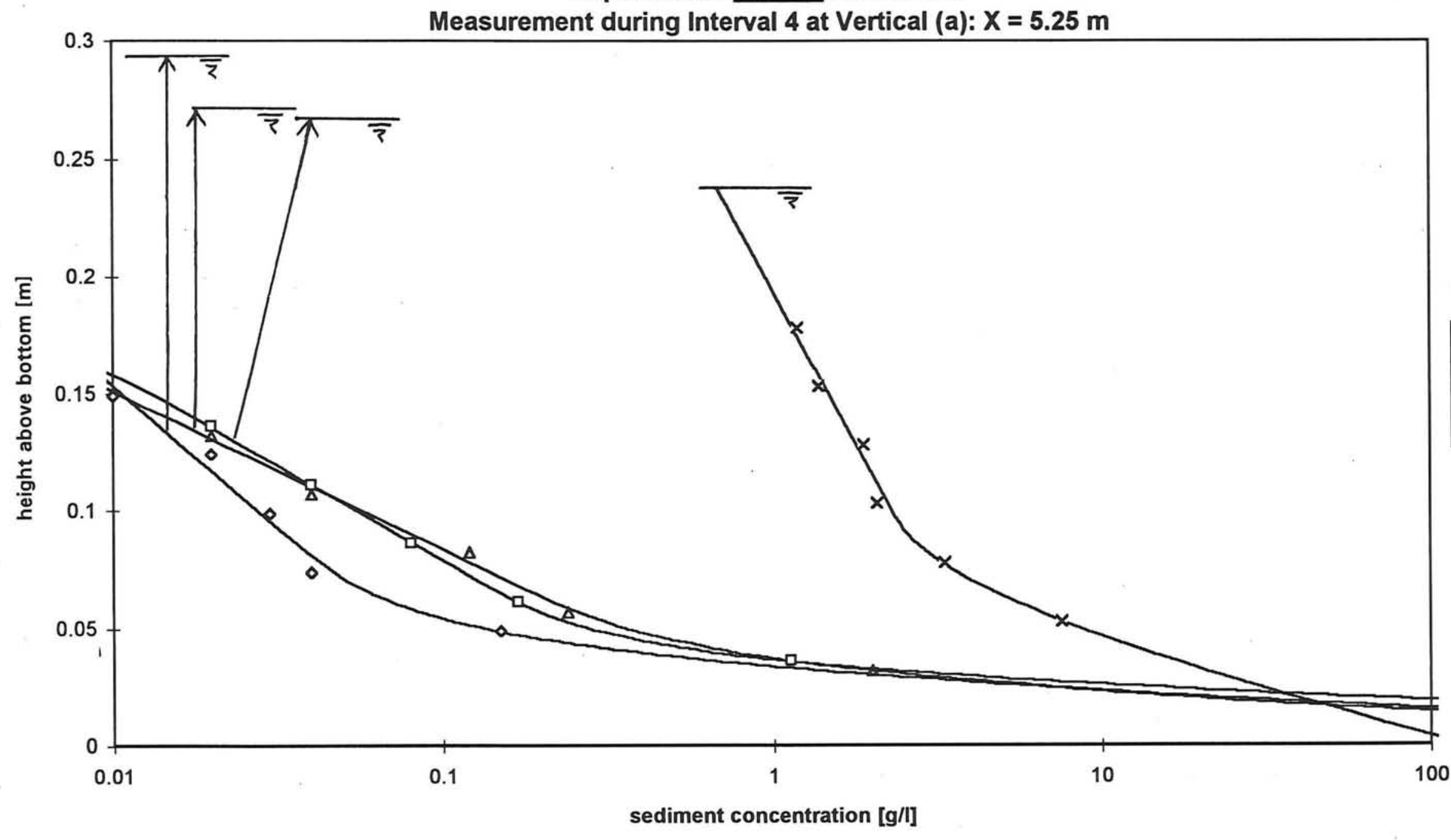




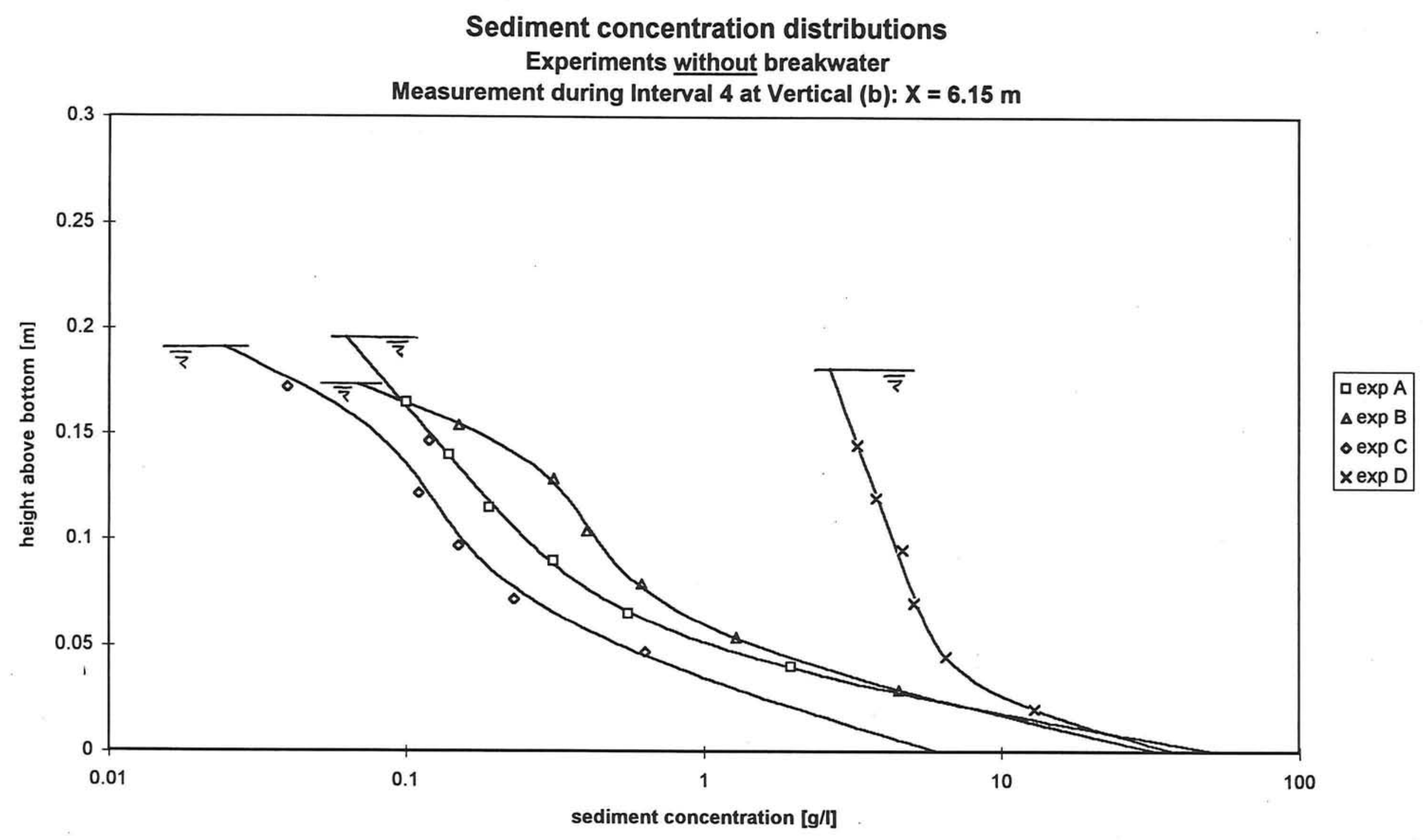

Graph 4.2.22 


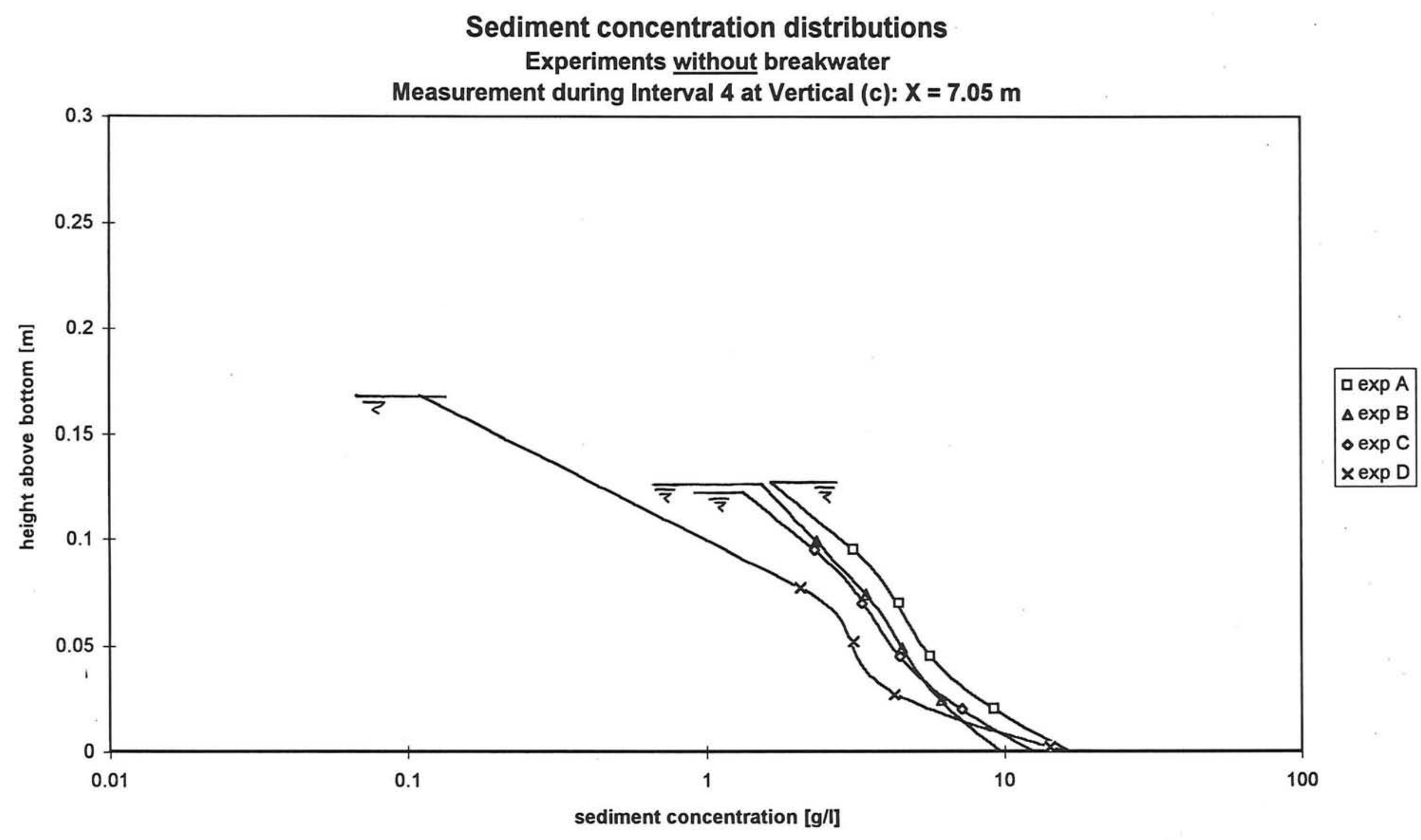

Graph 4.2.23 


\section{Sediment concentration distributions}

Experiments with breakwater

Measurement during Interval 4 at Vertical (a): $X=5.25 \mathrm{~m}$

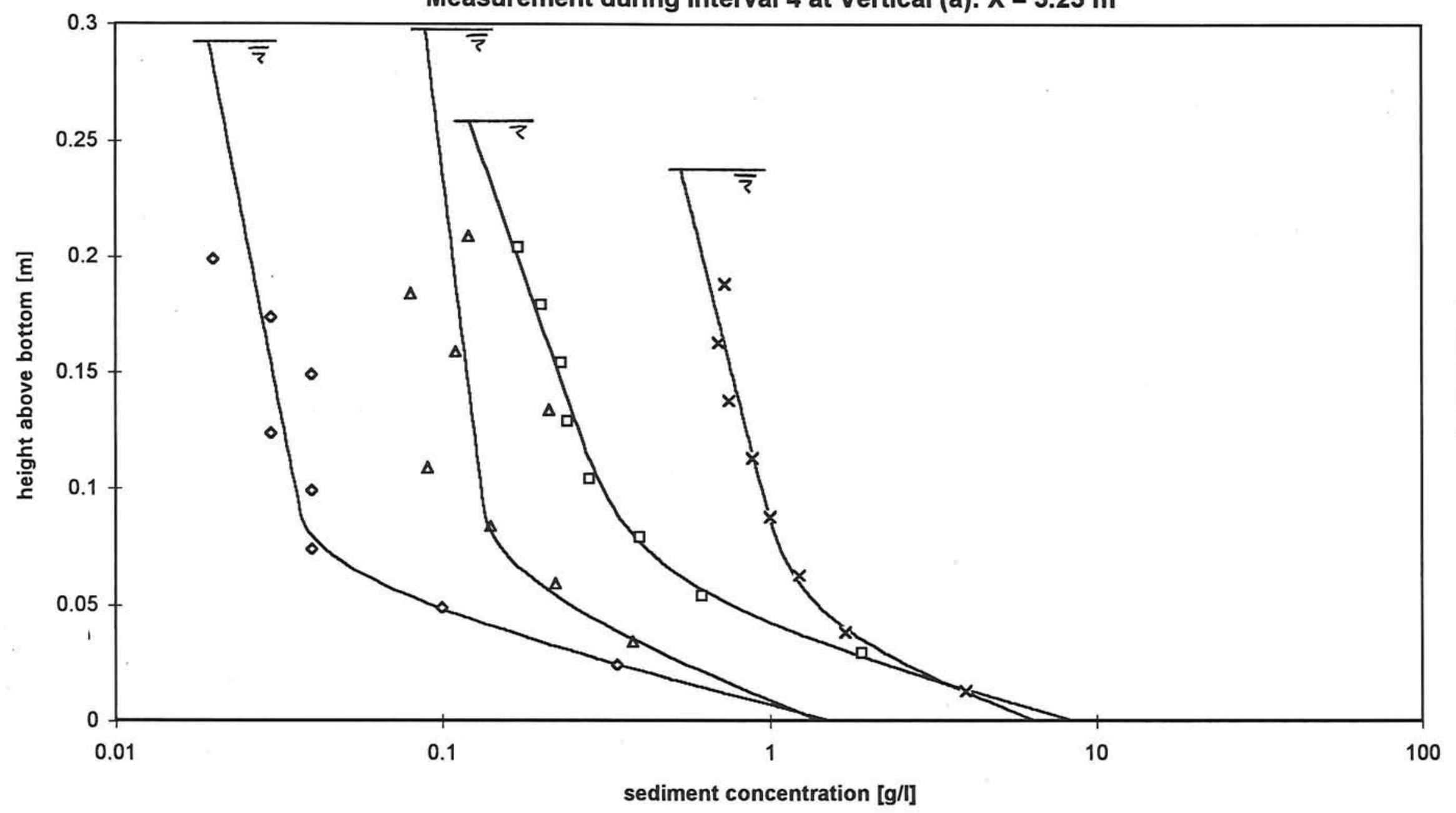




\section{Sediment concentration distributions}

Experiments with breakwater

Measurement during Interval 4 at Vertical (b): $X=6.15 \mathrm{~m}$

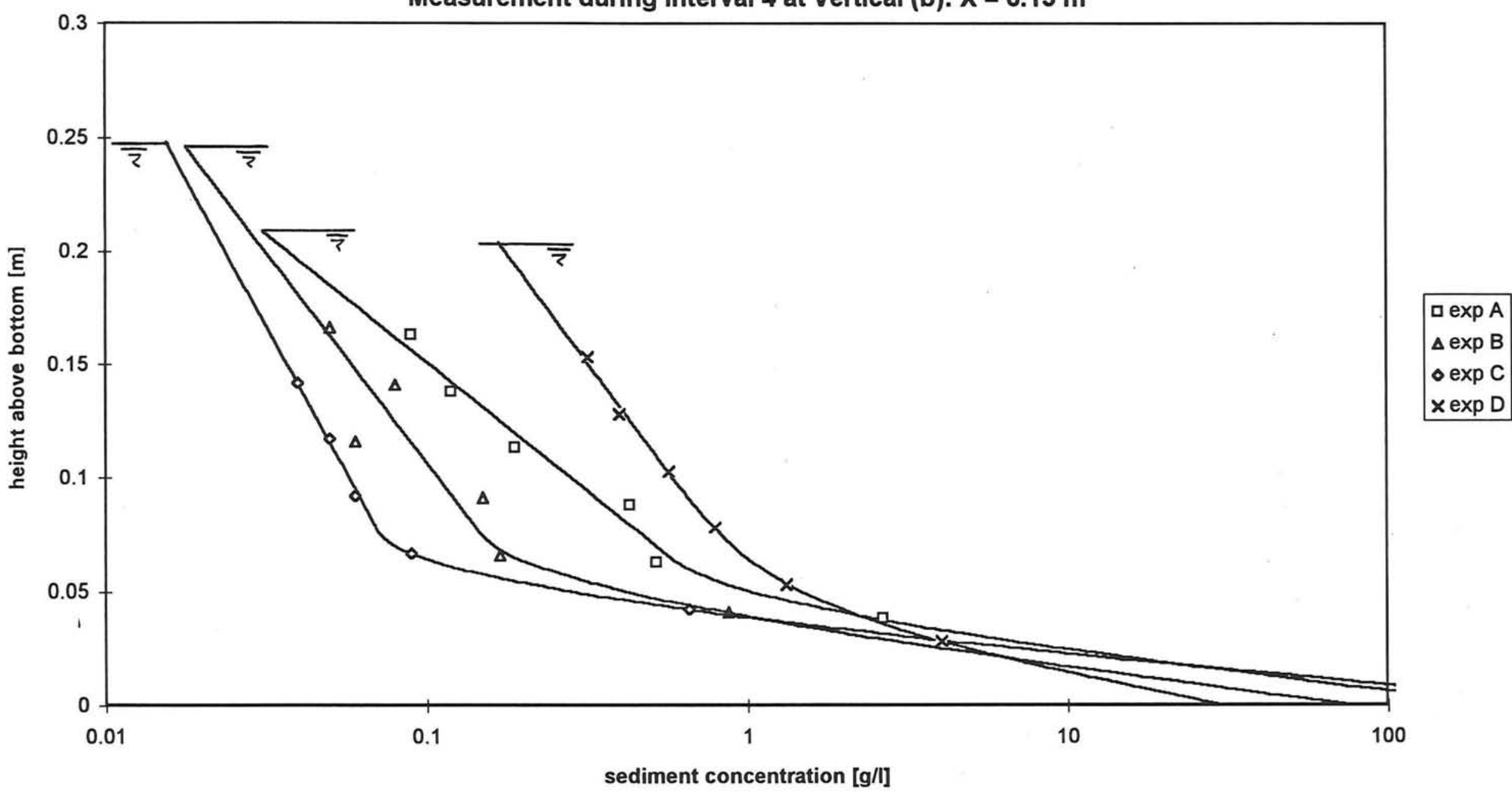

Graph 4.2.25 


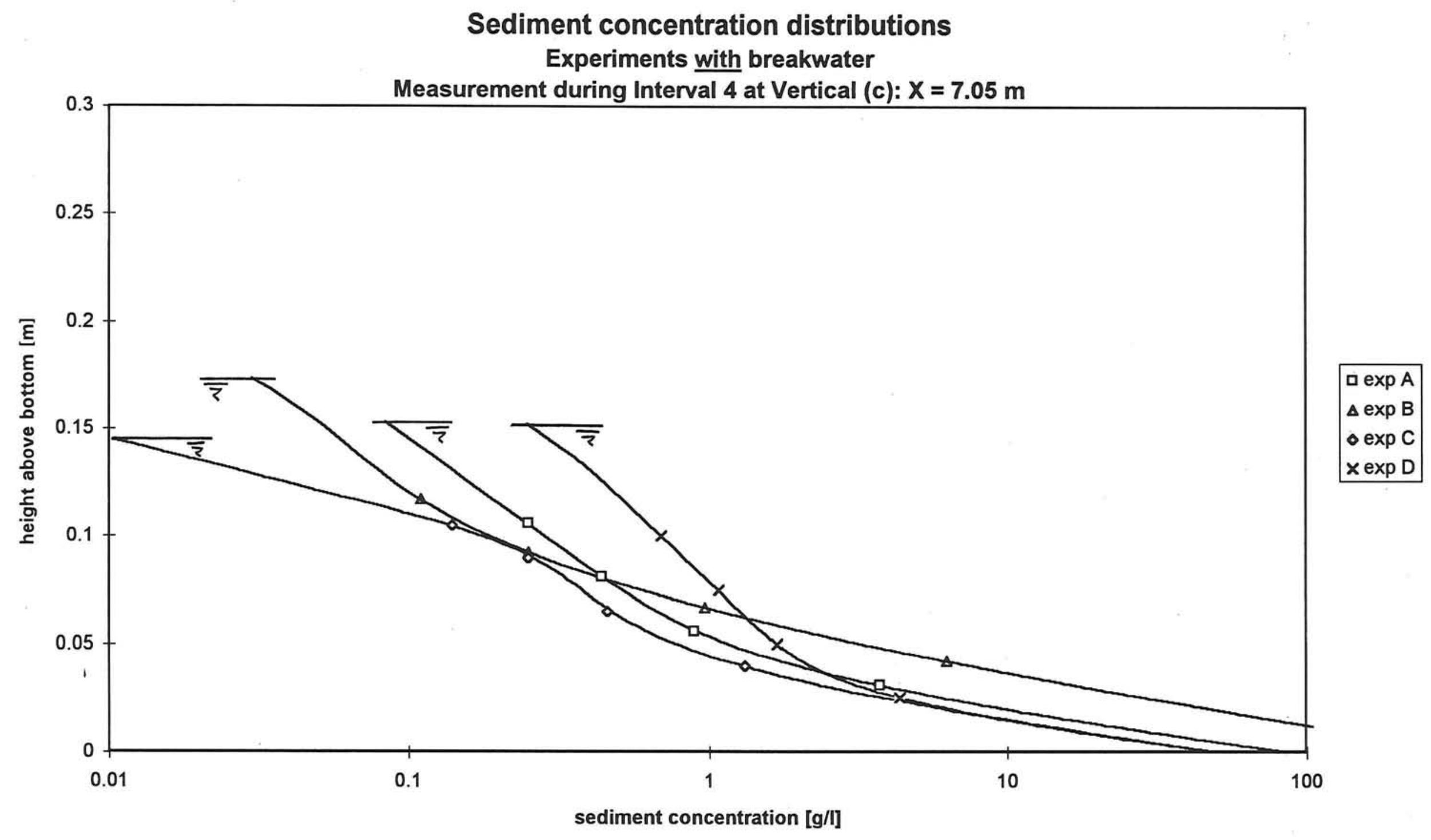

Graph 4.2.26 
Measured Hrms at WHM $1(x=-3.0 \mathrm{~m})$

Experiments without breakwater

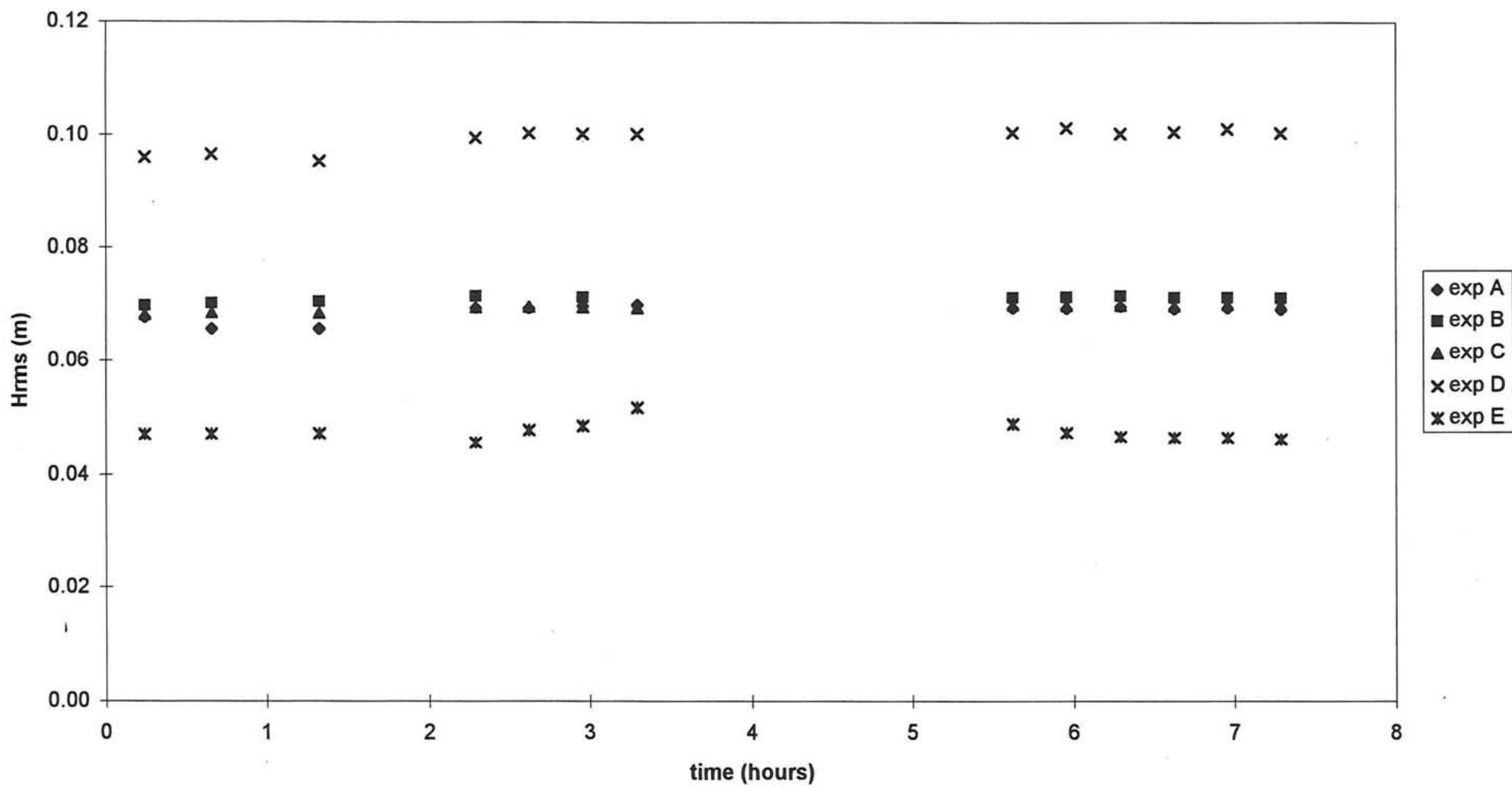

Graph 5.1 
Measured Hrms at WHM $1(x=-3.0 \mathrm{~m})$

Experiments with breakwater

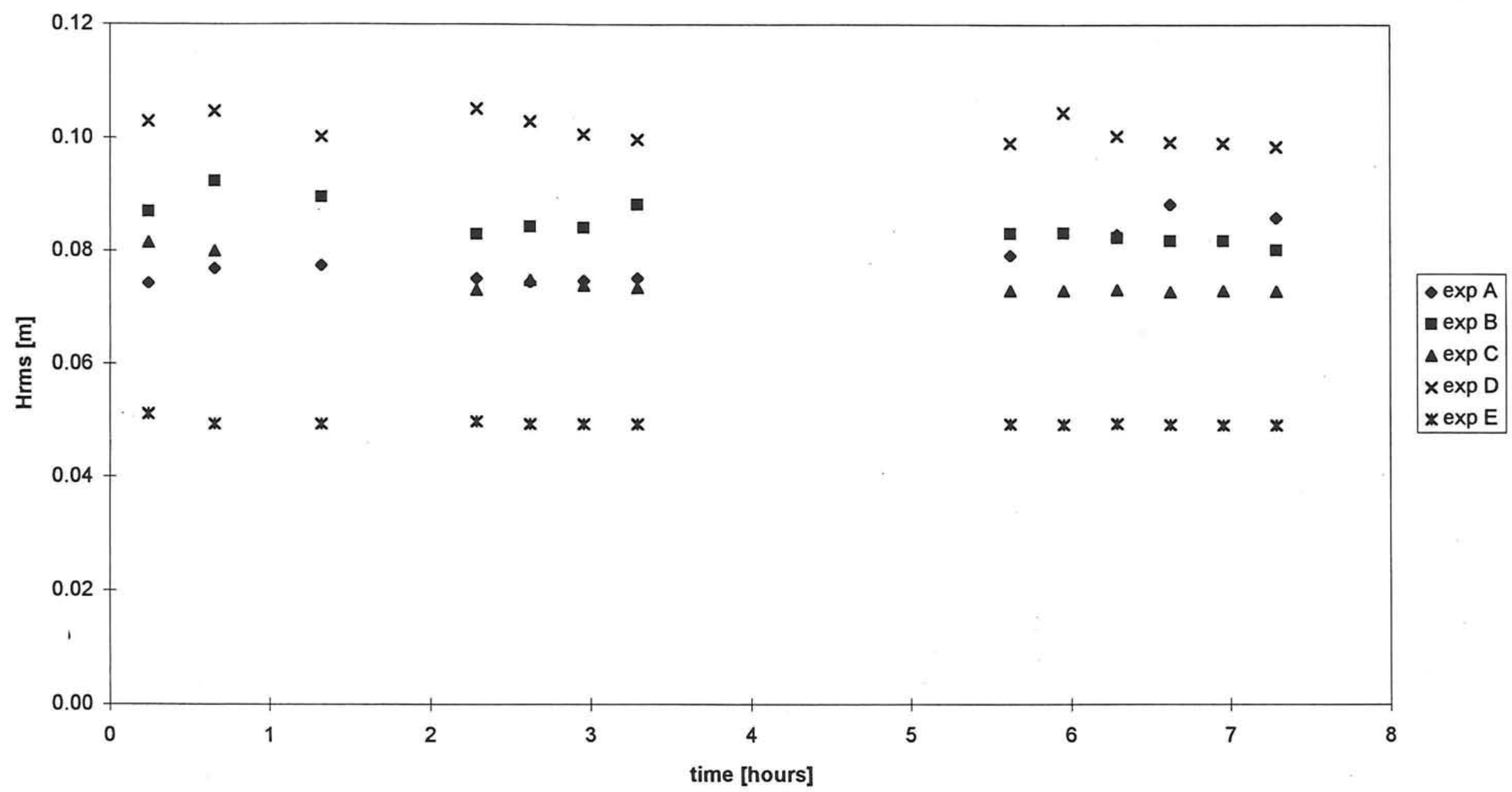

Graph 5.2 
Measured Hrms versus Hrms calculated by Unibest-TC

at Interval 1

Experiment A without breakwater ( $\mathrm{Hrms} 0=0.068 \mathrm{~m}, \mathrm{Tp}=2.07 \mathrm{~s}$ )

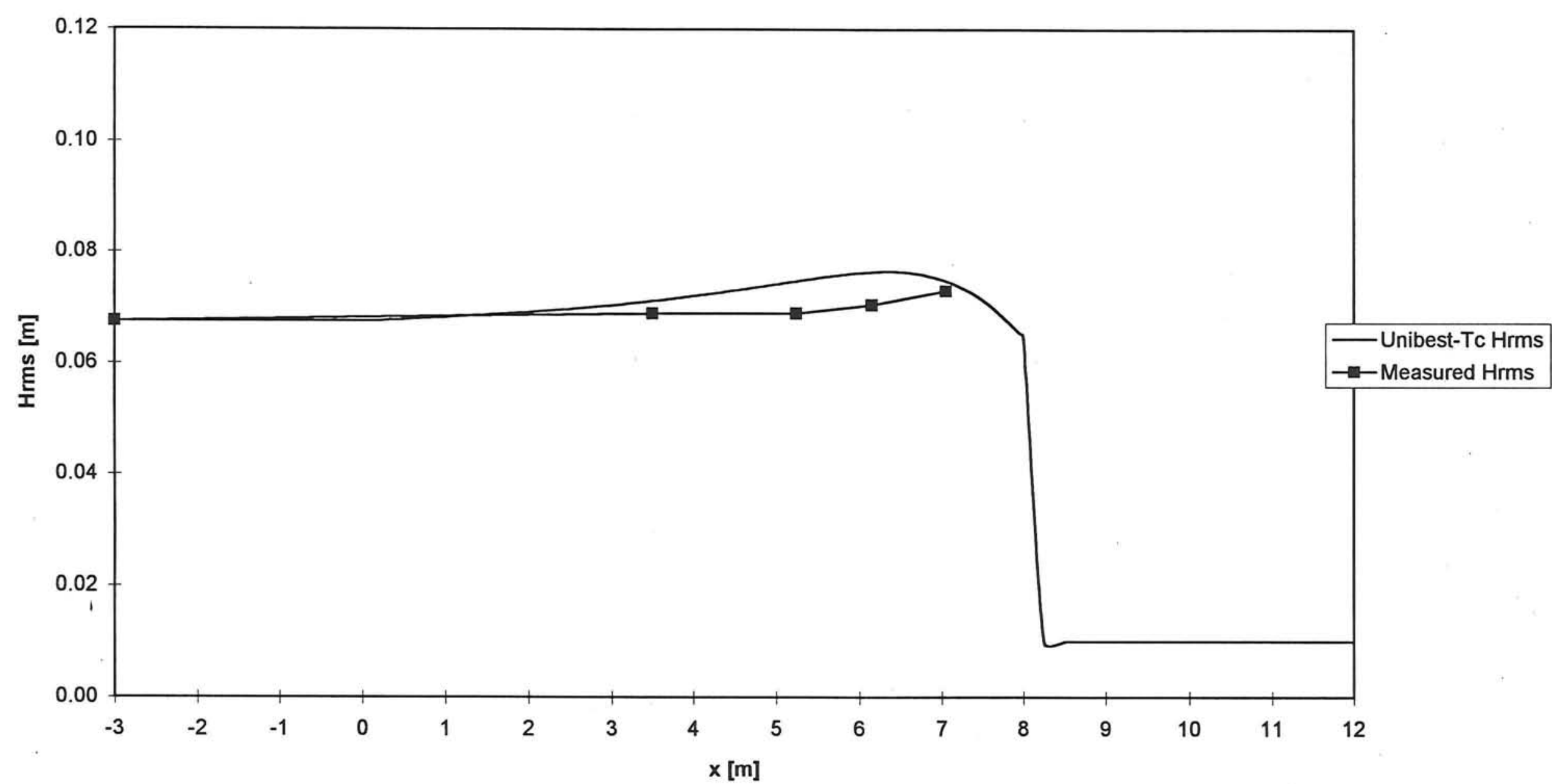

Graph 5.3 


\section{Measured Hrms versus Hrms calculated by Unibest-TC}

at Interval 1

Experiment $B$ without breakwater $(\mathrm{Hrms} 0=0.070 \mathrm{~m}, \mathrm{Tp}=1.55 \mathrm{~s})$

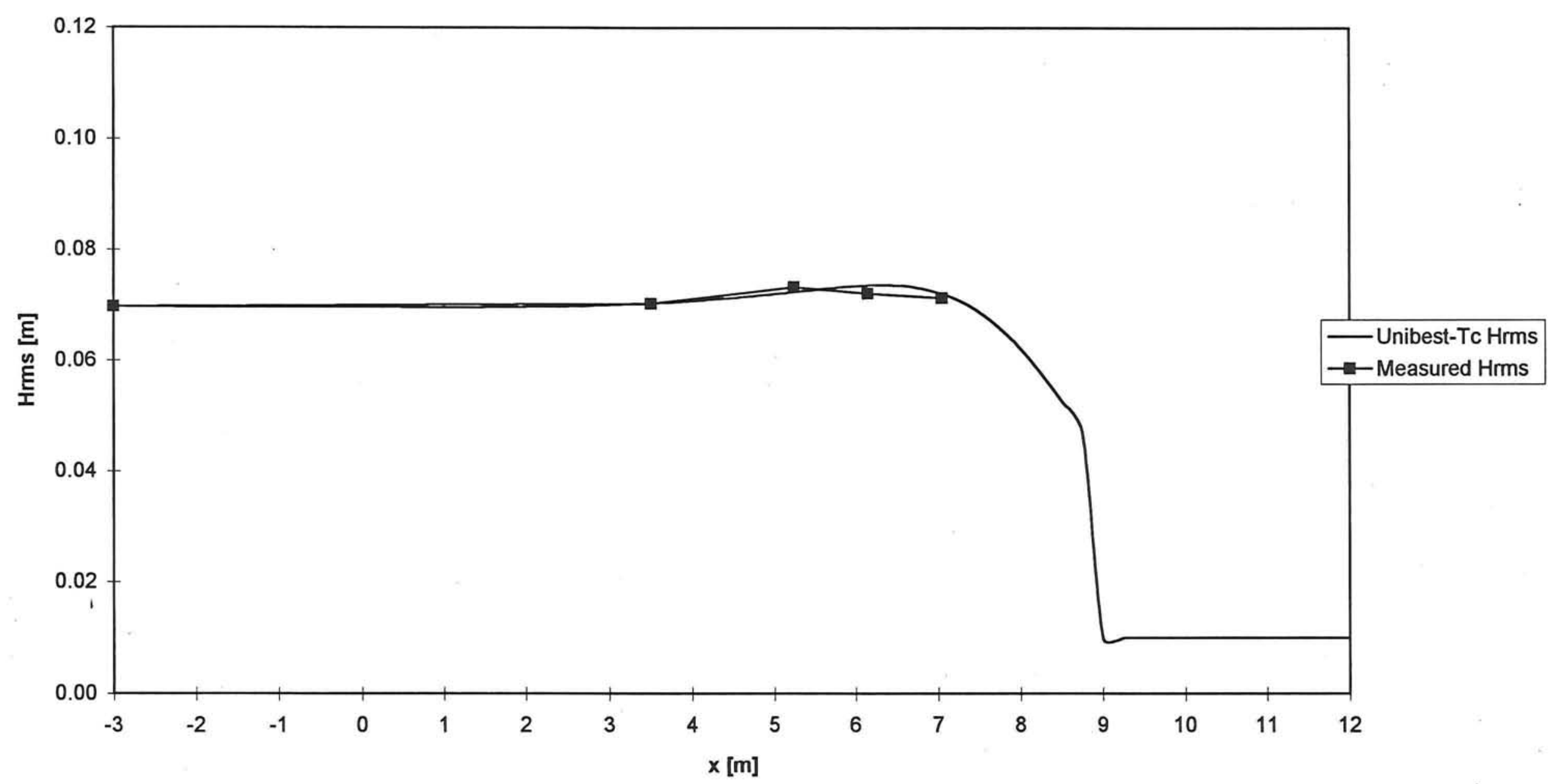

Graph 5.4 


\section{Measured Hrms versus Hrms calculated by Unibest-TC}

at Interval 1

Experiment $C$ without breakwater ( $\mathrm{Hrms} 0=0.068 \mathrm{~m}, \mathrm{Tp}=1.29 \mathrm{~s}$ )

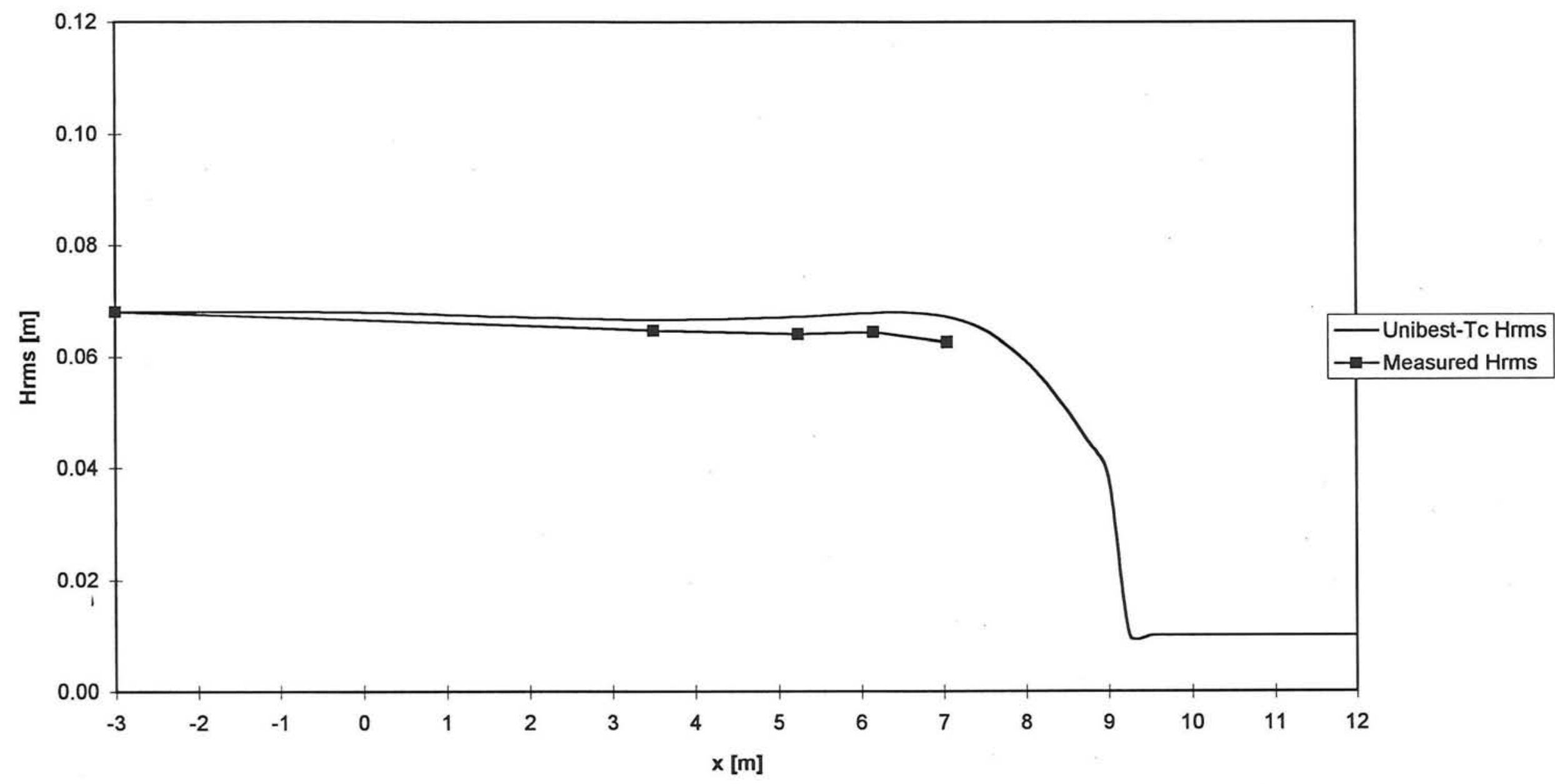

Graph 5.5 


\section{Measured Hrms versus Hrms calculated by Unibest-TC \\ at Interval 1}

Experiment $\mathrm{D}$ without breakwater (Hrms0 $=0.096 \mathrm{~m}, \mathrm{Tp}=1.81 \mathrm{~s}$ )

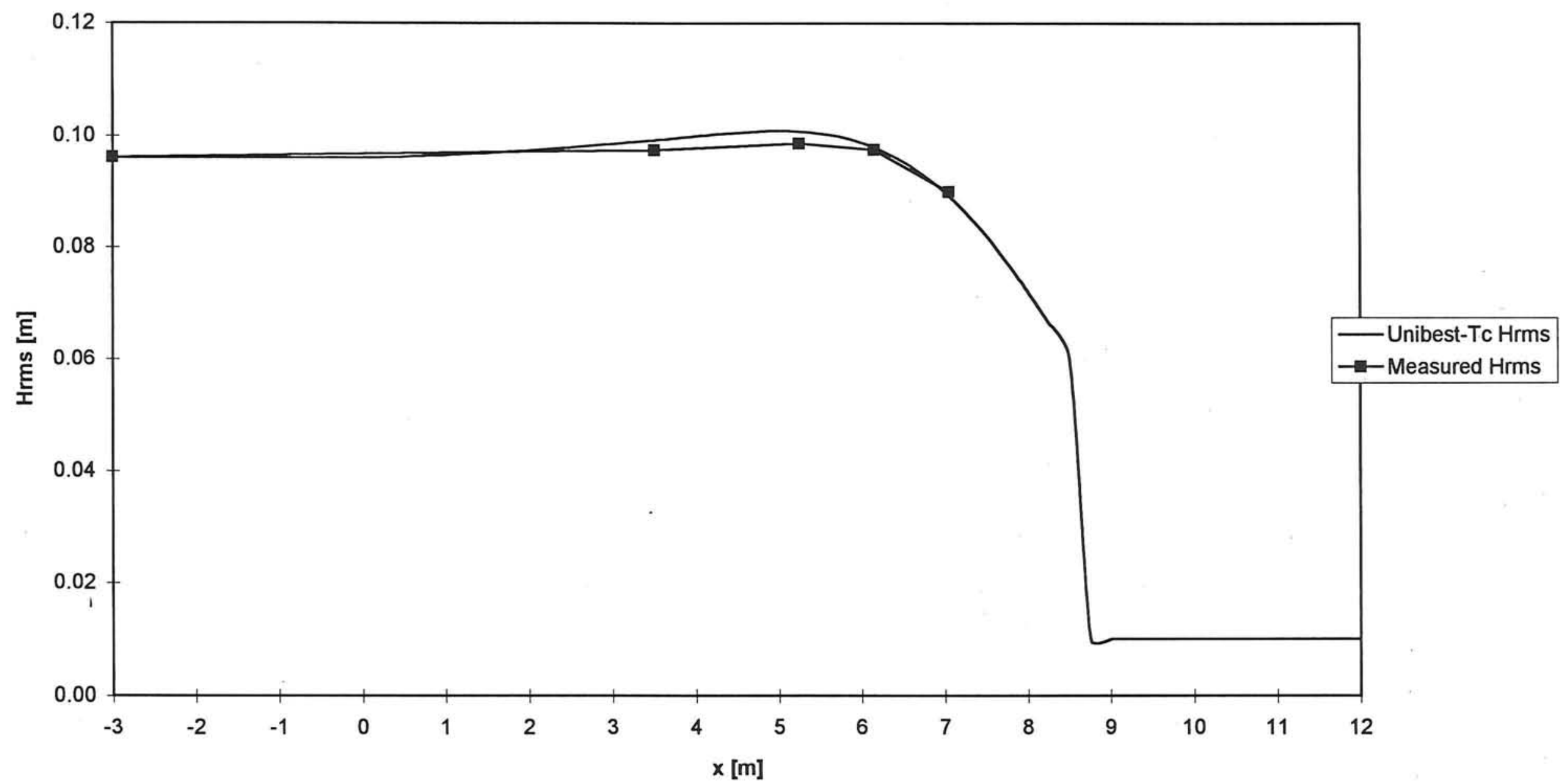

Graph 5.6 


\section{Measured Hrms versus Hrms calculated by Unibest-TC}

at Interval 1

Experiment $\mathrm{E}$ without breakwater ( $\mathrm{Hrms} \mathrm{O}=0.047 \mathrm{~m}, \mathrm{Tp}=1.29 \mathrm{~s}$ )

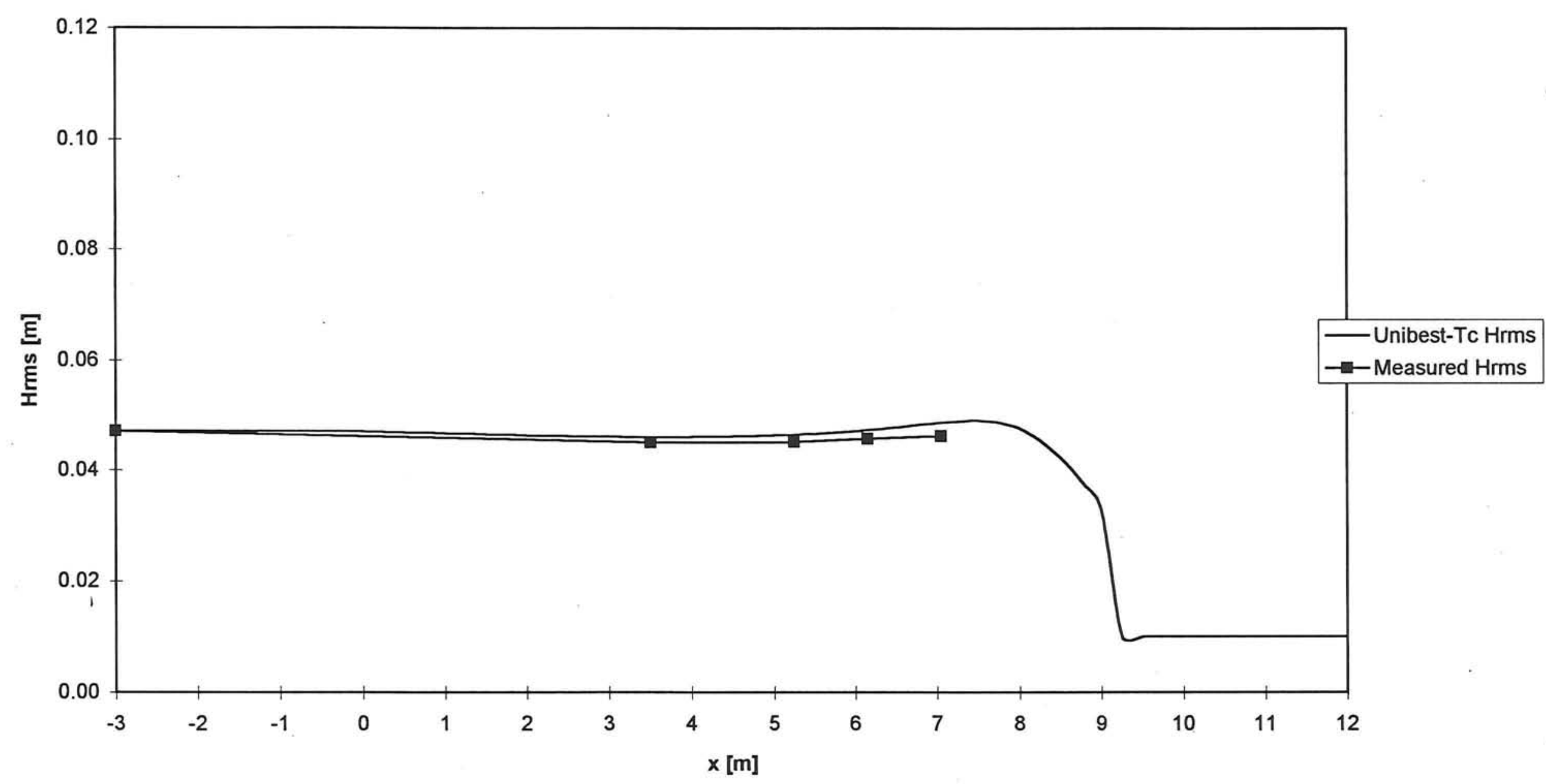

Graph 5.7 


\section{Measured Hrms versus Hrms calculated by Unibest-TC}

at Interval 1

Experiment $\mathrm{A}$ with breakwater ( $\mathrm{Hrms} 0=0.074 \mathrm{~m}, \mathrm{Tp}=2.07 \mathrm{~s}$ )

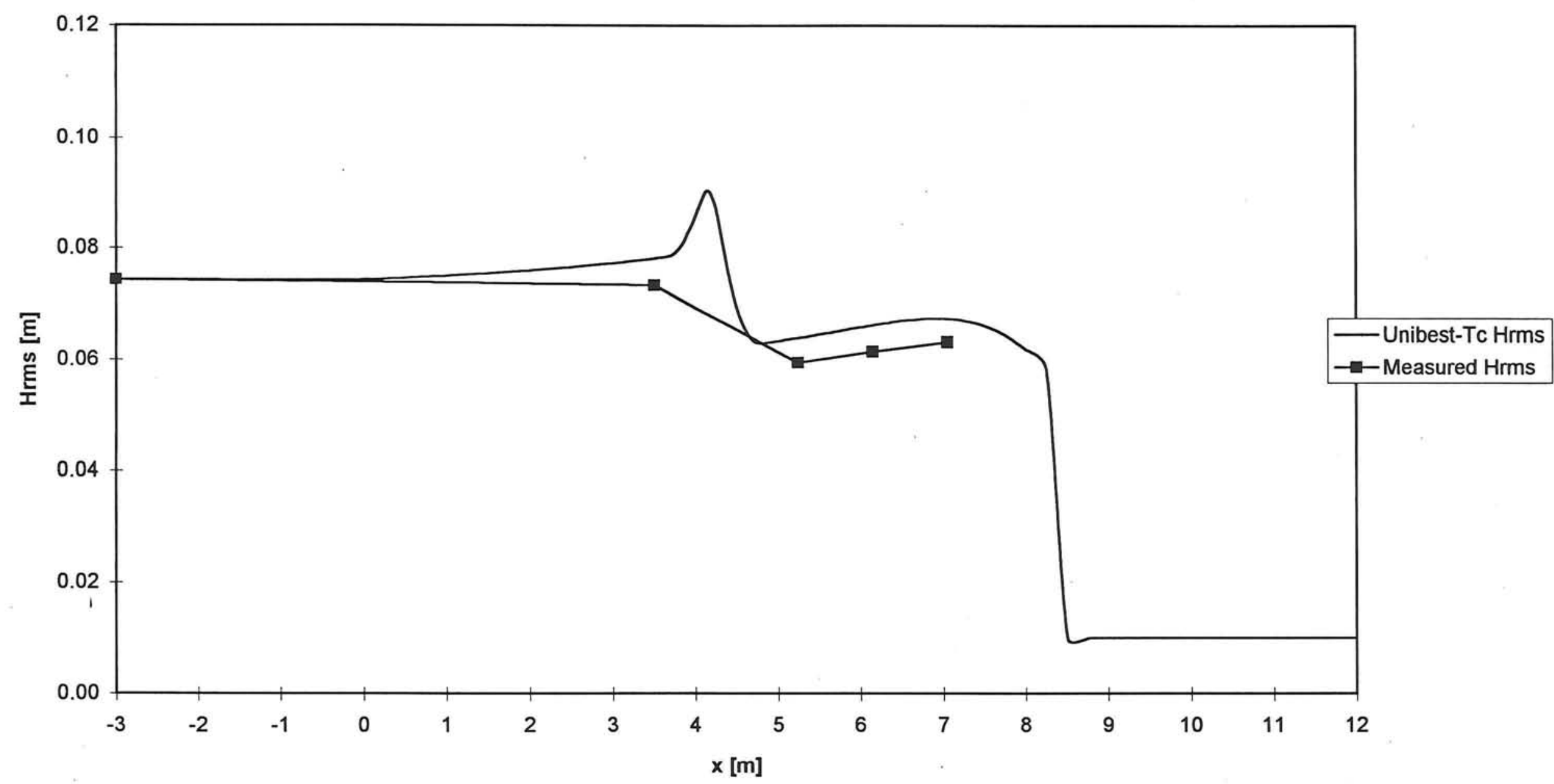

Graph 5.8 


\section{Measured Hrms versus Hrms calculated by Unibest-TC}

at Interval 1

Experiment $B$ with breakwater ( $\mathrm{Hrms} 0=0.087 \mathrm{~m}, \mathrm{Tp}=1.55 \mathrm{~s}$ )

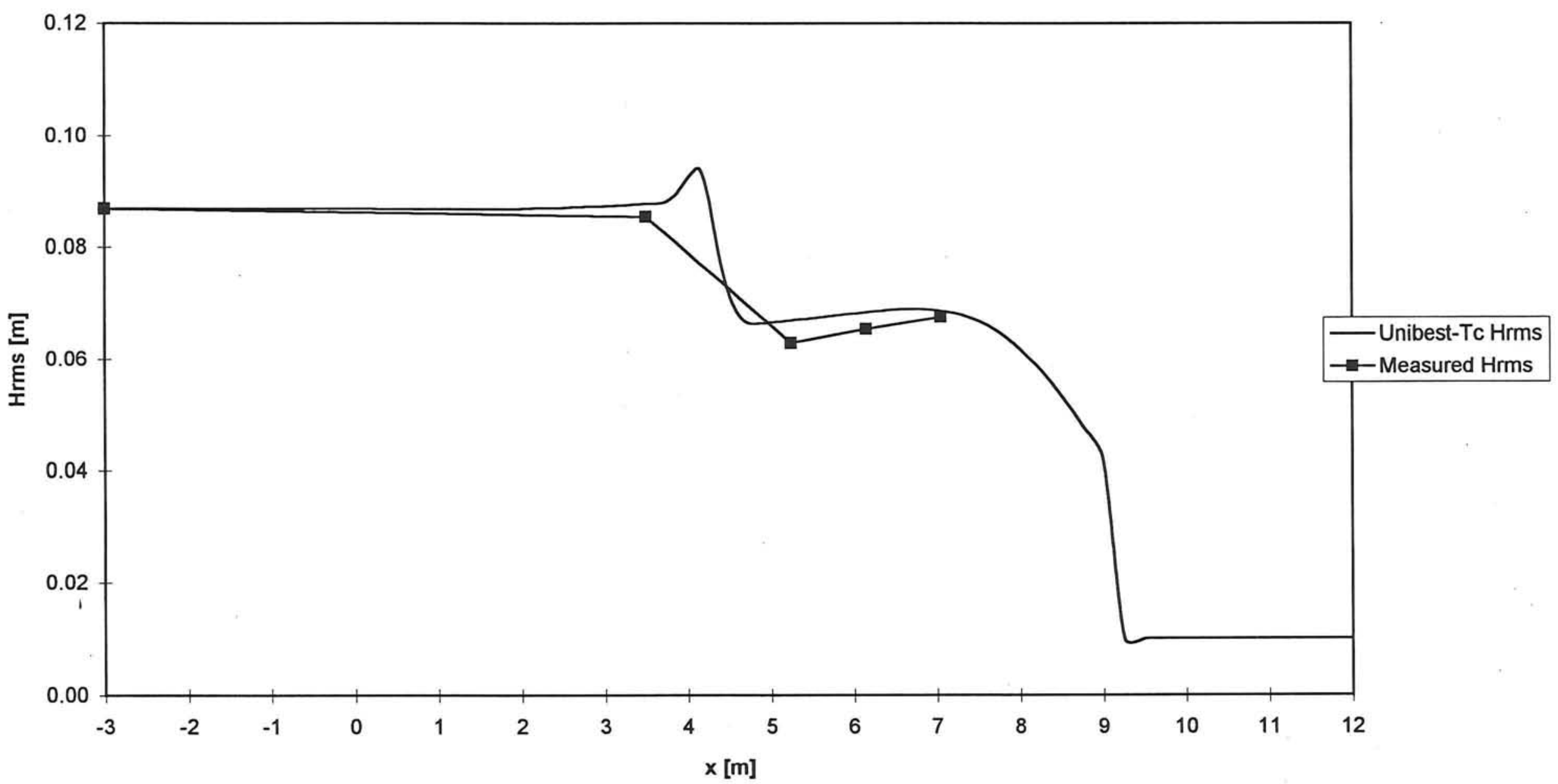

Graph 5.9 


\section{Measured Hrms versus Hrms calculated by Unibest-TC}

at Interval 1

Experiment $\mathrm{C}$ with breakwater $(\mathrm{Hrms} 0=0.081 \mathrm{~m}, \mathrm{Tp}=1.29 \mathrm{~s})$

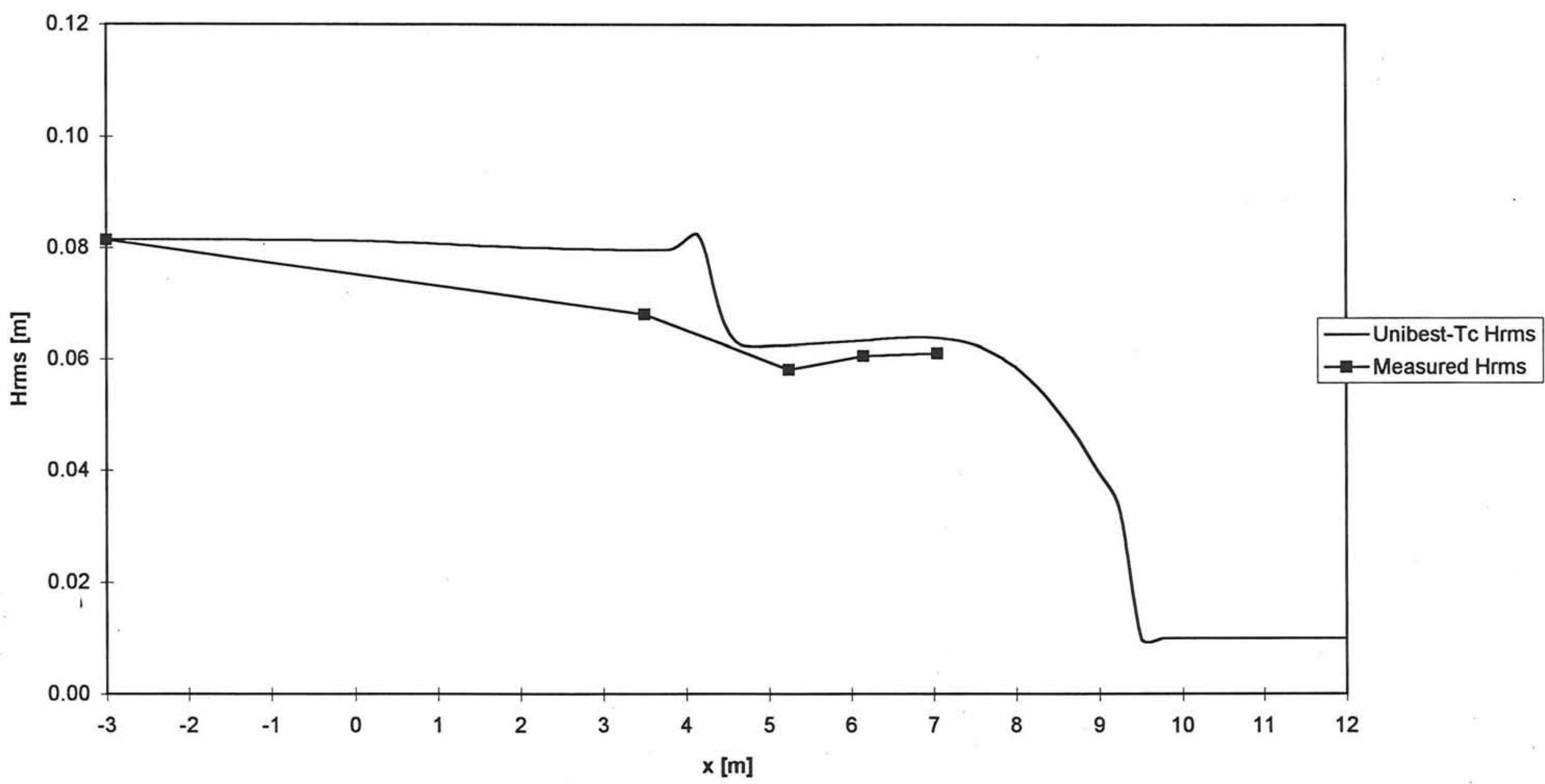

Graph 5.10 


\section{Measured Hrms versus Hrms calculated by Unibest-TC \\ at Interval 1}

Experiment $\mathrm{D}$ with breakwater $(\mathrm{Hrms} 0=0.103 \mathrm{~m}, \mathrm{Tp}=1.81 \mathrm{~s})$

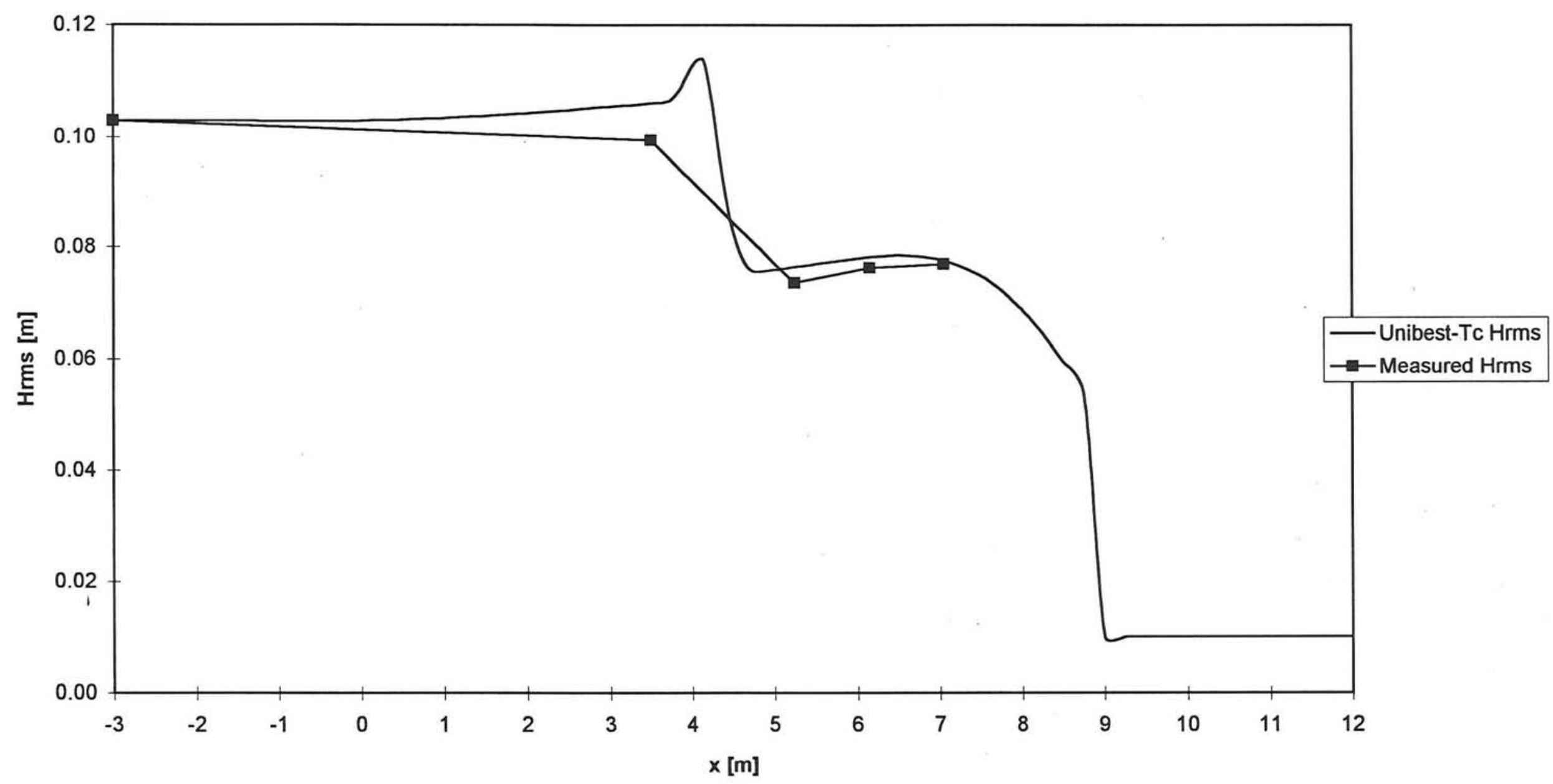

Graph 5.11 
Measured Hrms versus Hrms calculated by Unibest-TC

at Interval 1

Experiment $\mathrm{E}$ with breakwater ( $\mathrm{Hrms} 0=0.051 \mathrm{~m}, \mathrm{Tp}=1.29 \mathrm{~s}$ )

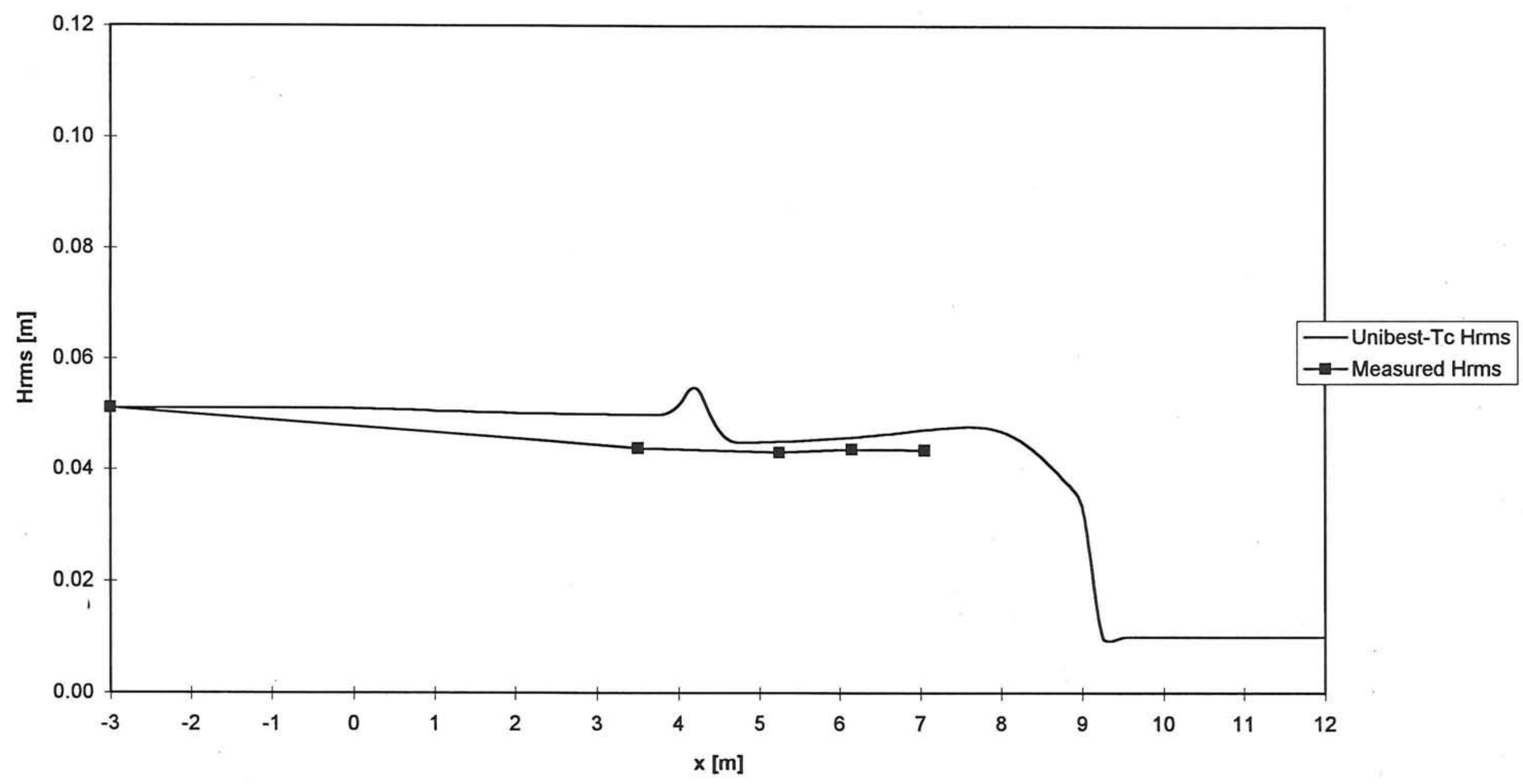

Graph 5.12 


\section{Measured profile versus profile calculated by Unibest-TC}

Elapsed time 7.5 hours

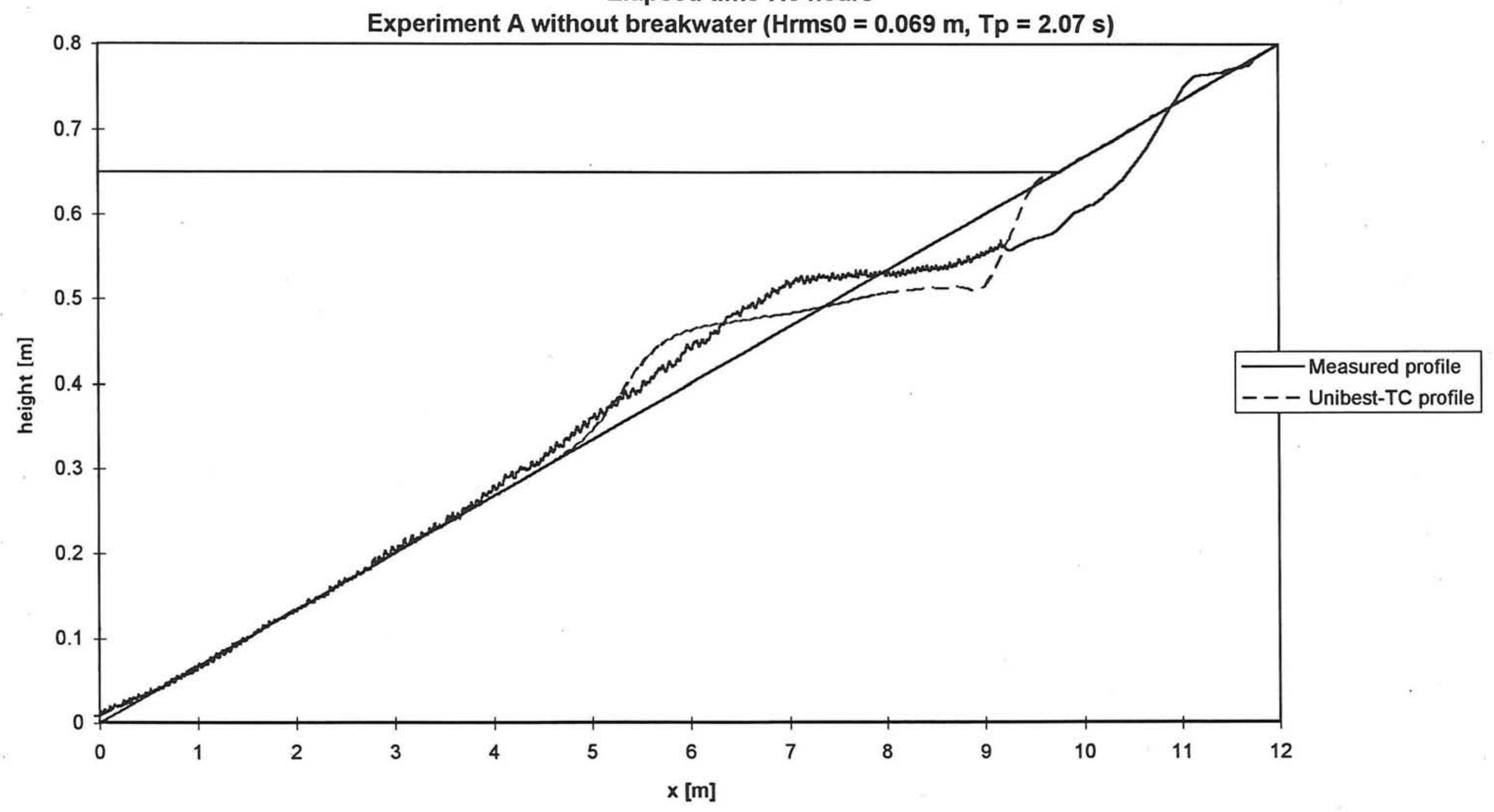

Graph 5.13 


\section{Measured profile versus profile calculated by Unibest-TC}

Elapsed time 7.5 hours

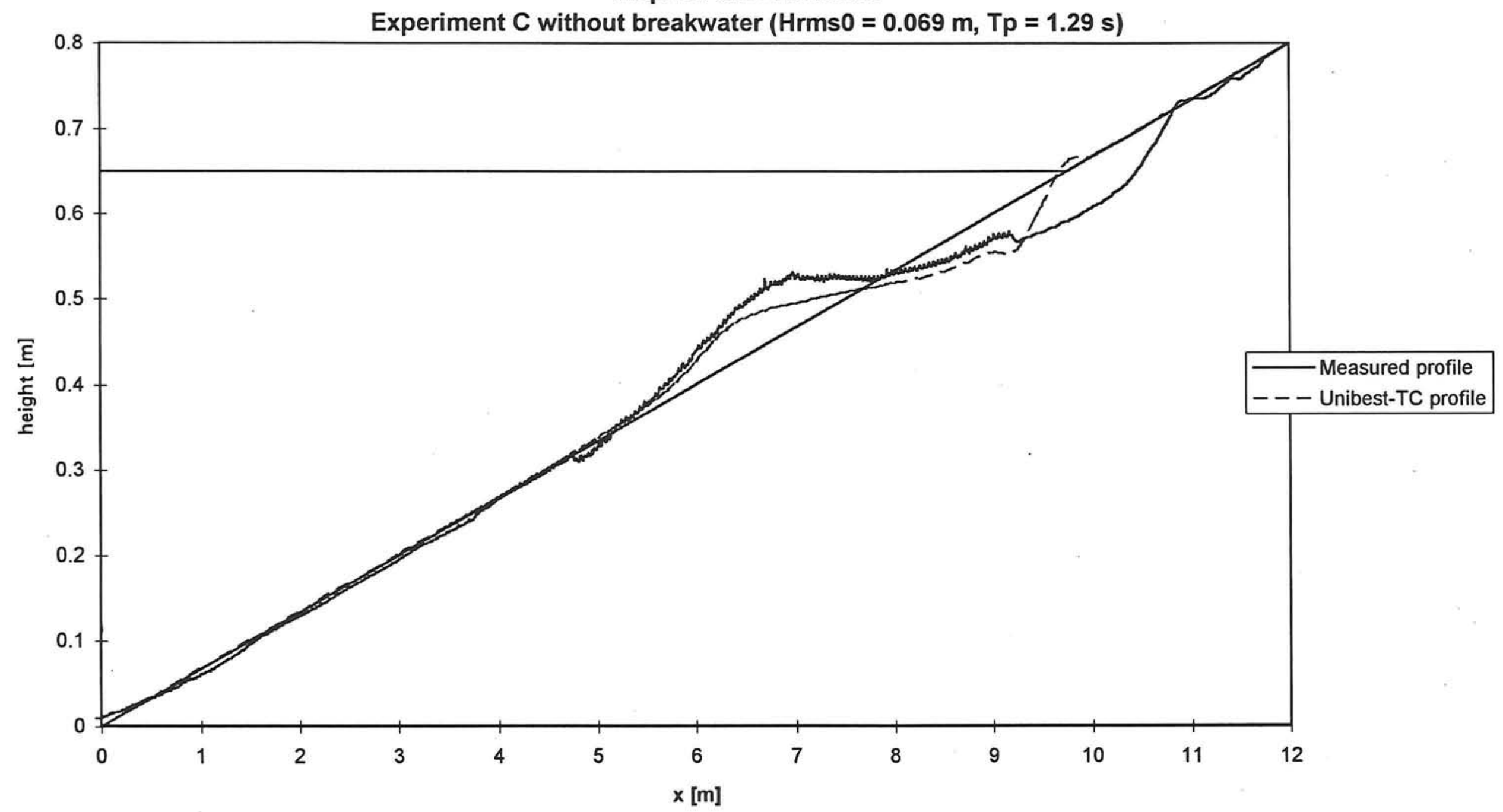

Graph 5.15 


\section{Measured profile versus profile calculated by Unibest-TC}

Elapsed time 7.5 hours

Experiment $\mathrm{D}$ without breakwater $(\mathrm{Hrms} 0=0.100 \mathrm{~m}, \mathrm{Tp}=1.81 \mathrm{~s})$

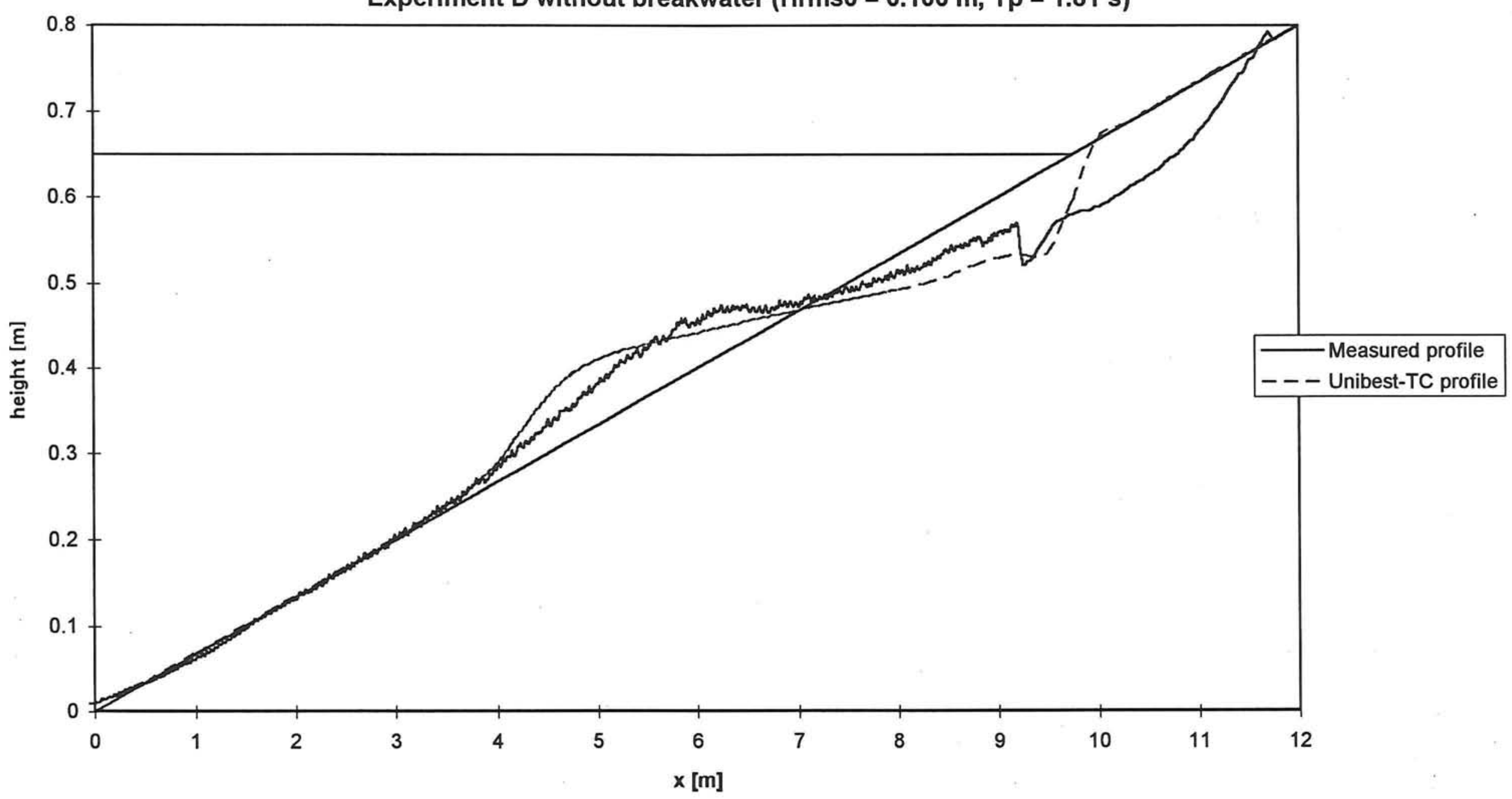

Graph 5.16 
Measured profile versus profile calculated by Unibest-TC

Elapsed time 7.5 hours

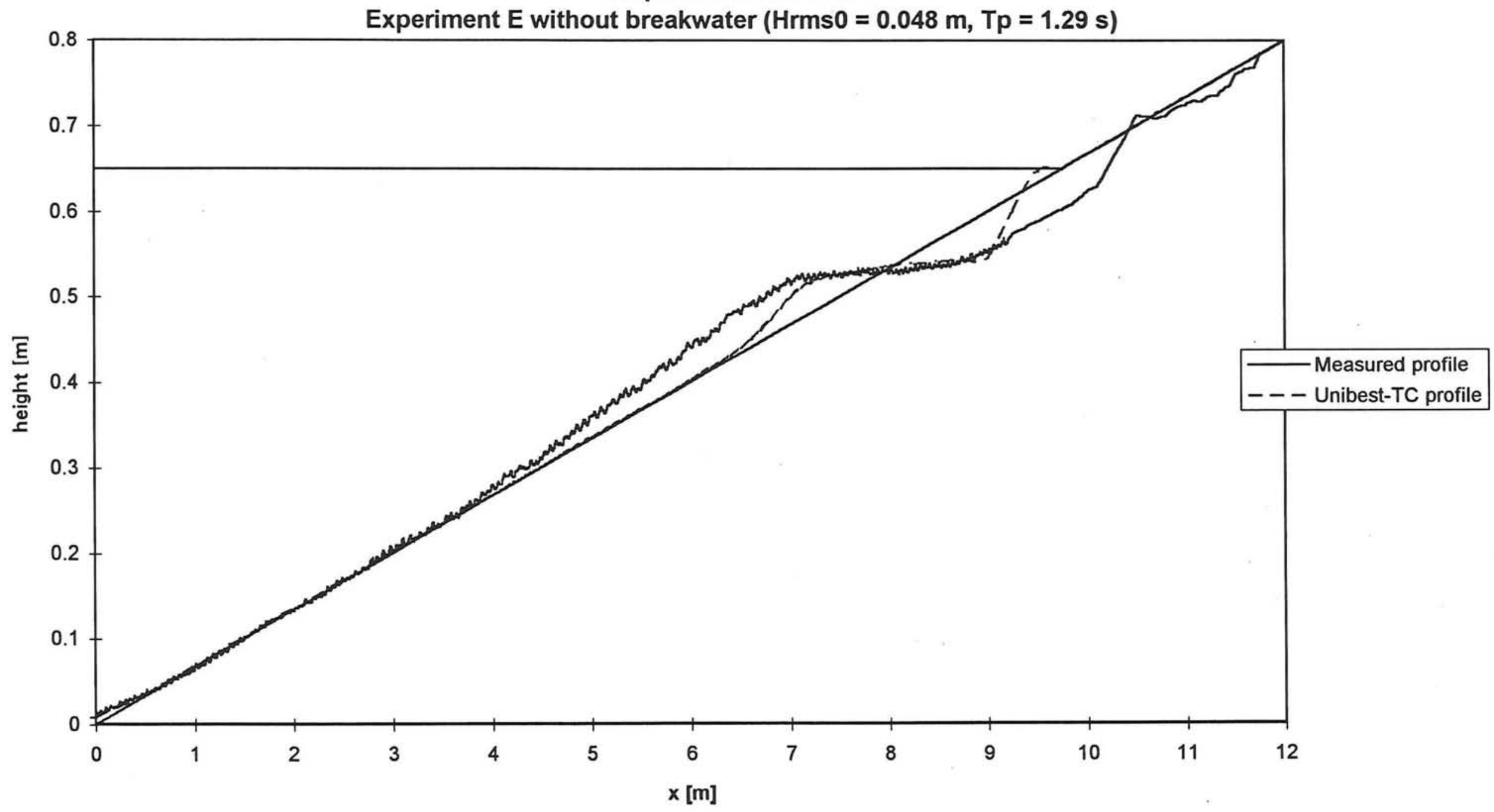

Graph 5.17 
Measured profile versus profile calculated by Unibest-TC

Elapsed time 7.5 hours

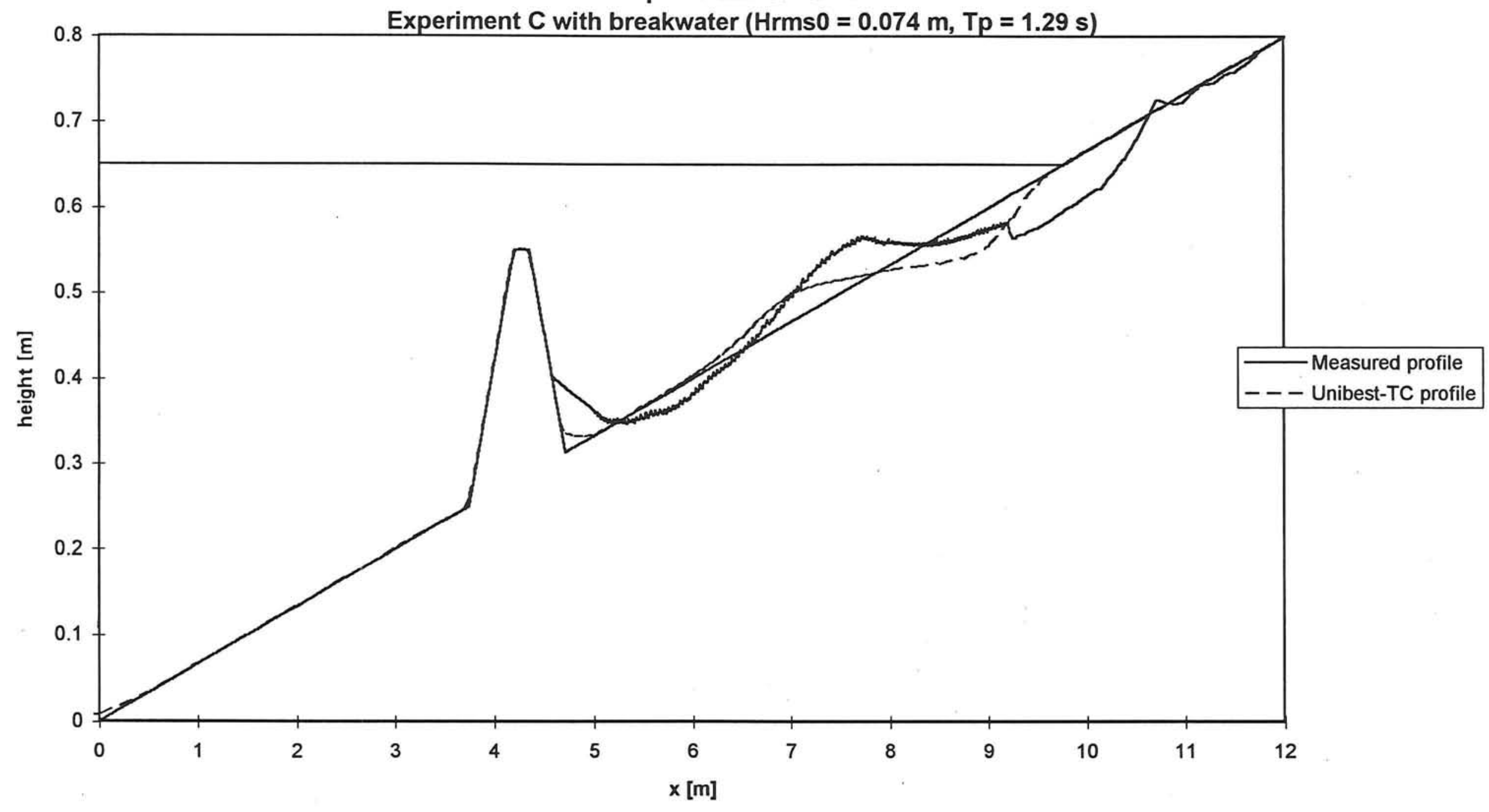

Graph 5.20 


\section{Measured profile versus profile calculated by Unibest-TC}

Elapsed time 7.5 hours

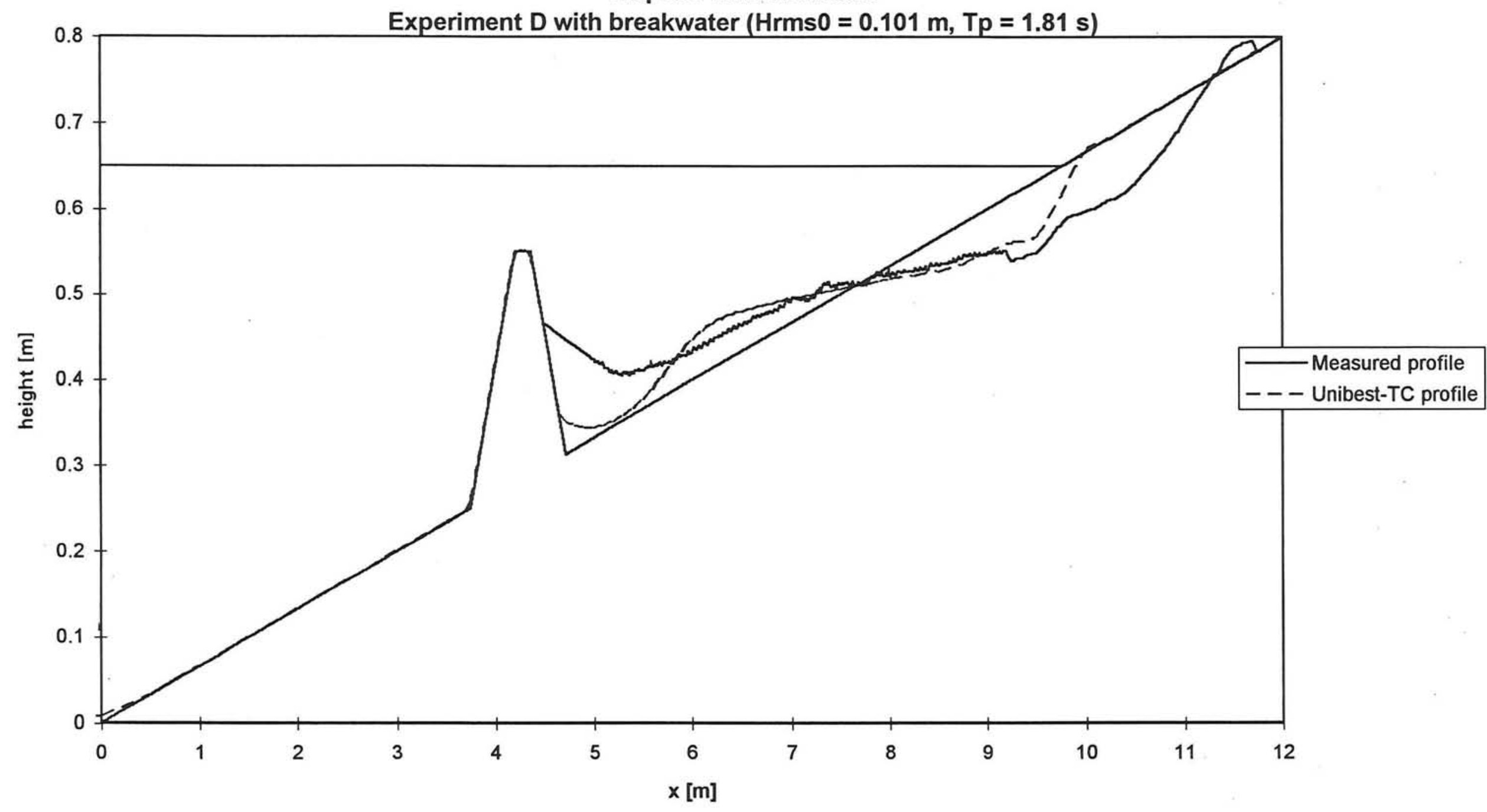

Graph 5.21 
Measured profile versus profile calculated by Unibest-TC

Elapsed time 7.5 hours

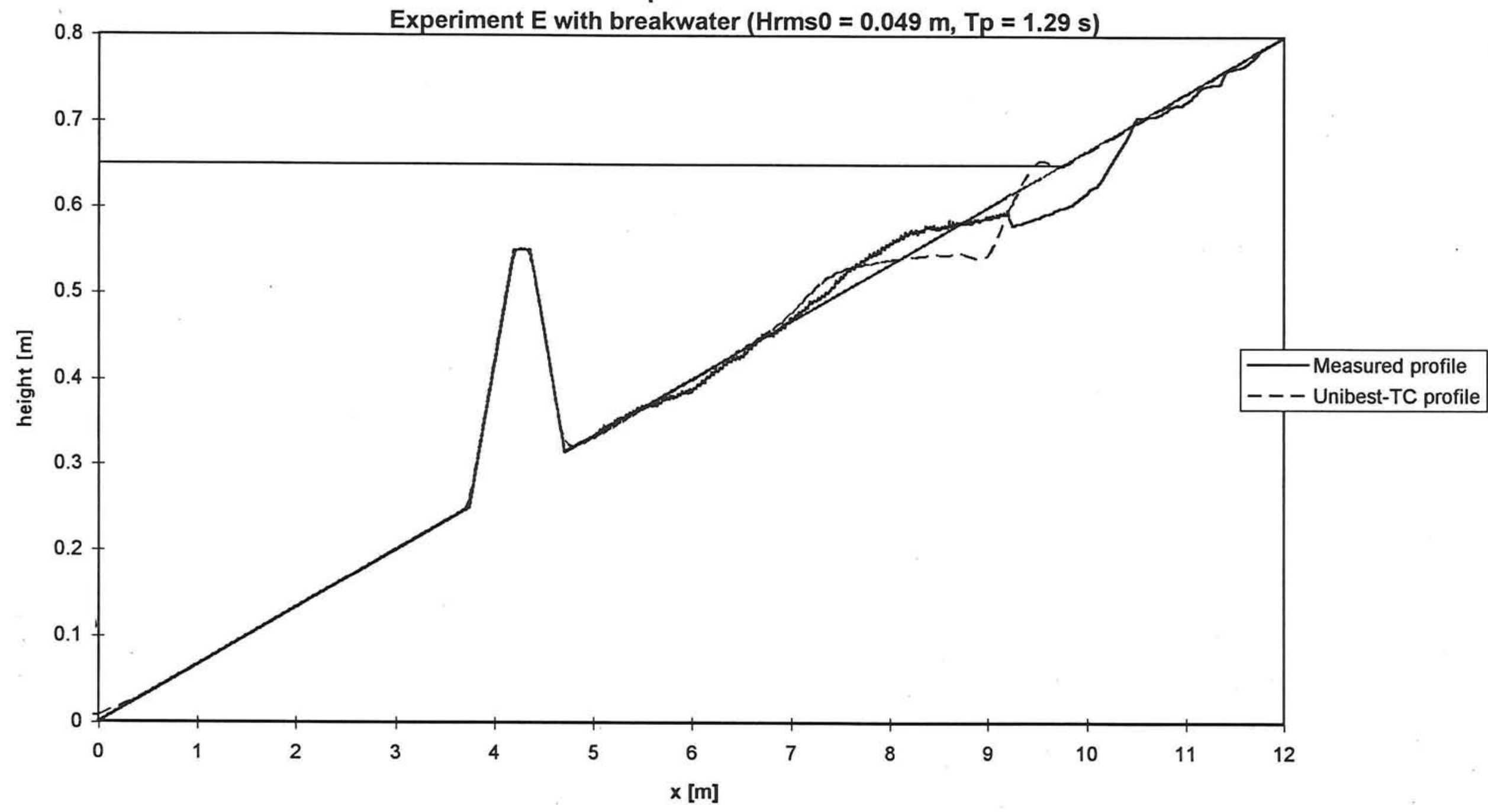

Graph 5.22 